Please note: An erratum has been published for this issue. To view the erratum, please click here.

\title{
Centers for Disease Control and Prevention
}

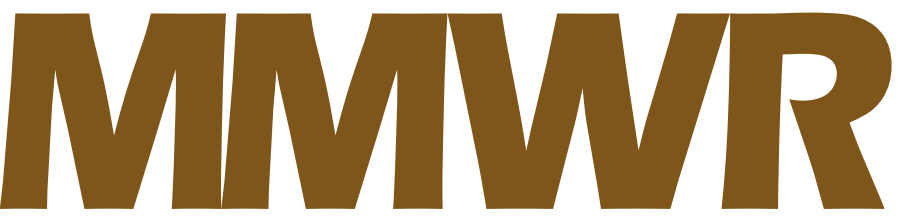

Morbidity and Mortality Weekly Report

\section{Youth Risk Behavior Surveillance - United States, 2015}




\section{CONTENTS}

Introduction Methods.

The MMWR series of publications is published by the Center for Surveillance, Epidemiology, and Laboratory Services, Centers for Disease Control and Prevention (CDC), U.S. Department of Health and Human Services, Atlanta, GA 30329-4027.

Suggested citation: [Author names; first three, then et al., if more than six.] [Title]. MMWR Surveill Summ 2016;65(No. SS-\#):[inclusive page numbers].

\section{Centers for Disease Control and Prevention}

Thomas R. Frieden, MD, MPH, Director

Harold W. Jaffe, MD, MA, Associate Director for Science

Joanne Cono, MD, ScM, Director, Office of Science Quality

Chesley L. Richards, MD, MPH, Deputy Director for Public Health Scientific Services

Michael F. Iademarco, MD, MPH, Director, Center for Surveillance, Epidemiology, and Laboratory Services

\section{MMWR Editorial and Production Staff (Serials)}

Sonja A. Rasmussen, MD, MS, Editor-in-Chief

Charlotte K. Kent, $\mathrm{PhD}$, MPH, Executive Editor

Christine G. Casey, MD, Editor

Teresa F. Rutledge, Managing Editor

David C. Johnson, Lead Technical Writer-Editor

Denise Williams, MBA, Project Editor
Martha F. Boyd, Lead Visual Information Specialist

Maureen A. Leahy, Julia C. Martinroe,

Stephen R. Spriggs, Moua Yang, Tong Yang, Visual Information Specialists

Quang M. Doan, MBA, Phyllis H. King, Terraye M. Starr, Information Technology Specialists
MMWR Editorial Board

Timothy F. Jones, MD, Chairman Matthew L. Boulton, MD, MPH Virginia A. Caine, MD

Katherine Lyon Daniel, $\mathrm{PhD}$

Jonathan E. Fielding, MD, MPH, MBA

David W. Fleming, MD
William E. Halperin, MD, DrPH, MPH

King K. Holmes, MD, PhD

Robin Ikeda, MD, MPH

Rima F. Khabbaz, MD

Phyllis Meadows, PhD, MSN, RN

Jewel Mullen, MD, MPH, MPA
Jeff Niederdeppe, PhD

Patricia Quinlisk, MD, MPH

Patrick L. Remington, MD, MPH Carlos Roig, MS, MA

William L. Roper, MD, MPH

William Schaffner, MD 


\title{
Youth Risk Behavior Surveillance — United States, 2015
}

\author{
Laura Kann, $\mathrm{PhD}^{1}$; Tim McManus, $\mathrm{MS}^{1}$; William A. Harris, MM${ }^{1}$; Shari L. Shanklin, $\mathrm{MPH}^{1}$; Katherine H. Flint, MA ${ }^{2}$; Joseph Hawkins, MA ${ }^{3}$; \\ Barbara Queen, MS ${ }^{3}$; Richard Lowry, $\mathrm{MD}^{1}$; Emily O’Malley Olsen, $\mathrm{MSPH}^{1}$; David Chyen, MS ${ }^{1}$; Lisa Whittle, MPH ${ }^{1}$; Jemekia Thornton, MPA ${ }^{1}$; \\ Connie Lim, MPA ${ }^{1}$; Yoshimi Yamakawa, $\mathrm{MPH}^{1}$; Nancy Brener, $\mathrm{PhD}^{1}$; Stephanie Zaza, MD ${ }^{1}$ \\ ${ }^{1}$ Division of Adolescent and School Health, National Center for HIVIAIDS, Viral Hepatitis, STD, and TB Prevention, CDC \\ 2ICF International, Rockville, Maryland \\ ${ }^{3}$ Westat, Rockville, Maryland
}

\begin{abstract}
Problem: Priority health-risk behaviors contribute to the leading causes of morbidity and mortality among youth and adults. Population-based data on these behaviors at the national, state, and local levels can help monitor the effectiveness of public health interventions designed to protect and promote the health of youth nationwide.
\end{abstract}

Reporting Period Covered: September 2014-December 2015.

Description of the System: The Youth Risk Behavior Surveillance System (YRBSS) monitors six categories of priority health behaviors among youth and young adults: 1) behaviors that contribute to unintentional injuries and violence; 2) tobacco use; 3) alcohol and other drug use; 4) sexual behaviors related to unintended pregnancy and sexually transmitted infections (STIs), including human immunodeficiency virus (HIV) infection; 5) unhealthy dietary behaviors; and 6) physical inactivity. In addition, YRBSS monitors the prevalence of obesity and asthma and other priority health behaviors. YRBSS includes a national school-based Youth Risk Behavior Survey (YRBS) conducted by CDC and state and large urban school district school-based YRBSs conducted by state and local education and health agencies. This report summarizes results for 118 health behaviors plus obesity, overweight, and asthma from the 2015 national survey, 37 state surveys, and 19 large urban school district surveys conducted among students in grades 9-12.

Results: Results from the 2015 national YRBS indicated that many high school students are engaged in priority health-risk behaviors associated with the leading causes of death among persons aged 10-24 years in the United States. During the 30 days before the survey, $41.5 \%$ of high school students nationwide among the $61.3 \%$ who drove a car or other vehicle during the 30 days before the survey had texted or e-mailed while driving, 32.8\% had drunk alcohol, and $21.7 \%$ had used marijuana. During the 12 months before the survey, $15.5 \%$ had been electronically bullied, $20.2 \%$ had been bullied on school property, and $8.6 \%$ had attempted suicide. Many high school students are engaged in sexual risk behaviors related to unintended pregnancies and STIs, including HIV infection. Nationwide, $41.2 \%$ of students had ever had sexual intercourse, $30.1 \%$ had had sexual intercourse during the 3 months before the survey (i.e., currently sexually active), and $11.5 \%$ had had sexual intercourse with four or more persons during their life. Among currently sexually active students, $56.9 \%$ had used a condom during their last sexual intercourse. Results from the 2015 national YRBS also indicated many high school students are engaged in behaviors associated with chronic diseases, such as cardiovascular disease, cancer, and diabetes. During the 30 days before the survey, $10.8 \%$ of high school students had smoked cigarettes and $7.3 \%$ had used smokeless tobacco. During the 7 days before the survey, $5.2 \%$ of high school students had not eaten fruit or drunk $100 \%$ fruit juices and $6.7 \%$ had not eaten vegetables. More than one third (41.7\%) had played video or computer games or used a computer for something that was not school work for 3 or more hours per day on an average school day and $14.3 \%$ had not participated in at least 60 minutes of any kind of physical activity that increased their heart rate and made them breathe hard on at least 1 day during the 7 days before the survey. Further, 13.9\% had obesity and 16.0\% were overweight.

Interpretation: Many high school students engage in behaviors that place them at risk for the leading causes of morbidity and mortality. The prevalence of most health behaviors varies by sex, race/ethnicity, and grade and across states and large urban school districts. Long-term temporal changes also have occurred. Since the earliest year of data collection, the prevalence of most healthrisk behaviors has decreased (e.g., riding with a driver who had been drinking alcohol, physical fighting, current cigarette use, current alcohol use, and current sexual activity), but the prevalence of other behaviors and health outcomes has not changed (e.g., suicide attempts treated by a doctor or nurse, smokeless tobacco use, having ever used marijuana, and attending physical education classes) or has increased (e.g., having not gone to school because of safety concerns, obesity, overweight, not eating vegetables, and

Corresponding author: Laura Kann, $\mathrm{PhD}$, Division of Adolescent and School Health, National Center for HIV/AIDS, Viral Hepatitis, STD, and TB Prevention. Telephone: 404-718-8132; E-mail: 1kk1@cdc.gov. not drinking milk). Monitoring emerging risk behaviors (e.g., texting and driving, bullying, and electronic vapor product use) is important to understand how they might vary over time. 
Public Health Action: YRBSS data are used widely to compare the prevalence of health behaviors among subpopulations of students; assess trends in health behaviors over time; monitor progress toward achieving 21 national health objectives for Healthy People 2020 and one of the 26 leading health indicators; provide comparable state and large urban school district data; and help develop and evaluate school and community policies, programs, and practices designed to decrease health-risk behaviors and improve health outcomes among youth.

\section{Introduction}

In the United States in $2014,71 \%$ of all deaths among persons aged 10-24 years resulted from four causes: motor vehicle crashes $(23 \%)$, other unintentional injuries (17\%), homicide (14\%), and suicide (17\%) (1). Among persons aged $15-19$ years, 273,105 births (2); 451,208 cases of chlamydia, gonorrhea, and syphilis (3); and 1,828 diagnoses of human immunodeficiency virus (HIV) (4) are reported annually. Among persons aged $\geq 25$ years, $54 \%$ of all deaths in the United States result from cardiovascular disease (31\%) and cancer (23\%) (1). These leading causes of mortality, morbidity, and social problems among youth and adults in the United States are related to six categories of priority health behaviors: 1) behaviors that contribute to unintentional injuries and violence; 2) tobacco use; 3) alcohol and other drug use; 4) sexual behaviors related to unintended pregnancy and sexually transmitted infections (STIs), including HIV infection; 5) unhealthy dietary behaviors; and 6) physical inactivity. These behaviors frequently are interrelated and are established during childhood and adolescence and extend into adulthood. To monitor priority health behaviors in each of these six categories, the prevalence of obesity, overweight and asthma, and other priority health behaviors among youth and young adults, CDC developed the Youth Risk Behavior Surveillance System (YRBSS) (5). YRBSS includes school-based national, state, and large urban school district Youth Risk Behavior Surveys (YRBS) conducted among representative samples of students in grades 9-12. National, state, and large urban school district surveys have been conducted biennially since 1991 (Table 1). Additional information about YRBSS is available at http://www.cdc.gov/ healthyyouth/data/yrbs/index.htm.

This report summarizes results for 118 health behaviors plus obesity, overweight, and asthma from the 2015 national YRBS and overall trends in health behaviors during 1991-2015. Data from the 37 state and 19 large urban school district surveys with weighted data for the 2015 YRBSS cycle (Figure) also are included in this report. Results from 10 state and two large urban school district surveys with unweighted data are not included. Among those with weighted data for 2015, three state and one large urban school district surveys were conducted during fall 2014; the national survey, 29 state, and 16 large urban school district surveys were conducted during spring 2015; and five state and two large urban school district surveys were conducted during fall 2015.

\section{Methods}

Detailed information about the methodology of the national, state, and large urban school district YRBSs has been described elsewhere (5).

\section{Sampling}

\section{National Youth Risk Behavior Survey}

The sampling frame for the 2015 national YRBS consisted of all regular public* and private ${ }^{\dagger}$ schools with students in at least one of grades 9-12 in the 50 states and the District of Columbia. The sampling frame was based on the Market Data Retrieval (MDR) database ( 6 ), which includes information on both public and private schools and the most recent data from the Common Core of Data from the National Center for Education Statistics (7). A three-stage cluster sample design produced a nationally representative sample of students in grades 9-12 who attend public and private schools. The first-stage sampling frame consisted of 1,259 primary sampling units (PSUs), consisting of counties, subareas of large counties, or groups of smaller, adjacent counties. The 1,259 PSUs were categorized into 16 strata according to their metropolitan statistical area (MSA) status (e.g., urban city) and the percentages of black and Hispanic students in the PSUs. From the 1,259 PSUs, 54 were sampled with probability proportional to overall school enrollment size for the PSU.

In the second stage of sampling, 180 schools with any of grades 9-12 were sampled with probability proportional to school enrollment size from within the 54 PSUs. The third stage of sampling consisted of random sampling in each of grades 9-12, one or two classrooms from either a required subject (e.g., English or social studies) or a required period (e.g., homeroom or second period). All students in sampled classes were eligible to participate. Schools, classes, and students that refused to participate were not replaced.

To enable a separate analysis of data for black and Hispanic students, two classes per grade, rather than one, were sampled

\footnotetext{
* Might include charter schools and public alternative, special education, or vocational schools.

$\dagger$ Might include religious and other private schools, but does not include private alternative, special education, or vocational schools.
} 
in schools with a high minority enrollment. Before the 2013 national YRBS, three strategies were used to oversample black and Hispanic students: 1) larger sampling rates were used to select PSUs that were in high-black and high-Hispanic strata; 2) a modified measure of size was used to increase the probability of sampling schools with a disproportionately high minority enrollment; and 3) two classes per grade, rather than one, were sampled in schools with a high minority enrollment. Because of increases in the proportions of black and Hispanic students in the population, only selection of two classes per grade was needed in 2013 and 2015 to balance the precision needed for subgroup estimates with minimum variance for overall estimates.

\section{State and Large Urban School District Youth Risk Behaviors}

In 2015, a two-stage cluster sample design was used to produce a representative sample of public $^{\S}$ school students in grades 9-12 in 36 states and 19 large urban school districts and of public and private school students in grades 9-12 in one state (South Dakota). In the first sampling stage, schools with any of grades 9-12 were sampled with probability proportional to school enrollment size in 34 states and three large urban school districts; all schools with any of grades 9-12 were invited to participate in three

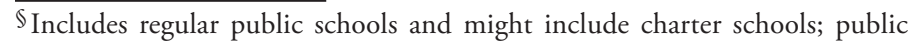
alternative, special education, or vocational schools; and schools overseen by the Bureau of Indian Education.

Might include religious and other private schools.
}

FIGURE. State and large urban school district Youth Risk Behavior Surveys — United States, 2015

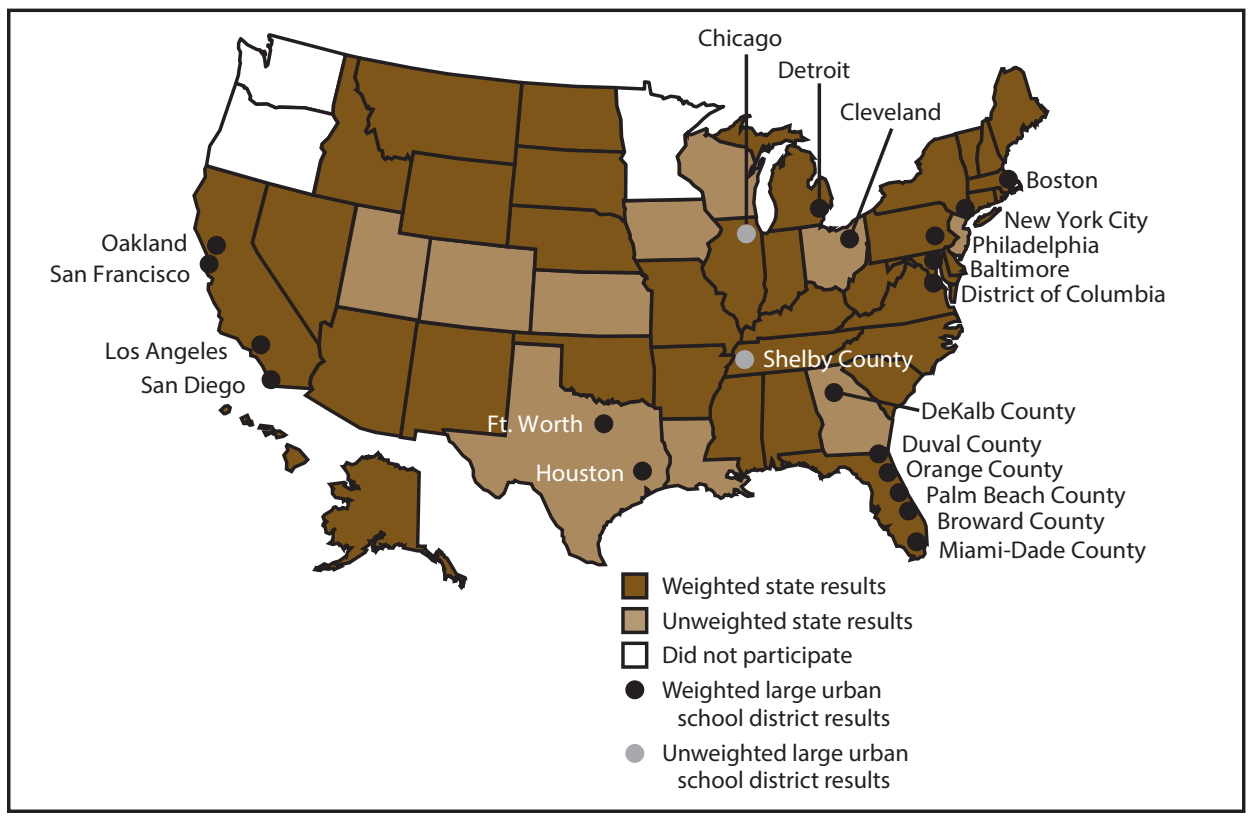

states and 16 large urban school districts. In the second sampling stage, intact classes from either a required subject (e.g., English or social studies) or a required period (e.g., homeroom or second period) were sampled randomly in 36 states and 18 large urban school districts, and all students in the sampled classes were eligible to participate. In one state and one large urban school district, all students in sampled schools were eligible to participate.

\section{Data Collection Procedures and Questionnaires}

Survey procedures for the national, state, and large urban school district surveys were designed to protect students' privacy by allowing for anonymous and voluntary participation. Before survey administration, local parental permission procedures were followed. Students completed the self-administered questionnaire during one class period and recorded their responses directly on a computer-scannable booklet or answer sheet. CDC's Institutional Review Board approved the protocol for the national YRBS.

The 2015 YRBS standard questionnaire contained 89 questions. This questionnaire was used as the starting point for the state and large urban school district questionnaires. States and large urban school districts could add and/or delete questions from the standard questionnaire. Only one state and three large urban school districts included in this report used the 2015 YRBS standard questionnaire without modifications. This report presents state and large urban school district results only from selected questions on the 2015 standard questionnaire.

The 2015 national YRBS questionnaire contained 99 questions, including all 89 questions on the standard questionnaire. This report presents national results (along with state and large urban school district results) for selected questions on the 2015 standard questionnaire plus national only results from eight additional questions measuring usual method of marijuana use, ever use of hallucinogenic drugs, consumption of sports drinks, consumption of water, muscle strengthening exercises, indoor tanning device use, having had a sunburn, and avoidance of foods because eating the food could cause an allergic reaction.

Except for six demographic questions (sex, grade in school, age, Hispanic ethnicity, race, and sexual identity) and 
three questions assessing height, weight, and asthma, all the remaining questions on the standard questionnaire and the national questionnaire measured behaviors practiced or experienced by the student (referred to as "behaviors"). Skip patterns, which occur when a particular response to one question indicates to the respondents that they should not answer one or more subsequent questions, were not included in any YRBS questionnaire to protect students' privacy by ensuring all students took about the same amount of time to complete the questionnaire. All questions (except for two questions assessing height and weight and the race question) were multiple choice with a maximum of eight mutually exclusive response options and only one possible answer per respondent. Information about the reliability of the standard questionnaire has been published elsewhere (8). The wording of each question, including recall periods, response options, and operational definitions are available in the 2015 standard and national YRBS questionnaires at http://www.cdc.gov/ healthyyouth/data/yrbs/index.htm.

Results from two new standard questions measuring sexual minority status (i.e., sexual identity and sex of sexual contacts) used by 25 states and 19 large urban school districts and included on the national questionnaire are not described in this report.

\section{Data Processing Procedures and Response Rates}

For the 2015 national YRBS, 15,713 questionnaires were completed in 125 public and private schools. The national data set was cleaned and edited for inconsistencies. Missing data were not statistically imputed. Among the 15,713 completed questionnaires, 89 failed quality control ${ }^{* *}$ and were excluded from analysis, resulting in 15,624 usable questionnaires (Table 2). The school response rate was 69\%, the student response rate was $86 \%$, and the overall response rate was $60 \%{ }^{\dagger \dagger}$ (Table 2).

Data from each state and large urban school district survey were cleaned and edited for inconsistencies with the same procedures used for the national data set. The percentage of completed questionnaires that failed quality control checks and were excluded from analysis ranged from $0.2 \%$ to $5.3 \%$ (median: $0.8 \%$ ) across the 37 states and from $0.3 \%$ to $6.4 \%$ (median: $1.6 \%)$ across the 19 large urban school districts. The student sample sizes ranged from 1,313 to 55,596 (median: 2,777) across the states and from 1,052 to 10,419 (median: 2,181) across

\footnotetext{
** A questionnaire that fails quality control has $<20$ remaining responses after editing or has the same answer to $\geq 15$ consecutive questions.

$\dagger \dagger$ Overall response rate $=$ (number of participating schools/number of eligible sampled schools) $\mathrm{x}$ (number of usable questionnaires/number of eligible students sampled).
}

the large urban school districts (Table 2). Among the states, the school response rates ranged from $70 \%$ to $100 \%$, student response rates ranged from $64 \%$ to $90 \%$, and overall response rates ranged from $60 \%$ to $84 \%$. Among the large urban school districts, the school response rates ranged from $90 \%$ to $100 \%$, student response rates ranged from $66 \%$ to $88 \%$, and overall response rates ranged from $64 \%$ to $88 \%$ (Table 2).

Race/ethnicity was computed from two questions: 1) "Are you Hispanic or Latino?" (response options were "yes" or "no"), and 2) "What is your race?" (response options were "American Indian or Alaska Native," "Asian," "black or African American," "Native Hawaiian or other Pacific Islander," or "white"). For the second question, students could select more than one response option. For this report, students were classified as "Hispanic/ Latino" and are referred to as "Hispanic" if they answered "yes" to the first question, regardless of how they answered the second question. Students who answered "no" to the first question and selected only "black or African American" to the second question were classified as "black or African American" and are referred to as "black." Students who answered "no" to the first question and selected only "white" to the second question were classified, and are referred to, as "white." Race/ethnicity was classified as missing for students who did not answer the first question and for students who answered "no" to the first question but did not answer the second question.

Students were classified as as having obesity or being overweight or overweight based on their body mass index $\left(\mathrm{kg} / \mathrm{m}^{2}\right)(\mathrm{BMI})$, which was calculated from self-reported height and weight. BMI values were compared with sex- and age-specific reference data from the 2000 CDC growth charts (9). Obesity was defined as a BMI of $\geq 95$ th percentile for age and sex. Overweight was defined as a BMI of $\geq 85$ th percentile and $<95$ th percentile for age and sex. These classifications are not intended to diagnose obesity or overweight in individual students, but to provide population-level estimates of obesity and overweight.

\section{Weighting}

For the national YRBS, a weight based on student sex, race/ethnicity, and grade was applied to each record to adjust for school and student nonresponse and oversampling of black and Hispanic students. The overall weights were scaled so that the weighted count of students equals the total sample size, and the weighted proportions of students in each grade match the national population proportions. Therefore, weighted estimates are representative of all students in grades 9-12 attending public and private schools in the United States.

Data from states and large urban school districts that had a representative sample of students, appropriate documentation, 
and an overall response rate of $\geq 60 \%$ were weighted. A weight was applied to each record to adjust for school and student nonresponse and the distribution of students by grade, sex, and race/ethnicity in each jurisdiction, such that the weighted count of students equals the student population in each jurisdiction. Data from 37 states and 19 large urban school districts were weighted. In 36 states and all large urban school districts, weighted estimates are representative of all students in grades 9-12 attending public schools in each jurisdiction. In one state (South Dakota), weighted estimates are representative of all students in grades 9-12 attending public and private schools.

\section{Analytic Methods}

Statistical analyses were conducted on weighted data using SAS (10) and SUDAAN (11) software to account for the complex sampling designs. Prevalence estimates and confidence intervals were computed for all variables and all data sets. In addition, for the national YRBS data, $t$ tests were used to determine pairwise differences between subpopulations (12). Differences between prevalence estimates were considered statistically significant if the test p value was $<0.05$ for main effects (sex, race/ethnicity, and grade) and for interactions (sex by race/ethnicity, sex by grade, race/ethnicity by sex, and grade by sex). In the results section, only statistically significant differences in national YRBS prevalence estimates are reported in the following order: sex, sex by race/ethnicity, sex by grade, race/ethnicity, race/ethnicity by sex, grade, and grade by sex.

To identify long-term temporal trends in health behaviors nationwide, prevalence estimates from the earliest year of data collection to 2015 for each variable assessed with identically worded questions in three or more survey years were examined. Logistic regression analyses were used to account for all available estimates; control for sex, grade, and racial/ ethnic changes over time; and assess long-term linear and quadratic trends (12). A p value associated with the regression coefficient that was $<0.05$ was considered statistically significant. Linear and quadratic time variables were treated as continuous and were coded using orthogonal coefficients calculated with PROC IML in SAS. Separate regression models were used to assess linear and quadratic trends for every variable. When a significant quadratic trend was identified, Joinpoint software (13) was used to automate identification of the year or "joinpoint" where the nonlinear (i.e., quadratic) trend changed and then regression models were used to identify linear trends occurring in each segment. Cubic and higher order trends were not assessed. A quadratic trend indicates a significant but nonlinear trend in prevalence over time. A long-term temporal change that includes a significant linear and quadratic trend demonstrates nonlinear variation (e.g., leveling off or change in direction) in addition to an overall increase or decrease over time.

To identify 2-year temporal changes in health behaviors nationwide, prevalence estimates from 2013 and 2015 were compared using $\mathrm{t}$ tests for each variable assessed with identically worded questions in both survey years. Prevalence estimates were considered statistically different if the $t$ test $\mathrm{p}$ value was $<0.05$.

In the results section, long-term linear and quadratic trends are described first followed by results from the $t$ tests used to assess 2-year temporal changes. Information about long-term temporal trends and 2-year temporal changes are not available because of changes in question or response option wording or because the question was asked for the first time during 2015 for the following variables: usually obtained their own cigarettes by buying them on the Internet; ever use of electronic vapor products; current use of electronic vapor products; current use of cigarettes, cigars, smokeless tobacco, or electronic vapor products; usual method of marijuana use; ever use of synthetic marijuana; sports drink consumption; water consumption; had a sunburn; having seen a dentist; and avoidance of foods because eating the food could cause an allergic reaction.

\section{Results}

\section{Behaviors that Contribute to Unintentional Injuries}

\section{Rarely or Never Wore a Bicycle Helmet}

Among the $68.0 \%$ of students nationwide who had ridden a bicycle during the 12 months before the survey, $81.4 \%$ had rarely or never worn a bicycle helmet (Table 3). The prevalence of having rarely or never worn a bicycle helmet was higher among 11 th-grade male $(85.4 \%)$ than 11 th-grade female $(78.5 \%)$ students. The prevalence of having rarely or never worn a bicycle helmet was higher among black (88.2\%) and Hispanic (90.1\%) than white $(76.4 \%)$ students, higher among Hispanic female (90.3\%) than white female (75.3\%) students, and higher among black male (91.6\%) and Hispanic male (90.0\%) than white male $(77.5 \%)$ students. The prevalence of having rarely or never worn a bicycle helmet was higher among 12th-grade (83.5\%) than 9th-grade $(79.4 \%)$ students, higher among 11 th-grade male $(85.4 \%)$ and 12 th-grade male $(84.9 \%)$ than 9 th-grade male $(80.2 \%)$ students, and higher among 11 th-grade male $(85.4 \%)$ than 10th-grade male (80.4\%) students.

During 1991-2015, a significant linear decrease occurred overall in the prevalence of having rarely or never worn a bicycle 
helmet (96.2\%-81.4\%). A significant quadratic trend also was identified. The prevalence of having rarely or never worn a bicycle helmet decreased during 1991-2001 (96.2\%-84.7\%) and then did not change significantly during 2001-2015 (84.7\%-81.4\%). The prevalence of having never or rarely worn a bicycle helmet decreased significantly from 2013 (87.9\%) to 2015 (81.4\%).

Across 28 states, the prevalence of having rarely or never worn a bicycle helmet ranged from $53.0 \%$ to $94.1 \%$ (median: $84.6 \%$ ) (Table 4). Across 16 large urban school districts, the prevalence ranged from $55.1 \%$ to $95.6 \%$ (median: $87.3 \%$ ).

\section{Rarely or Never Wore a Seat Belt}

Nationwide, $6.1 \%$ of students rarely or never wore a seat belt when riding in a car driven by someone else (Table 3). The prevalence of having rarely or never worn a seat belt was higher among male $(7.2 \%)$ than female (4.9\%) students; higher among white male $(5.3 \%)$ and black male $(12.4 \%)$ than white female $(3.5 \%)$ and black female $(7.6 \%)$ students, respectively; and higher among 10th-grade male (7.6\%) and 11th-grade male $(7.1 \%)$ than 10 th-grade female $(4.5 \%)$ and 11 th-grade female (4.1\%) students, respectively. The prevalence of having rarely or never worn a seat belt was higher among black (10.1\%) and Hispanic (6.5\%) than white (4.4\%) students, higher among black (10.1\%) than Hispanic (6.5\%) students, higher among black female (7.6\%) and Hispanic female (6.3\%) than white female $(3.5 \%)$ students, and higher among black male $(12.4 \%)$ than white male $(5.3 \%)$ and Hispanic male (6.8\%) students.

During 1991-2015, a significant linear decrease occurred overall in the prevalence of having rarely or never worn a seat belt $(25.9 \%-6.1 \%)$. A significant quadratic trend was not identified. The prevalence of having rarely or never worn a seat belt did not change significantly from 2013 (7.6\%) to $2015(6.1 \%)$.

Across 32 states, the prevalence of having rarely or never wore a seat belt ranged from $3.6 \%$ to $14.6 \%$ (median: $8.1 \%$ ) (Table 4). Across 17 large urban school districts, the prevalence ranged from $4.5 \%$ to $21.7 \%$ (median: $8.2 \%$ ).

\section{Rode with a Driver Who Had Been Drinking Alcohol}

During the 30 days before the survey, $20.0 \%$ of students nationwide had ridden in a car or other vehicle one or more times with a driver who had been drinking alcohol (Table 5). The prevalence of having ridden with a driver who had been drinking alcohol was higher among Hispanic (26.2\%) than white $(17.7 \%)$ and black $(21.1 \%)$ students, higher among Hispanic female (27.3\%) than white female (17.5\%) students, and higher among Hispanic male (25.3\%) than white male $(17.7 \%)$ and black male (20.6\%) students.
During 1991-2015, a significant linear decrease occurred overall in the prevalence of having ridden with a driver who had been drinking alcohol (39.9\%-20.0\%). A significant quadratic trend also was identified. The prevalence of having ridden with a driver who had been drinking alcohol decreased during 1991-2009 (39.9\%-28.3\%) and then decreased more gradually from 2009-2015 (28.3\%-20.0\%). The prevalence of having ridden with a driver who had been drinking alcohol did not change significantly from 2013 (21.9\%) to 2015 (20.0\%).

Across 33 states, the prevalence of having ridden with a driver who had been drinking alcohol ranged from $14.2 \%$ to $25.5 \%$ (median: $18.3 \%$ ) (Table 6). Across 18 large urban school districts, the prevalence ranged from $13.4 \%$ to $31.6 \%$ (median: 22.0\%).

\section{Drove When Drinking Alcohol}

Among the $61.4 \%$ of students nationwide who drove a car or other vehicle during the 30 days before the survey, ${ }^{\$ \$} 7.8 \%$ had driven a car or other vehicle one or more times when they had been drinking alcohol during the 30 days before the survey (Table 5). The prevalence of having driven a car or other vehicle when they had been drinking alcohol was higher among male $(9.5 \%)$ than female $(6.0 \%)$ students, higher among white male $(9.4 \%)$ than white female $(5.4 \%)$ students, and higher among 10 th-grade male $(8.2 \%)$ than 10 th-grade female $(2.2 \%)$ students. The prevalence of having driven a car or other vehicle when they had been drinking alcohol was higher among 12th-grade (9.9\%) than 9th-grade (5.6\%) students; higher among 11 th-grade $(8.7 \%)$ and 12 th-grade $(9.9 \%)$ than 10th-grade $(5.3 \%)$ students; higher among 9th-grade female $(5.5 \%)$, 11 th-grade female $(6.8 \%)$, and 12 th-grade female $(8.0 \%)$ than 10 th-grade female $(2.2 \%)$ students; and higher among 12 th-grade male (11.7\%) than 9th-grade male (5.7\%) students.

Because of changes in response options starting in 2013, long-term temporal trends are not available for the prevalence of having driven a car or other vehicle when they had been drinking. The prevalence of having driven a car or other vehicle when they had been drinking alcohol decreased significantly from $2013(10.0 \%)$ to 2015 (7.8\%).

Across 35 states, the prevalence of having driven a car or other vehicle when they had been drinking alcohol among students who drove a car or other vehicle during the 30 days before the survey ranged from $4.3 \%$ to $10.9 \%$ (median: $7.1 \%$ ) (Table 6). Across 18 large urban school districts, the prevalence ranged from $4.4 \%$ to $9.7 \%$ (median: $7.0 \%$ ).

\footnotetext{
$\$ \$$ The prevalence of driving a car or other vehicle during the 30 days before the survey varies slightly for driving when drinking alcohol and texting or e-mailing while driving because of differences in the number of usable responses to each question.
} 


\section{Texted or E-Mailed While Driving}

Among the $61.3 \%$ of students nationwide who drove a car or other vehicle during the 30 days before the survey, ${ }^{\$} 441.5 \%$ had texted or e-mailed while driving a car or other vehicle on at least 1 day during the 30 days before the survey (Table 7). The prevalence of having texted or e-mailed while driving was higher among Hispanic male (42.2\%) than Hispanic female $(28.2 \%)$ students. The prevalence of having texted or e-mailed while driving was higher among white $(45.2 \%)$ than black (32.8\%) and Hispanic (35.8\%) students, higher among white female (45.3\%) than black female (33.1\%) and Hispanic female (28.2\%) students, and higher among white male (45.0\%) and Hispanic male (42.2\%) than black male (33.0\%) students. The prevalence of having texted or e-mailed while driving was higher among 10th-grade (25.0\%), 11th-grade (47.9\%), and 12th-grade $(61.4 \%)$ than 9th-grade $(15.9 \%)$ students; higher among 11 th-grade $(47.9 \%)$ and 12 th-grade $(61.4 \%)$ than 10th-grade (25.0\%) students; higher among 12th-grade (61.4\%) than 11th-grade (47.9\%) students; higher among 10th-grade female $(24.7 \%)$, 11th-grade female $(45.1 \%)$, and 12th-grade female $(60.8 \%)$ than 9th-grade female (14.4\%) students; higher among 11th-grade female (45.1\%) and 12th-grade female $(60.8 \%)$ than 10th-grade female $(24.7 \%)$ students; higher among 12th-grade female $(60.8 \%)$ than 11 th-grade female (45.1\%) students; higher among 10th-grade male (25.2\%), 11 th-grade male $(50.1 \%)$, and 12 th-grade male $(61.9 \%)$ than 9th-grade male $(17.4 \%)$ students; higher among 11 th-grade male $(50.1 \%)$ and 12 th-grade male $(61.9 \%)$ than 10th-grade male (25.2\%) students; and higher among 12th-grade male (61.9\%) than 11th-grade male (50.1\%) students.

Because of changes in response options starting in 2013, long-term temporal trends are not available for the prevalence of having texted or e-mailed while driving. The prevalence of having texted or e-mailed while driving did not change significantly from 2013 (41.4\%) to 2015 (41.5\%).

Across 35 states, the prevalence of having texted or e-mailed while driving ranged from $26.1 \%$ to $63.2 \%$ (median: $39.3 \%$ ) (Table 8). Across 18 large urban school districts, the prevalence ranged from $14.1 \%$ to $38.7 \%$ (median: $32.1 \%$ ).

\section{Behaviors that Contribute to Violence}

\section{Carried a Weapon}

Nationwide, $16.2 \%$ of students had carried a weapon (e.g., gun, knife, or club) on at least 1 day during the 30 days before the survey (Table 9). The prevalence of having carried a weapon was higher among male $(24.3 \%)$ than female $(7.5 \%)$ students; higher among white male $(28.0 \%)$, black male $(17.6 \%)$, and Hispanic male $(20.2 \%)$ than white female $(8.1 \%)$, black female $(6.2 \%)$, and
Hispanic female (7.1\%) students, respectively; and higher among 9th-grade male (24.6\%), 10th-grade male (25.5\%), 11th-grade male $(23.0 \%)$, and 12 th-grade male $(23.4 \%)$ than 9th-grade female $(6.6 \%), 10$ th-grade female $(7.2 \%), 11$ th-grade female $(8.0 \%)$, and 12 th-grade female $(8.0 \%)$ students, respectively. The prevalence of having carried a weapon was higher among white (18.1\%) than black (12.4\%) and Hispanic (13.7\%) students and higher among white male (28.0\%) than black male (17.6\%) and Hispanic male (20.2\%) students.

During 1991-2015, a significant linear decrease occurred overall in the prevalence of having carried a weapon $(26.1 \%-16.2 \%)$. A significant quadratic trend also was identified. The prevalence of having carried a weapon decreased during 1991-1997 (26.1\%$18.3 \%)$ and then did not change significantly during 1997-2015 $(18.3 \%-16.2 \%)$. The prevalence of having carried a weapon also did not change significantly from 2013 (17.9\%) to 2015 (16.2\%).

Across 27 states, the prevalence of having carried a weapon ranged from $8.9 \%$ to $29.6 \%$ (median: $19.3 \%$ ) (Table 10 ). Across 19 large urban school districts, the prevalence ranged from $7.7 \%$ to $21.9 \%$ (median: $12.5 \%$ ).

\section{Carried a Gun}

Nationwide, $5.3 \%$ of students had carried a gun on at least 1 day during the 30 days before the survey (Table 9). The prevalence of having carried a gun was higher among male $(8.7 \%)$ than female $(1.6 \%)$ students; higher among white male (9.6\%), black male $(9.6 \%)$, and Hispanic male $(6.5 \%)$ than white female (1.4\%), black female (1.7\%), and Hispanic female $(1.9 \%)$ students, respectively; and higher among 9 th-grade male (7.0\%), 10th-grade male (8.8\%), 11th-grade male $(9.0 \%)$, and 12 th-grade male $(9.7 \%)$ than 9 th-grade female $(1.2 \%)$, 10th-grade female $(1.6 \%), 11$ th-grade female $(1.4 \%)$, and 12 th-grade female $(1.7 \%)$ students, respectively. The prevalence of having carried a gun was higher among white male (9.6\%) than Hispanic male (6.5\%) students. The prevalence of having carried a gun was higher among 12th-grade $(5.7 \%)$ than 9th-grade (4.4\%) students and higher among 12th-grade male $(9.0 \%)$ than 9 th-grade male $(7.0 \%)$ students.

During 1993-2015, a significant linear decrease occurred overall in the prevalence of having carried a gun $(7.9 \%-$ $5.3 \%)$. A significant quadratic trend also was identified. The prevalence of having carried a gun decreased during 1993$1997(7.9 \%-5.9 \%)$ and then did not change significantly during 1997-2015 (5.9\%-5.3\%). The prevalence of having carried a gun also did not change significantly from 2013 (5.5\%) to 2015 (5.3\%).

Across 19 states, the prevalence of having carried a gun ranged from $2.7 \%$ to $11.5 \%$ (median: $6.8 \%$ ) (Table 10 ). Across 15 large urban school districts, the prevalence ranged from $2.2 \%$ to $5.9 \%$ (median: $4.5 \%$ ). 


\section{Carried a Weapon on School Property}

Nationwide, $4.1 \%$ of students had carried a weapon (e.g., a gun, knife, or club) on school property on at least 1 day during the 30 days before the survey (Table 11). The prevalence of having carried a weapon on school property was higher among male $(5.9 \%)$ than female $(2.0 \%)$ students; higher among white male (5.7\%), black male (4.7\%), and Hispanic male (6.1\%) than white female (1.6\%), black female (2.1\%), and Hispanic female $(2.9 \%)$ students, respectively; and higher among 9th-grade male (4.6\%), 10th-grade male (6.1\%), 11th-grade male $(7.4 \%)$, and 12 th-grade male $(5.1 \%)$ than 9 th-grade female $(1.9 \%)$, 10 th-grade female $(2.2 \%), 11$ th-grade female $(1.9 \%)$, and 12 th-grade female $(2.0 \%)$ students, respectively. The prevalence of having carried a weapon on school property was higher among 11 th-grade $(4.8 \%)$ than 9 th-grade $(3.4 \%)$ students and higher among 11th-grade male (7.4\%) than 9th-grade male (4.6\%) students.

During 1993-2015, a significant linear decrease occurred overall in the prevalence of having carried a weapon on school property $(11.8 \%-4.1 \%)$. A significant quadratic trend also was identified. The prevalence of having carried a weapon on school property decreased during 1993-1997 (11.8\%-8.5\%) and then decreased more rapidly during 1997-2015 (8.5\%-4.1\%). The prevalence of having carried a weapon on school property also decreased significantly from 2013 (5.2\%) to 2015 (4.1\%).

Across 33 states, the prevalence of having carried a weapon on school property ranged from $2.0 \%$ to $10.7 \%$ (median: $5.2 \%$ ) (Table 12). Across 17 large urban school districts, the prevalence ranged from $2.4 \%$ to $9.8 \%$ (median: 3.3\%).

\section{Threatened or Injured with a Weapon on School Property}

Nationwide, $6.0 \%$ of students had been threatened or injured with a weapon (e.g., a gun, knife, or club) on school property one or more times during the 12 months before the survey (Table 11). The prevalence of having been threatened or injured with a weapon on school property was higher among male $(7.0 \%)$ than female (4.6\%) students; higher among black male $(8.9 \%)$ and Hispanic male $(8.4 \%)$ than black female (6.5\%) and Hispanic female (4.7\%) students, respectively; and higher among 11th-grade male $(7.3 \%)$ and 12 th-grade male $(5.7 \%)$ than 11 th-grade female $(2.9 \%)$ and 12 th-grade female (3.2\%) students, respectively. The prevalence of having been threatened or injured with a weapon on school property was higher among black (7.9\%) and Hispanic (6.6\%) than white $(4.9 \%)$ students and higher among black male $(8.9 \%)$ and Hispanic male (8.4\%) than white male $(5.4 \%)$ students. The prevalence of having been threatened or injured with a weapon on school property was higher among 9 th-grade $(7.2 \%)$ than
11 th-grade $(5.5 \%)$ students, higher among 9 th-grade $(7.2 \%)$ and 10th-grade $(6.2 \%)$ than 12 th-grade $(4.4 \%)$ students, higher among 9 th-grade female $(6.2 \%)$ and 10 th-grade female $(5.5 \%)$ than 11 th-grade female $(2.9 \%)$ students, and higher among 9 th-grade female (6.2\%) and 10th-grade female (5.5\%) than 12th-grade female (3.2\%) students.

During 1993-2015, a significant linear decrease occurred overall in the prevalence of having been threatened or injured with a weapon on school property $(7.3 \%-6.0 \%)$. A significant quadratic trend also was identified. The prevalence of having been threatened or injured with a weapon on school property did not change significantly from 1993-2003 (7.3\%-9.2\%) and then decreased during 2003-2015 (9.2\%-6.0\%). The prevalence of having been threatened or injured with a weapon on school property did not change significantly from 2013 (6.9\%) to 2015 (6.0\%).

Across 30 states, the prevalence of having been threatened or injured with a weapon ranged from $4.1 \%$ to $10.6 \%$ (median: 6.6\%) (Table 12). Across 18 large urban school districts, the prevalence ranged from $4.3 \%$ to $13.9 \%$ (median: 6.8\%).

\section{In a Physical Fight}

Nationwide, $22.6 \%$ of students had been in a physical fight one or more times during the 12 months before the survey (Table 13). The prevalence of having been in a physical fight was higher among male $(28.4 \%)$ than female (16.5\%) students; higher among white male (26.6\%), black male (38.6\%), and Hispanic male $(27.3 \%)$ than white female $(13.5 \%)$, black female $(25.4 \%)$, and Hispanic female $(18.6 \%)$ students, respectively; and higher among 9th-grade male (32.5\%), 10 th-grade male $(29.4 \%)$, 11 th-grade male $(27.1 \%)$, and 12th-grade male $(22.9 \%)$ than 9th-grade female $(22.6 \%)$, 10th-grade female $(17.6 \%), 11$ th-grade female $(12.8 \%)$, and 12th-grade female (12.0\%) students, respectively. The prevalence of having been in a physical fight was higher among black $(32.4 \%)$ than white $(20.1 \%)$ and Hispanic (23.0\%) students, higher among black female $(25.4 \%)$ and Hispanic female $(18.6 \%)$ than white female $(13.5 \%)$ students, higher among black female (25.4\%) than Hispanic female (18.6\%) students, and higher among black male $(38.6 \%)$ than white male $(26.6 \%)$ and Hispanic male $(27.3 \%)$ students. The prevalence of having been in a physical fight was higher among 9th-grade $(27.9 \%)$ than 10 th-grade $(23.4 \%), 11$ th-grade (20.5\%), and 12th-grade (17.4\%) students; higher among 10 th-grade $(23.4 \%)$ than 11 th-grade $(20.5 \%)$ and 12 th-grade (17.4\%) students; higher among 9th-grade female (22.6\%) than 10th-grade female (17.6\%), 11th-grade female (12.8\%), and 12th-grade female (12.0\%) students; higher among 10th-grade female (17.6\%) than 11th-grade female (12.8\%) and 12 th-grade female (12.0\%) students; and higher among 
9th-grade male $(32.5 \%)$ and 10th-grade male $(29.4 \%)$ than 12th-grade male $(22.9 \%)$ students.

During 1991-2015, a significant linear decrease occurred in the prevalence of having been in a physical fight $(42.5 \%$ $22.6 \%)$. A significant quadratic trend also was identified. The prevalence of having been in a physical fight decreased during 1991-2011 (42.5\%-32.8\%) and then decreased more gradually during 2011-2015 (32.8\%-22.6\%). The prevalence of having been in a physical fight did not change significantly from 2013 (24.7\%) to 2015 (22.6\%).

Across 31 states, the prevalence of having been in a physical fight ranged from $14.9 \%$ to $27.3 \%$ (median: 20.6\%) (Table 14). Across 19 large urban school districts, the prevalence ranged from $13.9 \%$ to $42.5 \%$ (median: $22.8 \%$ ).

\section{Injured in a Physical Fight}

During the 12 months before the survey, $2.9 \%$ of students nationwide had been in a physical fight one or more times in which they were injured and had to be treated by a doctor or nurse (Table 13). The prevalence of having been injured in a physical fight was higher among male $(3.7 \%)$ than female $(1.8 \%)$ students; higher among white male $(2.8 \%)$ than white female $(0.9 \%)$ students; and higher among 10th-grade male (3.3\%), 11th-grade male (4.3\%), and 12th-grade male (3.5\%) than 10th-grade female (1.4\%), 11th-grade female $(1.5 \%)$, and 12th-grade female (1.4\%) students, respectively. The prevalence of having been injured in a physical fight was higher among black (4.7\%) and Hispanic (3.8\%) than white (1.9\%) students, higher among black female (3.4\%) and Hispanic female $(3.0 \%)$ than white female $(0.9 \%)$ students, and higher among black male $(5.8 \%)$ than white male $(2.8 \%)$ students. The prevalence of having been injured in a physical fight was higher among 9 th-grade female $(2.5 \%)$ than 12 th-grade female $(1.4 \%)$ students.

During 1991-2015, a significant linear decrease occurred overall in the prevalence of having been injured in a physical fight (4.4\%-2.9\%). A significant quadratic trend was not identified. The prevalence of having been injured in a physical fight did not change significantly from 2013 (3.1\%) to 2015 (2.9\%).

Across 29 states, the prevalence of having been injured in a physical fight ranged from $1.9 \%$ to $6.5 \%$ (median: $2.8 \%$ ) (Table 14). Across 14 large urban school districts, the prevalence ranged from $2.2 \%$ to $9.4 \%$ (median: $3.5 \%$ ).

\section{In a Physical Fight on School Property}

Nationwide, $7.8 \%$ of students had been in a physical fight on school property one or more times during the 12 months before the survey (Table 15). The prevalence of having been in a physical fight on school property was higher among male $(10.3 \%)$ than female (5.0\%) students; higher among white male
(8.0\%), black male (15.4\%), and Hispanic male (10.7\%) than white female (3.2\%), black female (9.4\%), and Hispanic female (7.1\%) students, respectively; and higher among 9 th-grade male (14.7\%), 10th-grade male (10.0\%), 11th-grade male (8.3\%), and 12th-grade male (6.4\%) than 9th-grade female (8.2\%), 10 th-grade female $(4.6 \%)$, 11 th-grade female $(4.1 \%)$, and 12th-grade female $(2.5 \%)$ students, respectively. The prevalence of having been in a physical fight on school property was higher among black (12.6\%) and Hispanic (8.9\%) than white (5.6\%) students, higher among black female (9.4\%) and Hispanic female $(7.1 \%)$ than white female (3.2\%) students, and higher among black male (15.4\%) than white male (8.0\%) students. The prevalence of having been in a physical fight on school property was higher among 9 th-grade $(11.6 \%)$ than 10 th-grade (7.3\%), 11th-grade $(6.5 \%)$, and 12th-grade (4.5\%) students; higher among 10th-grade (7.3\%) and 11th-grade (6.5\%) than 12 th-grade $(4.5 \%)$ students; higher among 9th-grade female $(8.2 \%)$ than 10 th-grade female $(4.6 \%), 11$ th-grade female $(4.1 \%)$, and 12 th-grade female $(2.5 \%)$ students; higher among 10 th-grade female $(4.6 \%)$ than 12 th-grade female $(2.5 \%)$ students; higher among 9th-grade male (14.7\%) than 10th-grade male $(10.0 \%)$, 11 th-grade male $(8.3 \%)$, and 12 th-grade male (6.4\%) students; and higher among 10th-grade male (10.0\%) than 12th-grade male (6.4\%) students.

During 1993-2015, a significant linear decrease occurred overall in the prevalence of having been in a physical fight on school property $(16.2 \%-7.8 \%)$. A significant quadratic trend also was identified. The prevalence of having been in a physical fight on school property decreased during 1993-2011 (16.2\%-12.0\%) and then decreased more gradually from 2011-2015 (12.0\%-7.8\%). The prevalence of having been in a physical fight on school property did not change significantly from $2013(8.1 \%)$ to 2015 (7.8\%).

Across 33 states, the prevalence of having been in a physical fight on school property ranged from $4.9 \%$ to $12.2 \%$ (median: $7.2 \%$ ) (Table 16). Across 16 large urban school districts, the prevalence ranged from $5.7 \%$ to $17.5 \%$ (median: $8.1 \%$ ).

\section{Did Not Go to School Because of Safety Concerns}

Nationwide, $5.6 \%$ of students had not gone to school on at least 1 day during the 30 days before the survey because they felt they would be unsafe at school or on their way to or from school (i.e., did not go to school because of safety concerns) (Table 15). The prevalence of having not gone to school because of safety concerns was higher among female $(6.0 \%)$ than male $(5.0 \%)$ students; higher among white female $(5.4 \%)$ than white male $(2.9 \%)$ students; and higher among 9th-grade female $(7.7 \%)$ and 10th-grade female $(6.3 \%)$ than 9th-grade male (4.9\%) and 10th-grade male (4.4\%) students, respectively. The prevalence of having not gone to school 
because of safety concerns was higher among black (6.8\%) and Hispanic (7.6\%) than white (4.2\%) students and higher among black male $(6.9 \%)$ and Hispanic male $(7.6 \%)$ than white male $(2.9 \%)$ students. The prevalence of having not gone to school because of safety concerns was higher among 9 th-grade $(6.4 \%)$ than 11 th-grade $(4.6 \%)$ students and higher among 9 th-grade female $(7.7 \%)$ than 11 th-grade female $(5.3 \%)$ and 12 th-grade female $(4.3 \%)$ students.

During 1993-2015, a significant linear increase occurred overall in the prevalence of having not gone to school because of safety concerns $(4.4 \%-5.6 \%)$. A significant quadratic trend was not identified. The prevalence of having not gone to school because of safety concerns decreased significantly from 2013 (7.1\%) to $2015(5.6 \%)$.

Across 36 states, the prevalence of having not gone to school because of safety concerns ranged from $4.3 \%$ to 11.5\% (median: 6.2\%) (Table 16). Across 18 large urban school districts, the prevalence ranged from $4.5 \%$ to $12.8 \%$ (median: 9.1\%).

\section{Electronically Bullied}

Nationwide, $15.5 \%$ of students had been electronically bullied, counting being bullied through e-mail, chat rooms, instant messaging, websites, or texting, during the 12 months before the survey (Table 17). The prevalence of having been electronically bullied was higher among female $(21.7 \%)$ than male $(9.7 \%)$ students; higher among white female $(26.0 \%)$, black female (11.9\%), and Hispanic female (16.7\%) than white male $(10.8 \%)$, black male $(5.6 \%)$, and Hispanic male $(8.1 \%)$ students, respectively; and higher among 9th-grade female $(22.7 \%), 10$ th-grade female $(23.2 \%), 11$ th-grade female $(21.4 \%)$, and 12 th-grade female $(19.5 \%)$ than 9 th-grade male (11.0\%), 10th-grade male (9.9\%), 11th-grade male (8.4\%), and 12 th-grade male $(9.2 \%)$ students, respectively. The prevalence of having been electronically bullied was higher among white $(18.4 \%)$ and Hispanic $(12.4 \%)$ than black $(8.6 \%)$ students, higher among white $(18.4 \%)$ than Hispanic (12.4\%) students, higher among white female $(26.0 \%)$ and Hispanic female (16.7\%) than black female (11.9\%) students, higher among white female $(26.0 \%)$ than Hispanic female $(16.7 \%)$ students, and higher among white male $(10.8 \%)$ than black male (5.6\%) and Hispanic male (8.1\%) students. The prevalence of having been electronically bullied was higher among 10th-grade (16.6\%) than 12th-grade (14.3\%) students and higher among 10 th-grade female $(23.2 \%)$ than 12 th-grade female $(19.5 \%)$ students.

During 2011-2015, significant linear and quadratic trends were not identified in the prevalence of having been electronically bullied. The prevalence of having been electronically bullied did not change significantly from 2013 (14.8\%) to 2015 (15.5\%).

Across 36 states, the prevalence of having been electronically bullied ranged from $11.6 \%$ to $21.1 \%$ (median: $15.4 \%$ ) (Table 18). Across 19 large urban school districts, the prevalence ranged from $7.9 \%$ to $16.3 \%$ (median: $11.2 \%$ ).

\section{Bullied on School Property}

Nationwide, $20.2 \%$ of students had been bullied on school property during the 12 months before the survey (Table 17). The prevalence of having been bullied on school property was higher among female $(24.8 \%)$ than male $(15.8 \%)$ students; higher among white female (29.1\%), black female (15.1\%), and Hispanic female (19.3\%) than white male (18.1\%), black male (11.2\%), and Hispanic male (13.7\%) students, respectively; and higher among 9th-grade female (29.0\%), 10 th-grade female $(25.5 \%), 11$ th-grade female $(24.2 \%)$, and 12th-grade female $(19.8 \%)$ than 9th-grade male $(18.3 \%)$, 10 th-grade male $(16.1 \%)$, 11 th-grade male $(16.4 \%)$, and 12th-grade male (12.1\%) students, respectively. The prevalence of having been bullied on school property was higher among white $(23.5 \%)$ than black (13.2\%) and Hispanic (16.5\%) students, higher among white female $(29.1 \%)$ than black female (15.1\%) and Hispanic female (19.3\%) students, and higher among white male (18.1\%) than black male (11.2\%) and Hispanic male $(13.7 \%)$ students. The prevalence of having been bullied on school property was higher among 9th-grade (23.4\%), 10th-grade (20.8\%), and 11th-grade $(20.3 \%)$ than 12 th-grade $(15.9 \%)$ students; higher among 9 th-grade female $(29.0 \%)$ than 11 th-grade female $(24.2 \%)$ students; higher among 9th-grade female (29.0\%), 10th-grade female (25.5\%), and 11 th-grade female $(24.2 \%)$ than 12 th-grade female $(19.8 \%)$ students; and higher among 9th-grade male (18.3\%), 10 th-grade male $(16.1 \%)$, and 11 th-grade male $(16.4 \%)$ than 12th-grade male $(12.1 \%)$ students.

During 2009-2015, significant linear and quadratic trends were not identified in the prevalence of having been bullied on school property. The prevalence of having been bullied on school property did not change significantly from 2013 (19.6\%) to $2015(20.2 \%)$.

Across 35 states, the prevalence of having been bullied on school property ranged from $15.0 \%$ to $26.3 \%$ (median: 19.9\%) (Table 18). Across 19 large urban school districts, the prevalence ranged from $11.3 \%$ to $20.2 \%$ (median: $14.6 \%$ ).

\section{Forced to Have Sexual Intercourse}

Nationwide, $6.7 \%$ of students had ever been physically forced to have sexual intercourse when they did not want to (Table 19). The prevalence of having been forced to have 
sexual intercourse was higher among female (10.3\%) than male $(3.1 \%)$ students; higher among white female (9.9\%), black female $(10.3 \%)$, and Hispanic female $(10.1 \%)$ than white male (2.0\%), black male (4.4\%), and Hispanic male (4.0\%) students, respectively; and higher among 9th-grade female $(9.4 \%)$, 10th-grade female $(7.9 \%), 11$ th-grade female $(12.0 \%)$, and 12 th-grade female $(11.9 \%)$ than 9 th-grade male (2.1\%), 10th-grade male (3.9\%), 11th-grade male (2.8\%), and 12 th-grade male $(3.5 \%)$ students, respectively. The prevalence of having been forced to have sexual intercourse was higher among black male $(4.4 \%)$ and Hispanic male $(4.0 \%)$ than white male $(2.0 \%)$ students. The prevalence of having been forced to have sexual intercourse was higher among 11 th-grade (7.6\%) and 12th-grade (7.6\%) than 9th-grade (5.6\%) students, higher among 12 th-grade $(7.6 \%)$ than 10 th-grade $(5.9 \%)$ students, higher among 11 th-grade female $(12.0 \%)$ and 12th-grade female (11.9\%) than 10th-grade female $(7.9 \%)$ students, and higher among 10th-grade male $(3.9 \%)$ and 12th-grade male $(3.5 \%)$ than 9th-grade male $(2.1 \%)$ students.

During 2001-2015, a significant linear decrease occurred overall in the prevalence of having been forced to have sexual intercourse (7.7\%-6.7\%). A significant quadratic trend was not identified. The prevalence of having been forced to have sexual intercourse did not change significantly from 2013 (7.3\%) to $2015(6.7 \%)$.

Across 34 states, the prevalence of having been physically forced to have sexual intercourse ranged from $5.1 \%$ to 11.7\% (median: 7.9\%) (Table 20). Across 17 large urban school districts, the prevalence ranged from $5.5 \%$ to $12.6 \%$ (median: 8.2\%).

\section{Physical Dating Violence}

Among the $68.6 \%$ of students nationwide who dated or went out with someone during the 12 months before the survey, 99 9.6\% had been physically hurt on purpose (counting being hit, slammed into something, or injured with an object or weapon) by someone they were dating or going out with one or more times during the 12 months before the survey (i.e., physical dating violence) (Table 21). The prevalence of physical dating violence was higher among female (11.7\%) than male (7.4\%) students; higher among white female $(11.9 \%)$ and Hispanic female $(11.4 \%)$ than white male $(5.9 \%)$ and Hispanic male $(8.0 \%)$ students, respectively; and higher among 9th-grade female $(11.1 \%)$, 11th-grade female $(11.6 \%)$, and 12 th-grade female (12.9\%) than 9 th-grade male $(5.3 \%), 11$ th-grade male

\footnotetext{
99 The prevalence of dating or going out with someone during the 12 months before the survey varies slightly for physical dating violence and sexual dating violence because of differences in the number of usable responses to each question.
}

(7.9\%), and 12th-grade male (8.2\%) students, respectively. The prevalence of physical dating violence was higher among 11 th-grade $(10.1 \%)$ than 9 th-grade $(8.1 \%)$ students and higher among 11 th-grade male $(7.9 \%)$ and 12 th-grade male $(8.2 \%)$ than 9th-grade male $(5.3 \%)$ students.

Because of changes in the question and response options starting in 2013, long-term temporal trends are not available for the prevalence of physical dating violence. The prevalence of physical dating violence did not change significantly from $2013(10.3 \%)$ to $2015(9.6 \%)$.

Across 36 states, the prevalence of physical dating violence ranged from $6.7 \%$ to $14.6 \%$ (median: $9.0 \%$ ) (Table 22). Across 19 large urban school districts, the prevalence ranged from $6.4 \%$ to $12.7 \%$ (median: $10.1 \%$ ).

\section{Sexual Dating Violence}

Among the $69.1 \%$ of students nationwide who dated or went out with someone during the 12 months before the survey, ${ }^{91} 10.6 \%$ of students had been forced to do sexual things (counting being kissed, touched, or physically forced to have sexual intercourse) they did not want to do by someone they were dating or going out with one or more times during the 12 months before the survey (i.e., sexual dating violence) (Table 21). The prevalence of sexual dating violence was higher among female (15.6\%) than male $(5.4 \%)$ students; higher among white female (16.6\%) and Hispanic female $(14.2 \%)$ than white male $(3.5 \%)$ and Hispanic male $(7.0 \%)$ students, respectively; and higher among 9th-grade female (17.6\%), 10th-grade female (15.8\%), 11 th-grade female $(14.9 \%)$, and 12 th-grade female $(13.9 \%)$ than 9 th-grade male (4.5\%), 10th-grade male (7.4\%), 11 th-grade male $(5.1 \%)$, and 12 th-grade male $(4.6 \%)$ students, respectively. The prevalence of sexual dating violence was higher among black male (8.0\%) and Hispanic male $(7.0 \%)$ than white male $(3.5 \%)$ students. The prevalence of sexual dating violence was higher among 10th-grade $(11.8 \%)$ than 12 th-grade $(9.2 \%)$ students and higher among 10th-grade male (7.4\%) than 9th-grade male $(4.5 \%)$ and 12 th-grade male (4.6\%) students.

Because of changes in the question and response options starting in 2013, long-term temporal trends are not available for the prevalence of sexual dating violence. The prevalence of sexual dating violence did not change significantly from 2013 $(10.4 \%)$ to $2015(10.6 \%)$.

Across 30 states, the prevalence of sexual dating violence ranged from $7.5 \%$ to $14.7 \%$ (median: $10.1 \%$ ) (Table 22 ). Across 19 large urban school districts, the prevalence ranged from $6.8 \%$ to $14.4 \%$ (median: $10.5 \%$ ). 


\section{Felt Sad or Hopeless}

During the 12 months before the survey, 29.9\% of students nationwide had felt so sad or hopeless almost every day for 2 or more weeks in a row that they stopped doing somec usual activities (Table 23). The prevalence of having felt sad or hopeless was higher among female (39.8\%) than male (20.3\%) students; higher among white female (37.9\%), black female (33.9\%), and Hispanic female (46.7\%) than white male (19.2\%), black male (17.6\%), and Hispanic male (24.3\%) students, respectively; and higher among 9th-grade female (41.5\%), 10th-grade female (40.1\%), 11th-grade female (40.9\%), and 12th-grade female (36.3\%) than 9th-grade male (16.7\%), 10th-grade male (19.2\%), 11th-grade male (22.1\%), and 12 th-grade male (23.9\%) students, respectively. The prevalence of having felt sad or hopeless was higher among Hispanic (35.3\%) than white (28.6\%) and black (25.2\%) students, higher among Hispanic female (46.7\%) than white female (37.9\%) and black female (33.9\%) students, and higher among Hispanic male (24.3\%) than white male (19.2\%) and black male (17.6\%) students. The prevalence of having felt sad or hopeless was higher among 9 th-grade female (41.5\%) and 11th-grade female (40.9\%) than 12th-grade female (36.3\%) students, higher among 11th-grade male $(22.1 \%)$ and 12 th-grade male $(23.9 \%)$ than 9th-grade male (16.7\%) students, and higher among 12th-grade male (23.9\%) than 10th-grade male (19.2\%) students.

During 1999-2015, significant linear and quadratic trends were not identified in the prevalence of having felt sad or hopeless. The prevalence of having felt sad or hopeless did not change significantly from 2013 (29.9\%) to 2015 (29.9\%).

Across 37 states, the prevalence of having felt sad or hopeless ranged from $24.1 \%$ to $34.2 \%$ (median: $28.9 \%$ ) (Table 24 ). Across 19 large urban school districts, the prevalence ranged from $24.9 \%$ to $36.0 \%$ (median: $29.8 \%$ ).

\section{Seriously Considered Attempting Suicide}

Nationwide, $17.7 \%$ of students had seriously considered attempting suicide during the 12 months before the survey (Table 25). The prevalence of having seriously considered attempting suicide was higher among female (23.4\%) than male (12.2\%) students; higher among white female (22.8\%), black female $(18.7 \%)$, and Hispanic female $(25.6 \%)$ than white male (11.5\%), black male (11.0\%), and Hispanic male (12.4\%) students, respectively; and higher among 9th-grade female $(26.5 \%)$, 10th-grade female $(25.7 \%)$, 11th-grade female $(22.1 \%)$, and 12 th-grade female $(18.6 \%)$ than 9 th-grade male (10.7\%), 10th-grade male (10.8\%), 11th-grade male (13.3\%), and 12 th-grade male $(14.0 \%)$ students, respectively. The prevalence of having seriously considered attempting suicide was higher among Hispanic (18.8\%) than black (14.5\%) students and higher among Hispanic female (25.6\%) than black female
$(18.7 \%)$ students. The prevalence of having seriously considered attempting suicide was higher among 9th-grade female (26.5\%) and 10th-grade female (25.7\%) than 11th-grade female (22.1\%) students and 12th-grade female (22.1\%) students and higher among 11 th-grade male (13.3\%) and 12th-grade male (14.0\%) than 10th-grade male (10.8\%) students.

During 1991-2015, a significant linear decrease occurred overall in the prevalence of having seriously considered attempting suicide (29.0\%-17.7\%). A significant quadratic trend also was identified. The prevalence of having seriously considered attempting suicide decreased during 1991-2009 $(29.0 \%-13.8 \%)$ and then increased during 2009-2015 (13.8\%-17.7\%). The prevalence of having seriously considered attempting suicide did not change significantly from 2013 $(17.0 \%)$ to $2015(17.7 \%)$.

Across 36 states, the prevalence of having seriously considered attempting suicide ranged from $13.4 \%$ to $20.3 \%$ (median: 16.0\%) (Table 26). Across 19 large urban school districts, the prevalence ranged from $10.7 \%$ to $19.7 \%$ (median: $15.1 \%$ ).

\section{Made a Suicide Plan}

During the 12 months before the survey, $14.6 \%$ of students nationwide had made a plan about how they would attempt suicide (Table 25). The prevalence of having made a suicide plan was higher among female (19.4\%) than male (9.8\%) students; higher among white female (18.4\%), black female (17.3\%), and Hispanic female $(20.7 \%)$ than white male $(9.3 \%)$, black male (10.6\%), and Hispanic male (10.9\%) students, respectively; and higher among 9th-grade female (22.5\%), 10th-grade female $(21.6 \%)$, 11th-grade female $(17.2 \%)$, and 12 th-grade female $(15.7 \%)$ than 9 th-grade male $(8.1 \%)$, 10th-grade male $(9.2 \%)$, 11 th-grade male $(10.4 \%)$, and 12 th-grade male $(12.0 \%)$ students, respectively. The prevalence of having made a suicide plan was higher among 9th-grade female $(22.5 \%)$ and 10th-grade female (21.6\%) than 11th-grade female (17.2\%) and 12th-grade female $(15.7 \%)$ students, higher among 11 th-grade male $(10.4 \%)$ and 12 th-grade male $(12.0 \%)$ than 9th-grade male (8.1\%) students, and higher among 12th-grade male (12.0\%) than 10th-grade male $(9.2 \%)$ students.

During 1991-2015, a significant linear decrease occurred overall in the prevalence of having made a suicide plan (18.6\%-14.6\%). A significant quadratic trend also was identified. The prevalence of having made a suicide plan decreased during 1991-2009 (18.6\%$10.9 \%)$ and then increased during 2009-2015 (10.9\%-14.6\%). The prevalence of having made a suicide plan did not change significantly from 2013 (13.6\%) to 2015 (14.6\%).

Across 34 states, the prevalence of having made a suicide plan ranged from $11.0 \%$ to $18.2 \%$ (median: $14.3 \%$ ) (Table 26). Across 16 large urban school districts, the prevalence ranged from $8.7 \%$ to $19.3 \%$ (median: $13.8 \%$ ). 


\section{Attempted Suicide}

Nationwide, $8.6 \%$ of students had attempted suicide one or more times during the 12 months before the survey (Table 27). The prevalence of having attempted suicide was higher among female (11.6\%) than male (5.5\%) students; higher among white female $(9.8 \%)$ and Hispanic female (15.1\%) than white male (3.7\%) and Hispanic male (7.6\%) students, respectively; and higher among 9th-grade female (15.1\%), 10th-grade female $(13.0 \%)$, and 11 th-grade female $(10.2 \%)$ than 9 th-grade male (5.1\%), 10th-grade male (5.7\%), and 11th-grade male (5.8\%) students, respectively. The prevalence of having attempted suicide was higher among Hispanic (11.3\%) than white (6.8\%) students, higher among Hispanic female (15.1\%) than white female $(9.8 \%)$ and black female $(10.2 \%)$ students, and higher among black male (7.2\%) and Hispanic male (7.6\%) than white male $(3.7 \%)$ students. The prevalence of having attempted suicide was higher among 9th-grade (9.9\%) than 11 th-grade $(8.0 \%)$ students; higher among 9 th-grade $(9.9 \%)$ and 10 th-grade $(9.4 \%)$ than 12 th-grade $(6.2 \%)$ students; higher among 9th-grade female (15.1\%) than 11th-grade female (10.2\%) students; and higher among 9th-grade female $(15.1 \%), 10$ th-grade female $(13.0 \%)$, and 11 th-grade female (10.2\%) than 12 th-grade female $(7.2 \%)$ students.

During 1991-2015, a significant linear decrease occurred overall in the prevalence of having attempted suicide $(7.3 \%-8.6 \%) .^{* * *}$ A significant quadratic trend was not identified. The prevalence having attempted suicide did not change significantly from $2013(8.0 \%)$ to 2015 (8.6\%).

Across 35 states, the prevalence of having attempted suicide ranged from $5.9 \%$ to $12.7 \%$ (median: $9.6 \%$ ) (Table 28). Across 19 large urban school districts, the prevalence ranged from $6.4 \%$ to $20.7 \%$ (median: $9.9 \%$ ).

\section{Suicide Attempt Treated by a Doctor or Nurse}

During the 12 months before the survey, $2.8 \%$ of students nationwide had made a suicide attempt that resulted in an injury, poisoning, or overdose that had to be treated by a doctor or nurse (Table 27). The prevalence of having made a suicide attempt that resulted in an injury, poisoning, or overdose that had to be treated by a doctor or nurse was higher among female (3.7\%) than male (1.9\%) students; higher among white female (3.4\%) and Hispanic female (4.5\%) than white male (0.9\%) and Hispanic male (2.9\%) students, respectively; and higher among 9th-grade female (4.7\%) than 9th-grade male (1.9\%) students. The prevalence of having made a suicide attempt that resulted

\footnotetext{
*** Review of only the oldest and most recent data points are not necessarily indicative of long-term temporal trends because the logistic regression analyses take into account all data points and adjust for changes in sex, grade, and race/ethnicity over time.
}

in an injury, poisoning, or overdose that had to be treated by a doctor or nurse was higher among Hispanic (3.7\%) than white $(2.1 \%)$ students and higher among black male $(4.0 \%)$ and Hispanic male $(2.9 \%)$ than white male $(0.9 \%)$ students. The prevalence of having made a suicide attempt that resulted in an injury, poisoning, or overdose that had to be treated by a doctor or nurse was higher among 9th-grade (3.2\%) and 10th-grade (3.1\%) than 12th-grade (1.9\%) students and higher among 9th-grade female (4.7\%) and 10th-grade female (3.9\%) than 12th-grade female $(2.3 \%)$ students.

During 1991-2015, significant linear and quadratic trends were not identified in the prevalence of having made a suicide attempt that resulted in an injury, poisoning, or overdose that had to be treated by a doctor or nurse. The prevalence of having made a suicide attempt that resulted in an injury, poisoning, or overdose that had to be treated by a doctor or nurse did not change significantly from 2013 (2.7\%) to 2015 (2.8\%).

Across 33 states, the prevalence of having made a suicide attempt that resulted in an injury, poisoning, or overdose that had to be treated by a doctor or nurse ranged from $1.9 \%$ to $9.3 \%$ (median: $3.2 \%$ ) (Table 28). Across 17 large urban school districts, the prevalence ranged from $1.9 \%$ to $7.4 \%$ (median: 3.5\%).

\section{Tobacco Use}

\section{Ever Tried Cigarette Smoking}

Nationwide, $32.3 \%$ of students had ever tried cigarette smoking (even one or two puffs) (i.e., ever tried cigarette smoking) (Table 29). The prevalence of having ever tried cigarette smoking was higher among Hispanic male (37.8\%) than Hispanic female $(32.7 \%)$ students and higher among 11 th-grade male $(40.5 \%)$ than 11 th-grade female $(34.4 \%)$ students. The prevalence of having ever tried cigarette smoking was higher among Hispanic male (37.8\%) than white male (33.2\%) and black male (30.6\%) students. The prevalence of having ever tried cigarette smoking was higher among 11 th-grade $(37.5 \%)$ and 12 th-grade $(38.3 \%)$ than 9 th-grade $(25.1 \%)$ students and 10th-grade $(29.1 \%)$ students, higher among 11th-grade female (34.4\%) and 12th-grade female (36.3\%) than 9th-grade female (24.5\%) and 10th-grade female (28.2\%) students, and higher among 11th-grade male (40.5\%) and 12 th-grade male $(40.4 \%)$ than 9 th-grade male $(25.8 \%)$ and 10th-grade male $(30.0 \%)$ students.

During 1991-2015, a significant linear decrease occurred overall in the prevalence of having ever tried cigarette smoking (70.1\%-32.3\%). A significant quadratic trend also was identified. The prevalence of having ever tried cigarette smoking did not change significantly during 1991-1999 
(70.1\%-70.4\%) and then decreased during 1999-2015 (70.4\%-32.3\%). The prevalence of having ever tried cigarette smoking also decreased significantly from 2013 (41.1\%) to $2015(32.3 \%)$.

Across 31 states, the prevalence of having ever tried cigarette smoking ranged from $22.4 \%$ to $47.3 \%$ (median: $34.5 \%$ ) (Table 30). Across 15 large urban school districts, the prevalence ranged from $21.4 \%$ to $34.4 \%$ (median: $26.4 \%$ ).

\section{Smoked a Whole Cigarette Before Age 13 Years}

Nationwide, $6.6 \%$ of students had smoked a whole cigarette for the first time before age 13 years (Table 29). The prevalence of having smoked a whole cigarette before age 13 years was higher among male $(8.0 \%)$ than female $(5.0 \%)$ students; higher among black male (10.1\%) and Hispanic male $(9.2 \%)$ than black female $(3.8 \%)$ and Hispanic female $(4.9 \%)$ students, respectively; and higher among 9th-grade male $(8.2 \%)$, 10th-grade male $(9.1 \%), 11$ th-grade male $(6.8 \%)$, and 12 th-grade male $(7.3 \%)$ than 9 th-grade female $(6.1 \%), 10$ th-grade female $(6.0 \%), 11$ th-grade female $(4.5 \%)$, and 12 th-grade female $(3.0 \%)$ students, respectively. The prevalence of having smoked a whole cigarette before age 13 years was higher among black male (10.1\%) and Hispanic male $(9.2 \%)$ than white male $(6.6 \%)$ students. The prevalence of having smoked a whole cigarette before age 13 years was higher among 10th-grade (7.6\%) than 11th-grade $(5.6 \%)$ and 12 th-grade $(5.2 \%)$ students and higher among 9 th-grade female $(6.1 \%)$, 10th-grade female $(6.0 \%)$, and 11 th-grade female (4.5\%) than 12th-grade female (3.0\%) students.

During 1991-2015, a significant linear decrease occurred overall in the prevalence of having smoked a whole cigarette before age 13 years $(23.8 \%-6.6 \%)$. A significant quadratic trend also was identified. The prevalence of having smoked a whole cigarette before age 13 years did not change significantly during 1991-1999 (23.8\%-24.7\%) and then decreased during 1999-2015 (24.7\%-6.6\%). The prevalence of having smoked a whole cigarette before age 13 years also decreased significantly from $2013(9.3 \%)$ to 2015 (6.6\%).

Across 35 states, the prevalence of having smoked a whole cigarette before age 13 years ranged from $4.3 \%$ to 13.1\% (median: 7.8\%) (Table 30). Across 16 large urban school districts, the prevalence ranged from $3.8 \%$ to $13.7 \%$ (median: 5.8\%).

\section{Current Cigarette Use}

Nationwide, $10.8 \%$ of students had smoked cigarettes on at least 1 day during the 30 days before the survey (i.e., current cigarette use) (Table 31). The prevalence of current cigarette use was higher among male (11.8\%) than female (9.7\%) students; higher among black male (9.1\%) and Hispanic male $(11.3 \%)$ than black female $(3.7 \%)$ and Hispanic female (7.1\%) students, respectively; and higher among 11th-grade male $(15.8 \%)$ than 11 th-grade female $(10.1 \%)$ students. The prevalence of current cigarette use was higher among white (12.4\%) and Hispanic (9.2\%) than black (6.5\%) students, higher among white (12.4\%) than Hispanic (9.2\%) students, higher among white female (12.2\%) and Hispanic female $(7.1 \%)$ than black female $(3.7 \%)$ students, and higher among white female (12.2\%) than Hispanic female (7.1\%) students. The prevalence of current cigarette use was higher among 11th-grade (13.1\%) and 12th-grade (14.1\%) than 9th-grade $(7.6 \%)$ and 10th-grade $(8.8 \%)$ students, higher among 11th-grade female (10.1\%) than 9th-grade female $(6.7 \%)$ students; higher among 12th-grade female (13.3\%) than 9th-grade female $(6.7 \%)$, 10th-grade female $(9.1 \%)$, and 11 th-grade female $(10.1 \%)$ students; and higher among 11 th-grade male $(15.8 \%)$ and 12 th-grade male $(15.0 \%)$ than 9th-grade male $(8.3 \%)$ and 10 th-grade male $(8.5 \%)$ students.

During 1991-2015, a significant linear decrease occurred overall in the prevalence of current cigarette use (27.5\%-10.8\%). A significant quadratic trend also was identified. The prevalence of current cigarette use increased during 1991-1997 $(27.5 \%-36.4 \%)$ and then decreased during 1997-2015 $(36.4 \%-10.8 \%)$. The prevalence of current cigarette use also decreased significantly from 2013 (15.7\%) to 2015 (10.8\%).

Across 37 states, the prevalence of current cigarette use ranged from $4.8 \%$ to $18.8 \%$ (median: $10.8 \%$ ) (Table 32 ). Across 18 large urban school districts, the prevalence ranged from $4.2 \%$ to $9.0 \%$ (median: $5.8 \%$ ).

\section{Current Frequent Cigarette Use}

Nationwide, $3.4 \%$ of students had smoked cigarettes 20 or more days during the 30 days before the survey (i.e., current frequent cigarette use) (Table 31). The prevalence of current frequent cigarette use was higher among white $(4.0 \%)$ than black (1.8\%) and Hispanic $(2.1 \%)$ students, higher among white female (4.4\%) and Hispanic female $(2.1 \%)$ than black female $(0.8 \%)$ students, higher among white female (4.4\%) than Hispanic female (2.1\%) students, and higher among white male $(3.6 \%)$ than Hispanic male $(2.2 \%)$ students. The prevalence of current frequent cigarette use was higher among 12th-grade $(5.1 \%)$ than 9 th-grade $(2.2 \%)$, 10th-grade $(2.9 \%)$, and 11th-grade $(3.2 \%)$ students; higher among 12th-grade female $(4.9 \%)$ than 9 th-grade female $(2.5 \%)$ and 10 th-grade female $(2.7 \%)$ students; higher among 11 th-grade male $(3.5 \%)$ and 12th-grade male (5.4\%) than 9th-grade male (1.9\%) students; and higher among 12th-grade male (5.4\%) than 10th-grade male $(3.1 \%)$ students.

During 1991-2015, a significant linear decrease occurred overall in the prevalence of current frequent cigarette use 
$(12.7 \%-3.4 \%)$. A significant quadratic trend also was identified. The prevalence of current frequent cigarette use increased during 1991-1999 (12.7\%-16.8\%) and then decreased during 1999-2015 (16.8\%-3.4\%). The prevalence of current frequent cigarette use also decreased significantly from 2013 (5.6\%) to 2015 (3.4\%).

Across 37 states, the prevalence of current frequent cigarette use ranged from $1.5 \%$ to $7.4 \%$ (median: $3.6 \%$ ) (Table 32 ). Across 18 large urban school districts, the prevalence ranged from $0.6 \%$ to $2.0 \%$ (median: $1.3 \%$ ).

\section{Smoked More Than 10 Cigarettes per Day}

Among the $10.8 \%$ of students nationwide who currently smoked cigarettes, $7.9 \%$ of students had smoked more than 10 cigarettes per day on the days they smoked during the 30 days before the survey (Table 33). The prevalence of having smoked more than 10 cigarettes per day was higher among 10 th-grade $(10.4 \%)$ and 12 th-grade $(9.1 \%)$ than 11 th-grade (3.4\%) students, higher among 10th-grade male (14.7\%) than 9th-grade male (5.1\%) students, and higher among 10th-grade male $(14.7 \%)$ and 12 th-grade male $(12.5 \%)$ than 11 th-grade male $(3.6 \%)$ students.

During 1991-2015, a significant linear decrease occurred overall in the prevalence of having smoked more than 10 cigarettes per day (18.0\%-7.9\%). A significant quadratic trend was not identified. The prevalence of having smoked more than 10 cigarettes per day did not change significantly from 2013 (8.6\%) to 2015 (7.9\%).

Across 30 states, the prevalence of having smoked more than 10 cigarettes per day ranged from $2.0 \%$ to $14.6 \%$ (median: 8.3\%) (Table 34). Across 12 large urban school districts, the prevalence ranged from $0.9 \%$ to $12.8 \%$ (median: $8.2 \%$ ).

\section{Currently Smoked Cigarettes Daily}

Nationwide, $2.3 \%$ of students had smoked cigarettes on all 30 days during the 30 days before the survey (i.e., currently smoked cigarettes daily) (Table 33). The prevalence of having currently smoked cigarettes daily was higher among black male $(2.4 \%)$ than black female $(0.4 \%)$ students and higher among 10th-grade male (2.7\%) than 10th-grade female (1.4\%) students. The prevalence of having currently smoked cigarettes daily was higher among white (2.8\%) than black (1.4\%) and Hispanic (1.6\%) students, higher among white female (3.1\%) and Hispanic female (1.4\%) than black female (0.4\%) students, and higher among white female (3.1\%) than Hispanic female $(1.4 \%)$ students. The prevalence of having currently smoked cigarettes daily was higher among 12 th-grade $(3.4 \%)$ than 9th-grade $(1.7 \%)$, 10th-grade $(2.1 \%)$, and 11 th-grade $(1.9 \%)$ students; higher among 12th-grade female $(3.5 \%)$ than 10th-grade female (1.4\%) students; and higher among
12 th-grade male $(3.2 \%)$ than 9 th-grade male $(1.6 \%)$ and 11 th-grade male $(1.8 \%)$ students.

During 1991-2015, a significant linear decrease occurred overall in the prevalence of having currently smoked cigarettes daily $(9.8 \%-2.3 \%)$. A significant quadratic trend also was identified. The prevalence of having currently smoked cigarettes daily increased during 1991-1999 (9.8\%-12.8\%) and then decreased during 1999-2015 (12.8\%-2.3\%). The prevalence of having currently smoked cigarettes daily also decreased significantly from $2013(4.0 \%)$ to 2015 (2.3\%).

Across 37 states, the prevalence of having currently smoked cigarettes daily ranged from $1.0 \%$ to $5.4 \%$ (median: $2.6 \%$ ) (Table 34). Across 18 large urban school districts, the prevalence ranged from $0.3 \%$ to $1.6 \%$ (median: $0.9 \%$ ).

\section{Bought Cigarettes in a Store or Gas Station}

Among the $8.5 \%$ of students who currently smoked cigarettes and were aged $<18$ years, $12.6 \%$ had usually obtained their own cigarettes by buying them in a store (e.g., convenience store, supermarket, or discount store) or gas station during the 30 days before the survey (Table 35). The prevalence of usually obtaining their own cigarettes by buying them in a store or gas station was higher among male $(16.5 \%)$ than female $(7.7 \%)$ students; higher among white male (12.8\%) and Hispanic male $(21.9 \%)$ than white female $(6.6 \%)$ and Hispanic female $(9.8 \%)$ students, respectively; and higher among 11 th-grade male $(27.1 \%)$ than 11 th-grade female $(8.8 \%)$ students. The prevalence of usually obtaining their own cigarettes by buying them in a store or gas station was higher among Hispanic $(17.5 \%)$ than white $(9.7 \%)$ students and higher among Hispanic male (21.9\%) than white male (12.8\%) students. The prevalence of usually obtaining their own cigarettes by buying them in a store or gas station was higher among 11th-grade $(20.2 \%)$ and 12 th-grade $(16.5 \%)$ than 9 th-grade $(6.3 \%)$ students and 10th-grade (6.1\%) students and higher among 11 th-grade male $(27.1 \%)$ and 12 th-grade male $(22.8 \%)$ than 9th-grade male $(6.2 \%)$ and 10 th-grade male $(6.7 \%)$ students.

During 2001-2015, a significant linear decrease occurred overall in the prevalence of usually obtaining their own cigarettes by buying them in a store or gas station (19.0\%-12.6\%). A significant quadratic trend was not identified. The prevalence of usually obtaining their own cigarettes by buying them in a store or gas station also decreased significantly from 2013 (18.1\%) to 2015 (12.6\%).

Across 28 states, the prevalence of usually obtaining their own cigarettes by buying them in a store or gas station ranged from $4.4 \%$ to $20.6 \%$ (median: $11.6 \%$ ) (Table 36). Across 5 large urban school districts, the prevalence ranged from $14.9 \%$ to 64.5\% (median: $22.5 \%$ ). 


\section{Bought Cigarettes on the Internet}

Among the $8.5 \%$ of students who currently smoked cigarettes and were aged $<18$ years, $1.0 \%$ had usually obtained their own cigarettes by buying them on the Internet during the 30 days before the survey (Table 35). Because the response option "I got them on the Internet" was included for the first time in 2015 in the question on usual source of cigarettes, long-term temporal trends and 2-year temporal changes are not available.

Across 28 states, the prevalence of usually obtaining their own cigarettes by buying them on the Internet ranged from $0.0 \%$ to $6.0 \%$ (median: $1.1 \%$ ) (Table 36). Across 5 large urban school districts, the prevalence ranged from $1.7 \%$ to 5.9\% (median: $2.4 \%$ ).

\section{Tried to Quit Smoking Cigarettes}

Among the $10.8 \%$ of students nationwide who currently smoked cigarettes, $45.4 \%$ had tried to quit smoking cigarettes during the 12 months before the survey (Table 37). The prevalence of having tried to quit smoking cigarettes was higher among female $(52.8 \%)$ than male $(39.7 \%)$ students; higher among white female $(51.0 \%)$ than white male $(37.9 \%)$ students; and higher among 11th-grade female (52.2\%) and 12th-grade female $(54.1 \%)$ than 11 th-grade male $(29.9 \%)$ and 12th-grade male (42.0\%) students, respectively. The prevalence of having tried to quit smoking cigarettes was higher among 9th-grade $(47.8 \%)$, 10th-grade $(51.6 \%)$, and 12th-grade (47.7\%) than 11th-grade (37.9\%) students and higher among 9th-grade male (48.2\%) and 12th-grade male (42.0\%) than 11th-grade male $(29.9 \%)$ students.

During 2001-2015, a significant linear decrease occurred overall in the prevalence of having tried to quit smoking cigarettes $(57.4 \%-45.4 \%)$. A significant quadratic trend was not identified. The prevalence of having tried to quit smoking cigarettes did not change significantly from 2013 (48.0\%) to 2015 (45.4\%).

Across 29 states, the prevalence of having tried to quit smoking cigarettes ranged from $35.2 \%$ to $59.5 \%$ (median: 49.2\%) (Table 38). Across 9 large urban school districts, the prevalence ranged from $35.7 \%$ to $81.8 \%$ (median: $46.9 \%$ ).

\section{Current Smokeless Tobacco Use}

Nationwide, $7.3 \%$ of students had used smokeless tobacco (e.g., chewing tobacco, snuff, or dip) on at least 1 day during the 30 days before the survey (i.e., current smokeless tobacco use) (Table 39). The prevalence of current smokeless tobacco use was higher among male $(11.9 \%)$ than female $(2.3 \%)$ students; higher among white male (15.9\%), black male (5.6\%), and Hispanic male (6.4\%) than white female (2.5\%), black female (1.1\%), and Hispanic female $(2.5 \%)$ students, respectively; and higher among 9th-grade male (8.8\%), 10th-grade male (10.6\%), 11th-grade male (15.0\%), and 12th-grade male
$(13.1 \%)$ than 9th-grade female $(2.4 \%), 10$ th-grade female $(2.0 \%), 11$ th-grade female $(2.9 \%)$, and 12 th-grade female $(1.7 \%)$ students, respectively. The prevalence of current smokeless tobacco use was higher among white $(9.3 \%)$ than black $(3.7 \%)$ and Hispanic (4.5\%) students, higher among white female $(2.5 \%)$ and Hispanic female $(2.5 \%)$ than black female $(1.1 \%)$ students, and higher among white male (15.9\%) than black male (5.6\%) and Hispanic male (6.4\%) students. The prevalence of current smokeless tobacco use was higher among 11 th-grade $(9.3 \%)$ than 9 th-grade $(5.9 \%)$ and 10th-grade $(6.3 \%)$ students, higher among 11 th-grade male $(15.0 \%)$ and 12 th-grade male $(13.1 \%)$ than 9th-grade male $(8.8 \%)$ students, and higher among 11 th-grade male $(15.0 \%)$ than 10th-grade male (10.6\%) students.

During 1995-2015, a significant linear trend was not identified in the prevalence of current smokeless tobacco use. A significant quadratic trend was identified. The prevalence of current smokeless tobacco use decreased during 1995-1999 $(11.4 \%-7.8 \%)$ and then did not change significantly during 1999-2015 (7.8\%-7.3\%). The prevalence of current smokeless tobacco use also did not change significantly from 2013 (8.8\%) to $2015(7.3 \%)$.

Across 34 states, the prevalence of current smokeless tobacco use ranged from $3.0 \%$ to $13.4 \%$ (median: $8.6 \%$ ) (Table 40 ). Across 15 large urban school districts, the prevalence ranged from $2.4 \%$ to $7.8 \%$ (median: $3.7 \%$ ).

\section{Current Cigar Use}

Nationwide, $10.3 \%$ of students had smoked cigars, cigarillos, or little cigars on at least 1 day during the 30 days before the survey (i.e., current cigar use) (Table 39). The prevalence of current cigar use was higher among male (14.0\%) than female $(6.3 \%)$ students; higher among white male (14.8\%), black male $(12.9 \%)$, and Hispanic male $(12.4 \%)$ than white female (6.0\%), black female (8.5\%), and Hispanic female $(6.5 \%)$ students, respectively; and higher among 9th-grade male $(8.5 \%)$, 10th-grade male $(12.5 \%), 11$ th-grade male $(15.1 \%)$, and 12 th-grade male $(20.4 \%)$ than 9 th-grade female (4.1\%), 10th-grade female (6.6\%), 11 th-grade female (6.3\%), and 12 th-grade female $(8.1 \%)$ students, respectively. The prevalence of current cigar use was higher among 10th-grade (9.6\%), 11th-grade (11.0\%), and 12th-grade (14.3\%) than 9th-grade $(6.6 \%)$ students; higher among 12 th-grade $(14.3 \%)$ than 10th-grade $(9.6 \%)$ students; higher among 10th-grade female $(6.6 \%), 11$ th-grade female $(6.3 \%)$, and 12 th-grade female $(8.1 \%)$ than 9 th-grade female $(4.1 \%)$ students; higher among 10th-grade male (12.5\%), 11th-grade male (15.1\%), and 12th-grade male $(20.4 \%)$ than 9th-grade male $(8.5 \%)$ students; and higher among 12th-grade male (20.4\%) than 10th-grade male $(12.5 \%)$ students. 
During 1997-2015, a significant linear decrease occurred overall in the prevalence of current cigar use (22.0\%-10.3\%). A significant quadratic trend was not identified. The prevalence of current cigar use decreased significantly from 2013 (12.6\%) to $2015(10.3 \%)$.

Across 33 states, the prevalence of current cigar use ranged from $6.8 \%$ to $16.5 \%$ (median: $10.4 \%$ ) (Table 40 ). Across 16 large urban school districts, the prevalence ranged from $4.4 \%$ to $14.9 \%$ (median: $9.1 \%$ ).

\section{Ever Used Electronic Vapor Products}

Nationwide, $44.9 \%$ of students had ever used electronic vapor products (including e-cigarettes, e-cigars, e-pipes, vape pipes, vaping pens, e-hookahs, and hookah pens) (i.e, ever used electronic vapor products) (Table 41). The prevalence of having ever used electronic vapor products was higher among 10th-grade male $(45.3 \%)$ than 10th-grade female $(41.2 \%)$ students. The prevalence of having ever used electronic vapor products was higher among Hispanic $(51.9 \%)$ than white (43.2\%) and black (42.4\%) students, higher among Hispanic female $(51.2 \%)$ than white female $(42.3 \%)$ and black female (37.7\%) students, and higher among Hispanic male (52.6\%) than white male $(44.0 \%)$ students. The prevalence of having ever used electronic vapor products was higher among 10 th-grade $(43.3 \%), 11$ th-grade $(49.5 \%)$, and 12 th-grade $(50.9 \%)$ than 9th-grade $(37.2 \%)$ students; higher among 11 th-grade $(49.5 \%)$ and 12 th-grade $(50.9 \%)$ than 10 th-grade (43.3\%) students; higher among 11th-grade female (47.8\%) and 12 th-grade female $(49.0 \%)$ than 9 th-grade female $(37.4 \%)$ and 10th-grade female $(41.2 \%)$ students; higher among 10th-grade male (45.3\%), 11th-grade male $(51.1 \%)$, and 12 th-grade male $(52.6 \%)$ than 9th-grade male $(37.0 \%)$ students; and higher among 11th-grade male (51.1\%) than 10 th-grade male $(45.3 \%)$ students. Because the question measuring the prevalence of having ever used electronic vapor products was used for the first time in the 2015 national YRBS, long-term temporal trends and 2-year temporal changes are not available.

Across 31 states, the prevalence of having ever used electronic vapor products ranged from $30.4 \%$ to $54.4 \%$ (median: 42.9\%) (Table 42). Across 16 large urban school districts, the prevalence ranged from $32.2 \%$ to $49.9 \%$ (median: $39.9 \%$ ).

\section{Current Electronic Vapor Products Use}

Nationwide, $24.1 \%$ of students had used electronic vapor products (including e-cigarettes, e-cigars, e-pipes, vape pipes, vaping pens, e-hookahs, and hookah pens) on at least 1 day during the 30 days before the survey (i.e., current electronic vapor product use) (Table 41). The prevalence of current electronic vapor product use was higher among male (25.6\%) than female (22.6\%) students, higher among black male $(21.2 \%)$ than black female $(14.5 \%)$ students, and higher among 12th-grade male $(31.9 \%)$ than 12 th-grade female $(24.6 \%)$ students. The prevalence of current electronic vapor product use was higher among white $(25.2 \%)$ and Hispanic $(26.3 \%)$ than black (18.0\%) students, higher among white female $(24.2 \%)$ and Hispanic female $(25.0 \%)$ than black female (14.5\%) students, and higher among Hispanic male $(27.4 \%)$ than black male $(21.2 \%)$ students. The prevalence of current electronic vapor product use was higher among 11 th-grade $(25.9 \%)$ than 9 th-grade $(19.7 \%)$ students; higher among 12th-grade (28.2\%) than 9th-grade (19.7\%), 10 th-grade $(23.2 \%)$, and 11 th-grade $(25.9 \%)$ students; higher among 11 th-grade female $(24.1 \%)$ and 12 th-grade female $(24.6 \%)$ than 9 th-grade female $(19.8 \%)$ students; higher among 10th-grade male (24.2\%), 11th-grade male (27.4\%), and 12th-grade male $(31.9 \%)$ than 9th-grade male $(19.6 \%)$ students; and higher among 12th-grade male $(31.9 \%)$ than 10 th-grade male $(24.2 \%)$ and 11 th-grade male $(27.4 \%)$ students. Because the question measuring the prevalence of current electronic vapor product use was used for the first time in the 2015 national YRBS, long-term temporal trends and 2-year temporal changes are not available.

Across 35 states, the prevalence of current electronic vapor product use ranged from $15.3 \%$ to $31.2 \%$ (median: 23.5\%) (Table 42). Across 19 large urban school districts, the prevalence ranged from $13.0 \%$ to $27.4 \%$ (median: $17.6 \%$ ).

\section{Current Cigarette or Cigar Use}

Nationwide, $16.0 \%$ of students reported current cigarette or cigar use (Table 43). The prevalence of current cigarette or cigar use was higher among male $(19.0 \%)$ than female $(12.7 \%)$ students; higher among white male (20.4\%), black male $(16.8 \%)$, and Hispanic male $(16.5 \%)$ than white female (14.7\%), black female (10.1\%), and Hispanic female $(9.7 \%)$ students, respectively; and higher among 9th-grade male $(12.3 \%)$, 11th-grade male $(22.9 \%)$, and 12 th-grade male $(25.9 \%)$ than 9 th-grade female $(8.8 \%), 11$ th-grade female $(13.0 \%)$, and 12 th-grade female $(17.2 \%)$ students, respectively. The prevalence of current cigarette or cigar use was higher among white (17.5\%) than black (13.9\%) and Hispanic $(13.1 \%)$ students and higher among white female $(14.7 \%)$ than black female (10.1\%) and Hispanic female (9.7\%) students. The prevalence of current cigarette or cigar use was higher among 11 th-grade (18.3\%) and 12th-grade (21.6\%) than 9th-grade $(10.7 \%)$ and 10th-grade $(13.9 \%)$ students; higher among 11th-grade female $(13.0 \%)$ than 9th-grade female $(8.8 \%)$ students; higher among 12th-grade female $(17.2 \%)$ than 9 th-grade female $(8.8 \%)$, 10th-grade female $(12.2 \%)$, and 11th-grade female $(13.0 \%)$ students; 
and higher among 11 th-grade male $(22.9 \%)$ and 12th-grade male $(25.9 \%)$ than 9 th-grade male $(12.3 \%)$ and 10 th-grade male (15.6\%) students.

During 1997-2015, a significant linear decrease occurred overall in the prevalence of current cigarette or cigar use $(42.6 \%-16.0 \%)$. A significant quadratic trend was not identified. The prevalence of current cigarette or cigar use decreased significantly from $2013(21.0 \%)$ to 2015 (16.0\%).

Across 33 states, the prevalence of current cigarette or cigar use ranged from $10.7 \%$ to $24.8 \%$ (median: $15.7 \%$ ) (Table 44). Across 15 large urban school districts, the prevalence ranged from $6.8 \%$ to $18.1 \%$ (median: $12.0 \%$ ).

\section{Current Cigarette, Cigar, or Smokeless Tobacco Use}

Nationwide, $18.5 \%$ of students reported current cigarette, cigar, or smokeless tobacco use (Table 43). The prevalence of current cigarette, cigar, or smokeless tobacco use was higher among male (23.3\%) than female (13.4\%) students; higher among white male $(26.7 \%)$, black male $(18.6 \%)$, and Hispanic male $(18.1 \%)$ than white female $(15.6 \%)$, black female $(10.3 \%)$, and Hispanic female (10.3\%) students, respectively; and higher among 9th-grade male (15.8\%), 10 th-grade male $(19.6 \%)$, 11th-grade male $(28.8 \%)$, and 12th-grade male $(29.9 \%)$ than 9 th-grade female $(9.3 \%)$, 10th-grade female $(12.6 \%), 11$ th-grade female $(14.2 \%)$, and 12 th-grade female $(17.9 \%)$ students, respectively. The prevalence of current cigarette, cigar, or smokeless tobacco use was higher among white (21.2\%) than black (14.9\%) and Hispanic (14.2\%) students, higher among white female $(15.6 \%)$ than black female $(10.3 \%)$ and Hispanic female $(10.3 \%)$ students, and higher among white male $(26.7 \%)$ than black male (18.6\%) and Hispanic male (18.1\%) students. The prevalence of current cigarette, cigar, or smokeless tobacco use was higher among 11 th-grade $(21.9 \%)$ and 12 th-grade $(23.9 \%)$ than 9th-grade $(12.8 \%)$ and 10th-grade $(16.1 \%)$ students; higher among 11th-grade female (14.2\%) than 9th-grade female $(9.3 \%)$ students; higher among 12 th-grade female $(17.9 \%)$ than 9th-grade female $(9.3 \%)$, 10th-grade female $(12.6 \%)$, and 11 th-grade female $(14.2 \%)$ students; and higher among 11 th-grade male $(28.8 \%)$ and 12 th-grade male $(29.9 \%)$ than 9 th-grade male $(15.8 \%)$ and 10 th-grade male (19.6\%) students.

During 1997-2015, a significant linear decrease occurred in the prevalence of current cigarette, cigar, or smokeless tobacco use $(44.5 \%-18.5 \%)$. A significant quadratic trend was not identified. The prevalence of current cigarette, cigar, or smokeless tobacco use decreased significantly from 2013 $(24.0 \%)$ to $2015(18.5 \%)$.
Across 33 states, the prevalence of current cigarette, cigar, or smokeless tobacco use ranged from $11.9 \%$ to 29.2\% (median: 19.7\%) (Table 44). Across 15 large urban school districts, the prevalence ranged from $7.5 \%$ to $20.4 \%$ (median: 12.8\%).

\section{Current Cigarette, Cigar, Smokeless Tobacco, or Electronic Vapor Product Use}

Nationwide, $31.4 \%$ of students reported current cigarette, cigar, smokeless tobacco, or electronic vapor product use (Table 45). The prevalence of current cigarette, cigar, smokeless tobacco, or electronic vapor product use was higher among male $(34.9 \%)$ than female $(27.7 \%)$ students; higher among white male (36.6\%), black male (30.6\%), and Hispanic male (34.2\%) than white female $(29.4 \%)$, black female $(21.2 \%)$, and Hispanic female (29.4\%) students, respectively; and higher among 9th-grade male (26.9\%), 10 th-grade male $(31.7 \%), 11$ th-grade male $(39.4 \%)$, and 12th-grade male $(43.0 \%)$ than 9 th-grade female $(23.0 \%)$, 10 th-grade female $(26.3 \%), 11$ th-grade female $(30.0 \%)$, and 12 th-grade female (32.2\%) students, respectively. The prevalence of current cigarette, cigar, smokeless tobacco, or electronic vapor product use was higher among white (32.9\%) and Hispanic (31.8\%) than black (26.3\%) students, higher among white female (29.4\%) and Hispanic female (29.4\%) than black female $(21.2 \%)$ students, and higher among white male $(36.6 \%)$ than black male $(30.6 \%)$ students. The prevalence of current cigarette, cigar, smokeless tobacco, or electronic vapor product use was higher among 11 th-grade (34.9\%) and 12th-grade (37.5\%) than 9th-grade (25.1\%) and 10th-grade (28.9\%) students, higher among 11th-grade female $(30.0 \%)$ and 12 th-grade female $(32.2 \%)$ than 9th-grade female (23.0\%) students, higher among 12th-grade female $(32.2 \%)$ than 10 th-grade female $(26.3 \%)$ students, and higher among 11 th-grade male (39.4\%) and 12th-grade male $(43.0 \%)$ than 9 th-grade male $(26.9 \%)$ and 10 th-grade male $(31.7 \%)$ students. Because the question measuring the prevalence of current electronic vapor product use was used for the first time in the 2015 national YRBS, long-term temporal trends and 2-year temporal changes are not available for current cigarette, cigar, smokeless tobacco, or electronic vapor product use.

Across 33 states, the prevalence of current cigarette, cigar, smokeless tobacco, or electronic vapor product use ranged from $22.7 \%$ to $40.8 \%$ (median: $30.9 \%$ ) (Table 46). Across 15 large urban school districts, the prevalence ranged from $19.9 \%$ to 34.3\% (median: 25.5\%). 


\section{Alcohol and Other Drug Use}

\section{Ever Drank Alcohol}

Nationwide, $63.2 \%$ of students had had at least one drink of alcohol on at least 1 day during their life (i.e., ever drank alcohol) (Table 47). The prevalence of having ever drunk alcohol was higher among female (65.3\%) than male (61.4\%) students; higher among black female (57.9\%) and Hispanic female $(68.6 \%)$ than black male $(51.0 \%)$ and Hispanic male (63.4\%) students, respectively; and higher among 9th-grade female $(53.0 \%)$ than 9 th-grade male $(48.9 \%)$ students. The prevalence of having ever drunk alcohol was higher among white (65.3\%) and Hispanic (65.9\%) than black (54.4\%) students, higher among white female $(66.7 \%)$ and Hispanic female $(68.6 \%)$ than black female $(57.9 \%)$ students, and higher among white male (64.0\%) and Hispanic male (63.4\%) than black male $(51.0 \%)$ students. The prevalence of having ever drunk alcohol was higher among 10th-grade (60.8\%), 11 th-grade $(70.3 \%)$, and 12 th-grade $(73.3 \%)$ than 9 th-grade $(50.8 \%)$ students; higher among 11 th-grade $(70.3 \%)$ and 12 th-grade $(73.3 \%)$ than 10 th-grade $(60.8 \%)$ students; higher among 11th-grade female $(72.1 \%)$ and 12 th-grade female $(75.2 \%)$ than 9 th-grade female $(53.0 \%)$ and 10 th-grade female $(62.7 \%)$ students; higher among 10th-grade male (58.8\%), 11 th-grade male $(68.7 \%)$, and 12th-grade male $(71.5 \%)$ than 9th-grade male (48.9\%) students; and higher among 11 th-grade male $(68.7 \%)$ and 12 th-grade male $(71.5 \%)$ than 10 th-grade male $(58.8 \%)$ students.

During 1991-2015, a significant linear decrease occurred overall in the prevalence having ever drunk alcohol $(81.6 \%$ $63.2 \%)$. A significant quadratic trend also was identified. The prevalence of having ever drunk alcohol decreased during 1991-2007 (81.6\%-75.0\%) and then decreased more rapidly during 2007-2015 (75.0\%-63.2\%). The prevalence of having ever drunk alcohol did not change significantly from 2013 $(66.2 \%)$ to $2015(63.2 \%)$.

Across 26 states, the prevalence of having ever drunk alcohol ranged from 50.7\% to 69.9\% (median:59.8\%) (Table 48). Across 17 large urban school districts, the prevalence ranged from $43.5 \%$ to $62.8 \%$ (median: $56.1 \%$ ).

\section{Drank Alcohol Before Age 13 Years}

Nationwide, $17.2 \%$ of students had drunk alcohol (other than a few sips) for the first time before age 13 years (Table 47). The prevalence of having drunk alcohol for the first time before age 13 years was higher among male (19.7\%) than female $(14.6 \%)$ students; higher among white male $(17.3 \%)$ and Hispanic male $(23.6 \%)$ than white female $(11.7 \%)$ and Hispanic female (19.0\%) students, respectively; and higher among 10th-grade male (21.3\%), 11th-grade male (17.5\%), and 12th-grade male (17.0\%) than 10th-grade female (15.8\%), 11th-grade female $(12.9 \%)$, and 12th-grade female $(9.9 \%)$ students, respectively. The prevalence of having drunk alcohol for the first time before age 13 years was higher among black (18.0\%) and Hispanic (21.3\%) than white (14.5\%) students, higher among Hispanic (21.3\%) than black (18.0\%) students, higher among black female $(16.9 \%)$ and Hispanic female $(19.0 \%)$ than white female $(11.7 \%)$ students, and higher among Hispanic male $(23.6 \%)$ than white male $(17.3 \%)$ and black male (18.7\%) students. The prevalence of having drunk alcohol for the first time before age 13 years was higher among 9th-grade $(20.3 \%)$ and 10 th-grade $(18.6 \%)$ than 11 th-grade $(15.2 \%)$ and 12 th-grade (13.5\%) students, higher among 9th-grade female $(18.8 \%)$ than 11th-grade female (12.9\%) and 12th-grade female $(9.9 \%)$ students, higher among 10 th-grade female $(15.8 \%)$ than 12 th-grade female $(9.9 \%)$ students, and higher among 9 th-grade male $(21.5 \%)$ than 12 th-grade male (17.0\%) students.

During 1991-2015, a significant linear decrease occurred overall in the prevalence of having drunk alcohol for the first time before age 13 years $(32.7 \%-17.2 \%)$. A significant quadratic trend also was identified. The prevalence of having drunk alcohol for the first time before age 13 years did not change significantly during 1991-1999 (32.7\%-32.2\%) and then decreased during 1999-2015 (32.2\%-17.2\%). The prevalence of having drunk alcohol for the first time before age 13 years did not change significantly from 2013 (18.6\%) to $2015(17.2 \%)$.

Across 36 states, the prevalence of having drunk alcohol for the first time before age 13 years ranged from $10.6 \%$ to 24.5\% (median: 15.7\%) (Table 48). Across 19 large urban school districts, the prevalence ranged from $14.8 \%$ to $23.6 \%$ (median: 18.1\%).

\section{Current Alcohol Use}

Nationwide, $32.8 \%$ of students had had at least one drink of alcohol on at least 1 day during the 30 days before the survey (i.e., current alcohol use) (Table 49). The prevalence of current alcohol use was higher among white (35.2\%) and Hispanic (34.4\%) than black $(23.8 \%)$ students, higher among white female (35.3\%) and Hispanic female (35.6\%) than black female $(25.9 \%)$ students, and higher among white male (35.2\%) and Hispanic male (33.4\%) than black male (22.1\%) students. The prevalence of current alcohol use was higher among 11th-grade (38.0\%) and 12th-grade (42.4\%) than 9th-grade $(23.4 \%)$ and 10th-grade $(29.0 \%)$ students, higher among 12 th-grade $(42.4 \%)$ than 11 th-grade $(38.0 \%)$ students, higher among 11th-grade female $(38.3 \%)$ and 12th-grade female $(43.2 \%)$ than 9 th-grade female $(24.9 \%)$ and 10th-grade female $(28.8 \%)$ students; higher among 12 th-grade 
female (43.2\%) than 11th-grade female (38.3\%) students, and higher among 11 th-grade male $(37.7 \%)$ and 12 th-grade male (41.6\%) than 9th-grade male (22.1\%) students and 10th-grade male $(29.3 \%)$ students.

During 1991-2015, a significant linear decrease occurred overall in the prevalence of current alcohol use $(50.8 \%-$ $32.8 \%)$. A significant quadratic trend also was identified. The prevalence of current alcohol use decreased during 1991-2007 (50.8\%-44.7\%) and then decreased more rapidly during 2007-2015 (44.7\%-32.8\%). The prevalence of current alcohol use did not change significantly from 2013 $(34.9 \%)$ to 2015 (32.8\%).

Across 36 states, the prevalence of current alcohol use ranged from $22.0 \%$ to $34.8 \%$ (median: $29.8 \%$ ) (Table 50 ). Across 19 large urban school districts, the prevalence ranged from $18.4 \%$ to $38.2 \%$ (median: $24.8 \%$ ).

\section{Someone Gave Alcohol to Them}

Among the $32.8 \%$ of students nationwide who currently drank alcohol, $44.1 \%$ had usually obtained the alcohol they drank by someone giving it to them during the 30 days before the survey (Table 49). The prevalence of usually obtaining the alcohol they drank by someone giving it to them was higher among female $(48.5 \%)$ than male $(39.9 \%)$ students; higher among white female (50.7\%) and Hispanic female (45.9\%) than white male (41.6\%) and Hispanic male (36.5\%) students, respectively; and higher among 9th-grade female (50.4\%), 10th-grade female $(50.0 \%)$, and 11th-grade female $(49.7 \%)$ than 9th-grade male (39.4\%), 10th-grade male (34.5\%), and 11 th-grade male $(39.3 \%)$ students, respectively. The prevalence of usually obtaining the alcohol they drank by someone giving it to them was higher among white (46.1\%) than Hispanic (41.3\%) students.

During 2007-2015, significant linear and quadratic trends were not identified in the prevalence of usually obtaining the alcohol they drank by someone giving it to them. The prevalence of usually obtaining the alcohol they drank by someone giving it to them did not change significantly from $2013(41.8 \%)$ to 2015 (44.1\%).

Across 32 states, the prevalence of usually obtaining the alcohol they drank by someone giving it to them ranged from $32.2 \%$ to $45.3 \%$ (median: $39.8 \%$ ) (Table 44 ). Across 14 large urban school districts, the prevalence ranged from $26.9 \%$ to 44.8\% (median: 37.9\%).

\section{Consumed Five or More Drinks in a Row}

Nationwide, $17.7 \%$ of students had had five or more drinks of alcohol in a row (i.e., within a couple of hours) on at least 1 day during the 30 days before the survey (Table 51). The prevalence of having five or more drinks of alcohol in a row was higher among white (19.7\%) and Hispanic (17.7\%) than black (11.4\%) students, higher among white female (18.6\%) and Hispanic female (17.9\%) than black female (9.9\%) students, and higher among white male (21.0\%) and Hispanic male (17.5\%) than black male (12.8\%) students. The prevalence of having five or more drinks of alcohol in a row was higher among 10th-grade (15.1\%), 11th-grade (22.1\%), and 12 th-grade $(24.6 \%)$ than 9th-grade $(10.4 \%)$ students; higher among 11th-grade (22.1\%) and 12th-grade (24.6\%) than 10th-grade $(15.1 \%)$ students; higher among 10th-grade female (14.1\%), 11th-grade female (19.6\%), and 12th-grade female $(23.8 \%)$ than 9th-grade female $(10.5 \%)$ students; higher among 11th-grade female (19.6\%) and 12th-grade female $(23.8 \%)$ than 10th-grade female $(14.1 \%)$ students; higher among 12th-grade female (23.8\%) than 11th-grade female (19.6\%) students, higher among 10th-grade male $(16.2 \%), 11$ th-grade male $(24.4 \%)$, and 12 th-grade male (25.6\%) than 9th-grade male (10.2\%) students; and higher among 11 th-grade male (24.4\%) and 12th-grade male (25.6\%) than 10th-grade male (16.2\%) students.

During 1991-2015, a significant linear decrease occurred overall in the prevalence of having five or more drinks of alcohol in a row $(31.3 \%-17.7 \%)$. A significant quadratic trend also was identified. The prevalence of having five or more drinks of alcohol in a row increased during 1991-1999 (31.3\%-31.5\%) and then decreased during 1999-2015 (31.5\%-17.7\%). The prevalence of having five or more drinks of alcohol in a row also decreased significantly from 2013 (20.8\%) to 2015 (17.7\%).

Across 36 states, the prevalence of having five or more drinks of alcohol in a row ranged from $11.0 \%$ to $20.7 \%$ (median: 15.5\%) (Table 52). Across 19 large urban school districts, the prevalence ranged from $8.0 \%$ to $16.5 \%$ (median: $10.9 \%$ ).

\section{Largest Number of Drinks in a Row Was 10 or More}

Nationwide, $4.3 \%$ of students reported that the largest number of drinks that they had had in a row (i.e., within a couple of hours) during the 30 days before the survey was 10 or more (Table 51). The prevalence of reporting 10 or more as the largest number of drinks in a row was higher among male $(6.1 \%)$ than female $(2.5 \%)$ students; higher among white male (6.6\%), black male (3.2\%), and Hispanic male (6.5\%) than white female $(2.4 \%)$, black female (1.0\%), and Hispanic female $(3.6 \%)$ students, respectively; and higher among 10th-grade male $(6.3 \%), 11$ th-grade male $(7.3 \%)$, and 12 th-grade male $(8.8 \%)$ than 10 th-grade female $(2.2 \%), 11$ th-grade female $(2.5 \%)$, and 12 th-grade female $(3.0 \%)$ students, respectively. The prevalence of reporting 10 or more as the largest number of drinks in a row was higher among white $(4.5 \%)$ and Hispanic (5.1\%) than black (2.1\%) students, higher among white female (2.4\%) and Hispanic female (3.6\%) than black female (1.0\%) 
students, and higher among white male (6.6\%) and Hispanic male $(6.5 \%)$ than black male $(3.2 \%)$ students. The prevalence of reporting 10 or more as the largest number of drinks in a row was higher among 10 th-grade $(4.2 \%), 11$ th-grade $(5.0 \%)$, and 12 th-grade $(5.9 \%)$ than 9 th-grade $(2.4 \%)$ students and higher among 10th-grade male (6.3\%), 11th-grade male (7.3\%), and 12th-grade male $(8.8 \%)$ than 9th-grade male $(2.4 \%)$ students.

Because the question measuring the reporting of 10 or more as the largest number of drinks in a row was used for the first time in the 2013 national YRBS, long-term temporal trends are not available. The prevalence of reporting 10 or more as the largest number of drinks in a row decreased significantly from 2013 (6.1\%) to 2015 (4.3\%).

Across 20 states, the prevalence of reporting 10 or more as the largest number of drinks in a row ranged from $2.1 \%$ to $7.4 \%$ (median: $3.5 \%$ ) (Table 52). Across 13 large urban school districts, the prevalence ranged from $0.7 \%$ to $3.4 \%$ (median: 2.2\%).

\section{Ever Used Marijuana}

Nationwide, $38.6 \%$ of students had used marijuana one or more times during their life (i.e., ever used marijuana) (Table 53). The prevalence of having ever used marijuana was higher among black male (49.7\%) than black female (40.5\%) students. The prevalence of having ever used marijuana was higher among black (45.5\%) and Hispanic (45.6\%) than white (35.2\%) students, higher among black female (40.5\%) and Hispanic female (45.3\%) than white female (34.3\%) students, and higher among black male (49.7\%) and Hispanic male (46.0\%) than white male (36.2\%) students. The prevalence of having ever used marijuana was higher among 10th-grade (35.5\%), 11 th-grade $(45.2 \%)$, and 12 th-grade $(49.8 \%)$ than 9th-grade (25.9\%) students; higher among 11th-grade (45.2\%) and 12 th-grade $(49.8 \%)$ than 10 th-grade $(35.5 \%)$ students; higher among 12th-grade (49.8\%) than 11th-grade $(45.2 \%)$ students; higher among 10th-grade female (33.8\%), 11th-grade female (43.6\%), and 12th-grade female (48.8\%) than 9th-grade female $(25.3 \%)$ students; higher among 11th-grade female (43.6\%) and 12th-grade female (48.8\%) than 10th-grade female $(33.8 \%)$ students; higher among 12 th-grade female $(48.8 \%)$ than 11 th-grade female $(43.6 \%)$ students; higher among 10th-grade male $(37.1 \%), 11$ th-grade male $(46.9 \%)$, and 12 th-grade male $(50.9 \%)$ than 9th-grade male $(26.5 \%)$ students; and higher among 11th-grade male $(46.9 \%)$ and 12 th-grade male $(50.9 \%)$ than 10 th-grade male (37.1\%) students.

During 1991-2015, a significant linear trend was not identified in the prevalence of having ever used marijuana (31.3\%-38.6\%). A significant quadratic trend was identified. The prevalence of having ever used marijuana increased during 1991-1997 (31.3\%-47.1\%) and then decreased during 1997-2015 (47.1\%-38.6\%). The prevalence of having ever used marijuana did not change significantly from 2013 (40.7\%) to 2015 (38.6\%).

Across 29 states, the prevalence of having ever used marijuana ranged from $25.0 \%$ to $42.9 \%$ (median: $34.7 \%$ ) (Table 54). Across 15 large urban school districts, the prevalence ranged from $28.7 \%$ to $45.8 \%$ (median: $40.1 \%$ ).

\section{Tried Marijuana Before Age 13 Years}

Nationwide, $7.5 \%$ of students had tried marijuana for the first time before age 13 years (Table 53). The prevalence of having tried marijuana before age 13 years was higher among male $(9.2 \%)$ than female $(5.6 \%)$ students; higher among white male $(6.7 \%)$, black male $(13.0 \%)$, and Hispanic male $(13.6 \%)$ than white female (4.2\%), black female (7.4\%), and Hispanic female (8.2\%) students, respectively; and higher among 11th-grade male (8.9\%) and 12th-grade male (8.5\%) than 11 th-grade female (4.5\%) and 12th-grade female $(3.7 \%)$ students, respectively. The prevalence of having tried marijuana before age 13 years was higher among black (10.6\%) and Hispanic (10.9\%) than white (5.4\%) students, higher among black female (7.4\%) and Hispanic female (8.2\%) than white female (4.2\%) students, and higher among black male (13.0\%) and Hispanic male (13.6\%) than white male (6.7\%) students. The prevalence of having tried marijuana before age 13 years was higher among 9th-grade (8.5\%) and 10th-grade $(8.3 \%)$ than 12 th-grade $(6.1 \%)$ students and higher among 9 th-grade female $(6.8 \%)$ and 10 th-grade female $(7.2 \%)$ than 11 th-grade female $(4.5 \%)$ and 12 th-grade female (3.7\%) students.

During 1991-2015, a significant linear decrease occurred overall in the prevalence of having tried marijuana before age 13 years $(7.4 \%-7.5 \%)$. ${ }^{* *}$ A significant quadratic trend also was identified. The prevalence of having tried marijuana before age 13 years increased during $1991-1999(7.4 \%-11.3 \%)$ and then decreased during 1999-2015 (11.3\%-7.5\%). The prevalence of having tried marijuana before age 13 years did not change significantly from 2013 (8.6\%) to 2015 (7.5\%).

Across 35 states, the prevalence of having tried marijuana before age 13 years ranged from $5.3 \%$ to $16.5 \%$ (median: 7.2\%) (Table 54). Across 18 large urban school districts, the prevalence ranged from $5.7 \%$ to $15.7 \%$ (median: $8.9 \%$ ).

\section{Current Marijuana Use}

Nationwide, $21.7 \%$ of students had used marijuana one or more times during the 30 days before the survey (i.e., current marijuana use) (Table 55). The prevalence of current marijuana use was higher among male $(23.2 \%)$ than female $(20.1 \%)$ students, higher among black male $(31.3 \%)$ than black female $(22.1 \%)$ students, and higher among 10th-grade 
male $(22.6 \%)$ than 10 th-grade female $(17.5 \%)$ students. The prevalence of current marijuana use was higher among black (27.1\%) and Hispanic (24.5\%) than white (19.9\%) students and higher among black male $(31.3 \%)$ than white male $(21.2 \%)$ and Hispanic male (25.5\%) students. The prevalence of current marijuana use was higher among 10th-grade (20.0\%), 11 th-grade $(24.8 \%)$, and 12 th-grade $(27.6 \%)$ than 9 th-grade (15.2\%) students; higher among 11th-grade (24.8\%) and 12th-grade (27.6\%) than 10th-grade (20.0\%) students; higher among 11th-grade female (24.3\%) and 12th-grade female $(24.6 \%)$ than 9 th-grade female $(14.7 \%)$ and 10th-grade female (17.5\%) students; higher among 10th-grade male (22.6\%), 11 th-grade male $(25.5 \%)$, and 12 th-grade male $(30.5 \%)$ than 9th-grade male (15.5\%) students; and higher among 12th-grade male (30.5\%) than 10th-grade male (22.6\%) students.

During 1991-2015, a significant linear trend was not identified in the prevalence of current marijuana use $(14.7 \%$ $21.7 \%)$. A significant quadratic trend was identified. The prevalence of current marijuana use increased during 19911995 (14.7\%-25.3\%) and then decreased during 1995-2015 (25.3\%-21.7\%). The prevalence of current marijuana use did not change significantly from 2013 (23.4\%) to 2015 (21.7\%).

Across 36 states, the prevalence of current marijuana use ranged from $12.4 \%$ to $25.3 \%$ (median: $19.1 \%$ ) (Table 56). Across 19 large urban school districts, the prevalence ranged from $15.9 \%$ to $28.7 \%$ (median: $22.2 \%$ ).

\section{Usually Used Marijuana by Smoking It}

Among the $21.7 \%$ of students who currently used marijuana, $90.0 \%$ usually used marijuana by smoking it in a joint, bong, pipe, or blunt during the 30 days before the survey (Table 55). The prevalence of usually using marijuana by smoking it was higher among female $(92.5 \%)$ than male $(89.0 \%)$ students and higher among 12 th-grade female $(96.7 \%)$ than 12 th-grade male $(87.4 \%)$ students. The prevalence of usually using marijuana by smoking it was higher among 12 th-grade female (96.7\%) than 10th-grade female (91.4\%) students. Because the question measuring the prevalence of usually using marijuana by smoking it in a joint, bong, pipe, or blunt was used for the first time in the 2015 national YRBS, long-term temporal trends and 2-year temporal changes are not available.

Also, this question was not included in the standard questionnaire used in the state and large urban school district surveys in 2015. Consequently, the range and median prevalence estimates across states and large urban school districts for the prevalence of usually using marijuana by smoking it are not available.

\section{Ever Used Synthetic Marijuana}

Nationwide, $9.2 \%$ of students had used synthetic marijuana (also called "K2," "Spice," "fake weed," "King Kong," "Yucatan Fire," "Skunk," or "Moon Rocks") one or more times during their life (i.e., ever used synthetic marijuana) (Table 57). The prevalence of having ever used synthetic marijuana was higher among male $(10.3 \%)$ than female $(7.9 \%)$ students; higher among white male $(9.9 \%)$ and Hispanic male (12.9\%) than white female (7.6\%) and Hispanic female (10.3\%) students, respectively; and higher among 11 th-grade male $(11.1 \%)$ and 12 th-grade male $(14.2 \%)$ than 11 th-grade female $(8.8 \%)$ and 12 th-grade female $(7.6 \%)$ students, respectively. The prevalence of having ever used synthetic marijuana was higher among Hispanic (11.7\%) than white $(8.8 \%)$ and black (7.8\%) students, higher among Hispanic female (10.3\%) than black female $(5.9 \%)$ students, and higher among Hispanic male (12.9\%) than white male $(9.9 \%)$ and black male $(8.9 \%)$ students. The prevalence of having ever used synthetic marijuana was higher among 11 th-grade $(10.0 \%)$ and 12 th-grade $(11.0 \%)$ than 9 th-grade $(7.1 \%)$ students; higher among 10th-grade male (9.7\%), 11th-grade male (11.1\%), and 12th-grade male (14.2\%) than 9th-grade male (6.7\%) students; and higher among 12th-grade male (14.2\%) than 10th-grade male $(9.7 \%)$ students. Because the question measuring the prevalence of having ever used synthetic marijuana was used for the first time in the 2015 national YRBS, long-term temporal trends and 2-year temporal changes are not available.

Across 27 states, the prevalence of having ever used synthetic marijuana ranged from $5.9 \%$ to $14.6 \%$ (median: 9.2\%) (Table 58). Across 17 large urban school districts, the prevalence ranged from $4.9 \%$ to $13.3 \%$ (median: $8.6 \%$ ).

\section{Ever Used Hallucinogenic Drugs}

Nationwide, $6.4 \%$ of students had used hallucinogenic drugs (e.g., LSD, acid, PCP, angel dust, mescaline, or mushrooms) one or more times during their life (i.e., ever used hallucinogenic drugs) (Table 57). The prevalence of having ever used hallucinogenic drugs was higher among male $(8.0 \%)$ than female (4.6\%) students; higher among white male $(8.1 \%)$ and black male $(6.7 \%)$ than white female $(4.7 \%)$ and black female (1.9\%) students, respectively; and higher among 9th-grade male $(5.9 \%)$ and 12 th-grade male $(12.0 \%)$ than 9 th-grade female $(3.1 \%)$ and 12 th-grade female $(3.9 \%)$ students, respectively. The prevalence of having ever used hallucinogenic drugs was higher among Hispanic (6.8\%) than black $(4.7 \%)$ students and higher among white female (4.7\%) and Hispanic female (6.1\%) than black female (1.9\%) students. The prevalence of having 
ever used hallucinogenic drugs was higher among 11th-grade $(6.7 \%)$ and 12 th-grade $(8.0 \%)$ than 9 th-grade $(4.7 \%)$ students; higher among 10 th-grade female $(5.3 \%)$ and 11 th-grade female (5.9\%) than 9th-grade female (3.1\%) students; and higher among 12th-grade male (12.0\%) than 9th-grade male (5.9\%), 10th-grade male $(7.0 \%)$, and 11 th-grade male $(7.0 \%)$ students.

During 2001-2015, a significant linear decrease occurred overall in the prevalence of having ever used hallucinogenic drugs (13.3\%-6.4\%). A significant quadratic trend also was identified. The prevalence of having ever used hallucinogenic drugs decreased during 2001-2005 (13.3\%-8.5\%) and then decreased more gradually during 2005-2015 (8.5\%-6.4\%). The prevalence of having ever used hallucinogenic drugs did not change significantly from 2013 (7.1\%) to 2015 (6.4\%).

This question was not included in the standard questionnaire used in the state and large urban school district surveys in 2015. As a result, the range and median prevalence estimates across states and large urban school districts for the prevalence of having ever used hallucinogenic drugs are not available.

\section{Ever Used Cocaine}

Nationwide, $5.2 \%$ of students had used any form of cocaine (e.g., powder, crack, ${ }^{\dagger \dagger}$ or freebase ${ }^{\$ \$ \$}$ ) one or more times during their life (i.e., ever used cocaine) (Table 59). The prevalence of having ever used cocaine was higher among male (6.3\%) than female (3.8\%) students; higher among white male (5.0\%), black male (5.3\%), and Hispanic male (9.4\%) than white female (3.3\%), black female (1.8\%), and Hispanic female $(6.6 \%)$ students, respectively; and higher among 10th-grade male $(6.4 \%), 11$ th-grade male $(6.2 \%)$, and 12 th-grade male $(9.7 \%)$ than 10 th-grade female $(3.8 \%), 11$ th-grade female $(3.3 \%)$, and 12 th-grade female $(4.5 \%)$ students, respectively. The prevalence of having ever used cocaine was higher among Hispanic (8.0\%) than white (4.1\%) and black (3.8\%) students, higher among Hispanic female (6.6\%) than white female $(3.3 \%)$ and black female (1.8\%) students, and higher among Hispanic male (9.4\%) than white male $(5.0 \%)$ and black male $(5.3 \%)$ students. The prevalence of having ever used cocaine was higher among 10th-grade (5.1\%), 11th-grade (5.0\%), and 12 th-grade $(7.2 \%)$ than 9 th-grade $(3.4 \%)$ students; higher among 12th-grade (7.2\%) than 10th-grade (5.1\%) and 11 th-grade $(5.0 \%)$ students; higher among 10th-grade male $(6.4 \%), 11$ th-grade male $(6.2 \%)$, and 12 th-grade male (9.7\%) than 9th-grade male $(3.3 \%)$ students; and higher among 12 th-grade male $(9.7 \%)$ than 10th-grade male $(6.4 \%)$ and 11 th-grade male $(6.2 \%)$ students.

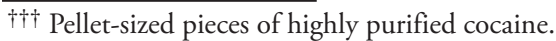

$\$ \$ \$$ A process in which cocaine is dissolved in ether or sodium hydroxide and the precipitate is filtered off.
}

During 1991-2015, a significant linear decrease occurred in the prevalence of having ever used cocaine (5.9\%-5.2\%). A significant quadratic trend also was identified. The prevalence of having ever used cocaine increased during 1991-1999 $(5.9 \%-9.5 \%)$ and then decreased during 1999-2015 (9.5\%$5.2 \%)$. The prevalence of having ever used cocaine did not change significantly from 2013 (5.5\%) to 2015 (5.2\%).

Across 32 states, the prevalence of having ever used cocaine ranged from $3.4 \%$ to $9.2 \%$ (median: $4.8 \%$ ) (Table 60 ). Across 16 large urban school districts, the prevalence ranged from $4.4 \%$ to $9.6 \%$ (median: $5.3 \%$ ).

\section{Ever Used Ecstasy}

Nationwide, $5.0 \%$ of students had used ecstasy (also called "MDMA") one or more times during their life (i.e., ever used ecstasy) (Table 59). The prevalence of having ever used ecstasy was higher among male (6.0\%) than female (3.9\%) students; higher among black male (5.9\%) and Hispanic male (7.8\%) than black female (2.5\%) and Hispanic female (4.1\%) students, respectively; and higher among 10th-grade male $(6.0 \%)$ and 12 th-grade male $(8.0 \%)$ than 10th-grade female (3.7\%) and 12th-grade female (4.0\%) students, respectively. The prevalence of having ever used ecstasy was higher among Hispanic (6.1\%) than white (4.3\%) and black (4.3\%) students and higher among Hispanic male (7.8\%) than white male (4.7\%) students. The prevalence of having ever used ecstasy was higher among 10th-grade (4.9\%), 11th-grade (5.7\%), and 12 th-grade $(6.1 \%)$ than 9 th-grade $(3.2 \%)$ students; higher among 11 th-grade female $(4.8 \%)$ than 9 th-grade female $(2.8 \%)$ students; and higher among 10th-grade male (6.0\%), 11 th-grade male $(6.2 \%)$, and 12 th-grade male $(8.0 \%)$ than 9th-grade male $(3.6 \%)$ students.

During 2001-2015, a significant linear decrease occurred overall in the prevalence of having ever used ecstasy $(11.1 \%-5.0 \%)$. A significant quadratic trend also was identified. The prevalence of having ever used ecstasy decreased during 2001-2005 $(11.1 \%-6.3 \%)$ and then did not change significantly during 2005-2015 (6.3\%-5.0\%). The prevalence of having ever used ecstasy decreased significantly from 2013 (6.6\%) to 2015 (5.0\%).

Across 27 states, the prevalence of having ever used ecstasy ranged from $3.4 \%$ to $8.0 \%$ (median: $5.1 \%$ ) (Table 60 ). Across 14 large urban school districts, the prevalence ranged from $4.2 \%$ to $9.1 \%$ (median: $5.6 \%$ ).

\section{Ever Used Heroin}

Nationwide, $2.1 \%$ of students had used heroin (also called "smack," "junk," or "China White") one or more times during their life (i.e., ever used heroin) (Table 61). The prevalence of having ever used heroin was higher among male $(2.7 \%)$ than 
female $(1.2 \%)$ students; higher among black male $(3.8 \%)$ and Hispanic male (3.2\%) than black female (1.5\%) and Hispanic female $(1.9 \%)$ students, respectively; and higher among 10th-grade male (3.3\%), 11th-grade male $(2.3 \%)$, and 12 th-grade male $(2.8 \%)$ than 10th-grade female $(1.5 \%)$, 11 th-grade female $(0.9 \%)$, and 12 th-grade female $(1.0 \%)$ students, respectively. The prevalence of having ever used heroin was higher among Hispanic (2.6\%) than white (1.3\%) students, higher among Hispanic female (1.9\%) than white female $(0.8 \%)$ students, and higher among Hispanic male $(3.2 \%)$ than white male $(1.7 \%)$ students. The prevalence of having ever used heroin was higher among 10th-grade male (3.3\%) than 9th-grade male (2.0\%) students.

During 1999-2015, a significant linear decrease occurred overall in the prevalence of having ever used heroin $(2.4 \%-$ $2.1 \%)$. A significant quadratic trend was not identified. The prevalence of having ever used heroin did not change significantly from 2013 (2.2\%) to 2015 (2.1\%).

Across 32 states, the prevalence of having ever used heroin ranged from $0.9 \%$ to $5.9 \%$ (median: $2.5 \%$ ) (Table 62 ). Across 17 large urban school districts, the prevalence ranged from $1.5 \%$ to $8.3 \%$ (median: $3.3 \%$ ).

\section{Ever Used Methamphetamines}

Nationwide, 3.0\% of students had used methamphetamines (also called "speed," "crystal," "crank," or "ice") one or more times during their life (i.e., ever used methamphetamines) (Table 61). The prevalence of having ever used methamphetamines was higher among male $(3.6 \%)$ than female $(2.3 \%)$ students, higher among black male $(3.9 \%)$ than black female (1.4\%) students, and higher among 12th-grade male (5.6\%) than 12th-grade female $(1.8 \%)$ students. The prevalence of having ever used methamphetamines was higher among Hispanic (4.4\%) than white $(2.1 \%)$ students, higher among Hispanic female $(4.0 \%)$ than white female (1.7\%) and black female (1.4\%) students, and higher among Hispanic male (4.7\%) than white male $(2.5 \%)$ students. The prevalence of having ever used methamphetamines was higher among 10th-grade (3.3\%) and 12 th-grade $(3.8 \%)$ than 9 th-grade $(2.0 \%)$ students, higher among 10th-grade male (4.2\%) and 12th-grade male (5.6\%) than 9th-grade male $(1.9 \%)$ students, and higher among 12th-grade male $(5.6 \%)$ than 11 th-grade male $(2.8 \%)$ students.

During 1999-2015, a significant linear decrease occurred overall in the prevalence of having ever used methamphetamines $(9.1 \%-3.0 \%)$. A significant quadratic trend was not identified. The prevalence of having ever used methamphetamines did not change significantly from 2013 (3.2\%) to 2015 (3.0\%).

Across 29 states, the prevalence of having ever used methamphetamines ranged from $1.7 \%$ to $6.0 \%$ (median:
3.2\%) (Table 62). Across 16 large urban school districts, the prevalence ranged from $2.4 \%$ to $7.9 \%$ (median: $3.7 \%$ ).

\section{Ever Took Steroids Without a Doctor's Prescription}

Nationwide, 3.5\% of students had taken steroid pills or shots without a doctor's prescription one or more times during their life (i.e., ever took steroids without a doctor's prescription) (Table 63). The prevalence of having ever taken steroids without a doctor's prescription was higher among male (4.0\%) than female $(2.7 \%)$ students, higher among white male (3.6\%) than white female (1.8\%) students, and higher among 12 th-grade male ( $4.8 \%)$ higher than 12 th-grade female $(1.6 \%)$ students. The prevalence of having ever taken steroids without a doctor's prescription was higher among Hispanic (4.1\%) than white $(2.7 \%)$ students and higher among Hispanic female (3.9\%) than white female $(1.8 \%)$ students. The prevalence of having ever taken steroids without a doctor's prescription was higher among 9th-grade female (3.4\%) and 10th-grade female $(3.4 \%)$ than 12 th-grade female $(2.0 \%)$ students and higher among 10th-grade male $(4.4 \%)$ and 12 th-grade male (4.8\%) than 11 th-grade male $(2.8 \%)$ students.

During 1991-2015, a significant linear trend was not identified in the prevalence of having ever taken steroids without a doctor's prescription. A significant quadratic trend was identified. The prevalence of having ever taken steroids without a doctor's prescription increased during 1991-2001 $(2.7 \%-5.0 \%)$ and then decreased during 2001-2015 (5.0\%$3.5 \%)$. The prevalence of having ever taken steroids without a doctor's prescription did not change significantly from 2013 (3.2\%) to 2015 (3.5\%).

Across 24 states, the prevalence of having ever taken steroids without a doctor's prescription ranged from $1.4 \%$ to $6.3 \%$ (median: 3.8\%) (Table 64). Across 12 large urban school districts, the prevalence ranged from $2.5 \%$ to $8.7 \%$ (median: $3.5 \%$ ).

\section{Ever Took Prescription Drugs Without a Doctor's Prescription}

Nationwide, $16.8 \%$ of students had taken prescription drugs (e.g., Oxycontin, Percocet, Vicodin, codeine, Adderall, Ritalin, or Xanax) without a doctor's prescription one or more times during their life (i.e., ever took prescription drugs without a doctor's prescription) (Table 63). The prevalence of having ever taken prescription drugs without a doctor's prescription was higher among male $(17.8 \%)$ than female $(15.6 \%)$ students, higher among black male (18.1\%) than black female (10.7\%) students, and higher among 11 th-grade male $(21.5 \%)$ higher than 11 th-grade female $(16.0 \%)$ students. The prevalence of having ever taken prescription drugs without a doctor's prescription was higher among Hispanic (17.5\%) than black $(14.8 \%)$ students and higher among white female (15.9\%) and 
Hispanic female (16.5\%) than black female (10.7\%) students. The prevalence of having ever taken prescription drugs without a doctor's prescription was higher among 11th-grade (18.9\%) and 12th-grade (20.3\%) than 9th-grade (13.0\%) and 10th-grade (15.3\%) students, higher among 11th-grade female (16.0\%) and 12th-grade female (18.8\%) than 9th-grade female $(12.5 \%)$ students, and higher among 11th-grade male $(21.5 \%)$ and 12 th-grade male $(21.7 \%)$ than 9th-grade male (13.3\%) and 10th-grade male (15.1\%) students.

During 2009-2015, a significant linear decrease occurred in the prevalence of having ever taken prescription drugs without a doctor's prescription (20.2\%-16.8\%). A significant quadratic trend was not identified. The prevalence of having ever taken prescription drugs without a doctor's prescription did not change significantly from 2013 (17.8\%) to 2015 (16.8\%).

Across 32 states, the prevalence of having ever taken prescription drugs without a doctor's prescription ranged from $10.8 \%$ to $19.2 \%$ (median: $14.4 \%$ ) (Table 64 ). Across 17 large urban school districts, the prevalence ranged from $7.9 \%$ to 16.1\% (median: 13.3\%).

\section{Ever Used Inhalants}

Nationwide, $7.0 \%$ of students had sniffed glue, breathed the contents of aerosol spray cans, or inhaled any paints or sprays to get high one or more times during their life (i.e., ever used inhalants) (Table 65). The prevalence of having ever used inhalants was higher among Hispanic female (8.3\%) than white female (5.9\%) students. The prevalence of having ever used inhalants was higher among 9 th-grade $(8.3 \%)$ than 11 th-grade $(5.9 \%)$ and 12 th-grade $(6.0 \%)$ students and higher among 9th-grade female $(8.5 \%)$ and 10th-grade female $(7.8 \%)$ than 11 th-grade female $(4.6 \%)$ and 12th-grade female (4.9\%) students.

During 1995-2015, a significant linear decrease occurred overall in the prevalence of having ever used inhalants $(20.3 \%-$ 7.0\%). A significant quadratic trend was not identified. The prevalence of having ever used inhalants without a doctor's prescription decreased significantly from 2013 (8.9\%) to $2015(7.0 \%)$.

Across 29 states, the prevalence of having ever used inhalants ranged from $3.2 \%$ to $11.6 \%$ (median: $7.7 \%$ ) (Table 66). Across 16 large urban school districts, the prevalence ranged from $4.7 \%$ to $12.7 \%$ (median: $7.7 \%$ ).

\section{Ever Injected Any Illegal Drug}

Nationwide, $1.8 \%$ of students had used a needle to inject any illegal drug into their body one or more times during their life (i.e., ever injected any illegal drug) (Table 67). The prevalence of having ever injected any illegal drug was higher among male $(2.3 \%)$ than female (1.0\%) students; higher among white male
(1.7\%) and Hispanic male (2.8\%) than white female $(0.7 \%)$ and Hispanic female (1.6\%) students, respectively; and higher among 10th-grade male (2.6\%) and 12th-grade male (3.4\%) than 10th-grade female $(0.7 \%)$ and 12 th-grade female $(0.8 \%)$ students, respectively. The prevalence of having ever injected any illegal drug was higher among black (2.5\%) and Hispanic (2.2\%) than white (1.2\%) students and higher among Hispanic female (1.6\%) than white female $(0.7 \%)$ students. The prevalence of having ever injected any illegal drug was higher among 9th-grade female $(1.5 \%)$ than 10 th-grade female $(0.7 \%)$ students.

During 1995-2015, a significant linear trend was not identified in the prevalence of having ever injected any illegal drug. A significant quadratic trend was identified. The prevalence of having ever injected any illegal drug did not change during 1995-2011 (2.1\%-2.3\%) and then decreased during 2011-2015 (2.3\%-1.8\%). The prevalence of having ever injected any illegal drug did not change significantly from $2013(1.7 \%)$ to $2015(1.8 \%)$.

Across 25 states, the prevalence of having ever injected any illegal drug ranged from $1.0 \%$ to $5.4 \%$ (median: $2.4 \%$ ) (Table 68). Across 13 large urban school districts, the prevalence ranged from $1.6 \%$ to $6.6 \%$ (median: $2.5 \%$ ).

\section{Offered, Sold, or Given an Illegal Drug on School Property}

Nationwide, $21.7 \%$ of students had been offered, sold, or given an illegal drug by someone on school property during the 12 months before the survey (Table 67). The prevalence of having been offered, sold, or given an illegal drug on school property was higher among male (24.2\%) than female (19.1\%) students; higher among white male (23.2\%) than white female (16.5\%) students; and higher among 10 th-grade male $(24.6 \%), 11$ th-grade male $(24.9 \%)$, and 12 th-grade male $(24.4 \%)$ than 10 th-grade female $(19.3 \%)$, 11th-grade female $(20.4 \%)$, and 12 th-grade female $(16.3 \%)$ students, respectively. The prevalence of having been offered, sold, or given an illegal drug on school property was higher among Hispanic (27.2\%) than white (19.8\%) and black (20.6\%) students, higher among Hispanic female (25.5\%) than white female $(16.5 \%)$ students, and higher among Hispanic male $(28.9 \%)$ than white male $(23.2 \%)$ and black male $(22.0 \%)$ students. The prevalence of having been offered, sold, or given an illegal drug on school property was higher among 9th-grade female $(20.0 \%)$ and 11 th-grade female $(20.4 \%)$ than 12 th-grade female (20.4\%) students.

During 1993-2015, a significant linear decrease occurred overall in the prevalence of having been offered, sold, or given an illegal drug on school property $(24.0 \%-21.7 \%)$. A significant quadratic trend also was identified. The prevalence of having been offered, sold, or given an illegal drug on school property increased during 1993-1997 (24.0\%-31.7\%) and then decreased during 
1997-2015 (31.7\%-21.7\%). The prevalence of having been offered, sold, or given an illegal drug on school property did not change significantly from 2013 (22.1\%) to 2015 (21.7\%).

Across 32 states, the prevalence of having been offered, sold, or given an illegal drug on school property ranged from $14.7 \%$ to $29.8 \%$ (median: 22.3\%) (Table 68). Across 16 large urban school districts, the prevalence ranged from $20.5 \%$ to $34.6 \%$ (median: 26.8\%).

\section{Sexual Behaviors Related to Unintended Pregnancy and Sexually Transmitted Infections, Including HIV Infection}

\section{Ever Had Sexual Intercourse}

Nationwide, $41.2 \%$ of students had ever had sexual intercourse (Table 69). The prevalence of having ever had sexual intercourse was higher among male $(43.2 \%)$ than female (39.2\%) students; higher among black male (58.8\%) and Hispanic male (45.1\%) than black female (37.4\%) and Hispanic female (39.8\%) students, respectively; and higher among 9th-grade male (27.3\%) than 9th-grade female (20.7\%) students. The prevalence of having ever had sexual intercourse was higher among black (48.5\%) than white $(39.9 \%)$ students and higher among black male $(58.8 \%)$ than white male (39.5\%) and Hispanic male (45.1\%) students. The prevalence of having ever had sexual intercourse was higher among 10 th-grade $(35.7 \%), 11$ th-grade $(49.6 \%)$, and 12 th-grade $(58.1 \%)$ than 9th-grade $(24.1 \%)$ students; higher among 11 th-grade $(49.6 \%)$ and 12 th-grade $(58.1 \%)$ than 10 th-grade $(35.7 \%)$ students; higher among 12 th-grade $(58.1 \%)$ than 11 th-grade $(49.6 \%)$ students; higher among 10 th-grade female $(33.5 \%), 11$ th-grade female $(48.2 \%)$, and 12 th-grade female $(57.2 \%)$ than 9th-grade female $(20.7 \%)$ students; higher among 11th-grade female (48.2\%) and 12th-grade female $(57.2 \%)$ than 10 th-grade female $(33.5 \%)$ students; higher among 12th-grade female (57.2\%) than 11th-grade female (48.2\%) students; higher among 10th-grade male (37.9\%), 11 th-grade male $(51.2 \%)$, and 12 th-grade male $(59.0 \%)$ than 9th-grade male $(27.3 \%)$ students; higher among 11th-grade male $(51.2 \%)$ and 12 th-grade male $(59.0 \%)$ than 10 th-grade male $(37.9 \%)$ students; and higher among 12th-grade male $(59.0 \%)$ than 11 th-grade male $(51.2 \%)$ students.

During 1991-2015, a significant linear decrease occurred overall in the prevalence of having ever had sexual intercourse (54.1\%-41.2\%). A significant quadratic trend was not identified. The prevalence of having ever had sexual intercourse decreased significantly from 2013 (46.8\%) to 2015 (41.2\%).

Across 33 states, the prevalence of having ever had sexual intercourse ranged from $30.4 \%$ to $48.0 \%$ (median: $39.0 \%$ )
(Table 70). Across 19 large urban school districts, the prevalence ranged from $25.9 \%$ to $52.4 \%$ (median: $39.5 \%$ ).

\section{Had First Sexual Intercourse Before Age 13 Years}

Nationwide, $3.9 \%$ of students had had sexual intercourse for the first time before age 13 years (Table 69). The prevalence of having had sexual intercourse before age 13 years was higher among male $(5.6 \%)$ than female $(2.2 \%)$ students; higher among white male (3.5\%), black male (12.1\%), and Hispanic male (6.8\%) than white female (1.6\%), black female (4.3\%), and Hispanic female (3.1\%) students, respectively; and higher among 9th-grade male (4.6\%), 10th-grade male (6.8\%), 11th-grade male (4.8\%), and 12th-grade male (5.5\%) than 9th-grade female $(2.5 \%)$, 10th-grade female $(2.7 \%)$, 11 th-grade female $(1.6 \%)$, and 12 th-grade female $(1.7 \%)$ students, respectively. The prevalence of having had sexual intercourse before age 13 years was higher among black (8.3\%) and Hispanic $(5.0 \%)$ than white $(2.5 \%)$ students, higher among black (8.3\%) than Hispanic $(5.0 \%)$ students, higher among black female (4.3\%) and Hispanic female $(3.1 \%)$ than white female $(1.6 \%)$ students, higher among black male (12.1\%) and Hispanic male (6.8\%) than white male (3.5\%) students, and higher among black male (12.1\%) than Hispanic male $(6.8 \%)$ students. The prevalence of having had sexual intercourse before age 13 years was higher among 10th-grade (4.7\%) than 11 th-grade $(3.2 \%)$ students and higher among 10th-grade male $(6.8 \%)$ than 9 th-grade male $(4.6 \%)$ and 11 th-grade male $(4.8 \%)$ students.

During 1991-2015, a significant linear decrease occurred overall in the prevalence of having had sexual intercourse before age 13 years $(10.2 \%-3.9 \%)$. A significant quadratic trend was not identified. The prevalence of having had sexual intercourse before age 13 years decreased significantly from 2013 (5.6\%) to $2015(3.9 \%)$.

Across 35 states, the prevalence of having had sexual intercourse before age 13 years ranged from $2.6 \%$ to $8.3 \%$ (median: $3.5 \%$ ) (Table 70). Across 19 large urban school districts, the prevalence ranged from $2.7 \%$ to $12.2 \%$ (median: $5.7 \%$ ).

\section{Had Sexual Intercourse with Four or More Persons During Their Life}

Nationwide, $11.5 \%$ of students had had sexual intercourse with four or more persons during their life (Table 71). The prevalence of having had sexual intercourse with four or more persons was higher among male (14.1\%) than female (8.8\%) students; higher among black male (28.2\%) and Hispanic male $(15.3 \%)$ than black female $(9.2 \%)$ and Hispanic female $(6.7 \%)$ students, respectively; and higher among 9th-grade male $(6.7 \%)$, 10th-grade male $(12.1 \%)$, 11th-grade male $(16.0 \%)$, and 12 th-grade male $(22.4 \%)$ than 9 th-grade 
female (3.0\%), 10th-grade female (6.0\%), 11th-grade female $(10.7 \%)$, and 12 th-grade female (16.1\%) students, respectively. The prevalence of having had sexual intercourse with four or more persons was higher among black (19.0\%) than white (9.9\%) and Hispanic (11.0\%) students, higher among black male $(28.2 \%)$ and Hispanic male (15.3\%) than white male $(10.6 \%)$ students, and higher among black male $(28.2 \%)$ than Hispanic male $(15.3 \%)$ students. The prevalence of having had sexual intercourse with four or more persons was higher among 10th-grade (9.0\%), 11th-grade (13.4\%), and 12th-grade $(19.2 \%)$ than 9th-grade $(4.9 \%)$ students; higher among 11 th-grade $(13.4 \%)$ and 12 th-grade $(19.2 \%)$ than 10th-grade $(9.0 \%)$ students; higher among 12th-grade (19.2\%) than 11th-grade (13.4\%) students, higher among 10th-grade female $(6.0 \%)$, 11th-grade female $(10.7 \%)$, and 12th-grade female (16.1\%) than 9th-grade female (3.0\%) students; higher among 11 th-grade female $(10.7 \%)$ and 12 th-grade female $(16.1 \%)$ than 10 th-grade female $(6.0 \%)$ students; higher among 12th-grade female $(16.1 \%)$ than 11th-grade female $(10.7 \%)$ students, and higher among 10th-grade male (12.1\%), 11 th-grade male $(16.0 \%)$, and 12 th-grade male $(22.4 \%)$ than 9th-grade male (6.7\%) students; higher among 11th-grade male $(16.0 \%)$ and 12 th-grade male $(22.4 \%)$ than 10th-grade male $(12.1 \%)$ students; and higher among 12th-grade male (22.4\%) than 11th-grade male (16.0\%) students.

During 1991-2015, a significant linear decrease occurred overall in the prevalence of having had sexual intercourse with four or more persons (18.7\%-11.5\%). A significant quadratic trend was not identified. The prevalence of having had sexual intercourse with four or more persons decreased significantly from $2013(15.0 \%)$ to 2015 (11.5\%).

Across 31 states, the prevalence of having had sexual intercourse with four or more persons ranged from $6.2 \%$ to 16.0\% (median: 10.0\%) (Table 72). Across 18 large urban school districts, the prevalence ranged from $5.7 \%$ to $19.4 \%$ (median: 11.2\%).

\section{Currently Sexually Active}

Nationwide, $30.1 \%$ of students had had sexual intercourse with at least one person during the 3 months before the survey (i.e., currently sexually active) (Table 71). The prevalence of being currently sexually active was higher among black male $(40.0 \%)$ than black female $(25.7 \%)$ students and higher among 9th-grade male (17.3\%) than 9th-grade female (14.0\%) students. The prevalence of being currently sexually active was higher among white female (31.4\%) than black female (25.7\%) students and higher among black male (40.0\%) than white male (29.1\%) and Hispanic male (30.5\%) students. The prevalence of being currently sexually active was higher among 10 th-grade $(25.5 \%), 11$ th-grade $(35.5 \%)$, and 12 th-grade
(46.0\%) than 9th-grade $(15.7 \%)$ students; higher among 11 th-grade $(35.5 \%)$ and 12 th-grade $(46.0 \%)$ than 10 th-grade $(25.5 \%)$ students; higher among 12 th-grade $(46.0 \%)$ than 11 th-grade $(35.5 \%)$ students; higher among 10 th-grade female $(24.7 \%), 11$ th-grade female $(36.7 \%)$, and 12 th-grade female $(46.5 \%)$ than 9 th-grade female $(14.0 \%)$ students; higher among 11th-grade female (36.7\%) and 12th-grade female (46.5\%) than 10th-grade female $(24.7 \%)$ students; higher among 12th-grade female (46.5\%) than 11th-grade female $(36.7 \%)$ students; higher among 10th-grade male (26.4\%), 11 th-grade male $(34.5 \%)$, and 12 th-grade male (45.4\%) than 9th-grade male (17.3\%) students; higher among 11th-grade male $(34.5 \%)$ and 12 th-grade male $(45.4 \%)$ than 10 th-grade male $(26.4 \%)$ students; and higher among 12th-grade male (45.4\%) than 11th-grade male (34.5\%) students.

During 1991-2015, a significant linear decrease occurred overall in the prevalence of being currently sexually active $(37.5 \%-30.1 \%)$. A significant quadratic trend was not identified. The prevalence of being currently sexually active decreased significantly from $2013(34.0 \%)$ to 2015 (30.1\%).

Across 35 states, the prevalence of being currently sexually active ranged from $22.3 \%$ to $35.5 \%$ (median: $28.5 \%$ ) (Table 72). Across 19 large urban school districts, the prevalence ranged from $18.7 \%$ to $37.2 \%$ (median: $26.3 \%$ ).

\section{Condom Use}

Among the $30.1 \%$ of currently sexually active students nationwide, $56.9 \%$ reported that either they or their partner had used a condom during last sexual intercourse (Table 73). The prevalence of having used a condom during last sexual intercourse was higher among male (61.5\%) than female (52.0\%) students; higher among black male (73.6\%) and Hispanic male (62.5\%) than black female (46.7\%) and Hispanic female (48.3\%) students, respectively; and higher among 10th-grade male (65.6\%), 11 th-grade male $(62.5 \%)$, and 12 th-grade male $(57.4 \%)$ than 10th-grade female $(54.0 \%)$, 11th-grade female $(52.9 \%)$, and 12 th-grade female (48.8\%) students, respectively. The prevalence of having used a condom during last sexual intercourse was higher among black (63.4\%) than Hispanic (55.6\%) students, higher among white female (55.9\%) than black female (46.7\%) students, and higher among black male (73.6\%) than white male (58.1\%) and Hispanic male (62.5\%) students. The prevalence of having used a condom during last sexual intercourse was higher among 9th-grade $(60.5 \%)$ and 10th-grade $(59.9 \%)$ than 12th-grade (52.9\%) students and higher among 9 th-grade female $(56.7 \%)$ than 12th-grade female (48.8\%) students.

Because the response options measuring the prevalence of using both a condom during last sexual intercourse and birth control pills; an IUD or implant; or a shot, patch, or birth control ring before last sexual intercourse were used for the first time in the 
2013 national YRBS, long-term temporal trends are not available. A significant quadratic trend also was identified. The prevalence of having used a condom during last sexual intercourse increased during 1991-2003 (46.2\%-63.0\%) and then decreased during 2003-2015 (63.0\%-56.9\%). The prevalence of having used a condom during last sexual intercourse did not change significantly from 2013 (59.1\%) to 2015 (56.9\%).

Across 35 states, the prevalence of having used a condom during last sexual intercourse ranged from $48.4 \%$ to $63.3 \%$ (median: 57.9\%) (Table 74). Across 19 large urban school districts, the prevalence ranged from $55.4 \%$ to $68.9 \%$ (median: 60.6\%).

\section{Birth Control Pill Use}

Among the $30.1 \%$ of currently sexually active students nationwide, $18.2 \%$ reported that either they or their partner had used birth control pills to prevent pregnancy before last sexual intercourse (Table 73). The prevalence of having used birth control pills before last sexual intercourse was higher among female $(21.3 \%)$ than male (15.2\%) students; higher among Hispanic female (15.4\%) than Hispanic male (8.1\%) students; and higher among 10th-grade female (20.2\%) and 12th-grade female (23.2\%) than 10th-grade male (11.6\%) and 12th-grade male $(16.9 \%)$ students, respectively. The prevalence of having used birth control pills before last sexual intercourse was higher among white $(23.5 \%)$ than black (9.0\%) and Hispanic (11.8\%) students, higher among white female (25.4\%) than black female (9.0\%) and Hispanic female (15.4\%) students, and higher among white male (21.4\%) than black male (9.0\%) and Hispanic male (8.1\%) students. The prevalence of having used birth control pills before last sexual intercourse was higher among 10th-grade (15.9\%), 11 th-grade $(21.5 \%)$, and 12 th-grade $(20.1 \%)$ than 9 th-grade $(10.9 \%)$ students; higher among 11 th-grade $(21.5 \%)$ than 10th-grade $(15.9 \%)$ students; higher among 10th-grade female (20.2\%), 11th-grade female (23.9\%), and 12th-grade female (23.2\%) than 9th-grade female (11.2\%) students; higher among 11th-grade male $(19.1 \%)$ and 12 th-grade male $(16.9 \%)$ than 9th-grade male (10.8\%) students; and higher among 11th-grade male (19.1\%) than 10th-grade male (11.6\%) students.

During 1991-2015, a significant linear trend was not identified in the prevalence of having used birth control pills before last sexual intercourse. A significant quadratic trend was identified. The prevalence of having used birth control pills before last sexual intercourse decreased during 1991-1995 (20.8\%-17.4\%) and then increased during 1995-2015 (17.4\%-18.2\%). The prevalence of having used birth control pills before last sexual intercourse did not change significantly from 2013 (19.0\%) to 2015 (18.2\%).

Across 33 states, the prevalence of having used birth control pills before last sexual intercourse ranged from $13.1 \%$ to $34.6 \%$ (median: 20.7\%) (Table 74). Across 18 large urban school districts, the prevalence ranged from $6.8 \%$ to $18.6 \%$ (median: $12.0 \%$ ).

\section{IUD or Implant Use}

Among the $30.1 \%$ of currently sexually active students nationwide, 3.3\% reported that either they or their partner had used an IUD (such as Mirena or ParaGard) or implant (such as Implanon or Nexplanon) to prevent pregnancy before last sexual intercourse (Table 75). The prevalence of having used an IUD or implant before last sexual intercourse was higher among female (4.5\%) than male $(2.2 \%)$ students.

Because the response option measuring the prevalence of having used an IUD or implant before last sexual intercourse was used for the first time in the 2013 national YRBS, long-term temporal trends are not available. The prevalence of having used an IUD or implant before last sexual intercourse increased from 2013 (1.6\%) to 2015 (3.3\%).

Across 33 states, the prevalence of having used an IUD or implant before last sexual intercourse ranged from $1.3 \%$ to $9.7 \%$ (median: 3.4\%) (Table 76). Across 18 large urban school districts, the prevalence ranged from $0.5 \%$ to $16.9 \%$ (median: $2.1 \%$ ).

\section{Shot, Patch, or Birth Control Ring Use}

Among the $30.1 \%$ of currently sexually active students nationwide, 5.3\% reported that either they or their partner had used a shot (such as Depo-Provera), patch (such as OrthoEvra), or birth control ring (such as NuvaRing) to prevent pregnancy before last sexual intercourse (Table 75). The prevalence of having used a shot, patch, or birth control ring before last sexual intercourse was higher among female (7.9\%) than male $(2.7 \%)$ students; higher among white female (8.9\%) and black female (7.8\%) than white male $(2.7 \%)$ and black male (3.1\%) students, respectively; and higher among 9th-grade female (5.8\%), 10th-grade female (9.3\%), 11th-grade female (7.3\%), and 12 th-grade female (8.3\%) than 9 th-grade male $(1.4 \%), 10$ th-grade male $(2.4 \%), 11$ th-grade male $(3.5 \%)$, and 12 th-grade male $(3.0 \%)$ students, respectively. The prevalence of having used a shot, patch, or birth control ring before last sexual intercourse was higher among white $(6.0 \%)$ than Hispanic (3.1\%) students and higher among white female (8.9\%) and black female (7.8\%) than Hispanic female (3.7\%) students.

Because the response option measuring the prevalence of having used a shot, patch, or birth control ring before last sexual intercourse was used for the first time in the 2013 national YRBS, long-term temporal trends are not available. The prevalence of having used a shot, patch, or birth control ring before last sexual intercourse did not change significantly from 2013 (4.7\%) to 2015 (5.3\%).

Across 33 states, the prevalence of having used a shot, patch, or birth control ring before last sexual intercourse ranged from $1.6 \%$ to $9.5 \%$ (median: $5.6 \%$ ) (Table 76 ). Across 18 large urban school districts, the prevalence ranged from $1.1 \%$ to $10.5 \%$ (median: 4.4\%). 


\section{Birth Control Pill; IUD or Implant; or Shot, Patch, or Birth Control Ring Use}

Among the $30.1 \%$ of currently sexually active students nationwide, $26.8 \%$ reported that either they or their partner had used birth control pills; an IUD (such as Mirena or ParaGard) or implant (such as Implanon or Nexplanon); or a shot (such as Depo-Provera), patch (such as OrthoEvra), or birth control ring (such as NuvaRing) to prevent pregnancy before last sexual intercourse (Table 77). The prevalence of having used birth control pills; an IUD or implant; or a shot, patch, or birth control ring before last sexual intercourse was higher among female $(33.7 \%)$ than male (20.2\%) students; higher among white female (39.2\%) and Hispanic female (23.2\%) than white male (27.0\%) and Hispanic male (12.3\%) students, respectively; and higher among 9 th-grade female $(20.8 \%), 10$ th-grade female $(33.6 \%), 11$ th-grade female $(36.2 \%)$, and 12th-grade female (36.2\%) than 9th-grade male (13.1\%), 10th-grade male (15.5\%), 11 th-grade male $(25.5 \%)$, and 12 th-grade male $(22.7 \%)$ students, respectively. The prevalence of having used birth control pills; an IUD or implant; or a shot, patch, or birth control ring before last sexual intercourse was higher among white (33.3\%) than black (15.9\%) and Hispanic (17.8\%) students, higher among white female (39.2\%) than black female (20.5\%) and Hispanic female (23.2\%) students, and higher among white male (27.0\%) than black male (13.1\%) and Hispanic male (12.3\%) students. The prevalence of having used birth control pills; an IUD or implant; or a shot, patch, or birth control ring before last sexual intercourse was higher among 10th-grade (24.4\%), 11 th-grade (30.9\%), and 12th-grade $(29.6 \%)$ than 9th-grade $(16.4 \%)$ students; higher among 11th-grade (30.9\%) than 10th-grade (24.4\%) students; higher among 10th-grade female (33.6\%), 11th-grade female $(36.2 \%)$, and 12 th-grade female $(36.2 \%)$ than 9 th-grade female (20.8\%) students; and higher among 11th-grade male (25.5\%) and 12th-grade male $(22.7 \%)$ than 9th-grade male (13.1\%) and 10th-grade male $(15.5 \%)$ students.

During 2011-2015, a significant linear increase occurred overall in the prevalence of having used birth control pills; an IUD or implant; or a shot, patch, or birth control ring before last sexual intercourse (23.3\%-26.8\%). A significant quadratic trend was not identified. The prevalence of having used birth control pills; an IUD or implant; or a shot, patch, or birth control ring before last sexual intercourse did not change significantly from 2013 (25.3\%) to 2015 (26.8\%).

Across 33 states, the prevalence of having used birth control pills; an IUD or implant; or a shot, patch, or birth control ring before last sexual intercourse ranged from $17.5 \%$ to $47.3 \%$ (median: 30.1\%) (Table 78). Across 18 large urban school districts, the prevalence ranged from $10.4 \%$ to $37.6 \%$ (median: $18.3 \%$ ).

\section{Condom Use and Birth Control Pill; IUD or Implant; or Shot, Patch, or Birth Control Ring Use}

Among the $30.1 \%$ of currently sexually active students nationwide, $8.8 \%$ reported that either they or their partner had used both a condom during last sexual intercourse and birth control pills; an IUD (such as Mirena or ParaGard) or implant (such as Implanon or Nexplanon); or a shot (such as Depo-Provera), patch (such as OrthoEvra), or birth control ring (such as NuvaRing) to prevent pregnancy before last sexual intercourse (Table 77). The prevalence of having used both a condom during last sexual intercourse and birth control pills; an IUD or implant; or a shot, patch, or birth control ring before last sexual intercourse was higher among female (11.8\%) than male $(5.9 \%)$ students; higher among white female $(15.9 \%)$ than white male $(7.7 \%)$ students; and higher among 10th-grade female $(12.4 \%)$ and 12th-grade female $(9.9 \%)$ than 10th-grade male (4.3\%) and 12th-grade male (5.5\%) students, respectively. The prevalence of having used both a condom during last sexual intercourse and birth control pills; an IUD or implant; or a shot, patch, or birth control ring before last sexual intercourse was higher among white (12.0\%) than black (4.7\%) and Hispanic (4.7\%) students, higher among white female $(15.9 \%)$ than black female $(5.7 \%)$ and Hispanic female (4.8\%) students, and higher among white male (7.7\%) than black male $(4.1 \%)$ students. The prevalence of having used both a condom during last sexual intercourse and birth control pills; an IUD or implant; or a shot, patch, or birth control ring before last sexual intercourse was higher among 11 th-grade $(12.2 \%)$ than 9 th-grade $(5.8 \%)$ and 12th-grade (7.7\%) students and higher among 11th-grade male (9.4\%) than 9th-grade male (3.2\%) students.

Because the response options measuring the prevalence of having used both a condom during last sexual intercourse and birth control pills; an IUD or implant; or a shot, patch, or birth control ring before last sexual intercourse were used for the first time in the 2013 national YRBS, long-term temporal trends are not available. The prevalence of having used both a condom during last sexual intercourse and birth control pills; an IUD or implant; or a shot, patch, or birth control ring before last sexual intercourse did not change significantly from 2013 (8.8\%) to 2015 (8.8\%).

Across 33 states, the prevalence of having used both a condom during last sexual intercourse and birth control pills; an IUD or implant; or a shot, patch, or birth control ring before last sexual intercourse ranged from $5.8 \%$ to $18.8 \%$ (median: $11.1 \%$ ) (Table 78). Across 18 large urban school districts, the prevalence ranged from $2.8 \%$ to $10.2 \%$ (median: $6.5 \%$ ). 


\section{Did Not Use Any Method to Prevent Pregnancy}

Among the $30.1 \%$ of currently sexually active students nationwide, $13.8 \%$ reported that neither they nor their partner had used any method to prevent pregnancy during last sexual intercourse (Table 79). The prevalence of not having used any method to prevent pregnancy was higher among black female (25.6\%) than black male (9.9\%) students and higher among 9th-grade female $(22.0 \%)$ than 9th-grade male $(12.1 \%)$ students. The prevalence of not having used any method to prevent pregnancy was higher among Hispanic $(20.0 \%)$ than white $(10.4 \%)$ students, higher among black female $(25.6 \%)$ and Hispanic female $(22.7 \%)$ than white female $(10.2 \%)$ students, and higher among Hispanic male (17.2\%) than white male (10.3\%) and black male (9.9\%) students. The prevalence of not having used any method to prevent pregnancy was higher among 9th-grade (16.5\%) and 12 th-grade $(15.5 \%)$ than 11 th-grade $(11.1 \%)$ students and higher among 9th-grade female $(22.0 \%)$ than 10th-grade female $(12.9 \%)$ and 11 th-grade female $(12.3 \%)$ students.

During 1991-2015, a significant linear decrease occurred overall in the prevalence of not having used any method to prevent pregnancy $(16.5 \%-13.8 \%)$. A significant quadratic trend also was identified. The prevalence of not having used any method to prevent pregnancy decreased during 1991-2007 (16.5\%-12.2\%) and then did not change significantly during 2007-2015 (12.2\%-13.8\%). The prevalence of not having used any method to prevent pregnancy also did not change significantly from 2013 (13.7\%) to 2015 (13.8\%).

Across 33 states, the prevalence of not having used any method to prevent pregnancy ranged from $7.2 \%$ to $20.0 \%$ (median: $12.8 \%$ ) (Table 80). Across 18 large urban school districts, the prevalence ranged from $10.5 \%$ to $22.0 \%$ (median: $17.1 \%$ ).

\section{Drank Alcohol or Used Drugs Before Last Sexual Intercourse}

Among the $30.1 \%$ of currently sexually active students nationwide, $20.6 \%$ had drunk alcohol or used drugs before last sexual intercourse (Table 79). The prevalence of having drunk alcohol or used drugs before last sexual intercourse was higher among male (24.6\%) than female (16.4\%) students; higher among white male $(24.4 \%)$ and Hispanic male $(27.7 \%)$ than white female (14.7\%) and Hispanic female (17.7\%) students, respectively; and higher among 9th-grade male (27.2\%) and 12 th-grade male $(25.6 \%)$ than 9 th-grade female $(16.8 \%)$ and 12th-grade female $(16.1 \%)$ students, respectively.

During 1991-2015, a significant linear trend in the prevalence of having drunk alcohol or used drugs before last sexual intercourse was not identified. A significant quadratic trend was identified. The prevalence of having drunk alcohol or used drugs before last sexual intercourse increased during 1991-1999 (21.6\%-24.8\%) and then decreased during 1999-2015 (24.8\%-20.6\%). The prevalence of having drunk alcohol or used drugs before last sexual intercourse did not change significantly from 2013 (22.4\%) to 2015 (20.6\%).

Across 34 states, the prevalence of having drunk alcohol or used drugs before last sexual intercourse ranged from 13.5\% to $24.6 \%$ (median: $18.9 \%$ ) (Table 80 ). Across 19 large urban school districts, the prevalence ranged from $13.2 \%$ to $24.8 \%$ (median: 19.8\%).

\section{Tested for HIV}

Nationwide, $10.2 \%$ of students had ever been tested for HIV, not counting tests done when donating blood (Table 81). The prevalence of having ever been tested for HIV was higher among 12th-grade female (16.8\%) than 12 th-grade male $(10.9 \%)$ students. The prevalence of having ever been tested for HIV was higher among black (16.6\%) and Hispanic (11.1\%) than white (8.0\%) students, higher among black female (16.2\%) and Hispanic female (12.3\%) than white female $(9.1 \%)$ students, and higher among black male $(17.1 \%)$ and Hispanic male $(10.1 \%)$ than white male $(7.0 \%)$ students. The prevalence of having ever been tested for HIV was higher among 12th-grade $(13.8 \%)$ than 9th-grade (7.8\%), 10th-grade $(9.8 \%)$, and 11 th-grade $(9.6 \%)$ students and higher among 12 th-grade female $(16.8 \%)$ than 9 th-grade female $(7.7 \%)$, 10th-grade female $(9.8 \%)$, and 11 th-grade female $(10.3 \%)$ students.

During 2005-2015, a significant linear decrease occurred overall in the prevalence of having ever been tested for HIV $(11.9 \%-10.2 \%)$. A significant quadratic trend also was identified. The prevalence of having ever been tested for HIV did not change significantly during 2005-2011 (11.9\%-12.9\%) and then decreased during 2011-2015 (12.9\%-10.2\%). The prevalence of having ever been tested for HIV also decreased significantly from 2013 (12.9\%) to 2015 (10.2\%).

Across 27 states, the prevalence of having ever been tested for HIV ranged from $7.4 \%$ to $18.0 \%$ (median: $11.4 \%$ ) (Table 82 ). Across 19 large urban school districts, the prevalence ranged from $7.0 \%$ to $37.4 \%$ (median: $18.9 \%$ ).

\section{Dietary Behaviors}

\section{Did Not Eat Fruit or Drink 100\% Fruit Juices}

Nationwide, $5.2 \%$ of students had not eaten fruit or drunk $100 \%$ fruit juices during the 7 days before the survey (Table 83). The prevalence of not having eaten fruit or drunk $100 \%$ fruit juices was higher among male $(5.9 \%)$ than female $(4.3 \%)$ students, higher among black male $(8.6 \%)$ than black female (5.2\%) students, and higher among 10th-grade 
male $(6.7 \%)$ than 10 th-grade female $(3.4 \%)$ students. The prevalence of not having eaten fruit or drunk $100 \%$ fruit juices was higher among 9 th-grade $(6.0 \%)$ than 11 th-grade (4.4\%) students and higher among 9th-grade male (6.7\%) than 11 th-grade male $(4.7 \%)$ students.

During 1999-2015, a significant linear decrease occurred overall in the prevalence of not having eaten fruit or drunk $100 \%$ fruit juices $(5.4 \%-5.2 \%)$. A significant quadratic trend was not identified. The prevalence of not having eaten fruit or drunk $100 \%$ fruit juices did not change significantly from 2013 (5.0\%) to 2015 (5.2\%).

Across 36 states, the prevalence of not having eaten fruit or drunk $100 \%$ fruit juices ranged from $3.2 \%$ to $11.7 \%$ (median: $7.0 \%$ ) (Table 84). Across 18 large urban school districts, the prevalence ranged from $3.8 \%$ to $11.4 \%$ (median: $7.1 \%$ ).

\section{Ate Fruit or Drank 100\% Fruit Juices One or More Times per Day}

Nationwide, $63.3 \%$ of students had eaten fruit or drunk $100 \%$ fruit juices one or more times per day during the 7 days before the survey (Table 83). The prevalence of having eaten fruit or drunk $100 \%$ fruit juices one or more times per day was higher among black male (67.0\%) and Hispanic male $(67.3 \%)$ than black female $(56.5 \%)$ and Hispanic female $(60.7 \%)$ students, respectively. The prevalence of having eaten fruit or drunk $100 \%$ fruit juices one or more times per day was higher among white female $(64.1 \%)$ than black female (56.5\%) students and higher among Hispanic male (67.3\%) than white male $(62.1 \%)$ students.

During 1999-2015, significant linear and quadratic trends were not identified in the prevalence of having eaten fruit or drunk $100 \%$ fruit juices one or more times per day. The prevalence of having eaten fruit or drunk 100\% fruit juices one or more times per day did not change significantly from $2013(62.6 \%)$ to 2015 (63.3\%).

Across 36 states, the prevalence of having eaten fruit or drunk $100 \%$ fruit juices one or more times per day ranged from $49.0 \%$ to $67.7 \%$ (median: $58.7 \%$ ) (Table 84 ). Across 18 large urban school districts, the prevalence ranged from $52.4 \%$ to $66.7 \%$ (median: $57.6 \%$ ).

\section{Ate Fruit or Drank 100\% Fruit Juices Two or More Times per Day}

Nationwide, $31.5 \%$ of students had eaten fruit or drunk $100 \%$ fruit juices two or more times per day during the 7 days before the survey (Table 85 ). The prevalence of having eaten fruit or drunk $100 \%$ fruit juices two or more times per day was higher among male $(33.1 \%)$ than female $(30.0 \%)$ students; higher among black male (38.2\%) and Hispanic male (36.7\%) than black female (27.8\%) and Hispanic female (29.9\%) students, respectively; and higher among 9th-grade male $(35.0 \%)$ than 9th-grade female $(30.0 \%)$ students. The prevalence of having eaten fruit or drunk $100 \%$ fruit juices two or more times per day was higher among Hispanic (33.4\%) than white (29.8\%) students and higher among black male (38.2\%) and Hispanic male $(36.7 \%)$ than white male $(29.9 \%)$ students.

During 1999-2015, significant linear and quadratic trends were not identified in the prevalence of having eaten fruit or drunk $100 \%$ fruit juices two or more times per day. The prevalence of having eaten fruit or drunk $100 \%$ fruit juices two or more times per day did not change significantly from 2013 (33.2\%) to 2015 (31.5\%).

Across 36 states, the prevalence of having eaten fruit or drunk $100 \%$ fruit juices two or more times per day ranged from $21.0 \%$ to $34.3 \%$ (median: $27.7 \%$ ) (Table 86 ). Across 18 large urban school districts, the prevalence ranged from $24.0 \%$ to $37.6 \%$ (median: $32.2 \%$ ).

\section{Ate Fruit or Drank 100\% Fruit Juices Three or More Times per Day}

Nationwide, $20.0 \%$ of students had eaten fruit or drunk $100 \%$ fruit juices three or more times per day during the 7 days before the survey (Table 85). The prevalence of having eaten fruit or drunk $100 \%$ fruit juices three or more times per day was higher among male $(22.1 \%)$ than female $(18.0 \%)$ students; higher among black male $(29.1 \%)$ and Hispanic male $(26.6 \%)$ than black female (20.5\%) and Hispanic female (20.5\%) students, respectively; and higher among 9th-grade male $(24.3 \%)$ than 9th-grade female $(16.9 \%)$ students. The prevalence of having eaten fruit or drunk $100 \%$ fruit juices three or more times per day was higher among black $(25.1 \%)$ and Hispanic $(23.6 \%)$ than white $(17.0 \%)$ students, higher among black female $(20.5 \%)$ and Hispanic female $(20.5 \%)$ than white female $(16.0 \%)$ students, and higher among black male $(29.1 \%)$ and Hispanic male (26.6\%) than white male (18.0\%) students.

During 1999-2015, a significant linear decrease occurred in the prevalence of having eaten fruit or drunk $100 \%$ fruit juices three or more times per day $(24.9 \%-20.0 \%)$. A significant quadratic trend was not identified. The prevalence of having eaten fruit or drunk $100 \%$ fruit juices three or more times per day decreased significantly from 2013 (21.9\%) to 2015 (20.0\%).

Across 36 states, the prevalence of having eaten fruit or drunk $100 \%$ fruit juices three or more times per day ranged from $13.1 \%$ to $22.5 \%$ (median: $17.0 \%$ ) (Table 86 ). Across 18 large urban school districts, the prevalence ranged from $17.3 \%$ to 24.6\% (median: 21.4\%). 


\section{Did Not Eat Vegetables}

Nationwide, $6.7 \%$ of students had not eaten vegetables 999 during the 7 days before the survey (Table 87). The prevalence of not having eaten vegetables was higher among male $(7.7 \%)$ than female $(5.6 \%)$ students; higher among white male $(6.0 \%)$ and black male (13.0\%) than white female $(3.7 \%)$ and black female (8.8\%) students, respectively; and higher among 11th-grade male (8.0\%) than 11th-grade female (4.5\%) students. The prevalence of not having eaten vegetables was higher among black (10.9\%) and Hispanic (8.5\%) than white (4.9\%) students, higher among black (10.9\%) than Hispanic (8.5\%) students, higher among black female $(8.8 \%)$ and Hispanic female (7.7\%) than white female (3.7\%) students, higher among black male (13.0\%) and Hispanic male (9.2\%) than white male $(6.0 \%)$ students, and higher among black male (13.0\%) than Hispanic male (9.2\%) students.

During 1999-2015, a significant linear increase occurred overall in the prevalence of not having eaten vegetables $(4.2 \%-6.7 \%)$. A significant quadratic trend was not identified. The prevalence of not having eaten vegetables did not change significantly from 2013 (6.6\%) to 2015 (6.7\%).

Across 32 states, the prevalence of not having eaten vegetables ranged from $3.3 \%$ to $11.3 \%$ (median: $7.0 \%$ ) (Table 88 ). Across 16 large urban school districts, the prevalence ranged from $5.6 \%$ to $11.7 \%$ (median: $10.7 \%$ ).

\section{Ate Vegetables One or More Times per Day}

Nationwide, $61.0 \%$ of students had eaten vegetables one or more times per day during the 7 days before the survey (Table 87). The prevalence of having eaten vegetables one or more times per day was higher among black male (56.5\%) than black female (48.0\%) students. The prevalence of having eaten vegetables one or more times per day was higher among white (64.2\%) and Hispanic (56.5\%) than black (52.5\%) students, higher among white (64.2\%) than Hispanic (56.5\%) students, higher among white female $(64.5 \%)$ and Hispanic female $(55.3 \%)$ than black female $(48.0 \%)$ students, higher among white female (64.5\%) than Hispanic female $(55.3 \%)$ students, and higher among white male $(64.0 \%)$ than black male $(56.5 \%)$ and Hispanic male $(57.7 \%)$ students. The prevalence of having eaten vegetables one or more times per day was higher among 11th-grade (62.5\%) and 12th-grade $(63.5 \%)$ than 9th-grade $(58.6 \%)$ students, higher among 12 th-grade $(63.5 \%)$ than 10 th-grade $(59.9 \%)$ students, and higher among 12th-grade female $(63.1 \%)$ than 9th-grade female $(57.3 \%)$ and 10 th-grade female $(58.6 \%)$ students.

\footnotetext{
999 Green salad, potatoes (excluding French fries, fried potatoes, or potato chips), carrots, or other vegetables.
}

During 1999-2015, significant linear and quadratic trends were not identified in the prevalence of having eaten vegetables one or more times per day. The prevalence of having eaten vegetables one or more times per day did not change significantly from 2013 (61.5\%) to 2015 (61.0\%).

Across 32 states, the prevalence of having eaten vegetables one or more times per day ranged from $46.7 \%$ to $72.3 \%$ (median: 59.1\%) (Table 88). Across 16 large urban school districts, the prevalence ranged from $48.7 \%$ to $61.1 \%$ (median: 54.3\%).

\section{Ate Vegetables Two or More Times per Day}

Nationwide, $28.0 \%$ of students had eaten vegetables two or more times per day during the 7 days before the survey (Table 89). The prevalence of having eaten vegetables two or more times per day was higher among male $(29.6 \%)$ than female $(26.5 \%)$ students and higher among black male $(25.7 \%)$ and Hispanic male (30.3\%) than black female (17.9\%) and Hispanic female (23.8\%) students, respectively. The prevalence of having eaten vegetables two or more times per day was higher among white (28.6\%) and Hispanic (27.2\%) than black $(22.0 \%)$ students, higher among white female $(29.1 \%)$ and Hispanic female (23.8\%) than black female (17.9\%) students, and higher among white female $(29.1 \%)$ than Hispanic female $(23.8 \%)$ students. The prevalence of having eaten vegetables two or more times per day was higher among 12 th-grade female (29.8\%) than 9th-grade female (24.2\%) students.

During 1999-2015, significant linear and quadratic trends were not identified in the prevalence of having eaten vegetables two or more times per day. The prevalence of having eaten vegetables two or more times per day did not change significantly from $2013(28.4 \%)$ to 2015 (28.0\%).

Across 32 states, the prevalence of having eaten vegetables two or more times per day ranged from $18.5 \%$ to $36.3 \%$ (median: 25.2\%) (Table 90). Across 16 large urban school districts, the prevalence ranged from $19.0 \%$ to $27.4 \%$ (median: $23.2 \%$ ).

\section{Ate Vegetables Three or More Times per Day}

Nationwide, $14.8 \%$ of students had eaten vegetables three or more times per day during the 7 days before the survey (Table 89). The prevalence of having eaten vegetables three or more times per day was higher among male (16.6\%) than female (12.9\%) students; higher among Hispanic male (18.8\%) than Hispanic female $(12.7 \%)$ students; and higher among 9th-grade male (17.1\%) and 10th-grade male (15.9\%) than 9th-grade female (11.6\%) and 10th-grade female (11.2\%), respectively. The prevalence of having eaten vegetables three or more times per day was higher among Hispanic (15.8\%) 
than white $(13.5 \%)$ students and higher among Hispanic male (18.8\%) than white male (13.9\%) students. The prevalence of having eaten vegetables three or more times per day was higher among 12 th-grade $(16.0 \%)$ than 10th-grade $(13.5 \%)$ students and higher among 11 th-grade female $(13.9 \%)$ than 10th-grade female $(11.2 \%)$ students.

During 1999-2015, a significant linear increase occurred in the prevalence of having eaten vegetables three or more times per day (14.0\%-14.8\%). A significant quadratic trend was not identified. The prevalence of having eaten vegetables three or more times per day did not change significantly from 2013 $(15.7 \%)$ to 2015 (14.8\%).

Across 32 states, the prevalence of having eaten vegetables three or more times per day ranged from $9.1 \%$ to $18.1 \%$ (median: 12.6\%) (Table 90). Across 16 large urban school districts, the prevalence ranged from $9.5 \%$ to $16.8 \%$ (median: $12.6 \%$ ).

\section{Did Not Drink Milk}

Nationwide, $21.5 \%$ of students had not drunk milk during the 7 days before the survey (Table 91). The prevalence of not having drunk milk was higher among female (28.4\%) than male $(15.1 \%)$ students; higher among white female $(24.4 \%)$, black female (44.6\%), and Hispanic female $(26.3 \%)$ than white male (12.1\%), black male (25.8\%), and Hispanic male (13.0\%) students, respectively; and higher among 9th-grade female (27.0\%), 10th-grade female (26.9\%), 11th-grade female $(31.7 \%)$, and 12 th-grade female $(28.2 \%)$ than 9 th-grade male (15.6\%), 10th-grade male (14.1\%), 11th-grade male (14.2\%), and 12th-grade male (16.6\%) students, respectively. The prevalence of not having drunk milk was higher among black (34.7\%) than white (18.3\%) and Hispanic (19.6\%) students, higher among black female (44.6\%) than white female (24.4\%) and Hispanic female (26.3\%) students, and higher among black male $(25.8 \%)$ than white male $(12.1 \%)$ and Hispanic male $(13.0 \%)$ students. The prevalence of not having drunk milk was higher among 11 th-grade female $(31.7 \%)$ than 9th-grade female $(27.0 \%)$ students.

During 1999-2015, a significant linear increase occurred overall in the prevalence of not having drunk milk $(17.0 \%$ $21.5 \%)$. A significant quadratic trend also was identified. The prevalence of not having drunk milk did not change significantly during 1999-2011 (17.0\%-17.3\%) and then increased during 2011-2015 (17.3\%-21.5\%). The prevalence of not having drunk milk did not change significantly from $2013(19.4 \%)$ to $2015(21.5 \%)$.

Across 28 states, the prevalence of not having drunk milk ranged from $13.9 \%$ to $31.7 \%$ (median: $22.6 \%$ ) (Table 92 ). Across 15 large urban school districts, the prevalence ranged from $20.7 \%$ to $36.9 \%$ (median: $27.5 \%$ ).

\section{Drank One or More Glasses of Milk per Day}

Nationwide, $37.5 \%$ of students had drunk one or more glasses of milk per day during the 7 days before the survey (Table 91). The prevalence of having drunk one or more glasses of milk per day was higher among male (46.2\%) than female $(28.2 \%)$ students; higher among white male (50.6\%), black male (31.8\%), and Hispanic male (45.1\%) than white female $(32.0 \%)$, black female $(17.7 \%)$, and Hispanic female $(27.2 \%)$ students, respectively; and higher among 9th-grade male $(47.0 \%)$, 10th-grade male $(49.2 \%), 11$ th-grade male $(44.7 \%)$, and 12 th-grade male $(43.8 \%)$ than 9 th-grade female $(29.3 \%), 10$ th-grade female $(30.1 \%), 11$ th-grade female (26.3\%), and 12 th-grade female $(26.5 \%)$ students, respectively. The prevalence of having drunk one or more glasses of milk per day was higher among white (41.2\%) and Hispanic (36.2\%) than black ( $25.1 \%)$ students, higher among white (41.2\%) than Hispanic (36.2\%) students, higher among white female (32.0\%) and Hispanic female (27.2\%) than black female $(17.7 \%)$ students, higher among white female $(32.0 \%)$ than Hispanic female (27.2\%) students, and higher among white male $(50.6 \%)$ and Hispanic male $(45.1 \%)$ than black male (31.8\%) students.

During 1999-2015, a significant linear decrease occurred overall in the prevalence of having drunk one or more glasses of milk per day (47.1\%-37.5\%). A significant quadratic trend was not identified. The prevalence of having drunk one or more glasses of milk per day did not change significantly from 2013 (40.3\%) to $2015(37.5 \%)$.

Across 28 states, the prevalence of having drunk one or more glasses of milk per day ranged from $23.0 \%$ to $51.6 \%$ (median: $34.0 \%$ ) (Table 92). Across 15 large urban school districts, the prevalence ranged from $16.2 \%$ to $34.1 \%$ (median: $26.1 \%$ ).

\section{Drank Two or More Glasses of Milk per Day}

Nationwide, $22.4 \%$ of students had drunk two or more glasses of milk per day during the 7 days before the survey (Table 93). The prevalence of having drunk two or more glasses of milk per day was higher among male $(29.6 \%)$ than female $(14.6 \%)$ students; higher among white male (32.9\%), black male $(21.0 \%)$, and Hispanic male $(28.3 \%)$ than white female (17.8\%), black female (5.8\%), and Hispanic female (12.8\%) students, respectively; and higher among 9th-grade male (32.5\%), 10th-grade male (30.8\%), 11th-grade male (29.2\%), and 12 th-grade male $(25.4 \%)$ than 9th-grade female (16.7\%), 10 th-grade female $(14.8 \%)$, 11 th-grade female $(13.7 \%)$, and 12th-grade female (12.5\%) students, respectively. The prevalence of having drunk two or more glasses of milk per day was higher among white (25.3\%) and Hispanic (20.6\%) than black (13.8\%) students, higher among white $(25.3 \%)$ 
than Hispanic (20.6\%) students, higher among white female $(17.8 \%)$ and Hispanic female $(12.8 \%)$ than black female (5.8\%) students, higher among white female $(17.8 \%)$ than Hispanic female (12.8\%) students, and higher among white male $(32.9 \%)$ and Hispanic male $(28.3 \%)$ than black male $(21.0 \%)$ students. The prevalence of having drunk two or more glasses of milk per day was higher among 9th-grade (25.1\%) than 11th-grade (21.9\%) and 12th-grade (19.0\%) students, higher among 9th-grade female (16.7\%) than 12th-grade female $(12.5 \%)$ students, and higher among 9th-grade male (32.5\%) than 12 th-grade male $(25.4 \%)$ students.

During 1999-2015, a significant linear decrease occurred overall in the prevalence of having drunk two or more glasses of milk per day (33.6\%-22.4\%). A significant quadratic trend was not identified. The prevalence of having drunk two or more glasses of milk per day decreased significantly from 2013 (25.9\%) to 2015 (22.4\%).

Across 28 states, the prevalence of having drunk two or more glasses of milk per day ranged from $12.6 \%$ to $35.8 \%$ (median: $20.0 \%$ ) (Table 94). Across 15 large urban school districts, the prevalence ranged from $8.2 \%$ to $20.2 \%$ (median: $14.6 \%$ ).

\section{Drank Three or More Glasses of Milk per Day}

Nationwide, $10.2 \%$ of students had drunk three or more glasses of milk per day during the 7 days before the survey (Table 93). The prevalence of having drunk three or more glasses of milk per day was higher among male (14.8\%) than female (5.4\%) students; higher among white male (16.1\%), black male (10.3\%), and Hispanic male $(15.0 \%)$ than white female $(6.4 \%)$, black female $(3.0 \%)$, and Hispanic female (4.9\%) students, respectively; and higher among 9 th-grade male (15.7\%), 10th-grade male (17.0\%), 11th-grade male (13.8\%), and 12th-grade male (12.1\%) than 9th-grade female (6.9\%), 10th-grade female $(5.1 \%), 11$ th-grade female $(4.6 \%)$, and 12th-grade female (4.6\%) students, respectively. The prevalence of having drunk three or more glasses of milk per day was higher among white (11.2\%) and Hispanic (10.0\%) than black (6.9\%) students, higher among white female $(6.4 \%)$ than black female (3.0\%) students, and higher among white male (16.1\%) and Hispanic male (15.0\%) than black male (10.3\%) students. The prevalence of having drunk three or more glasses of milk per day was higher among 9th-grade (11.5\%) and 10th-grade (11.0\%) than 12th-grade (8.4\%) students and higher among 9th-grade male $(15.7 \%)$ and 10 th-grade male $(17.0 \%)$ than 12 th-grade male (13.8\%) students.

During 1999-2015, a significant linear decrease occurred overall in the prevalence of having drunk three or more glasses of milk per day (18.0\%-10.2\%). A significant quadratic trend was not identified. The prevalence of having drunk three or more glasses of milk per day decreased significantly from 2013 (12.5\%) to $2015(10.2 \%)$.

Across 28 states, the prevalence of having drunk three or more glasses of milk per day ranged from $5.8 \%$ to $17.9 \%$ (median: 9.0\%) (Table 94). Across 15 large urban school districts, the prevalence ranged from $4.1 \%$ to $9.9 \%$ (median: $7.1 \%$ ).

\section{Did Not Drink Soda or Pop}

Nationwide, $26.2 \%$ of students had not drunk soda or pop (not counting diet soda or diet pop) during the 7 days before the survey (Table 95). The prevalence of not having drunk soda or pop was higher among female (31.0\%) than male (21.4\%) students; higher among white female $(33.3 \%)$ and Hispanic female $(26.3 \%)$ than white male $(21.6 \%)$ and Hispanic male $(19.0 \%)$ students, respectively; and higher among 9th-grade female (30.3\%), 10th-grade female (28.7\%), 11th-grade female $(33.3 \%)$, and 12 th-grade female $(31.9 \%)$ than 9 th-grade male (21.6\%), 10th-grade male (20.5\%), 11th-grade male (19.8\%), and 12 th-grade male $(23.6 \%)$ students, respectively. The prevalence of not having drunk soda or pop was higher among white $(27.5 \%)$ than black (22.8\%) and Hispanic (22.6\%) students and higher among white female $(33.3 \%)$ than black female $(22.6 \%)$ and Hispanic female $(26.3 \%)$ students. The prevalence of not having drunk soda or pop was higher among 12 th-grade $(27.7 \%)$ than 10 th-grade $(24.6 \%)$ students, higher among 11th-grade female (33.3\%) than 10th-grade female (28.7\%) students, and higher among 12th-grade male (23.6\%) than 10th-grade male (20.5\%) students.

During 2007-2015, a significant linear increase occurred overall in the prevalence of not having drunk soda or pop $(18.6 \%-26.2 \%)$. A significant quadratic trend was not identified. The prevalence of not having drunk soda or pop increased significantly from 2013 (22.3\%) to 2015 (26.2\%).

Across 36 states, the prevalence of not having drunk soda or pop ranged from $18.7 \%$ to $37.0 \%$ (median: $26.0 \%$ ) (Table 96 ). Across 18 large urban school districts, the prevalence ranged from $18.9 \%$ to $39.0 \%$ (median: $27.6 \%$ ).

\section{Drank Soda or Pop One or More Times per Day}

Nationwide, $20.4 \%$ of students had drunk a can, bottle, or glass of soda or pop (not counting diet soda or diet pop) one or more times per day during the 7 days before the survey (Table 95). The prevalence of having drunk soda or pop one or more times per day was higher among male $(24.3 \%)$ than female (16.4\%) students; higher among white male $(24.5 \%)$ and Hispanic male $(25.1 \%)$ than white female (15.0\%) and Hispanic female (18.1\%) students, respectively; and higher among 9th-grade male (22.4\%), 10th-grade male (25.4\%), 11 th-grade male $(25.6 \%)$, and 12 th-grade male $(24.2 \%)$ than 9 th-grade female $(16.1 \%)$, 10th-grade female $(16.2 \%)$, 
11 th-grade female $(15.1 \%)$, and 12 th-grade female $(17.9 \%)$ students, respectively. The prevalence of having drunk soda or pop one or more times per day was higher among black female (21.6\%) than white female (15.0\%) students.

During 2007-2015, a significant linear decrease occurred overall in the prevalence of having drunk soda or pop one or more times per day $(33.8 \%-20.4 \%)$. A significant quadratic trend was not identified. The prevalence of having drunk soda or pop one or more times per day decreased significantly from $2013(27.0 \%)$ to 2015 (20.4\%).

Across 36 states, the prevalence of having drunk soda or pop one or more times per day ranged from $11.9 \%$ to 32.4\% (median: 19.0\%) (Table 96). Across 18 large urban school districts, the prevalence ranged from $9.6 \%$ to $27.5 \%$ (median: 18.9\%).

\section{Drank Soda or Pop Two or More Times per Day}

Nationwide, $13.0 \%$ of students had drunk a can, bottle, or glass of soda or pop (not counting diet soda or diet pop) two or more times per day during the 7 days before the survey (Table 97). The prevalence of having drunk soda or pop two or more times per day was higher among male (15.6\%) than female (10.4\%) students; higher among white male (15.1\%) and Hispanic male (17.4\%) than white female (9.1\%) and Hispanic female (11.4\%) students, respectively; and higher among 9th-grade male (14.6\%), 10th-grade male (15.8\%), 11 th-grade male $(16.1 \%)$, and 12 th-grade male $(16.0 \%)$ than 9th-grade female $(10.0 \%)$, 10th-grade female $(10.2 \%)$, 11 th-grade female $(10.5 \%)$, and 12 th-grade female $(10.5 \%)$ students, respectively. The prevalence of having drunk soda or pop two or more times per day was higher among black female (15.0\%) than white female (9.1\%) students.

During 2007-2015, a significant linear decrease occurred overall in the prevalence of having drunk soda or pop two or more times per day (24.4\%-13.0\%). A significant quadratic trend was not identified. The prevalence of having drunk soda or pop two or more times per day decreased significantly from $2013(19.4 \%)$ to 2015 (13.0\%).

Across 36 states, the prevalence of having drunk soda or pop two or more times per day ranged from $7.3 \%$ to $23.3 \%$ (median: 11.8\%) (Table 98). Across 18 large urban school districts, the prevalence ranged from $6.0 \%$ to $19.2 \%$ (median: $12.0 \%$ ).

\section{Drank Soda or Pop Three or More Times per Day}

Nationwide, $7.1 \%$ of students had drunk a can, bottle, or glass of soda or pop (not counting diet soda or diet pop) three or more times per day during the 7 days before the survey (Table 97). The prevalence of having drunk soda or pop three or more times per day was higher among male $(8.3 \%)$ than female $(5.9 \%)$ students; higher among white male $(7.2 \%)$ and Hispanic male $(9.4 \%)$ than white female (4.7\%) and Hispanic female (6.7\%) students, respectively; and higher among 10 th-grade male $(9.1 \%)$ and 12 th-grade male $(8.4 \%)$ than 10 th-grade female $(6.0 \%)$ and 12 th-grade female $(5.5 \%)$ students, respectively. The prevalence of having drunk soda or pop three or more times per day was higher among black (9.5\%) and Hispanic (8.1\%) than white (5.9\%) students and higher among black female $(9.9 \%)$ than white female (4.7\%) students.

During 2007-2015, a significant linear decrease occurred overall in the prevalence of having drunk soda or pop three or more times per day $(14.4 \%-7.1 \%)$. A significant quadratic trend was not identified. The prevalence of having drunk soda or pop three or more times per day decreased significantly from 2013 (11.2\%) to 2015 (7.1\%).

Across 36 states, the prevalence of having drunk soda or pop three or more times per day ranged from $4.0 \%$ to $13.5 \%$ (median: 6.6\%) (Table 98). Across 18 large urban school districts, the prevalence ranged from $2.6 \%$ to $13.5 \%$ (median: $7.6 \%$ ).

\section{Did Not Drink Sports Drinks}

Nationwide, $42.4 \%$ of students had not drunk a can, bottle, or glass of a sports drink (not counting low-calorie sports drinks) during the 7 days before the survey (Table 99). The prevalence of not having drunk a sports drink was higher among female $(52.7 \%)$ than male $(32.3 \%)$ students; higher among white female $(55.8 \%)$, black female $(45.7 \%)$, and Hispanic female (45.2\%) than white male $(33.5 \%)$, black male (25.9\%), and Hispanic male (27.6\%) students, respectively; and higher among 9th-grade female (49.7\%), 10th-grade female $(51.4 \%), 11$ th-grade female $(53.4 \%)$, and 12 th-grade female $(57.1 \%)$ than 9 th-grade male $(31.3 \%)$, 10th-grade male $(31.2 \%)$, 11th-grade male $(29.8 \%)$, and 12 th-grade male $(36.9 \%)$ students, respectively. The prevalence of not having drunk a sports drink was higher among white (44.6\%) than black $(36.0 \%)$ and Hispanic (36.2\%) students, higher among white female (55.8\%) than black female (45.7\%) and Hispanic female $(45.2 \%)$ students, and higher among white male $(33.5 \%)$ than black male $(25.9 \%)$ and Hispanic male $(27.6 \%)$ students. The prevalence of not having drunk a sports drink was higher among 12 th-grade $(46.8 \%)$ than 9th-grade $(40.0 \%)$ and 10th-grade $(41.6 \%)$ students and higher among 12th-grade female $(57.1 \%)$ than 9th-grade female $(49.7 \%)$ and 10th-grade female (51.4\%) students. Because the question measuring the prevalence of not having drunk a sports drink was used for the first time in the 2015 national YRBS, long-term temporal trends and 2-year temporal changes are not available.

This question was not included in the standard questionnaire used in the state and large urban school district surveys in 2015. 
As a result, the range and median prevalence estimates across states and large urban school districts for the prevalence of not having drunk a sports drink are not available.

\section{Drank Sports Drinks One or More Times per Day}

Nationwide, $13.8 \%$ of students had drunk a can, bottle, or glass of a sports drink (not counting low-calorie sports drinks) one or more times per day during the 7 days before the survey (Table 99). The prevalence of having drunk a sports drink one or more times per day was higher among male $(18.7 \%)$ than female (8.8\%) students; higher among white male (18.1\%), black male (25.2\%), and Hispanic male (19.0\%) than white female (6.7\%), black female (14.3\%), and Hispanic female $(12.2 \%)$ students, respectively; and higher among 9th-grade male $(18.5 \%)$, 10th-grade male $(20.5 \%)$, 11th-grade male $(21.1 \%)$, and 12 th-grade male $(14.5 \%)$ than 9 th-grade female $(9.9 \%)$, 10th-grade female $(9.6 \%), 11$ th-grade female $(9.0 \%)$, and 12 th-grade female $(6.5 \%)$ students, respectively. The prevalence of having drunk a sports drink one or more times per day was higher among black (19.7\%) and Hispanic $(15.7 \%)$ than white (12.4\%) students, higher among black female $(14.3 \%)$ and Hispanic female $(12.2 \%)$ than white female $(6.7 \%)$ students, and higher among black male (25.2\%) than white male (18.1\%) students. The prevalence of having drunk a sports drink one or more times per day was higher among 9th-grade (14.4\%), 10th-grade (14.9\%), and 11 th-grade $(15.3 \%)$ than 12 th-grade $(10.6 \%)$ students, higher among 9 th-grade female $(9.9 \%)$ and 10 th-grade female $(9.6 \%)$ than 12 th-grade female $(6.5 \%)$ students, and higher among 10th-grade male (20.5\%) and 11th-grade male (21.1\%) than 12th-grade male (14.5\%) students. Because the question measuring the prevalence of having drunk a sports drink one or more times per day was used for the first time in the 2015 national YRBS, long-term temporal trends and 2-year temporal changes are not available.

This question was not included in the standard questionnaire used in the state and large urban school district surveys in 2015. As a result, the range and median prevalence estimates across states and large urban school districts for the prevalence of having drunk a sports drink one or more times per day are not available.

\section{Drank Sports Drinks Two or More Times per Day}

Nationwide, $8.3 \%$ of students had drunk a can, bottle, or glass of a sports drink (not counting low- calorie sports drinks) two or more times per day during the 7 days before the survey (Table 100). The prevalence of having drunk a sports drink two or more times per day was higher among male (11.2\%) than female (5.4\%) students; higher among white male (9.0\%), black male (21.4\%), and Hispanic male (13.0\%) than white female $(3.5 \%)$, black female $(10.1 \%)$, and Hispanic female (7.9\%) students, respectively; and higher among 9th-grade male $(10.6 \%)$, 10th-grade male $(12.8 \%), 11$ th-grade male $(12.4 \%)$, and 12 th-grade male $(9.0 \%)$ than 9 th-grade female $(5.4 \%), 10$ th-grade female $(5.8 \%), 11$ th-grade female (5.9\%), and 12th-grade female (4.1\%) students, respectively. The prevalence of having drunk a sports drink two or more times per day was higher among black (15.8\%) and Hispanic $(10.5 \%)$ than white $(6.3 \%)$ students, higher among black (15.8\%) than Hispanic (10.5\%) students, higher among black female (10.1\%) and Hispanic female (7.9\%) than white female (3.5\%) students, higher among black male (21.4\%) and Hispanic male (13.0\%) than white male (9.0\%) students, and higher among black male (21.4\%) than Hispanic male (13.0\%) students. The prevalence of having drunk a sports drink two or more times per day was higher among 10th-grade (9.2\%) and 11 th-grade $(9.3 \%)$ than 12 th-grade $(6.6 \%)$ students and higher among 10th-grade male $(12.8 \%)$ and 11 th-grade male $(12.4 \%)$ than 12 th-grade male $(9.0 \%)$ students. Because the question measuring the prevalence of having drunk a sports drink two or more times per day was used for the first time in the 2015 national YRBS, long-term temporal trends and 2-year temporal changes are not available.

This question was not included in the standard questionnaire used in the state and large urban school district surveys in 2015. As a result, the range and median prevalence estimates across states and large urban school districts for the prevalence of having drunk a sports drink two or more times per day are not available.

\section{Drank Sports Drinks Three or More Times per Day}

Nationwide, $4.8 \%$ of students had drunk a can, bottle, or glass of a sports drink (not counting low-calorie sports drinks) three or more times per day during the 7 days before the survey (Table 100). The prevalence of having drunk a sports drink three or more times per day was higher among male $(6.3 \%)$ than female $(3.1 \%)$ students; higher among white male (4.3\%), black male (13.1\%), and Hispanic male (8.7\%) than white female (1.9\%), black female (5.1\%), and Hispanic female $(5.4 \%)$ students, respectively; and higher among 10th-grade male $(8.3 \%), 11$ th-grade male $(6.6 \%)$, and 12 th-grade male $(5.5 \%)$ than 10 th-grade female $(3.4 \%)$, 11 th-grade female $(3.6 \%)$, and 12 th-grade female $(2.1 \%)$ students, respectively. The prevalence of having drunk a sports drink three or more times per day was higher among black (9.2\%) and Hispanic $(7.1 \%)$ than white $(3.1 \%)$ students, higher among black female (5.1\%) and Hispanic female (5.4\%) than white female (1.9\%) students, and higher among black male (13.1\%) and Hispanic male $(8.7 \%)$ than white male $(4.3 \%)$ students. The prevalence of having drunk a sports drink three or more times per day was higher among 10th-grade (5.8\%) than 9th-grade (4.2\%) and 
12th-grade (3.8\%) students, higher among 11th-grade (5.2\%) than 12th-grade (3.8\%) students, and higher among 10th-grade male $(8.3 \%)$ than 9 th-grade male $(5.0 \%)$ and 12 th-grade male $(5.5 \%)$ students. Because the question measuring the prevalence of having drunk a sports drink three or more times per day was used for the first time in the 2015 national YRBS, long-term temporal trends and 2-year temporal changes are not available.

This question was not included in the standard questionnaire used in the state and large urban school district surveys in 2015. As a result, the range and median prevalence estimates across states and large urban school districts for the prevalence of having drunk a sports drink three or more times per day are not available.

\section{Did Not Drink Water}

Nationwide, 3.5\% of students had not drunk water during the 7 days before the survey (Table 101). The prevalence of not having drunk water was higher among black $(8.7 \%)$ than white $(2.7 \%)$ and Hispanic $(3.3 \%)$ students, higher among black female $(9.0 \%)$ than white female $(2.5 \%)$ and Hispanic female $(2.8 \%)$ students, and higher among black male $(7.8 \%)$ than white male $(2.9 \%)$ and Hispanic male $(3.8 \%)$ students. The prevalence of not having drunk water was higher among 10th-grade female (4.4\%) than 11th-grade female $(2.0 \%)$ students. Because the question measuring the prevalence of not having drunk water was used for the first time in the 2015 national YRBS, long-term temporal trends and 2-year temporal changes are not available.

This question was not included in the standard questionnaire used in the state and large urban school district surveys in 2015. As a result, the range and median prevalence estimates across states and large urban school districts for the prevalence of not having drunk water are not available.

\section{Drank One or More Glasses of Water per Day}

Nationwide, $73.6 \%$ of students had drunk one or more glasses of water per day during the 7 days before the survey (Table 101). The prevalence of having drunk one or more glasses of water per day was higher among white $(76.6 \%)$ and Hispanic $(71.6 \%)$ than black $(60.2 \%)$ students, higher among white (76.6\%) than Hispanic (71.6\%) students, higher among white female (77.2\%) and Hispanic female (70.6\%) than black female $(58.4 \%)$ students, higher among white female $(77.2 \%)$ than Hispanic female $(70.6 \%)$ students, and higher among white male $(76.4 \%)$ and Hispanic male $(72.5 \%)$ than black male $(62.0 \%)$ students. The prevalence of having drunk one or more glasses of water per day was higher among 11th-grade $(75.0 \%)$ and 12 th-grade $(75.3 \%)$ than 9 th-grade $(71.2 \%)$ students and higher among 11th-grade female (76.0\%) than 9th-grade female $(70.9 \%)$ students. Because the question measuring the prevalence of having drunk one or more glasses of water per day was used for the first time in the 2015 national YRBS, long-term temporal trends and 2-year temporal changes are not available.

This question was not included in the standard questionnaire used in the state and large urban school district surveys in 2015. As a result, the range and median prevalence estimates across states and large urban school districts for the prevalence of having drunk one or more glasses of water per day are not available.

\section{Drank Two or More Glasses of Water per Day}

Nationwide, $64.3 \%$ of students had drunk two or more glasses of water per day during the 7 days before the survey (Table 102). The prevalence of having drunk two or more glasses of water per day was higher among 10th-grade male $(67.5 \%)$ than 10th-grade female $(60.6 \%)$ students. The prevalence of having drunk two or more glasses of water per day was higher among white (66.3\%) and Hispanic (63.7\%) than black (50.8\%) students, higher among white female $(65.7 \%)$ and Hispanic female $(62.7 \%)$ than black female (47.4\%) students, and higher among white male (67.2\%) and Hispanic male (64.7\%) than black male (54.1\%) students. The prevalence of having drunk two or more glasses of water per day was higher among 11th-grade $(65.8 \%)$ and 12th-grade $(66.6 \%)$ than 9th-grade $(61.3 \%)$ students, higher among 12th-grade (66.6\%) than 10th-grade $(63.9 \%)$ students, higher among 11 th-grade female $(66.1 \%)$ than 9 th-grade female $(61.3 \%)$ and 10th-grade female $(60.6 \%)$ students, and higher among 10th-grade male (67.5\%) and 12th-grade male (67.6\%) than 9th-grade male $(61.7 \%)$ students. Because the question measuring the prevalence of having drunk two or more glasses of water per day was used for the first time in the 2015 national YRBS, long-term temporal trends and 2-year temporal changes are not available.

This question was not included in the standard questionnaire used in the state and large urban school district surveys in 2015. As a result, the range and median prevalence estimates across states and large urban school districts for the prevalence of having drunk two or more glasses of water per day are not available.

\section{Drank Three or More Glasses of Water per Day}

Nationwide, $49.5 \%$ of students had drunk three or more glasses of water per day during the 7 days before the survey (Table 102). The prevalence of having drunk three or more glasses of water per day was higher among male $(51.0 \%)$ than female $(48.1 \%)$ students and higher among Hispanic male $(52.5 \%)$ than Hispanic female $(47.9 \%)$ students. The prevalence of having drunk three or more glasses of water per day was higher among white $(49.9 \%)$ and Hispanic $(50.3 \%)$ than black $(39.1 \%)$ students, higher among white female $(49.7 \%)$ and Hispanic female (47.9\%) than black female (35.7\%) students, and higher among Hispanic male (52.5\%) 
than black male (42.2\%) students. The prevalence of having drunk three or more glasses of water per day was higher among 11 th-grade female $(51.4 \%)$ than 10th-grade female (46.1\%) students. Because the question measuring the prevalence of having drunk three or more glasses of water per day was used for the first time in the 2015 national YRBS, long-term temporal trends and 2-year temporal changes are not available.

This question was not included in the standard questionnaire used in the state and large urban school district surveys in 2015. As a result, the range and median prevalence estimates across states and large urban school districts for the prevalence of having drunk three or more glasses of water per day are not available.

\section{Did Not Eat Breakfast}

Nationwide, $13.8 \%$ of students had not eaten breakfast during the 7 days before the survey (Table 103). The prevalence of having not eaten breakfast was higher among 9th-grade female $(14.8 \%)$ than 9 th-grade male $(10.9 \%)$ students. The prevalence of having not eaten breakfast was higher among black (18.0\%) and Hispanic (14.7\%) than white (12.0\%) students, higher among black female (19.3\%) than white female $(12.5 \%)$ students, and higher among black male $(16.8 \%)$ than white male $(11.3 \%)$ students. The prevalence of having not eaten breakfast was higher among 11th-grade male $(14.2 \%)$ and 12 th-grade male $(14.8 \%)$ than 9 th-grade male $(10.9 \%)$ students.

During 2011-2015, significant linear and quadratic trends were not identified. The prevalence of having not eaten breakfast did not change significantly from 2013 (13.7\%) to 2015 (13.8\%).

Across 33 states, the prevalence of having not eaten breakfast ranged from $10.3 \%$ to $20.1 \%$ (median: $14.6 \%$ ) (Table 104). Across 18 large urban school districts, the prevalence ranged from $11.6 \%$ to $23.8 \%$ (median: $16.4 \%$ ).

\section{Ate Breakfast on All 7 Days}

Nationwide, $36.3 \%$ of students had eaten breakfast on all 7 days before the survey (Table 103). The prevalence of having eaten breakfast on all 7 days was higher among male $(40.5 \%)$ than female $(32.1 \%)$ students; higher among white male $(43.3 \%)$, black male $(30.8 \%)$, and Hispanic male $(39.5 \%)$ than white female $(34.8 \%)$, black female $(24.7 \%)$, and Hispanic female (30.1\%) students, respectively; and higher among 9th-grade male (46.6\%), 10th-grade male $(42.3 \%)$, and 11 th-grade male $(37.1 \%)$ than 9 th-grade female $(31.9 \%)$, 10th-grade female $(31.1 \%)$, and 11 th-grade female (32.4\%) students, respectively. The prevalence of having eaten breakfast on all 7 days was higher among white (39.0\%) and Hispanic (34.8\%) than black (27.9\%) students, higher among white female $(34.8 \%)$ than black female $(24.7 \%)$ students, and higher among white male (43.3\%) and Hispanic male (39.5\%) than black male $(30.8 \%)$ students. The prevalence of having eaten breakfast on all 7 days was higher among 9th-grade $(39.6 \%)$ than 11 th-grade $(34.6 \%)$ and 12 th-grade $(33.8 \%)$ students; higher among 9th-grade male (46.6\%) than 10th-grade male (42.3\%), 11th-grade male (37.1\%), and 12th-grade male (34.7\%) students, and higher among 10th-grade male $(42.3 \%)$ than 11 th-grade male $(37.1 \%)$ and 12th-grade male $(34.7 \%)$ students.

During 2011-2015, significant linear and quadratic trends were not identified. The prevalence of having eaten breakfast on all 7 days did not change significantly from 2013 (38.1\%) to 2015 (36.3\%).

Across 33 states, the prevalence of having eaten breakfast on all 7 days ranged from $26.6 \%$ to $42.1 \%$ (median: $34.8 \%$ ) (Table 104). Across 18 large urban school districts, the prevalence ranged from $16.4 \%$ to $44.6 \%$ (median: $31.0 \%$ ).

\section{Physical Activity}

\section{Did Not Participate in at Least 60 Minutes of Physical Activity on at Least 1 Day}

Nationwide, $14.3 \%$ of students had not participated in at least 60 minutes of any kind of physical activity that increased their heart rate and made them breathe hard some of the time on at least 1 day during the 7 days before the survey (i.e., did not participate in at least 60 minutes of physical activity on at least 1 day) (Table 105). The prevalence of not having participated in at least 60 minutes of physical activity on at least 1 day was higher among female (17.5\%) than male (11.1\%) students; higher among white female (14.3\%), black female (25.2\%), and Hispanic female (19.2\%) than white male (8.8\%), black male (16.2\%), and Hispanic male (11.9\%) students, respectively; and higher among 9th-grade female (14.7\%), 10th-grade female $(15.8 \%), 11$ th-grade female $(18.2 \%)$, and 12 th-grade female $(21.4 \%)$ than 9 th-grade male $(9.5 \%)$, 10th-grade male $(10.4 \%), 11$ th-grade male $(12.4 \%)$, and 12 th-grade male $(12.4 \%)$ students, respectively. The prevalence of not having participated in at least 60 minutes of physical activity on at least 1 day was higher among black (20.4\%) and Hispanic $(15.6 \%)$ than white $(11.6 \%)$ students, higher among black (20.4\%) than Hispanic (15.6\%) students, higher among black female (25.2\%) and Hispanic female (19.2\%) than white female $(14.3 \%)$ students, higher among black female (25.2\%) than Hispanic female (19.2\%) students, and higher among black male $(16.2 \%)$ than white male (8.8\%) students. The prevalence of not having participated in at least 60 minutes of physical activity on at least 1 day was higher among 11th-grade (15.5\%) and 12th-grade $(16.9 \%)$ than 9th-grade $(12.0 \%)$ students, higher 
among 12th-grade (16.9\%) than 10th-grade (13.1\%) students, higher among 11 th-grade female $(18.2 \%)$ and 12 th-grade female (21.4\%) than 9th-grade female (14.7\%) students; higher among 12th-grade female $(21.4 \%)$ than 10th-grade female $(15.8 \%)$ students, and higher among 11 th-grade male $(12.4 \%)$ than 9th-grade male $(9.5 \%)$ students.

During 2011-2015, significant linear and quadratic trends were not identified in the prevalence of not having participated in at least 60 minutes of physical activity on at least 1 day. The prevalence of not having participated in at least 60 minutes of physical activity on at least 1 day did not change significantly from 2013 (15.2\%) to 2015 (14.3\%).

Across 37 states, the prevalence of not having participated in at least 60 minutes of physical activity on at least 1 day ranged from $10.7 \%$ to $22.9 \%$ (median: $15.9 \%$ ) (Table 106). Across 18 large urban school districts, the prevalence ranged from $13.2 \%$ to $30.1 \%$ (median: $21.6 \%$ ).

\section{Physically Active at Least 60 Minutes per Day on 5 or More Days}

Nationwide, $48.6 \%$ of students had been physically active doing any kind of physical activity that increased their heart rate and made them breathe hard some of the time for a total of at least 60 minutes per day on 5 or more days during the 7 days before the survey (i.e., physically active at least 60 minutes per day on 5 or more days) (Table 105). The prevalence of having been physically active at least 60 minutes per day on 5 or more days was higher among male (57.8\%) than female (39.1\%) students; higher among white male $(62.0 \%)$, black male $(52.2 \%)$, and Hispanic male (53.5\%) than white female (43.5\%), black female (33.4\%), and Hispanic female (33.1\%) students, respectively; and higher among 9th-grade male (62.3\%), 10th-grade male (58.7\%), 11th-grade male $(56.3 \%)$, and 12 th-grade male $(53.3 \%)$ than 9th-grade female (43.9\%), 10th-grade female (41.9\%), 11th-grade female (36.6\%), and 12th-grade female (33.4\%) students, respectively. The prevalence of having been physically active at least 60 minutes per day on 5 or more days was higher among white (52.7\%) than black (43.5\%) and Hispanic (43.4\%) students, higher among white female (43.5\%) than black female (33.4\%) and Hispanic female $(33.1 \%)$ students, and higher among white male (62.0\%) than black male (52.2\%) and Hispanic male (53.5\%) students. The prevalence of having been physically active at least 60 minutes per day on 5 or more days was higher among 9th-grade (53.7\%) than 11th-grade (46.5\%) and 12th-grade (43.5\%) students, higher among 10th-grade (50.2\%) than 12th-grade (43.5\%) students, higher among 9 th-grade female (43.9\%) than 11th-grade female (36.6\%) and 12th-grade female (33.4\%) students, higher among 10th-grade female $(41.9 \%)$ than 12th-grade female $(33.4 \%)$ students, and higher among 9th-grade male (62.3\%) than 11 th-grade male $(56.3 \%)$ and 12 th-grade male $(53.3 \%)$ students.
During 2011-2015, significant linear and quadratic trends were not identified in the prevalence of having been physically active at least 60 minutes per day on 5 or more days. The prevalence of having been physically active at least 60 minutes per day on 5 or more days did not change significantly from 2013 (47.3\%) to 2015 (48.6\%).

Across 37 states, the prevalence of having been physically active at least 60 minutes per day on 5 or more days ranged from $34.2 \%$ to $54.0 \%$ (median: $45.3 \%$ ) (Table 106). Across 18 large urban school districts, the prevalence ranged from $28.1 \%$ to $51.1 \%$ (median: 36.1\%).

\section{Physically Active at Least 60 Minutes per Day on All 7 Days}

Nationwide, $27.1 \%$ of students had been physically active doing any kind of physical activity that increased their heart rate and made them breathe hard some of the time for a total of at least 60 minutes per day on each of the 7 days before the survey (i.e., physically active at least 60 minutes per day on all 7 days) (Table 107). The prevalence of having been physically active at least 60 minutes per day on all 7 days was higher among male (36.0\%) than female (17.7\%) students; higher among white male (38.5\%), black male (30.8\%), and Hispanic male (34.2\%) than white female (19.5\%), black female (16.6\%), and Hispanic female (14.7\%) students, respectively; and higher among 9 th-grade male (40.1\%), 10th-grade male (36.7\%), 11th-grade male (34.3\%), and 12th-grade male (32.6\%) than 9th-grade female (20.9\%), 10 th-grade female $(19.0 \%), 11$ th-grade female $(16.0 \%)$, and 12 th-grade female $(14.3 \%)$ students, respectively. The prevalence of having been physically active at least 60 minutes per day on all 7 days was higher among white (29.0\%) than black (24.2\%) and Hispanic (24.6\%) students, higher among white female $(19.5 \%)$ than Hispanic female (14.7\%) students, and higher among white male $(38.5 \%)$ than black male $(30.8 \%)$ students. The prevalence of having been physically active at least 60 minutes per day on all 7 days was higher among 9th-grade (31.0\%) than 10th-grade $(27.8 \%), 11$ th-grade $(25.3 \%)$, and 12 th-grade $(23.5 \%)$ students; higher among 10th-grade $(27.8 \%)$ than 12th-grade $(25.3 \%)$ students; higher among 9th-grade female $(20.9 \%)$ than 11 th-grade female $(16.0 \%)$ and 12 th-grade female (14.3\%) students; higher among 10th-grade female (19.0\%) than 12th-grade female (14.3\%) students; and higher among 9th-grade male $(40.1 \%)$ than 11 th-grade male $(34.3 \%)$ and 12 th-grade male (32.6\%) students.

During 2011-2015, significant linear and quadratic trends were not identified in the prevalence of having been physically active at least 60 minutes per day on all 7 days. The prevalence of having been physically active at least 60 minutes per day on all 7 days did not change significantly from 2013 (27.1\%) to 2015 (27.1\%). 
Across 37 states, the prevalence of having been physically active at least 60 minutes per day on all 7 days ranged from $19.5 \%$ to 32.2\% (median: 25.3\%) (Table 108). Across 18 large urban school districts, the prevalence ranged from $15.8 \%$ to $27.0 \%$ (median: 19.7\%).

\section{Participated in Muscle Strengthening Activities}

Nationwide, $53.4 \%$ of students had participated in muscle strengthening exercises (e.g., push-ups, sit-ups, or weight lifting) on 3 or more days during the 7 days before the survey (Table 107). The prevalence of having participated in muscle strengthening exercises on 3 or more days was higher among male $(63.7 \%)$ than female (42.7\%) students; higher among white male (63.0\%), black male $(69.8 \%)$, and Hispanic male $(64.4 \%)$ than white female $(46.1 \%)$, black female $(34.5 \%)$, and Hispanic female (39.9\%) students, respectively; and higher among 9th-grade male (64.9\%), 10th-grade male (67.3\%), 11th-grade male (62.5\%), and 12th-grade male $(59.9 \%)$ than 9th-grade female (48.2\%), 10th-grade female (43.0\%), 11th-grade female $(39.3 \%)$, and 12th-grade female $(39.9 \%)$ students, respectively. The prevalence of having participated in muscle strengthening exercises on 3 or more days was higher among white female $(46.1 \%)$ than black female $(34.5 \%)$ and Hispanic female (39.9\%) students and higher among black male $(69.8 \%)$ than white male $(63.0 \%)$ students. The prevalence of having participated in muscle strengthening exercises on 3 or more days was higher among 9 th-grade $(56.9 \%)$ and 10 th-grade $(54.9 \%)$ than 11 th-grade $(51.1 \%)$ and 12 th-grade (50.0\%) students; higher among 9th-grade female (48.2\%) than 10th-grade female (43.0\%), 11th-grade female (39.3\%), and 12th-grade female (39.9\%) students; and higher among 10th-grade male $(67.3 \%)$ than 11th-grade male $(62.5 \%)$ and 12th-grade male (59.9\%) students.

During 1991-2015, a significant linear increase occurred overall in the prevalence of having participated in muscle strengthening exercises on 3 or more days (47.8\%-53.4\%). A significant quadratic trend was not identified. The prevalence of having participated in muscle strengthening exercises on 3 or more days did not change significantly from 2013 (51.7\%) to 2015 (53.4\%).

This question was not included in the standard questionnaire used in the state and large urban school district surveys in 2015. Consequently, the range and median prevalence estimates across states and large urban school districts for the prevalence of having participated in muscle strengthening exercises on 3 or more days are not available.

\section{Used Computers 3 or More Hours per Day}

Nationwide, $41.7 \%$ of students played video or computer games or used a computer for something that was not school work for 3 or more hours per day on an average school day (i.e., used computers 3 or more hours per day) (Table 109). The prevalence of having used computers 3 or more hours per day was higher among 9th-grade female (48.7\%) than 9th-grade male $(42.5 \%)$ students. The prevalence of having used computers 3 or more hours per day was higher among black (44.6\%) and Hispanic (46.2\%) than white (38.6\%) students, higher among black female (48.4\%) and Hispanic female (47.4\%) than white female $(38.3 \%)$ students, and higher among Hispanic male (45.1\%) than white male (38.9\%) students. The prevalence of having used computers 3 or more hours per day was higher among 9th-grade $(45.4 \%)$ than 11 th-grade $(37.2 \%)$ and 12 th-grade $(40.5 \%)$ students; higher among 10 th-grade $(43.4 \%)$ than 11th-grade (37.2\%) students; higher among 9th-grade female $(48.7 \%)$ than 10 th-grade female $(43.3 \%)$, 11th-grade female (38.1\%), and 12th-grade female (40.4\%) students; higher among 10th-grade female (43.3\%) than 11th-grade female (38.1\%) students; and higher among 9th-grade male (42.5\%), 10th-grade male $(43.4 \%)$, and 12 th-grade male $(40.8 \%)$ than 11 th-grade male $(36.1 \%)$ students.

During 2003-2015, a significant linear increase occurred overall in the prevalence of having used computers 3 or more hours per day $(22.1 \%-41.7 \%)$. A significant quadratic trend also was identified. The prevalence of having used computers 3 or more hours per day increased during 2003-2009 (22.1\%$24.9 \%)$ and then increased more rapidly during 2009-2015 $(24.9 \%-41.7 \%)$. The prevalence of having used computers 3 or more hours per day did not change significantly from 2013 (41.3\%) to 2015 (41.7\%).

Across 36 states, the prevalence of having used computers 3 or more hours per day ranged from $30.1 \%$ to $45.6 \%$ (median: $38.7 \%$ ) (Table 110). Across 18 large urban school districts, the prevalence ranged from $33.8 \%$ to $47.7 \%$ (median: $41.7 \%$ ).

\section{Watched Television 3 or More Hours per Day}

Nationwide, $24.7 \%$ of students watched television 3 or more hours per day on an average school day (Table 109). The prevalence of having watched television 3 or more hours per day was higher among black (39.2\%) and Hispanic (28.2\%) than white $(20.0 \%)$ students, higher among black (39.2\%) than Hispanic (28.2\%) students, higher among black female $(41.5 \%)$ and Hispanic female $(29.2 \%)$ than white female $(18.8 \%)$ students, higher among black female $(41.5 \%)$ than Hispanic female (29.2\%) students, higher among black male (37.0\%) and Hispanic male (27.4\%) than white male (21.4\%) students, and higher among black male (37.0\%) than Hispanic male $(27.4 \%)$ students.

During 1999-2015, a significant linear decrease occurred overall in the prevalence of having watched television 3 or more hours per day $(42.8 \%-24.7 \%)$. A significant quadratic trend was not identified. The prevalence of having watched 
television 3 or more hours per day decreased significantly from $2013(32.5 \%)$ to $2015(24.7 \%)$.

Across 35 states, the prevalence of having watched television 3 or more hours per day ranged from $18.9 \%$ to $33.4 \%$ (median: 23.1\%) (Table 110). Across 18 large urban school districts, the prevalence ranged from $19.7 \%$ to $35.9 \%$ (median: $29.3 \%$ ).

\section{Attended Physical Education Classes}

Nationwide, $51.6 \%$ of students went to physical education (PE) classes on 1 or more days in an average week when they were in school (i.e., attended PE classes) (Table 111). The prevalence of having attended PE classes was higher among male $(55.3 \%)$ than female $(47.8 \%)$ students; higher among black male (65.4\%) and Hispanic male $(60.5 \%)$ than black female $(52.2 \%)$ and Hispanic female $(50.1 \%)$ students, respectively; and higher among 10th-grade male (61.3\%) and 12th-grade male $(42.9 \%)$ than 10th-grade female $(53.9 \%)$ and 12 th-grade female $(29.1 \%)$ students, respectively. The prevalence of having attended PE classes was higher among black (59.2\%) than white (48.4\%) students and higher among black male (65.4\%) and Hispanic male (60.5\%) than white male $(51.0 \%)$ students. The prevalence of having attended PE classes was higher among 9th-grade $(71.4 \%)$ than 10th-grade $(57.5 \%)$, 11th-grade $(38.5 \%)$, and 12 th-grade $(36.1 \%)$ students; higher among 10th-grade $(57.5 \%)$ than 11 th-grade $(38.5 \%)$ and 12 th-grade $(36.1 \%)$ students; higher among 9th-grade female $(70.4 \%)$ than 10th-grade female $(53.9 \%)$, 11 th-grade female $(34.6 \%)$, and 12th-grade female $(29.1 \%)$ students; higher among 10th-grade female $(53.9 \%)$ than 11 th-grade female $(34.6 \%)$ and 12 th-grade female $(29.1 \%)$ students; higher among 11 th-grade female $(34.6 \%)$ than 12th-grade female $(29.1 \%)$ students; higher among 9 th-grade male $(72.2 \%)$ than 10 th-grade male $(61.3 \%)$, 11th-grade male $(42.2 \%)$, and 12 th-grade male $(42.9 \%)$ students; and higher among 10th-grade male $(61.3 \%)$ than 11 th-grade male $(42.2 \%)$ and 12 th-grade male $(42.9 \%)$ students.

During 1991-2015, linear and quadratic trends were not identified in the prevalence of having attended PE classes. The prevalence of having attended PE classes also did not change significantly from 2013 (48.0\%) to 2015 (51.6\%).

Across 35 states, the prevalence of having attended PE classes ranged from $29.9 \%$ to $87.8 \%$ (median: $44.3 \%$ ) (Table 112). Across 14 large urban school districts, the prevalence ranged from $33.4 \%$ to $84.0 \%$ (median: $45.3 \%$ ).

\section{Attended Physical Education Classes Daily}

Nationwide, $29.8 \%$ of students went to physical education (PE) classes on all 5 days in an average week when they were in school (i.e., attended PE classes daily) (Table 111). The prevalence of having attended PE classes daily was higher among male (33.8\%) than female (25.5\%) students; higher among white male (29.6\%), black male $(38.9 \%)$, and Hispanic male $(42.4 \%)$ than white female $(21.1 \%)$, black female (32.2\%), and Hispanic female (33.0\%) students, respectively; and higher among 10th-grade male $(36.1 \%)$, 11th-grade male $(25.2 \%)$, and 12 th-grade male $(27.9 \%)$ than 10 th-grade female $(27.0 \%), 11$ th-grade female (18.1\%), and 12th-grade female (16.0\%) students, respectively. The prevalence of having attended PE classes daily was higher among black (35.8\%) and Hispanic (37.7\%) than white (25.4\%) students, higher among black female (32.2\%) and Hispanic female (33.0\%) than white female (21.1\%) students, and higher among black male (38.9\%) and Hispanic male (42.4\%) than white male (29.6\%) students. The prevalence of having attended PE classes daily was higher among 9th-grade (42.2\%) than 10th-grade (31.5\%), 11th-grade (21.8\%), and 12th-grade (21.9\%) students; higher among 10th-grade (31.5\%) than 11 th-grade $(21.8 \%)$ and 12th-grade $(21.9 \%)$ students; higher among 9 th-grade female $(39.5 \%)$ than 10 th-grade female $(27.0 \%), 11$ th-grade female (18.1\%), and 12th-grade female (16.0\%) students; higher among 10th-grade female (27.0\%) than 11th-grade female (18.1\%) and 12th-grade female $(16.0 \%)$ students, higher among 9th-grade male $(44.6 \%)$ than 10th-grade male $(36.1 \%)$, 11th-grade male (25.2\%), and 12th-grade male (27.9\%) students; and higher among 10th-grade male (36.1\%) than 11th-grade male (25.2\%) and 12th-grade $(27.9 \%)$ male students.

During 1991-2015, a significant linear trend was not identified in the prevalence of having attended PE classes daily. A significant quadratic trend was identified. The prevalence of having attended PE classes daily decreased during 1991-1995 (41.6\%-25.4\%) and then did not change during 1995-2015 (25.4\%-29.8\%). The prevalence of having attended PE classes daily also did not change significantly from $2013(29.4 \%)$ to 2015 (29.8\%).

Across 35 states, the prevalence of having attended PE classes daily ranged from $5.8 \%$ to $60.7 \%$ (median: $23.0 \%$ ) (Table 112). Across 14 large urban school districts, the prevalence ranged from $8.6 \%$ to $42.7 \%$ (median: $22.9 \%$ ).

\section{Played on at Least One Sports Team}

Nationwide, $57.6 \%$ of students had played on at least one sports team (run by their school or community groups) during the 12 months before the survey (Table 113). The prevalence of having played on at least one sports team was higher among male $(62.2 \%)$ than female $(53.0 \%)$ students; higher among black male (66.5\%) and Hispanic male (56.3\%) than black female (47.7\%) and Hispanic female (40.7\%) students, respectively; and higher among 9th-grade male (68.1\%), 10th-grade male (63.5\%), and 11th-grade male $(62.3 \%)$ than 9th-grade female $(57.6 \%)$, 10th-grade female (55.1\%), and 11th-grade female (51.7\%) students, respectively. The prevalence of having played on at least one sports team was higher among white $(62.4 \%)$ and black $(57.6 \%)$ than Hispanic 
(48.5\%) students, higher among white female (60.7\%) than black female (47.7\%) and Hispanic female (40.7\%) students, and higher among white male (64.4\%) and black male (66.5\%) than Hispanic male (56.3\%) students. The prevalence of having played on at least one sports team was higher among 9th-grade (63.0\%) than 11th-grade $(57.0 \%)$ and 12 th-grade $(50.8 \%)$ students, higher among 10th-grade (59.2\%) and 11th-grade (57.0\%) than 12th-grade $(50.8 \%)$ students, higher among 9th-grade female $(57.6 \%)$ than 11 th-grade female $(51.7 \%)$ and 12 th-grade female (46.9\%) students, higher among 10th-grade female (55.1\%) than 12th-grade female $(46.9 \%)$ students, higher among 9th-grade male $(68.1 \%)$ than 11 th-grade male $(62.3 \%)$ and 12 th-grade male $(54.6 \%)$ students, and higher among 10 th-grade male $(63.5 \%)$ than 12th-grade male $(54.6 \%)$ students.

During 1999-2015, significant linear and quadratic trends were not identified in the prevalence of having played on at least one sports team. The prevalence of having played on at least one sports team also did not change significantly from 2013 (54.0\%) to 2015 (57.6\%).

Across 25 states, the prevalence of having played on at least one sports team ranged from $48.6 \%$ to $64.3 \%$ (median: $54.8 \%$ ) (Table 112). Across 15 large urban school districts, the prevalence ranged from $44.7 \%$ to $55.6 \%$ (median: $48.9 \%$ ).

\section{Obesity, Overweight, and Weight Control}

\section{Obesity}

Nationwide, $13.9 \%$ of students had obesity (Table 115). The prevalence of obesity was higher among male (16.8\%) than female $(10.8 \%)$ students; higher among white male (15.6\%) and Hispanic male (19.4\%) than white female (9.1\%) and Hispanic female (13.3\%) students, respectively; and higher among 9th-grade male (15.4\%), 10th-grade male (18.2\%), 11 th-grade male $(18.4 \%)$, and 12 th-grade male $(15.0 \%)$ than 9th-grade female $(10.3 \%)$, 10th-grade female $(12.1 \%)$, 11 th-grade female $(10.2 \%)$, and 12 th-grade female $(10.5 \%)$ students, respectively. The prevalence of obesity was higher among black (16.8\%) and Hispanic (16.4\%) than white (12.4\%) students, higher among black female (15.2\%) and Hispanic female (13.3\%) than white female (9.1\%) students, and higher among Hispanic male (19.4\%) than white male $(15.6 \%)$ students. The prevalence of obesity was higher among 10th-grade (15.2\%) than 12th-grade (12.7\%) students and higher among 11 th-grade male $(18.4 \%)$ than 9th-grade male $(15.4 \%)$ and 12 th-grade male (15.0\%) students.

During 1999-2015, a significant linear increase occurred in the prevalence of obesity $(10.6 \%-13.9 \%)$. A significant quadratic trend was not identified. The prevalence of obesity did not change significantly from 2013 (13.7\%) to 2015 (13.9\%).
Across 37 states, the prevalence of obesity ranged from $10.3 \%$ to $18.9 \%$ (median: $13.3 \%$ ) (Table 116). Across 19 large urban school districts, the prevalence ranged from $9.9 \%$ to $22.5 \%$ (median: 13.3\%).

\section{Overweight}

Nationwide, $16.0 \%$ of students were overweight (Table 115). The prevalence of overweight was higher among black female $(21.2 \%)$ than black male (13.6\%) students. The prevalence of overweight was higher among Hispanic (18.4\%) than white $(15.2 \%)$ students and higher among black female $(21.2 \%)$ and Hispanic female (20.0\%) than white female (14.6\%) students.

During 1999-2015, a significant linear increase occurred in the prevalence of overweight $(14.1 \%-16.0 \%)$. A significant quadratic trend was not identified. The prevalence of overweight did not change significantly from 2013 (16.6\%) to $2015(16.0 \%)$.

Across 37 states, the prevalence of overweight ranged from $13.3 \%$ to $18.2 \%$ (median: $15.3 \%$ ) (Table 116). Across 19 large urban school districts, the prevalence ranged from $13.2 \%$ to 21.6\% (median: 16.8\%).

\section{Described Themselves as Overweight}

Nationwide, $31.5 \%$ of students described themselves as slightly or very overweight (Table 117). The prevalence of students describing themselves as overweight was higher among female $(38.2 \%)$ than male $(25.3 \%)$ students; higher among white female $(35.7 \%)$, black female (34.9\%), and Hispanic female (45.3\%) than white male (24.9\%), black male (20.0\%), and Hispanic male (28.0\%) students, respectively; and higher among 9th-grade female (35.8\%), 10th-grade female (38.9\%), 11 th-grade female $(38.5 \%)$, and 12th-grade female $(39.6 \%)$ than 9th-grade male $(25.5 \%)$, 10th-grade male $(25.2 \%)$, 11 th-grade male $(25.2 \%)$, and 12 th-grade male $(25.0 \%)$ students, respectively. The prevalence of students describing themselves as overweight was higher among Hispanic (36.4\%) than white $(30.3 \%)$ and black $(27.0 \%)$ students, higher among Hispanic female (45.3\%) than white female (35.7\%) and black female $(34.9 \%)$ students, and higher among white male $(24.9 \%)$ and Hispanic male (28.0\%) than black male (20.0\%) students.

During 1991-2015, a significant linear decrease occurred overall in the prevalence of students describing themselves as overweight $(31.8 \%-31.5 \%)$. A significant quadratic trend also was identified. The prevalence of students describing themselves as overweight decreased during 1991-1995 (31.8\%-27.6\%) and then increased during 1995-2015 (27.6\%-31.5\%). The prevalence of students describing themselves as overweight did not change significantly from 2013 (31.1\%) to 2015 (31.5\%).

Across 32 states, the prevalence of students describing themselves as overweight ranged from $26.2 \%$ to $33.4 \%$ (median: 
30.6\%) (Table 118). Across 18 large urban school districts, the prevalence ranged from $24.2 \%$ to $35.9 \%$ (median: $29.8 \%$ ).

\section{Were Trying to Lose Weight}

Nationwide, $45.6 \%$ of students were trying to lose weight (Table 117). The prevalence of trying to lose weight was higher among female $(60.6 \%)$ than male $(31.4 \%)$ students; higher among white female (59.5\%), black female $(54.2 \%)$, and Hispanic female (66.4\%) than white male (28.8\%), black male (26.2\%), and Hispanic male (40.0\%) students, respectively; and higher among 9th-grade female (59.4\%), 10th-grade female $(59.3 \%), 11$ th-grade female $(61.7 \%)$, and 12 th-grade female $(62.6 \%)$ than 9 th-grade male $(31.1 \%), 10$ th-grade male $(32.0 \%)$, 11th-grade male $(31.0 \%)$, and 12 th-grade male $(31.9 \%)$ students, respectively. The prevalence of trying to lose weight was higher among white $(44.1 \%)$ and Hispanic (53.1\%) than black (39.4\%) students, higher among Hispanic $(53.1 \%)$ than white (44.1\%) students, higher among Hispanic female (66.4\%) than white female (59.5\%) and black female $(54.2 \%)$ students, and higher among Hispanic male (40.0\%) than white male $(28.8 \%)$ and black male $(26.2 \%)$ students.

During 1991-2015, a significant linear increase occurred overall in the prevalence of trying to lose weight $(41.8 \%$ 45.6\%). A significant quadratic trend was not identified. The prevalence of trying to lose weight did not change significantly from 2013 (47.7\%) to 2015 (45.6\%).

Across 30 states, the prevalence of trying to lose weight ranged from $40.7 \%$ to $49.5 \%$ (median: $45.2 \%$ ) (Table 118 ). Across 17 large urban school districts, the prevalence ranged from $41.1 \%$ to $55.1 \%$ (median: $45.9 \%$ ).

\section{Other Health-Related Topics}

\section{Ever Had Asthma}

Nationwide, $22.8 \%$ of students had ever been told by a doctor or nurse that they had asthma (i.e., ever had asthma) (Table 119). The prevalence of having ever had asthma was higher among white female (23.7\%) than white male (20.4\%) students. The prevalence of having ever had asthma was higher among black $(27.8 \%)$ than white $(22.1 \%)$ and Hispanic $(22.5 \%)$ students and higher among black male $(28.4 \%)$ than white male (20.4\%) and Hispanic male (23.0\%) students.

During 2003-2015, a significant linear increase occurred overall in the prevalence of having ever had asthma $(18.9 \%-$ $22.8 \%$ ). A significant quadratic trend was not identified. The prevalence of having ever had asthma also increased significantly from $2013(21.0 \%)$ to 2015 (22.8\%).

Across 29 states, the prevalence of having ever had asthma ranged from $19.3 \%$ to $31.3 \%$ (median: $24.3 \%$ ) (Table 120 ).
Across 17 large urban school districts, the prevalence ranged from $19.0 \%$ to $34.4 \%$ (median: $23.7 \%$ ).

\section{Saw a Dentist}

Nationwide, $74.4 \%$ of students saw a dentist for a check-up, exam, teeth cleaning, or other dental work during the 12 months before the survey (Table 119). The prevalence of having seen a dentist was higher among white (81.0\%) than black (62.5\%) and Hispanic (66.4\%) students, higher among white female $(80.9 \%)$ than black female $(64.2 \%)$ and Hispanic female $(68.1 \%)$ students and higher among white male $(81.3 \%)$ than black male (61.1\%) and Hispanic male (64.8\%) students. The prevalence of having seen a dentist was higher among 9 th-grade male $(76.5 \%)$ than 12 th-grade male $(70.5 \%)$ students. Because the question measuring the prevalence of having seen a dentist was used for the first time in the 2015 national YRBS, long-term temporal trends and 2-year temporal changes are not available.

Across 31 states, the prevalence of having seen a dentist ranged from $65.7 \%$ to $82.7 \%$ (median: $73.6 \%$ ) (Table 120 ). Across 17 large urban school districts, the prevalence ranged from $55.1 \%$ to $72.6 \%$ (median: $65.7 \%$ ).

\section{Eight or More Hours of Sleep}

Nationwide, $27.3 \%$ of students got 8 or more hours of sleep on an average school night (Table 121). The prevalence of getting 8 or more hours of sleep was higher among male (30.1\%) than female $(24.4 \%)$ students; higher among white male $(31.1 \%)$, black male $(25.6 \%)$, and Hispanic male $(32.9 \%)$ than white female $(24.9 \%)$, black female $(20.6 \%)$, and Hispanic female (26.8\%) students, respectively; and higher among 9th-grade male (39.3\%) and 10th-grade male $(33.8 \%)$ than 9 th-grade female $(29.1 \%)$ and 10 th-grade female $(23.1 \%)$ students, respectively. The prevalence of getting 8 or more hours of sleep was higher among white $(28.0 \%)$ and Hispanic (29.8\%) than black (23.5\%) students, higher among Hispanic female (26.8\%) than black female (20.6\%) students, and higher among white male $(31.1 \%)$ and Hispanic male $(32.9 \%)$ than black male $(25.6 \%)$ students. The prevalence of getting 8 or more hours of sleep was higher among 9 th-grade (34.4\%) than 10 th-grade $(28.4 \%)$, 11 th-grade $(22.9 \%)$, and 12th-grade (22.4\%) students; higher among 10th-grade (28.4\%) than 11th-grade (22.9\%) and 12th-grade (22.4\%) students; higher among 9th-grade female $(29.1 \%)$ than 10 th-grade female $(23.1 \%), 11$ th-grade female $(23.0 \%)$, and 12th-grade female (22.2\%) students; higher among 9 th-grade male $(39.3 \%)$ than 10 th-grade male $(33.8 \%)$, 11 th-grade male $(22.9 \%)$, and 12 th-grade male $(22.6 \%)$ students, and higher among 10 th-grade male $(33.8 \%)$ than 11 th-grade male $(22.9 \%)$ and 12 th-grade male $(22.6 \%)$ students. 
During 2007-2015, a significant linear decrease occurred overall in the prevalence of getting 8 or more hours of sleep $(31.1 \%-27.3 \%)$. A significant quadratic trend was not identified. The prevalence of getting 8 or more hours of sleep also decreased significantly from 2013 (31.7\%) to 2015 (27.3\%).

Across 30 states, the prevalence of getting 8 or more hours of sleep ranged from $17.5 \%$ to $38.2 \%$ (median: $26.5 \%$ ) (Table 122). Across 19 large urban school districts, the prevalence ranged from $14.4 \%$ to $31.3 \%$ (median: $20.6 \%$ ).

\section{Indoor Tanning Device Use}

Nationwide, $7.3 \%$ of students had used an indoor tanning device, such as a sunlamp, sunbed, or tanning booth (not counting getting a spray-on tan), one or more times during the 12 months before the survey (i.e., indoor tanning device use) (Table 123). The prevalence of indoor tanning device use was higher among female (10.6\%) than male (4.0\%) students; higher among white female $(15.2 \%)$ than white male $(3.7 \%)$ students; and higher among 9th-grade female (6.0\%), 10th-grade female (7.1\%), 11 th-grade female (14.0\%), and 12 th-grade female $(16.2 \%)$ than 9 th-grade male $(2.7 \%)$, 10 th-grade male $(3.5 \%)$, 11th-grade male $(3.9 \%)$, and 12th-grade male (5.8\%) students, respectively. The prevalence of indoor tanning device use was higher among white (9.4\%) than black (3.7\%) and Hispanic (4.7\%) students, higher among white female (15.2\%) and Hispanic female (5.8\%) than black female (2.1\%) students, and higher among white female $(15.2 \%)$ than Hispanic female $(5.8 \%)$ students. The prevalence of indoor tanning device use was higher among 11 th-grade $(9.0 \%)$ and 12 th-grade $(10.9 \%)$ than 9 th-grade (4.2\%) and 10th-grade (5.3\%) students, higher among 11 th-grade female $(14.0 \%)$ and 12th-grade female $(16.2 \%)$ than 9th-grade female $(6.0 \%)$ and 10th-grade female $(7.1 \%)$ students, and higher among 12th-grade male $(5.8 \%)$ than 9th-grade male $(2.7 \%)$ students.

During 2009-2015, a significant linear decreased occurred overall in the prevalence of indoor tanning device use $(15.6 \%-$ 7.3\%). A significant quadratic trend was not identified. The prevalence of indoor tanning device use also decreased significantly from 2013 (12.8\%) to 2015 (7.3\%).

This question was not included in the standard questionnaire used in the state and large urban school district surveys in 2015. As a result, the range and median prevalence estimates across states and large urban school districts for the prevalence of indoor tanning device use are not available.

\section{Had a Sunburn}

Nationwide, $55.8 \%$ of students had a sunburn (counting even a small part of their skin turning red or hurting for 12 hours or more after being outside in the sun or after using a sunlamp or other indoor tanning device) one or more times during the 12 months before the survey (i.e., had a sunburn) (Table 123). The prevalence of having had a sunburn was higher among female (59.8\%) than male $(52.0 \%)$ students; higher among white female $(77.7 \%)$ and Hispanic female (43.8\%) than white male (67.6\%) and Hispanic male (38.0\%) students, respectively; and higher among 9th-grade female $(60.0 \%), 10$ th-grade female $(58.9 \%)$, and 11 th-grade female $(60.7 \%)$ than 9 th-grade male $(50.4 \%)$, 10th-grade male $(52.9 \%)$, and 11 th-grade male (52.4\%) students, respectively. The prevalence of having had a sunburn was higher among white $(72.5 \%)$ and Hispanic (40.8\%) than black (15.0\%) students, higher among white (72.5\%) than Hispanic (40.8\%) students, higher among white female $(77.7 \%)$ and Hispanic female $(43.8 \%)$ than black female $(16.2 \%)$ students, higher among white female $(77.7 \%)$ than Hispanic female (43.8\%) students, higher among white male (67.6\%) and Hispanic male $(38.0 \%)$ than black male (13.4\%) students, and higher among white male (67.6\%) than Hispanic male (38.0\%) students. Because the question measuring the prevalence of having had a sunburn was used for the first time in the 2015 national YRBS, long-term temporal trends and 2-year temporal changes are not available.

This question was not included in the standard questionnaire used in the state and large urban school district surveys in 2015. As a result, the range and median prevalence estimates across states and large urban school districts for the prevalence of having had a sunburn are not available.

\section{Avoided Food Because of Allergic Reaction Risk}

Nationwide, $16.0 \%$ of students avoided some foods because eating the food could cause an allergic reaction (such as skin rashes, swelling, itching, vomiting, coughing, or trouble breathing) (Table 124). The prevalence of avoiding food because eating the food could cause an allergic reaction was higher among female (19.9\%) than male (12.0\%) students; higher among white female (19.5\%) and Hispanic female (18.2\%) than white male (11.3\%) and Hispanic male (10.2\%) students, respectively; and higher among 9th-grade female (24.3\%), 10th-grade female (17.6\%), 11 th-grade female $(19.0 \%)$, and 12 th-grade female $(18.8 \%)$ than 9th-grade male $(13.5 \%)$, 10th-grade male (12.5\%), 11th-grade male $(10.7 \%)$, and 12 th-grade male $(11.3 \%)$ students, respectively. The prevalence of avoiding food because eating the food could cause an allergic reaction was higher among black (20.7\%) than white (15.4\%) and Hispanic (14.1\%) students and higher among black male $(18.8 \%)$ than white male $(11.3 \%)$ and Hispanic male (10.2\%) students. The prevalence of avoiding food because eating the food could cause an allergic reaction was higher among 9th-grade (18.7\%) than 10th-grade (15.2\%), 11th-grade (14.8\%), 
and 12th-grade (14.9\%) students and higher among 9th-grade female $(24.3 \%)$ than 10th-grade female $(17.6 \%)$, 11th-grade female (19.0\%), and 12th-grade female (18.8\%) students. Because the question measuring the prevalence of avoiding food because eating the food could cause an allergic reaction was used for the first time in the 2015 national YRBS, long-term temporal trends and 2-year temporal changes are not available.

This question was not included in the standard questionnaire used in the state and large urban school district surveys in 2015. As a result, the range and median prevalence estimates across states and large urban school districts for the prevalence of avoiding food because eating the food could cause an allergic reaction are not available.

\section{Discussion}

YRBSS is the largest public health surveillance system in the United States monitoring a broad range of health behaviors among high school students. YRBSS data are used widely to compare the prevalence of health behaviors among subpopulations of students; assess trends in health behaviors over time; monitor progress toward achieving national health objectives; provide comparable state and large urban school district data; and help develop, assess, and improve school and community policies, programs, and practices designed to decrease health-risk behaviors and improve health outcomes among youth.

\section{Compare Health Behavior Prevalence Among Subpopulations of Students}

YRBSS is designed to identify how health behaviors vary by subpopulations of high school students defined by sex and race/ ethnicity. Understanding these variations (or lack of variation) in health behaviors might help design, target, and identify the impact of school and community policies, programs, and practices. However, YRBSS data cannot isolate the effects of sex and race/ethnicity from the effects of socioeconomic status (SES) or culture on the prevalence of health behaviors. For example, in a national study, the likelihood of behavioral cardiovascular disease risks, including obesity, sedentary behaviors, and tobacco exposure, increased among adolescents aged $12-17$ years as the SES based on poverty-income ratio decreased (14).

\section{Variations by Sex}

The prevalence of most health behaviors varies by sex. For example, the prevalence of two injury-related behaviors (rarely or never wearing a seatbelt and driving when drinking alcohol) was higher among male than female students. The prevalence of seven violence-related behaviors (carrying a weapon, carrying a gun, carrying a weapon on school property, being threatened or injured with a weapon on school property, being in a physical fight, being injured in a physical fight, and being in a physical fight on school property) was also higher among male than female students. However, the prevalence of having not gone to school because of safety concerns, being electronically bullied, being bullied on school property, being forced to have sexual intercourse, physical dating violence, and sexual dating violence was higher among female than male students. The prevalence of all five suicide-related behaviors (feeling sad or hopeless, seriously considering attempting suicide, having made a suicide plan, attempting suicide, and having made a suicide attempt resulting in an injury, poisoning, or overdose that had to be treated by a doctor or nurse) also was higher among female than male students. Rarely or never wearing a bicycle helmet, riding with a driver who had been drinking alcohol, and texting or e-mailing while driving did not vary by sex.

The prevalence of nine tobacco use risk behaviors (smoking a whole cigarette before age 13 years; current cigarette use; usually obtaining their own cigarettes by buying them in a store or gas station; current smokeless tobacco use; current cigar use; current electronic vapor product use; current cigarette or cigar use; current cigarette, cigar, or smokeless tobacco use; and current cigarette, cigar, smokeless tobacco, or electronic vapor product use) was higher among male than female students. Having tried to quit smoking cigarettes, the only health promoting tobacco use behavior, had a higher prevalence among female than male students. However, the prevalence of six tobacco use behaviors (ever trying cigarette smoking, current frequent cigarette use, smoking more than 10 cigarettes per day, currently smoking cigarettes daily, usually obtaining their own cigarettes by buying them on the Internet, and ever use of electronic vapor products) did not vary by sex.

The prevalence of 14 of the 21 alcohol and other drug use behaviors (drinking alcohol before age 13 years; having 10 or more as the largest number of drinks of alcohol in a row; trying marijuana before age 13 years; current marijuana use; ever using synthetic marijuana; ever using hallucinogenic drugs; ever using cocaine; ever using ecstasy; ever using heroin; ever using methamphetamines; ever taking steroids without a doctor's prescription; ever taking prescription drugs without a doctor's prescription; ever injecting any illegal drug; and being offered, sold, or given an illegal drug on school property) was higher among male than female students. However, the prevalence of ever drinking alcohol, usually obtaining the alcohol they drank by someone giving it to them, and usually using marijuana by smoking it was higher among female than male students. Four alcohol and other drug use behaviors (current alcohol use, having five or more drinks of alcohol in a row, ever using marijuana, and ever using inhalants) did not vary by sex.

The prevalence of four risk (ever having had sexual intercourse, having sexual intercourse before age 13 years, having sexual 
intercourse with four or more persons during their life, and drinking alcohol or using drugs before last sexual intercourse) and one protective (condom use) sexual behavior was higher among male than female students, while the prevalence of five protective sexual behaviors (birth control pill use; IUD or implant use; shot, patch, or birth control ring use; birth control pill, IUD or implant, or shot, patch, or birth control ring use; and both condom use and birth control pill; IUD or implant; or shot, patch, or birth control ring use) was higher among female than male students. Current sexual activity, not using any method to prevent pregnancy, and having ever been tested for HIV did not vary by sex.

The prevalence of 17 of the 26 dietary behaviors (not eating fruit or drinking 100\% fruit juices; eating fruit or drinking $100 \%$ fruit juices two or more times and three or more times per day; not eating vegetables; eating vegetables two or more times and three or more times per day; drinking one or more, two or more, and three or more glasses of milk per day; drinking soda or pop one or more, two or more, and three or more times per day; drinking sports drinks one or more, two or more, and three or more times per day; drinking three or more glasses of water per day; and eating breakfast on all 7 days) was higher among male than female students. In contrast, the prevalence of only three dietary behaviors (not drinking milk, not drinking soda or pop, and not drinking sports drinks) was higher among female than male students. Eating fruit or drinking 100\% fruit juices one or more times per day, eating vegetables one or more times per day, not drinking water, drinking one or more and two or more glasses of water per day, and not eating breakfast did not vary by sex.

The prevalence of six of the nine physical activity behaviors (being physically active at least 60 minutes per day on 5 or more days, being physically active at least 60 minutes per day on all 7 days, participating in muscle strengthening activities, attending physical education classes, attending physical education classes daily, and playing on at least one sports team) was higher among male than female students. Only one physical activity behavior (not participating in at least 60 minutes of physical activity on at least 1 day) had a higher prevalence among female than male students. Using computers 3 or more hours per day and watching television 3 or more hours per day did not vary by sex.

The prevalence of obesity was higher among male than female students, and the prevalence of overweight did not vary by sex. The prevalence of describing themselves as overweight and trying to lose weight was higher among female than male students. Male students had a higher prevalence than female students of getting eight or more hours of sleep on an average school night, while female students had a higher prevalence than male students of using an indoor tanning device, having had a sunburn, and avoiding foods because of the risk of allergic reactions. Ever having had asthma and having seen a dentist did not vary by sex.

\section{Variations by Race/Ethnicity}

The prevalence of most health behaviors varies by race/ethnicity. The prevalence of 24 behaviors (12 risk and 12 protective) was higher among white than black and Hispanic students, the prevalence of 12 risk behaviors was higher among black than white and Hispanic students, and the prevalence of 10 risk behaviors was higher among Hispanic than white and black students. Twentythree behaviors did not vary by race/ethnicity.

White students had a higher prevalence than black and Hispanic students of four injury-related risk behaviors (texting or e-mailing while driving, carrying a weapon, being electronically bullied, and bullying on school property), six tobacco-use related risk behaviors (current cigarette use; current frequent cigarette use; currently smoked cigarettes daily; current smokeless tobacco use; current cigarette or cigar use; and current cigarette, cigar, or smokeless tobacco use), three protective sexual behaviors (birth control pill use; birth control pill, IUD or implant, or shot, patch, or birth control ring use; and both condom use and birth control pill, IUD or implant, or shot, patch, or birth control ring use), six protective dietary behaviors (eating vegetables one or more times per day, drinking one or more and two or more glasses of milk per day, not drinking soda or pop, not drinking sports drinks, and drinking one or more glasses of water per day), two protective physical activity behaviors (being physically active at least 60 minutes per day on 5 or more days and being physically active at least 60 minutes per day on all 7 days), and three additional behaviors (having seen a dentist, indoor tanning device use, and having had a sunburn).

Black students had a higher prevalence than white and Hispanic students of two injury-related risk behaviors (rarely or never wearing a seatbelt and being in a physical fight), two sexual risk behaviors (having sexual intercourse before age 13 years and having sexual intercourse with four or more persons during their life), four dietary risk behaviors (not eating vegetables, not drinking milk, drinking sports drinks two or more times per day, and not drinking water), two physical activity risk behaviors (not participating in at least 60 minutes of physical activity on at least 1 day and watching television 3 or more hours per day), having had asthma, and avoiding food because eating the food could cause an allergic reaction.

Hispanic students had a higher prevalence than white and black students of riding with a driver who had been drinking alcohol, feeling sad or hopeless, ever using electronic vapor products, five alcohol or other drug use behaviors (drinking alcohol before age 13 years; ever using synthetic marijuana; ever using cocaine; ever using ecstasy; and being offered, sold or given an illegal drug on school property), describing themselves as overweight, and trying to lose weight. 
The prevalence of seven injury-related behaviors (driving when drinking alcohol, carrying a gun, carrying a weapon on school property, being forced to have sexual intercourse, physical dating violence, sexual dating violence, and having made a suicide plan), six tobacco-related behaviors (ever tried cigarette smoking, smoking a whole cigarette before age 13 years, smoking more than 10 cigarettes per day, usually obtaining their own cigarettes by buying them on the Internet, having tried to quit smoking cigarettes, and current cigar use), two drug-related behaviors (usually using marijuana by smoking it and ever using inhalants), three sexual behaviors (currently sexually active, IUD or implant use, and drinking alcohol or using drugs before last sexual intercourse), four dietary behaviors (not eating fruit or drinking $100 \%$ fruit juices, eating fruit or drinking $100 \%$ fruit juices one or more times per day, and drinking soda or pop one or more and two or more times per day), and participating in muscle strengthening exercises did not vary at all by race/ethnicity.

\section{Assess Trends in Health Behaviors Over Time}

Because YRBSS has been implemented since 1991, YRBSS data can be used to assess both long-term temporal trends (i.e., as long as 24 years) and more recent 2 -year temporal changes in health behaviors. This reports describes many long-term linear increases and decreases in prevalence, which reflect real reductions in risk behaviors and potential improvements in health outcomes among high school students nationwide.

Long-term linear decreases occurred overall in the prevalence of all three behaviors (for which long-term trend data were available) that contribute to unintentional injuries (rarely or never wore a bicycle helmet, rarely or never wore a seatbelt, and rode with a driver who had been drinking alcohol). However, based on significant quadratic trends, the prevalence of rarely or never wearing a bicycle helmet has not changed since 2001. Long-term linear decreases also occurred overall in the prevalence of eight of the 11 violence-related behaviors for which long-term trend data were available (carrying a weapon, carrying a gun, carrying a weapon on school property, being threatened or injured with a weapon on school property, being in a physical fight, being injured in a physical fight, being in a physical fight on school property, and being forced to have sexual intercourse). However, a long-term linear increase was identified in the prevalence of having not gone to school because of safety concerns; no long-term linear changes were identified in the prevalence of being electronically bullied and being bullied on school property; and, based on significant quadratic trends, the prevalence of carrying a weapon and carrying gun has not changed since 1997. In addition, long-term linear decreases occurred overall in the prevalence of three of the five suicide-related behaviors (seriously considering attempting suicide, having made a suicide plan, and attempting suicide). Based on significant quadratic trends, the prevalence of having made a suicide plan and attempting suicide increased since 2009 .

Long-term linear decreases occurred overall in the prevalence of nine of the 10 tobacco use-related risk behaviors (for which long-term trend data were available) (ever trying cigarette smoking; smoking a whole cigarette before age 13 years; current cigarette use; current frequent cigarette use; smoking more than 10 cigarettes per day; currently smoking cigarettes daily; current cigar use; current cigarette or cigar use; and current cigarette, cigar, or smokeless tobacco use). The prevalence of current smokeless tobacco use was the only tobacco-use related risk behavior for which a long-term linear decrease was not identified. In addition, a long-term linear decrease was identified in the prevalence of the one tobacco use-related protective behavior (having tried to quit smoking cigarettes).

Long-term linear decreases occurred overall in the prevalence of 13 of the 18 alcohol- and other drug use-related behaviors (for which long-term trend data were available) (ever drinking alcohol; drinking alcohol before age 13 years; current alcohol use; having five or more drinks of alcohol in a row; trying marijuana before age 13 years; ever using hallucinogenic drugs; ever using cocaine; ever using ecstasy; ever using heroin; ever using methamphetamines; ever taking prescription drugs without a doctor's prescription; ever using inhalants; and being offered, sold, or given an illegal drug on school property). No long-term linear trends were identified in the prevalence of usually obtaining the alcohol they drank by someone giving it to them, ever using marijuana, current marijuana use, ever taking steroids without a doctor's prescription, and ever injecting any illegal drug. However, based on significant quadratic trends, ever using marijuana decreased since 1997, current marijuana use decreased since 1995, ever taking steroids without a doctor's prescription decreased since 2001, and ever injecting any illegal drug decreased since 2011.

Long-term linear decreases occurred overall in the prevalence of five sexual risk behaviors (ever having had sexual intercourse, having sexual intercourse before age 13 years, having sexual intercourse with four or more partners, being currently sexually active, and not using any method to prevent pregnancy) and a long-term linear increase occurred overall in the prevalence of condom use; IUD or implant use; and birth control pill, IUD or implant, or shot, patch, or birth control ring use. However, although a long-term linear increase in the prevalence of condom use was identified, a significant quadratic trend indicated that the prevalence of condom use decreased since 2003. In addition, a long-term linear decrease occurred in the prevalence of having ever been tested for HIV.

Long-term linear decreases in the prevalence of not eating fruit or drinking $100 \%$ fruit juices and drinking soda or pop one or more, two or more, and three or more times per day and long-term 
linear increases in eating vegetables three or more times per day and not drinking soda or pop are positive trends. However, longterm linear decreases in the prevalence of eating fruit or drinking $100 \%$ fruit juices three or more times per day and long-term linear increases in not eating vegetables are negative trends. Similarly, the long-term linear decrease in the prevalence of watching television 3 or more hours per day which represents a decrease in sedentary behaviors is probably offset by the long-term linear increase in the prevalence of using computers three or more hours per day.

\section{Monitor Progress Toward Achieving National Health Objectives}

The national YRBS is the primary source of data to measure 21 Healthy People 2020 objectives, including one leading health indicator (15). The Healthy People 2020 objectives provide a comprehensive agenda for improving the health of all persons in the United States during 2011-2020. This report provides the Healthy People 2020 targets and data from the 2015 national YRBS for 18 of the 21 objectives measured using the national YRBS (Table 125). The data indicate that, as of 2015, seven of these 18 objectives have been achieved, which is nearly double the number met when the 2013 national YRBS data were reported in 2014 (16). Healthy People 2020 objective C-20.3 is to reduce the proportion of adolescents in grades $9-12$ who report using artificial sources of ultraviolet light for tanning to $\leq 14.0 \%$. During $2015,7.3 \%$ of high school students nationwide had used an indoor tanning device (e.g., sunlamp, sunbed, or tanning booth) one or more times during the 12 months before the survey. Healthy People 2020 objective IVP-34 is to reduce physical fighting among adolescents to $\leq 28.4 \%$. During 2015, 22.6\% of high school students nationwide had been in a physical fight one or more times during the 12 months before the survey. Healthy People 2020 objective IVP-36 is to reduce weapon carrying by adolescents on school property to $\leq 4.6 \%$. During 2015, 4.1\% of high school students nationwide had carried a weapon on school property on at least 1 day during the 30 days before the survey. Healthy People 2020 objective PA-8.2.3 is to increase the proportion of adolescents in grades 9 through 12 who view television, videos, or play video games for no more than 2 hours per day. During 2015,75.3\% of high school students nationwide watched television for no more than 2 hours per day on an average school day. Healthy People 2020 objective SA-1 is to reduce the proportion of adolescents who report that they rode, during the previous 30 days, with a driver who had been drinking alcohol to $\leq 25.5 \%$. During 2015, 20.0\% of high school students nationwide had ridden in a car or other vehicle driven by someone who had been drinking alcohol one or more times during the 30 days before the survey. Healthy People 2020 objective TU-2.1 is to reduce the proportion of adolescents who use tobacco products during the past 30 days to $\leq 21.0 \%$. During 2015, $17.0 \%$ of high school students nationwide smoked cigarettes; used chewing tobacco, snuff, or dip; or smoked cigars, cigarillos, or little cigars on at least one day during the 30 days before the survey. Healthy People 2020 objective TU-2.2 is to reduce the proportion of adolescents who use cigarettes during the past 30 days to $\leq 16.0 \%$. During $2015,10.8 \%$ of high school students smoked cigarettes on at least one day during the 30 days before the survey. Although Table 125 indicates that Healthy People 2020 objective PA-3.1 to increase the proportion of adolescents who meet current Federal physical activity guidelines for aerobic activity has been met, the 2015 YRBS prevalence estimate for aerobic physical activity is not comparable to the baseline prevalence estimate upon which the target was set because of a change in the context of the question starting with the 2011 national YRBS questionnaire.

To meet additional Healthy People 2020 objectives, positive changes in school and community policies, programs, and practices might be needed. For example, Healthy People 2020 objective IVP-35 is to reduce bullying among adolescents to $\leq 17.9 \%$. During 2015, 20.2\% of high school students nationwide were bullied on school property during the 12 months before the survey. Similarly, Healthy People 2020 objective SH-3 is to increase the proportion of students in grades 9 through 12 who get sufficient sleep to $\geq 33.2 \%$. During 2015, $27.3 \%$ of high school students nationwide got 8 or more hours of sleep on an average school night.

\section{Provide Comparable State and Large Urban School District Data}

One of the strengths of YRBSS is that it provides not just national but state and large urban school district data. These data are more likely to be used to develop, improve, and evaluate state and local policies, programs, and practices because they reflect a more relevant population. It is also possible to compare data from the state and large urban school district surveys because they share similar sample designs, questionnaires, data collection procedures, and data processing procedures.

Across states, a range of 25 or more percentage points or a fivefold variation or greater was identified for the following 15 behaviors:

- rarely or never wore a bicycle helmet (minimum: 53.0\%; maximum: 94.1\%);

- texted or e-mailed while driving (minimum: $26.1 \%$; maximum: 63.2\%);

- carried a weapon on school property (minimum: $2.0 \%$; maximum: $10.7 \%$ );

- smoked more than 10 cigarettes per day (minimum: 2.0\%; maximum: $14.6 \%$ ); 
- currently smoked cigarettes daily (minimum: $1.0 \%$; maximum: $5.4 \%$ );

- usually obtained their own cigarettes by buying them on the Internet (minimum: 0.0\%; maximum: 6.0\%);

- ever used heroin (minimum: 0.9\%; maximum: 5.9\%);

- ever injected any illegal drug (minimum: 1.0\%; maximum: $5.4 \%$ );

- IUD or implant use (minimum: 1.3\%; maximum: 9.7\%);

- shot, patch, or birth control ring use (minimum: 1.6\%; maximum: 9.5\%);

- birth control pill; IUD or implant; or shot, patch, or birth control ring use (minimum: 17.5\%; maximum: 47.3\%);

- ate vegetables one or more times per day (minimum: 46.7\%; maximum: 72.3\%);

- drank one or more glasses of milk per day (minimum: 23.0\%; maximum: 51.6\%);

- attended physical education classes (minimum: 29.9\%; maximum: 87.8\%); and

- attended physical education classes daily (minimum: 5.8\%; maximum: 60.7\%).

Across large urban school districts, a range of 25 or more percentage points or a fivefold variation or greater was identified for the following 16 behaviors:

- rarely or never wore a bicycle helmet (minimum: 55.1\%; maximum: 95.6\%);

- being in a physical fight (minimum: 13.9\%; maximum: $42.5 \%$ );

- smoked more than 10 cigarettes per day (minimum: 0.9\%; maximum: $12.8 \%$ );

- currently smoked cigarettes daily (minimum: 0.3\%; maximum: $1.6 \%$ );

- usually obtained their own cigarettes by buying them in a store or gas station (minimum: 14.9\%; maximum: 64.5\%);

- tried to quit smoking cigarettes (minimum: $35.7 \%$; maximum: $81.8 \%$ );

- ever used heroin (minimum: 1.5\%; maximum: 8.3\%);

- ever had sexual intercourse (minimum: 25.9\%; maximum: 52.4\%);

- IUD or implant use (minimum: 0.5\%; maximum: $16.9 \%$ );

- shot, patch, or birth control ring use (minimum: 1.1\%; maximum: $10.5 \%$ );

- birth control pill; IUD or implant; or shot, patch, or birth control ring use (minimum: 10.4\%; maximum: 37.6\%);

- ever been tested for HIV (minimum: 7.0\%; maximum: 37.4\%);

- drank soda or pop three or more times per day (minimum: 2.6\%; maximum: $13.5 \%$ );

- ate breakfast on all 7 days (minimum: 16.4\%; maximum: $44.6 \%$ );
- attended physical education classes (minimum: 33.4\%; maximum: $84.0 \%$ ); and

- attended physical education classes daily (minimum: 8.6\%; maximum: $42.7 \%$ ).

Across states, the health behaviors with a range of 25 or more percentage points or a five-fold variation or greater were fairly evenly distributed across the following categories of behaviors: injury and violence (three), cigarette use (three), other drug use (two), birth control use (three), dietary behaviors (two), and physical activity (two). In contrast, across the large urban school districts, $81 \%$ of the health behaviors with a range of 25 or more percentage points or a five-fold variation or greater were distributed across just three categories of behaviors: cigarette use (four), sexual behaviors (four), and dietary behaviors and physical activity (four). The variations in ranges might reflect differences in state and local laws and policies, enforcement practices, access to drugs, availability of effective school and community interventions, prevailing behavioral and social norms, demographic characteristics of the population, and adult practices and health behaviors and also highlight how changes in one or more of these factors might contribute to reductions in health-risk behaviors among high school students.

\section{Develop, Assess, and Improve Health- Related Policies, Programs, and Practices}

In addition to measuring progress toward achieving Healthy People 2020 objectives, CDC and other federal agencies use YRBS data in a variety or reports and publications to stimulate support for and improvements in public health interventions, including 2015 NCHHSTP State Health Profiles (17), Indicators for Chronic Disease Surveillance (18), America's Children: Key National Indicators of Well-Being, 2015 (19), and Prevention Status Reports (20). CDC also uses YRBS data to assess the impact of funding initiatives. More specifically, YRBS data are a primary data source for monitoring the impact of two major CDC cooperative agreements: Promoting Adolescent Health Through SchoolBased HIV/STD Prevention and School-Based Surveillance (http://www.cdc.gov/healthyyouth/fundedpartners/1308/ pdf/rfa-1308.pdf) and State Public Health Actions to Prevent and Control Diabetes, Heart Disease, Obesity and Associated Risk Factors and Promote School Health (http://www.cdc.gov/ chronicdisease/about/state-public-health-actions.htm).

At the state and local level, health and education agencies and nongovernmental organizations use YRBS data in a variety of ways to improve health-related policies, programs, and practices. For example, Connecticut's YRBS data on sexual behaviors were used by the Connecticut State Board of Education to support inclusion of comprehensive sexuality education in their position 
statement on coordinated school health and by the Connecticut Department of Education to help develop Guidelines for the Sexual Health Education Component of Comprehensive Health Education for local school districts on best practice policies, programs, and instruction in sexual health education. Boston Public Schools used their YRBS data on sexual behaviors in an annual presentation to the Boston City Council to demonstrate the need for sexual health education and services and to garner support for the District Wellness Policy requiring schools to teach comprehensive sexual health education and provide sexual health services, including distribution of condoms at the high school level. The Hawaii Department of Health used the dietary behavior data from their YRBS to support their public education campaign (on television, in malls, and in movie theatres) to reduce youth consumption of sugar-sweetened beverages and to track the impact of school wellness policies which restrict access to sugarsweetened beverages on campus and at school-sponsored events. The Los Angeles Unified School District used their YRBS data to support a new Los Angeles County ordinance on electronic vapor products and to support passage of a city-wide ordinance banning electronic vapor products from public spaces, bars, and restaurants. Montana YRBS data on bullying were used to support passage of a new Student Protections Procedures rule requiring schools to address bullying and threatening behavior in schools, on school buses, at school-sponsored activities, and online and to create a BullyFree Montana website and toolkit. The Bureau of Children, Youth, and Families used New York City YRBS data on attempted suicide to support a mental health awareness campaign for teens that included videos and other online resources on New York City's dedicated web site for teens and that is available as part of the Teen Talk Toolkit distributed to health educators in New York City public middle and high schools.

\section{Limitations}

The findings in this report are subject to at least four limitations. First, these data apply only to youth who attend school and, therefore, are not representative of all persons in this age group. Nationwide, in 2012, of persons aged 16-17 years, approximately $3 \%$ were not enrolled in a high school program and had not completed high school (21). Second, the extent of underreporting or overreporting of behaviors cannot be determined, although the survey questions demonstrate good test-retest reliability $(5,8)$. Third, BMI is calculated on the basis of self-reported height and weight, and, therefore, tends to underestimate the prevalence of obesity and overweight (22). Fourth, not all states and large urban school districts include all of the standard questions on their YRBS questionnaire.

\section{Conclusions}

YRBSS is an ongoing source of high-quality data at the national, state, and large urban school district levels for monitoring health behaviors that contribute to the leading causes of mortality and morbidity among youth and adults in the United States. In 2015, in addition to the national data, 37 states and 19 large urban school districts obtained data representative of their high school students. YRBSS data are an important tool for planning, implementing, and evaluating public health policies, programs, and practices in each of these jurisdictions. Although beyond the scope of this report, a particular strength of YRBSS (as compared to more narrowly focused surveys) is that it allows analysis of the interrelationships among health behaviors (e.g., how alcohol and other drug use is associated with sexual behaviors). Similarly, because of its long history and consistent methodology, YRBSS can identify not only national long-term temporal trends in health behaviors overall as described in this report, but also long-term trends among subgroups of students (e.g., by sex or race/ethnicity) and long-term temporal trends at the state and large urban school district levels. These trend analyses are particularly valuable for understanding the impact of broad public health and school health policies and practices designed to improve the health outcomes of students.

To maintain the quality of YRBSS data, increased support for YRBSS, enhanced training and technical assistance for participating state and local health and education agencies, an increase in the number of states with representative data, more substate surveys at the large urban school district and countyor school-district level, and more universal use of all standard questions are needed. Further, to sustain the surveillance system over time, it is important to understand the impact of new education and public health laws, policies, and practices related to data collection that might threaten the quality of YRBSS and its ability to generate critical data for informing future efforts to protect and promote the health of youth nationwide.

\section{References}

1. CDC. Underlying cause of death 1999-2014 on CDC WONDER online database, released 2015. Data are from the Multiple Cause of Death Files, 1999-2014, as compiled from data provided by the 57 vital statistics jurisdictions through the Vital Statistics Cooperative Program. http://wonder.cdc.gov/ucd-icd10.html

2. Martin JA, Hamilton BE, Osterman MJK, Curtin SC, Mathews TJ. Births: final data for 2013. National Vital Statistics Reports, 2015;64:166. http://www.cdc.gov/nchs/data/nvsr/nvsr64/nvsr64_01.pdf

3. CDC. Sexually transmitted disease surveillance 2014. Atlanta: US Department of Health and Human Services; 2015. http://www.cdc. gov/std/stats14/surv-2014-print.pdf

4. CDC. HIV surveillance report, 2014; vol. 26. http://www.cdc.gov/hiv/ library/reports/surveillance

5. Brener ND, Kann L, Kinchen S, et al. Methodology of the Youth Risk Behavior Surveillance System-2013. MMWR Recomm Rep 2013;62(No RR-1). 
6. US Department of Education, National Center for Education Statistics. Common core of data public elementary/secondary school universe survey: school year 2013-14. Washington, DC: US Department of Education, National Center for Education Statistics. http://nces.ed.gov/ccd

7. MDR National Education Database Master Extract, Shelton, CT: Market Data Retrieval, Inc.: April 29, 2014.

8. Brener ND, Kann L, McManus T, et al. Reliability of the 1999 Youth Risk Behavior Survey questionnaire. J Adolesc Health 2002;31:336-42.

9. Kuczmarski RJ, Ogden CL, Grummer-Strawn LM, et al. CDC growth charts: United States. In: Advance data from vital and health statistics, no. 314. Hyattsville, MD: National Center for Health Statistics; 2000.

10. SAS Institute, Inc. SAS, version 9.3 [software and documentation]. Cary, NC: SAS Institute; 2010.

11. Research Triangle Institute. SUDAAN, version 11.0.0 [software and documentation]. Research Triangle Park, NC: Research Triangle Institute; 2012.

12. Hinkle DE, Wiersma W, Jurs SG. Applied statistics for the behavioral sciences. 5th ed. Boston, MA: Houghton Mifflin Co; 2003.

13. National Cancer Institute. Joinpoint Regression Program, 2013. http:// surveillance.cancer.gov/joinpoint

14. Ali MK, McKeever Bullard K, Beckles GL, et al. Household income and cardiovascular disease risks in U.S. children and young adults. Diabetes Care 2011;34:1998-2004.
15. US Department of Health and Human Services. Office of Disease Prevention Health Promotion. Healthy people 2020. Washington, DC. http://www.healthypeople.gov

16. Kann L, Kinchen S, Shanklin SL, et al. Youth risk behavior surveillanceUnited States, 2013. MMWR Surveill Summ 2014;63(No. SS-4).

17. CDC. 2015 NCHHSTP State health profiles. http://www.cdc.gov/ nchhstp/stateprofiles/default.htm

18. CDC. Indicators for chronic disease surveillance-United States, 2013. MMWR Recomm Rep 2015;64(No. RR-1).

19. Federal Interagency Forum on Child and Family Statistics. America's children: key national indicators of well-being, 2015. Washington, DC: U.S. Government Printing Office; 2015. http://www.childstats.gov/ americaschildren/index.asp

20. CDC. Prevention status reports 2013. http://www.cdc.gov/psr/overview.html

21. Stark P, Noel AM. Trends in high school dropout and completion rates in the United States: 1972-2012 (NCES 2015-015). US Department of Education. Washington, DC: National Center for Education Statistics; 2015. http://nces.ed.gov/pubs2015/2015015.pdf

22. Brener ND, McManus T, Galuska DA, Lowry R, Wechsler H. Reliability and validity of self-reported height and weight among high school students. J Adolesc Health 2003;32:281-7.

\section{State and Large Urban School District Youth Risk Behavior Survey Coordinators}

States: Alabama, Sarah Khalidi, MPH, Department of Public Health; Alaska, Kathleen Oliver, Department of Health and Social Service; Arizona, Tori Havins, MPA, Department of Education; Arkansas, Kathleen Courtney, MS, Department of Education; California, Daniela Torres, MPH, Department of Education; Connecticut, Celeste Jorge, MPH, Department of Public Health; Delaware, Fred Breukelman, Department of Health and Social Services; Florida, Thomas Troelstrup, MPH, Department of Health; Hawaii, Robert Hesia, MA, Department of Education; Idaho, Lisa Kramer, Department of Education; Illinois, Jessica Gerdes, MS, State Board of Education; Indiana, Robyn Matthews, MPH, Department of Health; Kentucky, Stephanie Bunge, MEd, Department of Education; Maine, Jean Zimmerman, MS, Department of Education; Maryland, Robert Fiedler, JD, Department of Health \& Mental Hygiene; Massachusetts, Chiniqua Milligan, MPH, Department of Elementary and Secondary Education; Michigan, Kim Kovalchick, MPH, Department of Education; Mississippi, LaTina Morgan, MSEd, Department of Education; Missouri, Janet S. Wilson, MEd, Department of Health and Senior Services; Montana, Susan Court, Office of Public Instruction; Nebraska, Julane Hill, Department of Education; Nevada, Amberlee Baxa, MPH, Division of Public and Behavioral Health; New Hampshire, Irene Koffink, Department of Education; New Mexico, Cris Kimbrough, MA, Public Education Department; New York, Martha Morrissey, MA, State Department of Education; North Carolina, Ellen Essick, PhD, Department of Public Instruction; North Dakota, Valerie Fischer, MSEd, Department of Public Instruction; Oklahoma, Thad Burk, MPH, Department of Health; Pennsylvania, Nicholas Slotterback, MEd, Department of Education; Rhode Island, Tara Cooper, MPH, Department of Health; South Carolina, Sabrina Moore, PhD, Department of Education; South Dakota, Deni Hollingsworth, Department of Health; Tennessee, Mark Bloodworth, EdS (abd), Department of Education; Vermont, Kristen Murray, PhD, Department of Health; Virginia, Sarah Conklin, PhD, Department of Health; West Virginia, Birgit Shanholtzer, MA, Department of Education; Wyoming, Donal Mattimoe, Department of Education.

Large Urban School Districts: Baltimore, MD, Alexia Lotts-McCain, MEd, Baltimore City Public Schools; Boston, MA, Katia Miller, MPH, Boston Public Schools; Broward County, FL, Sebrina James, EdS, Broward County Public Schools; Cleveland, OH, Deborah Aloshen, MEd, Cleveland Metropolitan School District; DeKalb County, GA, Jessica Grippo, MPH, DeKalb County Board of Health; Detroit, MI, Arlene Richardson, EdD, Detroit Public Schools; District of Columbia, Omotunde Sowole-West, MPH, Office of the State Superintendent of Education; Duval County, FL, Jamie Wells, MSH, Duval County Public Schools; Fort Worth, TX, Edward Patterson, MS, Fort Worth Independent School District; Houston, TX, Felicia Ceasar-White, MS, Houston Independent School District; Los Angeles, CA, Timothy Kordic, MA, Los Angeles Unified School District; Miami, FL, Jonathan Carbone, Miami-Dade County Public Schools; New York City, NY, Lauren Murray, New York City Department of Health and Mental Hygiene; Oakland, CA, Ilsa Bertolini, Oakland Unified School District; Orange County, FL, Brenda Christopher-Muench, Orange County Public Schools; Palm Beach, FL, William Stewart, MPH, School District of Palm Beach County; Philadelphia, PA, Judith Peters, MBA, School District of Philadelphia; San Diego, CA, Rachel Miller, MEd, San Diego Unified School District; San Francisco, CA, Kim Levine, MHA, San Francisco Unified School District. 
TABLE 1. Number of states and large urban school districts that conducted a Youth Risk Behavior Survey and number with weighted and unweighted data, by year of survey — United States, Youth Risk Behavior Surveillance System, 1991-2015

\begin{tabular}{|c|c|c|c|c|c|c|}
\hline \multirow[b]{2}{*}{ Year } & \multicolumn{3}{|c|}{ Number of states } & \multicolumn{3}{|c|}{ Number of large urban school districts } \\
\hline & Total & Weighted & Unweighted & Total & Weighted & Unweighted \\
\hline 1991 & 26 & 9 & 17 & 11 & 7 & 4 \\
\hline 1993 & 40 & 22 & 18 & 14 & 9 & 5 \\
\hline 1995 & 39 & 22 & 17 & 17 & 12 & 5 \\
\hline 1997 & 38 & 24 & 14 & 17 & 15 & 2 \\
\hline 1999 & 41 & 22 & 19 & 17 & 14 & 3 \\
\hline 2001 & 37 & 22 & 15 & 19 & 14 & 5 \\
\hline 2003 & 43 & 32 & 11 & 22 & 20 & 2 \\
\hline 2005 & 44 & 40 & 4 & 23 & 21 & 2 \\
\hline 2007 & 44 & 39 & 5 & 22 & 22 & 0 \\
\hline 2009 & 47 & 42 & 5 & 23 & 20 & 3 \\
\hline 2011 & 47 & 43 & 4 & 22 & 21 & 1 \\
\hline 2013 & 47 & 42 & 5 & 22 & 21 & 1 \\
\hline 2015 & 47 & 37 & 10 & 21 & 19 & 2 \\
\hline
\end{tabular}


TABLE 2. Sample sizes, response rates, and demographic characteristics* — United States and selected U.S. sites, Youth Risk Behavior Survey, 2015

\begin{tabular}{|c|c|c|c|c|c|c|c|c|c|c|c|c|c|c|}
\hline \multirow[b]{2}{*}{ Site } & \multirow{2}{*}{$\begin{array}{c}\text { Student } \\
\text { sample size }\end{array}$} & \multicolumn{3}{|c|}{ Response rate (\%) } & \multicolumn{2}{|c|}{ Sex $(\%)$} & \multicolumn{4}{|c|}{ Grade (\%) } & \multicolumn{4}{|c|}{ Race/Ethnicity (\%) } \\
\hline & & School & Student & Overall & Female & Male & 9 & 10 & 11 & 12 & White $^{\dagger}$ & Black $^{\dagger}$ & Hispanic & Other $§$ \\
\hline National survey & 15,624 & 69 & 86 & 60 & 48.7 & 51.3 & 27.2 & 25.7 & 23.9 & 23.1 & 54.5 & 13.6 & 22.3 & 9.7 \\
\hline \multicolumn{15}{|l|}{ State surveys } \\
\hline Alabama & 1,565 & 92 & 74 & 68 & 49.4 & 50.6 & 27.2 & 26.1 & 23.7 & 22.8 & 57.2 & 33.9 & 5.3 & 3.7 \\
\hline Alaska & 1,418 & 98 & 64 & 62 & 48.1 & 51.9 & 27.5 & 25.8 & 23.7 & 22.5 & 48.4 & 2.8 & 7.6 & 41.2 \\
\hline Arizona & 2,582 & 75 & 83 & 62 & 49.2 & 50.8 & 27.2 & 25.6 & 23.9 & 23.0 & 42.0 & 5.4 & 42.4 & 10.1 \\
\hline Arkansas & 2,880 & 78 & 79 & 62 & 49.1 & 50.9 & 26.6 & 26.0 & 23.8 & 23.0 & 63.6 & 21.4 & 10.4 & 4.6 \\
\hline California & 1,943 & 75 & 89 & 66 & 48.8 & 51.2 & 26.7 & 25.8 & 24.1 & 23.3 & 26.5 & 3.3 & 50.6 & 19.7 \\
\hline Connecticut & 2,398 & 82 & 76 & 62 & 49.1 & 50.9 & 26.6 & 25.2 & 24.4 & 23.6 & 61.3 & 13.0 & 19.2 & 6.5 \\
\hline Delaware & 2,777 & 86 & 86 & 73 & 49.0 & 51.0 & 29.4 & 25.7 & 22.6 & 22.0 & 48.8 & 30.0 & 15.3 & 5.9 \\
\hline Florida & 6,359 & 95 & 75 & 72 & 49.6 & 50.4 & 27.1 & 26.0 & 24.2 & 22.4 & 42.3 & 21.8 & 29.8 & 6.2 \\
\hline Hawaii & 6,089 & 100 & 78 & 78 & 50.1 & 49.9 & 28.8 & 25.0 & 22.7 & 23.2 & 14.4 & 0.6 & 8.9 & 76.1 \\
\hline Idaho & 1,760 & 94 & 81 & 77 & 48.4 & 51.6 & 27.2 & 26.0 & 24.2 & 22.5 & 78.3 & 1.0 & 15.8 & 5.0 \\
\hline Illinois & 3,282 & 76 & 80 & 61 & 49.1 & 50.9 & 26.8 & 25.4 & 24.1 & 23.4 & 55.9 & 14.8 & 22.4 & 6.8 \\
\hline Indiana & 1,912 & 73 & 82 & 60 & 49.1 & 50.9 & 26.0 & 25.4 & 24.8 & 23.8 & 74.6 & 10.4 & 8.8 & 6.2 \\
\hline Kentucky & 2,577 & 91 & 85 & 77 & 49.1 & 50.9 & 27.6 & 26.0 & 23.4 & 22.7 & 81.8 & 10.9 & 3.6 & 3.6 \\
\hline Maine & 9,605 & 85 & 78 & 66 & 48.4 & 51.6 & 25.3 & 25.0 & 25.0 & 24.3 & 91.3 & 1.4 & 2.3 & 5.0 \\
\hline Maryland & 55,596 & 100 & 82 & 82 & 49.2 & 50.8 & 28.1 & 25.1 & 23.5 & 22.8 & 41.9 & 34.7 & 12.8 & 10.5 \\
\hline Massachusetts & 3,120 & 75 & 81 & 61 & 49.6 & 50.4 & 26.3 & 25.2 & 24.6 & 23.6 & 66.8 & 8.8 & 16.0 & 8.4 \\
\hline Michigan & 4,816 & 86 & 77 & 66 & 49.5 & 50.5 & 26.7 & 26.0 & 23.7 & 23.2 & 71.7 & 16.0 & 5.9 & 6.3 \\
\hline Mississippi & 2,154 & 90 & 79 & 71 & 50.1 & 49.9 & 28.8 & 26.2 & 23.3 & 21.4 & 50.0 & 46.6 & 1.4 & 2.0 \\
\hline Missouri & 1,502 & 73 & 87 & 63 & 51.2 & 48.8 & 26.5 & 25.2 & 24.6 & 23.6 & 74.9 & 16.0 & 4.6 & 4.5 \\
\hline Montana & 4,486 & 91 & 87 & 79 & 48.2 & 51.8 & 26.7 & 25.4 & 24.3 & 23.5 & 82.3 & 0.6 & 3.8 & 13.3 \\
\hline Nebraska & 1,688 & 84 & 74 & 62 & 48.6 & 51.4 & 25.2 & 24.8 & 24.2 & 25.5 & 70.3 & 6.4 & 16.5 & 6.8 \\
\hline Nevada & 1,452 & 100 & 66 & 66 & 48.8 & 51.2 & 25.9 & 25.8 & 24.7 & 23.5 & 35.9 & 9.8 & 40.1 & 14.2 \\
\hline New Hampshire & 14,837 & 83 & 85 & 70 & 48.2 & 51.8 & 26.5 & 25.4 & 24.4 & 23.5 & 88.2 & 1.5 & 5.2 & 5.1 \\
\hline New Mexico & 8,304 & 94 & 78 & 73 & 49.1 & 50.9 & 29.4 & 26.1 & 23.2 & 21.0 & 24.4 & 1.6 & 60.4 & 13.6 \\
\hline New York & 10,834 & 84 & 76 & 64 & 49.3 & 50.7 & 27.2 & 25.6 & 23.2 & 23.4 & 51.2 & 16.8 & 21.4 & 10.6 \\
\hline North Carolina & 6,178 & 78 & 77 & 60 & 49.1 & 50.9 & 29.0 & 26.1 & 23.6 & 21.3 & 53.1 & 26.9 & 12.5 & 7.5 \\
\hline North Dakota & 2,121 & 90 & 90 & 81 & 48.5 & 51.5 & 25.4 & 25.4 & 24.6 & 24.4 & 81.7 & 1.6 & 3.8 & 12.9 \\
\hline Oklahoma & 1,611 & 82 & 85 & 69 & 51.2 & 48.8 & 27.3 & 25.9 & 24.0 & 21.9 & 53.2 & 8.9 & 13.2 & 24.7 \\
\hline Pennsylvania & 2,899 & 80 & 80 & 64 & 49.0 & 51.0 & 25.9 & 25.0 & 24.3 & 24.2 & 71.8 & 14.7 & 8.6 & 4.9 \\
\hline Rhode Island & 3,462 & 88 & 77 & 68 & 48.9 & 51.1 & 25.7 & 23.8 & 25.5 & 24.4 & 63.2 & 7.9 & 22.1 & 6.8 \\
\hline South Carolina & 1,358 & 74 & 85 & 63 & 49.4 & 50.6 & 30.0 & 25.9 & 22.0 & 22.0 & 54.4 & 37.0 & 6.1 & 2.6 \\
\hline South Dakota & 1,313 & 84 & 79 & 67 & 48.7 & 51.3 & 27.3 & 26.0 & 23.4 & 22.9 & 76.8 & 1.2 & 4.4 & 17.6 \\
\hline Tennessee & 4,138 & 100 & 82 & 82 & 48.0 & 52.0 & 27.5 & 25.8 & 24.1 & 22.3 & 64.6 & 24.3 & 8.6 & 2.5 \\
\hline Vermont & 21,013 & 99 & 78 & 77 & 49.1 & 50.9 & 24.9 & 24.0 & 26.1 & 24.7 & 84.2 & 2.4 & 4.6 & 8.8 \\
\hline Virginia & 5,195 & 100 & 84 & 84 & 48.2 & 51.8 & 26.6 & 25.6 & 23.6 & 23.9 & 52.5 & 22.7 & 12.9 & 11.9 \\
\hline West Virginia & 1,622 & 100 & 77 & 77 & 49.1 & 50.9 & 28.0 & 25.4 & 23.7 & 22.7 & 91.2 & 5.2 & 1.5 & 2.0 \\
\hline Wyoming & 2,424 & 70 & 83 & 64 & 48.7 & 51.3 & 26.7 & 25.8 & 23.7 & 23.3 & 78.3 & 0.5 & 12.0 & 9.2 \\
\hline \multicolumn{15}{|c|}{ Large urban school district surveys } \\
\hline Baltimore, MD & 1,052 & 100 & 67 & 67 & 48.8 & 51.2 & 32.0 & 24.8 & 22.7 & 20.4 & 6.2 & 86.9 & 5.3 & 1.6 \\
\hline Boston, MA & 1,669 & 100 & 80 & 80 & 48.9 & 51.1 & 28.1 & 24.4 & 25.0 & 21.5 & 12.8 & 38.0 & 35.4 & 13.8 \\
\hline Broward County, FL & 1,413 & 97 & 74 & 72 & 48.7 & 51.3 & 26.0 & 25.0 & 24.5 & 24.2 & 26.0 & 38.2 & 29.2 & 6.7 \\
\hline Cleveland, $\mathrm{OH}$ & 1,705 & 100 & 66 & 66 & 47.5 & 52.5 & 30.3 & 27.4 & 21.4 & 20.5 & 8.7 & 59.4 & 20.9 & 11.1 \\
\hline DeKalb County, GA & 1,868 & 100 & 82 & 82 & 49.5 & 50.5 & 30.6 & 24.8 & 22.2 & 21.7 & 11.7 & 69.8 & 11.1 & 7.4 \\
\hline Detroit, MI & 1,699 & 100 & 67 & 67 & 54.5 & 45.5 & 28.6 & 27.4 & 22.0 & 21.9 & 0.3 & 85.5 & 10.5 & 3.7 \\
\hline District of Columbia & 10,419 & 92 & 69 & 64 & 50.8 & 49.2 & 33.7 & 24.0 & 22.0 & 19.8 & 5.5 & 72.5 & 15.7 & 6.3 \\
\hline Duval County, FL & 3,608 & 100 & 75 & 75 & 51.8 & 48.2 & 27.4 & 26.4 & 23.0 & 22.5 & 37.1 & 43.9 & 9.2 & 9.8 \\
\hline Ft. Worth, TX & 2,604 & 100 & 75 & 75 & 49.6 & 50.4 & 31.5 & 26.5 & 22.2 & 19.6 & 12.3 & 21.9 & 62.0 & 3.8 \\
\hline Houston, TX & 3,077 & 100 & 86 & 86 & 48.3 & 51.7 & 31.9 & 25.1 & 22.3 & 20.5 & 8.6 & 25.4 & 60.1 & 5.9 \\
\hline Los Angeles, CA & 2,336 & 100 & 81 & 81 & 51.1 & 48.9 & 30.1 & 24.9 & 22.8 & 22.1 & 7.8 & 10.1 & 73.8 & 8.4 \\
\hline Miami-Dade County, FL & 2,728 & 97 & 80 & 78 & 49.9 & 50.1 & 25.7 & 25.9 & 24.3 & 23.7 & 7.2 & 21.8 & 68.7 & 2.3 \\
\hline New York City, NY & 8,522 & 90 & 78 & 70 & 49.1 & 50.9 & 29.9 & 26.6 & 21.8 & 21.4 & 13.4 & 29.9 & 37.8 & 18.9 \\
\hline Oakland, CA & 1,669 & 100 & 72 & 72 & 46.5 & 53.5 & 25.3 & 24.9 & 24.7 & 24.8 & 7.4 & 33.7 & 38.7 & 20.2 \\
\hline Orange County, FL & 1,458 & 100 & 79 & 79 & 49.9 & 50.1 & 27.5 & 26.2 & 23.4 & 22.2 & 31.0 & 25.7 & 35.5 & 7.8 \\
\hline Palm Beach County, FL & 2,490 & 96 & 74 & 71 & 49.1 & 50.9 & 26.5 & 25.4 & 23.5 & 24.3 & 38.0 & 26.3 & 29.0 & 6.8 \\
\hline Philadelphia, PA & 1,717 & 100 & 68 & 68 & 49.9 & 50.1 & 28.4 & 27.2 & 22.1 & 22.0 & 15.3 & 51.4 & 20.1 & 13.2 \\
\hline San Diego, CA & 2,333 & 100 & 88 & 88 & 48.7 & 51.3 & 27.9 & 25.4 & 23.7 & 23.0 & 24.1 & 8.9 & 42.6 & 24.4 \\
\hline San Francisco, CA & 2,181 & 100 & 82 & 82 & 47.7 & 52.3 & 25.5 & 25.2 & 24.5 & 24.5 & 7.7 & 8.2 & 24.8 & 59.3 \\
\hline
\end{tabular}

* Weighted population estimates for the United States and each site.

+ Non-Hispanic.

$\S$ American Indian or Alaska Native, Asian, Native Hawaiian or other Pacific Islander, and multiple race (non-Hispanic). 
TABLE 3. Percentage of high school students who rarely or never wore a bicycle helmet* and who rarely or never wore a seat belt, ${ }^{\dagger}$ by sex, race/ethnicity, and grade - United States, Youth Risk Behavior Survey, 2015

\begin{tabular}{|c|c|c|c|c|c|c|c|c|c|c|c|c|}
\hline \multirow[b]{3}{*}{ Category } & \multicolumn{6}{|c|}{ Rarely or never wore a bicycle helmet } & \multicolumn{6}{|c|}{ Rarely or never wore a seat belt } \\
\hline & \multicolumn{2}{|c|}{ Female } & \multicolumn{2}{|c|}{ Male } & \multicolumn{2}{|c|}{ Total } & \multicolumn{2}{|c|}{ Female } & \multicolumn{2}{|c|}{ Male } & \multicolumn{2}{|c|}{ Total } \\
\hline & $\%$ & $\mathrm{Cl}^{\S}$ & $\%$ & $\mathrm{Cl}$ & $\%$ & $\mathrm{Cl}$ & $\%$ & $\mathrm{Cl}$ & $\%$ & $\mathrm{Cl}$ & $\%$ & $\mathrm{Cl}$ \\
\hline \multicolumn{13}{|c|}{ Race/Ethnicity } \\
\hline White & 75.3 & $(69.4-80.4)$ & 77.5 & (71.4-82.7) & 76.4 & (70.5-81.4) & 3.5 & $(2.3-5.5)$ & 5.3 & $(3.6-7.6)$ & 4.4 & $(3.1-6.3)$ \\
\hline Black ${ }^{9}$ & 82.6 & $(68.1-91.3)$ & 91.6 & $(86.9-94.7)$ & 88.2 & $(80.6-93.1)$ & 7.6 & $(4.7-12.1)$ & 12.4 & $(8.8-17.2)$ & 10.1 & $(7.1-14.2)$ \\
\hline Hispanic & 90.3 & (87.2-92.8) & 90.0 & (87.0-92.4) & 90.1 & $(87.6-92.1)$ & 6.3 & $(4.8-8.2)$ & 6.8 & $(5.7-8.1)$ & 6.5 & $(5.5-7.8)$ \\
\hline \multicolumn{13}{|l|}{ Grade } \\
\hline 9 & 78.3 & $(72.4-83.2)$ & 80.2 & $(74.3-85.1)$ & 79.4 & (74.3-83.7) & 5.5 & $(4.1-7.5)$ & 7.0 & $(5.7-8.7)$ & 6.3 & $(5.2-7.6)$ \\
\hline 10 & 81.9 & (76.5-86.3) & 80.4 & $(73.4-86.0)$ & 81.1 & (75.3-85.8) & 4.5 & $(3.0-6.6)$ & 7.6 & (5.4-10.7) & 6.0 & $(4.3-8.3)$ \\
\hline 11 & 78.5 & $(71.5-84.2)$ & 85.4 & $(81.0-88.9)$ & 82.3 & (76.6-86.8) & 4.1 & $(3.0-5.6)$ & 7.1 & $(4.6-10.8)$ & 5.8 & $(4.0-8.2)$ \\
\hline 12 & 82.1 & (76.8-86.4) & 84.9 & $(80.6-88.4)$ & 83.5 & (79.8-86.7) & 5.1 & $(3.5-7.4)$ & 6.1 & $(4.1-9.0)$ & 5.6 & $(4.0-7.7)$ \\
\hline Total & 80.1 & $(75.2-84.3)$ & 82.4 & $(78.2-86.0)$ & 81.4 & $(77.0-85.1)$ & 4.9 & $(3.8-6.3)$ & 7.2 & $(5.7-9.0)$ & 6.1 & $(4.9-7.6)$ \\
\hline
\end{tabular}

* Among the $68.0 \%$ of students nationwide who had ridden a bicycle during the 12 months before the survey.

t When riding in a car driven by someone else.

$\S 95 \%$ confidence interval.

" Non-Hispanic.

TABLE 4. Percentage of high school students who rarely or never wore a bicycle helmet* and who rarely or never wore a seat belt, ${ }^{\dagger}$ by sex selected U.S. sites, Youth Risk Behavior Survey, 2015

\begin{tabular}{|c|c|c|c|c|c|c|c|c|c|c|c|c|}
\hline \multirow[b]{3}{*}{ Site } & \multicolumn{6}{|c|}{ Rarely or never wore a bicycle helmet } & \multicolumn{6}{|c|}{ Rarely or never wore a seat belt } \\
\hline & \multicolumn{2}{|c|}{ Female } & \multicolumn{2}{|c|}{ Male } & \multicolumn{2}{|c|}{ Total } & \multicolumn{2}{|c|}{ Female } & \multicolumn{2}{|c|}{ Male } & \multicolumn{2}{|c|}{ Total } \\
\hline & $\%$ & $\mathrm{Cl}^{\S}$ & $\%$ & $\mathrm{Cl}$ & $\%$ & $\mathrm{Cl}$ & $\%$ & $\mathrm{Cl}$ & $\%$ & $\mathrm{Cl}$ & $\%$ & $\mathrm{Cl}$ \\
\hline \multicolumn{13}{|l|}{ State surveys } \\
\hline Alabama & 90.3 & $(86.1-93.3)$ & 89.6 & $(84.6-93.1)$ & 89.6 & $(86.5-92.0)$ & 6.3 & $(4.4-8.9)$ & 11.6 & $(8.8-15.1)$ & 9.1 & $(7.2-11.4)$ \\
\hline Alaska & 57.3 & $(51.3-63.0)$ & 70.6 & $(65.9-75.0)$ & 64.6 & (59.9-69.1) & 8.5 & $(6.5-11.0)$ & 10.2 & $(8.4-12.5)$ & 9.4 & (7.8-11.3) \\
\hline Arizona & -q & - & - & - & - & - & 8.1 & $(4.9-13.1)$ & 10.7 & $(7.5-15.1)$ & 9.5 & $(6.6-13.5)$ \\
\hline Arkansas & 90.9 & (83.6-95.1) & 92.3 & (88.0-95.2) & 91.7 & (87.5-94.5) & 6.1 & $(5.0-7.4)$ & 14.4 & $(9.2-22.1)$ & 10.4 & $(7.7-14.1)$ \\
\hline California & 67.3 & (55.3-77.4) & 68.3 & (57.0-77.8) & 67.6 & $(57.5-76.3)$ & 4.1 & $(2.8-5.9)$ & 3.1 & $(2.0-4.7)$ & 3.6 & $(2.5-5.1)$ \\
\hline Connecticut & - & - & - & - & - & - & 7.0 & $(5.4-9.0)$ & 9.4 & (7.2-12.3) & 8.3 & $(6.5-10.5)$ \\
\hline Delaware & 78.8 & $(74.8-82.3)$ & 83.6 & $(79.4-87.0)$ & 81.7 & (78.4-84.6) & 4.9 & $(3.7-6.6)$ & 7.1 & $(5.4-9.4)$ & 6.3 & $(5.1-7.7)$ \\
\hline Florida & 85.8 & (84.0-87.4) & 89.7 & $(88.1-91.1)$ & 87.9 & $(86.6-89.1)$ & 7.0 & $(6.2-7.8)$ & 9.8 & $(8.2-11.6)$ & 8.5 & (7.4-9.7) \\
\hline Hawaii & - & - & - & - & - & - & - & - & - & - & - & - \\
\hline Idaho & 83.1 & (79.9-85.9) & 80.9 & (74.9-85.8) & 81.9 & (78.0-85.2) & 4.4 & $(3.3-5.8)$ & 6.8 & $(4.8-9.6)$ & 5.7 & $(4.3-7.5)$ \\
\hline Illinois & 89.0 & (82.2-93.4) & 91.1 & (87.3-93.8) & 90.1 & (85.5-93.4) & 5.2 & $(3.7-7.2)$ & 7.1 & $(5.6-9.1)$ & 6.3 & $(4.9-8.0)$ \\
\hline Indiana & 91.5 & (87.2-94.4) & 86.2 & (78.8-91.3) & 88.7 & (83.4-92.5) & 4.1 & $(2.6-6.6)$ & 7.6 & $(5.4-10.6)$ & 5.9 & $(4.3-8.0)$ \\
\hline Kentucky & - & - & - & - & - & - & 6.6 & $(5.0-8.8)$ & 11.5 & $(8.9-14.8)$ & 9.1 & $(7.4-11.2)$ \\
\hline Maine & - & - & - & - & - & - & 4.6 & $(4.1-5.2)$ & 7.7 & $(6.4-9.1)$ & 6.3 & $(5.4-7.2)$ \\
\hline Maryland & - & - & - & - & - & - & - & - & - & - & - & - \\
\hline Massachusetts & - & - & - & - & - & - & - & - & - & - & - & - \\
\hline Michigan & 86.4 & (84.0-88.4) & 90.2 & (86.7-92.9) & 88.4 & (86.0-90.5) & 6.7 & $(5.2-8.8)$ & 6.5 & $(4.8-8.7)$ & 6.6 & $(5.5-7.9)$ \\
\hline Mississippi & 93.5 & (89.8-96.0) & 94.8 & (91.6-96.9) & 94.1 & (91.7-95.8) & 8.2 & $(6.5-10.4)$ & 13.6 & $(10.8-17.1)$ & 11.1 & $(9.2-13.3)$ \\
\hline Missouri & 82.9 & (74.5-88.9) & 88.1 & (84.1-91.2) & 85.7 & $(80.2-89.8)$ & 4.4 & $(3.3-6.0)$ & 11.9 & (8.4-16.8) & 8.1 & $(6.1-10.9)$ \\
\hline Montana & 81.0 & $(78.0-83.7)$ & 79.8 & (76.9-82.4) & 80.3 & (78.1-82.4) & 7.6 & (6.4-8.9) & 11.4 & $(9.5-13.6)$ & 9.5 & $(8.2-11.0)$ \\
\hline Nebraska & 83.5 & $(79.1-87.2)$ & 85.7 & $(82.0-88.8)$ & 84.8 & $(82.2-87.1)$ & 8.5 & $(6.0-12.0)$ & 13.8 & $(11.5-16.6)$ & 11.3 & (9.1-13.9) \\
\hline Nevada & 83.8 & (77.0-88.9) & 85.1 & (81.9-87.8) & 84.5 & (80.9-87.4) & 4.4 & $(3.1-6.1)$ & 7.6 & (5.9-9.6) & 6.2 & $(5.2-7.4)$ \\
\hline New Hampshire & 50.8 & $(48.1-53.5)$ & 60.5 & $(58.0-62.9)$ & 56.3 & $(54.3-58.2)$ & 6.6 & $(5.8-7.6)$ & 9.4 & $(8.6-10.4)$ & 8.2 & (7.5-8.8) \\
\hline New Mexico & 84.0 & $(81.6-86.1)$ & 84.9 & $(82.2-87.2)$ & 84.4 & $(82.3-86.3)$ & 4.4 & $(3.9-5.1)$ & 7.4 & $(6.5-8.4)$ & 6.0 & $(5.4-6.6)$ \\
\hline New York & 76.9 & $(70.1-82.5)$ & 79.3 & (75.8-82.4) & 78.1 & (73.5-82.0) & - & - & - & - & - & - \\
\hline North Carolina & 82.4 & (71.4-89.8) & 86.9 & (83.3-89.8) & 84.7 & (78.5-89.4) & 4.8 & $(2.8-8.3)$ & 8.0 & $(4.9-12.7)$ & 6.5 & $(3.9-10.4)$ \\
\hline North Dakota & - & - & - & - & - & - & 6.9 & $(4.9-9.6)$ & 10.1 & $(7.8-12.8)$ & 8.5 & $(7.0-10.3)$ \\
\hline Oklahoma & 89.6 & (84.1-93.4) & 93.9 & (91.7-95.6) & 92.1 & (88.8-94.5) & 4.4 & $(3.1-6.3)$ & 6.9 & (4.9-9.6) & 5.7 & $(4.4-7.4)$ \\
\hline Pennsylvania & 81.3 & (76.2-85.5) & 84.0 & (80.5-87.0) & 82.7 & (79.1-85.7) & 8.6 & $(6.7-11.0)$ & 13.0 & $(10.7-15.9)$ & 10.9 & $(9.2-12.9)$ \\
\hline Rhode Island & - & - & - & - & - & - & 4.1 & $(2.4-7.0)$ & 7.6 & $(5.8-9.8)$ & 5.9 & $(4.3-8.1)$ \\
\hline South Carolina & 92.2 & (88.2-95.0) & 91.6 & (88.8-93.7) & 91.9 & (89.2-93.9) & 4.3 & $(3.0-6.1)$ & 6.3 & $(4.3-9.2)$ & 5.3 & $(4.1-6.9)$ \\
\hline South Dakota & 92.5 & (86.3-96.0) & 91.4 & (86.2-94.7) & 91.9 & (87.6-94.8) & 10.0 & $(6.8-14.6)$ & 19.1 & $(14.3-25.1)$ & 14.6 & $(11.0-19.3)$ \\
\hline Tennessee & 86.7 & (81.8-90.5) & 89.4 & (87.1-91.4) & 88.1 & (85.4-90.3) & 5.3 & $(3.7-7.6)$ & 8.9 & (7.6-10.4) & 7.3 & $(6.0-8.7)$ \\
\hline Vermont & 49.3 & $(48.1-50.5)$ & 55.8 & $(54.7-56.9)$ & 53.0 & (52.2-53.8) & - & - & - & - & - & - \\
\hline Virginia & 76.0 & $(70.0-81.1)$ & 76.8 & $(71.9-81.1)$ & 76.5 & $(71.5-80.9)$ & 4.8 & $(3.9-6.0)$ & 7.6 & $(6.0-9.8)$ & 6.3 & $(5.3-7.6)$ \\
\hline
\end{tabular}

See table footnotes on the next page. 
TABLE 4. (Continued) Percentage of high school students who rarely or never wore a bicycle helmet* and who rarely or never wore a seat belt, ${ }^{\dagger}$ by sex — selected U.S. sites, Youth Risk Behavior Survey, 2015

\begin{tabular}{|c|c|c|c|c|c|c|c|c|c|c|c|c|}
\hline \multirow[b]{3}{*}{ Site } & \multicolumn{6}{|c|}{ Rarely or never wore a bicycle helmet } & \multicolumn{6}{|c|}{ Rarely or never wore a seat belt } \\
\hline & \multicolumn{2}{|c|}{ Female } & \multicolumn{2}{|c|}{ Male } & \multicolumn{2}{|c|}{ Total } & \multicolumn{2}{|c|}{ Female } & \multicolumn{2}{|c|}{ Male } & \multicolumn{2}{|c|}{ Total } \\
\hline & $\%$ & $\mathrm{Cl}^{\S}$ & $\%$ & $\mathrm{Cl}$ & $\%$ & $\mathrm{Cl}$ & $\%$ & $\mathrm{Cl}$ & $\%$ & $\mathrm{Cl}$ & $\%$ & $\mathrm{Cl}$ \\
\hline West Virginia & 85.3 & (78.4-90.3) & 83.4 & $(75.5-89.1)$ & 84.2 & (77.8-89.1) & 9.0 & $(6.5-12.2)$ & 13.4 & $(10.9-16.3)$ & 11.2 & $(9.4-13.4)$ \\
\hline Wyoming & 80.4 & $(75.0-84.9)$ & 79.8 & $(75.3-83.7)$ & 80.1 & (76.3-83.5) & 9.5 & $(7.6-11.9)$ & 11.7 & $(9.2-14.8)$ & 10.7 & $(9.1-12.6)$ \\
\hline Median & \multirow{2}{*}{\multicolumn{2}{|c|}{$\begin{array}{c}83.6 \\
(49.3-93.5)\end{array}$}} & \multirow{2}{*}{\multicolumn{2}{|c|}{$\begin{array}{c}85.4 \\
(55.8-94.8)\end{array}$}} & \multirow{2}{*}{\multicolumn{2}{|c|}{$\begin{array}{c}84.6 \\
(53.0-94.1)\end{array}$}} & \multirow{2}{*}{\multicolumn{2}{|c|}{$\begin{array}{c}6.2 \\
(4.1-10.0)\end{array}$}} & \multirow{2}{*}{\multicolumn{2}{|c|}{$\begin{array}{c}9.4 \\
(3.1-19.1)\end{array}$}} & \multirow{2}{*}{\multicolumn{2}{|c|}{$\begin{array}{c}8.1 \\
(3.6-14.6)\end{array}$}} \\
\hline Range & & & & & & & & & & & & \\
\hline \multicolumn{13}{|c|}{ Large urban school district surveys } \\
\hline Baltimore, MD & 91.6 & $(87.2-94.6)$ & 90.8 & (87.2-93.5) & 90.1 & $(87.1-92.5)$ & 12.8 & $(10.1-16.0)$ & 15.4 & $(12.2-19.3)$ & 14.3 & $(12.0-17.0)$ \\
\hline Boston, MA & 74.9 & $(68.8-80.1)$ & 83.3 & $(79.6-86.5)$ & 79.9 & (76.3-83.0) & 18.0 & $(15.5-20.8)$ & 21.2 & $(17.9-24.9)$ & 19.8 & $(17.5-22.2)$ \\
\hline Broward County, FL & 87.8 & (83.9-90.9) & 88.2 & $(84.4-91.2)$ & 87.9 & $(85.1-90.3)$ & 5.1 & $(3.6-7.1)$ & 9.4 & $(7.0-12.4)$ & 7.3 & $(5.8-9.2)$ \\
\hline Cleveland, $\mathrm{OH}$ & 96.2 & $(94.0-97.6)$ & 96.0 & $(93.9-97.4)$ & 95.6 & $(94.2-96.7)$ & 16.6 & $(13.8-19.7)$ & 23.6 & $(20.8-26.7)$ & 20.4 & $(18.3-22.7)$ \\
\hline DeKalb County, GA & 74.3 & $(66.6-80.7)$ & 83.8 & (79.1-87.6) & 80.1 & (75.4-84.1) & 7.2 & $(5.6-9.3)$ & 7.7 & $(5.7-10.5)$ & 7.6 & $(6.2-9.3)$ \\
\hline Detroit, MI & 83.2 & (78.4-87.1) & 90.7 & $(86.6-93.6)$ & 86.8 & (83.3-89.7) & 7.1 & $(5.7-8.9)$ & 11.9 & $(9.0-15.5)$ & 9.4 & $(7.7-11.4)$ \\
\hline District of Columbia & - & - & - & - & - & - & - & - & - & - & - & - \\
\hline Duval County, FL & 90.3 & (87.2-92.7) & 90.7 & (88.2-92.7) & 90.1 & $(87.6-92.1)$ & 7.9 & $(5.6-11.0)$ & 10.9 & $(8.7-13.7)$ & 9.7 & $(7.6-12.3)$ \\
\hline Ft. Worth, TX & 87.1 & $(84.0-89.7)$ & 91.8 & $(89.1-93.9)$ & 89.8 & $(87.7-91.5)$ & 4.7 & $(3.6-6.1)$ & 6.2 & $(4.8-8.0)$ & 5.5 & $(4.5-6.7)$ \\
\hline Houston, TX & 85.5 & $(82.8-87.8)$ & 88.2 & $(85.4-90.5)$ & 86.8 & (84.6-88.6) & 6.9 & $(5.8-8.3)$ & 9.3 & $(7.8-11.0)$ & 8.2 & $(7.2-9.4)$ \\
\hline Los Angeles, CA & 88.0 & $(83.0-91.7)$ & 84.0 & $(79.8-87.5)$ & 85.8 & $(83.0-88.1)$ & 5.4 & $(4.0-7.2)$ & 5.9 & $(3.8-9.1)$ & 5.6 & $(4.1-7.8)$ \\
\hline Miami-Dade County, FL & 89.6 & $(86.7-92.0)$ & 90.9 & $(88.8-92.6)$ & 90.4 & (88.7-91.9) & 7.0 & $(5.5-8.9)$ & 9.9 & $(8.0-12.2)$ & 8.7 & $(7.4-10.2)$ \\
\hline New York City, NY & 84.1 & $(79.1-88.1)$ & 86.7 & $(84.5-88.5)$ & 85.6 & (83.1-87.8) & - & - & - & - & - & - \\
\hline Oakland, CA & - & - & - & - & - & - & 9.8 & $(7.3-13.1)$ & 9.4 & $(7.2-12.0)$ & 9.8 & $(8.1-11.7)$ \\
\hline Orange County, FL & 86.5 & $(81.7-90.2)$ & 90.8 & $(87.7-93.1)$ & 88.9 & $(86.0-91.3)$ & 6.3 & $(4.5-8.7)$ & 9.1 & $(6.8-12.1)$ & 7.9 & $(6.1-10.0)$ \\
\hline Palm Beach County, FL & - & - & - & - & - & - & 7.5 & $(6.0-9.4)$ & 7.4 & $(6.2-8.8)$ & 7.7 & $(6.6-9.0)$ \\
\hline Philadelphia, PA & 88.0 & $(82.3-92.0)$ & 89.9 & $(87.5-91.8)$ & 89.0 & $(86.1-91.4)$ & 21.8 & $(17.7-26.5)$ & 21.8 & $(19.0-24.9)$ & 21.7 & $(18.8-25.0)$ \\
\hline San Diego, CA & 68.2 & $(62.1-73.7)$ & 73.8 & $(69.7-77.5)$ & 71.4 & $(67.5-75.1)$ & 2.6 & $(1.7-4.1)$ & 6.3 & $(4.7-8.5)$ & 4.5 & $(3.5-5.7)$ \\
\hline San Francisco, CA & 49.6 & $(43.7-55.5)$ & 58.6 & $(52.6-64.3)$ & 55.1 & $(50.4-59.6)$ & 6.6 & $(3.9-11.1)$ & 9.6 & $(7.1-12.9)$ & 8.2 & $(5.7-11.7)$ \\
\hline Median & \multicolumn{2}{|c|}{86.8} & \multirow{2}{*}{\multicolumn{2}{|c|}{89.0}} & \multirow{2}{*}{\multicolumn{2}{|c|}{87.3}} & \multirow{2}{*}{\multicolumn{2}{|c|}{$\begin{array}{c}7.1 \\
(2.6-21.8)\end{array}$}} & \multirow{2}{*}{\multicolumn{2}{|c|}{$\begin{array}{c}9.4 \\
(5.9-23.6)\end{array}$}} & \multirow{2}{*}{\multicolumn{2}{|c|}{$\begin{array}{c}8.2 \\
(4.5-21.7)\end{array}$}} \\
\hline Range & (49. & $5-96.2)$ & $(58.6$ & & & & & & & & & \\
\hline
\end{tabular}

* Among students who had ridden a bicycle during the 12 months before the survey.

+ When riding in a car driven by someone else.

$\S 95 \%$ confidence interval.

n Not available.

TABLE 5. Percentage of high school students who rode with a driver who had been drinking alcohol* and who drove when they had been drinking alcohol, ${ }^{*}+$ by sex, race/ethnicity, and grade — United States, Youth Risk Behavior Survey, 2015

\begin{tabular}{|c|c|c|c|c|c|c|c|c|c|c|c|c|}
\hline \multirow[b]{3}{*}{ Category } & \multicolumn{6}{|c|}{ Rode with a driver who had been drinking alcohol } & \multicolumn{6}{|c|}{ Drove when drinking alcohol } \\
\hline & \multicolumn{2}{|c|}{ Female } & \multicolumn{2}{|c|}{ Male } & \multicolumn{2}{|c|}{ Total } & \multicolumn{2}{|c|}{ Female } & \multicolumn{2}{|c|}{ Male } & \multicolumn{2}{|c|}{ Total } \\
\hline & $\%$ & $\mathrm{Cl}^{\S}$ & $\%$ & $\mathrm{Cl}$ & $\%$ & $\mathrm{Cl}$ & $\%$ & $\mathrm{Cl}$ & $\%$ & $\mathrm{Cl}$ & $\%$ & $\mathrm{Cl}$ \\
\hline \multicolumn{13}{|c|}{ Race/Ethnicity } \\
\hline White & 17.5 & $(14.8-20.7)$ & 17.7 & (15.7-19.8) & 17.7 & $(15.9-19.6)$ & 5.4 & $(3.9-7.5)$ & 9.4 & $(7.6-11.6)$ & 7.4 & $(5.9-9.3)$ \\
\hline Black 9 & 21.2 & $(15.9-27.9)$ & 20.6 & $(16.9-24.9)$ & 21.1 & $(17.2-25.6)$ & 5.1 & $(2.6-9.5)$ & 8.3 & $(6.0-11.5)$ & 6.9 & $(5.2-9.1)$ \\
\hline Hispanic & 27.3 & $(25.2-29.4)$ & 25.3 & $(22.8-27.9)$ & 26.2 & $(24.4-28.2)$ & 8.0 & $(6.0-10.6)$ & 10.7 & $(8.7-13.1)$ & 9.4 & $(7.8-11.3)$ \\
\hline \multicolumn{13}{|l|}{ Grade } \\
\hline 9 & 21.3 & (18.5-24.3) & 19.1 & (15.9-22.6) & 20.2 & (17.8-22.7) & 5.5 & $(3.5-8.3)$ & 5.7 & (3.9-8.2) & 5.6 & $(4.3-7.4)$ \\
\hline 10 & 18.4 & $(15.1-22.2)$ & 19.0 & $(16.4-21.8)$ & 18.7 & $(16.3-21.3)$ & 2.2 & $(1.3-3.8)$ & 8.2 & $(6.1-10.9)$ & 5.3 & $(3.9-7.1)$ \\
\hline 11 & 20.1 & $(17.6-22.9)$ & 20.4 & $(17.7-23.5)$ & 20.6 & (18.6-22.7) & 6.8 & $(4.9-9.3)$ & 10.3 & $(6.1-16.7)$ & 8.7 & $(6.2-12.1)$ \\
\hline 12 & 21.0 & $(17.9-24.4)$ & 19.9 & $(17.3-22.9)$ & 20.4 & $(18.2-22.8)$ & 8.0 & $(5.6-11.2)$ & 11.7 & $(9.0-15.0)$ & 9.9 & (7.9-12.3) \\
\hline Total & 20.2 & $(17.9-22.8)$ & 19.6 & $(18.2-21.1)$ & 20.0 & (18.4-21.6) & 6.0 & $(4.7-7.7)$ & 9.5 & (8.3-10.9) & 7.8 & $(6.8-9.0)$ \\
\hline
\end{tabular}

* In a car or other vehicle one or more times during the 30 days before the survey.

† Among the $61.4 \%$ of students nationwide who had driven a car or other vehicle during the 30 days before the survey.

$\S 95 \%$ confidence interval.

"Non-Hispanic. 
TABLE 6. Percentage of high school students who rode with a driver who had been drinking alcohol* and who drove when they had been drinking alcohol, ${ }^{*}+$ by sex - selected U.S. sites, Youth Risk Behavior Survey, 2015

\begin{tabular}{|c|c|c|c|c|c|c|c|c|c|c|c|c|}
\hline \multirow[b]{3}{*}{ Site } & \multicolumn{6}{|c|}{ Rode with a driver who had been drinking alcohol } & \multicolumn{6}{|c|}{ Drove when drinking alcohol } \\
\hline & \multicolumn{2}{|c|}{ Female } & \multicolumn{2}{|c|}{ Male } & \multicolumn{2}{|c|}{ Total } & \multicolumn{2}{|c|}{ Female } & \multicolumn{2}{|c|}{ Male } & \multicolumn{2}{|c|}{ Total } \\
\hline & $\%$ & $\mathrm{Cl}^{\S}$ & $\%$ & $\mathrm{Cl}$ & $\%$ & $\mathrm{Cl}$ & $\%$ & $\mathrm{Cl}$ & $\%$ & $\mathrm{Cl}$ & $\%$ & $\mathrm{Cl}$ \\
\hline \multicolumn{13}{|l|}{ State surveys } \\
\hline Alabama & 26.1 & (22.7-29.9) & 23.8 & (20.7-27.3) & 25.5 & (22.6-28.6) & 9.3 & $(6.7-12.9)$ & 11.6 & $(7.8-16.8)$ & 10.8 & $(8.0-14.4)$ \\
\hline Alaska & 15.2 & $(12.7-18.1)$ & 13.3 & $(11.2-15.8)$ & 14.3 & $(12.7-16.1)$ & 5.1 & $(3.3-7.7)$ & 5.7 & $(3.9-8.1)$ & 5.6 & $(4.3-7.3)$ \\
\hline Arizona & -9 & - & - & - & - & - & 7.7 & (3.4-16.5) & 10.9 & $(8.6-13.8)$ & 9.4 & $(6.1-14.1)$ \\
\hline Arkansas & 19.4 & $(16.2-23.2)$ & 22.8 & $(19.6-26.4)$ & 21.1 & $(19.0-23.5)$ & 4.9 & $(2.1-10.9)$ & 11.4 & $(8.0-15.9)$ & 8.2 & $(5.7-11.6)$ \\
\hline California & 22.6 & $(19.4-26.2)$ & 19.4 & $(15.7-23.7)$ & 20.9 & (18.5-23.5) & 6.2 & (3.9-9.8) & 7.6 & $(4.6-12.4)$ & 6.9 & $(4.5-10.6)$ \\
\hline Connecticut & 19.0 & $(16.5-21.9)$ & 17.9 & (15.3-20.8) & 18.7 & $(16.5-21.1)$ & 4.3 & $(2.7-6.7)$ & 9.8 & $(7.4-12.8)$ & 7.4 & $(5.9-9.2)$ \\
\hline Delaware & 16.4 & $(13.8-19.5)$ & 16.6 & (13.9-19.7) & 17.0 & (14.6-19.7) & 4.8 & $(3.3-7.0)$ & 8.0 & $(5.1-12.2)$ & 6.5 & $(4.7-8.8)$ \\
\hline Florida & 21.5 & (19.6-23.6) & 20.1 & (18.4-21.9) & 21.0 & (19.6-22.4) & 6.8 & $(5.7-8.2)$ & 10.3 & $(8.7-12.0)$ & 8.8 & $(7.7-10.0)$ \\
\hline Hawaii & - & - & - & - & - & - & - & - & - & - & - & - \\
\hline Idaho & 17.1 & $(14.0-20.7)$ & 15.7 & $(12.9-18.8)$ & 16.4 & $(14.1-19.1)$ & 3.5 & $(2.1-5.8)$ & 6.5 & $(4.3-9.6)$ & 5.1 & $(3.7-6.8)$ \\
\hline Illinois & 21.9 & $(18.0-26.4)$ & 22.7 & $(18.9-27.1)$ & 22.4 & (19.5-25.5) & 6.6 & $(4.8-9.0)$ & 9.7 & $(6.9-13.7)$ & 8.2 & $(6.4-10.4)$ \\
\hline Indiana & 18.7 & $(15.5-22.3)$ & 17.0 & $(14.0-20.5)$ & 17.9 & (15.5-20.6) & 6.2 & (3.6-10.4) & 6.6 & $(4.0-10.6)$ & 6.3 & (4.5-8.9) \\
\hline Kentucky & 12.9 & $(10.7-15.5)$ & 15.3 & $(13.1-17.7)$ & 14.2 & $(12.6-16.1)$ & 6.1 & $(4.4-8.3)$ & 5.4 & $(3.6-8.0)$ & 5.9 & $(4.6-7.5)$ \\
\hline Maine & - & - & - & - & - & - & 2.8 & $(2.2-3.6)$ & 5.7 & $(4.4-7.3)$ & 4.6 & $(3.8-5.5)$ \\
\hline Maryland & 18.6 & $(18.0-19.2)$ & 17.6 & $(17.0-18.2)$ & 18.2 & $(17.8-18.7)$ & 5.0 & $(4.5-5.6)$ & 8.6 & $(8.0-9.3)$ & 7.1 & $(6.6-7.6)$ \\
\hline Massachusetts & 18.5 & (16.2-21.0) & 17.7 & (15.3-20.4) & 18.2 & (16.3-20.4) & 6.5 & $(4.6-9.2)$ & 12.1 & $(9.3-15.7)$ & 9.4 & (7.4-11.7) \\
\hline Michigan & 18.2 & $(15.5-21.2)$ & 19.3 & (16.9-21.9) & 18.7 & $(17.0-20.6)$ & 3.0 & $(1.3-6.8)$ & 7.8 & $(4.3-13.6)$ & 5.4 & (3.3-8.9) \\
\hline Mississippi & 22.5 & (18.7-26.8) & 25.9 & $(22.8-29.3)$ & 24.3 & $(22.0-26.8)$ & 6.5 & $(3.7-11.3)$ & 7.8 & $(6.2-9.8)$ & 7.2 & (5.3-9.5) \\
\hline Missouri & 16.8 & (13.4-20.9) & 19.9 & (16.5-23.9) & 18.5 & (15.7-21.6) & 5.8 & $(2.7-12.0)$ & 7.7 & $(5.0-11.6)$ & 6.8 & $(4.0-11.4)$ \\
\hline Montana & 22.9 & (20.3-25.7) & 23.1 & $(21.2-25.1)$ & 23.0 & $(21.2-24.9)$ & 9.3 & $(7.5-11.5)$ & 12.3 & $(10.2-14.8)$ & 10.9 & $(9.2-12.8)$ \\
\hline Nebraska & 23.2 & (19.2-27.8) & 21.4 & (18.3-24.8) & 22.3 & (19.7-25.2) & 9.1 & $(6.0-13.5)$ & 11.2 & $(8.2-15.0)$ & 10.1 & $(7.6-13.3)$ \\
\hline Nevada & 25.1 & (20.9-29.7) & 20.9 & $(17.6-24.7)$ & 23.0 & $(20.0-26.4)$ & 6.0 & $(3.7-9.6)$ & 10.2 & $(7.0-14.6)$ & 8.2 & (5.8-11.3) \\
\hline New Hampshire & 15.9 & $(14.2-17.8)$ & 15.6 & $(14.4-16.9)$ & 15.8 & $(14.9-16.9)$ & 4.8 & $(4.0-5.8)$ & 7.6 & $(6.7-8.5)$ & 6.3 & $(5.7-7.1)$ \\
\hline New Mexico & 19.5 & $(17.8-21.3)$ & 19.7 & $(18.5-21.0)$ & 19.7 & (18.5-20.9) & 6.4 & $(5.0-8.1)$ & 8.2 & $(7.2-9.5)$ & 7.4 & $(6.5-8.4)$ \\
\hline New York & - & - & - & - & - & - & 5.5 & $(3.9-7.6)$ & 10.1 & $(7.3-13.7)$ & 8.0 & $(6.6-9.7)$ \\
\hline North Carolina & 15.3 & $(11.6-20.0)$ & 18.4 & $(14.6-23.1)$ & 16.9 & $(13.9-20.5)$ & 2.5 & $(1.1-5.5)$ & 6.0 & $(4.6-7.9)$ & 4.3 & $(3.3-5.6)$ \\
\hline North Dakota & 18.2 & $(15.7-21.1)$ & 17.2 & (14.9-19.8) & 17.7 & (15.8-19.8) & 5.5 & $(4.1-7.5)$ & 9.9 & $(7.5-12.9)$ & 7.8 & $(6.3-9.5)$ \\
\hline Oklahoma & 14.7 & (11.6-18.4) & 20.4 & $(16.6-24.8)$ & 17.4 & (14.7-20.4) & 4.0 & $(2.1-7.5)$ & 9.0 & $(6.0-13.2)$ & 6.4 & $(4.3-9.5)$ \\
\hline Pennsylvania & 18.0 & $(15.3-21.0)$ & 18.5 & (15.5-21.9) & 18.3 & (15.9-21.0) & 4.4 & $(2.7-7.0)$ & 6.3 & $(4.3-9.2)$ & 5.4 & $(4.1-7.2)$ \\
\hline Rhode Island & 18.8 & (15.4-22.8) & 15.9 & (12.9-19.5) & 17.5 & $(15.0-20.3)$ & - & - & - & - & - & - \\
\hline South Carolina & 18.2 & $(14.1-23.0)$ & 17.9 & $(13.0-24.0)$ & 18.0 & $(14.4-22.3)$ & 3.5 & $(1.0-11.3)$ & 5.5 & $(2.7-10.8)$ & 4.5 & $(2.2-9.1)$ \\
\hline South Dakota & 19.7 & $(15.5-24.7)$ & 20.5 & (15.6-26.4) & 20.1 & $(16.0-25.0)$ & 5.9 & $(3.8-8.8)$ & 9.7 & $(6.6-14.0)$ & 7.9 & $(5.8-10.8)$ \\
\hline Tennessee & 16.5 & $(14.8-18.4)$ & 16.2 & $(14.1-18.5)$ & 16.3 & $(14.7-18.1)$ & 4.2 & $(3.2-5.6)$ & 9.5 & $(7.3-12.3)$ & 7.1 & $(5.8-8.7)$ \\
\hline Vermont & 20.0 & (19.3-20.8) & 19.4 & $(18.6-20.1)$ & 19.8 & (19.2-20.3) & 5.0 & $(4.5-5.6)$ & 8.9 & $(8.2-9.6)$ & 7.2 & $(6.7-7.6)$ \\
\hline Virginia & 17.0 & (15.1-19.0) & 14.3 & $(12.7-16.1)$ & 15.6 & $(14.3-17.0)$ & 5.6 & $(4.0-7.8)$ & 8.2 & (6.4-10.4) & 7.0 & $(5.8-8.5)$ \\
\hline West Virginia & 16.5 & (14.6-18.7) & 16.9 & (13.9-20.5) & 16.7 & $(14.7-18.9)$ & 4.8 & (3.1-7.3) & 7.7 & $(4.8-12.2)$ & 6.3 & $(4.6-8.6)$ \\
\hline Wyoming & 20.2 & $(17.2-23.6)$ & 22.0 & (18.9-25.6) & 21.2 & $(18.8-23.8)$ & 8.3 & $(5.5-12.4)$ & 11.5 & $(8.4-15.5)$ & 9.9 & $(7.8-12.4)$ \\
\hline Median & \multicolumn{2}{|c|}{18.6} & \multicolumn{2}{|c|}{18.5} & \multicolumn{2}{|c|}{18.3} & \multirow{2}{*}{\multicolumn{2}{|c|}{$\begin{array}{c}5.5 \\
(2.5-9.3)\end{array}$}} & & & & 1 \\
\hline Range & $(12.9$ & $-26.1)$ & & $-25.9)$ & & $-25.5)$ & & & & -12.3) & & \\
\hline Large urban school di & & & & & & & & & & & & \\
\hline Baltimore, MD & 20.3 & $(16.8-24.4)$ & 23.5 & $(19.9-27.4)$ & 22.3 & (19.9-24.9) & 2.2 & $(0.9-5.4)$ & 7.6 & $(3.8-14.9)$ & 5.4 & $(3.1-9.2)$ \\
\hline Boston, MA & 20.0 & $(17.6-22.6)$ & 16.9 & (14.3-19.9) & 18.5 & (16.7-20.4) & - & - & - & - & - & - \\
\hline Broward County, FL & 20.8 & $(16.9-25.2)$ & 23.0 & (19.2-27.4) & 22.1 & $(19.1-25.3)$ & 5.0 & $(3.0-8.2)$ & 8.2 & $(5.6-11.9)$ & 6.8 & $(5.0-9.3)$ \\
\hline Cleveland, $\mathrm{OH}$ & 28.6 & $(25.3-32.2)$ & 26.8 & $(23.2-30.6)$ & 28.2 & $(25.6-31.0)$ & 5.7 & $(3.4-9.6)$ & 11.2 & $(7.7-16.1)$ & 9.3 & $(7.0-12.3)$ \\
\hline DeKalb County, GA & 19.0 & $(16.7-21.6)$ & 19.8 & $(17.1-22.8)$ & 19.5 & $(17.7-21.3)$ & 4.2 & $(2.8-6.3)$ & 6.0 & $(3.9-9.2)$ & 5.2 & $(3.7-7.2)$ \\
\hline Detroit, MI & 31.8 & $(28.2-35.6)$ & 30.9 & $(26.8-35.3)$ & 31.6 & $(28.7-34.6)$ & 2.5 & $(1.4-4.6)$ & 5.5 & $(3.5-8.6)$ & 4.4 & $(3.1-6.4)$ \\
\hline District of Columbia & 20.7 & $(18.4-20.7)$ & 19.5 & $(18.4-20.7)$ & 20.4 & $(19.6-21.2)$ & 6.1 & $(5.0-7.4)$ & 8.6 & $(7.4-10.0)$ & 7.8 & (6.9-8.8) \\
\hline Duval County, FL & 25.5 & (23.2-28.0) & 24.0 & $(21.1-27.1)$ & 25.3 & $(19.6-27.3)$ & 7.9 & $(5.9-7.4)$ & 8.4 & $(6.1-11.3)$ & 8.6 & $(7.0-10.7)$ \\
\hline Ft. Worth, TX & 28.9 & $(26.2-31.7)$ & 23.5 & $(21.0-26.3)$ & 26.2 & $(24.1-28.4)$ & 7.7 & $(5.9-10.5)$ & 11.6 & $(8.7-15.3)$ & 9.7 & $(7.8-12.1)$ \\
\hline Houston, TX & 28.4 & $(26.0-30.9)$ & 27.5 & $(25.0-30.1)$ & 28.1 & $(26.4-30.0)$ & 5.6 & $(4.1-7.6)$ & 7.8 & $(6.0-10.1)$ & 7.0 & $(5.7-8.6)$ \\
\hline Los Angeles, CA & 19.1 & $(16.4-22.2)$ & 20.6 & $(16.9-24.9)$ & 19.8 & (17.4-22.5) & 4.4 & $(1.7-11.0)$ & 7.6 & $(4.3-13.2)$ & 6.4 & $(3.4-11.5)$ \\
\hline Miami-Dade County, FL & 23.7 & $(20.6-27.1)$ & 23.4 & $(20.3-26.9)$ & 23.6 & $(21.0-26.3)$ & 6.1 & $(4.4-8.3)$ & 10.9 & $(8.6-13.7)$ & 8.6 & (7.0-10.4) \\
\hline New York City, NY & - & - & - & - & - & - & 5.6 & $(3.7-8.2)$ & 7.7 & $(6.1-9.6)$ & 7.1 & $(5.9-8.4)$ \\
\hline
\end{tabular}

See table footnotes on the next page. 
TABLE 6. (Continued) Percentage of high school students who rode with a driver who had been drinking alcohol* and who drove when they had been drinking alcohol, ${ }^{*}+$ by sex — selected U.S. sites, Youth Risk Behavior Survey, 2015

\begin{tabular}{|c|c|c|c|c|c|c|c|c|c|c|c|c|}
\hline \multirow[b]{3}{*}{ Site } & \multicolumn{6}{|c|}{ Rode with a driver who had been drinking alcohol } & \multicolumn{6}{|c|}{ Drove when drinking alcohol } \\
\hline & \multicolumn{2}{|c|}{ Female } & \multicolumn{2}{|c|}{ Male } & \multicolumn{2}{|c|}{ Total } & \multicolumn{2}{|c|}{ Female } & \multicolumn{2}{|c|}{ Male } & \multicolumn{2}{|c|}{ Total } \\
\hline & $\%$ & $\mathrm{Cl}^{\S}$ & $\%$ & $\mathrm{Cl}$ & $\%$ & $\mathrm{Cl}$ & $\%$ & $\mathrm{Cl}$ & $\%$ & $\mathrm{Cl}$ & $\%$ & $\mathrm{Cl}$ \\
\hline Oakland, CA & 21.2 & $(18.3-24.4)$ & 24.9 & $(21.6-28.4)$ & 23.1 & $(20.7-25.7)$ & 4.3 & $(2.6-7.2)$ & 6.0 & $(3.6-9.7)$ & 5.5 & $(3.9-7.8)$ \\
\hline Orange County, FL & 22.9 & $(19.8-26.3)$ & 21.0 & $(18.0-24.3)$ & 21.9 & (19.7-24.3) & 6.7 & $(4.4-10.1)$ & 9.5 & $(6.5-13.8)$ & 8.2 & $(6.0-11.0)$ \\
\hline Palm Beach County, FL & 20.5 & $(18.0-23.2)$ & 21.6 & $(19.2-24.2)$ & 21.6 & (19.9-23.4) & 8.0 & $(6.0-10.6)$ & 9.4 & $(7.4-11.8)$ & 9.0 & (7.4-10.9) \\
\hline Philadelphia, PA & 21.9 & $(18.1-26.2)$ & 19.4 & $(16.1-23.2)$ & 20.8 & (18.4-23.4) & 5.8 & $(3.3-10.2)$ & 4.8 & $(3.1-7.3)$ & 5.7 & $(3.8-8.4)$ \\
\hline San Diego, CA & 20.9 & (18.1-23.9) & 18.8 & $(16.6-21.3)$ & 19.8 & (17.8-22.0) & 4.0 & $(2.5-6.5)$ & 10.3 & $(7.9-13.2)$ & 7.4 & $(6.0-9.3)$ \\
\hline San Francisco, CA & 13.8 & $(11.3-16.6)$ & 13.0 & $(11.3-15.1)$ & 13.4 & $(11.8-15.3)$ & 4.6 & $(2.3-9.0)$ & 5.1 & $(2.9-9.0)$ & 4.9 & $(3.1-7.6)$ \\
\hline Median & \multicolumn{2}{|c|}{21.0} & \multicolumn{2}{|c|}{22.3} & \multicolumn{2}{|r|}{22.0} & \multicolumn{2}{|c|}{5.6} & \multirow{2}{*}{\multicolumn{2}{|c|}{$\begin{array}{c}8.0 \\
(4.8-11.6)\end{array}$}} & \multirow{2}{*}{\multicolumn{2}{|c|}{$\begin{array}{c}7.0 \\
(4.4-9.7)\end{array}$}} \\
\hline Range & & $-31.8)$ & & $-30.9)$ & & 4-31.6) & & $-8.0)$ & & & & \\
\hline
\end{tabular}

* In a car or other vehicle one or more times during the 30 days before the survey.

† Among students who had driven a car or other vehicle during the 30 days before the survey.

$\S 95 \%$ confidence interval.

" Not available.

TABLE 7. Percentage of high school students who texted or e-mailed while driving a car or other vehicle, ${ }^{*}$ by sex, race/ethnicity, and grade United States, Youth Risk Behavior Survey, 2015

\begin{tabular}{|c|c|c|c|c|c|c|}
\hline \multirow[b]{2}{*}{ Category } & \multicolumn{2}{|c|}{ Female } & \multicolumn{2}{|c|}{ Male } & \multicolumn{2}{|c|}{ Total } \\
\hline & $\%$ & $\mathrm{Cl}^{\dagger}$ & $\%$ & $\mathrm{Cl}$ & $\%$ & $\mathrm{Cl}$ \\
\hline \multicolumn{7}{|c|}{ Race/Ethnicity } \\
\hline White ${ }^{\S}$ & 45.3 & (41.3-49.4) & 45.0 & (39.8-50.3) & 45.2 & $(42.1-48.3)$ \\
\hline Black $^{\S}$ & 33.1 & $(28.3-38.3)$ & 33.0 & $(27.7-38.7)$ & 32.8 & $(28.5-37.5)$ \\
\hline Hispanic & 28.2 & $(22.9-34.1)$ & 42.2 & $(37.0-47.6)$ & 35.8 & $(31.2-40.7)$ \\
\hline \multicolumn{7}{|l|}{ Grade } \\
\hline 9 & 14.4 & $(11.2-18.3)$ & 17.4 & $(13.8-21.5)$ & 15.9 & (13.4-18.9) \\
\hline 10 & 24.7 & $(20.0-30.1)$ & 25.2 & $(21.7-29.1)$ & 25.0 & (21.4-28.9) \\
\hline 11 & 45.1 & $(38.7-51.6)$ & 50.1 & $(44.4-55.8)$ & 47.9 & $(43.9-52.0)$ \\
\hline 12 & 60.8 & $(55.4-66.0)$ & 61.9 & $(56.1-67.3)$ & 61.4 & $(57.0-65.6)$ \\
\hline Total & 40.4 & (37.1-43.7) & 42.4 & (38.5-46.3) & 41.5 & $(38.9-44.1)$ \\
\hline
\end{tabular}

* On at least 1 day during the 30 days before the survey, among the $61.3 \%$ of students nationwide who had driven a car or other vehicle during the 30 days before the survey.

† $95 \%$ confidence interval.

$\S$ Non-Hispanic. 
TABLE 8. Percentage of high school students who texted or e-mailed while driving a car or other vehicle, ${ }^{*}$ by sex — selected U.S. sites, Youth Risk Behavior Survey, 2015

\begin{tabular}{|c|c|c|c|c|c|c|}
\hline \multirow[b]{2}{*}{ Site } & \multicolumn{2}{|c|}{ Female } & \multicolumn{2}{|c|}{ Male } & \multicolumn{2}{|c|}{ Total } \\
\hline & $\%$ & $\mathrm{Cl}^{\dagger}$ & $\%$ & $\mathrm{Cl}$ & $\%$ & $\mathrm{Cl}$ \\
\hline \multicolumn{7}{|l|}{ State surveys } \\
\hline Alabama & 45.6 & (39.4-51.8) & 41.8 & $(35.6-48.2)$ & 43.9 & (38.9-48.9) \\
\hline Alaska & 37.1 & $(30.4-44.3)$ & 33.0 & $(27.7-38.8)$ & 35.1 & $(30.5-40.0)$ \\
\hline Arizona & $\ldots \S$ & - & - & - & - & - \\
\hline Arkansas & 40.4 & (35.2-45.8) & 40.6 & $(34.8-46.8)$ & 40.6 & (35.5-45.9) \\
\hline California & 29.9 & $(24.1-36.5)$ & 34.4 & $(26.2-43.7)$ & 32.4 & (25.7-39.8) \\
\hline Connecticut & 29.4 & $(24.6-34.7)$ & 29.4 & $(24.3-35.1)$ & 29.6 & $(25.3-34.4)$ \\
\hline Delaware & 33.4 & $(28.0-39.2)$ & 37.9 & $(31.1-45.2)$ & 35.8 & (30.7-41.2) \\
\hline Florida & 35.3 & (32.1-38.7) & 37.0 & $(34.0-40.1)$ & 36.3 & (33.6-39.2) \\
\hline Hawaii & 42.0 & $(38.0-46.1)$ & 37.9 & $(34.5-41.5)$ & 40.3 & (37.7-43.0) \\
\hline Idaho & 49.3 & $(41.1-57.4)$ & 48.2 & $(40.4-56.2)$ & 48.7 & $(41.5-56.0)$ \\
\hline Illinois & 41.6 & (33.9-49.8) & 41.2 & $(33.7-49.2)$ & 41.5 & $(36.1-47.0)$ \\
\hline Indiana & 41.2 & $(32.8-50.1)$ & 44.6 & $(39.8-49.5)$ & 43.1 & (38.1-48.3) \\
\hline Kentucky & 37.1 & (31.4-43.3) & 36.1 & $(30.4-42.2)$ & 36.5 & $(32.1-41.2)$ \\
\hline Maine & - & - & - & - & - & - \\
\hline Maryland & 22.6 & $(21.5-23.8)$ & 28.6 & $(27.5-29.8)$ & 26.1 & $(25.2-27.1)$ \\
\hline Massachusetts & 38.2 & $(32.8-43.9)$ & 40.4 & $(36.1-44.7)$ & 39.3 & (35.3-43.3) \\
\hline Michigan & 36.8 & $(29.1-45.2)$ & 40.9 & $(34.0-48.2)$ & 39.0 & (33.5-44.7) \\
\hline Mississippi & 44.1 & $(37.9-50.4)$ & 43.5 & $(38.4-48.9)$ & 44.0 & (39.5-48.6) \\
\hline Missouri & 47.5 & $(36.5-58.8)$ & 46.7 & $(40.5-52.9)$ & 47.0 & $(41.2-53.0)$ \\
\hline Montana & 55.6 & $(51.4-59.7)$ & 53.8 & $(50.2-57.3)$ & 54.6 & $(51.2-58.0)$ \\
\hline Nebraska & 49.8 & $(44.5-55.1)$ & 49.1 & $(43.8-54.3)$ & 49.4 & $(45.0-53.8)$ \\
\hline Nevada & 39.0 & $(34.0-44.3)$ & 39.5 & $(33.8-45.5)$ & 39.3 & $(36.1-42.7)$ \\
\hline New Hampshire & 46.0 & $(43.1-48.9)$ & 41.3 & $(38.8-43.9)$ & 43.7 & $(41.6-45.8)$ \\
\hline New Mexico & 37.7 & $(34.7-40.9)$ & 38.0 & $(34.9-41.2)$ & 37.9 & $(35.2-40.6)$ \\
\hline New York & 26.5 & $(21.5-32.3)$ & 30.6 & $(24.8-37.1)$ & 28.9 & $(24.5-33.7)$ \\
\hline North Carolina & 38.4 & $(33.8-43.2)$ & 36.5 & $(31.1-42.2)$ & 37.6 & (33.5-41.8) \\
\hline North Dakota & 59.5 & $(54.5-64.2)$ & 55.8 & $(51.2-60.3)$ & 57.6 & (53.9-61.2) \\
\hline Oklahoma & 43.0 & $(36.1-50.2)$ & 45.0 & $(35.1-55.3)$ & 44.2 & (36.7-52.0) \\
\hline Pennsylvania & 37.3 & $(30.1-45.2)$ & 32.9 & $(29.6-36.4)$ & 35.0 & (30.7-39.7) \\
\hline Rhode Island & 45.0 & $(38.2-51.9)$ & 46.0 & $(38.4-53.8)$ & 45.7 & (39.7-51.7) \\
\hline South Carolina & 39.1 & $(32.8-45.7)$ & 36.3 & $(28.9-44.5)$ & 37.6 & $(32.7-42.7)$ \\
\hline South Dakota & 66.9 & $(59.0-74.0)$ & 59.5 & $(52.4-66.2)$ & 63.2 & (56.6-69.4) \\
\hline Tennessee & 33.6 & $(29.5-37.9)$ & 37.1 & $(33.1-41.2)$ & 35.4 & (32.4-38.5) \\
\hline Vermont & 32.7 & (31.5-33.9) & 33.3 & $(32.1-34.4)$ & 33.1 & $(32.2-33.9)$ \\
\hline Virginia & 31.0 & $(26.8-35.6)$ & 31.5 & $(27.4-35.9)$ & 31.3 & $(27.9-35.0)$ \\
\hline West Virginia & 34.8 & $(29.6-40.4)$ & 35.4 & $(29.5-41.8)$ & 35.1 & (30.1-40.5) \\
\hline Wyoming & 53.1 & $(47.0-59.1)$ & 50.3 & $(43.9-56.5)$ & 51.8 & $(46.3-57.2)$ \\
\hline Median & & & & & & \\
\hline Range & & & & & & \\
\hline \multicolumn{7}{|c|}{ Large urban school district surveys } \\
\hline Baltimore, MD & 23.1 & $(18.1-29.0)$ & 30.6 & $(25.4-36.5)$ & 27.9 & $(24.4-31.7)$ \\
\hline Boston, MA & 27.1 & $(20.7-34.6)$ & 35.2 & $(29.5-41.4)$ & 31.8 & $(27.3-36.7)$ \\
\hline Broward County, FL & 38.8 & $(32.8-45.1)$ & 38.6 & $(33.8-43.6)$ & 38.7 & $(34.3-43.2)$ \\
\hline Cleveland, $\mathrm{OH}$ & 23.8 & $(19.3-29.0)$ & 29.8 & $(24.8-35.4)$ & 28.6 & $(24.8-32.7)$ \\
\hline DeKalb County, GA & 23.9 & $(18.7-30.1)$ & 28.5 & $(23.3-34.4)$ & 26.6 & $(22.4-31.1)$ \\
\hline Detroit, MI & 33.5 & $(27.3-40.4)$ & 31.9 & $(25.3-39.3)$ & 33.0 & $(27.5-39.0)$ \\
\hline District of Columbia & - & - & - & - & - & - \\
\hline Duval County, FL & 35.7 & $(31.5-40.1)$ & 32.7 & $(29.2-36.4)$ & 34.8 & (31.5-38.3) \\
\hline Ft. Worth, TX & 36.0 & $(31.4-40.8)$ & 36.2 & $(31.9-40.7)$ & 36.1 & $(33.0-39.3)$ \\
\hline Houston, TX & 32.0 & $(27.5-36.8)$ & 37.0 & $(33.2-41.0)$ & 35.0 & $(31.9-38.3)$ \\
\hline Los Angeles, CA & 16.1 & $(10.9-23.1)$ & 25.1 & $(18.9-32.5)$ & 21.3 & $(15.6-28.4)$ \\
\hline Miami-Dade County, FL & 25.9 & $(21.0-31.4)$ & 38.2 & $(34.1-42.6)$ & 32.4 & $(28.8-36.3)$ \\
\hline New York City, NY & 7.7 & $(5.8-10.1)$ & 17.5 & $(15.5-19.8)$ & 14.1 & $(12.3-16.0)$ \\
\hline
\end{tabular}

See table footnotes on the next page. 
TABLE 8. (Continued) Percentage of high school students who texted or e-mailed while driving a car or other vehicle,* by sex — selected U.S. sites, Youth Risk Behavior Survey, 2015

\begin{tabular}{|c|c|c|c|c|c|c|}
\hline \multirow[b]{2}{*}{ Site } & \multicolumn{2}{|c|}{ Female } & \multicolumn{2}{|c|}{ Male } & \multicolumn{2}{|c|}{ Total } \\
\hline & $\%$ & $\mathrm{Cl}^{\dagger}$ & $\%$ & $\mathrm{Cl}$ & $\%$ & $\mathrm{Cl}$ \\
\hline Oakland, CA & 12.6 & $(9.3-16.9)$ & 25.4 & $(21.4-29.9)$ & 20.9 & $(17.8-24.3)$ \\
\hline Orange County, FL & 32.3 & $(26.3-39.1)$ & 33.5 & $(28.5-39.0)$ & 33.1 & $(28.3-38.2)$ \\
\hline Palm Beach County, FL & 37.3 & $(32.0-43.0)$ & 39.3 & $(34.2-44.6)$ & 38.3 & (34.1-42.7) \\
\hline Philadelphia, PA & 18.1 & $(13.1-24.5)$ & 24.9 & $(20.1-30.4)$ & 21.8 & $(18.6-25.4)$ \\
\hline San Diego, CA & 32.5 & $(25.5-40.3)$ & 33.8 & $(27.9-40.2)$ & 33.2 & $(27.4-39.5)$ \\
\hline San Francisco, CA & 20.3 & $(13.7-29.1)$ & 22.7 & $(16.4-30.6)$ & 21.5 & $(16.0-28.2)$ \\
\hline Median & \multicolumn{2}{|c|}{26.5} & \multicolumn{2}{|c|}{32.3} & \multicolumn{2}{|c|}{32.1} \\
\hline Range & & & & & & \\
\hline
\end{tabular}

* On at least 1 day during the 30 days before the survey, among students who had driven a car or other vehicle during the 30 days before the survey.

† $95 \%$ confidence interval.

$\S$ Not available.

TABLE 9. Percentage of high school students who carried a weapon ${ }^{*}{ }^{\dagger}$ and who carried a gun, ${ }^{\dagger}$ by sex, race/ethnicity, and grade - United States, Youth Risk Behavior Survey, 2015

\begin{tabular}{|c|c|c|c|c|c|c|c|c|c|c|c|c|}
\hline \multirow[b]{3}{*}{ Category } & \multicolumn{6}{|c|}{ Carried a weapon } & \multicolumn{6}{|c|}{ Carried a gun } \\
\hline & \multicolumn{2}{|c|}{ Female } & \multicolumn{2}{|c|}{ Male } & \multicolumn{2}{|c|}{ Total } & \multicolumn{2}{|c|}{ Female } & \multicolumn{2}{|c|}{ Male } & \multicolumn{2}{|c|}{ Total } \\
\hline & $\%$ & $\mathrm{Cl}^{\S}$ & $\%$ & $\mathrm{Cl}$ & $\%$ & $\mathrm{Cl}$ & $\%$ & $\mathrm{Cl}$ & $\%$ & $\mathrm{Cl}$ & $\%$ & $\mathrm{Cl}$ \\
\hline \multicolumn{13}{|c|}{ Race/Ethnicity } \\
\hline White & 8.1 & $(6.0-10.9)$ & 28.0 & $(24.7-31.5)$ & 18.1 & $(15.5-21.0)$ & 1.4 & $(0.9-2.0)$ & 9.6 & $(7.8-11.9)$ & 5.5 & $(4.7-6.6)$ \\
\hline Black ${ }^{\Uparrow}$ & 6.2 & $(3.4-10.9)$ & 17.6 & $(11.9-25.2)$ & 12.4 & $(9.9-15.4)$ & 1.7 & $(0.8-3.6)$ & 9.6 & $(6.1-14.6)$ & 6.0 & $(4.3-8.3)$ \\
\hline Hispanic & 7.1 & $(5.6-8.9)$ & 20.2 & $(17.2-23.6)$ & 13.7 & $(11.6-16.2)$ & 1.9 & $(1.1-3.2)$ & 6.5 & $(5.2-8.1)$ & 4.3 & $(3.4-5.4)$ \\
\hline \multicolumn{13}{|l|}{ Grade } \\
\hline 9 & 6.6 & $(5.2-8.5)$ & 24.6 & $(21.3-28.2)$ & 16.1 & $(14.0-18.5)$ & 1.2 & $(0.8-2.0)$ & 7.0 & $(5.0-9.8)$ & 4.4 & $(3.5-5.6)$ \\
\hline 10 & 7.2 & $(5.2-10.0)$ & 25.5 & $(21.2-30.3)$ & 16.3 & $(13.5-19.5)$ & 1.6 & $(0.9-2.7)$ & 8.8 & (7.0-10.9) & 5.2 & $(4.2-6.3)$ \\
\hline 11 & 8.0 & (5.9-10.8) & 23.0 & $(20.2-26.1)$ & 16.0 & $(13.7-18.6)$ & 1.4 & $(0.9-2.3)$ & 9.0 & $(7.2-11.1)$ & 5.5 & $(4.3-7.0)$ \\
\hline 12 & 8.0 & $(5.7-11.2)$ & 23.4 & $(19.5-27.8)$ & 15.8 & $(13.4-18.5)$ & 1.7 & $(1.0-2.8)$ & 9.7 & $(6.9-13.4)$ & 5.7 & $(4.5-7.3)$ \\
\hline Total & 7.5 & $(6.1-9.3)$ & 24.3 & $(21.9-27.0)$ & 16.2 & $(14.4-18.1)$ & 1.6 & $(1.2-2.0)$ & 8.7 & $(7.3-10.3)$ & 5.3 & $(4.6-6.1)$ \\
\hline
\end{tabular}

* Such as, a gun, knife, or club.

$\dagger$ On at least 1 day during the 30 days before the survey.

$\S 95 \%$ confidence interval.

" Non-Hispanic. 
TABLE 10. Percentage of high school students who carried a weapon ${ }^{*}{ }^{\dagger}$ and who carried a gun, ${ }^{\dagger}$ by sex - selected U.S. sites, Youth Risk Behavior Survey, 2015

\begin{tabular}{|c|c|c|c|c|c|c|c|c|c|c|c|c|}
\hline \multirow[b]{3}{*}{ Site } & \multicolumn{6}{|c|}{ Carried a weapon } & \multicolumn{6}{|c|}{ Carried a gun } \\
\hline & \multicolumn{2}{|c|}{ Female } & \multicolumn{2}{|c|}{ Male } & \multicolumn{2}{|c|}{ Total } & \multicolumn{2}{|c|}{ Female } & \multicolumn{2}{|c|}{ Male } & \multicolumn{2}{|c|}{ Total } \\
\hline & $\%$ & $\mathrm{Cl}^{\S}$ & $\%$ & $\mathrm{Cl}$ & $\%$ & $\mathrm{Cl}$ & $\%$ & $\mathrm{Cl}$ & $\%$ & $\mathrm{Cl}$ & $\%$ & $\mathrm{Cl}$ \\
\hline \multicolumn{13}{|l|}{ State surveys } \\
\hline Alabama & 10.2 & $(7.1-14.3)$ & 34.6 & $(29.7-39.7)$ & 22.5 & (18.7-26.7) & 3.4 & $(2.0-5.7)$ & 15.9 & (12.7-19.7) & 10.0 & $(8.0-12.5)$ \\
\hline Alaska & - & - & - & - & - & - & - & - & - & - & - & - \\
\hline Arizona & 10.2 & $(7.5-13.6)$ & 25.5 & $(22.2-29.1)$ & 18.0 & (15.6-20.7) & 2.8 & $(1.7-4.4)$ & 6.9 & $(5.5-8.5)$ & 4.9 & $(4.0-6.0)$ \\
\hline Arkansas & 9.3 & $(7.5-11.6)$ & 32.1 & (27.8-36.8) & 21.0 & (18.3-23.9) & 3.6 & $(2.2-5.9)$ & 15.7 & $(12.6-19.3)$ & 9.8 & $(8.1-11.7)$ \\
\hline California & 4.6 & $(2.8-7.4)$ & 13.2 & $(9.4-18.2)$ & 8.9 & $(6.6-12.0)$ & 0.7 & $(0.4-1.5)$ & 4.8 & $(3.4-6.5)$ & 2.8 & $(2.1-3.7)$ \\
\hline Connecticut & - & - & - & - & - & - & - & - & - & - & - & - \\
\hline Delaware & 6.0 & $(4.4-8.2)$ & 19.6 & $(17.0-22.6)$ & 13.0 & (11.3-14.9) & 1.1 & $(0.5-2.7)$ & 8.2 & $(6.3-10.6)$ & 4.7 & $(3.6-6.3)$ \\
\hline Florida & 8.1 & $(7.0-9.3)$ & 22.3 & $(19.6-25.2)$ & 15.4 & $(13.6-17.3)$ & - & - & - & - & - & - \\
\hline Hawaii & 6.1 & $(5.0-7.3)$ & 15.1 & $(13.6-16.8)$ & 10.7 & $(9.6-11.9)$ & - & - & - & - & - & - \\
\hline Idaho & 14.7 & $(11.8-18.3)$ & 40.9 & $(36.3-45.7)$ & 28.2 & $(25.2-31.4)$ & - & - & 一 & - & - & - \\
\hline Illinois & 8.2 & $(6.2-10.8)$ & 22.5 & $(18.7-26.8)$ & 15.4 & (12.8-18.5) & 2.1 & $(1.3-3.3)$ & 9.0 & $(7.3-11.2)$ & 5.6 & $(4.5-6.9)$ \\
\hline Indiana & 8.4 & $(6.1-11.5)$ & 30.2 & $(25.3-35.6)$ & 19.6 & $(16.1-23.6)$ & 1.6 & $(1.0-2.4)$ & 10.6 & (7.4-14.8) & 6.2 & $(4.4-8.7)$ \\
\hline Kentucky & 11.6 & $(9.4-14.3)$ & 34.0 & $(28.8-39.6)$ & 23.1 & $(20.0-26.5)$ & - & - & - & - & - & - \\
\hline Maine & - & - & - & - & - & - & - & - & - & - & - & - \\
\hline Maryland & 8.3 & $(7.9-8.8)$ & 20.9 & (20.2-21.6) & 14.9 & $(14.4-15.3)$ & - & - & - & - & - & - \\
\hline Massachusetts & 5.0 & $(3.7-6.8)$ & 19.9 & $(16.7-23.5)$ & 12.6 & $(10.4-15.3)$ & 0.9 & $(0.5-1.8)$ & 4.5 & $(3.6-5.7)$ & 2.7 & $(2.1-3.5)$ \\
\hline Michigan & 8.1 & $(6.5-10.0)$ & 24.8 & $(20.3-30.1)$ & 16.6 & (13.8-19.8) & 2.2 & $(1.3-3.8)$ & 6.9 & $(5.5-8.5)$ & 4.6 & $(3.8-5.6)$ \\
\hline Mississippi & 10.0 & $(7.7-12.8)$ & 32.4 & $(27.6-37.6)$ & 21.0 & (18.2-24.2) & 2.7 & $(1.7-4.1)$ & 14.3 & $(11.8-17.2)$ & 8.5 & $(7.2-10.0)$ \\
\hline Missouri & 10.8 & $(7.6-15.2)$ & 33.8 & (29.9-38.0) & 22.1 & $(18.6-26.0)$ & - & - & - & - & - & - \\
\hline Montana & 14.2 & $(12.3-16.2)$ & 37.8 & (35.4-40.3) & 26.4 & $(24.5-28.3)$ & 4.4 & $(3.7-5.3)$ & 17.2 & $(15.6-19.0)$ & 11.1 & $(10.2-12.0)$ \\
\hline Nebraska & - & - & - & - & - & - & - & - & - & - & - & - \\
\hline Nevada & 9.5 & $(7.6-11.9)$ & 26.7 & $(21.6-32.4)$ & 18.3 & $(15.2-21.8)$ & 3.1 & $(1.9-5.0)$ & 8.0 & $(6.1-10.6)$ & 5.7 & $(4.2-7.6)$ \\
\hline New Hampshire & - & - & - & - & - & - & - & - & - & - & - & - \\
\hline New Mexico & 12.8 & (11.2-14.6) & 32.1 & (29.7-34.5) & 22.5 & $(20.9-24.2)$ & 3.3 & $(2.6-4.3)$ & 11.9 & $(10.3-13.7)$ & 7.7 & $(6.6-8.8)$ \\
\hline New York & 6.6 & (5.3-8.1) & 19.0 & $(15.8-22.8)$ & 13.0 & $(11.2-15.0)$ & 1.8 & $(1.2-2.6)$ & 6.3 & $(5.2-7.6)$ & 4.1 & $(3.4-5.1)$ \\
\hline North Carolina & 9.7 & $(7.3-12.9)$ & 28.5 & $(24.8-32.4)$ & 19.3 & $(16.8-22.1)$ & - & - & - & - & - & - \\
\hline North Dakota & - & - & - & - & - & - & - & - & - & - & - & - \\
\hline Oklahoma & 9.0 & $(6.7-11.9)$ & 30.5 & $(26.2-35.2)$ & 19.5 & $(16.3-23.1)$ & 3.3 & $(2.0-5.4)$ & 10.5 & $(7.6-14.4)$ & 6.8 & $(4.9-9.4)$ \\
\hline Pennsylvania & 8.1 & $(6.1-10.5)$ & 26.4 & $(22.9-30.2)$ & 17.4 & $(15.0-20.2)$ & 2.7 & $(1.6-4.6)$ & 12.7 & $(10.6-15.3)$ & 7.9 & $(6.4-9.7)$ \\
\hline Rhode Island & - & - & - & - & - & - & - & - & - & - & - & - \\
\hline South Carolina & 9.6 & $(7.9-11.6)$ & 31.3 & $(25.1-38.2)$ & 20.5 & $(16.7-25.0)$ & 3.1 & $(2.0-4.8)$ & 13.5 & $(9.3-19.1)$ & 8.4 & $(5.8-12.1)$ \\
\hline South Dakota & - & - & - & - & - & - & - & - & - & - & - & - \\
\hline Tennessee & - & - & - & - & - & - & - & - & - & - & - & - \\
\hline Vermont & - & - & - & - & - & - & - & - & - & - & - & - \\
\hline Virginia & 6.7 & (5.4-8.2) & 22.5 & $(20.2-25.0)$ & 15.0 & (13.6-16.6) & - & - & - & - & - & - \\
\hline West Virginia & 13.4 & $(10.5-17.0)$ & 38.3 & (33.9-42.9) & 26.1 & (22.9-29.5) & 2.4 & $(1.6-3.6)$ & 12.7 & $(10.5-15.2)$ & 7.6 & $(6.3-9.3)$ \\
\hline Wyoming & 18.1 & $(15.6-20.8)$ & 40.8 & $(36.2-45.7)$ & 29.6 & $(27.0-32.3)$ & 6.9 & $(5.5-8.5)$ & 15.9 & $(13.1-19.2)$ & 11.5 & (9.7-13.5) \\
\hline Median & & 9.3 & & 28.5 & & 9.3 & & & & 0.6 & & 5.8 \\
\hline Range & & $-18.1)$ & & 2-40.9) & & $-29.6)$ & & & & $-17.2)$ & & $-11.5)$ \\
\hline \multicolumn{13}{|c|}{ Large urban school district surveys } \\
\hline Baltimore, MD & 14.9 & $(12.1-18.2)$ & 28.1 & $(23.5-33.1)$ & 21.9 & $(19.0-25.0)$ & 2.1 & $(1.1-3.9)$ & 7.0 & $(4.7-10.3)$ & 5.4 & $(3.8-7.6)$ \\
\hline Boston, MA & 5.4 & (3.9-7.5) & 17.7 & $(14.7-21.2)$ & 11.7 & $(9.7-13.9)$ & - & - & - & - & - & - \\
\hline Broward County, FL & 7.6 & $(5.8-9.9)$ & 16.7 & $(13.7-20.2)$ & 12.4 & $(10.4-14.7)$ & 1.1 & $(0.6-2.0)$ & 4.9 & $(3.3-7.1)$ & 3.1 & $(2.3-4.3)$ \\
\hline Cleveland, $\mathrm{OH}$ & 13.8 & $(10.7-17.7)$ & 23.4 & $(20.3-26.9)$ & 19.2 & $(16.6-22.0)$ & - & - & - & - & - & - \\
\hline DeKalb County, GA & 6.0 & $(4.6-7.9)$ & 15.3 & $(12.5-18.6)$ & 10.7 & $(9.0-12.7)$ & 1.7 & $(1.0-2.7)$ & 6.9 & $(5.1-9.4)$ & 4.3 & $(3.3-5.7)$ \\
\hline Detroit, MI & 10.2 & $(8.2-12.5)$ & 19.5 & $(16.7-22.6)$ & 14.4 & $(12.5-16.5)$ & 2.4 & $(1.4-4.0)$ & 8.7 & $(6.7-11.1)$ & 5.4 & $(4.4-6.7)$ \\
\hline District of Columbia & 12.5 & $(11.7-13.5)$ & 23.6 & $(22.4-24.9)$ & 18.1 & (17.4-18.9) & - & - & - & - & - & - \\
\hline Duval County, FL & 12.2 & $(10.2-14.5)$ & 26.2 & $(23.4-29.2)$ & 19.3 & (17.4-21.4) & - & - & - & - & - & - \\
\hline Ft. Worth, TX & 6.6 & $(5.2-8.2)$ & 18.4 & $(15.7-21.4)$ & 12.5 & $(10.9-14.5)$ & 1.6 & $(0.9-2.7)$ & 7.5 & $(5.9-9.4)$ & 4.5 & $(3.6-5.6)$ \\
\hline Houston, TX & 7.7 & $(6.3-9.3)$ & 18.1 & $(16.3-20.1)$ & 13.2 & $(11.9-14.6)$ & 3.3 & $(2.6-4.2)$ & 7.3 & $(6.0-8.8)$ & 5.5 & $(4.7-6.4)$ \\
\hline Los Angeles, CA & 4.0 & $(3.1-5.3)$ & 11.8 & $(9.4-14.6)$ & 7.8 & $(6.4-9.6)$ & 0.9 & $(0.4-1.8)$ & 3.5 & $(2.6-4.6)$ & 2.2 & $(1.7-2.8)$ \\
\hline Miami-Dade County, FL & 4.7 & $(3.7-6.0)$ & 13.6 & $(11.4-16.2)$ & 9.1 & $(7.9-10.5)$ & 1.9 & $(1.3-2.6)$ & 6.5 & $(5.1-8.4)$ & 4.2 & $(3.4-5.1)$ \\
\hline New York City, NY & 4.6 & $(3.8-5.6)$ & 10.4 & $(8.7-12.3)$ & 7.7 & $(6.6-8.9)$ & 1.0 & $(0.6-1.5)$ & 3.3 & $(2.6-4.2)$ & 2.3 & $(1.9-2.8)$ \\
\hline
\end{tabular}

See table footnotes on the next page. 
TABLE 10. (Continued) Percentage of high school students who carried a weapon*, ${ }^{\dagger}$ and who carried a gun, ${ }^{\dagger}$ by sex - selected U.S. sites, Youth Risk Behavior Survey, 2015

\begin{tabular}{|c|c|c|c|c|c|c|c|c|c|c|c|c|}
\hline \multirow[b]{3}{*}{ Site } & \multicolumn{6}{|c|}{ Carried a weapon } & \multicolumn{6}{|c|}{ Carried a gun } \\
\hline & \multicolumn{2}{|c|}{ Female } & \multicolumn{2}{|c|}{ Male } & \multicolumn{2}{|c|}{ Total } & \multicolumn{2}{|c|}{ Female } & \multicolumn{2}{|c|}{ Male } & \multicolumn{2}{|c|}{ Total } \\
\hline & $\%$ & $\mathrm{Cl}^{\S}$ & $\%$ & $\mathrm{Cl}$ & $\%$ & $\mathrm{Cl}$ & $\%$ & $\mathrm{Cl}$ & $\%$ & $\mathrm{Cl}$ & $\%$ & $\mathrm{Cl}$ \\
\hline Oakland, CA & 9.6 & $(7.5-12.1)$ & 18.3 & $(15.5-21.4)$ & 14.4 & $(12.6-16.4)$ & 2.2 & $(1.3-3.8)$ & 9.2 & $(7.2-11.6)$ & 5.9 & $(4.7-7.3)$ \\
\hline Orange County, FL & 5.1 & $(3.8-6.9)$ & 18.3 & $(15.3-21.8)$ & 11.7 & $(10.0-13.6)$ & 1.6 & $(0.9-2.9)$ & 7.8 & $(5.9-10.4)$ & 4.7 & $(3.5-6.2)$ \\
\hline Palm Beach County, FL & 7.9 & $(6.2-10.1)$ & 20.3 & $(17.4-23.6)$ & 14.5 & (12.7-16.5) & 1.1 & $(0.5-2.3)$ & 7.4 & $(5.8-9.4)$ & 4.7 & $(3.7-6.0)$ \\
\hline Philadelphia, PA & 8.5 & $(6.5-11.1)$ & 17.0 & $(12.8-22.1)$ & 12.7 & $(10.2-15.7)$ & 1.8 & $(1.0-3.1)$ & 8.0 & $(5.4-11.8)$ & 5.0 & $(3.5-7.0)$ \\
\hline San Diego, CA & 4.7 & $(3.3-6.6)$ & 16.1 & $(14.1-18.4)$ & 10.5 & $(9.0-12.1)$ & 0.4 & $(0.2-1.0)$ & 4.9 & $(3.6-6.7)$ & 2.7 & $(2.0-3.7)$ \\
\hline San Francisco, CA & 6.1 & $(4.6-7.9)$ & 12.2 & $(9.5-15.5)$ & 9.2 & (7.4-11.5) & 1.2 & $(0.6-2.4)$ & 3.7 & $(2.2-6.0)$ & 2.6 & $(1.8-3.9)$ \\
\hline Median & \multicolumn{2}{|c|}{7.6} & \multicolumn{2}{|c|}{18.1} & \multicolumn{2}{|c|}{12.5} & \multicolumn{2}{|c|}{1.6} & \multirow{2}{*}{\multicolumn{2}{|c|}{$\begin{array}{c}7.0 \\
(3.3-9.2)\end{array}$}} & \multirow{2}{*}{\multicolumn{2}{|c|}{$\begin{array}{c}4.5 \\
(2.2-5.9)\end{array}$}} \\
\hline Range & & 14.9) & $(10$ & $4-28.1)$ & & $-21.9)$ & & & & & & \\
\hline
\end{tabular}

* Such as, a gun, knife, or club.

+ On at least 1 day during the 30 days before the survey.

$\S 95 \%$ confidence interval.

" Not available.

TABLE 11. Percentage of high school students who carried a weapon* on school property ${ }^{\dagger}$ and who were threatened or injured with a weapon* on school property, $\S$ by sex, race/ethnicity, and grade — United States, Youth Risk Behavior Survey, 2015

\begin{tabular}{|c|c|c|c|c|c|c|c|c|c|c|c|c|}
\hline \multirow[b]{3}{*}{ Category } & \multicolumn{6}{|c|}{ Carried a weapon on school property } & \multicolumn{6}{|c|}{ Threatened or injured with a weapon on school property } \\
\hline & \multicolumn{2}{|c|}{ Female } & \multicolumn{2}{|c|}{ Male } & \multicolumn{2}{|c|}{ Total } & \multicolumn{2}{|c|}{ Female } & \multicolumn{2}{|c|}{ Male } & \multicolumn{2}{|c|}{ Total } \\
\hline & $\%$ & $\mathrm{Cl}^{\uparrow}$ & $\%$ & $\mathrm{Cl}$ & $\%$ & $\mathrm{Cl}$ & $\%$ & $\mathrm{Cl}$ & $\%$ & $\mathrm{Cl}$ & $\%$ & $\mathrm{Cl}$ \\
\hline \multicolumn{13}{|c|}{ Race/Ethnicity } \\
\hline White** & 1.6 & $(1.1-2.4)$ & 5.7 & $(4.5-7.2)$ & 3.7 & $(2.9-4.6)$ & 4.3 & $(3.4-5.5)$ & 5.4 & $(4.2-6.9)$ & 4.9 & $(4.0-6.0)$ \\
\hline Black** & 2.1 & $(0.8-4.9)$ & 4.7 & (3.3-6.6) & 3.4 & $(2.3-5.1)$ & 6.5 & $(4.6-9.0)$ & 8.9 & $(6.4-12.2)$ & 7.9 & $(6.0-10.4)$ \\
\hline Hispanic & 2.9 & $(1.9-4.3)$ & 6.1 & $(4.6-8.0)$ & 4.5 & $(3.5-5.8)$ & 4.7 & $(3.3-6.6)$ & 8.4 & $(6.9-10.0)$ & 6.6 & $(5.4-8.0)$ \\
\hline \multicolumn{13}{|l|}{ Grade } \\
\hline 9 & 1.9 & $(1.3-2.7)$ & 4.6 & $(3.5-6.0)$ & 3.4 & $(2.8-4.0)$ & 6.2 & $(4.8-8.1)$ & 7.8 & $(6.4-9.5)$ & 7.2 & $(6.2-8.3)$ \\
\hline 10 & 2.2 & $(1.3-3.6)$ & 6.1 & $(4.4-8.2)$ & 4.1 & $(3.1-5.4)$ & 5.5 & $(4.3-7.2)$ & 6.8 & $(5.3-8.8)$ & 6.2 & $(5.1-7.4)$ \\
\hline 11 & 1.9 & $(1.2-3.0)$ & 7.4 & $(6.1-9.0)$ & 4.8 & $(3.9-6.0)$ & 2.9 & $(2.0-4.2)$ & 7.3 & $(5.3-10.0)$ & 5.5 & $(4.3-7.0)$ \\
\hline 12 & 2.0 & $(1.3-3.2)$ & 5.1 & $(3.5-7.4)$ & 3.6 & $(2.6-4.9)$ & 3.2 & $(2.0-5.0)$ & 5.7 & $(4.0-8.0)$ & 4.4 & $(3.2-6.1)$ \\
\hline Total & 2.0 & $(1.5-2.7)$ & 5.9 & $(5.0-6.9)$ & 4.1 & $(3.5-4.7)$ & 4.6 & $(3.9-5.5)$ & 7.0 & $(6.1-8.1)$ & 6.0 & $(5.2-6.8)$ \\
\hline
\end{tabular}

* Such as a gun, knife, or club.

† On at least 1 day during the 30 days preceding the survey.

$\S$ One or more times during the 12 months preceding the survey.

१ $95 \%$ confidence interval.

** Non-Hispanic. 
TABLE 12. Percentage of high school students who carried a weapon* on school property ${ }^{\dagger}$ and who were threatened or injured with a weapon* on school property, ${ }^{\S}$ by sex — selected U.S. sites, Youth Risk Behavior Survey, 2015

\begin{tabular}{|c|c|c|c|c|c|c|c|c|c|c|c|c|}
\hline \multirow[b]{3}{*}{ Site } & \multicolumn{6}{|c|}{ Carried a weapon on school property } & \multicolumn{6}{|c|}{ Threatened or injured with a weapon on school property } \\
\hline & \multicolumn{2}{|c|}{ Female } & \multicolumn{2}{|c|}{ Male } & \multicolumn{2}{|c|}{ Total } & \multicolumn{2}{|c|}{ Female } & \multicolumn{2}{|c|}{ Male } & \multicolumn{2}{|c|}{ Total } \\
\hline & $\%$ & $\mathrm{Cl}^{\text {กी }}$ & $\%$ & $\mathrm{Cl}$ & $\%$ & $\mathrm{Cl}$ & $\%$ & $\mathrm{Cl}$ & $\%$ & $\mathrm{Cl}$ & $\%$ & $\mathrm{Cl}$ \\
\hline \multicolumn{13}{|l|}{ State surveys } \\
\hline Alabama & 2.6 & $(1.4-4.8)$ & 8.2 & $(5.4-12.3)$ & 5.6 & $(3.6-8.5)$ & 6.2 & $(4.9-7.8)$ & 10.7 & $(8.1-14.1)$ & 8.7 & $(7.0-10.8)$ \\
\hline Alaska & 4.2 & $(3.0-5.8)$ & 11.3 & (8.9-14.4) & 8.2 & $(6.6-10.1)$ & —** & - & - & - & - & - \\
\hline Arizona & 2.8 & $(1.5-5.2)$ & 6.0 & $(3.8-9.3)$ & 4.5 & $(3.0-6.8)$ & 5.3 & (3.4-8.1) & 9.5 & $(7.4-12.0)$ & 7.5 & $(5.7-9.6)$ \\
\hline Arkansas & 2.7 & $(1.5-4.9)$ & 7.8 & $(5.4-11.1)$ & 5.4 & (3.8-7.5) & 6.9 & $(5.2-9.0)$ & 13.8 & $(11.8-16.0)$ & 10.6 & $(9.4-12.0)$ \\
\hline California & 1.8 & $(0.8-3.7)$ & 3.7 & $(2.7-5.2)$ & 2.7 & $(1.9-4.0)$ & 4.3 & $(3.1-6.0)$ & 5.8 & $(4.0-8.3)$ & 5.2 & (3.8-6.9) \\
\hline Connecticut & 3.3 & $(2.5-4.3)$ & 8.6 & $(6.5-11.4)$ & 6.2 & $(5.1-7.5)$ & 5.2 & $(3.6-7.5)$ & 7.9 & (6.4-9.6) & 6.7 & (5.4-8.3) \\
\hline Delaware & 2.6 & $(1.7-4.1)$ & 5.2 & (3.7-7.3) & 4.0 & $(3.1-5.2)$ & 3.8 & $(2.5-5.6)$ & 7.8 & $(5.9-10.2)$ & 6.2 & $(4.6-8.2)$ \\
\hline Florida & - & - & - & - & - & - & 5.1 & $(4.3-6.0)$ & 9.1 & (7.9-10.5) & 7.3 & $(6.5-8.2)$ \\
\hline Hawaii & - & - & - & - & - & - & - & - & - & - & - & - \\
\hline Idaho & 3.5 & $(2.1-5.8)$ & 9.9 & (7.3-13.4) & 6.8 & $(5.0-9.2)$ & 4.8 & (3.4-6.8) & 7.4 & $(5.9-9.2)$ & 6.1 & $(5.2-7.2)$ \\
\hline Illinois & 2.5 & $(1.8-3.4)$ & 6.0 & $(4.6-7.6)$ & 4.3 & (3.4-5.5) & 3.6 & $(2.6-5.1)$ & 9.4 & (7.1-12.4) & 6.6 & (5.2-8.5) \\
\hline Indiana & 2.6 & $(1.8-3.7)$ & 8.3 & (5.0-13.3) & 5.6 & (3.7-8.4) & 4.7 & (3.0-7.5) & 8.3 & $(5.5-12.3)$ & 6.6 & $(4.8-9.0)$ \\
\hline Kentucky & 4.1 & $(2.7-6.2)$ & 8.8 & $(6.0-12.6)$ & 6.5 & $(4.7-8.9)$ & 6.7 & $(4.9-9.1)$ & 7.6 & $(5.8-9.9)$ & 7.2 & $(5.7-9.2)$ \\
\hline Maine & 2.7 & $(2.2-3.2)$ & 8.5 & (7.3-9.9) & 5.8 & $(5.1-6.6)$ & 3.6 & $(2.9-4.4)$ & 6.4 & $(5.4-7.5)$ & 5.2 & $(4.5-6.0)$ \\
\hline Maryland & 2.8 & $(2.5-3.1)$ & 5.4 & $(5.0-5.8)$ & 4.3 & $(4.0-4.5)$ & 5.3 & $(4.9-5.7)$ & 8.7 & $(8.2-9.2)$ & 7.2 & $(6.9-7.6)$ \\
\hline Massachusetts & 1.6 & $(1.0-2.6)$ & 4.6 & $(3.5-5.8)$ & 3.2 & $(2.5-4.1)$ & 2.8 & $(2.0-4.0)$ & 5.0 & $(3.9-6.4)$ & 4.1 & $(3.2-5.1)$ \\
\hline Michigan & 1.6 & $(0.8-2.9)$ & 5.5 & $(3.6-8.4)$ & 3.6 & $(2.6-5.0)$ & 5.0 & $(3.7-6.6)$ & 8.0 & $(6.1-10.3)$ & 6.6 & $(5.4-8.0)$ \\
\hline Mississippi & 2.8 & $(1.7-4.6)$ & 7.2 & $(5.8-8.9)$ & 5.2 & $(4.3-6.3)$ & 8.0 & (5.9-10.7) & 11.4 & (8.9-14.4) & 10.1 & $(8.2-12.2)$ \\
\hline Missouri & 2.9 & $(1.7-4.9)$ & 9.1 & $(7.2-11.4)$ & 5.9 & $(4.6-7.5)$ & - & - & - & - & - & - \\
\hline Montana & 5.4 & $(4.3-6.8)$ & 15.5 & (13.2-18.0) & 10.6 & $(9.1-12.3)$ & 4.3 & $(3.3-5.7)$ & 6.6 & $(5.3-8.1)$ & 5.5 & $(4.7-6.6)$ \\
\hline Nebraska & 5.3 & $(3.5-8.0)$ & 10.2 & $(8.1-12.8)$ & 8.1 & $(6.4-10.2)$ & 5.1 & (3.5-7.4) & 8.3 & (6.4-10.7) & 7.1 & $(5.6-8.9)$ \\
\hline Nevada & 2.5 & $(1.6-3.9)$ & 4.7 & $(2.8-8.0)$ & 3.7 & $(2.6-5.2)$ & 6.0 & $(4.6-7.8)$ & 7.5 & $(5.2-10.5)$ & 6.9 & $(5.4-8.7)$ \\
\hline New Hampshire & - & - & - & - & - & - & - & - & - & - & - & - \\
\hline New Mexico & 2.2 & $(1.7-2.8)$ & 6.9 & $(5.9-7.9)$ & 4.6 & $(4.0-5.3)$ & - & - & - & - & - & - \\
\hline New York & 2.6 & $(1.9-3.6)$ & 5.8 & $(4.3-7.9)$ & 4.5 & $(3.6-5.6)$ & 6.0 & $(4.5-7.9)$ & 9.9 & $(8.1-12.1)$ & 8.3 & (7.1-9.8) \\
\hline North Carolina & 2.3 & $(1.2-4.2)$ & 5.4 & $(4.2-6.8)$ & 3.9 & $(3.0-5.2)$ & 3.6 & $(2.6-5.1)$ & 5.9 & $(4.1-8.3)$ & 4.9 & (3.7-6.5) \\
\hline North Dakota & 2.1 & $(1.4-3.3)$ & 8.0 & $(6.5-9.8)$ & 5.2 & $(4.3-6.2)$ & - & - & - & - & - & - \\
\hline Oklahoma & 2.2 & $(1.3-3.9)$ & 7.6 & $(5.3-10.7)$ & 4.8 & $(3.4-6.8)$ & 3.6 & $(2.2-5.8)$ & 6.8 & $(4.8-9.6)$ & 5.1 & $(3.7-7.0)$ \\
\hline Pennsylvania & 0.9 & $(0.5-1.6)$ & 3.0 & $(1.9-4.7)$ & 2.0 & $(1.3-3.1)$ & 3.3 & (2.4-4.4) & 6.4 & $(5.1-8.2)$ & 5.0 & $(4.1-6.0)$ \\
\hline Rhode Island & 2.1 & $(1.1-3.8)$ & 7.0 & $(5.0-9.7)$ & 4.8 & $(3.3-6.9)$ & - & - & - & - & - & - \\
\hline South Carolina & 1.8 & $(0.7-4.7)$ & 4.0 & $(2.7-5.9)$ & 2.9 & $(2.0-4.1)$ & 3.4 & $(2.2-5.4)$ & 7.2 & $(4.8-10.6)$ & 5.3 & $(3.9-7.2)$ \\
\hline South Dakota & 2.4 & $(1.2-4.9)$ & 11.5 & (7.9-16.5) & 7.1 & $(4.9-10.3)$ & 5.4 & $(3.2-9.1)$ & 8.9 & $(6.5-12.0)$ & 7.3 & $(5.3-9.9)$ \\
\hline Tennessee & - & - & - & - & - & - & 8.8 & $(6.6-11.8)$ & 11.1 & $(9.2-13.4)$ & 10.2 & $(8.2-12.5)$ \\
\hline Vermont & 3.7 & (3.3-4.0) & 11.2 & (10.6-11.9) & 7.7 & $(7.3-8.0)$ & 4.0 & $(3.7-4.4)$ & 6.3 & $(5.8-6.8)$ & 5.3 & $(5.0-5.6)$ \\
\hline Virginia & 1.0 & $(0.5-1.8)$ & 4.0 & $(2.8-5.6)$ & 2.6 & $(1.9-3.7)$ & 4.6 & $(3.5-6.0)$ & 8.0 & $(6.7-9.6)$ & 6.4 & $(5.3-7.7)$ \\
\hline West Virginia & 3.8 & $(2.5-5.7)$ & 9.2 & $(6.1-13.7)$ & 6.5 & $(4.9-8.6)$ & 6.1 & $(4.3-8.6)$ & 7.7 & $(5.7-10.3)$ & 6.9 & $(5.8-8.2)$ \\
\hline Wyoming & 5.7 & $(4.0-8.1)$ & 15.3 & (12.9-17.9) & 10.7 & $(9.2-12.4)$ & 5.2 & $(3.9-6.8)$ & 7.9 & (5.9-10.4) & 6.6 & $(5.3-8.2)$ \\
\hline Median & \multirow{2}{*}{\multicolumn{2}{|c|}{$\begin{array}{l}2.6 \\
9-5.7)\end{array}$}} & \multirow{2}{*}{\multicolumn{2}{|c|}{$\begin{array}{c}7.6 \\
(3.0-15.5)\end{array}$}} & \multirow{2}{*}{\multicolumn{2}{|c|}{$\begin{array}{c}5.2 \\
(2.0-10.7)\end{array}$}} & \multirow{2}{*}{\multicolumn{2}{|c|}{$\begin{array}{c}5.0 \\
(2.8-8.8)\end{array}$}} & \multirow{2}{*}{\multicolumn{2}{|c|}{$\begin{array}{c}7.9 \\
(5.0-13.8)\end{array}$}} & & .6 \\
\hline Range & & & & & & & & & & & & -10.6) \\
\hline Large urban sch & & & & & & & & & & & & \\
\hline Baltimore, MD & 6.3 & $(4.5-8.6)$ & 12.7 & $(9.2-17.3)$ & 9.8 & $(7.5-12.7)$ & 6.9 & $(4.9-9.5)$ & 11.9 & (8.5-16.5) & 10.0 & $(7.9-12.7)$ \\
\hline Boston, MA & 2.0 & $(1.3-3.2)$ & 5.6 & (3.8-8.0) & 3.8 & $(2.8-5.1)$ & 3.0 & $(2.0-4.5)$ & 5.4 & (3.7-7.8) & 4.3 & $(3.3-5.5)$ \\
\hline Broward County, FL & 2.5 & $(1.6-3.8)$ & 4.2 & $(2.8-6.2)$ & 3.5 & $(2.6-4.6)$ & 5.8 & $(4.2-8.1)$ & 7.4 & $(5.8-9.5)$ & 6.7 & $(5.3-8.5)$ \\
\hline Cleveland, $\mathrm{OH}$ & - & - & - & - & - & - & - & - & - & - & - & - \\
\hline DeKalb County, GA & 2.0 & $(1.1-3.3)$ & 4.1 & $(2.8-5.8)$ & 3.0 & $(2.2-4.1)$ & 6.0 & $(4.5-7.8)$ & 9.1 & $(7.0-11.8)$ & 7.7 & $(6.3-9.2)$ \\
\hline Detroit, MI & 3.8 & $(2.7-5.3)$ & 5.2 & $(3.7-7.2)$ & 4.6 & $(3.7-5.6)$ & 11.7 & $(7.9-16.9)$ & 16.3 & $(12.3-21.1)$ & 13.9 & $(10.5-18.3)$ \\
\hline District of Columbia & - & - & - & - & - & - & 6.0 & $(5.4-6.7)$ & 8.7 & $(7.9-9.5)$ & 7.6 & $(7.1-8.1)$ \\
\hline Duval County, FL & 4.2 & $(3.0-5.9)$ & 6.8 & $(4.8-9.6)$ & 5.8 & $(4.3-7.8)$ & 9.5 & (7.4-12.1) & 12.3 & $(9.5-15.8)$ & 11.5 & $(9.2-14.2)$ \\
\hline Ft. Worth, TX & 2.3 & $(1.5-3.5)$ & 3.7 & $(2.7-4.9)$ & 3.0 & $(2.3-3.8)$ & 4.7 & $(3.6-6.0)$ & 6.1 & $(4.7-7.8)$ & 5.4 & $(4.5-6.5)$ \\
\hline Houston, TX & 3.0 & $(2.1-4.3)$ & 4.3 & $(3.4-5.4)$ & 3.9 & $(3.0-4.9)$ & 6.1 & $(4.7-7.9)$ & 10.0 & $(8.3-12.0)$ & 8.5 & $(7.2-9.9)$ \\
\hline Los Angeles, CA & 1.2 & $(0.6-2.2)$ & 4.1 & $(2.6-6.2)$ & 2.6 & $(1.8-3.9)$ & 3.1 & $(1.9-5.1)$ & 6.6 & $(4.5-9.7)$ & 4.9 & $(3.4-7.0)$ \\
\hline Miami-Dade County, FL & 1.1 & $(0.7-1.8)$ & 3.6 & $(2.5-5.2)$ & 2.4 & $(1.7-3.2)$ & 3.9 & $(2.9-5.3)$ & 9.4 & $(7.7-11.5)$ & 6.7 & $(5.6-8.0)$ \\
\hline
\end{tabular}

See table footnotes on the next page. 
TABLE 12. (Continued) Percentage of high school students who carried a weapon* on school property ${ }^{\dagger}$ and who were threatened or injured with a weapon* on school property, ${ }^{\S}$ by sex — selected U.S. sites, Youth Risk Behavior Survey, 2015

\begin{tabular}{|c|c|c|c|c|c|c|c|c|c|c|c|c|}
\hline \multirow[b]{3}{*}{ Site } & \multicolumn{6}{|c|}{ Carried a weapon on school property } & \multicolumn{6}{|c|}{ Threatened or injured with a weapon on school property } \\
\hline & \multicolumn{2}{|c|}{ Female } & \multicolumn{2}{|c|}{ Male } & \multicolumn{2}{|c|}{ Total } & \multicolumn{2}{|c|}{ Female } & \multicolumn{2}{|c|}{ Male } & \multicolumn{2}{|c|}{ Total } \\
\hline & $\%$ & $\mathrm{Cl}^{\uparrow}$ & $\%$ & $\mathrm{Cl}$ & $\%$ & $\mathrm{Cl}$ & $\%$ & $\mathrm{Cl}$ & $\%$ & $\mathrm{Cl}$ & $\%$ & $\mathrm{Cl}$ \\
\hline New York City, NY & 2.0 & $(1.6-2.7)$ & 3.9 & $(2.9-5.3)$ & 3.1 & $(2.5-3.9)$ & 4.4 & $(3.4-5.7)$ & 8.0 & $(6.5-9.7)$ & 6.3 & $(5.4-7.5)$ \\
\hline Oakland, CA & 4.8 & $(3.4-6.8)$ & 8.2 & $(6.1-11.1)$ & 6.7 & $(5.5-8.2)$ & 5.3 & $(3.9-7.2)$ & 10.6 & $(8.2-13.5)$ & 8.2 & $(6.8-9.9)$ \\
\hline Orange County, FL & 1.7 & $(1.0-3.0)$ & 4.2 & $(2.5-7.0)$ & 3.0 & $(1.9-4.5)$ & 5.0 & $(3.5-7.2)$ & 8.7 & $(6.1-12.3)$ & 6.9 & $(5.1-9.1)$ \\
\hline Palm Beach County, FL & 2.0 & $(1.2-3.4)$ & 3.8 & $(2.8-5.2)$ & 3.2 & $(2.4-4.4)$ & 5.4 & $(4.0-7.4)$ & 11.1 & $(9.1-13.5)$ & 9.0 & $(7.5-10.8)$ \\
\hline Philadelphia, PA & 1.9 & $(1.0-3.6)$ & 3.6 & $(2.1-6.1)$ & 2.9 & $(1.8-4.6)$ & 4.9 & $(3.2-7.6)$ & 8.6 & $(5.5-13.1)$ & 6.8 & $(4.7-9.9)$ \\
\hline San Diego, CA & 1.6 & $(0.8-3.2)$ & 4.8 & $(3.7-6.2)$ & 3.3 & $(2.5-4.2)$ & 3.0 & $(2.1-4.3)$ & 6.1 & $(4.9-7.5)$ & 4.6 & $(3.8-5.6)$ \\
\hline San Francisco, CA & 3.4 & $(2.3-5.1)$ & 6.0 & $(4.4-8.0)$ & 4.9 & $(3.8-6.3)$ & 4.2 & $(2.5-7.0)$ & 6.8 & $(4.9-9.2)$ & 5.9 & $(4.3-8.1)$ \\
\hline Median & \multicolumn{2}{|c|}{2.0} & \multicolumn{2}{|c|}{4.2} & \multicolumn{2}{|c|}{3.3} & \multicolumn{2}{|c|}{5.1} & \multicolumn{2}{|r|}{7} & \multirow{2}{*}{\multicolumn{2}{|c|}{$\begin{array}{c}6.8 \\
(4.3-13.9)\end{array}$}} \\
\hline Range & \multicolumn{2}{|c|}{$(1.1-6.3)$} & \multicolumn{2}{|c|}{$(3.6-12.7)$} & \multicolumn{2}{|c|}{$(2.4-9.8)$} & \multicolumn{2}{|c|}{ (3.0-11.7) } & \multicolumn{2}{|c|}{$(5.4-16.3)$} & & \\
\hline
\end{tabular}

* Such as a gun, knife, or club.

† On at least 1 day during the 30 days before the survey.

$\$$ One or more times during the 12 months before the survey.

$95 \%$ confidence interval.

** Not available.

TABLE 13. Percentage of high school students who were in a physical fight* and who were injured in a physical fight, ${ }^{*},+$ by sex, race/ethnicity, and grade - United States, Youth Risk Behavior Survey, 2015

\begin{tabular}{|c|c|c|c|c|c|c|c|c|c|c|c|c|}
\hline \multirow[b]{3}{*}{ Category } & \multicolumn{6}{|c|}{ In a physical fight } & \multicolumn{6}{|c|}{ Injured in a physical fight } \\
\hline & \multicolumn{2}{|c|}{ Female } & \multicolumn{2}{|r|}{ Male } & \multicolumn{2}{|r|}{ Total } & \multicolumn{2}{|c|}{ Female } & \multicolumn{2}{|c|}{ Male } & \multicolumn{2}{|c|}{ Total } \\
\hline & $\%$ & $\mathrm{Cl}^{\S}$ & $\%$ & $\mathrm{Cl}$ & $\%$ & $\mathrm{Cl}$ & $\%$ & $\mathrm{Cl}$ & $\%$ & $\mathrm{Cl}$ & $\%$ & $\mathrm{Cl}$ \\
\hline \multicolumn{13}{|c|}{ Race/Ethnicity } \\
\hline White & 13.5 & $(10.8-16.7)$ & 26.6 & $(24.3-29.0)$ & 20.1 & $(17.9-22.5)$ & 0.9 & $(0.6-1.6)$ & 2.8 & $(2.0-3.8)$ & 1.9 & $(1.4-2.6)$ \\
\hline Black" & 25.4 & $(20.1-31.5)$ & 38.6 & (33.9-43.5) & 32.4 & $(28.3-36.8)$ & 3.4 & $(2.1-5.6)$ & 5.8 & $(4.1-8.0)$ & 4.7 & $(3.5-6.3)$ \\
\hline Hispanic & 18.6 & $(16.1-21.4)$ & 27.3 & $(24.2-30.6)$ & 23.0 & $(20.8-25.3)$ & 3.0 & $(2.3-3.9)$ & 4.6 & $(3.2-6.8)$ & 3.8 & $(3.0-4.8)$ \\
\hline \multicolumn{13}{|l|}{ Grade } \\
\hline 9 & 22.6 & $(19.6-26.0)$ & 32.5 & $(28.5-36.9)$ & 27.9 & $(25.0-31.1)$ & 2.5 & $(1.8-3.5)$ & 3.5 & $(2.5-4.9)$ & 3.1 & $(2.5-3.8)$ \\
\hline 10 & 17.6 & $(14.2-21.7)$ & 29.4 & $(26.0-32.9)$ & 23.4 & $(20.6-26.5)$ & 1.4 & $(0.9-2.2)$ & 3.3 & $(2.1-5.0)$ & 2.4 & $(1.7-3.4)$ \\
\hline 11 & 12.8 & $(10.5-15.4)$ & 27.1 & (23.9-30.6) & 20.5 & $(18.1-23.1)$ & 1.5 & $(0.9-2.8)$ & 4.3 & $(3.0-6.0)$ & 3.3 & $(2.3-4.6)$ \\
\hline 12 & 12.0 & $(9.4-15.1)$ & 22.9 & $(19.2-27.0)$ & 17.4 & $(15.1-20.1)$ & 1.4 & $(0.8-2.5)$ & 3.5 & $(2.3-5.2)$ & 2.5 & $(1.8-3.5)$ \\
\hline Total & 16.5 & $(14.5-18.7)$ & 28.4 & $(26.3-30.5)$ & 22.6 & (20.9-24.4) & 1.8 & $(1.4-2.3)$ & 3.7 & $(3.1-4.5)$ & 2.9 & $(2.5-3.4)$ \\
\hline
\end{tabular}

* One or more times during the 12 months before the survey.

${ }^{\dagger}$ Injuries had to be treated by a doctor or nurse.

$\S 95 \%$ confidence interval.

"Non-Hispanic. 
TABLE 14. Percentage of high school students who were in a physical fight* and who were injured in a physical fight, ${ }^{*}+{ }^{*}$ by sex - selected U.S. sites, Youth Risk Behavior Survey, 2015

\begin{tabular}{|c|c|c|c|c|c|c|c|c|c|c|c|c|}
\hline \multirow[b]{3}{*}{ Site } & \multicolumn{6}{|c|}{ In a physical fight } & \multicolumn{6}{|c|}{ Injured in a physical fight } \\
\hline & \multicolumn{2}{|c|}{ Female } & \multicolumn{2}{|r|}{ Male } & \multicolumn{2}{|r|}{ Total } & \multicolumn{2}{|c|}{ Female } & \multicolumn{2}{|r|}{ Male } & \multicolumn{2}{|r|}{ Total } \\
\hline & $\%$ & $\mathrm{Cl}^{\S}$ & $\%$ & $\mathrm{Cl}$ & $\%$ & $\mathrm{Cl}$ & $\%$ & $\mathrm{Cl}$ & $\%$ & $\mathrm{Cl}$ & $\%$ & $\mathrm{Cl}$ \\
\hline \multicolumn{13}{|l|}{ State surveys } \\
\hline Alabama & 17.2 & $(14.4-20.4)$ & 31.2 & $(27.4-35.2)$ & 24.3 & (21.4-27.4) & 2.7 & $(1.7-4.2)$ & 3.7 & $(2.4-5.4)$ & 3.3 & $(2.3-4.7)$ \\
\hline Alaska & 14.8 & $(11.7-18.4)$ & 24.9 & $(21.4-28.7)$ & 20.1 & $(17.4-23.1)$ & 2.2 & $(1.2-3.8)$ & 3.7 & $(2.6-5.4)$ & 3.0 & $(2.2-4.1)$ \\
\hline Arizona & 16.6 & $(13.5-20.2)$ & 28.7 & $(25.0-32.8)$ & 22.8 & (20.4-25.4) & - & - & - & - & - & - \\
\hline Arkansas & 15.6 & (13.2-18.3) & 32.9 & $(29.9-36.0)$ & 24.4 & $(22.8-26.1)$ & 2.5 & $(1.3-4.8)$ & 4.8 & $(3.4-6.9)$ & 3.8 & $(2.7-5.3)$ \\
\hline California & 12.9 & $(9.6-17.0)$ & 19.8 & $(15.5-24.9)$ & 16.3 & (13.3-19.9) & 1.0 & $(0.5-2.1)$ & 4.0 & $(2.5-6.2)$ & 2.5 & $(1.7-3.6)$ \\
\hline Connecticut & 13.2 & $(11.0-15.9)$ & 22.9 & $(20.1-26.0)$ & 18.4 & (16.4-20.5) & - & - & - & - & - & - \\
\hline Delaware & 15.9 & $(13.6-18.6)$ & 25.4 & $(22.0-29.0)$ & 21.2 & $(18.9-23.8)$ & 2.9 & $(1.8-4.5)$ & 4.4 & $(3.2-6.0)$ & 3.8 & $(2.8-5.1)$ \\
\hline Florida & 14.8 & $(13.2-16.6)$ & 26.7 & (24.7-28.9) & 20.9 & $(19.3-22.7)$ & 2.3 & $(1.7-3.0)$ & 4.3 & $(3.4-5.4)$ & 3.4 & $(2.8-4.1)$ \\
\hline Hawaii & 10.9 & $(8.8-13.3)$ & 18.5 & $(16.8-20.5)$ & 14.9 & (13.2-16.9) & 1.5 & $(1.2-2.0)$ & 4.1 & $(3.0-5.6)$ & 2.9 & $(2.3-3.8)$ \\
\hline Idaho & 18.5 & $(16.3-20.9)$ & 27.5 & $(24.5-30.6)$ & 23.2 & $(21.1-25.4)$ & 2.9 & $(2.1-4.2)$ & 2.6 & $(1.7-3.9)$ & 2.8 & $(2.2-3.6)$ \\
\hline Illinois & 15.6 & $(12.8-18.7)$ & 29.8 & $(26.3-33.5)$ & 22.7 & (19.8-25.9) & 2.1 & $(1.4-3.1)$ & 4.1 & $(3.2-5.2)$ & 3.2 & $(2.5-4.0)$ \\
\hline Indiana & 12.9 & $(9.8-16.8)$ & 22.7 & (19.2-26.6) & 18.1 & $(15.0-21.6)$ & 1.1 & $(0.5-2.2)$ & 3.1 & $(1.9-5.1)$ & 2.2 & (1.4-3.3) \\
\hline Kentucky & 16.1 & $(12.9-19.7)$ & 23.6 & (20.2-27.3) & 19.9 & $(17.8-22.2)$ & 1.6 & $(0.8-3.0)$ & 2.4 & $(1.5-3.9)$ & 2.2 & $(1.5-3.3)$ \\
\hline Maine & 9.1 & $(7.9-10.5)$ & 20.5 & $(19.0-22.0)$ & 15.1 & $(13.8-16.4)$ & 1.0 & $(0.8-1.3)$ & 2.7 & $(2.4-3.0)$ & 1.9 & $(1.8-2.1)$ \\
\hline Maryland & - & - & - & - & - & - & - & - & - & - & - & - \\
\hline Massachusetts & 11.4 & $(9.3-14.0)$ & 26.8 & $(23.7-30.1)$ & 19.2 & $(16.7-22.0)$ & 1.2 & $(0.6-2.3)$ & 2.7 & $(1.8-3.9)$ & 2.0 & $(1.4-2.8)$ \\
\hline Michigan & 14.3 & (11.7-17.4) & 26.4 & $(22.8-30.4)$ & 20.4 & $(17.8-23.1)$ & 2.3 & $(1.4-3.7)$ & 2.9 & $(1.9-4.5)$ & 2.7 & $(1.8-3.9)$ \\
\hline Mississippi & 19.9 & $(16.5-23.7)$ & 34.5 & $(29.7-39.7)$ & 27.3 & $(23.9-31.1)$ & 2.6 & $(1.7-3.9)$ & 5.7 & $(3.9-8.2)$ & 4.4 & (3.3-5.9) \\
\hline Missouri & - & - & - & - & - & - & 3.8 & $(2.7-5.5)$ & 8.7 & $(6.6-11.5)$ & 6.3 & $(4.9-8.1)$ \\
\hline Montana & 16.4 & $(14.5-18.5)$ & 28.1 & $(25.8-30.5)$ & 22.4 & $(20.9-24.1)$ & 1.7 & $(1.3-2.3)$ & 2.9 & $(2.3-3.7)$ & 2.3 & $(1.9-2.8)$ \\
\hline Nebraska & 16.1 & (13.3-19.4) & 22.8 & (19.9-26.0) & 19.7 & $(17.7-22.0)$ & 2.4 & $(1.3-4.5)$ & 2.9 & $(1.7-5.0)$ & 2.8 & $(1.9-4.2)$ \\
\hline Nevada & 16.0 & $(12.9-19.6)$ & 24.0 & (20.9-27.4) & 20.1 & $(17.7-22.7)$ & 1.8 & $(0.9-3.3)$ & 5.2 & $(2.9-9.3)$ & 3.6 & $(2.2-5.8)$ \\
\hline New Hampshire & - & - & - & - & - & - & 2.8 & $(2.3-3.4)$ & 5.3 & $(4.6-6.0)$ & 4.2 & $(3.7-4.6)$ \\
\hline New Mexico & 20.5 & $(18.6-22.6)$ & 31.1 & $(28.8-33.4)$ & 25.9 & $(24.2-27.6)$ & - & - & - & - & - & - \\
\hline New York & 17.3 & (15.3-19.5) & 22.8 & $(20.3-25.4)$ & 20.2 & $(18.5-22.0)$ & - & - & - & - & - & - \\
\hline North Carolina & 16.2 & $(12.8-20.3)$ & 24.8 & (19.9-30.4) & 20.7 & $(17.7-24.1)$ & 2.0 & $(1.1-3.8)$ & 3.3 & $(2.1-5.3)$ & 2.7 & $(1.8-4.1)$ \\
\hline North Dakota & - & - & - & - & - & - & - & - & - & - & - & - \\
\hline Oklahoma & 14.2 & $(11.1-18.1)$ & 28.1 & $(24.2-32.4)$ & 21.0 & $(18.0-24.4)$ & 1.4 & $(0.6-3.5)$ & 2.8 & $(1.6-4.7)$ & 2.1 & $(1.2-3.7)$ \\
\hline Pennsylvania & 16.5 & (13.2-20.5) & 26.6 & (23.6-29.9) & 21.7 & (18.9-24.7) & 2.2 & $(1.5-3.3)$ & 3.6 & $(2.5-5.1)$ & 2.9 & $(2.2-3.9)$ \\
\hline Rhode Island & - & - & - & - & - & - & - & - & - & - & - & - \\
\hline South Carolina & 19.4 & $(15.1-24.5)$ & 32.1 & $(28.2-36.2)$ & 25.8 & (21.7-30.4) & 1.5 & $(0.7-3.2)$ & 3.8 & $(2.7-5.3)$ & 2.7 & $(2.1-3.3)$ \\
\hline South Dakota & 14.4 & (10.4-19.5) & 28.8 & $(22.6-35.8)$ & 21.7 & $(17.0-27.2)$ & 1.1 & $(0.5-2.4)$ & 2.8 & $(1.4-5.4)$ & 2.0 & $(1.2-3.3)$ \\
\hline Tennessee & - & - & - & - & - & - & 4.7 & $(3.6-6.0)$ & 8.0 & $(6.8-9.4)$ & 6.5 & $(5.6-7.5)$ \\
\hline Vermont & 12.3 & $(11.7-12.9)$ & 24.2 & $(23.4-25.0)$ & 18.4 & $(17.9-18.9)$ & - & - & - & - & - & - \\
\hline Virginia & 13.5 & $(11.6-15.6)$ & 27.3 & $(24.8-30.1)$ & 20.6 & $(18.7-22.7)$ & 1.9 & $(1.3-2.8)$ & 3.6 & $(2.8-4.6)$ & 2.9 & $(2.3-3.5)$ \\
\hline West Virginia & 14.0 & $(11.1-17.5)$ & 26.9 & $(23.5-30.7)$ & 20.5 & $(17.7-23.6)$ & 2.3 & $(1.5-3.5)$ & 3.1 & $(2.3-4.2)$ & 2.7 & $(2.1-3.6)$ \\
\hline Wyoming & 15.7 & (12.9-18.9) & 23.4 & $(20.6-26.4)$ & 19.7 & $(17.4-22.2)$ & 2.5 & $(1.7-3.6)$ & 2.9 & $(1.9-4.5)$ & 2.7 & $(2.0-3.7)$ \\
\hline Median & \multicolumn{2}{|r|}{15.6} & \multicolumn{2}{|r|}{26.6} & \multicolumn{2}{|r|}{20.6} & \multicolumn{2}{|c|}{2.2} & \multicolumn{2}{|r|}{3.6} & & 2.8 \\
\hline Range & & $-20.5)$ & & $3.5-34.5)$ & & $.9-27.3)$ & & $-4.7)$ & & (1-8.7) & & 9-6.5) \\
\hline Large urban school dist & Irveys & & & & & & & & & & & \\
\hline Baltimore, MD & 34.4 & $(28.8-40.4)$ & 40.0 & $(35.1-45.1)$ & 37.8 & (34.3-41.4) & 7.0 & $(4.2-11.2)$ & 7.1 & $(4.8-10.4)$ & 7.7 & $(5.5-10.7)$ \\
\hline Boston, MA & 17.0 & $(14.1-20.3)$ & 22.9 & $(19.9-26.2)$ & 20.0 & $(17.7-22.4)$ & - & - & - & - & - & - \\
\hline Broward County, FL & 17.0 & (13.8-20.8) & 28.1 & $(24.1-32.5)$ & 22.8 & (19.7-26.2) & 3.3 & $(2.1-5.2)$ & 4.2 & $(2.8-6.2)$ & 3.8 & $(2.8-5.0)$ \\
\hline Cleveland, $\mathrm{OH}$ & 40.3 & $(36.2-44.5)$ & 44.0 & $(40.0-48.1)$ & 42.5 & $(39.5-45.4)$ & - & - & - & - & - & - \\
\hline DeKalb County, GA & 20.2 & $(17.1-23.7)$ & 30.9 & $(27.5-34.5)$ & 25.7 & $(23.4-28.2)$ & 1.5 & $(0.9-2.5)$ & 4.5 & $(2.9-6.8)$ & 3.0 & $(2.2-4.2)$ \\
\hline Detroit, MI & 31.3 & $(27.1-35.7)$ & 39.7 & (32.9-46.8) & 35.2 & $(31.1-39.5)$ & 9.6 & $(6.4-14.3)$ & 9.0 & $(5.8-13.6)$ & 9.4 & $(6.4-13.5)$ \\
\hline District of Columbia & 30.6 & $(29.3-31.9)$ & 34.0 & $(32.5-35.4)$ & 32.4 & $(31.4-33.3)$ & - & - & - & - & - & - \\
\hline Duval County, FL & 23.8 & $(21.4-26.4)$ & 31.1 & $(27.9-34.5)$ & 27.4 & $(25.1-29.7)$ & - & - & - & - & - & - \\
\hline Ft. Worth, TX & 18.3 & $(15.8-21.2)$ & 29.5 & $(26.6-32.7)$ & 23.8 & $(21.7-26.1)$ & 1.6 & $(0.9-2.6)$ & 4.6 & $(3.5-6.1)$ & 3.1 & (2.4-3.9) \\
\hline Houston, TX & 20.0 & $(17.8-22.3)$ & 28.7 & $(26.3-31.1)$ & 24.6 & $(22.7-26.5)$ & 3.1 & $(2.2-4.2)$ & 4.7 & $(3.6-6.1)$ & 4.1 & $(3.3-5.0)$ \\
\hline Los Angeles, CA & 11.7 & $(9.4-14.6)$ & 21.1 & $(16.9-26.0)$ & 16.3 & $(13.9-19.2)$ & 1.3 & $(0.7-2.5)$ & 3.2 & $(2.3-4.5)$ & 2.2 & $(1.7-2.9)$ \\
\hline Miami-Dade County, FL & 12.4 & $(10.5-14.6)$ & 27.3 & $(24.6-30.2)$ & 19.8 & $(17.9-21.7)$ & 1.2 & $(0.7-2.1)$ & 4.7 & $(3.5-6.3)$ & 3.0 & $(2.2-4.0)$ \\
\hline
\end{tabular}

See table footnotes on the next page. 
TABLE 14. (Continued) Percentage of high school students who were in a physical fight* and who were injured in a physical fight, ${ }^{*}, \dagger$ by sex selected U.S. sites, Youth Risk Behavior Survey, 2015

\begin{tabular}{|c|c|c|c|c|c|c|c|c|c|c|c|c|}
\hline \multirow[b]{3}{*}{ Site } & \multicolumn{6}{|c|}{ In a physical fight } & \multicolumn{6}{|c|}{ Injured in a physical fight } \\
\hline & \multicolumn{2}{|c|}{ Female } & \multicolumn{2}{|r|}{ Male } & \multicolumn{2}{|r|}{ Total } & \multicolumn{2}{|c|}{ Female } & \multicolumn{2}{|c|}{ Male } & \multicolumn{2}{|r|}{ Total } \\
\hline & $\%$ & $\mathrm{Cl}^{\S}$ & $\%$ & $\mathrm{Cl}$ & $\%$ & $\mathrm{Cl}$ & $\%$ & $\mathrm{Cl}$ & $\%$ & $\mathrm{Cl}$ & $\%$ & $\mathrm{Cl}$ \\
\hline New York City, NY & 18.8 & $(16.2-21.7)$ & 25.6 & $(23.6-27.8)$ & 22.4 & $(21.0-24.0)$ & - & - & - & - & - & - \\
\hline Oakland, CA & 16.0 & $(13.1-19.5)$ & 21.4 & $(18.2-25.0)$ & 19.1 & $(16.7-21.9)$ & 2.2 & $(1.3-3.5)$ & 4.3 & $(2.7-6.9)$ & 3.4 & $(2.3-4.8)$ \\
\hline Orange County, FL & 13.5 & $(10.6-16.9)$ & 24.4 & $(20.1-29.2)$ & 18.7 & $(15.8-22.1)$ & 2.9 & $(1.7-4.9)$ & 4.8 & $(3.0-7.6)$ & 3.8 & $(2.6-5.5)$ \\
\hline Palm Beach County, FL & 14.0 & $(11.9-16.3)$ & 26.9 & $(24.2-29.8)$ & 21.1 & $(19.2-23.2)$ & 1.8 & $(1.0-3.0)$ & 4.6 & $(3.5-6.1)$ & 3.6 & $(2.7-4.8)$ \\
\hline Philadelphia, PA & 30.0 & $(24.1-36.7)$ & 41.6 & $(36.0-47.5)$ & 35.9 & $(31.2-41.0)$ & 3.8 & $(2.6-5.4)$ & 6.9 & $(5.1-9.3)$ & 5.4 & $(4.3-6.8)$ \\
\hline San Diego, CA & 11.9 & $(9.6-14.6)$ & 27.4 & $(24.2-30.9)$ & 19.8 & $(17.6-22.3)$ & 1.3 & $(0.7-2.5)$ & 3.4 & $(2.5-4.7)$ & 2.4 & $(1.8-3.1)$ \\
\hline San Francisco, CA & 11.7 & (8.9-15.1) & 16.0 & $(13.3-19.1)$ & 13.9 & $(11.8-16.4)$ & 1.4 & $(0.8-2.7)$ & 2.7 & $(1.7-4.5)$ & 2.2 & $(1.4-3.4)$ \\
\hline Median & \multicolumn{2}{|c|}{18.3} & \multicolumn{2}{|r|}{28.1} & \multicolumn{2}{|r|}{22.8} & \multicolumn{2}{|c|}{2.0} & \multicolumn{2}{|c|}{4.6} & \multicolumn{2}{|r|}{3.5} \\
\hline Range & \multicolumn{2}{|c|}{$(11.7-40.3)$} & \multicolumn{2}{|r|}{$(16.0-44.0)$} & \multicolumn{2}{|c|}{$(13.9-42.5)$} & \multicolumn{2}{|c|}{$(1.2-9.6)$} & \multicolumn{2}{|c|}{$(2.7-9.0)$} & \multicolumn{2}{|c|}{$(2.2-9.4)$} \\
\hline
\end{tabular}

* One or more times during the 12 months before the survey.

$\dagger$ Injuries had to be treated by a doctor or nurse.

$\S 95 \%$ confidence interval.

१ Not available.

TABLE 15. Percentage of high school students who were in a physical fight on school property* and who did not go to school because they felt unsafe at school or on their way to or from school, ${ }^{\dagger}$ by sex, race/ethnicity, and grade - United States, Youth Risk Behavior Survey, 2015

\begin{tabular}{|c|c|c|c|c|c|c|c|c|c|c|c|c|}
\hline \multirow[b]{3}{*}{ Category } & \multicolumn{6}{|c|}{ In a physical fight on school property } & \multicolumn{6}{|c|}{ Did not go to school because of safety concerns } \\
\hline & \multicolumn{2}{|c|}{ Female } & \multicolumn{2}{|r|}{ Male } & \multicolumn{2}{|r|}{ Total } & \multicolumn{2}{|c|}{ Female } & \multicolumn{2}{|c|}{ Male } & \multicolumn{2}{|c|}{ Total } \\
\hline & $\%$ & $\mathrm{Cl}^{\S}$ & $\%$ & $\mathrm{Cl}$ & $\%$ & $\mathrm{Cl}$ & $\%$ & $\mathrm{Cl}$ & $\%$ & $\mathrm{Cl}$ & $\%$ & $\mathrm{Cl}$ \\
\hline \multicolumn{13}{|c|}{ Race/Ethnicity } \\
\hline Whiteq & 3.2 & $(2.2-4.6)$ & 8.0 & $(6.8-9.4)$ & 5.6 & $(5.0-6.4)$ & 5.4 & $(4.1-7.1)$ & 2.9 & $(2.0-4.1)$ & 4.2 & $(3.2-5.4)$ \\
\hline Black" & 9.4 & $(6.7-13.1)$ & 15.4 & $(10.9-21.2)$ & 12.6 & $(9.1-17.1)$ & 6.4 & $(4.7-8.5)$ & 6.9 & $(5.0-9.4)$ & 6.8 & $(5.4-8.5)$ \\
\hline Hispanic & 7.1 & $(5.5-9.1)$ & 10.7 & $(8.6-13.2)$ & 8.9 & $(7.3-10.9)$ & 7.4 & $(5.8-9.3)$ & 7.6 & $(6.2-9.4)$ & 7.6 & $(6.3-9.0)$ \\
\hline \multicolumn{13}{|l|}{ Grade } \\
\hline 9 & 8.2 & $(6.7-9.9)$ & 14.7 & $(12.6-17.2)$ & 11.6 & $(10.1-13.4)$ & 7.7 & $(6.0-9.9)$ & 4.9 & $(3.5-6.9)$ & 6.4 & $(5.1-7.8)$ \\
\hline 10 & 4.6 & $(3.3-6.4)$ & 10.0 & $(7.8-12.8)$ & 7.3 & $(5.9-9.0)$ & 6.3 & $(5.0-8.0)$ & 4.4 & $(2.9-6.6)$ & 5.4 & $(4.2-6.9)$ \\
\hline 11 & 4.1 & $(2.8-5.9)$ & 8.3 & $(6.0-11.4)$ & 6.5 & $(5.0-8.4)$ & 5.3 & $(4.0-7.1)$ & 3.7 & $(2.7-5.0)$ & 4.6 & $(3.7-5.9)$ \\
\hline 12 & 2.5 & $(1.7-3.7)$ & 6.4 & $(4.7-8.6)$ & 4.5 & $(3.5-5.6)$ & 4.3 & $(3.0-6.2)$ & 6.9 & $(4.3-10.8)$ & 5.7 & $(4.0-7.9)$ \\
\hline Total & 5.0 & $(4.1-6.0)$ & 10.3 & $(8.8-12.0)$ & 7.8 & $(6.7-8.9)$ & 6.0 & $(5.1-7.1)$ & 5.0 & $(4.2-5.9)$ & 5.6 & $(4.8-6.5)$ \\
\hline
\end{tabular}

* One or more times during the 12 months before the survey.

+ On at least 1 day during the 30 days before the survey.

$\S 95 \%$ confidence interval.

I Non-Hispanic. 
TABLE 16. Percentage of high school students who were in a physical fight on school property* and who did not go to school because they felt unsafe at school or on their way to or from school, ${ }^{\dagger}$ by sex — selected U.S. sites, Youth Risk Behavior Survey, 2015

\begin{tabular}{|c|c|c|c|c|c|c|c|c|c|c|c|c|}
\hline \multirow[b]{3}{*}{ Site } & \multicolumn{6}{|c|}{ In a physical fight on school property } & \multicolumn{6}{|c|}{ Did not go to school because of safety concerns } \\
\hline & \multicolumn{2}{|r|}{ Female } & \multicolumn{2}{|c|}{ Male } & \multicolumn{2}{|r|}{ Total } & \multicolumn{2}{|c|}{ Female } & \multicolumn{2}{|c|}{ Male } & \multicolumn{2}{|c|}{ Total } \\
\hline & $\%$ & $\mathrm{Cl}^{\S}$ & $\%$ & $\mathrm{Cl}$ & $\%$ & $\mathrm{Cl}$ & $\%$ & $\mathrm{Cl}$ & $\%$ & $\mathrm{Cl}$ & $\%$ & $\mathrm{Cl}$ \\
\hline \multicolumn{13}{|l|}{ State surveys } \\
\hline Alabama & 6.1 & $(4.5-8.2)$ & 12.2 & $(9.9-15.1)$ & 9.3 & $(7.7-11.2)$ & 8.6 & $(6.2-11.8)$ & 7.7 & $(5.7-10.4)$ & 8.1 & $(6.2-10.5)$ \\
\hline Alaska & 3.2 & $(1.9-5.5)$ & 7.8 & $(6.2-9.7)$ & 5.8 & $(4.6-7.3)$ & 9.0 & $(7.0-11.6)$ & 8.2 & $(6.2-10.7)$ & 8.8 & $(7.3-10.6)$ \\
\hline Arizona & 4.8 & $(3.2-7.2)$ & 9.4 & $(7.5-11.8)$ & 7.2 & $(5.5-9.3)$ & 6.8 & $(5.2-8.8)$ & 5.6 & $(4.1-7.5)$ & 6.3 & $(5.3-7.5)$ \\
\hline Arkansas & 5.3 & $(3.4-8.1)$ & 16.9 & $(14.7-19.3)$ & 11.2 & $(9.9-12.7)$ & 8.4 & $(7.0-10.2)$ & 6.7 & $(5.0-8.9)$ & 7.7 & $(6.6-8.9)$ \\
\hline California & 4.3 & $(3.0-6.1)$ & 8.8 & $(6.9-11.4)$ & 6.6 & $(5.5-7.8)$ & 7.4 & $(5.2-10.5)$ & 4.4 & $(3.3-6.0)$ & 6.1 & $(4.6-8.0)$ \\
\hline Connecticut & -q & - & - & - & - & - & 6.2 & $(4.5-8.6)$ & 7.2 & $(5.3-9.9)$ & 6.9 & $(5.4-8.8)$ \\
\hline Delaware & 5.9 & $(4.2-8.1)$ & 10.1 & $(8.0-12.6)$ & 8.1 & $(6.7-9.8)$ & 3.9 & $(2.6-5.8)$ & 6.1 & $(4.8-7.8)$ & 5.3 & $(4.3-6.5)$ \\
\hline Florida & 5.6 & $(4.7-6.7)$ & 9.5 & $(8.2-11.0)$ & 7.6 & $(6.6-8.8)$ & 8.2 & (7.1-9.4) & 7.8 & $(6.3-9.6)$ & 8.1 & (7.1-9.4) \\
\hline Hawaii & - & - & - & - & - & - & 8.9 & $(7.4-10.6)$ & 8.8 & $(7.3-10.7)$ & 9.1 & $(7.8-10.6)$ \\
\hline Idaho & 3.5 & $(2.2-5.5)$ & 8.2 & $(6.3-10.6)$ & 6.0 & $(4.9-7.3)$ & 6.7 & $(5.0-9.0)$ & 4.0 & $(2.8-5.9)$ & 5.4 & (4.1-6.9) \\
\hline Illinois & 5.5 & $(3.9-7.8)$ & 9.7 & $(7.8-12.2)$ & 7.7 & $(6.0-9.8)$ & 5.8 & $(4.4-7.6)$ & 6.1 & $(4.5-8.3)$ & 6.0 & $(4.6-7.8)$ \\
\hline Indiana & 3.4 & $(2.1-5.6)$ & 7.2 & $(5.4-9.5)$ & 5.5 & $(4.2-7.2)$ & 6.8 & $(4.6-9.9)$ & 6.3 & $(3.7-10.5)$ & 6.7 & $(4.6-9.6)$ \\
\hline Kentucky & 4.8 & $(3.3-7.0)$ & 10.3 & $(8.1-12.9)$ & 7.8 & (6.4-9.4) & 5.8 & $(4.5-7.6)$ & 5.1 & $(3.3-7.7)$ & 5.7 & $(4.5-7.3)$ \\
\hline Maine & 2.3 & $(1.9-2.8)$ & 7.1 & $(6.1-8.2)$ & 4.9 & $(4.3-5.5)$ & 6.0 & $(5.3-6.8)$ & 4.5 & $(3.6-5.5)$ & 5.4 & $(4.7-6.1)$ \\
\hline Maryland & 8.5 & $(7.9-9.1)$ & 15.1 & $(14.4-15.9)$ & 12.2 & $(11.6-12.8)$ & 5.5 & $(5.1-6.0)$ & 6.0 & $(5.6-6.4)$ & 6.0 & $(5.7-6.4)$ \\
\hline Massachusetts & 3.3 & $(2.1-5.2)$ & 7.7 & $(6.4-9.2)$ & 5.6 & $(4.5-7.0)$ & 5.8 & $(4.6-7.3)$ & 3.8 & $(2.9-5.0)$ & 4.8 & (3.9-5.9) \\
\hline Michigan & 4.8 & $(3.2-7.2)$ & 9.9 & $(7.6-12.7)$ & 7.4 & $(5.8-9.5)$ & 6.6 & $(5.0-8.7)$ & 4.8 & $(3.7-6.2)$ & 5.8 & $(4.8-6.9)$ \\
\hline Mississippi & 5.6 & $(3.9-8.1)$ & 11.4 & $(8.6-15.0)$ & 8.7 & $(6.8-11.2)$ & 8.7 & $(6.7-11.4)$ & 8.5 & $(6.0-12.0)$ & 9.0 & $(6.9-11.8)$ \\
\hline Missouri & - & - & - & - & - & - & 4.1 & $(3.2-5.2)$ & 8.0 & $(5.7-11.3)$ & 6.1 & $(4.8-7.8)$ \\
\hline Montana & 4.8 & $(3.8-6.2)$ & 10.2 & $(8.6-11.9)$ & 7.6 & $(6.6-8.7)$ & 5.3 & $(4.4-6.4)$ & 4.6 & $(3.6-6.0)$ & 5.0 & $(4.2-6.0)$ \\
\hline Nebraska & 3.1 & $(2.0-4.9)$ & 7.3 & $(5.4-9.8)$ & 5.5 & $(4.4-6.9)$ & 6.8 & $(4.8-9.4)$ & 5.1 & (3.2-8.1) & 6.2 & $(4.6-8.4)$ \\
\hline Nevada & 5.7 & $(3.9-8.4)$ & 7.7 & $(5.2-11.2)$ & 6.8 & $(5.2-8.8)$ & 8.5 & $(6.1-11.9)$ & 8.1 & $(6.4-10.3)$ & 8.5 & $(7.0-10.4)$ \\
\hline New Hampshire & 3.5 & $(3.0-4.1)$ & 8.9 & $(8.1-9.8)$ & 6.4 & $(5.9-7.0)$ & 6.2 & $(5.5-6.9)$ & 4.5 & $(3.9-5.2)$ & 5.4 & $(4.9-5.9)$ \\
\hline New Mexico & 5.8 & $(4.9-6.9)$ & 11.1 & $(9.7-12.6)$ & 8.5 & $(7.5-9.5)$ & 7.7 & $(5.3-11.3)$ & 7.8 & $(6.3-9.6)$ & 7.8 & $(5.9-10.2)$ \\
\hline New York & - & - & - & - & - & - & 6.3 & $(4.9-8.0)$ & 8.4 & $(6.9-10.1)$ & 7.6 & $(6.5-8.8)$ \\
\hline North Carolina & 4.9 & $(3.5-6.9)$ & 8.7 & $(6.3-11.9)$ & 6.9 & $(5.6-8.5)$ & 5.9 & $(4.2-8.5)$ & 6.2 & $(4.7-8.2)$ & 6.2 & $(4.9-8.0)$ \\
\hline North Dakota & 3.2 & $(1.9-5.2)$ & 7.5 & $(5.9-9.4)$ & 5.4 & $(4.3-6.8)$ & - & - & - & - & - & - \\
\hline Oklahoma & 4.4 & $(2.7-7.2)$ & 9.9 & $(7.7-12.5)$ & 7.1 & $(5.3-9.6)$ & 5.0 & $(3.3-7.4)$ & 3.8 & $(1.9-7.2)$ & 4.3 & $(2.9-6.5)$ \\
\hline Pennsylvania & 4.3 & $(3.0-6.2)$ & 9.2 & $(7.1-11.8)$ & 6.8 & $(5.3-8.7)$ & 8.3 & $(5.3-12.8)$ & 6.7 & $(4.3-10.3)$ & 7.6 & $(5.1-11.2)$ \\
\hline Rhode Island & 6.6 & $(4.6-9.3)$ & 11.1 & $(8.8-13.9)$ & 9.1 & $(7.1-11.5)$ & 4.9 & $(3.2-7.3)$ & 6.7 & $(5.4-8.4)$ & 6.0 & $(4.9-7.3)$ \\
\hline South Carolina & 5.4 & $(3.5-8.2)$ & 12.8 & $(9.0-18.0)$ & 9.1 & $(6.5-12.6)$ & 14.2 & $(8.1-23.7)$ & 8.8 & $(6.5-11.7)$ & 11.5 & (7.7-16.8) \\
\hline South Dakota & 3.3 & $(1.9-5.5)$ & 10.2 & $(5.9-17.1)$ & 6.8 & $(4.5-10.2)$ & 4.8 & $(2.9-7.8)$ & 4.7 & $(2.5-8.8)$ & 4.7 & $(2.8-7.8)$ \\
\hline Tennessee & 7.7 & $(6.1-9.7)$ & 13.5 & $(10.9-16.4)$ & 10.8 & $(9.4-12.4)$ & 9.9 & $(8.0-12.3)$ & 8.6 & $(7.1-10.3)$ & 9.3 & $(7.7-11.2)$ \\
\hline Vermont & 4.0 & $(3.7-4.4)$ & 10.4 & $(9.9-11.0)$ & 7.4 & $(7.0-7.7)$ & 7.2 & $(6.7-7.7)$ & 4.4 & $(4.0-4.8)$ & 5.9 & $(5.6-6.2)$ \\
\hline Virginia & 4.6 & $(3.6-6.0)$ & 10.4 & $(8.7-12.4)$ & 7.7 & $(6.5-9.0)$ & 6.6 & $(5.0-8.6)$ & 5.6 & $(4.5-7.0)$ & 6.1 & $(5.0-7.4)$ \\
\hline West Virginia & 4.2 & $(3.0-5.7)$ & 10.3 & $(7.0-14.9)$ & 7.2 & $(5.1-10.1)$ & 9.4 & $(6.9-12.7)$ & 8.4 & $(5.8-12.0)$ & 8.9 & $(7.0-11.2)$ \\
\hline Wyoming & 3.7 & $(2.6-5.1)$ & 8.4 & $(6.8-10.3)$ & 6.1 & $(5.1-7.4)$ & 7.1 & $(5.6-9.1)$ & 6.2 & $(4.7-8.2)$ & 6.7 & $(5.5-8.2)$ \\
\hline Median & \multirow{2}{*}{\multicolumn{2}{|c|}{$\begin{array}{c}4.8 \\
(2.3-8.5)\end{array}$}} & \multirow{2}{*}{\multicolumn{2}{|c|}{$\begin{array}{c}9.9 \\
(7.1-16.9)\end{array}$}} & \multirow{2}{*}{\multicolumn{2}{|c|}{$\begin{array}{c}7.2 \\
(4.9-12.2)\end{array}$}} & \multirow{2}{*}{\multicolumn{2}{|c|}{$\begin{array}{c}6.7 \\
(3.9-14.2)\end{array}$}} & \multirow{2}{*}{\multicolumn{2}{|c|}{$\begin{array}{c}6.2 \\
(3.8-8.8)\end{array}$}} & & 6.2 \\
\hline Range & & & & & & & & & & & & $.3-11.5)$ \\
\hline Large urban school dist & Irveys & & & & & & & & & & & \\
\hline Baltimore, MD & 12.6 & $(9.7-16.3)$ & 18.0 & $(14.0-22.9)$ & 15.9 & $(13.4-18.7)$ & 8.6 & $(6.2-11.8)$ & 12.4 & $(8.7-17.3)$ & 11.5 & $(9.3-14.3)$ \\
\hline Boston, MA & 6.3 & $(4.7-8.5)$ & 7.9 & $(6.1-10.2)$ & 7.1 & $(5.9-8.6)$ & 6.4 & $(4.6-8.7)$ & 5.7 & $(4.1-7.9)$ & 6.1 & $(4.9-7.6)$ \\
\hline Broward County, FL & 6.3 & $(4.5-8.8)$ & 9.7 & $(7.6-12.3)$ & 8.1 & $(6.5-10.1)$ & 7.9 & $(5.9-10.5)$ & 7.6 & $(5.8-10.0)$ & 7.9 & (6.4-9.6) \\
\hline Cleveland, $\mathrm{OH}$ & - & - & - & - & - & - & 10.8 & $(8.1-14.3)$ & 11.6 & $(9.2-14.5)$ & 12.0 & $(10.0-14.2)$ \\
\hline DeKalb County, GA & - & - & - & - & - & - & 10.3 & $(8.2-12.8)$ & 9.5 & $(7.2-12.4)$ & 9.9 & $(8.1-12.1)$ \\
\hline Detroit, MI & 15.9 & $(12.1-20.5)$ & 18.9 & $(14.9-23.6)$ & 17.5 & $(14.4-21.0)$ & 11.5 & $(8.8-14.9)$ & 11.2 & $(8.6-14.6)$ & 11.5 & (9.1-14.4) \\
\hline District of Columbia & 12.8 & (11.9-13.6) & 14.6 & $(13.5-15.7)$ & 13.8 & $(13.1-14.5)$ & 6.3 & $(5.7-7.0)$ & 7.8 & $(7.1-8.7)$ & 7.3 & $(6.8-7.8)$ \\
\hline Duval County, FL & 8.7 & $(7.0-10.9)$ & 13.9 & $(11.4-16.8)$ & 11.4 & $(9.7-13.5)$ & 11.5 & $(9.5-13.9)$ & 12.8 & $(10.2-15.9)$ & 12.8 & $(10.7-15.1)$ \\
\hline Ft. Worth, TX & 7.2 & $(5.6-9.3)$ & 11.6 & $(9.7-13.8)$ & 9.4 & $(8.1-10.8)$ & 7.2 & $(5.6-9.1)$ & 6.8 & (5.3-8.7) & 7.0 & (5.9-8.4) \\
\hline Houston, TX & 8.4 & $(6.8-10.4)$ & 12.0 & $(10.2-14.2)$ & 10.4 & $(8.9-12.0)$ & 10.5 & $(8.9-12.4)$ & 11.2 & $(9.5-13.2)$ & 11.1 & $(9.8-12.6)$ \\
\hline Los Angeles, CA & 4.5 & $(2.9-7.0)$ & 8.1 & $(5.7-11.5)$ & 6.3 & $(4.6-8.7)$ & 5.8 & $(3.8-8.7)$ & 6.3 & $(4.2-9.4)$ & 6.2 & $(4.5-8.4)$ \\
\hline Miami-Dade County, FL & 3.5 & $(2.6-4.8)$ & 11.0 & $(8.9-13.6)$ & 7.3 & $(6.1-8.8)$ & 6.6 & $(5.2-8.4)$ & 8.1 & $(6.4-10.1)$ & 7.4 & $(6.1-8.9)$ \\
\hline
\end{tabular}

See table footnotes on the next page. 
TABLE 16. (Continued) Percentage of high school students who were in a physical fight on school property* and who did not go to school because they felt unsafe at school or on their way to or from school, ${ }^{\dagger}$ by sex - selected U.S. sites, Youth Risk Behavior Survey, 2015

\begin{tabular}{|c|c|c|c|c|c|c|c|c|c|c|c|c|}
\hline \multirow[b]{3}{*}{ Site } & \multicolumn{6}{|c|}{ In a physical fight on school property } & \multicolumn{6}{|c|}{ Did not go to school because of safety concerns } \\
\hline & \multicolumn{2}{|c|}{ Female } & \multicolumn{2}{|r|}{ Male } & \multicolumn{2}{|r|}{ Total } & \multicolumn{2}{|r|}{ Female } & \multicolumn{2}{|r|}{ Male } & \multicolumn{2}{|r|}{ Total } \\
\hline & $\%$ & $\mathrm{Cl}^{\S}$ & $\%$ & $\mathrm{Cl}$ & $\%$ & $\mathrm{Cl}$ & $\%$ & $\mathrm{Cl}$ & $\%$ & $\mathrm{Cl}$ & $\%$ & $\mathrm{Cl}$ \\
\hline New York City, NY & - & - & - & - & - & - & 5.7 & $(4.8-6.8)$ & 5.9 & $(5.0-7.0)$ & 6.0 & $(5.2-6.8)$ \\
\hline Oakland, CA & 5.4 & $(4.0-7.4)$ & 8.7 & $(6.2-12.1)$ & 7.3 & $(5.7-9.3)$ & 8.8 & $(6.5-11.9)$ & 8.4 & $(6.5-11.0)$ & 9.0 & $(7.5-10.8)$ \\
\hline Orange County, FL & 5.3 & $(3.6-7.5)$ & 11.3 & $(8.2-15.3)$ & 8.2 & $(6.2-10.7)$ & 10.1 & $(8.0-12.7)$ & 8.4 & $(5.6-12.3)$ & 9.3 & $(7.3-11.9)$ \\
\hline Palm Beach County, FL & 3.7 & $(2.7-5.1)$ & 7.6 & $(5.9-9.9)$ & 6.0 & $(4.9-7.4)$ & 8.8 & $(6.9-11.1)$ & 9.6 & $(7.6-12.0)$ & 9.7 & $(8.0-11.6)$ \\
\hline Philadelphia, PA & 11.7 & $(8.5-15.8)$ & 18.5 & $(15.4-22.1)$ & 15.2 & $(12.4-18.5)$ & 11.0 & $(9.1-13.2)$ & 8.3 & $(5.7-12.0)$ & 9.9 & $(7.9-12.4)$ \\
\hline San Diego, CA & 2.8 & $(2.0-3.9)$ & 10.6 & $(8.7-12.9)$ & 6.8 & (5.6-8.1) & 4.9 & $(3.7-6.4)$ & 4.1 & $(2.8-6.1)$ & 4.5 & $(3.5-5.6)$ \\
\hline San Francisco, CA & 3.8 & $(2.8-5.3)$ & 7.1 & $(5.5-9.1)$ & 5.7 & $(4.7-6.9)$ & - & - & - & - & - & - \\
\hline Median & \multicolumn{2}{|c|}{6.3} & \multicolumn{2}{|r|}{11.1} & \multirow{2}{*}{\multicolumn{2}{|c|}{$\begin{array}{c}8.1 \\
(5.7-17.5)\end{array}$}} & \multirow{2}{*}{\multicolumn{2}{|c|}{$\begin{array}{c}8.7 \\
(4.9-11.5)\end{array}$}} & \multirow{2}{*}{\multicolumn{2}{|c|}{$\begin{array}{c}8.3 \\
(4.1-12.8)\end{array}$}} & \multirow{2}{*}{\multicolumn{2}{|c|}{$\begin{array}{c}9.1 \\
(4.5-12.8)\end{array}$}} \\
\hline Range & & $8-15.9)$ & & (7.1-18.9) & & & & & & & & \\
\hline
\end{tabular}

* One or more times during the 12 months before the survey.

$\dagger$ On at least 1 day during the 30 days before the survey.

$\S 95 \%$ confidence interval.

" Not available.

TABLE 17. Percentage of high school students who were electronically bullied, ${ }^{*}{ }^{\dagger}$ and who were bullied on school property, ${ }^{\dagger}$ by sex, race/ ethnicity, and grade - United States, Youth Risk Behavior Survey, 2015

\begin{tabular}{|c|c|c|c|c|c|c|c|c|c|c|c|c|}
\hline \multirow[b]{3}{*}{ Category } & \multicolumn{6}{|c|}{ Electronically bullied } & \multicolumn{6}{|c|}{ Bullied on school property } \\
\hline & \multicolumn{2}{|r|}{ Female } & \multicolumn{2}{|c|}{ Male } & \multicolumn{2}{|r|}{ Total } & \multicolumn{2}{|c|}{ Female } & \multicolumn{2}{|r|}{ Male } & \multicolumn{2}{|r|}{ Total } \\
\hline & $\%$ & $\mathrm{Cl}^{\S}$ & $\%$ & $\mathrm{Cl}$ & $\%$ & $\mathrm{Cl}$ & $\%$ & $\mathrm{Cl}$ & $\%$ & $\mathrm{Cl}$ & $\%$ & $\mathrm{Cl}$ \\
\hline \multicolumn{13}{|c|}{ Race/Ethnicity } \\
\hline White? & 26.0 & $(24.0-28.1)$ & 10.8 & $(8.8-13.3)$ & 18.4 & $(16.8-20.0)$ & 29.1 & $(26.5-31.8)$ & 18.1 & $(16.3-20.0)$ & 23.5 & $(21.7-25.3)$ \\
\hline Black" & 11.9 & $(9.1-15.3)$ & 5.6 & $(3.8-8.2)$ & 8.6 & $(6.8-10.8)$ & 15.1 & $(11.9-18.9)$ & 11.2 & $(8.4-14.9)$ & 13.2 & $(10.7-16.3)$ \\
\hline Hispanic & 16.7 & $(14.0-19.9)$ & 8.1 & $(6.4-10.3)$ & 12.4 & $(10.5-14.5)$ & 19.3 & $(15.9-23.3)$ & 13.7 & $(11.6-16.2)$ & 16.5 & $(14.2-19.1)$ \\
\hline \multicolumn{13}{|l|}{ Grade } \\
\hline 9 & 22.7 & $(19.8-26.0)$ & 11.0 & $(8.1-14.7)$ & 16.5 & $(14.6-18.6)$ & 29.0 & $(25.8-32.4)$ & 18.3 & $(15.2-21.7)$ & 23.4 & $(20.7-26.3)$ \\
\hline 10 & 23.2 & $(20.6-25.9)$ & 9.9 & $(7.9-12.4)$ & 16.6 & $(14.7-18.6)$ & 25.5 & $(21.9-29.4)$ & 16.1 & $(13.7-18.9)$ & 20.8 & (18.4-23.5) \\
\hline 11 & 21.4 & $(18.7-24.4)$ & 8.4 & $(6.3-11.0)$ & 14.7 & $(12.5-17.2)$ & 24.2 & $(21.8-26.8)$ & 16.4 & $(14.2-18.8)$ & 20.3 & $(18.6-22.1)$ \\
\hline 12 & 19.5 & $(16.8-22.4)$ & 9.2 & $(7.2-11.8)$ & 14.3 & $(12.6-16.1)$ & 19.8 & $(16.6-23.4)$ & 12.1 & $(10.2-14.3)$ & 15.9 & $(14.1-17.9)$ \\
\hline Total & 21.7 & $(20.1-23.4)$ & 9.7 & $(8.4-11.1)$ & 15.5 & $(14.5-16.6)$ & 24.8 & $(22.8-26.9)$ & 15.8 & $(14.5-17.2)$ & 20.2 & $(18.8-21.7)$ \\
\hline
\end{tabular}

* Counting being bullied through e-mail, chat rooms, instant messaging, Web sites, or texting.

† During the 12 months before the survey.

$\S 95 \%$ confidence interval.

१ Non-Hispanic. 
TABLE 18. Percentage of high school students who were electronically bullied, ${ }^{*},{ }^{\dagger}$ and who were bullied on school property, ${ }^{\dagger}$ by sex - selected U.S. sites, Youth Risk Behavior Survey, 2015

\begin{tabular}{|c|c|c|c|c|c|c|c|c|c|c|c|c|}
\hline \multirow[b]{3}{*}{ Site } & \multicolumn{6}{|c|}{ Electronically bullied } & \multicolumn{6}{|c|}{ Bullied on school property } \\
\hline & \multicolumn{2}{|c|}{ Female } & \multicolumn{2}{|r|}{ Male } & \multicolumn{2}{|r|}{ Total } & \multicolumn{2}{|c|}{ Female } & \multicolumn{2}{|r|}{ Male } & \multicolumn{2}{|r|}{ Total } \\
\hline & $\%$ & $\mathrm{Cl}^{\S}$ & $\%$ & $\mathrm{Cl}$ & $\%$ & $\mathrm{Cl}$ & $\%$ & $\mathrm{Cl}$ & $\%$ & $\mathrm{Cl}$ & $\%$ & $\mathrm{Cl}$ \\
\hline \multicolumn{13}{|l|}{ State surveys } \\
\hline Alabama & 19.6 & $(16.7-22.8)$ & 7.3 & $(5.7-9.2)$ & 13.5 & $(11.7-15.5)$ & 21.6 & (17.9-25.8) & 16.2 & (13.5-19.4) & 19.0 & (16.8-21.5) \\
\hline Alaska & 22.9 & $(19.9-26.2)$ & 12.6 & $(10.1-15.5)$ & 17.7 & $(15.7-19.9)$ & 26.5 & $(23.2-30.2)$ & 19.5 & $(16.9-22.4)$ & 22.8 & (20.4-25.4) \\
\hline Arizona & - & - & - & - & - & - & - & - & - & - & - & - \\
\hline Arkansas & 24.8 & $(21.5-28.4)$ & 11.8 & $(8.5-16.3)$ & 18.2 & $(15.8-20.9)$ & 25.8 & $(21.7-30.4)$ & 19.9 & $(16.3-24.1)$ & 22.9 & $(20.3-25.7)$ \\
\hline California & 18.1 & $(13.9-23.3)$ & 8.3 & $(5.6-12.2)$ & 13.5 & $(10.0-18.0)$ & 22.2 & $(18.6-26.2)$ & 14.3 & $(11.6-17.5)$ & 18.5 & $(15.3-22.2)$ \\
\hline Connecticut & 17.4 & (15.4-19.6) & 10.3 & (8.6-12.4) & 13.9 & (12.3-15.6) & 20.8 & $(17.6-24.3)$ & 16.2 & (13.9-18.7) & 18.6 & (16.9-20.5) \\
\hline Delaware & 16.0 & (13.7-18.6) & 7.3 & $(5.8-9.0)$ & 11.7 & (10.4-13.1) & 18.6 & (15.9-21.8) & 13.6 & (11.7-15.8) & 16.4 & (14.5-18.5) \\
\hline Florida & 15.2 & $(14.1-16.3)$ & 7.9 & $(7.0-8.9)$ & 11.6 & $(10.9-12.3)$ & 17.4 & $(15.8-19.1)$ & 12.5 & $(11.6-13.5)$ & 15.0 & $(14.0-16.0)$ \\
\hline Hawaii & 17.5 & $(15.7-19.4)$ & 11.5 & $(9.8-13.5)$ & 14.7 & $(13.3-16.2)$ & 19.9 & $(17.3-22.8)$ & 17.0 & $(15.1-19.1)$ & 18.6 & $(16.7-20.6)$ \\
\hline Idaho & 31.4 & $(28.1-34.8)$ & 11.4 & $(9.1-14.1)$ & 21.1 & $(18.8-23.6)$ & 33.8 & $(30.7-37.1)$ & 18.6 & $(16.0-21.4)$ & 26.0 & $(23.9-28.2)$ \\
\hline Illinois & 18.7 & $(16.3-21.3)$ & 11.8 & $(9.5-14.7)$ & 15.3 & (13.3-17.5) & 19.8 & $(17.3-22.5)$ & 19.4 & $(16.0-23.2)$ & 19.6 & $(17.6-21.8)$ \\
\hline Indiana & 20.6 & (17.4-24.1) & 11.0 & $(8.7-13.7)$ & 15.7 & $(14.0-17.7)$ & 22.5 & (18.8-26.7) & 14.8 & (11.8-18.3) & 18.7 & (16.1-21.5) \\
\hline Kentucky & 25.0 & $(21.1-29.5)$ & 9.2 & (7.1-11.9) & 17.0 & $(14.5-19.9)$ & 28.6 & $(25.5-32.0)$ & 15.5 & (12.4-19.2) & 22.0 & (19.4-25.0) \\
\hline Maine & 25.3 & (23.4-27.3) & 12.6 & (11.8-13.6) & 18.9 & $(17.7-20.1)$ & 26.7 & $(24.8-28.7)$ & 19.6 & (18.3-20.9) & 23.2 & (21.9-24.5) \\
\hline Maryland & 17.2 & (16.6-17.8) & 10.2 & $(9.8-10.7)$ & 13.8 & (13.4-14.2) & 19.8 & (19.2-20.4) & 15.5 & $(15.0-16.1)$ & 17.7 & (17.3-18.2) \\
\hline Massachusetts & 17.4 & (15.2-19.9) & 8.8 & $(7.3-10.5)$ & 13.0 & $(11.5-14.6)$ & 18.7 & $(16.2-21.4)$ & 12.6 & $(10.6-14.9)$ & 15.6 & $(14.0-17.4)$ \\
\hline Michigan & 25.0 & $(21.5-28.8)$ & 12.8 & $(10.8-15.0)$ & 18.8 & $(16.7-21.2)$ & 28.4 & $(24.2-33.1)$ & 22.6 & $(19.3-26.3)$ & 25.6 & $(22.8-28.6)$ \\
\hline Mississippi & 20.3 & $(17.0-24.2)$ & 10.3 & $(7.9-13.2)$ & 15.5 & $(13.2-18.2)$ & 24.8 & $(22.0-27.9)$ & 13.7 & $(10.7-17.5)$ & 19.5 & (17.3-21.8) \\
\hline Missouri & 21.4 & $(18.0-25.1)$ & 11.7 & $(9.8-14.0)$ & 16.6 & $(14.3-19.3)$ & 22.9 & (18.6-27.8) & 19.6 & $(16.5-23.2)$ & 21.4 & (18.1-25.1) \\
\hline Montana & 26.6 & $(24.5-28.8)$ & 10.9 & $(9.4-12.6)$ & 18.5 & $(17.2-19.8)$ & 29.0 & $(26.9-31.1)$ & 22.0 & $(19.7-24.6)$ & 25.3 & $(23.4-27.4)$ \\
\hline Nebraska & 25.1 & (21.1-29.4) & 13.0 & $(10.4-16.2)$ & 18.9 & (16.5-21.5) & 28.0 & $(23.7-32.8)$ & 24.4 & $(21.6-27.5)$ & 26.3 & (23.8-28.9) \\
\hline Nevada & 20.8 & $(18.2-23.7)$ & 8.2 & $(6.2-10.7)$ & 14.6 & $(12.8-16.5)$ & 20.4 & $(18.2-22.7)$ & 16.7 & $(13.3-20.7)$ & 18.6 & $(16.7-20.7)$ \\
\hline New Hampshire & 26.0 & $(24.6-27.5)$ & 11.3 & $(10.2-12.5)$ & 18.6 & $(17.7-19.4)$ & 27.3 & $(25.7-29.0)$ & 16.8 & $(15.7-17.9)$ & 22.1 & $(21.2-23.0)$ \\
\hline New Mexico & 17.4 & (15.8-19.2) & 9.9 & $(8.8-11.1)$ & 13.7 & $(12.6-14.8)$ & 20.5 & $(18.8-22.4)$ & 16.3 & $(15.2-17.6)$ & 18.4 & $(17.2-19.7)$ \\
\hline New York & 19.8 & (17.5-22.4) & 11.5 & $(9.7-13.6)$ & 15.7 & (14.3-17.3) & 23.4 & $(21.0-26.0)$ & 17.8 & (15.8-19.9) & 20.6 & $(19.1-22.3)$ \\
\hline North Carolina & 16.2 & $(12.1-21.5)$ & 7.9 & $(5.8-10.8)$ & 12.1 & $(9.5-15.3)$ & 16.8 & $(13.6-20.7)$ & 14.0 & $(10.0-19.2)$ & 15.6 & $(12.6-19.2)$ \\
\hline North Dakota & 22.9 & (20.3-25.7) & 9.3 & (7.7-11.3) & 15.9 & (14.4-17.5) & 29.3 & $(25.9-33.1)$ & 19.0 & $(16.3-21.9)$ & 24.0 & $(21.9-26.3)$ \\
\hline Oklahoma & 20.8 & (17.2-24.9) & 8.1 & $(6.2-10.5)$ & 14.5 & (12.3-17.0) & 26.0 & (22.4-30.0) & 14.7 & (11.7-18.4) & 20.4 & (17.6-23.5) \\
\hline Pennsylvania & 20.1 & $(16.6-24.0)$ & 8.7 & $(7.2-10.3)$ & 14.3 & $(12.4-16.4)$ & 24.0 & $(20.7-27.6)$ & 15.9 & $(13.6-18.5)$ & 19.9 & $(17.7-22.2)$ \\
\hline Rhode Island & 15.3 & (12.4-18.7) & 9.5 & (7.4-12.2) & 12.4 & $(10.3-14.8)$ & 16.0 & (13.0-19.7) & 15.0 & (12.5-17.9) & 15.5 & (13.6-17.6) \\
\hline South Carolina & 20.4 & $(15.7-26.1)$ & 8.0 & $(5.7-11.0)$ & 14.1 & $(11.4-17.3)$ & 25.0 & $(22.3-28.0)$ & 14.5 & $(11.2-18.6)$ & 19.7 & $(17.1-22.6)$ \\
\hline South Dakota & 26.6 & $(21.6-32.4)$ & 10.5 & $(7.8-13.9)$ & 18.4 & $(15.3-21.9)$ & 24.9 & $(18.9-32.1)$ & 18.5 & $(14.2-23.6)$ & 21.6 & $(17.1-27.0)$ \\
\hline Tennessee & 21.7 & $(19.6-23.9)$ & 9.3 & $(8.3-10.5)$ & 15.3 & (14.2-16.5) & 29.4 & $(26.8-32.1)$ & 19.2 & (17.7-20.8) & 24.1 & $(22.7-25.6)$ \\
\hline Vermont & 23.2 & $(22.4-24.0)$ & 9.9 & $(9.4-10.5)$ & 16.5 & $(16.0-17.0)$ & - & - & - & - & - & - \\
\hline Virginia & 18.8 & (16.5-21.4) & 9.0 & $(7.8-10.4)$ & 13.8 & (12.5-15.1) & 23.3 & (20.4-26.3) & 15.9 & (13.7-18.5) & 19.5 & (17.6-21.6) \\
\hline West Virginia & 27.6 & $(22.9-32.9)$ & 13.1 & $(10.5-16.3)$ & 20.2 & $(17.0-23.8)$ & 30.1 & $(26.9-33.4)$ & 19.0 & $(16.3-22.0)$ & 24.4 & $(22.0-27.0)$ \\
\hline Wyoming & 23.2 & $(19.8-27.0)$ & 11.9 & $(9.6-14.7)$ & 17.5 & $(15.7-19.5)$ & 28.8 & $(25.4-32.5)$ & 18.7 & $(16.3-21.4)$ & 23.7 & $(21.7-25.9)$ \\
\hline Median & & 20.7 & & 10.2 & & 15.4 & & 24.0 & & 16.7 & & 19.9 \\
\hline Range & & $5.2-31.4)$ & & .3-13.1) & & $1.6-21.1)$ & & $6.0-33.8)$ & & $2.5-24.4)$ & & $5.0-26.3)$ \\
\hline \multicolumn{13}{|c|}{ Large urban school district surveys } \\
\hline Baltimore, MD & 10.3 & $(7.9-13.4)$ & 8.2 & $(6.0-11.0)$ & 9.6 & $(7.8-11.7)$ & 11.5 & $(8.2-15.7)$ & 11.6 & $(8.6-15.5)$ & 11.9 & $(9.7-14.6)$ \\
\hline Boston, MA & 10.0 & $(7.9-12.7)$ & 5.9 & $(4.5-7.8)$ & 8.1 & $(6.7-9.6)$ & 14.0 & $(11.6-16.8)$ & 9.3 & $(7.4-11.7)$ & 11.8 & $(10.2-13.5)$ \\
\hline Broward County, FL & 15.8 & $(12.9-19.3)$ & 9.1 & $(6.7-12.2)$ & 12.5 & $(10.3-15.1)$ & 19.5 & $(17.0-22.3)$ & 12.4 & $(9.7-15.7)$ & 15.9 & $(14.0-18.1)$ \\
\hline Cleveland, $\mathrm{OH}$ & 17.7 & $(14.5-21.5)$ & 9.3 & $(7.2-12.0)$ & 13.8 & $(11.8-16.0)$ & 18.6 & $(15.4-22.4)$ & 13.0 & $(10.5-15.9)$ & 16.1 & $(14.0-18.4)$ \\
\hline DeKalb County, GA & 10.6 & $(8.9-12.7)$ & 6.6 & $(4.8-9.1)$ & 8.8 & $(7.4-10.3)$ & 15.2 & $(12.8-18.0)$ & 13.0 & $(10.5-15.9)$ & 14.1 & $(12.1-16.4)$ \\
\hline Detroit, MI & 19.4 & $(15.2-24.5)$ & 12.6 & $(9.6-16.4)$ & 16.3 & $(13.0-20.3)$ & 17.9 & $(13.6-23.3)$ & 16.1 & $(12.5-20.4)$ & 17.3 & $(13.7-21.6)$ \\
\hline District of Columbia & 9.2 & $(8.4-10.0)$ & 6.2 & $(5.6-7.0)$ & 7.9 & $(7.3-8.4)$ & 13.1 & $(12.2-14.0)$ & 10.8 & $(9.9-11.8)$ & 12.1 & $(11.4-12.8)$ \\
\hline Duval County, FL & 18.7 & $(16.3-21.4)$ & 11.6 & $(9.2-14.5)$ & 15.4 & $(13.3-17.8)$ & 23.5 & $(20.8-26.5)$ & 16.3 & $(13.3-19.8)$ & 20.2 & $(17.8-22.9)$ \\
\hline Ft. Worth, TX & 12.1 & $(10.1-14.3)$ & 5.6 & $(4.5-7.0)$ & 8.8 & $(7.6-10.1)$ & 14.6 & $(12.5-16.9)$ & 11.2 & $(9.2-13.5)$ & 12.9 & $(11.4-14.5)$ \\
\hline Houston, TX & 13.5 & $(11.9-15.2)$ & 9.0 & $(7.6-10.6)$ & 11.2 & $(10.2-12.4)$ & 14.5 & $(12.7-16.5)$ & 11.7 & $(9.8-13.8)$ & 13.1 & $(11.8-14.4)$ \\
\hline Los Angeles, CA & 11.7 & $(9.3-14.6)$ & 6.9 & $(4.8-9.9)$ & 9.3 & $(7.6-11.5)$ & 16.3 & $(13.2-19.8)$ & 12.6 & $(10.5-15.0)$ & 14.6 & $(12.8-16.5)$ \\
\hline Miami-Dade County, FL & 11.6 & $(9.4-14.2)$ & 7.8 & $(6.2-9.7)$ & 9.8 & (8.4-11.5) & 11.9 & $(9.9-14.2)$ & 10.5 & $(8.4-12.9)$ & 11.3 & $(9.9-12.7)$ \\
\hline
\end{tabular}

\footnotetext{
See table footnotes on the next page.
} 
TABLE 18. (Continued) Percentage of high school students who were electronically bullied, ${ }^{*}{ }^{\dagger}$ and who were bullied on school property, ${ }^{\dagger}$ by sex - selected U.S. sites, Youth Risk Behavior Survey, 2015

\begin{tabular}{|c|c|c|c|c|c|c|c|c|c|c|c|c|}
\hline \multirow[b]{3}{*}{ Site } & \multicolumn{6}{|c|}{ Electronically bullied } & \multicolumn{6}{|c|}{ Bullied on school property } \\
\hline & \multicolumn{2}{|r|}{ Female } & \multicolumn{2}{|c|}{ Male } & \multicolumn{2}{|r|}{ Total } & \multicolumn{2}{|c|}{ Female } & \multicolumn{2}{|r|}{ Male } & \multicolumn{2}{|r|}{ Total } \\
\hline & $\%$ & $\mathrm{Cl}^{\S}$ & $\%$ & $\mathrm{Cl}$ & $\%$ & $\mathrm{Cl}$ & $\%$ & $\mathrm{Cl}$ & $\%$ & $\mathrm{Cl}$ & $\%$ & $\mathrm{Cl}$ \\
\hline New York City, NY & 15.2 & $(13.6-16.9)$ & 8.9 & $(7.9-10.1)$ & 12.1 & $(11.1-13.0)$ & 17.9 & $(15.7-20.4)$ & 11.7 & $(10.4-13.1)$ & 14.8 & $(13.3-16.5)$ \\
\hline Oakland, CA & 12.0 & $(9.7-14.8)$ & 9.5 & $(7.2-12.4)$ & 10.6 & $(9.1-12.4)$ & 17.9 & $(14.4-22.1)$ & 16.0 & $(12.6-20.2)$ & 16.9 & (14.4-19.7) \\
\hline Orange County, FL & 15.8 & $(13.1-18.8)$ & 7.9 & $(5.3-11.7)$ & 11.8 & $(10.1-13.9)$ & 18.7 & $(15.4-22.5)$ & 12.8 & $(10.2-15.9)$ & 15.7 & (13.4-18.4) \\
\hline Palm Beach County, FL & 17.1 & $(14.8-19.6)$ & 10.2 & $(8.2-12.5)$ & 13.5 & $(11.9-15.3)$ & 19.1 & $(16.4-22.1)$ & 14.7 & $(12.3-17.4)$ & 16.9 & (15.2-18.8) \\
\hline Philadelphia, PA & 11.0 & $(8.3-14.5)$ & 8.1 & $(5.9-11.0)$ & 9.6 & $(7.6-12.0)$ & 15.2 & $(11.3-20.3)$ & 12.6 & $(9.8-16.0)$ & 13.9 & (11.2-17.2) \\
\hline San Diego, CA & 17.4 & $(15.0-20.0)$ & 10.7 & $(8.8-13.0)$ & 14.0 & $(12.5-15.8)$ & 19.2 & $(16.5-22.2)$ & 14.3 & $(11.9-17.0)$ & 16.7 & (14.9-18.6) \\
\hline San Francisco, CA & 12.8 & $(10.3-15.7)$ & 9.2 & $(7.3-11.6)$ & 11.2 & $(9.4-13.2)$ & 13.9 & $(11.3-16.9)$ & 12.3 & $(9.8-15.2)$ & 13.3 & (11.3-15.4) \\
\hline Median & \multicolumn{2}{|r|}{12.8} & \multicolumn{2}{|c|}{8.9} & \multicolumn{2}{|r|}{11.2} & \multicolumn{2}{|r|}{16.3} & \multicolumn{2}{|r|}{12.6} & \multicolumn{2}{|r|}{14.6} \\
\hline Range & \multicolumn{2}{|c|}{$(9.2-19.4)$} & \multicolumn{2}{|c|}{$(5.6-12.6)$} & \multicolumn{2}{|c|}{ (7.9-16.3) } & \multicolumn{2}{|c|}{$(11.5-23.5)$} & \multicolumn{2}{|r|}{$(9.3-16.3)$} & \multicolumn{2}{|c|}{$(11.3-20.2)$} \\
\hline
\end{tabular}

* Counting being bullied through e-mail, chat rooms, instant messaging, Web sites, or texting.

† During the 12 months before the survey.

$\S 95 \%$ confidence interval.

" Not available.

TABLE 19. Percentage of high school students who were ever physically forced to have sexual intercourse,* by sex, race/ethnicity, and grade United States, Youth Risk Behavior Survey, 2015

\begin{tabular}{|c|c|c|c|c|c|c|}
\hline \multirow[b]{2}{*}{ Category } & \multicolumn{2}{|c|}{ Female } & \multicolumn{2}{|c|}{ Male } & \multicolumn{2}{|c|}{ Total } \\
\hline & $\%$ & $\mathrm{Cl}^{\dagger}$ & $\%$ & $\mathrm{Cl}$ & $\%$ & $\mathrm{Cl}$ \\
\hline \multicolumn{7}{|c|}{ Race/Ethnicity } \\
\hline White $§$ & 9.9 & $(7.4-13.0)$ & 2.0 & $(1.4-2.8)$ & 6.0 & $(4.7-7.5)$ \\
\hline Black $^{\S}$ & 10.3 & $(7.4-14.1)$ & 4.4 & $(2.7-6.9)$ & 7.3 & $(5.6-9.6)$ \\
\hline Hispanic & 10.1 & $(8.0-12.7)$ & 4.0 & $(2.8-5.7)$ & 7.0 & $(5.6-8.6)$ \\
\hline \multicolumn{7}{|l|}{ Grade } \\
\hline 9 & 9.4 & $(7.3-12.1)$ & 2.1 & $(1.4-3.1)$ & 5.6 & $(4.4-7.1)$ \\
\hline 10 & 7.9 & $(5.7-10.7)$ & 3.9 & $(2.7-5.5)$ & 5.9 & $(4.5-7.7)$ \\
\hline 11 & 12.0 & $(9.3-15.4)$ & 2.8 & $(1.9-4.1)$ & 7.6 & $(6.0-9.5)$ \\
\hline 12 & 11.9 & $(9.3-15.1)$ & 3.5 & $(2.4-5.1)$ & 7.6 & (6.4-9.1) \\
\hline Total & 10.3 & $(8.4-12.6)$ & 3.1 & $(2.5-4.0)$ & 6.7 & $(5.6-8.0)$ \\
\hline
\end{tabular}

* When they did not want to.

† $95 \%$ confidence interval.

$\S$ Non-Hispanic. 
TABLE 20. Percentage of high school students who were ever physically forced to have sexual intercourse,* by sex- selected U.S. sites, Youth Risk Behavior Survey, 2015

\begin{tabular}{|c|c|c|c|c|c|c|}
\hline \multirow[b]{2}{*}{ Site } & \multicolumn{2}{|c|}{ Female } & \multicolumn{2}{|c|}{ Male } & \multicolumn{2}{|c|}{ Total } \\
\hline & $\%$ & $\mathrm{Cl}^{\dagger}$ & $\%$ & $\mathrm{Cl}$ & $\%$ & $\mathrm{Cl}$ \\
\hline \multicolumn{7}{|l|}{ State surveys } \\
\hline Alabama & 12.9 & $(9.7-16.8)$ & 7.8 & $(5.8-10.5)$ & 10.4 & $(8.4-12.7)$ \\
\hline Alaska & 11.3 & $(9.0-14.0)$ & 4.0 & $(2.7-5.9)$ & 7.5 & $(6.0-9.4)$ \\
\hline Arizona & 11.9 & $(8.8-15.8)$ & 6.0 & $(4.3-8.4)$ & 9.0 & $(7.1-11.3)$ \\
\hline Arkansas & 16.4 & $(12.5-21.2)$ & 7.1 & $(4.9-10.1)$ & 11.7 & $(9.4-14.4)$ \\
\hline California & 7.8 & $(4.4-13.2)$ & 4.3 & $(3.0-6.1)$ & 6.0 & $(4.2-8.3)$ \\
\hline Connecticut & 9.3 & $(7.2-12.0)$ & 6.2 & $(4.7-8.2)$ & 7.8 & $(6.4-9.4)$ \\
\hline Delaware & 8.6 & $(6.6-11.1)$ & 4.4 & $(3.0-6.3)$ & 6.6 & $(5.2-8.3)$ \\
\hline Florida & 8.3 & $(7.3-9.5)$ & 4.2 & $(3.5-5.1)$ & 6.4 & $(5.8-7.1)$ \\
\hline Hawaii & 10.9 & $(9.3-12.8)$ & 6.5 & $(5.3-7.8)$ & 8.8 & $(7.9-9.8)$ \\
\hline Idaho & 9.0 & $(6.9-11.7)$ & 4.4 & $(3.0-6.4)$ & 6.7 & (5.5-8.2) \\
\hline Illinois & 11.5 & $(8.7-15.1)$ & 6.4 & $(4.7-8.8)$ & 8.9 & $(6.8-11.6)$ \\
\hline Indiana & 13.4 & $(10.2-17.3)$ & 6.4 & $(4.5-9.2)$ & 10.0 & $(8.0-12.4)$ \\
\hline Kentucky & 14.1 & $(11.2-17.5)$ & 6.5 & $(5.0-8.5)$ & 10.3 & (8.9-11.9) \\
\hline Maine & 10.0 & $(8.9-11.3)$ & 4.2 & $(3.5-5.0)$ & 7.1 & (6.4-7.9) \\
\hline Maryland & 9.9 & $(9.4-10.4)$ & 6.2 & $(5.8-6.7)$ & 8.1 & $(7.8-8.5)$ \\
\hline Massachusetts & 7.6 & $(6.0-9.6)$ & 3.3 & $(2.3-4.8)$ & 5.5 & $(4.4-6.8)$ \\
\hline Michigan & 12.2 & $(10.3-14.3)$ & 4.4 & $(3.3-5.9)$ & 8.3 & $(7.3-9.6)$ \\
\hline Mississippi & 13.9 & $(11.5-16.8)$ & 8.7 & $(6.3-11.9)$ & 11.4 & (9.4-13.6) \\
\hline Missouri & 11.9 & $(9.3-15.0)$ & 4.7 & $(3.1-6.9)$ & 8.4 & $(6.8-10.3)$ \\
\hline Montana & 12.7 & $(11.3-14.4)$ & 5.0 & $(4.1-6.1)$ & 8.7 & (7.9-9.7) \\
\hline Nebraska & 11.4 & $(9.0-14.3)$ & 5.4 & $(3.8-7.5)$ & 8.3 & $(6.8-10.0)$ \\
\hline Nevada & 11.1 & $(8.0-15.2)$ & 5.6 & $(4.5-6.8)$ & 8.4 & $(6.8-10.4)$ \\
\hline New Hampshire & 9.8 & $(8.6-11.0)$ & 3.0 & $(2.5-3.6)$ & 6.3 & $(5.7-7.0)$ \\
\hline New Mexico & 10.6 & $(9.4-12.0)$ & 4.1 & $(3.5-4.8)$ & 7.3 & $(6.5-8.1)$ \\
\hline New York & —§ & - & - & - & - & - \\
\hline North Carolina & 8.8 & $(5.9-12.9)$ & 4.4 & $(2.8-6.9)$ & 6.6 & $(5.2-8.5)$ \\
\hline North Dakota & 9.8 & $(7.8-12.2)$ & 3.0 & $(2.0-4.5)$ & 6.3 & $(5.2-7.7)$ \\
\hline Oklahoma & 10.1 & $(8.1-12.5)$ & 3.2 & $(2.1-5.0)$ & 6.7 & $(5.4-8.2)$ \\
\hline Pennsylvania & 9.5 & $(7.5-11.9)$ & 3.4 & $(2.3-5.0)$ & 6.4 & $(5.1-8.0)$ \\
\hline Rhode Island & 9.8 & $(8.0-11.8)$ & 6.4 & $(5.3-7.6)$ & 8.1 & $(6.9-9.4)$ \\
\hline South Carolina & 8.4 & $(5.3-12.9)$ & 6.0 & $(4.0-8.9)$ & 7.2 & $(5.3-9.7)$ \\
\hline South Dakota & 6.1 & $(3.7-9.8)$ & 4.2 & $(2.1-8.0)$ & 5.1 & $(3.2-8.0)$ \\
\hline Tennessee & - & - & - & - & - & - \\
\hline Vermont & 10.2 & $(9.6-10.8)$ & 3.1 & $(2.7-3.4)$ & 6.6 & $(6.3-7.0)$ \\
\hline Virginia & - & - & - & - & - & - \\
\hline West Virginia & 12.7 & $(9.5-16.7)$ & 7.5 & $(5.2-10.7)$ & 10.0 & $(7.8-12.8)$ \\
\hline Wyoming & 13.2 & $(10.2-16.9)$ & 6.8 & $(5.2-8.7)$ & 10.1 & $(8.2-12.5)$ \\
\hline Median & \multirow{2}{*}{\multicolumn{2}{|c|}{$\begin{array}{c}10.4 \\
(6.1-16.4)\end{array}$}} & \multirow{2}{*}{\multicolumn{2}{|c|}{$\begin{array}{c}4.8 \\
(3.0-8.7)\end{array}$}} & \multirow{2}{*}{\multicolumn{2}{|c|}{$\begin{array}{c}7.9 \\
(5.1-11.7)\end{array}$}} \\
\hline Range & & & & & & \\
\hline \multicolumn{7}{|c|}{ Large urban school district surveys } \\
\hline Baltimore, MD & 9.9 & $(8.1-12.1)$ & 10.7 & $(7.9-14.3)$ & 10.6 & $(8.7-12.9)$ \\
\hline Boston, MA & 8.9 & $(7.0-11.2)$ & 6.1 & $(4.6-8.2)$ & 7.6 & $(6.1-9.3)$ \\
\hline Broward County, FL & 9.9 & $(7.8-12.6)$ & 7.7 & $(5.5-10.8)$ & 8.9 & (7.3-10.9) \\
\hline Cleveland, $\mathrm{OH}$ & 14.8 & $(11.3-19.1)$ & 8.5 & $(5.7-12.5)$ & 12.0 & $(9.3-15.4)$ \\
\hline DeKalb County, GA & 13.1 & $(10.8-15.8)$ & 10.6 & $(8.2-13.5)$ & 11.9 & $(10.0-13.9)$ \\
\hline Detroit, MI & 11.4 & $(9.3-14.0)$ & 9.5 & $(7.5-12.1)$ & 10.6 & $(9.1-12.4)$ \\
\hline District of Columbia & 9.7 & $(8.9-10.6)$ & 6.3 & $(5.6-7.1)$ & 8.2 & $(7.6-8.7)$ \\
\hline Duval County, FL & 14.3 & $(12.6-16.2)$ & 10.2 & $(8.3-12.4)$ & 12.6 & $(11.2-14.3)$ \\
\hline Ft. Worth, TX & 7.5 & $(6.0-9.3)$ & 3.5 & $(2.5-4.7)$ & 5.5 & $(4.6-6.6)$ \\
\hline Houston, TX & 11.0 & $(9.3-12.9)$ & 8.3 & $(7.0-9.7)$ & 9.6 & $(8.5-10.8)$ \\
\hline Los Angeles, CA & 6.8 & $(5.4-8.7)$ & 6.0 & $(4.5-8.1)$ & 6.5 & $(5.2-8.1)$ \\
\hline Miami-Dade County, FL & 7.1 & $(5.5-9.1)$ & 4.7 & $(3.5-6.4)$ & 6.0 & $(5.0-7.2)$ \\
\hline
\end{tabular}

\footnotetext{
See table footnotes on the next page.
} 
TABLE 20. (Continued) Percentage of high school students who were ever physically forced to have sexual intercourse, ${ }^{*}$ by sex- selected U.S. sites, Youth Risk Behavior Survey, 2015

\begin{tabular}{|c|c|c|c|c|c|c|}
\hline \multirow[b]{2}{*}{ Site } & \multicolumn{2}{|c|}{ Female } & \multicolumn{2}{|c|}{ Male } & \multicolumn{2}{|c|}{ Total } \\
\hline & $\%$ & $\mathrm{Cl}^{\dagger}$ & $\%$ & $\mathrm{Cl}$ & $\%$ & $\mathrm{Cl}$ \\
\hline New York City, NY & - & - & - & - & - & - \\
\hline Oakland, CA & - & - & - & - & - & - \\
\hline Orange County, FL & 8.4 & $(6.2-11.3)$ & 4.4 & $(2.7-7.0)$ & 6.4 & (5.1-7.9) \\
\hline Palm Beach County, FL & 11.8 & $(9.7-14.3)$ & 8.6 & $(6.8-10.9)$ & 10.5 & $(8.8-12.5)$ \\
\hline Philadelphia, PA & 11.2 & $(8.8-14.0)$ & 4.9 & $(3.5-6.8)$ & 8.0 & (6.4-10.0) \\
\hline San Diego, CA & 8.8 & $(7.0-11.0)$ & 5.9 & $(4.6-7.5)$ & 7.4 & $(6.3-8.6)$ \\
\hline San Francisco, CA & 9.2 & $(7.2-11.6)$ & 5.7 & $(4.3-7.7)$ & 7.6 & $(6.2-9.4)$ \\
\hline Median & \multirow{2}{*}{\multicolumn{2}{|c|}{$\begin{array}{c}9.9 \\
(6.8-14.8)\end{array}$}} & \multirow{2}{*}{\multicolumn{2}{|c|}{$\begin{array}{c}6.3 \\
(3.5-10.7)\end{array}$}} & \multirow{2}{*}{\multicolumn{2}{|c|}{$\begin{array}{c}8.2 \\
(5.5-12.6)\end{array}$}} \\
\hline Range & & & & & & \\
\hline
\end{tabular}

* When they did not want to.

† $95 \%$ confidence interval.

$\S$ Not available.

TABLE 21. Percentage of high school students who experienced physical dating violence ${ }^{*, \dagger}$ and sexual dating violence, ${ }^{*, \S}$ by sex, race/ethnicity, and grade - United States, Youth Risk Behavior Survey, 2015

\begin{tabular}{|c|c|c|c|c|c|c|c|c|c|c|c|c|}
\hline \multirow[b]{3}{*}{ Category } & \multicolumn{6}{|c|}{ Physical dating violence } & \multicolumn{6}{|c|}{ Sexual dating violence } \\
\hline & \multicolumn{2}{|c|}{ Female } & \multicolumn{2}{|c|}{ Male } & \multicolumn{2}{|c|}{ Total } & \multicolumn{2}{|c|}{ Female } & \multicolumn{2}{|c|}{ Male } & \multicolumn{2}{|r|}{ Total } \\
\hline & $\%$ & $\mathrm{Cl}^{\uparrow}$ & $\%$ & $\mathrm{Cl}$ & $\%$ & $\mathrm{Cl}$ & $\%$ & $\mathrm{Cl}$ & $\%$ & $\mathrm{Cl}$ & $\%$ & $\mathrm{Cl}$ \\
\hline \multicolumn{13}{|c|}{ Race/Ethnicity } \\
\hline White ${ }^{* *}$ & 11.9 & $(9.2-15.2)$ & 5.9 & $(4.8-7.2)$ & 9.0 & $(7.5-10.7)$ & 16.6 & (13.8-19.7) & 3.5 & $(2.8-4.4)$ & 10.1 & $(8.7-11.7)$ \\
\hline Black** & 12.2 & $(9.1-16.2)$ & 9.0 & $(6.0-13.4)$ & 10.5 & $(8.4-13.0)$ & 11.7 & $(8.0-16.8)$ & 8.0 & $(4.7-13.1)$ & 10.0 & $(7.0-14.0)$ \\
\hline Hispanic & 11.4 & $(9.2-14.1)$ & 8.0 & $(6.2-10.2)$ & 9.7 & $(8.0-11.7)$ & 14.2 & $(11.3-17.6)$ & 7.0 & $(5.6-8.8)$ & 10.6 & $(8.8-12.6)$ \\
\hline \multicolumn{13}{|l|}{ Grade } \\
\hline 9 & 11.1 & $(8.3-14.7)$ & 5.3 & $(3.5-7.9)$ & 8.1 & $(6.8-9.5)$ & 17.6 & $(14.0-21.9)$ & 4.5 & $(3.4-6.1)$ & 10.8 & $(9.0-12.9)$ \\
\hline 10 & 10.9 & $(8.7-13.6)$ & 8.2 & $(6.3-10.7)$ & 9.6 & $(8.0-11.5)$ & 15.8 & (13.0-19.0) & 7.4 & $(5.4-10.0)$ & 11.8 & $(10.0-13.8)$ \\
\hline 11 & 11.6 & $(9.0-14.7)$ & 7.9 & $(6.6-9.5)$ & 10.1 & $(8.6-11.8)$ & 14.9 & $(12.1-18.2)$ & 5.1 & $(3.1-8.4)$ & 10.3 & $(8.3-12.7)$ \\
\hline 12 & 12.9 & $(9.9-16.5)$ & 8.2 & $(6.4-10.6)$ & 10.5 & $(8.6-12.7)$ & 13.9 & $(11.3-17.0)$ & 4.6 & $(3.1-6.7)$ & 9.2 & $(7.8-10.8)$ \\
\hline Total & 11.7 & $(9.9-13.8)$ & 7.4 & $(6.5-8.5)$ & 9.6 & $(8.8-10.6)$ & 15.6 & $(13.7-17.6)$ & 5.4 & (4.4-6.7) & 10.6 & $(9.5-11.7)$ \\
\hline
\end{tabular}

* One or more times during the 12 months before the survey.

+ Among the $68.6 \%$ of students nationwide who dated or went out with someone during the 12 months before the survey, being physically hurt on purpose (counting being hit, slammed into something, or injured with an object or weapon) by someone they were dating or going out with.

$\S$ Among the $69.1 \%$ of students nationwide who dated or went out with someone during the 12 months before the survey, being forced to do sexual things (counting kissing, touching, or being physically forced to have sexual intercourse) they did not want to do by someone they were dating or going out with.

१ 95\% confidence interval.

** Non-Hispanic.

Note: The prevalence of dating or going out with someone during the 12 months before the survey varies slightly for physical dating violence and sexual dating violence because of differences in the number of usable responses to each question. 
TABLE 22. Percentage of high school students who experienced physical dating violence ${ }^{*, \dagger}$ and sexual dating violence, ${ }^{*, \$}$ by sex - selected U.S. sites, Youth Risk Behavior Survey, 2015

\begin{tabular}{|c|c|c|c|c|c|c|c|c|c|c|c|c|}
\hline \multirow[b]{3}{*}{ Site } & \multicolumn{6}{|c|}{ Physical dating violence } & \multicolumn{6}{|c|}{ Sexual dating violence } \\
\hline & \multicolumn{2}{|r|}{ Female } & \multicolumn{2}{|c|}{ Male } & \multicolumn{2}{|r|}{ Total } & \multicolumn{2}{|c|}{ Female } & \multicolumn{2}{|c|}{ Male } & \multicolumn{2}{|r|}{ Total } \\
\hline & $\%$ & $\mathrm{Cl}^{\pi}$ & $\%$ & $\mathrm{Cl}$ & $\%$ & $\mathrm{Cl}$ & $\%$ & $\mathrm{Cl}$ & $\%$ & $\mathrm{Cl}$ & $\%$ & $\mathrm{Cl}$ \\
\hline \multicolumn{13}{|l|}{ State surveys } \\
\hline Alabama & 13.9 & (11.8-16.4) & 8.4 & $(5.7-12.0)$ & 11.4 & $(9.0-14.4)$ & 13.4 & $(11.2-16.0)$ & 6.1 & $(4.2-8.7)$ & 10.0 & (8.7-11.5) \\
\hline Alaska & 10.5 & $(7.3-14.9)$ & 8.2 & $(5.6-11.8)$ & 9.5 & $(7.5-12.1)$ & 14.9 & $(11.3-19.3)$ & 5.2 & $(3.1-8.7)$ & 10.1 & $(7.7-13.2)$ \\
\hline Arizona & -** & - & - & - & - & - & - & - & - & - & - & - \\
\hline Arkansas & 18.2 & $(15.1-21.9)$ & 10.5 & $(7.2-15.1)$ & 14.6 & (13.5-15.8) & 16.0 & $(13.7-18.6)$ & 7.1 & $(4.2-11.7)$ & 11.8 & $(9.7-14.3)$ \\
\hline California & 11.7 & $(9.0-15.1)$ & 7.4 & $(4.4-12.2)$ & 9.7 & $(7.5-12.5)$ & 18.1 & $(14.1-22.9)$ & 5.3 & $(3.0-9.3)$ & 11.8 & $(8.9-15.6)$ \\
\hline Connecticut & 8.7 & $(6.7-11.2)$ & 6.8 & $(4.9-9.4)$ & 8.0 & $(6.7-9.5)$ & 16.4 & $(12.6-21.0)$ & 6.4 & $(4.4-9.2)$ & 11.5 & $(9.2-14.3)$ \\
\hline Delaware & 9.8 & $(7.8-12.3)$ & 7.2 & $(5.2-9.8)$ & 8.6 & $(7.1-10.4)$ & 9.8 & $(7.8-12.2)$ & 5.7 & $(3.8-8.5)$ & 7.9 & $(6.5-9.4)$ \\
\hline Florida & 11.2 & $(9.5-13.1)$ & 10.5 & $(9.0-12.3)$ & 11.0 & $(9.8-12.2)$ & 13.8 & $(12.4-15.4)$ & 8.5 & $(7.2-10.0)$ & 11.3 & $(10.5-12.3)$ \\
\hline Hawaii & 10.7 & $(8.2-13.7)$ & 8.1 & $(6.6-10.0)$ & 9.8 & $(8.0-11.9)$ & 15.4 & $(12.7-18.5)$ & 8.9 & $(7.2-10.9)$ & 12.7 & $(11.0-14.6)$ \\
\hline Idaho & 12.6 & $(9.0-17.4)$ & 5.1 & $(3.4-7.5)$ & 8.9 & $(6.6-11.9)$ & 21.6 & $(18.3-25.3)$ & 3.2 & $(1.7-5.9)$ & 12.4 & $(10.3-14.8)$ \\
\hline Illinois & 12.7 & (10.3-15.4) & 9.7 & $(6.4-14.4)$ & 11.3 & $(9.6-13.2)$ & 15.0 & $(12.7-17.7)$ & 6.9 & $(4.6-10.3)$ & 11.1 & $(9.5-12.8)$ \\
\hline Indiana & 11.9 & $(9.0-15.6)$ & 7.7 & $(5.0-11.7)$ & 10.0 & (8.4-11.9) & 16.4 & $(13.1-20.4)$ & 8.6 & $(6.1-12.0)$ & 12.6 & $(10.6-15.0)$ \\
\hline Kentucky & 12.1 & $(8.7-16.6)$ & 5.3 & $(3.7-7.4)$ & 8.9 & $(6.8-11.6)$ & 13.8 & $(10.9-17.2)$ & 6.1 & $(4.4-8.4)$ & 10.1 & $(8.3-12.3)$ \\
\hline Maine & 9.9 & $(8.9-11.0)$ & 6.4 & $(5.3-7.7)$ & 8.3 & (7.4-9.2) & - & - & - & - & - & - \\
\hline Maryland & 11.2 & $(10.6-11.9)$ & 8.5 & $(7.9-9.1)$ & 10.1 & $(9.7-10.6)$ & 13.1 & (12.4-13.7) & 6.9 & $(6.4-7.5)$ & 10.3 & $(9.8-10.7)$ \\
\hline Massachusetts & 9.3 & $(7.3-11.8)$ & 4.1 & $(2.7-6.3)$ & 6.7 & $(5.1-8.6)$ & 11.2 & $(8.9-14.0)$ & 3.7 & $(2.5-5.7)$ & 7.5 & $(6.2-9.0)$ \\
\hline Michigan & 9.2 & $(7.1-11.9)$ & 6.8 & $(4.4-10.3)$ & 8.2 & $(6.7-10.0)$ & 17.5 & (14.9-20.5) & 5.9 & $(3.9-8.9)$ & 11.9 & $(10.3-13.6)$ \\
\hline Mississippi & 12.3 & $(9.0-16.6)$ & 8.2 & $(5.5-12.1)$ & 10.6 & $(8.7-12.9)$ & 14.8 & $(11.8-18.4)$ & 7.7 & $(5.4-10.8)$ & 11.4 & $(9.5-13.6)$ \\
\hline Missouri & 10.2 & $(8.1-12.6)$ & 12.9 & $(8.8-18.7)$ & 11.7 & $(8.9-15.3)$ & - & - & - & - & - & - \\
\hline Montana & 10.8 & $(9.3-12.7)$ & 5.5 & $(4.3-7.1)$ & 8.2 & $(7.3-9.3)$ & 14.4 & $(12.4-16.8)$ & 5.6 & $(4.3-7.2)$ & 10.0 & $(8.8-11.4)$ \\
\hline Nebraska & 11.7 & $(8.5-16.0)$ & 3.7 & $(2.1-6.4)$ & 8.1 & $(6.0-10.8)$ & 13.8 & $(10.6-17.8)$ & 3.2 & $(1.7-5.8)$ & 8.8 & $(7.0-11.1)$ \\
\hline Nevada & 10.5 & $(7.9-13.9)$ & 8.8 & $(6.1-12.5)$ & 9.6 & $(8.0-11.6)$ & 14.4 & $(11.7-17.7)$ & 8.5 & $(5.3-13.2)$ & 11.5 & $(9.1-14.5)$ \\
\hline New Hampshire & 9.5 & $(8.4-10.7)$ & 6.1 & $(5.2-7.2)$ & 7.8 & $(7.1-8.7)$ & 17.3 & $(15.5-19.2)$ & 6.0 & $(5.1-7.1)$ & 11.7 & $(10.7-12.7)$ \\
\hline New Mexico & 9.6 & $(8.5-10.9)$ & 7.6 & $(6.4-8.9)$ & 8.6 & $(7.7-9.6)$ & 12.7 & (11.1-14.4) & 5.8 & $(4.8-6.9)$ & 9.2 & $(8.2-10.3)$ \\
\hline New York & 11.9 & $(9.9-14.4)$ & 10.3 & $(8.0-13.2)$ & 11.5 & $(9.7-13.6)$ & 16.6 & $(13.7-20.1)$ & 11.9 & $(9.5-14.8)$ & 14.7 & $(12.7-17.0)$ \\
\hline North Carolina & 9.2 & $(6.1-13.6)$ & 7.3 & $(5.4-9.9)$ & 8.3 & $(6.4-10.7)$ & 11.1 & (8.3-14.8) & 4.4 & $(2.8-6.8)$ & 7.8 & $(6.1-10.0)$ \\
\hline North Dakota & 9.6 & $(7.4-12.4)$ & 5.6 & $(4.0-7.9)$ & 7.6 & $(6.2-9.2)$ & - & - & - & - & - & - \\
\hline Oklahoma & 8.9 & $(7.2-10.9)$ & 5.3 & $(3.0-9.1)$ & 7.2 & $(5.7-9.0)$ & 13.1 & $(10.5-16.3)$ & 3.9 & $(2.3-6.7)$ & 8.6 & (7.0-10.5) \\
\hline Pennsylvania & 9.4 & $(7.3-12.0)$ & 5.0 & $(3.3-7.5)$ & 7.2 & $(5.7-9.1)$ & 14.2 & $(11.5-17.3)$ & 4.3 & $(2.8-6.6)$ & 9.3 & $(7.7-11.2)$ \\
\hline Rhode Island & 9.5 & $(7.8-11.4)$ & 7.9 & $(6.0-10.3)$ & 8.8 & $(7.5-10.2)$ & 12.8 & $(10.5-15.6)$ & 5.9 & $(3.8-8.8)$ & 9.6 & (8.4-10.9) \\
\hline South Carolina & 8.4 & (5.9-11.7) & 7.7 & $(5.1-11.3)$ & 8.0 & $(6.0-10.6)$ & 9.8 & $(6.2-15.2)$ & 5.3 & $(3.5-7.9)$ & 7.6 & $(5.0-11.4)$ \\
\hline South Dakota & 9.8 & $(7.0-13.7)$ & 7.8 & $(4.2-14.2)$ & 8.8 & $(6.2-12.4)$ & 10.1 & $(7.0-14.4)$ & 6.2 & $(3.1-12.2)$ & 8.1 & $(5.5-12.0)$ \\
\hline Tennessee & 12.9 & $(11.1-15.0)$ & 9.5 & $(7.5-12.1)$ & 11.2 & $(9.8-12.8)$ & - & - & - & - & - & - \\
\hline Vermont & 10.7 & (10.0-11.5) & 7.2 & $(6.6-7.9)$ & 9.1 & $(8.6-9.6)$ & - & - & - & - & - & - \\
\hline Virginia & 12.3 & $(9.8-15.2)$ & 9.5 & $(7.4-12.1)$ & 10.9 & $(9.1-12.9)$ & - & - & - & - & - & - \\
\hline West Virginia & 11.2 & $(8.5-14.6)$ & 8.7 & $(6.0-12.4)$ & 10.1 & $(8.2-12.3)$ & 11.7 & $(9.3-14.6)$ & 6.1 & $(4.3-8.6)$ & 9.0 & (7.5-10.9) \\
\hline Wyoming & 9.9 & $(7.8-12.6)$ & 8.1 & $(5.6-11.6)$ & 9.1 & (7.2-11.4) & 10.7 & $(8.8-13.0)$ & 5.1 & $(3.6-7.2)$ & 8.0 & $(6.8-9.5)$ \\
\hline Median & \multirow{2}{*}{\multicolumn{2}{|c|}{$\begin{array}{c}10.6 \\
(8.4-18.2)\end{array}$}} & \multirow{2}{*}{\multicolumn{2}{|c|}{$\begin{array}{c}7.7 \\
(3.7-12.9)\end{array}$}} & \multirow{2}{*}{\multicolumn{2}{|c|}{$\begin{array}{c}9.0 \\
(6.7-14.6)\end{array}$}} & \multirow{2}{*}{\multicolumn{2}{|c|}{$\begin{array}{c}14.0 \\
(9.8-21.6)\end{array}$}} & \multirow{2}{*}{\multicolumn{2}{|c|}{$\begin{array}{c}5.9 \\
(3.2-11.9)\end{array}$}} & & 10.1 \\
\hline Range & & & & & & & & & & & & 7.5-14.7) \\
\hline Large urban scl & rveys & & & & & & & & & & & \\
\hline Baltimore, MD & 10.0 & $(7.8-12.8)$ & 12.1 & $(8.3-17.4)$ & 11.8 & $(9.6-14.3)$ & 11.5 & $(8.3-15.6)$ & 12.1 & $(8.5-17.0)$ & 12.1 & $(9.6-15.0)$ \\
\hline Boston, MA & 7.0 & $(5.0-9.9)$ & 5.5 & $(3.6-8.4)$ & 6.4 & $(4.8-8.4)$ & 8.3 & $(6.1-11.4)$ & 5.1 & $(3.2-8.0)$ & 6.8 & $(5.0-9.1)$ \\
\hline Broward County, FL & 10.6 & $(7.8-14.2)$ & 7.5 & $(5.0-11.1)$ & 9.2 & $(7.3-11.5)$ & 14.4 & $(10.8-19.0)$ & 6.6 & $(4.6-9.3)$ & 10.5 & $(8.6-12.8)$ \\
\hline Cleveland, $\mathrm{OH}$ & 11.6 & $(9.1-14.8)$ & 12.5 & $(9.8-15.7)$ & 12.7 & $(10.8-15.0)$ & 8.3 & $(6.5-10.6)$ & 11.9 & $(9.1-15.3)$ & 10.7 & $(9.0-12.6)$ \\
\hline DeKalb County, GA & 11.1 & $(8.5-14.5)$ & 7.7 & $(5.4-10.9)$ & 9.4 & $(7.6-11.5)$ & 10.3 & $(7.8-13.5)$ & 7.9 & $(5.5-11.2)$ & 9.1 & $(7.3-11.3)$ \\
\hline Detroit, MI & 12.2 & $(9.4-15.6)$ & 8.6 & $(6.2-11.8)$ & 10.8 & $(8.6-13.6)$ & 8.2 & $(5.9-11.4)$ & 9.0 & $(6.5-12.4)$ & 8.8 & $(7.0-11.0)$ \\
\hline District of Columbia & 11.8 & $(10.7-12.9)$ & 8.4 & $(7.4-9.5)$ & 10.3 & $(9.6-11.1)$ & 8.3 & $(7.4-9.2)$ & 6.5 & $(5.7-7.5)$ & 7.7 & $(7.1-8.4)$ \\
\hline Duval County, FL & 12.3 & $(10.3-14.7)$ & 11.3 & $(9.1-14.0)$ & 12.4 & $(10.8-14.1)$ & 17.3 & (14.9-19.9) & 10.0 & $(8.0-12.4)$ & 14.4 & $(12.8-16.2)$ \\
\hline Ft. Worth, TX & 10.4 & $(8.4-12.9)$ & 6.2 & $(4.7-8.2)$ & 8.3 & $(6.9-10.0)$ & 10.0 & $(8.1-12.3)$ & 7.1 & $(5.3-9.6)$ & 8.6 & $(7.3-10.1)$ \\
\hline Houston, TX & 11.7 & $(9.6-14.2)$ & 8.1 & $(6.4-10.3)$ & 10.1 & $(8.8-11.7)$ & 11.1 & $(9.1-13.4)$ & 5.9 & $(4.6-7.5)$ & 8.8 & $(7.6-10.1)$ \\
\hline Los Angeles, CA & 6.6 & $(4.9-8.9)$ & 7.5 & $(5.6-10.0)$ & 7.2 & $(6.0-8.6)$ & 10.8 & $(7.7-15.0)$ & 6.3 & $(4.9-8.0)$ & 8.6 & $(6.6-11.0)$ \\
\hline Miami-Dade County, FL & 7.6 & $(5.6-10.3)$ & 7.0 & $(5.5-8.9)$ & 7.4 & $(6.0-9.2)$ & 9.5 & $(7.4-12.2)$ & 6.8 & $(4.9-9.4)$ & 8.2 & $(6.8-10.0)$ \\
\hline
\end{tabular}

See table footnotes on the next page. 
TABLE 22. (Continued) Percentage of high school students who experienced physical dating violence ${ }^{*, \dagger}$ and sexual dating violence, ${ }^{*}, \S$ by sex selected U.S. sites, Youth Risk Behavior Survey, 2015

\begin{tabular}{|c|c|c|c|c|c|c|c|c|c|c|c|c|}
\hline \multirow[b]{3}{*}{ Site } & \multicolumn{6}{|c|}{ Physical dating violence } & \multicolumn{6}{|c|}{ Sexual dating violence } \\
\hline & \multicolumn{2}{|c|}{ Female } & \multicolumn{2}{|r|}{ Male } & \multicolumn{2}{|r|}{ Total } & \multicolumn{2}{|r|}{ Female } & \multicolumn{2}{|r|}{ Male } & \multicolumn{2}{|r|}{ Total } \\
\hline & $\%$ & $\mathrm{Cl}^{\Uparrow}$ & $\%$ & $\mathrm{Cl}$ & $\%$ & $\mathrm{Cl}$ & $\%$ & $\mathrm{Cl}$ & $\%$ & $\mathrm{Cl}$ & $\%$ & $\mathrm{Cl}$ \\
\hline New York City, NY & 12.5 & $(11.2-13.9)$ & 11.1 & $(9.1-13.5)$ & 12.0 & $(10.7-13.5)$ & 13.6 & $(11.8-15.6)$ & 8.6 & $(7.1-10.4)$ & 11.4 & $(10.4-12.6)$ \\
\hline Oakland, CA & 13.4 & $(9.9-17.9)$ & 9.7 & $(6.8-13.7)$ & 11.6 & $(8.9-14.8)$ & 12.4 & $(9.1-16.6)$ & 8.7 & $(5.9-12.7)$ & 10.8 & $(8.6-13.5)$ \\
\hline Orange County, FL & 8.9 & $(6.5-12.0)$ & 9.0 & $(5.2-15.0)$ & 9.0 & $(6.6-12.1)$ & 16.3 & $(12.8-20.6)$ & 6.9 & $(3.9-11.8)$ & 11.4 & $(9.0-14.4)$ \\
\hline Palm Beach County, FL & 10.8 & $(8.8-13.1)$ & 8.5 & $(6.4-11.1)$ & 10.2 & $(8.5-12.3)$ & 13.1 & $(10.6-16.1)$ & 8.7 & $(6.6-11.3)$ & 11.6 & (9.6-13.8) \\
\hline Philadelphia, PA & 13.6 & $(9.9-18.5)$ & 7.7 & $(5.2-11.2)$ & 10.8 & $(8.1-14.2)$ & 14.4 & $(10.5-19.5)$ & 6.4 & $(3.9-10.4)$ & 10.6 & $(8.3-13.4)$ \\
\hline San Diego, CA & 9.1 & $(6.7-12.4)$ & 6.4 & $(4.7-8.8)$ & 7.8 & $(6.2-9.7)$ & 16.0 & $(12.3-20.4)$ & 5.9 & $(4.4-7.9)$ & 10.7 & (8.7-13.3) \\
\hline San Francisco, CA & 7.5 & $(4.8-11.5)$ & 5.6 & $(3.4-9.1)$ & 6.6 & $(4.8-8.9)$ & 12.1 & $(8.7-16.5)$ & 4.7 & $(3.0-7.2)$ & 8.3 & $(6.3-10.8)$ \\
\hline Median & \multicolumn{2}{|r|}{10.8} & \multicolumn{2}{|r|}{8.1} & \multicolumn{2}{|r|}{10.1} & \multicolumn{2}{|r|}{11.5} & \multicolumn{2}{|r|}{6.9} & \multicolumn{2}{|r|}{10.5} \\
\hline Range & \multicolumn{2}{|c|}{$(6.6-13.6)$} & \multicolumn{2}{|c|}{$(5.5-12.5)$} & \multicolumn{2}{|c|}{$(6.4-12.7)$} & \multicolumn{2}{|r|}{$(8.2-17.3)$} & \multicolumn{2}{|c|}{$(4.7-12.1)$} & \multicolumn{2}{|c|}{$(6.8-14.4)$} \\
\hline
\end{tabular}

* One or more times during the 12 months before the survey, among students who dated or went out with someone during the 12 months before the survey.

† Being physically hurt on purpose (counting being hit, slammed into something, or injured with an object or weapon) by someone they were dating or going out with.

$\S$ Being forced to do sexual things (counting kissing, touching, or being physically forced to have sexual intercourse) they did not want to do by someone they were

dating or going out with.

१ $95 \%$ confidence interval.

** Not available.

TABLE 23. Percentage of high school students who felt sad or hopeless, * by sex, race/ethnicity, and grade — United States, Youth Risk Behavior Survey, 2015

\begin{tabular}{|c|c|c|c|c|c|c|}
\hline \multirow[b]{2}{*}{ Category } & \multicolumn{2}{|c|}{ Female } & \multicolumn{2}{|c|}{ Male } & \multicolumn{2}{|c|}{ Total } \\
\hline & $\%$ & $\mathrm{Cl}^{\dagger}$ & $\%$ & $\mathrm{Cl}$ & $\%$ & $\mathrm{Cl}$ \\
\hline \multicolumn{7}{|c|}{ Race/Ethnicity } \\
\hline White ${ }^{\S}$ & 37.9 & (32.5-43.6) & 19.2 & $(17.2-21.5)$ & 28.6 & $(25.8-31.5)$ \\
\hline Black ${ }^{\S}$ & 33.9 & $(28.5-39.8)$ & 17.6 & $(13.7-22.4)$ & 25.2 & $(21.7-29.1)$ \\
\hline Hispanic & 46.7 & $(42.6-50.9)$ & 24.3 & $(21.6-27.2)$ & 35.3 & $(32.3-38.4)$ \\
\hline \multicolumn{7}{|l|}{ Grade } \\
\hline 9 & 41.5 & (37.7-45.4) & 16.7 & $(14.5-19.1)$ & 28.4 & $(25.9-31.0)$ \\
\hline 10 & 40.1 & (35.1-45.4) & 19.2 & $(16.1-22.8)$ & 29.8 & $(26.6-33.1)$ \\
\hline 11 & 40.9 & (37.3-44.7) & 22.1 & $(19.1-25.4)$ & 31.4 & $(28.3-34.8)$ \\
\hline 12 & 36.3 & $(32.3-40.5)$ & 23.9 & $(21.2-26.8)$ & 30.0 & $(27.5-32.6)$ \\
\hline Total & 39.8 & $(36.5-43.2)$ & 20.3 & $(18.9-21.8)$ & 29.9 & $(28.0-31.8)$ \\
\hline
\end{tabular}

* Almost every day for $\geq 2$ weeks in a row so that they stopped doing some usual activities during the 12 months before the survey.

† $95 \%$ confidence interval.

$\S$ Non-Hispanic. 
TABLE 24. Percentage of high school students who felt sad or hopeless,* by sex — selected U.S. sites, Youth Risk Behavior Survey, 2015

\begin{tabular}{|c|c|c|c|c|c|c|}
\hline \multirow[b]{2}{*}{ Site } & \multicolumn{2}{|c|}{ Female } & \multicolumn{2}{|c|}{ Male } & \multicolumn{2}{|c|}{ Total } \\
\hline & $\%$ & $\mathrm{Cl}^{\dagger}$ & $\%$ & $\mathrm{Cl}$ & $\%$ & $\mathrm{Cl}$ \\
\hline \multicolumn{7}{|l|}{ State surveys } \\
\hline Alabama & 38.1 & $(33.8-42.7)$ & 20.5 & $(17.6-23.8)$ & 29.3 & $(26.2-32.7)$ \\
\hline Alaska & 42.7 & $(37.4-48.2)$ & 25.0 & $(21.6-28.8)$ & 33.6 & $(30.1-37.4)$ \\
\hline Arizona & 44.0 & $(40.0-48.0)$ & 24.6 & $(20.8-28.8)$ & 34.2 & $(30.7-37.8)$ \\
\hline Arkansas & 38.1 & (35.3-40.9) & 23.4 & $(18.4-29.3)$ & 30.7 & $(27.6-33.9)$ \\
\hline California & 38.8 & $(35.4-42.3)$ & 20.9 & $(19.1-22.9)$ & 29.7 & $(26.9-32.8)$ \\
\hline Connecticut & 34.7 & (30.9-38.7) & 18.5 & $(15.9-21.6)$ & 26.6 & (23.9-29.5) \\
\hline Delaware & 32.5 & $(29.3-35.8)$ & 15.3 & $(13.2-17.7)$ & 24.2 & $(22.1-26.5)$ \\
\hline Florida & 35.4 & (33.2-37.6) & 17.3 & $(15.6-19.1)$ & 26.4 & $(25.0-27.9)$ \\
\hline Hawaii & 36.2 & $(33.5-39.0)$ & 22.4 & $(20.4-24.6)$ & 29.5 & $(27.7-31.4)$ \\
\hline Idaho & 42.7 & (39.0-46.5) & 21.2 & $(18.3-24.5)$ & 31.6 & $(29.1-34.3)$ \\
\hline Illinois & 36.8 & $(32.6-41.3)$ & 21.7 & $(18.2-25.7)$ & 29.3 & $(26.1-32.7)$ \\
\hline Indiana & 39.2 & $(33.6-45.0)$ & 19.8 & $(17.5-22.3)$ & 29.3 & $(27.0-31.9)$ \\
\hline Kentucky & 42.5 & $(37.2-48.0)$ & 20.5 & $(17.5-24.0)$ & 31.3 & $(28.0-34.9)$ \\
\hline Maine & 35.5 & $(33.5-37.5)$ & 17.8 & $(16.6-19.0)$ & 26.4 & $(25.3-27.6)$ \\
\hline Maryland & 35.0 & $(34.2-35.9)$ & 18.7 & $(18.1-19.3)$ & 26.8 & $(26.3-27.4)$ \\
\hline Massachusetts & 34.8 & $(32.1-37.7)$ & 19.9 & $(17.5-22.6)$ & 27.4 & $(25.5-29.4)$ \\
\hline Michigan & 40.7 & $(35.6-46.0)$ & 22.7 & $(20.0-25.5)$ & 31.7 & $(28.6-34.9)$ \\
\hline Mississippi & 38.3 & $(35.2-41.6)$ & 20.1 & $(16.4-24.5)$ & 29.5 & $(26.5-32.6)$ \\
\hline Missouri & 31.3 & $(26.2-36.9)$ & 23.6 & (19.4-28.5) & 27.5 & $(23.6-31.8)$ \\
\hline Montana & 38.8 & (36.2-41.4) & 20.4 & $(18.7-22.3)$ & 29.3 & $(27.7-30.9)$ \\
\hline Nebraska & 31.4 & $(26.7-36.6)$ & 17.1 & $(14.1-20.5)$ & 24.1 & $(21.2-27.3)$ \\
\hline Nevada & 44.2 & (38.7-49.9) & 21.6 & $(19.3-24.1)$ & 32.7 & $(30.0-35.6)$ \\
\hline New Hampshire & 36.9 & $(34.8-39.0)$ & 17.9 & $(16.7-19.2)$ & 27.2 & $(26.2-28.3)$ \\
\hline New Mexico & 42.3 & (39.9-44.7) & 23.0 & $(21.2-24.9)$ & 32.5 & $(30.8-34.3)$ \\
\hline New York & 36.0 & (33.4-38.7) & 21.0 & $(18.7-23.5)$ & 28.6 & $(26.1-31.2)$ \\
\hline North Carolina & 35.2 & $(30.3-40.5)$ & 17.5 & $(14.7-20.7)$ & 26.4 & $(23.5-29.4)$ \\
\hline North Dakota & 35.2 & $(32.1-38.4)$ & 19.6 & $(17.1-22.4)$ & 27.2 & $(25.1-29.4)$ \\
\hline Oklahoma & 37.9 & $(33.3-42.8)$ & 19.7 & $(17.0-22.8)$ & 28.9 & $(25.8-32.1)$ \\
\hline Pennsylvania & 36.7 & $(33.1-40.5)$ & 19.2 & $(16.8-21.7)$ & 27.8 & $(25.3-30.5)$ \\
\hline Rhode Island & 35.8 & $(33.9-37.8)$ & 17.2 & $(14.8-20.0)$ & 26.4 & $(24.3-28.5)$ \\
\hline South Carolina & 39.6 & $(33.7-45.7)$ & 20.4 & $(17.5-23.7)$ & 29.9 & $(26.1-33.9)$ \\
\hline South Dakota & 34.7 & $(27.6-42.4)$ & 16.5 & $(12.6-21.3)$ & 25.4 & $(21.0-30.4)$ \\
\hline Tennessee & 38.0 & $(34.6-41.5)$ & 18.5 & $(16.8-20.3)$ & 28.0 & $(26.0-30.1)$ \\
\hline Vermont & 33.5 & $(32.6-34.5)$ & 15.4 & $(14.7-16.1)$ & 24.4 & $(23.8-25.0)$ \\
\hline Virginia & 37.1 & $(34.5-39.7)$ & 17.3 & $(15.3-19.5)$ & 26.9 & $(25.1-28.8)$ \\
\hline West Virginia & 43.8 & (38.8-49.0) & 22.4 & $(18.5-26.8)$ & 32.9 & $(29.5-36.4)$ \\
\hline Wyoming & 39.1 & $(35.3-42.9)$ & 22.8 & $(19.9-25.9)$ & 30.8 & $(28.3-33.4)$ \\
\hline Median & \multirow{2}{*}{\multicolumn{2}{|c|}{$\begin{array}{c}37.1 \\
(31.3-44.2)\end{array}$}} & \multirow{2}{*}{\multicolumn{2}{|c|}{$\begin{array}{c}20.1 \\
(15.3-25.0)\end{array}$}} & \multirow{2}{*}{\multicolumn{2}{|c|}{$\begin{array}{c}28.9 \\
(24.1-34.2)\end{array}$}} \\
\hline Range & & & & & & \\
\hline \multicolumn{7}{|c|}{ Large urban school district surveys } \\
\hline Baltimore, MD & 36.5 & (31.9-41.4) & 23.0 & $(17.8-29.2)$ & 29.8 & $(26.6-33.2)$ \\
\hline Boston, MA & 32.6 & $(29.4-36.0)$ & 20.7 & $(17.8-23.9)$ & 26.7 & $(24.6-28.8)$ \\
\hline Broward County, FL & 38.2 & $(34.1-42.4)$ & 22.2 & $(18.9-26.0)$ & 30.0 & $(27.0-33.2)$ \\
\hline Cleveland, $\mathrm{OH}$ & 47.0 & $(43.4-50.6)$ & 25.1 & $(21.9-28.5)$ & 36.0 & (33.3-38.9) \\
\hline DeKalb County, GA & 34.1 & $(31.1-37.3)$ & 21.0 & $(18.4-24.0)$ & 27.5 & $(25.3-29.9)$ \\
\hline Detroit, MI & 37.7 & $(34.0-41.5)$ & 21.7 & $(18.2-25.6)$ & 30.6 & $(27.8-33.5)$ \\
\hline District of Columbia & 33.5 & $(32.2-34.8)$ & 19.7 & $(18.6-21.0)$ & 27.0 & $(26.0-27.9)$ \\
\hline Duval County, FL & 39.9 & (37.4-42.5) & 22.2 & $(19.8-24.9)$ & 31.4 & (29.5-33.4) \\
\hline Ft. Worth, TX & 40.2 & $(37.3-43.2)$ & 17.7 & (15.8-19.9) & 29.0 & $(27.2-30.9)$ \\
\hline Houston, TX & 37.4 & $(34.8-40.0)$ & 23.8 & $(21.9-25.7)$ & 30.3 & $(28.8-32.0)$ \\
\hline Los Angeles, CA & 37.1 & (33.4-40.8) & 23.3 & $(20.7-26.3)$ & 30.4 & $(27.8-33.1)$ \\
\hline Miami-Dade County, FL & 30.8 & $(27.6-34.2)$ & 18.8 & $(16.5-21.4)$ & 25.0 & $(22.8-27.4)$ \\
\hline
\end{tabular}

See table footnotes on the next page. 
TABLE 24. (Continued) Percentage of high school students who felt sad or hopeless,* by sex - selected U.S. sites, Youth Risk Behavior Survey, 2015

\begin{tabular}{|c|c|c|c|c|c|c|}
\hline \multirow[b]{2}{*}{ Site } & \multicolumn{2}{|c|}{ Female } & \multicolumn{2}{|c|}{ Male } & \multicolumn{2}{|c|}{ Total } \\
\hline & $\%$ & $\mathrm{Cl}^{\dagger}$ & $\%$ & $\mathrm{Cl}$ & $\%$ & $\mathrm{Cl}$ \\
\hline New York City, NY & 37.7 & $(35.5-39.9)$ & 21.2 & $(18.8-23.8)$ & 29.4 & $(27.6-31.2)$ \\
\hline Oakland, CA & 33.2 & $(29.4-37.3)$ & 21.9 & $(18.8-25.4)$ & 27.3 & $(24.9-29.8)$ \\
\hline Orange County, FL & 39.4 & $(35.7-43.2)$ & 20.6 & $(16.8-25.1)$ & 30.1 & $(26.9-33.4)$ \\
\hline Palm Beach County, FL & 38.3 & $(34.9-41.9)$ & 21.3 & $(18.8-23.9)$ & 29.9 & $(27.7-32.1)$ \\
\hline Philadelphia, PA & 43.1 & $(39.5-46.7)$ & 21.4 & $(18.3-25.0)$ & 32.4 & $(29.8-35.0)$ \\
\hline San Diego, CA & 38.9 & $(35.1-42.9)$ & 19.9 & $(17.5-22.6)$ & 29.1 & $(26.5-31.9)$ \\
\hline San Francisco, CA & 30.6 & $(27.0-34.4)$ & 19.6 & $(16.8-22.7)$ & 24.9 & $(22.8-27.1)$ \\
\hline Median & \multirow{2}{*}{\multicolumn{2}{|c|}{$\begin{array}{c}37.7 \\
(30.6-47.0)\end{array}$}} & \multirow{2}{*}{\multicolumn{2}{|c|}{$\begin{array}{c}21.3 \\
(17.7-25.1)\end{array}$}} & \multirow{2}{*}{\multicolumn{2}{|c|}{$\begin{array}{c}29.8 \\
(24.9-36.0)\end{array}$}} \\
\hline Range & & & & & & \\
\hline
\end{tabular}

* Almost every day for $\geq 2$ weeks in a row so that they stopped doing some usual activities during the 12 months before the survey.

† $95 \%$ confidence interval.

TABLE 25. Percentage of high school students who seriously considered attempting suicide* and who made a plan about how they would attempt suicide,* by sex, race/ethnicity, and grade — United States, Youth Risk Behavior Survey, 2015

\begin{tabular}{|c|c|c|c|c|c|c|c|c|c|c|c|c|}
\hline \multirow[b]{3}{*}{ Category } & \multicolumn{6}{|c|}{ Seriously considered attempting suicide } & \multicolumn{6}{|c|}{ Made a suicide plan } \\
\hline & \multicolumn{2}{|c|}{ Female } & \multicolumn{2}{|r|}{ Male } & \multicolumn{2}{|r|}{ Total } & \multicolumn{2}{|c|}{ Female } & \multicolumn{2}{|c|}{ Male } & \multicolumn{2}{|r|}{ Total } \\
\hline & $\%$ & $\mathrm{Cl}^{\dagger}$ & $\%$ & $\mathrm{Cl}$ & $\%$ & $\mathrm{Cl}$ & $\%$ & $\mathrm{Cl}$ & $\%$ & $\mathrm{Cl}$ & $\%$ & $\mathrm{Cl}$ \\
\hline \multicolumn{13}{|c|}{ Race/Ethnicity } \\
\hline White ${ }^{\S}$ & 22.8 & $(19.9-26.1)$ & 11.5 & $(9.9-13.4)$ & 17.2 & $(15.4-19.2)$ & 18.4 & $(15.6-21.7)$ & 9.3 & $(7.7-11.1)$ & 13.9 & $(12.1-15.9)$ \\
\hline Black $^{\S}$ & 18.7 & $(14.4-23.8)$ & 11.0 & $(9.2-13.1)$ & 14.5 & $(12.3-17.1)$ & 17.3 & $(12.7-23.2)$ & 10.6 & $(8.0-14.0)$ & 13.7 & $(10.8-17.2)$ \\
\hline Hispanic & 25.6 & $(22.9-28.5)$ & 12.4 & $(10.5-14.6)$ & 18.8 & $(17.1-20.7)$ & 20.7 & $(17.7-24.1)$ & 10.9 & $(9.5-12.5)$ & 15.7 & $(14.2-17.4)$ \\
\hline \multicolumn{13}{|l|}{ Grade } \\
\hline 9 & 26.5 & $(23.3-30.0)$ & 10.7 & $(8.6-13.2)$ & 18.2 & $(16.4-20.2)$ & 22.5 & $(19.2-26.1)$ & 8.1 & $(6.6-10.1)$ & 15.0 & $(13.4-16.8)$ \\
\hline 10 & 25.7 & $(22.2-29.5)$ & 10.8 & $(8.8-13.2)$ & 18.3 & $(16.2-20.5)$ & 21.6 & $(18.7-24.7)$ & 9.2 & $(7.3-11.4)$ & 15.4 & $(13.6-17.4)$ \\
\hline 11 & 22.1 & $(19.0-25.4)$ & 13.3 & (11.3-15.5) & 17.7 & $(15.7-19.9)$ & 17.2 & $(14.4-20.3)$ & 10.4 & $(8.8-12.3)$ & 13.9 & (12.2-15.9) \\
\hline 12 & 18.6 & $(16.1-21.4)$ & 14.0 & $(11.5-17.1)$ & 16.3 & $(14.7-18.0)$ & 15.7 & $(13.6-18.0)$ & 12.0 & $(9.8-14.5)$ & 13.8 & (12.4-15.4) \\
\hline Total & 23.4 & (21.5-25.4) & 12.2 & (11.2-13.3) & 17.7 & (16.7-18.8) & 19.4 & $(17.5-21.5)$ & 9.8 & $(8.8-11.0)$ & 14.6 & (13.4-15.8) \\
\hline
\end{tabular}

* During the 12 months before the survey.

† $95 \%$ confidence interval.

$\S$ Non-Hispanic. 
TABLE 26. Percentage of high school students who seriously considered attempting suicide* and who made a plan about how they would attempt suicide, ${ }^{*}$ by sex — selected U.S. sites, Youth Risk Behavior Survey, 2015

\begin{tabular}{|c|c|c|c|c|c|c|c|c|c|c|c|c|}
\hline \multirow[b]{3}{*}{ Site } & \multicolumn{6}{|c|}{ Seriously considered attempting suicide } & \multicolumn{6}{|c|}{ Made a suicide plan } \\
\hline & \multicolumn{2}{|c|}{ Female } & \multicolumn{2}{|r|}{ Male } & \multicolumn{2}{|r|}{ Total } & \multicolumn{2}{|c|}{ Female } & \multicolumn{2}{|r|}{ Male } & \multicolumn{2}{|r|}{ Total } \\
\hline & $\%$ & $\mathrm{Cl}^{\dagger}$ & $\%$ & $\mathrm{Cl}$ & $\%$ & $\mathrm{Cl}$ & $\%$ & $\mathrm{Cl}$ & $\%$ & $\mathrm{Cl}$ & $\%$ & $\mathrm{Cl}$ \\
\hline \multicolumn{13}{|l|}{ State surveys } \\
\hline Alabama & 23.1 & $(18.6-28.2)$ & 11.8 & $(9.5-14.6)$ & 17.5 & $(14.5-21.0)$ & 18.7 & $(15.2-22.8)$ & 8.5 & $(6.0-11.7)$ & 13.7 & (11.4-16.3) \\
\hline Alaska & 23.3 & $(19.6-27.5)$ & 17.0 & $(14.1-20.4)$ & 20.1 & $(17.2-23.4)$ & 18.4 & $(15.5-21.7)$ & 14.9 & $(11.9-18.4)$ & 16.7 & $(14.2-19.5)$ \\
\hline Arizona & 23.5 & $(20.2-27.2)$ & 13.2 & $(10.5-16.4)$ & 18.6 & $(16.3-21.1)$ & 21.3 & $(17.5-25.7)$ & 12.3 & $(9.8-15.4)$ & 17.0 & $(15.2-18.9)$ \\
\hline Arkansas & 23.2 & $(20.6-26.0)$ & 14.4 & $(10.7-19.0)$ & 18.9 & $(16.6-21.5)$ & 18.9 & $(16.6-21.5)$ & 14.1 & $(10.8-18.3)$ & 16.7 & $(15.1-18.4)$ \\
\hline California & 24.0 & $(20.2-28.4)$ & 11.9 & $(8.4-16.5)$ & 17.9 & $(14.4-22.1)$ & 21.8 & (18.4-25.8) & 8.5 & $(5.8-12.3)$ & 15.2 & $(12.2-18.7)$ \\
\hline Connecticut & 16.3 & $(13.7-19.2)$ & 10.4 & $(8.6-12.5)$ & 13.4 & $(11.9-15.1)$ & —§ & - & - & - & - & - \\
\hline Delaware & 17.5 & $(14.8-20.6)$ & 10.0 & $(8.4-11.9)$ & 14.0 & $(12.7-15.5)$ & 14.8 & (11.9-18.3) & 7.2 & $(5.8-8.9)$ & 11.0 & $(9.4-12.8)$ \\
\hline Florida & 17.9 & $(16.7-19.3)$ & 9.5 & $(8.3-10.8)$ & 13.8 & $(13.0-14.7)$ & 14.3 & $(13.1-15.7)$ & 7.7 & $(6.9-8.5)$ & 11.1 & (10.3-11.9) \\
\hline Hawaii & 20.1 & $(17.8-22.6)$ & 11.7 & $(9.8-13.8)$ & 16.0 & $(14.2-18.1)$ & 18.8 & $(17.1-20.6)$ & 12.1 & $(10.5-13.9)$ & 15.6 & $(14.3-17.0)$ \\
\hline Idaho & 27.2 & $(23.7-30.9)$ & 12.9 & $(10.5-15.6)$ & 19.8 & $(17.5-22.3)$ & 23.3 & $(20.0-26.9)$ & 10.8 & $(8.8-13.1)$ & 17.0 & $(14.8-19.3)$ \\
\hline Illinois & 18.8 & (15.8-22.4) & 12.9 & (10.0-16.4) & 15.9 & (13.9-18.2) & 16.4 & $(14.1-18.9)$ & 12.6 & (10.4-15.3) & 14.5 & $(12.9-16.4)$ \\
\hline Indiana & 26.0 & $(22.2-30.1)$ & 13.7 & $(10.5-17.6)$ & 19.8 & (17.9-21.7) & 20.6 & $(18.5-22.8)$ & 13.6 & $(10.7-17.2)$ & 17.0 & $(15.3-19.0)$ \\
\hline Kentucky & 20.6 & $(16.9-24.9)$ & 10.6 & $(8.6-13.1)$ & 15.7 & (13.8-17.8) & 18.1 & $(14.5-22.5)$ & 9.8 & $(7.8-12.2)$ & 13.9 & (11.9-16.3) \\
\hline Maine & 19.8 & $(18.5-21.2)$ & 10.5 & $(9.3-11.9)$ & 15.2 & $(14.1-16.3)$ & 16.1 & (14.9-17.4) & 8.7 & $(7.9-9.6)$ & 12.4 & $(11.6-13.1)$ \\
\hline Maryland & 20.7 & $(20.1-21.3)$ & 10.9 & $(10.4-11.4)$ & 15.9 & (15.4-16.3) & 15.9 & (15.3-16.4) & 9.4 & $(8.9-9.9)$ & 12.7 & $(12.3-13.1)$ \\
\hline Massachusetts & 17.4 & $(15.2-19.9)$ & 12.2 & $(10.4-14.3)$ & 14.9 & $(13.1-16.8)$ & 14.0 & (11.9-16.3) & 9.8 & $(8.3-11.6)$ & 11.9 & $(10.6-13.4)$ \\
\hline Michigan & 21.4 & $(18.7-24.4)$ & 13.0 & $(11.3-15.0)$ & 17.3 & $(15.9-18.7)$ & 17.5 & (15.4-19.8) & 12.5 & $(10.2-15.2)$ & 15.0 & $(13.5-16.6)$ \\
\hline Mississippi & 20.9 & $(18.5-23.6)$ & 13.0 & (10.7-15.9) & 17.0 & $(15.5-18.6)$ & 17.6 & (14.8-20.9) & 12.4 & (9.9-15.4) & 15.1 & $(13.2-17.3)$ \\
\hline Missouri & 18.9 & (15.4-23.0) & 13.2 & $(10.1-17.2)$ & 16.2 & (13.9-18.9) & 15.8 & $(12.2-20.1)$ & 11.0 & $(9.4-12.7)$ & 13.4 & $(11.2-16.0)$ \\
\hline Montana & 25.5 & $(23.4-27.7)$ & 12.7 & $(11.3-14.3)$ & 18.8 & $(17.6-20.1)$ & 19.8 & $(17.8-22.0)$ & 11.5 & $(10.2-12.9)$ & 15.5 & $(14.3-16.8)$ \\
\hline Nebraska & 18.0 & $(14.6-21.8)$ & 11.3 & $(9.2-13.8)$ & 14.6 & $(12.8-16.8)$ & 17.0 & $(13.5-21.2)$ & 9.8 & $(7.6-12.5)$ & 13.3 & $(11.5-15.4)$ \\
\hline Nevada & 23.8 & $(20.9-27.0)$ & 10.6 & $(7.9-14.2)$ & 17.2 & $(14.8-20.0)$ & 21.7 & $(18.7-24.9)$ & 9.8 & $(7.1-13.3)$ & 15.8 & $(13.8-18.1)$ \\
\hline New Hampshire & 20.1 & $(18.8-21.5)$ & 10.7 & $(9.8-11.7)$ & 15.3 & $(14.5-16.2)$ & - & - & - & - & - & - \\
\hline New Mexico & 21.4 & $(19.1-23.8)$ & 11.6 & $(10.6-12.7)$ & 16.5 & $(15.1-17.9)$ & 19.0 & $(17.4-20.6)$ & 10.3 & $(9.3-11.4)$ & 14.6 & $(13.6-15.7)$ \\
\hline New York & 19.4 & $(16.9-22.1)$ & 11.8 & $(9.7-14.3)$ & 15.7 & (13.7-17.8) & - & - & - & - & - & - \\
\hline North Carolina & 20.5 & $(17.3-24.1)$ & 10.7 & $(8.7-13.2)$ & 15.9 & (13.8-18.4) & 17.3 & $(14.1-21.0)$ & 10.5 & $(7.6-14.3)$ & 14.1 & $(12.5-15.8)$ \\
\hline North Dakota & 20.4 & (17.6-23.6) & 12.2 & (10.0-14.8) & 16.2 & (14.2-18.4) & 16.1 & (13.8-18.7) & 10.9 & $(8.9-13.3)$ & 13.5 & $(11.8-15.3)$ \\
\hline Oklahoma & 19.9 & $(17.2-22.9)$ & 10.2 & (7.4-13.9) & 15.1 & (12.9-17.6) & 19.1 & $(16.2-22.4)$ & 10.0 & (7.3-13.5) & 14.6 & $(12.3-17.1)$ \\
\hline Pennsylvania & 20.3 & $(18.1-22.6)$ & 11.2 & $(9.2-13.6)$ & 15.7 & (14.2-17.3) & 17.1 & (14.9-19.6) & 9.9 & $(7.8-12.5)$ & 13.5 & $(11.9-15.2)$ \\
\hline Rhode Island & 19.3 & (17.2-21.6) & 8.9 & $(7.3-10.9)$ & 14.1 & (12.4-15.9) & 15.8 & (13.9-17.9) & 8.4 & $(6.4-10.9)$ & 12.1 & (10.4-14.0) \\
\hline South Carolina & 21.3 & $(16.9-26.5)$ & 13.3 & $(10.3-17.0)$ & 17.3 & $(14.2-20.9)$ & 19.7 & $(16.4-23.5)$ & 9.7 & $(7.2-13.0)$ & 14.7 & $(12.6-17.2)$ \\
\hline South Dakota & 23.3 & $(17.6-30.0)$ & 9.3 & $(6.4-13.2)$ & 16.1 & (12.4-20.7) & 16.7 & $(11.9-23.0)$ & 7.1 & $(4.8-10.4)$ & 11.8 & $(8.8-15.6)$ \\
\hline Tennessee & 21.7 & $(19.0-24.6)$ & 10.6 & $(9.3-12.1)$ & 16.0 & $(14.5-17.6)$ & 18.6 & $(16.8-20.4)$ & 9.9 & $(8.2-12.0)$ & 14.2 & $(13.1-15.3)$ \\
\hline Vermont & - & - & - & - & - & - & 16.2 & $(15.5-16.9)$ & 8.0 & $(7.5-8.5)$ & 12.1 & $(11.7-12.6)$ \\
\hline Virginia & 18.6 & $(16.3-21.1)$ & 9.6 & $(8.1-11.3)$ & 14.0 & $(12.6-15.5)$ & 15.4 & $(13.6-17.5)$ & 8.1 & $(6.9-9.5)$ & 11.7 & $(10.5-13.0)$ \\
\hline West Virginia & 22.8 & $(18.5-27.7)$ & 14.8 & $(12.8-17.0)$ & 18.7 & $(16.3-21.4)$ & 17.6 & $(14.2-21.6)$ & 13.1 & $(11.2-15.4)$ & 15.4 & $(13.2-17.7)$ \\
\hline Wyoming & 24.7 & $(21.3-28.4)$ & 15.9 & $(13.2-18.9)$ & 20.3 & $(18.1-22.7)$ & 20.9 & $(18.2-23.9)$ & 15.6 & $(13.4-17.9)$ & 18.2 & $(16.5-20.1)$ \\
\hline Median & & 20.6 & & 11.7 & & 16.0 & & 17.6 & & 9.9 & & 14.3 \\
\hline Range & & $6.3-27.2)$ & & $3.9-17.0)$ & & $3.4-20.3)$ & & $4.0-23.3)$ & & 7.1-15.6) & & $1.0-18.2)$ \\
\hline \multicolumn{13}{|c|}{ Large urban school district surveys } \\
\hline Baltimore, MD & 20.3 & $(16.1-25.3)$ & 14.8 & $(11.0-19.7)$ & 17.8 & $(14.6-21.5)$ & 16.6 & $(12.9-21.1)$ & 11.8 & $(8.4-16.5)$ & 14.4 & (11.6-17.8) \\
\hline Boston, MA & 14.1 & $(11.5-17.2)$ & 7.3 & $(5.5-9.8)$ & 11.0 & $(9.2-13.0)$ & 12.8 & $(10.6-15.4)$ & 6.5 & $(4.8-8.8)$ & 9.7 & $(8.1-11.6)$ \\
\hline Broward County, FL & 21.0 & $(18.2-24.2)$ & 12.6 & $(10.2-15.4)$ & 16.8 & $(15.0-18.8)$ & 16.5 & $(14.0-19.4)$ & 10.9 & $(9.0-13.0)$ & 13.7 & $(12.0-15.5)$ \\
\hline Cleveland, $\mathrm{OH}$ & 25.8 & $(22.8-29.1)$ & 12.2 & $(9.7-15.2)$ & 19.2 & $(17.2-21.4)$ & - & - & - & - & - & - \\
\hline DeKalb County, GA & 22.1 & $(19.5-24.9)$ & 10.6 & $(8.7-12.9)$ & 16.3 & $(14.5-18.2)$ & 19.1 & $(16.3-22.2)$ & 12.6 & $(10.2-15.6)$ & 15.8 & $(13.9-18.0)$ \\
\hline Detroit, MI & 18.7 & $(15.6-22.2)$ & 14.3 & $(11.6-17.4)$ & 16.7 & $(14.6-19.0)$ & 16.7 & $(14.1-19.7)$ & 15.6 & $(12.8-19.0)$ & 16.4 & $(14.5-18.6)$ \\
\hline District of Columbia & 18.5 & $(17.4-19.6)$ & 8.9 & $(8.1-9.8)$ & 13.9 & $(13.2-14.6)$ & 18.9 & $(17.8-20.0)$ & 10.2 & $(9.3-11.2)$ & 14.8 & $(14.1-15.5)$ \\
\hline Duval County, FL & 24.2 & $(21.9-26.7)$ & 14.3 & $(12.0-17.1)$ & 19.7 & $(17.9-21.6)$ & 22.0 & $(17.8-20.0)$ & 15.9 & $(13.4-18.8)$ & 19.3 & $(17.3-21.4)$ \\
\hline Ft. Worth, TX & 19.8 & $(17.3-22.6)$ & 7.7 & $(6.3-9.4)$ & 13.8 & $(12.3-15.5)$ & 16.2 & $(13.9-18.9)$ & 7.1 & $(5.7-8.8)$ & 11.6 & $(10.2-13.2)$ \\
\hline Houston, TX & 19.3 & $(17.0-21.8)$ & 11.1 & $(9.4-13.2)$ & 15.1 & $(13.7-16.7)$ & 17.4 & $(15.4-19.6)$ & 11.7 & $(9.9-13.7)$ & 14.6 & $(13.3-16.0)$ \\
\hline Los Angeles, CA & 17.6 & $(15.1-20.4)$ & 10.4 & $(8.6-12.6)$ & 14.1 & $(12.7-15.5)$ & 14.1 & $(12.0-16.4)$ & 11.6 & $(9.9-13.5)$ & 12.9 & $(11.3-14.7)$ \\
\hline Miami-Dade County, FL & 12.5 & $(10.9-14.2)$ & 8.9 & $(7.5-10.6)$ & 10.7 & $(9.5-12.0)$ & 9.7 & $(8.2-11.5)$ & 7.6 & $(6.1-9.5)$ & 8.7 & $(7.6-10.0)$ \\
\hline
\end{tabular}

See table footnotes on the next page. 
TABLE 26. (Continued) Percentage of high school students who seriously considered attempting suicide* and who made a plan about how they would attempt suicide, ${ }^{*}$ by sex — selected U.S. sites, Youth Risk Behavior Survey, 2015

\begin{tabular}{|c|c|c|c|c|c|c|c|c|c|c|c|c|}
\hline \multirow[b]{3}{*}{ Site } & \multicolumn{6}{|c|}{ Seriously considered attempting suicide } & \multicolumn{6}{|c|}{ Made a suicide plan } \\
\hline & \multicolumn{2}{|c|}{ Female } & \multicolumn{2}{|c|}{ Male } & \multicolumn{2}{|r|}{ Total } & \multicolumn{2}{|r|}{ Female } & \multicolumn{2}{|r|}{ Male } & \multicolumn{2}{|r|}{ Total } \\
\hline & $\%$ & $\mathrm{Cl}^{\dagger}$ & $\%$ & $\mathrm{Cl}$ & $\%$ & $\mathrm{Cl}$ & $\%$ & $\mathrm{Cl}$ & $\%$ & $\mathrm{Cl}$ & $\%$ & $\mathrm{Cl}$ \\
\hline New York City, NY & 18.3 & $(16.3-20.4)$ & 9.0 & $(7.7-10.6)$ & 13.7 & $(12.2-15.4)$ & - & - & - & - & - & - \\
\hline Oakland, CA & 18.8 & $(15.7-22.2)$ & 10.6 & $(8.3-13.5)$ & 14.4 & $(12.6-16.3)$ & 16.3 & $(13.6-19.4)$ & 9.1 & $(6.9-12.0)$ & 12.5 & $(10.8-14.5)$ \\
\hline Orange County, FL & 20.1 & $(17.2-23.3)$ & 10.2 & $(7.9-12.9)$ & 15.1 & $(13.2-17.3)$ & 15.0 & $(12.5-18.0)$ & 9.9 & $(7.6-12.7)$ & 12.3 & $(10.6-14.3)$ \\
\hline Palm Beach County, FL & 20.8 & $(18.2-23.6)$ & 10.0 & $(8.2-12.2)$ & 15.5 & $(13.7-17.4)$ & 17.9 & $(15.2-21.0)$ & 11.3 & $(9.0-14.0)$ & 14.6 & $(12.7-16.8)$ \\
\hline Philadelphia, PA & 18.0 & $(15.5-20.9)$ & 9.9 & $(7.2-13.4)$ & 14.0 & $(11.7-16.8)$ & 16.6 & $(14.3-19.2)$ & 9.0 & $(6.3-12.9)$ & 12.8 & (11.0-14.9) \\
\hline San Diego, CA & 21.1 & $(18.0-24.6)$ & 12.1 & $(9.8-14.7)$ & 16.5 & $(14.7-18.5)$ & - & - & - & - & - & - \\
\hline San Francisco, CA & 15.6 & $(12.9-18.7)$ & 9.5 & $(7.2-12.4)$ & 12.6 & $(10.8-14.6)$ & 16.3 & $(13.5-19.7)$ & 11.3 & (9.2-13.6) & 13.9 & (11.9-16.2) \\
\hline Median & \multicolumn{2}{|c|}{19.3} & \multicolumn{2}{|c|}{10.4} & \multicolumn{2}{|c|}{15.1} & \multicolumn{2}{|r|}{16.5} & \multicolumn{2}{|c|}{11.1} & \multicolumn{2}{|r|}{13.8} \\
\hline Range & \multicolumn{2}{|c|}{$(12.5-25.8)$} & \multicolumn{2}{|c|}{ (7.3-14.8) } & \multicolumn{2}{|c|}{ (10.7-19.7) } & \multicolumn{2}{|c|}{$(9.7-22.0)$} & \multicolumn{2}{|c|}{$(6.5-15.9)$} & \multicolumn{2}{|c|}{ (8.7-19.3) } \\
\hline
\end{tabular}

* During the 12 months before the survey.

† $95 \%$ confidence interval.

$\S$ Not available.

TABLE 27. Percentage of high school students who attempted suicide ${ }^{*, \dagger}$ and whose suicide attempt resulted in an injury, poisoning, or overdose that had to be treated by a doctor or nurse, ${ }^{\dagger}$ by sex, race/ethnicity, and grade - United States, Youth Risk Behavior Survey, 2015

\begin{tabular}{|c|c|c|c|c|c|c|c|c|c|c|c|c|}
\hline \multirow[b]{3}{*}{ Category } & \multicolumn{6}{|c|}{ Attempted suicide } & \multicolumn{6}{|c|}{ Suicide attempt treated by a doctor or nurse } \\
\hline & \multicolumn{2}{|c|}{ Female } & \multicolumn{2}{|c|}{ Male } & \multicolumn{2}{|c|}{ Total } & \multicolumn{2}{|c|}{ Female } & \multicolumn{2}{|c|}{ Male } & \multicolumn{2}{|c|}{ Total } \\
\hline & $\%$ & $\mathrm{Cl}^{\S}$ & $\%$ & $\mathrm{Cl}$ & $\%$ & $\mathrm{Cl}$ & $\%$ & $\mathrm{Cl}$ & $\%$ & $\mathrm{Cl}$ & $\%$ & $\mathrm{Cl}$ \\
\hline \multicolumn{13}{|c|}{ Race/Ethnicity } \\
\hline White & 9.8 & $(7.5-12.8)$ & 3.7 & $(2.9-4.7)$ & 6.8 & $(5.5-8.4)$ & 3.4 & $(2.3-4.8)$ & 0.9 & $(0.5-1.4)$ & 2.1 & $(1.5-2.9)$ \\
\hline Black ${ }^{9}$ & 10.2 & $(6.8-15.1)$ & 7.2 & $(5.3-9.8)$ & 8.9 & $(6.7-11.9)$ & 3.6 & $(2.4-5.6)$ & 4.0 & $(2.0-7.8)$ & 3.8 & $(2.2-6.3)$ \\
\hline Hispanic & 15.1 & $(12.8-17.7)$ & 7.6 & $(5.8-9.9)$ & 11.3 & $(9.9-13.0)$ & 4.5 & $(3.1-6.4)$ & 2.9 & $(1.9-4.4)$ & 3.7 & $(2.7-5.1)$ \\
\hline \multicolumn{13}{|l|}{ Grade } \\
\hline 9 & 15.1 & $(12.2-18.5)$ & 5.1 & $(3.7-7.0)$ & 9.9 & $(8.5-11.5)$ & 4.7 & (3.3-6.6) & 1.9 & $(1.1-3.0)$ & 3.2 & (2.4-4.2) \\
\hline 10 & 13.0 & $(9.8-17.2)$ & 5.7 & $(4.1-7.8)$ & 9.4 & $(7.6-11.6)$ & 3.9 & $(2.6-5.6)$ & 2.2 & $(1.2-4.0)$ & 3.1 & $(2.2-4.3)$ \\
\hline 11 & 10.2 & $(8.2-12.5)$ & 5.8 & $(4.6-7.3)$ & 8.0 & $(6.8-9.5)$ & 3.4 & $(2.2-5.2)$ & 2.0 & $(1.1-3.5)$ & 2.6 & $(1.8-3.9)$ \\
\hline 12 & 7.2 & $(5.3-9.6)$ & 5.2 & $(3.4-7.8)$ & 6.2 & $(4.9-7.9)$ & 2.3 & $(1.5-3.6)$ & 1.4 & $(0.7-2.6)$ & 1.9 & $(1.3-2.8)$ \\
\hline Total & 11.6 & $(9.7-13.7)$ & 5.5 & $(4.7-6.4)$ & 8.6 & $(7.6-9.6)$ & 3.7 & $(2.9-4.7)$ & 1.9 & $(1.3-2.8)$ & 2.8 & $(2.2-3.5)$ \\
\hline
\end{tabular}

* One or more times.

† During the 12 months before the survey.

$\S 95 \%$ confidence interval.

१ Non-Hispanic. 
TABLE 28. Percentage of high school students who attempted suicide ${ }^{*, t}$ and whose suicide attempt resulted in an injury, poisoning, or overdose that had to be treated by a doctor or nurse, ${ }^{\dagger}$ by sex — selected U.S. sites, Youth Risk Behavior Survey, 2015

\begin{tabular}{|c|c|c|c|c|c|c|c|c|c|c|c|c|}
\hline \multirow[b]{3}{*}{ Site } & \multicolumn{6}{|c|}{ Attempted suicide } & \multicolumn{6}{|c|}{ Suicide attempt treated by a doctor or nurse } \\
\hline & \multicolumn{2}{|c|}{ Female } & \multicolumn{2}{|c|}{ Male } & \multicolumn{2}{|r|}{ Total } & \multicolumn{2}{|c|}{ Female } & \multicolumn{2}{|c|}{ Male } & \multicolumn{2}{|r|}{ Total } \\
\hline & $\%$ & $\mathrm{Cl}^{\S}$ & $\%$ & $\mathrm{Cl}$ & $\%$ & $\mathrm{Cl}$ & $\%$ & $\mathrm{Cl}$ & $\%$ & $\mathrm{Cl}$ & $\%$ & $\mathrm{Cl}$ \\
\hline \multicolumn{13}{|l|}{ State surveys } \\
\hline Alabama & 13.0 & (10.3-16.4) & 8.7 & $(6.6-11.4)$ & 11.2 & (9.4-13.3) & 4.8 & $(3.6-6.5)$ & 3.3 & $(2.0-5.4)$ & 4.3 & $(3.3-5.6)$ \\
\hline Alaska & 11.0 & $(8.7-14.0)$ & 10.4 & $(8.2-13.1)$ & 10.7 & $(8.9-12.8)$ & 4.0 & $(2.7-6.1)$ & 3.0 & $(1.9-4.7)$ & 3.5 & $(2.6-4.7)$ \\
\hline Arizona & 12.6 & $(9.5-16.6)$ & 6.2 & $(4.5-8.6)$ & 9.6 & $(7.9-11.5)$ & 3.7 & $(2.6-5.5)$ & 1.5 & $(0.6-3.6)$ & 2.7 & $(2.0-3.6)$ \\
\hline Arkansas & 13.8 & $(11.5-16.5)$ & 10.2 & $(7.7-13.5)$ & 12.1 & $(10.2-14.4)$ & 4.6 & $(3.9-5.5)$ & 4.4 & $(3.2-5.9)$ & 4.5 & $(3.7-5.4)$ \\
\hline California & 11.8 & $(9.9-14.0)$ & 4.7 & $(3.2-7.0)$ & 8.2 & $(6.8-9.9)$ & 2.8 & $(2.0-3.9)$ & 1.0 & $(0.4-2.7)$ & 1.9 & $(1.2-2.9)$ \\
\hline Connecticut & 8.7 & $(6.9-10.8)$ & 6.6 & $(5.3-8.1)$ & 7.9 & $(6.6-9.4)$ & - & - & - & - & - & - \\
\hline Delaware & 10.2 & $(7.5-13.6)$ & 4.8 & $(3.4-6.8)$ & 7.6 & (6.1-9.5) & 2.4 & $(1.4-4.2)$ & 2.1 & $(1.3-3.4)$ & 2.3 & $(1.7-3.2)$ \\
\hline Florida & 9.6 & $(8.6-10.7)$ & 5.2 & $(4.3-6.4)$ & 7.6 & $(6.8-8.5)$ & 2.8 & $(2.2-3.6)$ & 1.9 & $(1.3-2.7)$ & 2.5 & $(1.9-3.2)$ \\
\hline Hawaii & 11.0 & $(9.6-12.7)$ & 9.3 & (7.4-11.6) & 10.5 & $(9.3-11.9)$ & 3.1 & $(2.2-4.2)$ & 3.5 & $(2.6-4.7)$ & 3.4 & $(2.8-4.1)$ \\
\hline Idaho & 14.0 & (11.0-17.7) & 5.8 & $(4.8-7.0)$ & 9.8 & $(8.2-11.7)$ & 4.4 & (3.1-6.4) & 1.7 & $(1.0-2.9)$ & 3.1 & $(2.4-3.9)$ \\
\hline Illinois & 9.5 & $(7.2-12.4)$ & 9.8 & $(7.8-12.2)$ & 9.8 & $(8.1-11.7)$ & 2.9 & $(1.9-4.4)$ & 5.7 & $(3.8-8.3)$ & 4.3 & $(3.2-5.8)$ \\
\hline Indiana & 10.9 & $(8.3-14.1)$ & 8.7 & $(6.0-12.5)$ & 9.9 & (7.7-12.7) & 4.4 & $(2.7-7.0)$ & 3.3 & $(1.9-5.6)$ & 3.9 & $(2.5-6.0)$ \\
\hline Kentucky & 12.9 & $(9.4-17.3)$ & 5.7 & $(3.8-8.5)$ & 9.4 & $(7.0-12.4)$ & 5.3 & $(3.5-7.9)$ & 2.5 & $(1.4-4.3)$ & 3.9 & $(2.6-5.7)$ \\
\hline Maine & 11.2 & $(10.2-12.2)$ & 8.4 & $(7.5-9.4)$ & 9.9 & $(9.3-10.6)$ & - & - & - & - & - & - \\
\hline Maryland & - & - & - & - & - & - & - & - & - & - & - & - \\
\hline Massachusetts & 8.2 & $(6.5-10.3)$ & 5.4 & $(4.1-7.0)$ & 7.0 & $(6.0-8.2)$ & 2.9 & $(2.0-4.2)$ & 2.5 & $(1.7-3.8)$ & 2.8 & $(2.2-3.6)$ \\
\hline Michigan & 11.1 & $(9.6-12.8)$ & 7.1 & $(5.3-9.5)$ & 9.2 & $(7.9-10.7)$ & 3.0 & $(2.0-4.4)$ & 2.4 & $(1.6-3.8)$ & 2.7 & $(2.0-3.7)$ \\
\hline Mississippi & 13.4 & $(10.9-16.5)$ & 11.2 & $(8.7-14.4)$ & 12.7 & $(10.5-15.2)$ & 5.5 & $(3.6-8.4)$ & 5.9 & $(4.0-8.6)$ & 5.7 & $(4.3-7.6)$ \\
\hline Missouri & 10.3 & $(7.4-14.1)$ & 9.2 & (7.4-11.4) & 9.8 & $(7.8-12.2)$ & 3.2 & $(1.7-5.9)$ & 3.1 & $(2.1-4.5)$ & 3.2 & $(2.3-4.4)$ \\
\hline Montana & 11.5 & $(10.1-13.2)$ & 6.3 & $(5.1-7.8)$ & 8.9 & $(7.8-10.1)$ & 4.0 & $(3.1-5.0)$ & 2.4 & $(1.6-3.4)$ & 3.1 & $(2.4-4.0)$ \\
\hline Nebraska & 9.4 & $(7.0-12.5)$ & 7.7 & $(5.8-10.1)$ & 8.9 & (7.4-10.5) & 3.0 & $(1.8-5.0)$ & 3.5 & $(2.2-5.6)$ & 3.3 & $(2.5-4.5)$ \\
\hline Nevada & 13.1 & $(10.3-16.6)$ & 8.0 & $(6.3-10.1)$ & 10.7 & $(9.1-12.6)$ & 3.2 & $(2.1-4.8)$ & 2.3 & $(1.4-3.8)$ & 2.8 & $(2.0-3.7)$ \\
\hline New Hampshire & 8.9 & $(8.0-9.9)$ & 4.6 & $(3.9-5.3)$ & 6.8 & $(6.2-7.4)$ & 3.5 & $(2.8-4.2)$ & 1.5 & $(1.2-1.9)$ & 2.5 & $(2.1-2.9)$ \\
\hline New Mexico & 12.4 & (11.0-13.9) & 6.4 & $(5.4-7.5)$ & 9.4 & $(8.6-10.4)$ & 3.8 & $(3.1-4.7)$ & 2.5 & $(2.0-3.3)$ & 3.2 & $(2.7-3.8)$ \\
\hline New York & 9.9 & $(8.2-11.9)$ & 9.3 & $(7.3-11.8)$ & 9.9 & $(8.4-11.7)$ & 4.2 & $(3.2-5.5)$ & 4.4 & $(3.2-6.0)$ & 4.4 & $(3.6-5.3)$ \\
\hline North Carolina & - & - & - & - & - & - & 8.8 & $(5.9-12.9)$ & 9.6 & $(7.9-11.7)$ & 9.3 & $(8.0-10.7)$ \\
\hline North Dakota & 9.6 & $(7.6-12.1)$ & 9.3 & $(7.4-11.5)$ & 9.4 & $(7.9-11.1)$ & - & - & - & - & - & - \\
\hline Oklahoma & 10.2 & $(8.1-12.7)$ & 4.5 & $(2.8-7.2)$ & 7.4 & $(6.2-8.9)$ & 2.4 & $(1.6-3.6)$ & 1.6 & $(0.9-3.0)$ & 2.0 & $(1.5-2.8)$ \\
\hline Pennsylvania & 9.6 & $(7.5-12.2)$ & 5.3 & $(4.0-7.0)$ & 7.5 & $(6.1-9.1)$ & 3.0 & $(2.0-4.6)$ & 2.2 & $(1.4-3.4)$ & 2.6 & $(1.9-3.7)$ \\
\hline Rhode Island & 12.5 & $(9.0-17.2)$ & 8.3 & $(6.4-10.6)$ & 10.5 & (7.9-13.8) & 4.4 & $(3.0-6.5)$ & 3.8 & $(2.5-5.7)$ & 4.1 & $(3.0-5.6)$ \\
\hline South Carolina & 12.6 & (8.4-18.4) & 9.4 & $(7.0-12.5)$ & 11.0 & (8.4-14.3) & 3.5 & $(2.1-5.8)$ & 3.3 & $(1.9-5.8)$ & 3.4 & $(2.3-5.2)$ \\
\hline South Dakota & 10.0 & $(6.2-15.6)$ & 6.8 & $(4.0-11.3)$ & 8.4 & $(5.7-12.3)$ & 4.2 & $(2.1-8.2)$ & 3.2 & $(1.7-6.0)$ & 3.8 & $(2.2-6.6)$ \\
\hline Tennessee & 12.5 & $(11.2-13.8)$ & 7.3 & $(5.7-9.2)$ & 9.9 & $(8.9-10.9)$ & 4.2 & $(3.4-5.3)$ & 3.3 & $(2.3-4.7)$ & 3.8 & $(3.1-4.7)$ \\
\hline Vermont & 8.0 & (7.5-8.5) & 3.7 & $(3.3-4.1)$ & 5.9 & $(5.6-6.2)$ & 2.6 & $(2.3-3.0)$ & 1.2 & $(1.0-1.5)$ & 2.0 & $(1.8-2.2)$ \\
\hline Virginia & 8.7 & $(7.1-10.5)$ & 4.6 & $(3.7-5.6)$ & 6.7 & $(5.8-7.6)$ & 2.4 & $(1.6-3.7)$ & 1.3 & $(0.8-2.0)$ & 1.9 & $(1.3-2.6)$ \\
\hline West Virginia & 11.3 & $(8.9-14.2)$ & 8.5 & $(6.5-11.0)$ & 9.9 & $(8.5-11.5)$ & 3.7 & $(2.3-6.1)$ & 2.6 & $(1.5-4.7)$ & 3.2 & $(2.1-4.8)$ \\
\hline Wyoming & 14.7 & (12.0-17.8) & 7.7 & $(5.9-9.9)$ & 11.1 & $(9.5-13.0)$ & 4.5 & (3.2-6.3) & 3.7 & $(2.3-5.8)$ & 4.1 & $(3.2-5.3)$ \\
\hline Median & & 1.0 & & 7.3 & & 9.6 & & 3.7 & & .6 & & 3.2 \\
\hline Range & & $-14.7)$ & & $-11.2)$ & & $9-12.7)$ & & $-8.8)$ & & -9.6) & & .9-9.3) \\
\hline \multicolumn{13}{|c|}{ Large urban school district surveys } \\
\hline Baltimore, MD & 16.2 & $(12.0-21.5)$ & 20.5 & $(14.4-28.4)$ & 18.7 & $(14.6-23.6)$ & 4.9 & $(2.9-8.0)$ & 9.4 & $(5.9-14.5)$ & 7.3 & $(5.2-10.1)$ \\
\hline Boston, MA & 8.2 & $(6.3-10.7)$ & 7.9 & $(6.2-10.1)$ & 8.1 & $(6.7-9.8)$ & 2.4 & $(1.4-3.9)$ & 2.4 & $(1.5-4.0)$ & 2.4 & $(1.7-3.4)$ \\
\hline Broward County, FL & 11.8 & $(9.6-14.4)$ & 6.2 & $(4.6-8.4)$ & 9.3 & (7.9-10.9) & 4.3 & $(2.8-6.4)$ & 2.6 & $(1.6-4.4)$ & 3.5 & $(2.6-4.8)$ \\
\hline Cleveland, $\mathrm{OH}$ & 22.6 & $(19.5-26.0)$ & 17.7 & $(14.4-21.5)$ & 20.7 & $(18.3-23.3)$ & - & - & - & - & - & - \\
\hline DeKalb County, GA & 11.2 & $(8.5-14.5)$ & 8.6 & $(6.7-10.9)$ & 9.9 & $(8.1-12.0)$ & 4.1 & $(2.8-6.1)$ & 5.1 & $(3.4-7.5)$ & 4.6 & $(3.4-6.1)$ \\
\hline Detroit, MI & 15.1 & $(12.3-18.3)$ & 16.4 & $(12.0-22.1)$ & 16.0 & $(13.4-18.9)$ & 6.4 & $(4.8-8.6)$ & 8.4 & $(6.0-11.8)$ & 7.4 & $(5.9-9.3)$ \\
\hline District of Columbia & 13.7 & $(12.6-14.8)$ & 11.1 & $(10.0-12.2)$ & 12.7 & $(11.9-13.4)$ & 4.8 & $(4.2-5.5)$ & 4.9 & $(4.2-5.8)$ & 5.0 & $(4.5-5.5)$ \\
\hline Duval County, FL & 18.9 & $(16.2-21.9)$ & 17.9 & $(15.0-21.1)$ & 18.9 & $(16.4-21.5)$ & - & - & - & - & - & - \\
\hline Ft. Worth, TX & 11.8 & $(9.7-14.3)$ & 3.9 & $(2.9-5.3)$ & 7.8 & $(6.6-9.3)$ & 3.9 & $(2.8-5.4)$ & 1.7 & $(1.0-2.8)$ & 2.9 & $(2.3-3.7)$ \\
\hline Houston, TX & 14.1 & $(12.3-16.2)$ & 11.2 & $(9.4-13.4)$ & 13.0 & $(11.5-14.6)$ & 5.2 & $(4.1-6.6)$ & 3.9 & $(3.0-5.0)$ & 4.6 & $(3.8-5.6)$ \\
\hline Los Angeles, CA & 10.1 & $(8.2-12.2)$ & 6.5 & $(4.6-9.1)$ & 8.4 & $(7.1-9.9)$ & 2.2 & $(1.1-4.3)$ & 2.1 & $(1.4-3.2)$ & 2.1 & $(1.5-3.1)$ \\
\hline Miami-Dade County, FL & 7.4 & $(5.9-9.2)$ & 5.6 & $(4.0-7.7)$ & 6.4 & $(5.3-7.9)$ & 2.2 & $(1.5-3.3)$ & 1.9 & $(1.1-3.1)$ & 2.1 & $(1.5-2.8)$ \\
\hline
\end{tabular}

See table footnotes on the next page. 
TABLE 28. (Continued) Percentage of high school students who attempted suicide ${ }^{*}$, and whose suicide attempt resulted in an injury, poisoning, or overdose that had to be treated by a doctor or nurse, ${ }^{\dagger}$ by sex - selected U.S. sites, Youth Risk Behavior Survey, 2015

\begin{tabular}{|c|c|c|c|c|c|c|c|c|c|c|c|c|}
\hline \multirow[b]{3}{*}{ Site } & \multicolumn{6}{|c|}{ Attempted suicide } & \multicolumn{6}{|c|}{ Suicide attempt treated by a doctor or nurse } \\
\hline & \multicolumn{2}{|c|}{ Female } & \multicolumn{2}{|c|}{ Male } & \multicolumn{2}{|r|}{ Total } & \multicolumn{2}{|c|}{ Female } & \multicolumn{2}{|c|}{ Male } & \multicolumn{2}{|c|}{ Total } \\
\hline & $\%$ & $\mathrm{Cl}^{\S}$ & $\%$ & $\mathrm{Cl}$ & $\%$ & $\mathrm{Cl}$ & $\%$ & $\mathrm{Cl}$ & $\%$ & $\mathrm{Cl}$ & $\%$ & $\mathrm{Cl}$ \\
\hline New York City, NY & 10.1 & $(8.5-11.9)$ & 6.2 & $(5.3-7.2)$ & 8.3 & $(7.4-9.3)$ & 2.9 & $(2.1-3.8)$ & 2.6 & $(2.0-3.4)$ & 2.8 & $(2.3-3.3)$ \\
\hline Oakland, CA & 12.8 & $(9.8-16.5)$ & 9.8 & $(7.4-12.8)$ & 11.3 & $(9.5-13.3)$ & 2.8 & $(1.8-4.5)$ & 3.9 & $(2.5-6.2)$ & 3.4 & $(2.4-4.7)$ \\
\hline Orange County, FL & 12.1 & $(9.8-14.8)$ & 6.1 & $(3.8-9.8)$ & 9.1 & $(7.4-11.2)$ & 4.5 & $(3.1-6.4)$ & 2.2 & $(1.0-5.1)$ & 3.4 & $(2.2-5.1)$ \\
\hline Palm Beach County, FL & 11.1 & $(9.2-13.4)$ & 8.1 & $(6.4-10.2)$ & 10.1 & $(8.6-11.8)$ & 4.2 & $(3.0-5.7)$ & 3.0 & $(2.0-4.5)$ & 3.7 & $(3.0-4.7)$ \\
\hline Philadelphia, PA & 12.6 & $(10.9-14.6)$ & 8.8 & $(5.7-13.4)$ & 11.0 & $(8.6-14.1)$ & 3.8 & $(2.4-5.9)$ & 3.3 & $(1.8-5.7)$ & 3.6 & $(2.5-5.0)$ \\
\hline San Diego, CA & 11.0 & $(8.9-13.4)$ & 6.9 & $(5.3-8.8)$ & 8.9 & $(7.6-10.5)$ & 2.2 & $(1.4-3.5)$ & 1.7 & $(1.0-2.8)$ & 1.9 & $(1.3-2.8)$ \\
\hline San Francisco, CA & 11.3 & $(8.7-14.7)$ & 7.8 & $(5.7-10.5)$ & 9.8 & $(7.7-12.5)$ & 4.3 & $(2.8-6.6)$ & 3.7 & $(2.3-5.8)$ & 4.3 & $(3.1-6.0)$ \\
\hline Median & \multicolumn{2}{|c|}{11.8} & \multicolumn{2}{|r|}{8.1} & \multicolumn{2}{|r|}{9.9} & \multicolumn{2}{|c|}{4.1} & \multicolumn{2}{|c|}{3.0} & \multirow{2}{*}{\multicolumn{2}{|c|}{$\begin{array}{c}3.5 \\
(1.9-7.4)\end{array}$}} \\
\hline Range & & $4-22.6)$ & & 9-20.5) & & (4-20.7) & & $.2-6.4)$ & & 7-9.4) & & \\
\hline
\end{tabular}

* One or more times.

${ }^{\dagger}$ During the 12 months before the survey.

$\S 95 \%$ confidence interval.

" Not available.

TABLE 29. Percentage of high school students who ever tried cigarette smoking* and who smoked a whole cigarette for the first time before age 13 years, by sex, race/ethnicity, and grade — United States, Youth Risk Behavior Survey, 2015

\begin{tabular}{|c|c|c|c|c|c|c|c|c|c|c|c|c|}
\hline \multirow[b]{3}{*}{ Category } & \multicolumn{6}{|c|}{ Ever tried cigarette smoking } & \multicolumn{6}{|c|}{ Smoked a whole cigarette before age 13 years } \\
\hline & \multicolumn{2}{|c|}{ Female } & \multicolumn{2}{|c|}{ Male } & \multicolumn{2}{|r|}{ Total } & \multicolumn{2}{|c|}{ Female } & \multicolumn{2}{|c|}{ Male } & \multicolumn{2}{|c|}{ Total } \\
\hline & $\%$ & $\mathrm{Cl}^{\dagger}$ & $\%$ & $\mathrm{Cl}$ & $\%$ & $\mathrm{Cl}$ & $\%$ & $\mathrm{Cl}$ & $\%$ & $\mathrm{Cl}$ & $\%$ & $\mathrm{Cl}$ \\
\hline \multicolumn{13}{|c|}{ Race/Ethnicity } \\
\hline White $\S$ & 30.4 & $(23.5-38.2)$ & 33.2 & $(29.3-37.3)$ & 31.8 & $(26.8-37.2)$ & 5.3 & (3.8-7.4) & 6.6 & $(4.9-8.7)$ & 6.0 & (4.5-7.9) \\
\hline Black $^{\S}$ & 29.5 & $(24.2-35.5)$ & 30.6 & $(24.6-37.3)$ & 30.1 & $(25.0-35.7)$ & 3.8 & $(2.1-6.8)$ & 10.1 & $(7.8-13.0)$ & 7.0 & $(5.2-9.4)$ \\
\hline Hispanic & 32.7 & $(29.7-35.7)$ & 37.8 & $(34.8-41.0)$ & 35.2 & $(32.7-37.9)$ & 4.9 & $(3.9-6.2)$ & 9.2 & $(7.8-10.8)$ & 7.1 & $(6.1-8.3)$ \\
\hline \multicolumn{13}{|l|}{ Grade } \\
\hline 9 & 24.5 & $(20.2-29.4)$ & 25.8 & (22.4-29.4) & 25.1 & $(21.8-28.7)$ & 6.1 & $(4.4-8.5)$ & 8.2 & $(6.3-10.5)$ & 7.2 & $(5.5-9.3)$ \\
\hline 10 & 28.2 & $(22.1-35.2)$ & 30.0 & $(25.3-35.1)$ & 29.1 & $(24.2-34.6)$ & 6.0 & $(4.3-8.5)$ & 9.1 & $(7.0-11.9)$ & 7.6 & (5.9-9.7) \\
\hline 11 & 34.4 & $(29.9-39.3)$ & 40.5 & $(37.5-43.6)$ & 37.5 & $(34.5-40.7)$ & 4.5 & $(3.6-5.5)$ & 6.8 & $(5.0-9.2)$ & 5.6 & $(4.6-7.0)$ \\
\hline 12 & 36.3 & $(30.3-42.7)$ & 40.4 & (33.8-47.4) & 38.3 & (33.1-43.9) & 3.0 & $(2.1-4.3)$ & 7.3 & $(5.3-9.9)$ & 5.2 & (3.9-6.9) \\
\hline Total & 30.7 & $(26.3-35.4)$ & 33.8 & $(30.7-37.1)$ & 32.3 & $(28.9-35.8)$ & 5.0 & $(4.0-6.3)$ & 8.0 & $(6.6-9.6)$ & 6.6 & $(5.5-7.9)$ \\
\hline
\end{tabular}

*Even one or two puffs.

† $95 \%$ confidence interval.

$\S$ Non-Hispanic. 
TABLE 30. Percentage of high school students who ever tried cigarette smoking* and who smoked a whole cigarette for the first time before age 13 years, by sex - selected U.S. sites, Youth Risk Behavior Survey, 2015

\begin{tabular}{|c|c|c|c|c|c|c|c|c|c|c|c|c|}
\hline \multirow[b]{3}{*}{ Site } & \multicolumn{6}{|c|}{ Ever tried cigarette smoking } & \multicolumn{6}{|c|}{ Smoked a whole cigarette before age 13 years } \\
\hline & \multicolumn{2}{|c|}{ Female } & \multicolumn{2}{|r|}{ Male } & \multicolumn{2}{|r|}{ Total } & \multicolumn{2}{|c|}{ Female } & \multicolumn{2}{|r|}{ Male } & \multicolumn{2}{|r|}{ Total } \\
\hline & $\%$ & $\mathrm{Cl}^{\dagger}$ & $\%$ & $\mathrm{Cl}$ & $\%$ & $\mathrm{Cl}$ & $\%$ & $\mathrm{Cl}$ & $\%$ & $\mathrm{Cl}$ & $\%$ & $\mathrm{Cl}$ \\
\hline \multicolumn{13}{|l|}{ State surveys } \\
\hline Alabama & 39.4 & (33.4-45.7) & 41.4 & (36.4-46.6) & 40.5 & (35.8-45.4) & 7.8 & $(5.5-10.8)$ & 12.4 & $(9.7-15.8)$ & 10.3 & $(8.1-13.2)$ \\
\hline Alaska & 28.5 & $(25.0-32.3)$ & 36.0 & (32.2-40.0) & 32.5 & $(29.7-35.5)$ & 7.8 & $(5.8-10.4)$ & 9.7 & $(7.1-13.2)$ & 8.9 & $(7.0-11.2)$ \\
\hline Arizona & 36.9 & (33.0-41.0) & 36.8 & (31.5-42.5) & 37.0 & (32.8-41.4) & 6.6 & $(4.8-9.0)$ & 6.9 & $(5.1-9.4)$ & 7.0 & $(5.7-8.6)$ \\
\hline Arkansas & 37.3 & $(32.6-42.1)$ & 49.2 & $(44.6-53.7)$ & 43.1 & (39.4-47.0) & 8.0 & $(5.9-10.8)$ & 18.3 & (15.5-21.5) & 13.1 & (10.9-15.7) \\
\hline California & 26.9 & $(22.9-31.4)$ & 29.1 & $(23.3-35.7)$ & 28.1 & $(23.9-32.7)$ & 5.3 & $(3.8-7.4)$ & 5.1 & (3.3-7.8) & 5.2 & (3.7-7.1) \\
\hline Connecticut & —§ & - & - & - & - & - & - & - & - & - & - & - \\
\hline Delaware & 30.1 & $(26.6-33.8)$ & 29.8 & $(25.9-34.0)$ & 30.2 & $(27.1-33.5)$ & 5.6 & $(4.1-7.5)$ & 7.2 & $(5.3-9.7)$ & 6.6 & $(5.2-8.4)$ \\
\hline Florida & - & - & - & - & - & - & 4.8 & $(3.9-5.7)$ & 7.9 & $(6.6-9.4)$ & 6.5 & $(5.5-7.6)$ \\
\hline Hawaii & 25.7 & $(23.8-27.7)$ & 24.3 & (22.1-26.5) & 25.0 & $(23.6-26.5)$ & 5.8 & $(4.5-7.5)$ & 6.5 & $(5.2-8.0)$ & 6.3 & $(5.2-7.6)$ \\
\hline Idaho & 27.9 & $(24.0-32.2)$ & 33.7 & (28.3-39.7) & 30.9 & $(27.2-35.0)$ & 4.0 & $(2.5-6.2)$ & 8.2 & $(5.6-11.8)$ & 6.2 & $(4.8-7.9)$ \\
\hline Illinois & 31.6 & $(27.1-36.5)$ & 36.1 & (30.7-41.8) & 33.8 & (29.6-38.3) & 3.4 & $(2.3-4.8)$ & 10.1 & $(7.7-13.0)$ & 6.7 & $(5.6-8.1)$ \\
\hline Indiana & 34.1 & $(29.1-39.4)$ & 39.4 & $(33.0-46.1)$ & 36.9 & (31.8-42.4) & 7.9 & $(5.6-11.1)$ & 8.2 & $(6.1-10.9)$ & 8.2 & $(6.4-10.6)$ \\
\hline Kentucky & 46.8 & $(41.7-51.9)$ & 41.4 & $(36.5-46.5)$ & 44.1 & $(40.0-48.2)$ & 12.8 & $(10.4-15.7)$ & 12.0 & $(10.2-13.9)$ & 12.5 & $(11.1-14.0)$ \\
\hline Maine & 28.5 & $(26.2-31.1)$ & 29.5 & $(27.5-31.6)$ & 29.1 & $(27.2-31.1)$ & 4.6 & $(3.9-5.5)$ & 6.3 & $(5.3-7.5)$ & 5.5 & (4.8-6.4) \\
\hline Maryland & - & - & - & - & - & - & 5.2 & $(4.9-5.6)$ & 8.5 & $(8.0-9.0)$ & 7.1 & $(6.7-7.5)$ \\
\hline Massachusetts & 24.9 & $(21.3-28.9)$ & 30.6 & $(26.8-34.7)$ & 27.8 & (24.4-31.5) & 3.1 & $(2.2-4.3)$ & 5.3 & $(4.0-7.0)$ & 4.3 & $(3.3-5.6)$ \\
\hline Michigan & 31.5 & $(27.0-36.3)$ & 33.6 & $(29.2-38.4)$ & 32.5 & $(29.0-36.3)$ & 6.5 & $(5.1-8.1)$ & 9.1 & $(6.5-12.6)$ & 7.8 & $(6.4-9.5)$ \\
\hline Mississippi & 38.3 & (33.0-43.8) & 47.0 & $(43.5-50.6)$ & 42.7 & (39.2-46.3) & 8.2 & $(6.2-10.8)$ & 14.7 & $(12.0-17.8)$ & 11.6 & $(9.8-13.8)$ \\
\hline Missouri & 31.3 & $(25.8-37.4)$ & 37.9 & $(32.7-43.4)$ & 34.5 & $(29.8-39.4)$ & 6.6 & $(4.6-9.6)$ & 9.2 & $(7.1-12.0)$ & 8.0 & $(6.2-10.2)$ \\
\hline Montana & 38.7 & (34.7-42.8) & 39.6 & $(36.5-42.7)$ & 39.1 & $(36.0-42.3)$ & 7.8 & $(6.2-9.6)$ & 8.2 & (7.1-9.5) & 8.0 & (7.0-9.2) \\
\hline Nebraska & 32.7 & $(28.1-37.7)$ & 30.1 & $(25.3-35.4)$ & 31.4 & $(27.6-35.6)$ & 8.7 & $(6.5-11.7)$ & 8.5 & $(6.5-11.0)$ & 8.8 & $(6.9-11.0)$ \\
\hline Nevada & 33.6 & $(28.0-39.7)$ & 32.8 & $(28.9-36.8)$ & 33.3 & $(29.8-37.0)$ & 4.9 & $(3.0-7.8)$ & 8.3 & $(6.6-10.4)$ & 6.7 & (5.5-8.0) \\
\hline New Hampshire & - & - & - & - & - & - & 3.7 & $(3.2-4.3)$ & 7.0 & (6.2-7.9) & 5.5 & $(5.0-6.0)$ \\
\hline New Mexico & 39.4 & (37.0-41.8) & 40.0 & (37.8-42.3) & 39.7 & (37.8-41.7) & 8.8 & $(7.8-9.9)$ & 11.6 & $(10.6-12.8)$ & 10.3 & $(9.5-11.1)$ \\
\hline New York & 26.7 & (23.4-30.3) & 28.4 & $(24.8-32.2)$ & 27.6 & (24.4-31.1) & - & - & - & - & - & - \\
\hline North Carolina & - & - & - & - & - & - & 7.3 & $(5.2-10.2)$ & 10.2 & $(7.6-13.5)$ & 8.8 & $(6.9-11.3)$ \\
\hline North Dakota & 32.3 & $(28.4-36.5)$ & 37.8 & $(34.1-41.5)$ & 35.1 & $(32.1-38.2)$ & 5.9 & $(4.5-7.7)$ & 8.5 & $(7.0-10.3)$ & 7.2 & $(6.0-8.6)$ \\
\hline Oklahoma & 35.8 & $(31.7-40.1)$ & 41.0 & $(36.0-46.2)$ & 38.6 & (35.0-42.2) & 6.6 & $(4.6-9.5)$ & 10.8 & $(7.8-14.7)$ & 8.8 & $(6.8-11.3)$ \\
\hline Pennsylvania & 33.4 & $(28.0-39.3)$ & 36.4 & (31.8-41.3) & 34.9 & (30.5-39.6) & 5.7 & $(4.3-7.7)$ & 8.2 & $(6.6-10.2)$ & 7.0 & $(5.6-8.6)$ \\
\hline Rhode Island & 20.2 & $(16.7-24.2)$ & 24.4 & $(20.8-28.5)$ & 22.4 & (19.6-25.5) & 4.0 & $(2.7-5.8)$ & 6.7 & $(4.8-9.2)$ & 5.5 & $(4.3-7.0)$ \\
\hline South Carolina & 38.7 & $(32.4-45.5)$ & 34.9 & (29.6-40.4) & 36.9 & $(32.0-42.1)$ & 9.1 & $(6.5-12.8)$ & 10.7 & $(6.8-16.3)$ & 10.0 & $(7.4-13.2)$ \\
\hline South Dakota & 31.6 & $(24.5-39.6)$ & 34.9 & (30.4-39.8) & 33.3 & $(28.5-38.5)$ & 7.3 & $(4.2-12.4)$ & 10.9 & $(8.1-14.5)$ & 9.2 & $(6.9-12.3)$ \\
\hline Tennessee & 35.1 & $(31.6-38.7)$ & 36.8 & (33.9-39.8) & 36.1 & (33.4-38.8) & 7.5 & $(6.1-9.3)$ & 11.7 & $(10.1-13.6)$ & 9.8 & $(8.6-11.1)$ \\
\hline Vermont & - & - & - & - & - & - & 4.8 & $(4.4-5.3)$ & 7.4 & $(7.0-8.0)$ & 6.2 & (5.9-6.6) \\
\hline Virginia & 24.2 & $(21.0-27.7)$ & 27.0 & $(24.1-30.1)$ & 25.7 & $(23.2-28.4)$ & 4.2 & $(3.3-5.2)$ & 6.5 & $(5.2-8.1)$ & 5.4 & $(4.5-6.5)$ \\
\hline West Virginia & 45.8 & $(41.6-50.1)$ & 49.0 & $(43.4-54.5)$ & 47.3 & $(43.2-51.5)$ & 11.5 & $(8.5-15.2)$ & 14.5 & $(11.1-18.8)$ & 13.0 & $(10.2-16.4)$ \\
\hline Wyoming & 39.4 & $(34.6-44.4)$ & 39.3 & $(34.9-44.0)$ & 39.4 & $(35.3-43.6)$ & 10.0 & $(7.3-13.5)$ & 12.4 & $(10.1-15.2)$ & 11.4 & $(9.2-14.0)$ \\
\hline Median & & 32.7 & & 36.1 & & 34.5 & & 6.6 & & 8.5 & & 7.8 \\
\hline Range & & $0.2-46.8)$ & & 4.3-49.2) & & 2.4-47.3) & & . $1-12.8)$ & & $.1-18.3)$ & & 4.3-13.1) \\
\hline \multicolumn{13}{|c|}{ Large urban school district surveys } \\
\hline Baltimore, MD & 28.6 & $(23.5-34.4)$ & 26.7 & $(21.4-32.8)$ & 27.9 & $(23.6-32.6)$ & 7.5 & $(5.3-10.5)$ & 9.9 & $(7.0-14.0)$ & 9.7 & $(7.4-12.8)$ \\
\hline Boston, MA & - & - & - & - & - & - & 3.7 & $(2.7-5.1)$ & 6.1 & $(4.3-8.5)$ & 5.1 & $(4.0-6.5)$ \\
\hline Broward County, FL & 17.8 & $(14.3-21.9)$ & 25.3 & $(21.6-29.5)$ & 21.7 & $(18.9-24.7)$ & 3.5 & $(2.1-5.7)$ & 5.0 & $(3.7-6.9)$ & 4.4 & $(3.3-5.7)$ \\
\hline Cleveland, $\mathrm{OH}$ & 31.5 & $(27.8-35.3)$ & 32.8 & $(29.5-36.3)$ & 32.5 & $(29.9-35.2)$ & - & - & - & - & - & - \\
\hline DeKalb County, GA & 23.9 & $(21.0-27.0)$ & 24.8 & $(21.3-28.6)$ & 24.3 & $(22.0-26.8)$ & 3.4 & $(2.2-5.2)$ & 7.8 & $(5.9-10.1)$ & 5.6 & $(4.3-7.2)$ \\
\hline Detroit, MI & 32.8 & $(28.4-37.6)$ & 33.5 & $(28.7-38.6)$ & 33.1 & $(29.3-37.2)$ & 13.1 & $(9.0-18.6)$ & 14.1 & $(10.5-18.6)$ & 13.7 & $(10.2-18.1)$ \\
\hline District of Columbia & - & - & - & - & - & - & 5.4 & $(4.8-6.1)$ & 8.3 & $(7.5-9.2)$ & 7.0 & $(6.5-7.7)$ \\
\hline Duval County, FL & - & - & - & - & - & - & 7.2 & $(5.2-9.9)$ & 11.5 & $(8.9-14.6)$ & 9.6 & $(7.4-12.2)$ \\
\hline Ft. Worth, TX & 30.0 & $(27.0-33.1)$ & 35.7 & $(32.5-39.0)$ & 32.7 & $(30.2-35.3)$ & 4.1 & $(3.1-5.4)$ & 7.8 & $(6.1-9.9)$ & 5.9 & $(4.9-7.1)$ \\
\hline Houston, TX & 31.8 & $(28.8-35.0)$ & 36.7 & $(33.5-40.0)$ & 34.4 & $(31.9-36.9)$ & 6.9 & $(5.5-8.5)$ & 11.0 & $(8.9-13.6)$ & 9.3 & $(7.8-11.0)$ \\
\hline Los Angeles, CA & 21.8 & $(18.8-25.2)$ & 26.6 & $(22.2-31.6)$ & 24.1 & $(21.1-27.4)$ & 2.8 & $(1.8-4.4)$ & 5.4 & $(3.3-8.6)$ & 4.0 & $(2.7-6.1)$ \\
\hline Miami-Dade County, FL & 20.6 & $(18.0-23.6)$ & 24.0 & $(21.5-26.6)$ & 22.2 & $(20.3-24.3)$ & 2.4 & $(1.4-3.8)$ & 5.2 & $(3.7-7.2)$ & 3.8 & $(2.8-5.0)$ \\
\hline
\end{tabular}

See table footnotes on the next page. 
TABLE 30. (Continued) Percentage of high school students who ever tried cigarette smoking* and who smoked a whole cigarette for the first time before age 13 years, by sex - selected U.S. sites, Youth Risk Behavior Survey, 2015

\begin{tabular}{|c|c|c|c|c|c|c|c|c|c|c|c|c|}
\hline \multirow[b]{3}{*}{ Site } & \multicolumn{6}{|c|}{ Ever tried cigarette smoking } & \multicolumn{6}{|c|}{ Smoked a whole cigarette before age 13 years } \\
\hline & \multicolumn{2}{|c|}{ Female } & \multicolumn{2}{|r|}{ Male } & \multicolumn{2}{|r|}{ Total } & \multicolumn{2}{|c|}{ Female } & \multicolumn{2}{|c|}{ Male } & \multicolumn{2}{|c|}{ Total } \\
\hline & $\%$ & $\mathrm{Cl}^{\dagger}$ & $\%$ & $\mathrm{Cl}$ & $\%$ & $\mathrm{Cl}$ & $\%$ & $\mathrm{Cl}$ & $\%$ & $\mathrm{Cl}$ & $\%$ & $\mathrm{Cl}$ \\
\hline New York City, NY & 20.5 & $(18.3-22.9)$ & 23.3 & $(21.1-25.7)$ & 22.0 & $(20.3-23.8)$ & - & - & - & - & - & - \\
\hline Oakland, CA & - & - & - & - & - & - & - & - & - & - & - & - \\
\hline Orange County, FL & 19.6 & $(16.7-22.9)$ & 22.9 & $(19.6-26.7)$ & 21.4 & $(18.9-24.0)$ & 2.8 & $(1.7-4.5)$ & 6.2 & $(4.4-8.6)$ & 4.5 & $(3.4-5.9)$ \\
\hline Palm Beach County, FL & 28.6 & $(25.0-32.5)$ & 31.4 & $(28.2-34.8)$ & 30.4 & $(27.6-33.3)$ & 4.9 & $(3.6-6.6)$ & 7.0 & $(5.5-9.0)$ & 6.4 & $(5.1-7.9)$ \\
\hline Philadelphia, PA & 33.4 & (29.2-37.9) & 32.7 & (28.2-37.4) & 33.1 & $(30.6-35.6)$ & 6.0 & $(4.5-7.8)$ & 7.8 & $(5.9-10.4)$ & 7.0 & $(5.8-8.5)$ \\
\hline San Diego, CA & 24.8 & $(21.4-28.6)$ & 27.7 & $(24.5-31.1)$ & 26.4 & $(23.8-29.1)$ & 3.7 & $(2.4-5.5)$ & 7.4 & $(6.1-9.0)$ & 5.7 & $(4.7-6.8)$ \\
\hline San Francisco, CA & 22.5 & $(19.4-25.9)$ & 26.0 & $(21.6-31.1)$ & 24.4 & $(21.3-27.7)$ & 5.1 & $(3.3-7.8)$ & 4.9 & $(3.5-7.0)$ & 5.4 & $(4.1-7.0)$ \\
\hline Median & & 24.8 & & 26.7 & & 26.4 & & .5 & & 7.6 & & 5.8 \\
\hline Range & & 7.8-33.4) & & $2.9-36.7)$ & & (1.4-34.4) & & $-13.1)$ & & 9-14.1) & & $-13.7)$ \\
\hline
\end{tabular}

* Even one or two puffs.

† $95 \%$ confidence interval.

$\S$ Not available.

TABLE 31. Percentage of high school students who currently smoked cigarettes* and who currently frequently smoked cigarettes, ${ }^{\dagger}$ by sex, $^{*}$ race/ethnicity, and grade — United States, Youth Risk Behavior Survey, 2015

\begin{tabular}{|c|c|c|c|c|c|c|c|c|c|c|c|c|}
\hline \multirow[b]{3}{*}{ Category } & \multicolumn{6}{|c|}{ Current cigarette use } & \multicolumn{6}{|c|}{ Current frequent cigarette use } \\
\hline & \multicolumn{2}{|c|}{ Female } & \multicolumn{2}{|c|}{ Male } & \multicolumn{2}{|r|}{ Total } & \multicolumn{2}{|c|}{ Female } & \multicolumn{2}{|c|}{ Male } & \multicolumn{2}{|c|}{ Total } \\
\hline & $\%$ & $\mathrm{Cl}^{\S}$ & $\%$ & $\mathrm{Cl}$ & $\%$ & $\mathrm{Cl}$ & $\%$ & $\mathrm{Cl}$ & $\%$ & $\mathrm{Cl}$ & $\%$ & $\mathrm{Cl}$ \\
\hline \multicolumn{13}{|c|}{ Race/Ethnicity } \\
\hline White & 12.2 & $(9.1-16.1)$ & 12.7 & $(10.6-15.0)$ & 12.4 & $(10.1-15.2)$ & 4.4 & $(3.0-6.3)$ & 3.6 & $(2.7-4.9)$ & 4.0 & $(2.9-5.4)$ \\
\hline Black & 3.7 & $(2.5-5.6)$ & 9.1 & $(6.4-12.7)$ & 6.5 & $(4.8-8.7)$ & 0.8 & $(0.4-1.7)$ & 2.6 & $(1.3-5.2)$ & 1.8 & $(1.0-3.0)$ \\
\hline Hispanic & 7.1 & $(5.7-8.9)$ & 11.3 & $(9.6-13.4)$ & 9.2 & $(7.9-10.7)$ & 2.1 & $(1.2-3.5)$ & 2.2 & $(1.5-3.2)$ & 2.1 & $(1.6-2.9)$ \\
\hline \multicolumn{13}{|l|}{ Grade } \\
\hline 9 & 6.7 & $(4.7-9.4)$ & 8.3 & $(6.5-10.6)$ & 7.6 & $(5.8-9.8)$ & 2.5 & $(1.3-4.9)$ & 1.9 & $(1.1-3.2)$ & 2.2 & $(1.3-3.8)$ \\
\hline 10 & 9.1 & $(6.9-12.1)$ & 8.5 & $(6.6-10.9)$ & 8.8 & $(7.0-11.1)$ & 2.7 & $(1.7-4.2)$ & 3.1 & $(2.1-4.5)$ & 2.9 & $(2.0-4.1)$ \\
\hline 11 & 10.1 & $(7.7-13.2)$ & 15.8 & (13.6-18.4) & 13.1 & $(11.3-15.0)$ & 2.9 & $(2.0-4.2)$ & 3.5 & $(2.3-5.2)$ & 3.2 & $(2.3-4.4)$ \\
\hline 12 & 13.3 & $(10.8-16.2)$ & 15.0 & $(11.5-19.2)$ & 14.1 & $(11.8-16.8)$ & 4.9 & $(3.3-7.2)$ & 5.4 & $(3.7-7.9)$ & 5.1 & $(3.8-6.8)$ \\
\hline Total & 9.7 & $(8.1-11.7)$ & 11.8 & $(10.4-13.4)$ & 10.8 & $(9.4-12.4)$ & 3.3 & (2.4-4.4) & 3.4 & $(2.6-4.6)$ & 3.4 & $(2.6-4.3)$ \\
\hline
\end{tabular}

* On at least 1 day during the 30 days before the survey.

† On 20 or more days during the 30 days before the survey.

$\S 95 \%$ confidence interval.

१ Non-Hispanic. 


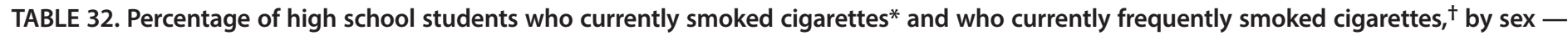
selected U.S. sites, Youth Risk Behavior Survey, 2015

\begin{tabular}{|c|c|c|c|c|c|c|c|c|c|c|c|c|}
\hline \multirow[b]{3}{*}{ Site } & \multicolumn{6}{|c|}{ Current cigarette use } & \multicolumn{6}{|c|}{ Current frequent cigarette use } \\
\hline & \multicolumn{2}{|c|}{ Female } & \multicolumn{2}{|r|}{ Male } & \multicolumn{2}{|r|}{ Total } & \multicolumn{2}{|c|}{ Female } & \multicolumn{2}{|r|}{ Male } & \multicolumn{2}{|r|}{ Total } \\
\hline & $\%$ & $\mathrm{Cl}^{\S}$ & $\%$ & $\mathrm{Cl}$ & $\%$ & $\mathrm{Cl}$ & $\%$ & $\mathrm{Cl}$ & $\%$ & $\mathrm{Cl}$ & $\%$ & $\mathrm{Cl}$ \\
\hline \multicolumn{13}{|l|}{ State surveys } \\
\hline Alabama & 14.3 & $(11.2-18.1)$ & 13.4 & $(10.7-16.6)$ & 14.0 & $(11.6-16.8)$ & 3.9 & $(2.6-5.8)$ & 4.4 & $(3.1-6.1)$ & 4.2 & $(3.1-5.5)$ \\
\hline Alaska & 8.4 & (6.0-11.7) & 13.3 & $(10.6-16.7)$ & 11.1 & (9.1-13.5) & 2.6 & $(1.2-5.7)$ & 4.6 & $(2.8-7.3)$ & 3.7 & $(2.2-6.1)$ \\
\hline Arizona & 10.2 & $(7.3-14.0)$ & 9.9 & (7.4-13.2) & 10.1 & (7.9-12.7) & 2.2 & $(1.1-4.1)$ & 2.3 & $(1.4-3.6)$ & 2.3 & $(1.4-3.7)$ \\
\hline Arkansas & 13.4 & $(10.0-17.6)$ & 18.2 & $(15.1-21.8)$ & 15.7 & $(12.4-19.7)$ & 4.4 & $(2.3-8.4)$ & 7.8 & $(5.3-11.5)$ & 6.1 & $(3.7-9.9)$ \\
\hline California & 6.8 & (4.3-10.7) & 8.4 & (5.3-13.1) & 7.7 & (5.2-11.3) & 1.5 & $(0.3-6.2)$ & 1.6 & $(0.7-3.5)$ & 1.5 & $(0.5-4.3)$ \\
\hline Connecticut & 8.2 & $(6.3-10.5)$ & 12.3 & $(10.1-14.8)$ & 10.3 & $(8.6-12.3)$ & 1.0 & $(0.4-2.4)$ & 2.4 & $(1.6-3.7)$ & 1.7 & $(1.1-2.6)$ \\
\hline Delaware & 9.2 & $(6.5-12.9)$ & 10.3 & (8.1-13.1) & 9.9 & $(7.7-12.6)$ & 2.9 & $(1.4-5.8)$ & 5.0 & $(3.4-7.2)$ & 4.0 & $(2.6-6.2)$ \\
\hline Florida & 7.8 & $(6.8-9.0)$ & 11.5 & $(9.9-13.4)$ & 9.9 & $(8.6-11.3)$ & 2.3 & $(1.8-3.0)$ & 3.8 & $(3.1-4.5)$ & 3.2 & $(2.7-3.7)$ \\
\hline Hawaii & 8.8 & $(7.4-10.4)$ & 10.2 & $(8.9-11.6)$ & 9.7 & $(8.7-10.9)$ & 1.4 & $(0.8-2.3)$ & 2.4 & $(1.8-3.3)$ & 1.9 & $(1.5-2.6)$ \\
\hline Idaho & 9.3 & $(6.9-12.5)$ & 10.1 & $(7.9-12.8)$ & 9.7 & $(8.1-11.7)$ & 2.6 & $(1.5-4.5)$ & 2.7 & $(1.5-4.8)$ & 2.7 & $(1.7-4.0)$ \\
\hline Illinois & 8.4 & $(6.8-10.5)$ & 11.7 & $(8.6-15.7)$ & 10.1 & $(8.6-11.9)$ & 1.8 & $(0.8-4.1)$ & 3.3 & $(2.0-5.4)$ & 2.6 & $(1.6-4.0)$ \\
\hline Indiana & 10.4 & (7.9-13.5) & 11.7 & (7.8-17.1) & 11.2 & $(8.3-14.8)$ & 3.4 & $(2.1-5.4)$ & 3.3 & $(2.1-5.2)$ & 3.4 & $(2.3-5.0)$ \\
\hline Kentucky & 18.0 & $(14.0-22.8)$ & 15.7 & $(13.2-18.6)$ & 16.9 & $(14.2-19.9)$ & 6.8 & $(4.8-9.4)$ & 4.5 & $(2.9-7.0)$ & 5.7 & $(4.1-7.8)$ \\
\hline Maine & 10.5 & (9.3-11.8) & 11.7 & $(10.2-13.5)$ & 11.2 & $(10.1-12.4)$ & 3.8 & $(3.3-4.5)$ & 5.3 & $(4.3-6.4)$ & 4.6 & $(4.0-5.3)$ \\
\hline Maryland & 7.7 & $(7.1-8.2)$ & 9.3 & (8.8-9.8) & 8.7 & $(8.2-9.1)$ & 1.9 & $(1.7-2.2)$ & 2.8 & $(2.5-3.1)$ & 2.4 & $(2.2-2.6)$ \\
\hline Massachusetts & 6.7 & $(5.2-8.6)$ & 8.6 & $(6.5-11.3)$ & 7.7 & $(6.1-9.7)$ & 1.8 & $(1.1-2.9)$ & 2.8 & $(1.8-4.3)$ & 2.3 & $(1.5-3.5)$ \\
\hline Michigan & 7.5 & $(5.5-10.2)$ & 12.5 & $(9.5-16.2)$ & 10.0 & $(7.8-12.6)$ & 2.0 & $(1.2-3.3)$ & 4.1 & $(2.4-7.0)$ & 3.0 & $(1.9-4.7)$ \\
\hline Mississippi & 12.9 & $(9.1-17.9)$ & 17.5 & $(14.1-21.6)$ & 15.2 & $(12.5-18.4)$ & 4.3 & $(2.5-7.3)$ & 5.4 & $(3.5-8.2)$ & 4.8 & $(3.4-6.9)$ \\
\hline Missouri & 8.1 & $(5.3-12.2)$ & 13.7 & $(10.4-17.8)$ & 11.0 & $(8.3-14.3)$ & 2.8 & $(1.5-5.3)$ & 5.7 & $(3.6-9.0)$ & 4.2 & $(2.7-6.5)$ \\
\hline Montana & 13.2 & $(11.1-15.6)$ & 13.0 & $(10.9-15.3)$ & 13.1 & $(11.3-15.1)$ & 3.4 & $(2.5-4.6)$ & 3.9 & $(2.9-5.2)$ & 3.6 & $(2.9-4.6)$ \\
\hline Nebraska & 12.2 & $(9.3-15.9)$ & 14.2 & $(10.8-18.4)$ & 13.3 & $(10.5-16.7)$ & 3.6 & $(2.4-5.6)$ & 3.6 & $(2.2-5.8)$ & 3.7 & $(2.5-5.4)$ \\
\hline Nevada & 7.6 & $(5.1-11.1)$ & 7.3 & $(5.2-10.0)$ & 7.5 & $(5.8-9.6)$ & 1.5 & $(0.6-3.6)$ & 2.5 & $(1.5-4.3)$ & 2.0 & $(1.2-3.5)$ \\
\hline New Hampshire & 8.0 & (7.2-8.9) & 10.2 & $(9.3-11.1)$ & 9.3 & $(8.6-10.0)$ & 2.8 & $(2.3-3.4)$ & 4.4 & $(3.9-5.0)$ & 3.7 & $(3.3-4.2)$ \\
\hline New Mexico & 9.8 & $(8.5-11.2)$ & 12.8 & $(11.5-14.3)$ & 11.4 & $(10.3-12.5)$ & 2.0 & $(1.5-2.6)$ & 3.4 & $(2.8-4.2)$ & 2.7 & $(2.3-3.2)$ \\
\hline New York & 7.2 & $(6.2-8.5)$ & 10.0 & (7.3-13.5) & 8.8 & (7.1-10.9) & 2.3 & $(1.5-3.6)$ & 3.5 & $(2.2-5.6)$ & 2.9 & $(2.1-4.0)$ \\
\hline North Carolina & 10.9 & $(8.9-13.4)$ & 14.9 & $(12.3-17.9)$ & 13.1 & $(11.7-14.6)$ & 2.9 & $(1.9-4.5)$ & 4.5 & $(3.2-6.3)$ & 3.8 & $(2.9-5.0)$ \\
\hline North Dakota & 11.5 & $(8.8-14.8)$ & 11.9 & (9.7-14.5) & 11.7 & (9.8-13.9) & 4.5 & $(3.2-6.4)$ & 4.1 & $(2.9-5.9)$ & 4.3 & $(3.3-5.7)$ \\
\hline Oklahoma & 11.3 & $(7.7-16.2)$ & 15.1 & $(11.9-19.0)$ & 13.1 & $(10.4-16.4)$ & 2.5 & $(1.4-4.3)$ & 4.1 & $(2.5-6.5)$ & 3.2 & $(2.1-4.9)$ \\
\hline Pennsylvania & 10.9 & $(8.2-14.4)$ & 14.9 & $(11.8-18.5)$ & 12.9 & $(10.4-16.0)$ & 3.7 & $(2.5-5.5)$ & 5.7 & $(4.0-8.1)$ & 4.7 & $(3.6-6.1)$ \\
\hline Rhode Island & 4.5 & $(3.2-6.5)$ & 5.0 & $(4.0-6.1)$ & 4.8 & $(3.9-5.9)$ & 1.6 & $(1.0-2.6)$ & 1.4 & $(0.9-2.1)$ & 1.5 & $(1.1-2.2)$ \\
\hline South Carolina & 9.6 & $(7.8-11.7)$ & 9.4 & $(6.6-13.1)$ & 9.6 & $(7.6-12.0)$ & 2.6 & $(1.5-4.2)$ & 2.8 & $(1.7-4.7)$ & 2.8 & $(1.9-4.1)$ \\
\hline South Dakota & 9.5 & $(5.7-15.4)$ & 10.5 & (7.4-14.6) & 10.1 & $(6.8-14.6)$ & 5.0 & $(2.6-9.2)$ & 4.2 & $(2.7-6.5)$ & 4.7 & $(2.9-7.5)$ \\
\hline Tennessee & 10.1 & $(8.3-12.2)$ & 12.5 & $(10.7-14.5)$ & 11.5 & $(10.0-13.1)$ & 3.6 & $(2.5-5.2)$ & 4.1 & $(3.1-5.5)$ & 3.9 & $(3.2-4.8)$ \\
\hline Vermont & 9.7 & $(9.1-10.3)$ & 11.7 & $(11.1-12.4)$ & 10.8 & $(10.4-11.2)$ & 3.2 & $(2.9-3.6)$ & 4.6 & $(4.2-5.0)$ & 3.9 & $(3.7-4.2)$ \\
\hline Virginia & 7.9 & $(6.2-10.0)$ & 8.6 & (6.9-10.6) & 8.2 & (6.8-9.9) & 1.7 & $(1.0-2.7)$ & 2.8 & $(1.7-4.4)$ & 2.2 & $(1.6-3.2)$ \\
\hline West Virginia & 18.4 & $(14.5-23.0)$ & 19.2 & $(15.5-23.4)$ & 18.8 & $(15.5-22.5)$ & 7.6 & $(5.8-9.8)$ & 7.3 & $(5.5-9.6)$ & 7.4 & $(6.1-9.0)$ \\
\hline Wyoming & 15.9 & $(12.8-19.7)$ & 15.4 & $(12.2-19.2)$ & 15.7 & $(13.1-18.6)$ & 5.4 & $(3.7-7.9)$ & 4.5 & $(3.2-6.3)$ & 5.0 & $(3.7-6.6)$ \\
\hline Median & & 9.6 & & 11.7 & & 10.8 & & 2.8 & & 4.1 & & 3.6 \\
\hline Range & & $.5-18.4)$ & & $5.0-19.2)$ & & $4.8-18.8)$ & & $0-7.6)$ & & $(1.4-7.8)$ & & $1.5-7.4)$ \\
\hline \multicolumn{13}{|c|}{ Large urban school district surveys } \\
\hline Baltimore, MD & 4.7 & $(2.8-7.8)$ & 7.0 & $(4.1-11.8)$ & 5.9 & $(4.0-8.8)$ & 0.5 & $(0.1-2.0)$ & 1.9 & $(0.9-4.1)$ & 1.3 & $(0.7-2.4)$ \\
\hline Boston, MA & 3.4 & $(2.4-4.8)$ & 6.0 & $(4.1-8.7)$ & 4.8 & $(3.6-6.3)$ & 0.6 & $(0.3-1.3)$ & 1.6 & $(0.8-3.1)$ & 1.1 & $(0.7-1.9)$ \\
\hline Broward County, FL & 3.3 & $(1.9-5.6)$ & 5.0 & (3.2-7.7) & 4.2 & $(2.9-5.9)$ & 0.5 & $(0.1-1.7)$ & 0.6 & $(0.2-2.0)$ & 0.6 & $(0.3-1.0)$ \\
\hline Cleveland, $\mathrm{OH}$ & 5.7 & $(4.0-8.1)$ & 9.7 & $(7.5-12.5)$ & 8.5 & $(6.8-10.6)$ & 1.4 & $(0.8-2.5)$ & 2.2 & $(1.4-3.4)$ & 1.9 & $(1.3-2.8)$ \\
\hline DeKalb County, GA & 4.5 & $(3.3-6.2)$ & 5.4 & $(3.6-7.9)$ & 5.0 & $(3.9-6.4)$ & 0.4 & $(0.2-1.0)$ & 1.9 & $(1.0-3.5)$ & 1.1 & $(0.6-2.0)$ \\
\hline Detroit, MI & 8.7 & $(5.3-13.9)$ & 8.5 & $(5.5-12.9)$ & 8.6 & $(5.6-12.9)$ & 0.3 & $(0.1-1.5)$ & 1.4 & $(0.7-2.8)$ & 0.8 & $(0.4-1.5)$ \\
\hline District of Columbia & —** & - & - & - & - & - & - & - & - & - & - & - \\
\hline Duval County, FL & 6.2 & $(4.9-7.9)$ & 7.3 & $(6.0-9.0)$ & 7.1 & $(6.0-8.4)$ & 1.3 & $(0.8-2.2)$ & 1.6 & $(0.9-2.7)$ & 1.6 & $(1.1-2.3)$ \\
\hline Ft. Worth, TX & 7.1 & $(5.5-9.1)$ & 10.9 & $(8.7-13.7)$ & 9.0 & $(7.5-10.6)$ & 1.2 & $(0.7-2.1)$ & 2.2 & $(1.4-3.4)$ & 1.7 & $(1.2-2.4)$ \\
\hline Houston, TX & 8.0 & $(6.3-10.0)$ & 8.9 & $(7.2-10.9)$ & 8.6 & $(7.2-10.2)$ & 1.2 & $(0.7-2.3)$ & 2.1 & $(1.5-3.1)$ & 1.8 & $(1.2-2.5)$ \\
\hline Los Angeles, CA & 3.0 & $(1.6-5.5)$ & 6.0 & $(4.1-8.9)$ & 4.5 & $(3.2-6.2)$ & 0.7 & $(0.3-1.9)$ & 1.3 & $(0.8-1.8)$ & 1.0 & $(0.6-1.7)$ \\
\hline Miami-Dade County, FL & 4.0 & $(2.8-5.6)$ & 6.8 & $(5.2-8.8)$ & 5.4 & $(4.3-6.6)$ & 0.3 & $(0.1-0.8)$ & 1.5 & $(0.8-2.6)$ & 0.9 & $(0.6-1.5)$ \\
\hline
\end{tabular}

See table footnotes on the next page. 
TABLE 32. (Continued) Percentage of high school students who currently smoked cigarettes* and who currently frequently smoked cigarettes, ${ }^{\dagger}$ by sex — selected U.S. sites, Youth Risk Behavior Survey, 2015

\begin{tabular}{|c|c|c|c|c|c|c|c|c|c|c|c|c|}
\hline \multirow[b]{3}{*}{ Site } & \multicolumn{6}{|c|}{ Current cigarette use } & \multicolumn{6}{|c|}{ Current frequent cigarette use } \\
\hline & \multicolumn{2}{|c|}{ Female } & \multicolumn{2}{|c|}{ Male } & \multicolumn{2}{|c|}{ Total } & \multicolumn{2}{|c|}{ Female } & \multicolumn{2}{|r|}{ Male } & \multicolumn{2}{|r|}{ Total } \\
\hline & $\%$ & $\mathrm{Cl}^{\S}$ & $\%$ & $\mathrm{Cl}$ & $\%$ & $\mathrm{Cl}$ & $\%$ & $\mathrm{Cl}$ & $\%$ & $\mathrm{Cl}$ & $\%$ & $\mathrm{Cl}$ \\
\hline New York City, NY & 4.7 & $(3.9-5.7)$ & 6.6 & $(5.1-8.4)$ & 5.8 & $(4.7-7.0)$ & 1.0 & $(0.7-1.5)$ & 1.8 & $(1.3-2.5)$ & 1.4 & $(1.1-1.8)$ \\
\hline Oakland, CA & 4.9 & $(3.3-7.2)$ & 6.2 & $(4.5-8.5)$ & 5.8 & $(4.4-7.5)$ & 1.4 & $(0.7-2.9)$ & 1.5 & $(0.8-2.5)$ & 1.4 & $(0.9-2.4)$ \\
\hline Orange County, FL & 5.2 & $(3.6-7.3)$ & 6.2 & $(4.3-9.0)$ & 5.8 & $(4.4-7.6)$ & 0.7 & $(0.2-2.4)$ & 3.0 & $(1.7-5.3)$ & 1.9 & $(1.1-3.2)$ \\
\hline Palm Beach County, FL & 6.5 & $(5.1-8.4)$ & 7.8 & $(6.3-9.7)$ & 7.6 & $(6.3-9.2)$ & 1.5 & $(0.9-2.6)$ & 2.1 & $(1.3-3.2)$ & 1.8 & $(1.3-2.6)$ \\
\hline Philadelphia, PA & 6.8 & $(4.8-9.6)$ & 7.0 & $(5.1-9.8)$ & 7.2 & $(5.6-9.1)$ & 2.2 & $(1.2-4.0)$ & 1.8 & $(1.0-3.2)$ & 2.0 & $(1.3-2.9)$ \\
\hline San Diego, CA & 6.0 & $(4.4-8.0)$ & 8.3 & $(6.8-10.1)$ & 7.1 & $(6.0-8.4)$ & 0.5 & $(0.2-1.1)$ & 2.2 & $(1.3-3.8)$ & 1.3 & $(0.8-2.2)$ \\
\hline San Francisco, CA & 5.0 & (3.3-7.4) & 5.6 & $(3.7-8.3)$ & 5.4 & $(4.0-7.2)$ & 0.8 & $(0.4-1.7)$ & 1.3 & $(0.6-2.9)$ & 1.1 & $(0.6-2.1)$ \\
\hline Median & \multirow{2}{*}{\multicolumn{2}{|c|}{$\begin{array}{l}5.1 \\
(30-87)\end{array}$}} & \multirow{2}{*}{\multicolumn{2}{|c|}{$\begin{array}{c}6.9 \\
(5.0-10.9)\end{array}$}} & \multirow{2}{*}{\multicolumn{2}{|c|}{$\begin{array}{c}5.8 \\
(4.2-9.0)\end{array}$}} & \multirow{2}{*}{\multicolumn{2}{|c|}{$\begin{array}{c}0.7 \\
(0.3-2.2)\end{array}$}} & \multirow{2}{*}{\multicolumn{2}{|c|}{$\begin{array}{c}1.8 \\
(0.6-3.0)\end{array}$}} & \multirow{2}{*}{\multicolumn{2}{|c|}{$\begin{array}{c}1.3 \\
(0.6-2.0)\end{array}$}} \\
\hline Range & & & & & & & & & & & & \\
\hline
\end{tabular}

* On at least 1 day during the 30 days before the survey.

† On 20 or more days during the 30 days before the survey.

$\S 95 \%$ confidence interval.

** Not available.

TABLE 33. Percentage of high school students who smoked more than 10 cigarettes/day* and who currently smoked cigarettes daily, ${ }^{\dagger}$ by sex, race/ethnicity, and grade - United States, Youth Risk Behavior Survey, 2015

\begin{tabular}{|c|c|c|c|c|c|c|c|c|c|c|c|c|}
\hline \multirow[b]{3}{*}{ Category } & \multicolumn{6}{|c|}{ Smoked more than 10 cigarettes/day } & \multicolumn{6}{|c|}{ Currently smoked cigarettes daily } \\
\hline & \multicolumn{2}{|c|}{ Female } & \multicolumn{2}{|c|}{ Male } & \multicolumn{2}{|r|}{ Total } & \multicolumn{2}{|c|}{ Female } & \multicolumn{2}{|c|}{ Male } & \multicolumn{2}{|c|}{ Total } \\
\hline & $\%$ & $\mathrm{Cl}^{\S}$ & $\%$ & $\mathrm{Cl}$ & $\%$ & $\mathrm{Cl}$ & $\%$ & $\mathrm{Cl}$ & $\%$ & $\mathrm{Cl}$ & $\%$ & $\mathrm{Cl}$ \\
\hline \multicolumn{13}{|c|}{ Race/Ethnicity } \\
\hline Whitef & 5.7 & $(2.8-11.0)$ & 5.6 & $(3.5-8.9)$ & 5.7 & (4.3-7.6) & 3.1 & $(2.1-4.6)$ & 2.4 & $(1.6-3.6)$ & 2.8 & $(1.9-4.0)$ \\
\hline Black" & 一** & - & - & - & 7.5 & (3.4-15.7) & 0.4 & $(0.1-1.3)$ & 2.4 & $(1.2-4.8)$ & 1.4 & $(0.8-2.5)$ \\
\hline Hispanic & 9.7 & $(4.0-21.8)$ & 9.0 & $(5.6-14.2)$ & 9.3 & $(5.4-15.7)$ & 1.4 & $(0.8-2.4)$ & 1.8 & $(1.2-2.6)$ & 1.6 & $(1.1-2.2)$ \\
\hline \multicolumn{13}{|l|}{ Grade } \\
\hline 9 & 8.4 & $(3.0-21.6)$ & 5.1 & $(2.2-11.4)$ & 6.8 & $(3.3-13.3)$ & 1.9 & $(0.9-3.7)$ & 1.6 & $(0.8-3.0)$ & 1.7 & $(1.0-3.1)$ \\
\hline 10 & 6.6 & $(2.7-15.1)$ & 14.7 & $(8.8-23.5)$ & 10.4 & $(7.0-15.3)$ & 1.4 & $(0.8-2.4)$ & 2.7 & $(1.8-4.0)$ & 2.1 & $(1.4-3.1)$ \\
\hline 11 & 2.7 & $(0.7-9.5)$ & 3.6 & $(1.9-6.9)$ & 3.4 & $(1.8-6.5)$ & 1.8 & $(1.3-2.7)$ & 1.8 & $(1.1-3.0)$ & 1.9 & $(1.3-2.7)$ \\
\hline 12 & 5.4 & $(2.1-13.6)$ & 12.5 & $(7.7-19.6)$ & 9.1 & $(5.6-14.5)$ & 3.5 & $(2.2-5.6)$ & 3.2 & $(2.2-4.7)$ & 3.4 & $(2.4-4.7)$ \\
\hline Total & 5.9 & $(3.7-9.4)$ & 9.2 & $(6.8-12.4)$ & 7.9 & $(6.2-9.9)$ & 2.2 & $(1.6-2.9)$ & 2.4 & $(1.8-3.2)$ & 2.3 & $(1.7-3.0)$ \\
\hline
\end{tabular}

* On the days they smoked during the 30 days before the survey, among the $10.8 \%$ of students nationwide who currently smoked cigarettes.

$\dagger$ On all 30 days during the 30 days before the survey.

$\S 95 \%$ confidence interval.

I Non-Hispanic.

** Not available. 
TABLE 34. Percentage of high school students who smoked more than 10 cigarettes/day* and who currently smoked cigarettes daily, ${ }^{\dagger}$ by sex selected U.S. sites, Youth Risk Behavior Survey, 2015

\begin{tabular}{|c|c|c|c|c|c|c|c|c|c|c|c|c|}
\hline \multirow[b]{3}{*}{ Site } & \multicolumn{6}{|c|}{ Smoked more than 10 cigarettes/day } & \multicolumn{6}{|c|}{ Currently smoked cigarettes daily } \\
\hline & \multicolumn{2}{|c|}{ Female } & \multicolumn{2}{|c|}{ Male } & \multicolumn{2}{|r|}{ Total } & \multicolumn{2}{|c|}{ Female } & \multicolumn{2}{|r|}{ Male } & \multicolumn{2}{|r|}{ Total } \\
\hline & $\%$ & $\mathrm{Cl}^{\S}$ & $\%$ & $\mathrm{Cl}$ & $\%$ & $\mathrm{Cl}$ & $\%$ & $\mathrm{Cl}$ & $\%$ & $\mathrm{Cl}$ & $\%$ & $\mathrm{Cl}$ \\
\hline \multicolumn{13}{|l|}{ State surveys } \\
\hline Alabama & - & - & - & - & 7.1 & $(3.6-13.5)$ & 2.8 & $(1.8-4.4)$ & 3.8 & $(2.5-5.6)$ & 3.3 & $(2.4-4.6)$ \\
\hline Alaska & - & - & - & - & 4.7 & $(2.0-10.5)$ & 1.4 & $(0.5-3.8)$ & 3.5 & $(2.1-5.7)$ & 2.6 & $(1.5-4.3)$ \\
\hline Arizona & 5.6 & $(1.6-17.4)$ & 6.1 & $(1.8-18.8)$ & 5.8 & (3.9-8.7) & 1.4 & $(0.7-2.6)$ & 1.5 & $(1.0-2.4)$ & 1.5 & $(1.0-2.3)$ \\
\hline Arkansas & 1.9 & $(0.8-4.9)$ & 10.6 & $(6.4-16.9)$ & 6.8 & $(4.4-10.3)$ & 4.0 & $(1.9-8.1)$ & 5.7 & $(2.7-11.6)$ & 4.8 & $(2.4-9.7)$ \\
\hline California & - & - & - & - & 4.6 & $(2.0-10.3)$ & 1.0 & $(0.2-5.6)$ & 1.0 & $(0.4-2.5)$ & 1.0 & $(0.3-3.2)$ \\
\hline Connecticut & - & - & - & - & - & - & 0.8 & $(0.3-2.0)$ & 1.7 & $(1.1-2.7)$ & 1.2 & $(0.8-2.0)$ \\
\hline Delaware & 8.6 & $(3.8-18.6)$ & 12.3 & $(7.3-20.0)$ & 11.9 & $(8.6-16.3)$ & 2.4 & $(1.1-5.5)$ & 3.2 & $(2.1-4.9)$ & 2.9 & $(1.8-4.7)$ \\
\hline Florida & - & - & - & - & - & - & 2.0 & $(1.5-2.6)$ & 3.0 & $(2.5-3.7)$ & 2.6 & $(2.2-3.1)$ \\
\hline Hawaii & - & - & - & - & - & - & 1.0 & $(0.6-1.8)$ & 1.5 & $(0.9-2.3)$ & 1.2 & $(0.8-1.8)$ \\
\hline Idaho & - & - & - & - & 2.0 & $(0.6-6.0)$ & 1.9 & $(0.9-3.8)$ & 2.1 & $(1.2-4.0)$ & 2.0 & $(1.2-3.2)$ \\
\hline Illinois & 3.5 & $(1.3-8.7)$ & 11.4 & $(6.8-18.4)$ & 8.6 & $(5.9-12.3)$ & 1.1 & $(0.5-2.4)$ & 2.9 & $(1.7-5.0)$ & 2.0 & $(1.3-3.2)$ \\
\hline Indiana & - & - & - & - & 8.7 & $(4.4-16.7)$ & 2.8 & $(1.7-4.7)$ & 2.6 & $(1.3-4.9)$ & 2.8 & $(1.7-4.5)$ \\
\hline Kentucky & 5.2 & $(2.3-11.4)$ & 8.6 & $(5.5-13.1)$ & 6.8 & $(4.6-9.9)$ & 5.5 & $(3.7-8.1)$ & 3.6 & $(2.2-5.9)$ & 4.6 & $(3.2-6.7)$ \\
\hline Maine & 8.8 & $(6.1-12.4)$ & 18.9 & $(14.6-24.0)$ & 14.6 & $(12.2-17.3)$ & 2.8 & $(2.4-3.3)$ & 4.2 & $(3.3-5.2)$ & 3.6 & $(3.1-4.1)$ \\
\hline Maryland & 7.4 & $(6.0-9.1)$ & 14.4 & $(12.8-16.2)$ & 11.3 & $(10.3-12.5)$ & 1.4 & $(1.2-1.5)$ & 2.1 & $(1.9-2.4)$ & 1.8 & $(1.6-1.9)$ \\
\hline Massachusetts & - & - & - & - & - & - & 1.2 & $(0.6-2.3)$ & 2.1 & $(1.3-3.3)$ & 1.7 & $(1.0-2.7)$ \\
\hline Michigan & 7.3 & $(3.0-17.0)$ & 9.8 & $(4.1-21.7)$ & 8.8 & $(5.0-15.2)$ & 1.6 & $(0.9-3.1)$ & 2.4 & $(1.2-4.6)$ & 2.0 & $(1.2-3.3)$ \\
\hline Mississippi & 3.3 & $(1.2-8.7)$ & 11.3 & $(6.4-19.2)$ & 7.7 & $(4.5-12.9)$ & 3.3 & $(1.7-6.3)$ & 3.9 & $(2.4-6.2)$ & 3.6 & $(2.3-5.6)$ \\
\hline Missouri & - & - & - & - & - & - & 1.8 & $(0.9-3.8)$ & 4.4 & $(2.5-7.6)$ & 3.1 & $(1.9-5.1)$ \\
\hline Montana & 2.2 & $(1.0-4.7)$ & 8.7 & $(5.8-12.7)$ & 5.5 & $(3.9-7.5)$ & 1.9 & $(1.3-2.9)$ & 2.5 & $(1.8-3.4)$ & 2.2 & $(1.7-2.9)$ \\
\hline Nebraska & - & - & 8.5 & $(4.0-17.2)$ & 8.0 & $(4.6-13.4)$ & 2.1 & $(1.2-3.6)$ & 2.9 & $(1.7-5.0)$ & 2.6 & $(1.6-4.0)$ \\
\hline Nevada & - & - & - & - & 7.1 & $(3.2-15.0)$ & 1.2 & $(0.4-3.5)$ & 2.2 & $(1.2-4.0)$ & 1.7 & $(0.9-3.1)$ \\
\hline New Hampshire & 9.1 & $(5.7-14.3)$ & 17.3 & (14.4-20.5) & 14.2 & $(11.9-17.0)$ & 2.0 & $(1.6-2.5)$ & 3.4 & $(2.9-3.9)$ & 2.8 & $(2.4-3.2)$ \\
\hline New Mexico & 1.9 & $(1.1-3.3)$ & 10.0 & $(7.3-13.6)$ & 6.5 & $(4.8-8.7)$ & 1.5 & $(1.1-2.0)$ & 2.8 & $(2.3-3.4)$ & 2.1 & $(1.8-2.6)$ \\
\hline New York & 7.2 & $(2.6-18.3)$ & 15.2 & $(8.8-25.0)$ & 11.6 & $(7.6-17.5)$ & 1.2 & $(0.8-2.0)$ & 2.4 & $(1.3-4.3)$ & 1.8 & $(1.2-2.7)$ \\
\hline North Carolina & - & - & - & - & - & - & 2.1 & $(1.3-3.3)$ & 3.8 & $(2.6-5.5)$ & 3.1 & $(2.3-4.1)$ \\
\hline North Dakota & - & - & - & - & - & - & 3.0 & $(1.8-4.8)$ & 3.4 & $(2.2-5.1)$ & 3.2 & $(2.3-4.5)$ \\
\hline Oklahoma & - & - & 3.5 & $(1.2-10.3)$ & 2.4 & $(1.0-6.1)$ & 1.5 & $(0.7-3.1)$ & 3.3 & $(1.9-5.6)$ & 2.4 & (1.4-3.9) \\
\hline Pennsylvania & 6.2 & $(3.1-12.0)$ & 9.3 & $(4.7-17.5)$ & 8.0 & $(4.9-13.0)$ & 2.3 & $(1.3-4.1)$ & 3.7 & $(2.5-5.5)$ & 3.0 & $(2.2-4.2)$ \\
\hline Rhode Island & - & - & - & - & 11.7 & $(5.8-22.0)$ & 1.0 & $(0.5-1.9)$ & 1.1 & $(0.6-2.2)$ & 1.1 & $(0.7-1.7)$ \\
\hline South Carolina & - & - & - & - & 8.5 & $(4.9-14.4)$ & 2.1 & $(1.2-3.6)$ & 2.0 & $(1.0-3.7)$ & 2.0 & $(1.3-3.0)$ \\
\hline South Dakota & - & - & - & - & 11.5 & $(5.9-21.1)$ & 3.7 & $(1.9-7.3)$ & 3.0 & $(1.9-4.7)$ & 3.3 & $(2.0-5.6)$ \\
\hline Tennessee & 4.1 & $(2.2-7.7)$ & 15.2 & $(9.8-22.8)$ & 10.8 & $(7.6-15.1)$ & 2.3 & $(1.5-3.6)$ & 3.2 & $(2.2-4.5)$ & 2.8 & $(2.1-3.7)$ \\
\hline Vermont & 9.2 & $(7.5-11.3)$ & 13.0 & $(11.2-15.1)$ & 11.7 & $(10.4-13.2)$ & 2.4 & $(2.1-2.8)$ & 3.5 & $(3.1-3.8)$ & 3.0 & $(2.7-3.2)$ \\
\hline Virginia & 6.1 & $(3.2-11.4)$ & 4.9 & $(2.4-9.9)$ & 5.5 & $(3.4-8.8)$ & 1.4 & $(0.9-2.4)$ & 1.8 & $(1.1-3.0)$ & 1.6 & $(1.1-2.3)$ \\
\hline West Virginia & 8.8 & $(5.4-14.1)$ & 11.3 & $(7.0-17.8)$ & 10.1 & $(7.2-14.0)$ & 6.1 & $(4.6-7.9)$ & 4.7 & $(3.3-6.7)$ & 5.4 & $(4.4-6.6)$ \\
\hline Wyoming & 9.0 & $(4.7-16.5)$ & 12.7 & $(7.5-20.5)$ & 10.9 & $(6.9-16.8)$ & 4.3 & $(2.8-6.6)$ & 3.2 & $(2.1-5.1)$ & 3.8 & $(2.6-5.4)$ \\
\hline Median & & 6.2 & & 11.3 & & 8.3 & & 2.0 & & 3.0 & & 2.6 \\
\hline Range & & 1.9-9.2) & & 5-18.9) & & $2.0-14.6)$ & & 8-6.1) & & 1.0-5.7) & & $.0-5.4)$ \\
\hline \multicolumn{13}{|c|}{ Large urban school district surveys } \\
\hline Baltimore, MD & - & - & - & - & - & - & 0.5 & $(0.1-2.0)$ & 1.1 & $(0.4-3.2)$ & 0.9 & $(0.4-1.9)$ \\
\hline Boston, MA & - & - & - & - & - & - & 0.4 & $(0.2-0.9)$ & 1.1 & $(0.5-2.3)$ & 0.8 & $(0.4-1.4)$ \\
\hline Broward County, FL & - & - & - & - & 一 & - & 0.2 & $(0.0-1.1)$ & 0.5 & $(0.1-2.0)$ & 0.3 & $(0.1-1.1)$ \\
\hline Cleveland, $\mathrm{OH}$ & - & - & - & - & 一 & - & 1.3 & $(0.7-2.5)$ & 1.6 & $(0.9-2.8)$ & 1.6 & $(1.0-2.4)$ \\
\hline DeKalb County, GA & - & - & - & - & - & - & 0.4 & $(0.2-1.0)$ & 1.2 & $(0.5-2.5)$ & 0.8 & $(0.4-1.5)$ \\
\hline Detroit, MI & - & - & - & - & 0.9 & $(0.1-6.5)$ & 0.2 & $(0.0-0.7)$ & 0.6 & $(0.2-1.4)$ & 0.4 & $(0.2-0.8)$ \\
\hline District of Columbia & - & - & - & - & - & - & - & - & - & - & - & - \\
\hline Duval County, FL & 7.7 & $(3.2-17.4)$ & - & - & 9.8 & $(6.3-14.7)$ & 0.9 & $(0.5-1.6)$ & 1.4 & $(0.8-2.5)$ & 1.1 & $(0.7-1.8)$ \\
\hline Ft. Worth, TX & - & - & 3.6 & $(1.3-9.4)$ & 3.1 & $(1.4-6.7)$ & 0.6 & $(0.3-1.2)$ & 1.8 & $(1.1-3.0)$ & 1.2 & $(0.8-1.8)$ \\
\hline Houston, TX & 9.0 & $(4.4-17.4)$ & 6.5 & $(3.1-13.0)$ & 8.3 & $(4.8-14.1)$ & 0.7 & $(0.3-1.6)$ & 1.2 & $(0.7-1.9)$ & 1.0 & $(0.6-1.7)$ \\
\hline Los Angeles, CA & - & - & - & - & 12.8 & $(6.5-23.5)$ & 0.6 & $(0.2-1.6)$ & 1.0 & $(0.6-1.5)$ & 0.8 & $(0.4-1.4)$ \\
\hline Miami-Dade County, FL & - & - & - & - & 11.2 & $(5.2-22.5)$ & 0.3 & $(0.1-0.8)$ & 1.2 & $(0.6-2.3)$ & 0.8 & $(0.4-1.4)$ \\
\hline
\end{tabular}

See table footnotes on the next page. 
TABLE 34. (Continued) Percentage of high school students who smoked more than 10 cigarettes/day* and who currently smoked cigarettes daily, ${ }^{\dagger}$ by sex — selected U.S. sites, Youth Risk Behavior Survey, 2015

\begin{tabular}{|c|c|c|c|c|c|c|c|c|c|c|c|c|}
\hline \multirow[b]{3}{*}{ Site } & \multicolumn{6}{|c|}{ Smoked more than 10 cigarettes/day } & \multicolumn{6}{|c|}{ Currently smoked cigarettes daily } \\
\hline & \multicolumn{2}{|r|}{ Female } & \multicolumn{2}{|c|}{ Male } & \multicolumn{2}{|c|}{ Total } & \multicolumn{2}{|c|}{ Female } & \multicolumn{2}{|r|}{ Male } & \multicolumn{2}{|r|}{ Total } \\
\hline & $\%$ & $\mathrm{Cl}^{\S}$ & $\%$ & $\mathrm{Cl}$ & $\%$ & $\mathrm{Cl}$ & $\%$ & $\mathrm{Cl}$ & $\%$ & $\mathrm{Cl}$ & $\%$ & $\mathrm{Cl}$ \\
\hline New York City, NY & 6.9 & $(2.4-18.0)$ & 10.7 & $(6.9-16.0)$ & 8.8 & $(5.9-13.0)$ & 0.6 & $(0.4-1.1)$ & 1.2 & $(0.8-1.7)$ & 0.9 & $(0.7-1.2)$ \\
\hline Oakland, CA & - & - & - & - & 5.5 & $(2.2-13.2)$ & 1.2 & $(0.5-2.6)$ & 0.7 & $(0.3-1.7)$ & 0.9 & $(0.5-1.8)$ \\
\hline Orange County, FL & - & - & - & - & - & - & 0.7 & $(0.2-2.4)$ & 2.3 & $(1.3-4.1)$ & 1.5 & $(0.9-2.7)$ \\
\hline Palm Beach County, FL & - & - & - & - & 10.5 & $(5.8-18.5)$ & 1.2 & $(0.6-2.2)$ & 1.7 & $(1.1-2.7)$ & 1.5 & $(1.0-2.2)$ \\
\hline Philadelphia, PA & - & - & - & - & 8.2 & $(3.8-16.6)$ & 1.6 & $(0.8-3.2)$ & 1.1 & $(0.5-2.3)$ & 1.3 & $(0.8-2.1)$ \\
\hline San Diego, CA & - & - & - & - & 7.2 & $(3.5-14.3)$ & 0.3 & $(0.1-1.0)$ & 1.5 & $(0.9-2.5)$ & 0.9 & $(0.6-1.5)$ \\
\hline San Francisco, CA & - & - & - & - & 2.9 & $(1.2-6.5)$ & 0.3 & $(0.1-0.9)$ & 1.0 & $(0.5-2.1)$ & 0.7 & $(0.3-1.3)$ \\
\hline Median & \multirow{2}{*}{\multicolumn{2}{|c|}{$\begin{array}{c}7.7 \\
(6.9-9.0)\end{array}$}} & \multirow{2}{*}{\multicolumn{2}{|c|}{$\begin{array}{c}6.5 \\
(3.6-10.7)\end{array}$}} & \multicolumn{2}{|r|}{8.2} & \multicolumn{2}{|r|}{0.6} & \multicolumn{2}{|r|}{1.2} & \multicolumn{2}{|r|}{0.9} \\
\hline Range & & & & & & $9-12.8)$ & & .2-1.6) & & $(0.5-2.3)$ & & $0.3-1.6)$ \\
\hline
\end{tabular}

* On the days they smoked during the 30 days before the survey, among students who currently smoked cigarettes.

† On all 30 days during the 30 days before the survey.

$\S 95 \%$ confidence interval.

I Not available.

TABLE 35. Percentage of high school students who usually obtained their own cigarettes by buying them in a store or gas station* and who usually obtained their own cigarettes by buying them on the internet,* by sex, race/ethnicity, and grade — United States, Youth Risk Behavior Survey, 2015

\begin{tabular}{|c|c|c|c|c|c|c|c|c|c|c|c|c|}
\hline \multirow[b]{3}{*}{ Category } & \multicolumn{6}{|c|}{ Bought cigarettes in a store or gas station } & \multicolumn{6}{|c|}{ Bought cigarettes on the internet } \\
\hline & \multicolumn{2}{|c|}{ Female } & \multicolumn{2}{|r|}{ Male } & \multicolumn{2}{|r|}{ Total } & \multicolumn{2}{|c|}{ Female } & \multicolumn{2}{|c|}{ Male } & \multicolumn{2}{|c|}{ Total } \\
\hline & $\%$ & $\mathrm{Cl}^{\dagger}$ & $\%$ & $\mathrm{Cl}$ & $\%$ & $\mathrm{Cl}$ & $\%$ & $\mathrm{Cl}$ & $\%$ & $\mathrm{Cl}$ & $\%$ & $\mathrm{Cl}$ \\
\hline \multicolumn{13}{|c|}{ Race/Ethnicity } \\
\hline White ${ }^{\S}$ & 6.6 & $(3.6-11.8)$ & 12.8 & $(8.8-18.3)$ & 9.7 & $(6.9-13.6)$ & 0.1 & $(0.0-0.4)$ & 0.2 & $(0.0-1.2)$ & 0.1 & $(0.0-0.6)$ \\
\hline Black $^{\S}$ & -? & - & - & - & - & - & - & - & - & - & - & - \\
\hline Hispanic & 9.8 & $(5.4-17.1)$ & 21.9 & $(14.9-31.0)$ & 17.5 & $(12.7-23.7)$ & 0.6 & $(0.1-4.7)$ & 0.9 & $(0.3-2.6)$ & 0.8 & $(0.3-2.0)$ \\
\hline \multicolumn{13}{|l|}{ Grade } \\
\hline 9 & 6.4 & $(2.4-16.3)$ & 6.2 & $(2.8-13.2)$ & 6.3 & $(3.4-11.4)$ & 0.0 & (.-.) & 0.3 & $(0.0-2.5)$ & 0.2 & $(0.0-1.5)$ \\
\hline 10 & 5.6 & $(2.2-13.1)$ & 6.7 & $(3.3-12.9)$ & 6.1 & $(3.2-11.2)$ & 0.4 & $(0.1-1.8)$ & 2.5 & $(0.4-15.2)$ & 1.3 & $(0.3-6.7)$ \\
\hline 11 & 8.8 & $(4.6-16.3)$ & 27.1 & $(18.6-37.7)$ & 20.2 & $(14.2-27.7)$ & 0.0 & (.-.) & 0.5 & $(0.2-1.7)$ & 0.5 & $(0.2-1.6)$ \\
\hline 12 & 10.7 & $(5.0-21.6)$ & 22.8 & $(13.9-35.0)$ & 16.5 & (11.2-23.7) & 0.9 & $(0.1-6.3)$ & 3.8 & $(0.9-15.0)$ & 2.3 & $(0.7-7.6)$ \\
\hline Total & 7.7 & $(4.8-12.2)$ & 16.5 & $(12.7-21.2)$ & 12.6 & $(9.7-16.1)$ & 0.3 & $(0.1-1.0)$ & 1.4 & $(0.5-3.7)$ & 1.0 & $(0.4-2.1)$ \\
\hline
\end{tabular}

* During the 30 days before the survey, among the $8.5 \%$ of students nationwide who currently smoked cigarettes and who were aged $<18$ years.

† $95 \%$ confidence interval.

$\S$ Non-Hispanic.

I Not available. 
TABLE 36. Percentage of high school students who usually obtained their own cigarettes by buying them in a store or gas station* and who usually obtained their own cigarettes by buying them on the internet,* by sex - selected U.S. sites, Youth Risk Behavior Survey, 2015

\begin{tabular}{|c|c|c|c|c|c|c|c|c|c|c|c|c|}
\hline \multirow[b]{3}{*}{ Site } & \multicolumn{6}{|c|}{ Bought cigarettes in a store or gas station } & \multicolumn{6}{|c|}{ Bought cigarettes on the internet } \\
\hline & \multicolumn{2}{|r|}{ Female } & \multicolumn{2}{|c|}{ Male } & \multicolumn{2}{|r|}{ Total } & \multicolumn{2}{|c|}{ Female } & \multicolumn{2}{|c|}{ Male } & \multicolumn{2}{|r|}{ Total } \\
\hline & $\%$ & $\mathrm{Cl}^{\dagger}$ & $\%$ & $\mathrm{Cl}$ & $\%$ & $\mathrm{Cl}$ & $\%$ & $\mathrm{Cl}$ & $\%$ & $\mathrm{Cl}$ & $\%$ & $\mathrm{Cl}$ \\
\hline \multicolumn{13}{|l|}{ State surveys } \\
\hline Alabama & —§ & - & - & - & 13.5 & $(7.4-23.4)$ & - & - & - & - & 3.9 & $(1.4-10.5)$ \\
\hline Alaska & - & - & - & - & 4.4 & $(1.9-10.0)$ & - & - & - & - & 0.9 & $(0.1-6.5)$ \\
\hline Arizona & 2.9 & $(0.8-10.4)$ & - & - & 10.0 & $(4.2-21.9)$ & 1.0 & $(0.1-6.9)$ & - & - & 0.5 & $(0.1-3.6)$ \\
\hline Arkansas & 1.1 & $(0.4-3.0)$ & 14.6 & $(8.5-24.0)$ & 8.4 & $(5.3-13.2)$ & 0.8 & $(0.2-3.7)$ & 0.4 & $(0.1-1.2)$ & 0.6 & $(0.2-1.7)$ \\
\hline California & - & - & - & - & - & - & - & - & - & - & - & - \\
\hline Connecticut & - & - & - & - & - & - & - & - & - & - & - & - \\
\hline Delaware & - & - & - & - & 20.6 & $(12.4-32.3)$ & - & - & - & - & 0.0 & - \\
\hline Florida & - & - & - & - & - & - & - & - & - & - & - & - \\
\hline Hawaii & - & - & - & - & - & - & - & - & - & - & - & - \\
\hline Idaho & - & - & - & - & 11.6 & $(6.3-20.4)$ & - & - & - & - & 1.0 & $(0.2-5.8)$ \\
\hline Illinois & - & - & 12.2 & $(5.9-23.6)$ & 11.3 & $(6.4-19.1)$ & - & - & 0.6 & $(0.1-2.6)$ & 0.3 & $(0.1-1.3)$ \\
\hline Indiana & - & - & - & - & 6.0 & $(2.7-12.7)$ & - & - & - & - & 0.0 & - \\
\hline Kentucky & 14.5 & $(7.9-25.2)$ & 26.8 & $(18.7-36.7)$ & 20.2 & $(13.9-28.5)$ & 1.2 & $(0.2-7.2)$ & 0.7 & $(0.1-4.1)$ & 1.0 & $(0.3-3.7)$ \\
\hline Maine & 4.3 & $(2.6-7.0)$ & 9.6 & $(6.7-13.5)$ & 6.9 & $(5.3-8.9)$ & 1.0 & $(0.4-2.8)$ & 3.0 & $(1.5-6.0)$ & 2.2 & $(1.3-3.7)$ \\
\hline Maryland & 10.4 & $(8.9-12.2)$ & 23.9 & $(21.6-26.3)$ & 17.4 & $(16.0-18.9)$ & 2.8 & $(2.0-3.9)$ & 5.6 & $(4.3-7.2)$ & 4.9 & $(4.0-6.0)$ \\
\hline Massachusetts & - & - & - & - & - & - & - & - & - & - & - & - \\
\hline Michigan & 15.2 & $(6.5-31.4)$ & 18.8 & $(9.1-34.9)$ & 17.4 & $(9.6-29.4)$ & 2.5 & $(0.4-14.4)$ & 5.0 & $(1.4-16.3)$ & 4.0 & $(1.4-10.7)$ \\
\hline Mississippi & - & - & 29.4 & $(21.2-39.3)$ & 19.2 & $(14.1-25.5)$ & - & - & 9.5 & $(4.3-19.9)$ & 6.0 & $(2.7-12.6)$ \\
\hline Missouri & - & - & - & - & 14.4 & $(8.8-22.5)$ & - & - & - & - & 1.6 & $(0.4-5.9)$ \\
\hline Montana & 3.5 & $(1.5-8.1)$ & 13.8 & $(10.4-18.1)$ & 8.4 & $(6.2-11.4)$ & 0.0 & - & 1.7 & $(0.5-5.0)$ & 0.8 & $(0.3-2.3)$ \\
\hline Nebraska & - & - & - & - & 9.7 & $(6.0-15.5)$ & - & - & - & - & 2.0 & $(0.5-8.1)$ \\
\hline Nevada & - & - & - & - & - & - & - & - & - & - & - & - \\
\hline New Hampshire & 7.3 & $(5.0-10.5)$ & 18.8 & $(15.3-22.7)$ & 13.6 & $(11.4-16.0)$ & 1.7 & $(0.8-3.9)$ & 2.7 & $(1.6-4.5)$ & 2.4 & $(1.5-3.6)$ \\
\hline New Mexico & - & - & - & - & - & - & - & - & - & - & - & - \\
\hline New York & - & - & - & - & - & - & - & - & - & - & - & - \\
\hline North Carolina & - & - & - & - & - & - & - & - & - & - & - & - \\
\hline North Dakota & - & - & - & - & 16.9 & $(10.8-25.4)$ & - & - & - & - & 0.6 & $(0.2-2.5)$ \\
\hline Oklahoma & - & - & - & - & 9.0 & $(4.8-16.4)$ & - & - & - & - & 0.0 & - \\
\hline Pennsylvania & 3.1 & $(1.1-8.7)$ & 23.4 & $(15.2-34.2)$ & 15.1 & $(10.0-22.1)$ & 2.1 & $(0.4-9.3)$ & 1.6 & $(0.3-8.8)$ & 1.8 & $(0.5-5.7)$ \\
\hline Rhode Island & - & - & - & - & 20.5 & $(8.9-40.3)$ & - & - & - & - & 1.2 & $(0.6-2.6)$ \\
\hline South Carolina & - & - & - & - & 13.9 & $(6.3-28.0)$ & - & - & - & - & 2.4 & $(0.4-12.0)$ \\
\hline South Dakota & - & - & - & - & 5.1 & $(2.2-11.5)$ & - & - & - & - & 0.5 & $(0.1-4.0)$ \\
\hline Tennessee & 7.6 & $(4.9-11.5)$ & 14.4 & $(8.6-23.2)$ & 11.2 & $(7.6-16.2)$ & 1.2 & $(0.3-4.4)$ & 2.2 & $(0.9-5.0)$ & 2.0 & $(1.0-4.3)$ \\
\hline Vermont & 4.2 & $(3.0-5.9)$ & 12.1 & $(10.0-14.5)$ & 8.5 & $(7.2-10.0)$ & 1.4 & $(0.8-2.5)$ & 2.1 & $(1.3-3.3)$ & 1.7 & $(1.2-2.5)$ \\
\hline Virginia & 11.6 & $(5.5-22.8)$ & 19.4 & $(12.4-29.1)$ & 15.7 & $(10.5-22.9)$ & 0.0 & - & 1.1 & $(0.2-6.1)$ & 0.6 & $(0.1-3.2)$ \\
\hline West Virginia & 7.6 & $(3.6-15.3)$ & 15.9 & $(9.3-25.9)$ & 11.7 & $(8.1-16.7)$ & 1.7 & $(0.5-5.4)$ & 6.0 & $(1.1-26.5)$ & 3.8 & $(1.1-12.4)$ \\
\hline Wyoming & 2.8 & $(1.2-6.5)$ & 11.6 & $(7.1-18.5)$ & 7.3 & $(4.6-11.2)$ & 0.9 & $(0.2-3.7)$ & 0.0 & - & 0.5 & $(0.1-1.9)$ \\
\hline Median & & 5.8 & & 5.9 & & 11.6 & & 1.2 & & 2.1 & & 1.1 \\
\hline Range & & $-15.2)$ & & $-29.4)$ & & 4-20.6) & & $-2.8)$ & & $0-9.5)$ & & $.0-6.0)$ \\
\hline \multicolumn{13}{|c|}{ Large urban school district surveys } \\
\hline Baltimore, MD & - & - & - & - & - & - & - & - & - & - & - & - \\
\hline Boston, MA & - & - & - & - & - & - & - & - & - & - & - & 一 \\
\hline Broward County, FL & - & - & - & - & - & - & - & - & - & - & - & 一 \\
\hline Cleveland, $\mathrm{OH}$ & - & - & - & - & - & - & - & - & - & - & - & 一 \\
\hline DeKalb County, GA & - & - & - & - & - & - & - & - & - & - & - & - \\
\hline Detroit, MI & - & - & - & - & 64.5 & $(52.2-75.2)$ & - & - & - & - & 2.4 & $(0.8-7.3)$ \\
\hline District of Columbia & - & - & - & - & - & - & - & - & - & - & - & - \\
\hline Duval County, FL & - & - & - & - & 14.9 & $(9.6-22.4)$ & - & - & - & - & 5.5 & $(2.7-10.8)$ \\
\hline Ft. Worth, TX & - & - & - & - & 31.7 & $(23.9-40.7)$ & - & - & - & - & 1.7 & $(0.4-6.9)$ \\
\hline Houston, TX & - & - & - & - & 22.0 & $(15.6-30.0)$ & - & - & - & - & 5.9 & $(2.8-11.8)$ \\
\hline Los Angeles, CA & - & - & - & - & - & - & - & - & - & - & - & - \\
\hline Miami-Dade County, FL & - & - & - & - & 22.5 & $(14.9-32.6)$ & - & - & - & - & 2.4 & $(0.8-6.9)$ \\
\hline
\end{tabular}

See table footnotes on the next page. 
TABLE 36. (Continued) Percentage of high school students who usually obtained their own cigarettes by buying them in a store or gas station* and who usually obtained their own cigarettes by buying them on the internet, * by sex - selected U.S. sites, Youth Risk Behavior Survey, 2015

\begin{tabular}{|c|c|c|c|c|c|c|c|c|c|c|c|c|}
\hline \multirow[b]{3}{*}{ Site } & \multicolumn{6}{|c|}{ Bought cigarettes in a store or gas station } & \multicolumn{6}{|c|}{ Bought cigarettes on the internet } \\
\hline & \multicolumn{2}{|c|}{ Female } & \multicolumn{2}{|c|}{ Male } & \multicolumn{2}{|c|}{ Total } & \multicolumn{2}{|c|}{ Female } & \multicolumn{2}{|c|}{ Male } & \multicolumn{2}{|c|}{ Total } \\
\hline & $\%$ & $\mathrm{Cl}^{\dagger}$ & $\%$ & $\mathrm{Cl}$ & $\%$ & $\mathrm{Cl}$ & $\%$ & $\mathrm{Cl}$ & $\%$ & $\mathrm{Cl}$ & $\%$ & $\mathrm{Cl}$ \\
\hline New York City, NY & - & - & - & - & - & - & - & - & - & - & - & - \\
\hline Oakland, CA & - & - & - & - & - & - & - & - & - & - & - & - \\
\hline Orange County, FL & - & - & - & - & - & - & - & - & - & - & - & - \\
\hline Palm Beach County, FL & - & - & - & - & - & - & - & - & - & - & - & - \\
\hline Philadelphia, PA & - & - & - & - & - & - & - & - & - & - & - & - \\
\hline San Diego, CA & - & - & - & - & - & - & - & - & - & - & - & - \\
\hline San Francisco, CA & - & - & - & - & - & - & - & - & - & - & - & - \\
\hline Median & \multicolumn{2}{|c|}{ - } & \multicolumn{2}{|c|}{ - } & \multicolumn{2}{|c|}{22.5} & \multicolumn{2}{|c|}{ - } & \multicolumn{2}{|c|}{ - } & \multicolumn{2}{|c|}{$\begin{array}{c}2.4 \\
(17-59)\end{array}$} \\
\hline Range & \multicolumn{2}{|c|}{ - } & \multicolumn{2}{|c|}{ - } & \multicolumn{2}{|c|}{ (14.9-64.5) } & \multicolumn{2}{|c|}{ - } & \multicolumn{2}{|c|}{ - } & \multicolumn{2}{|c|}{$(1.7-5.9)$} \\
\hline
\end{tabular}

* During the 30 days before the survey, among students who currently smoked cigarettes and who were aged $<18$ years.

† $95 \%$ confidence interval.

$\S$ Not available.

TABLE 37. Percentage of high school students who tried to quit smoking cigarettes,* by sex, race/ethnicity, and grade — United States, Youth Risk Behavior Survey, 2015

\begin{tabular}{|c|c|c|c|c|c|c|}
\hline \multirow[b]{2}{*}{ Category } & \multicolumn{2}{|c|}{ Female } & \multicolumn{2}{|c|}{ Male } & \multicolumn{2}{|c|}{ Total } \\
\hline & $\%$ & $\mathrm{Cl}^{\dagger}$ & $\%$ & $\mathrm{Cl}$ & $\%$ & $\mathrm{Cl}$ \\
\hline \multicolumn{7}{|c|}{ Race/Ethnicity } \\
\hline White ${ }^{\S}$ & 51.0 & $(45.7-56.3)$ & 37.9 & (31.2-45.0) & 44.1 & $(38.8-49.5)$ \\
\hline Black ${ }^{\S}$ & - & - & - & - & - & - \\
\hline Hispanic & 56.4 & $(46.3-66.1)$ & 45.3 & $(37.8-53.0)$ & 49.6 & $(44.2-55.0)$ \\
\hline \multicolumn{7}{|l|}{ Grade } \\
\hline 9 & 47.3 & (36.7-58.1) & 48.2 & $(41.1-55.5)$ & 47.8 & $(41.2-54.5)$ \\
\hline 10 & 57.0 & $(46.1-67.2)$ & 45.7 & (32.2-59.9) & 51.6 & (41.4-61.7) \\
\hline 11 & 52.2 & $(41.4-62.8)$ & 29.9 & $(22.6-38.3)$ & 37.9 & $(30.7-45.6)$ \\
\hline 12 & 54.1 & $(46.0-61.9)$ & 42.0 & $(34.4-50.1)$ & 47.7 & $(42.4-53.1)$ \\
\hline Total & 52.8 & $(48.5-57.0)$ & 39.7 & (34.6-45.0) & 45.4 & $(41.3-49.5)$ \\
\hline
\end{tabular}

* During the 12 months before the survey, among the $10.8 \%$ of students nationwide who currently smoked cigarettes.

+ $95 \%$ confidence interval.

$\S$ Non-Hispanic.

" Not available. 
TABLE 38. Percentage of high school students who tried to quit smoking cigarettes, ${ }^{*}$ by sex — selected U.S. sites, Youth Risk Behavior Survey, 2015

\begin{tabular}{|c|c|c|c|c|c|c|}
\hline \multirow[b]{2}{*}{ Site } & \multicolumn{2}{|c|}{ Female } & \multicolumn{2}{|c|}{ Male } & \multicolumn{2}{|c|}{ Total } \\
\hline & $\%$ & $\mathrm{Cl}^{\dagger}$ & $\%$ & $\mathrm{Cl}$ & $\%$ & $\mathrm{Cl}$ \\
\hline \multicolumn{7}{|l|}{ State surveys } \\
\hline Alabama & — & - & - & - & 49.3 & $(41.4-57.3)$ \\
\hline Alaska & - & - & - & - & 59.5 & $(47.4-70.5)$ \\
\hline Arizona & 51.3 & (37.9-64.5) & 46.3 & $(33.9-59.2)$ & 49.2 & (39.4-59.0) \\
\hline Arkansas & 62.1 & $(51.2-72.0)$ & 55.6 & $(45.6-65.1)$ & 58.4 & (50.5-65.9) \\
\hline California & - & - & - & - & 35.2 & $(25.8-45.8)$ \\
\hline Connecticut & - & - & - & - & - & - \\
\hline Delaware & - & - & 45.1 & $(34.4-56.3)$ & 49.2 & $(40.8-57.6)$ \\
\hline Florida & - & - & - & - & - & - \\
\hline Hawaii & - & - & - & - & - & - \\
\hline Idaho & - & - & - & - & 48.0 & $(38.7-57.5)$ \\
\hline Illinois & 59.0 & $(45.3-71.5)$ & 49.8 & $(41.4-58.3)$ & 53.8 & $(46.1-61.2)$ \\
\hline Indiana & - & - & - & - & 49.7 & (39.9-59.4) \\
\hline Kentucky & 53.0 & $(44.8-60.9)$ & 44.7 & $(35.1-54.8)$ & 49.0 & $(42.9-55.1)$ \\
\hline Maine & - & - & - & - & - & - \\
\hline Maryland & - & - & - & - & - & - \\
\hline Massachusetts & - & - & - & - & - & - \\
\hline Michigan & 63.2 & $(53.5-72.0)$ & 45.2 & $(35.7-55.0)$ & 52.0 & $(44.7-59.1)$ \\
\hline Mississippi & 51.9 & $(39.3-64.3)$ & 47.6 & $(38.5-56.9)$ & 49.6 & $(41.1-58.1)$ \\
\hline Missouri & - & - & - & - & 46.0 & $(36.6-55.7)$ \\
\hline Montana & 59.5 & $(50.2-68.1)$ & 40.7 & $(35.0-46.7)$ & 50.0 & (43.5-56.5) \\
\hline Nebraska & - & - & - & - & 48.7 & $(40.6-56.9)$ \\
\hline Nevada & - & - & - & - & - & - \\
\hline New Hampshire & 49.8 & $(44.7-54.9)$ & 44.2 & $(40.2-48.4)$ & 46.2 & $(43.3-49.1)$ \\
\hline New Mexico & 54.2 & $(46.5-61.8)$ & 50.8 & $(46.3-55.3)$ & 52.1 & $(47.8-56.4)$ \\
\hline New York & - & - & - & - & - & - \\
\hline North Carolina & 38.7 & $(23.7-56.2)$ & 50.3 & $(36.5-64.0)$ & 45.5 & $(34.2-57.4)$ \\
\hline North Dakota & 53.1 & $(43.0-62.9)$ & 41.8 & $(31.6-52.7)$ & 47.4 & $(40.9-53.9)$ \\
\hline Oklahoma & - & - & 47.7 & $(36.0-59.7)$ & 52.3 & $(43.3-61.2)$ \\
\hline Pennsylvania & 53.7 & $(44.3-62.8)$ & 48.2 & $(40.0-56.5)$ & 50.5 & $(44.2-56.8)$ \\
\hline Rhode Island & - & - & - & - & 46.0 & $(32.9-59.6)$ \\
\hline South Carolina & - & - & - & - & 50.3 & $(40.3-60.2)$ \\
\hline South Dakota & - & - & - & - & 50.6 & $(39.3-61.8)$ \\
\hline Tennessee & 50.4 & $(42.3-58.4)$ & 47.7 & (38.9-56.6) & 48.9 & $(43.2-54.6)$ \\
\hline Vermont & 43.4 & $(40.3-46.7)$ & 41.3 & (38.4-44.2) & 42.2 & $(40.1-44.3)$ \\
\hline Virginia & 52.2 & $(43.1-61.1)$ & 45.3 & $(35.6-55.3)$ & 48.5 & $(41.2-55.8)$ \\
\hline West Virginia & 50.8 & $(40.6-61.0)$ & 45.9 & $(34.9-57.2)$ & 48.4 & $(41.2-55.6)$ \\
\hline Wyoming & 51.5 & $(42.3-60.6)$ & 53.9 & $(43.5-64.0)$ & 52.8 & $(47.2-58.3)$ \\
\hline Median & & & & & & \\
\hline Range & & & & & & \\
\hline \multicolumn{7}{|c|}{ Large urban school district surveys } \\
\hline Baltimore, MD & - & - & - & - & - & - \\
\hline Boston, MA & - & - & - & - & - & - \\
\hline Broward County, FL & - & - & - & - & - & - \\
\hline Cleveland, $\mathrm{OH}$ & - & - & - & - & - & - \\
\hline DeKalb County, GA & - & - & - & - & - & - \\
\hline Detroit, MI & - & - & - & - & 81.8 & $(69.0-90.0)$ \\
\hline District of Columbia & - & - & - & - & - & - \\
\hline Duval County, FL & - & - & - & - & 51.5 & $(43.2-59.7)$ \\
\hline Ft. Worth, TX & - & - & 44.1 & $(32.5-56.4)$ & 48.7 & $(39.6-57.9)$ \\
\hline Houston, TX & 58.9 & $(48.1-68.9)$ & 57.8 & $(46.3-68.6)$ & 58.4 & $(50.1-66.2)$ \\
\hline Los Angeles, CA & - & - & - & - & 46.9 & (35.5-58.7) \\
\hline Miami-Dade County, FL & - & - & - & - & 40.1 & $(30.6-50.4)$ \\
\hline
\end{tabular}

See table footnotes on the next page. 
TABLE 38. (Continued) Percentage of high school students who tried to quit smoking cigarettes, ${ }^{*}$ by sex — selected U.S. sites, Youth Risk Behavior Survey, 2015

\begin{tabular}{|c|c|c|c|c|c|c|}
\hline \multirow[b]{2}{*}{ Site } & \multicolumn{2}{|c|}{ Female } & \multicolumn{2}{|c|}{ Male } & \multicolumn{2}{|c|}{ Total } \\
\hline & $\%$ & $\mathrm{Cl}^{\dagger}$ & $\%$ & $\mathrm{Cl}$ & $\%$ & $\mathrm{Cl}$ \\
\hline New York City, NY & - & - & - & - & - & - \\
\hline Oakland, CA & - & - & - & - & - & - \\
\hline Orange County, FL & - & - & - & - & - & - \\
\hline Palm Beach County, FL & - & - & - & - & 39.9 & $(29.1-51.8)$ \\
\hline Philadelphia, PA & - & - & - & - & - & - \\
\hline San Diego, CA & - & - & - & - & 35.7 & (27.9-44.3) \\
\hline San Francisco, CA & - & - & - & - & 41.6 & $(30.1-54.1)$ \\
\hline Median & \multirow{2}{*}{\multicolumn{2}{|c|}{$\begin{array}{c}58.9 \\
(58.9-58.9)\end{array}$}} & \multirow{2}{*}{\multicolumn{2}{|c|}{$\begin{array}{c}50.9 \\
(44.1-57.8)\end{array}$}} & \multirow{2}{*}{\multicolumn{2}{|c|}{$\begin{array}{c}46.9 \\
(35.7-81.8)\end{array}$}} \\
\hline Range & & & & & & \\
\hline
\end{tabular}

* During the 12 months before the survey, among students who currently smoked cigarettes.

† $95 \%$ confidence interval.

$\S$ Not available.

TABLE 39. Percentage of high school students who currently used smokeless tobacco* and who currently smoked cigars, ${ }^{\dagger}$ by sex, race/ethnicity, and grade - United States, Youth Risk Behavior Survey, 2015

\begin{tabular}{|c|c|c|c|c|c|c|c|c|c|c|c|c|}
\hline \multirow[b]{3}{*}{ Category } & \multicolumn{6}{|c|}{ Current smokeless tobacco use } & \multicolumn{6}{|c|}{ Current cigar use } \\
\hline & \multicolumn{2}{|c|}{ Female } & \multicolumn{2}{|r|}{ Male } & \multicolumn{2}{|r|}{ Total } & \multicolumn{2}{|c|}{ Female } & \multicolumn{2}{|r|}{ Male } & \multicolumn{2}{|r|}{ Total } \\
\hline & $\%$ & $\mathrm{Cl}^{\S}$ & $\%$ & $\mathrm{Cl}$ & $\%$ & $\mathrm{Cl}$ & $\%$ & $\mathrm{Cl}$ & $\%$ & $\mathrm{Cl}$ & $\%$ & $\mathrm{Cl}$ \\
\hline \multicolumn{13}{|c|}{ Race/Ethnicity } \\
\hline White & 2.5 & $(1.6-4.0)$ & 15.9 & (13.3-18.9) & 9.3 & $(7.5-11.4)$ & 6.0 & $(4.4-8.1)$ & 14.8 & (12.5-17.5) & 10.4 & $(8.7-12.5)$ \\
\hline Black" & 1.1 & $(0.5-2.3)$ & 5.6 & $(3.5-8.9)$ & 3.7 & $(2.4-5.5)$ & 8.5 & $(6.2-11.5)$ & 12.9 & $(9.3-17.6)$ & 11.0 & $(8.4-14.4)$ \\
\hline Hispanic & 2.5 & $(1.6-4.0)$ & 6.4 & $(4.6-9.0)$ & 4.5 & $(3.3-6.2)$ & 6.5 & $(4.9-8.8)$ & 12.4 & $(10.0-15.2)$ & 9.5 & $(7.7-11.7)$ \\
\hline \multicolumn{13}{|l|}{ Grade } \\
\hline 9 & 2.4 & $(1.5-4.0)$ & 8.8 & $(6.8-11.3)$ & 5.9 & $(4.6-7.5)$ & 4.1 & $(3.0-5.6)$ & 8.5 & $(6.9-10.6)$ & 6.6 & (5.4-7.9) \\
\hline 10 & 2.0 & $(1.2-3.3)$ & 10.6 & $(7.9-13.9)$ & 6.3 & $(4.7-8.3)$ & 6.6 & $(4.8-9.1)$ & 12.5 & $(9.6-16.0)$ & 9.6 & $(7.6-12.1)$ \\
\hline 11 & 2.9 & $(1.9-4.3)$ & 15.0 & $(12.5-17.8)$ & 9.3 & $(7.8-11.0)$ & 6.3 & $(4.7-8.4)$ & 15.1 & $(12.6-18.0)$ & 11.0 & $(9.2-13.2)$ \\
\hline 12 & 1.7 & $(1.0-2.9)$ & 13.1 & $(9.9-17.1)$ & 7.5 & $(5.8-9.5)$ & 8.1 & $(6.0-10.9)$ & 20.4 & $(16.3-25.2)$ & 14.3 & $(11.6-17.5)$ \\
\hline Total & 2.3 & $(1.7-3.2)$ & 11.9 & $(10.0-14.0)$ & 7.3 & $(6.1-8.6)$ & 6.3 & $(5.1-7.9)$ & 14.0 & (12.3-15.8) & 10.3 & $(9.0-11.8)$ \\
\hline
\end{tabular}

* Chewing tobacco, snuff, or dip on at least 1 day during the 30 days before the survey.

† Cigars, cigarillos, or little cigars on at least 1 day during the 30 days before the survey.

$\S 95 \%$ confidence interval.

" Non-Hispanic. 
TABLE 40. Percentage of high school students who currently used smokeless tobacco* and who currently smoked cigars, ${ }^{\dagger}$ by sex - selected U.S. sites, Youth Risk Behavior Survey, 2015

\begin{tabular}{|c|c|c|c|c|c|c|c|c|c|c|c|c|}
\hline \multirow[b]{3}{*}{ Site } & \multicolumn{6}{|c|}{ Current smokeless tobacco use } & \multicolumn{6}{|c|}{ Current cigar use } \\
\hline & \multicolumn{2}{|c|}{ Female } & \multicolumn{2}{|r|}{ Male } & \multicolumn{2}{|r|}{ Total } & \multicolumn{2}{|c|}{ Female } & \multicolumn{2}{|r|}{ Male } & \multicolumn{2}{|r|}{ Total } \\
\hline & $\%$ & $\mathrm{Cl}^{\S}$ & $\%$ & $\mathrm{Cl}$ & $\%$ & $\mathrm{Cl}$ & $\%$ & $\mathrm{Cl}$ & $\%$ & $\mathrm{Cl}$ & $\%$ & $\mathrm{Cl}$ \\
\hline \multicolumn{13}{|l|}{ State surveys } \\
\hline Alabama & 4.5 & $(2.8-7.2)$ & 19.6 & $(16.2-23.6)$ & 12.5 & $(9.7-15.8)$ & 10.7 & $(8.3-13.6)$ & 15.5 & $(13.1-18.3)$ & 13.4 & (11.3-15.9) \\
\hline Alaska & 8.2 & $(5.4-12.2)$ & 14.7 & $(11.2-19.1)$ & 11.7 & $(8.8-15.5)$ & 3.7 & $(2.2-6.0)$ & 9.9 & $(7.6-12.8)$ & 7.1 & $(5.6-8.9)$ \\
\hline Arizona & 2.8 & $(1.7-4.7)$ & 9.5 & $(6.5-13.7)$ & 6.2 & $(4.2-9.0)$ & 6.5 & $(4.3-9.9)$ & 13.4 & $(10.5-17.0)$ & 10.1 & $(7.8-12.9)$ \\
\hline Arkansas & 3.5 & $(2.4-5.0)$ & 17.2 & $(12.9-22.6)$ & 10.6 & $(8.5-13.2)$ & 10.6 & $(7.8-14.3)$ & 17.5 & $(13.7-22.1)$ & 14.2 & $(11.9-17.0)$ \\
\hline California & 1.1 & $(0.5-2.2)$ & 4.8 & (2.7-8.4) & 3.0 & $(1.8-4.8)$ & 4.6 & $(2.7-7.7)$ & 8.9 & $(6.4-12.3)$ & 6.8 & $(4.9-9.2)$ \\
\hline Connecticut & - & - & - & - & - & - & - & - & - & - & - & - \\
\hline Delaware & 1.2 & $(0.7-2.0)$ & 7.7 & $(5.7-10.2)$ & 4.5 & $(3.5-5.7)$ & 8.0 & $(5.8-11.0)$ & 13.3 & $(10.8-16.2)$ & 10.9 & $(8.9-13.3)$ \\
\hline Florida & - & - & - & - & - & - & - & - & - & - & - & - \\
\hline Hawaii & - & - & - & - & - & - & - & - & - & - & - & - \\
\hline Idaho & 2.0 & $(1.1-3.6)$ & 14.3 & $(11.3-18.0)$ & 8.3 & $(6.7-10.3)$ & 5.2 & $(3.3-8.1)$ & 11.1 & $(8.7-14.0)$ & 8.2 & $(6.6-10.2)$ \\
\hline Illinois & 2.5 & $(1.6-3.9)$ & 8.4 & $(6.2-11.2)$ & 5.6 & $(4.3-7.3)$ & 5.3 & $(4.0-7.0)$ & 13.2 & $(10.4-16.5)$ & 9.4 & $(7.9-11.0)$ \\
\hline Indiana & 2.6 & $(1.6-4.2)$ & 15.7 & $(9.8-24.3)$ & 9.4 & $(5.9-14.7)$ & 7.7 & $(5.8-10.2)$ & 14.8 & (11.6-18.8) & 11.4 & $(9.1-14.3)$ \\
\hline Kentucky & 3.6 & $(2.2-5.8)$ & 21.1 & $(17.2-25.6)$ & 12.6 & $(10.4-15.2)$ & 10.2 & $(7.4-13.9)$ & 17.1 & $(14.6-19.9)$ & 14.0 & $(11.7-16.6)$ \\
\hline Maine & 1.6 & $(1.1-2.2)$ & 8.3 & (7.1-9.6) & 5.1 & $(4.5-5.9)$ & 4.8 & $(4.1-5.7)$ & 12.3 & $(10.7-14.1)$ & 8.8 & (7.8-9.9) \\
\hline Maryland & 2.7 & $(2.4-2.9)$ & 8.3 & $(7.7-8.8)$ & 5.8 & $(5.4-6.1)$ & 7.8 & $(7.3-8.4)$ & 12.3 & $(11.7-12.9)$ & 10.3 & $(9.9-10.8)$ \\
\hline Massachusetts & 1.9 & $(1.1-3.2)$ & 9.0 & $(7.0-11.4)$ & 5.5 & $(4.4-7.0)$ & 5.3 & $(4.1-6.7)$ & 15.4 & $(12.6-18.6)$ & 10.4 & $(8.6-12.5)$ \\
\hline Michigan & 2.0 & $(1.2-3.2)$ & 10.4 & $(7.3-14.6)$ & 6.2 & $(4.5-8.6)$ & 5.0 & (3.4-7.4) & 13.2 & $(10.3-16.9)$ & 9.2 & $(7.4-11.4)$ \\
\hline Mississippi & 4.2 & $(2.8-6.2)$ & 18.4 & (15.4-21.8) & 11.6 & $(9.8-13.6)$ & 12.6 & $(10.1-15.7)$ & 20.2 & $(16.6-24.5)$ & 16.5 & $(14.2-18.9)$ \\
\hline Missouri & 2.9 & $(1.8-4.6)$ & 17.0 & $(12.6-22.7)$ & 10.0 & $(7.1-13.8)$ & 7.1 & $(4.6-10.7)$ & 17.1 & $(13.4-21.7)$ & 12.1 & $(9.1-16.0)$ \\
\hline Montana & 4.5 & $(3.6-5.6)$ & 19.6 & $(17.4-21.9)$ & 12.3 & $(11.0-13.7)$ & 8.2 & $(6.9-9.6)$ & 16.8 & $(14.9-18.9)$ & 12.6 & $(11.4-14.0)$ \\
\hline Nebraska & 3.2 & $(2.0-5.1)$ & 14.9 & $(11.9-18.6)$ & 9.3 & $(7.4-11.7)$ & 4.9 & $(3.0-7.7)$ & 11.0 & $(8.4-14.2)$ & 8.1 & $(6.3-10.3)$ \\
\hline Nevada & 1.8 & $(1.0-3.2)$ & 8.2 & (6.8-9.9) & 5.1 & $(4.2-6.2)$ & 5.3 & $(3.7-7.6)$ & 8.6 & $(6.6-11.0)$ & 7.1 & $(5.7-8.8)$ \\
\hline New Hampshire & 1.6 & $(1.2-2.0)$ & 9.9 & $(8.7-11.2)$ & 6.0 & $(5.3-6.7)$ & 5.8 & $(5.1-6.7)$ & 15.6 & $(14.3-17.1)$ & 11.0 & $(10.0-12.0)$ \\
\hline New Mexico & 2.9 & $(2.3-3.8)$ & 14.2 & $(12.1-16.6)$ & 8.7 & $(7.4-10.1)$ & 7.3 & $(6.4-8.3)$ & 13.5 & $(12.0-15.2)$ & 10.5 & $(9.5-11.5)$ \\
\hline New York & 3.3 & $(2.5-4.3)$ & 9.5 & $(8.0-11.1)$ & 6.7 & $(5.9-7.6)$ & 6.6 & $(4.9-8.8)$ & 13.4 & $(10.9-16.2)$ & 10.2 & $(8.6-12.1)$ \\
\hline North Carolina & 1.8 & $(1.0-3.3)$ & 14.9 & $(10.8-20.2)$ & 8.6 & $(6.4-11.4)$ & - & - & - & - & - & - \\
\hline North Dakota & 3.1 & $(2.0-4.6)$ & 17.6 & $(15.0-20.6)$ & 10.6 & $(9.0-12.3)$ & 4.9 & $(3.7-6.6)$ & 13.2 & $(10.9-15.9)$ & 9.2 & (7.8-10.8) \\
\hline Oklahoma & 2.2 & $(1.4-3.4)$ & 16.2 & $(12.7-20.3)$ & 9.0 & $(7.0-11.5)$ & 4.7 & $(3.2-7.0)$ & 14.0 & $(10.8-18.1)$ & 9.4 & $(7.3-12.1)$ \\
\hline Pennsylvania & 2.6 & $(1.7-4.0)$ & 16.0 & $(12.4-20.5)$ & 9.5 & $(7.4-12.1)$ & 6.7 & $(5.0-8.9)$ & 18.2 & $(15.1-21.8)$ & 12.5 & $(10.6-14.7)$ \\
\hline Rhode Island & 2.2 & $(1.2-4.3)$ & 8.0 & $(5.8-11.0)$ & 5.3 & $(3.9-7.2)$ & 4.7 & $(3.7-6.0)$ & 11.7 & $(9.3-14.6)$ & 8.4 & (7.3-9.8) \\
\hline South Carolina & 3.0 & $(2.1-4.4)$ & 11.0 & $(8.2-14.7)$ & 7.2 & $(5.3-9.6)$ & 9.6 & $(6.7-13.5)$ & 12.6 & $(9.0-17.3)$ & 11.2 & $(8.7-14.4)$ \\
\hline South Dakota & 4.0 & $(2.7-5.8)$ & 18.9 & $(14.1-24.9)$ & 11.7 & $(9.0-15.2)$ & 7.1 & $(4.8-10.5)$ & 11.7 & $(8.2-16.4)$ & 9.6 & $(6.9-13.2)$ \\
\hline Tennessee & 2.7 & $(1.9-3.7)$ & 18.5 & $(16.6-20.6)$ & 11.0 & $(9.8-12.3)$ & 7.9 & $(6.8-9.3)$ & 13.5 & $(11.8-15.5)$ & 11.0 & $(9.9-12.1)$ \\
\hline Vermont & 2.5 & $(2.2-2.8)$ & 10.8 & $(10.3-11.5)$ & 6.9 & $(6.5-7.2)$ & 6.1 & $(5.6-6.6)$ & 14.5 & $(13.8-15.2)$ & 10.4 & $(10.0-10.9)$ \\
\hline Virginia & 2.0 & $(1.5-2.8)$ & 8.7 & $(7.1-10.7)$ & 5.5 & $(4.5-6.7)$ & 5.5 & $(4.3-6.9)$ & 9.2 & $(7.7-11.0)$ & 7.4 & (6.4-8.7) \\
\hline West Virginia & 3.5 & $(2.4-5.2)$ & 22.8 & $(18.6-27.7)$ & 13.4 & $(10.9-16.3)$ & 10.2 & $(8.0-12.9)$ & 17.5 & $(14.7-20.7)$ & 13.9 & $(11.6-16.5)$ \\
\hline Wyoming & 5.5 & $(4.1-7.3)$ & 17.2 & $(14.3-20.6)$ & 11.6 & $(9.8-13.7)$ & 9.6 & $(7.6-11.9)$ & 15.4 & $(12.3-19.1)$ & 12.6 & $(10.6-15.0)$ \\
\hline Median & \multicolumn{2}{|c|}{2.7} & \multicolumn{2}{|c|}{14.5} & \multicolumn{2}{|c|}{8.6} & \multicolumn{2}{|r|}{6.6} & \multicolumn{2}{|r|}{13.4} & & 10.4 \\
\hline Range & & 1-8.2) & & 8-22.8) & & $.0-13.4)$ & & 7-12.6) & & $3.6-20.2)$ & & (.8-16.5) \\
\hline Large urban school dist & reys & & & & & & & & & & & \\
\hline Baltimore, MD & 3.5 & $(2.2-5.5)$ & 8.9 & $(5.9-13.3)$ & 7.5 & $(5.2-10.7)$ & 13.2 & $(9.1-18.9)$ & 14.6 & $(10.5-20.0)$ & 14.9 & $(11.6-19.0)$ \\
\hline Boston, MA & 0.8 & $(0.3-2.3)$ & 3.7 & $(2.5-5.3)$ & 2.4 & $(1.7-3.3)$ & 3.8 & $(2.6-5.7)$ & 6.8 & $(5.1-9.1)$ & 5.4 & $(4.2-6.9)$ \\
\hline Broward County, FL & 1.7 & $(0.9-3.4)$ & 5.5 & $(3.6-8.4)$ & 3.7 & $(2.6-5.2)$ & 4.0 & $(2.7-6.0)$ & 9.8 & $(7.3-13.1)$ & 7.1 & $(5.6-9.1)$ \\
\hline Cleveland, $\mathrm{OH}$ & - & - & - & - & - & - & - & - & - & - & - & - \\
\hline DeKalb County, GA & 1.0 & $(0.5-2.1)$ & 5.7 & $(4.1-7.9)$ & 3.4 & $(2.4-4.7)$ & 7.7 & $(5.8-10.1)$ & 11.6 & $(9.6-14.0)$ & 9.7 & $(8.1-11.7)$ \\
\hline Detroit, MI & 3.6 & $(2.5-5.1)$ & 8.1 & $(6.0-10.8)$ & 6.1 & $(4.9-7.6)$ & 6.7 & $(5.2-8.6)$ & 12.0 & $(9.5-15.1)$ & 9.3 & $(7.7-11.2)$ \\
\hline District of Columbia & - & - & - & - & - & - & 9.6 & $(8.9-10.5)$ & 12.9 & $(11.9-13.9)$ & 11.5 & $(10.8-12.1)$ \\
\hline Duval County, FL & 4.5 & $(3.5-5.9)$ & 10.5 & $(8.3-13.2)$ & 7.8 & $(6.4-9.5)$ & 9.8 & $(7.8-12.2)$ & 14.8 & $(12.0-18.1)$ & 12.8 & $(10.6-15.3)$ \\
\hline Ft. Worth, TX & 1.4 & $(0.8-2.6)$ & 5.0 & $(3.7-6.8)$ & 3.2 & $(2.4-4.2)$ & 7.8 & $(6.2-9.7)$ & 10.4 & $(8.5-12.7)$ & 9.0 & $(7.8-10.5)$ \\
\hline Houston, TX & 5.6 & $(4.2-7.5)$ & 7.8 & $(6.4-9.4)$ & 7.1 & $(5.9-8.5)$ & 11.6 & $(10.1-13.4)$ & 14.3 & $(12.4-16.4)$ & 13.3 & $(12.0-14.8)$ \\
\hline Los Angeles, CA & 1.8 & $(1.1-3.1)$ & 3.5 & $(2.2-5.5)$ & 2.6 & $(1.7-4.1)$ & 3.3 & $(2.1-5.1)$ & 5.5 & $(3.7-8.0)$ & 4.4 & $(3.0-6.4)$ \\
\hline Miami-Dade County, FL & 1.4 & $(0.8-2.3)$ & 4.8 & $(3.5-6.7)$ & 3.1 & $(2.2-4.4)$ & 3.0 & $(2.1-4.3)$ & 9.2 & $(7.3-11.5)$ & 6.1 & $(5.0-7.4)$ \\
\hline
\end{tabular}

See table footnotes on the next page. 
TABLE 40. (Continued) Percentage of high school students who currently used smokeless tobacco* and who currently smoked cigars, ${ }^{\dagger}$ by sex — selected U.S. sites, Youth Risk Behavior Survey, 2015

\begin{tabular}{|c|c|c|c|c|c|c|c|c|c|c|c|c|}
\hline \multirow[b]{3}{*}{ Site } & \multicolumn{6}{|c|}{ Current smokeless tobacco use } & \multicolumn{6}{|c|}{ Current cigar use } \\
\hline & \multicolumn{2}{|c|}{ Female } & \multicolumn{2}{|c|}{ Male } & \multicolumn{2}{|c|}{ Total } & \multicolumn{2}{|c|}{ Female } & \multicolumn{2}{|r|}{ Male } & \multicolumn{2}{|c|}{ Total } \\
\hline & $\%$ & $\mathrm{Cl}^{\S}$ & $\%$ & $\mathrm{Cl}$ & $\%$ & $\mathrm{Cl}$ & $\%$ & $\mathrm{Cl}$ & $\%$ & $\mathrm{Cl}$ & $\%$ & $\mathrm{Cl}$ \\
\hline New York City, NY & 1.6 & $(1.2-2.1)$ & 4.4 & $(3.6-5.3)$ & 3.1 & $(2.7-3.7)$ & 3.4 & $(2.8-4.2)$ & 7.7 & $(6.5-9.2)$ & 5.7 & $(4.9-6.7)$ \\
\hline Oakland, CA & 1.2 & $(0.7-2.1)$ & 4.0 & $(2.3-7.1)$ & 2.9 & $(1.9-4.5)$ & 4.1 & $(2.9-5.9)$ & 8.1 & $(6.1-10.6)$ & 6.3 & $(5.1-7.8)$ \\
\hline Orange County, FL & 2.7 & $(1.7-4.2)$ & 7.8 & $(5.3-11.3)$ & 5.4 & $(3.9-7.3)$ & 5.3 & $(3.8-7.3)$ & 10.5 & $(8.0-13.8)$ & 8.0 & $(6.4-9.9)$ \\
\hline Palm Beach County, FL & 2.8 & $(1.6-4.9)$ & 8.1 & $(6.2-10.6)$ & 6.4 & $(4.8-8.4)$ & 6.8 & $(5.0-9.1)$ & 12.5 & $(10.2-15.3)$ & 10.4 & $(8.8-12.2)$ \\
\hline Philadelphia, PA & 3.7 & $(2.5-5.5)$ & 7.4 & $(4.9-11.2)$ & 5.8 & $(4.2-8.1)$ & 8.9 & $(6.3-12.3)$ & 11.8 & $(8.6-15.8)$ & 10.5 & $(8.1-13.6)$ \\
\hline San Diego, CA & - & - & - & - & - & - & - & - & - & - & - & - \\
\hline San Francisco, CA & - & - & - & - & - & - & - & - & - & - & - & - \\
\hline Median & \multicolumn{2}{|c|}{1.8} & \multicolumn{2}{|c|}{5.7} & \multicolumn{2}{|c|}{3.7} & \multicolumn{2}{|c|}{6.7} & \multicolumn{2}{|r|}{11.0} & \multicolumn{2}{|r|}{9.1} \\
\hline Range & \multicolumn{2}{|c|}{$(0.8-5.6)$} & \multicolumn{2}{|c|}{$(3.5-10.5)$} & \multicolumn{2}{|c|}{$(2.4-7.8)$} & \multicolumn{2}{|c|}{$(3.0-13.2)$} & \multicolumn{2}{|c|}{$(5.5-14.8)$} & \multicolumn{2}{|c|}{$(4.4-14.9)$} \\
\hline
\end{tabular}

${ }^{*}$ Chewing tobacco, snuff, or dip on at least 1 day during the 30 days before the survey.

${ }^{\dagger}$ Cigars, cigarillos, or little cigars on at least 1 day during the 30 days before the survey.

$\S 95 \%$ confidence interval.

I Not available.

TABLE 41. Percentage of high school students who ever used electronic vapor products* and who currently used electronic vapor products,,+ by sex, race/ethnicity, and grade - United States, Youth Risk Behavior Survey, 2015

\begin{tabular}{|c|c|c|c|c|c|c|c|c|c|c|c|c|}
\hline \multirow[b]{3}{*}{ Category } & \multicolumn{6}{|c|}{ Ever used electronic vapor products } & \multicolumn{6}{|c|}{ Current electronic vapor products use } \\
\hline & \multicolumn{2}{|c|}{ Female } & \multicolumn{2}{|r|}{ Male } & \multicolumn{2}{|r|}{ Total } & \multicolumn{2}{|c|}{ Female } & \multicolumn{2}{|r|}{ Male } & \multicolumn{2}{|r|}{ Total } \\
\hline & $\%$ & $\mathrm{Cl}^{\S}$ & $\%$ & $\mathrm{Cl}$ & $\%$ & $\mathrm{Cl}$ & $\%$ & $\mathrm{Cl}$ & $\%$ & $\mathrm{Cl}$ & $\%$ & $\mathrm{Cl}$ \\
\hline \multicolumn{13}{|c|}{ Race/Ethnicity } \\
\hline White? & 42.3 & $(36.6-48.3)$ & 44.0 & $(39.9-48.2)$ & 43.2 & (38.8-47.6) & 24.2 & (20.6-28.3) & 26.3 & (23.3-29.6) & 25.2 & $(22.2-28.5)$ \\
\hline Black & 37.7 & $(32.0-43.8)$ & 46.5 & $(38.6-54.5)$ & 42.4 & $(37.1-47.8)$ & 14.5 & $(11.8-17.7)$ & 21.2 & $(16.2-27.1)$ & 18.0 & $(14.9-21.7)$ \\
\hline Hispanic & 51.2 & $(47.7-54.8)$ & 52.6 & $(49.0-56.1)$ & 51.9 & $(49.2-54.6)$ & 25.0 & $(21.9-28.5)$ & 27.4 & $(24.0-31.1)$ & 26.3 & $(23.6-29.1)$ \\
\hline \multicolumn{13}{|l|}{ Grade } \\
\hline 9 & 37.4 & $(33.0-42.1)$ & 37.0 & $(33.3-40.9)$ & 37.2 & (33.7-40.7) & 19.8 & $(16.6-23.3)$ & 19.6 & $(17.1-22.5)$ & 19.7 & $(17.4-22.2)$ \\
\hline 10 & 41.2 & $(34.8-47.9)$ & 45.3 & $(40.3-50.5)$ & 43.3 & $(37.8-49.0)$ & 22.3 & (19.4-25.6) & 24.2 & $(20.5-28.3)$ & 23.2 & $(20.3-26.4)$ \\
\hline 11 & 47.8 & $(42.7-53.1)$ & 51.1 & $(47.0-55.2)$ & 49.5 & $(45.7-53.2)$ & 24.1 & $(20.7-27.9)$ & 27.4 & $(24.1-31.0)$ & 25.9 & $(23.1-28.9)$ \\
\hline 12 & 49.0 & $(44.9-53.0)$ & 52.6 & $(46.7-58.5)$ & 50.9 & $(47.6-54.1)$ & 24.6 & $(21.2-28.2)$ & 31.9 & $(27.0-37.3)$ & 28.2 & $(25.1-31.5)$ \\
\hline Total & 43.6 & (39.8-47.5) & 46.1 & $(42.8-49.4)$ & 44.9 & $(41.9-48.0)$ & 22.6 & $(20.6-24.8)$ & 25.6 & $(23.0-28.4)$ & 24.1 & $(22.1-26.2)$ \\
\hline
\end{tabular}

* Including e-cigarettes, e-cigars, e-pipes, vape pipes, vaping pens, e-hookahs, and hookah pens.

${ }^{\dagger}$ On at least 1 day during the 30 days before the survey.

$\S 95 \%$ confidence interval.

"Non-Hispanic. 
TABLE 42. Percentage of high school students who ever used electronic vapor products* and who currently used electronic vapor products, ${ }^{*},+$ by sex — selected U.S. sites, Youth Risk Behavior Survey, 2015

\begin{tabular}{|c|c|c|c|c|c|c|c|c|c|c|c|c|}
\hline \multirow[b]{3}{*}{ Site } & \multicolumn{6}{|c|}{ Ever used electronic vapor products } & \multicolumn{6}{|c|}{ Current electronic vapor products use } \\
\hline & \multicolumn{2}{|c|}{ Female } & \multicolumn{2}{|r|}{ Male } & \multicolumn{2}{|r|}{ Total } & \multicolumn{2}{|c|}{ Female } & \multicolumn{2}{|r|}{ Male } & \multicolumn{2}{|r|}{ Total } \\
\hline & $\%$ & $\mathrm{Cl}^{\S}$ & $\%$ & $\mathrm{Cl}$ & $\%$ & $\mathrm{Cl}$ & $\%$ & $\mathrm{Cl}$ & $\%$ & $\mathrm{Cl}$ & $\%$ & $\mathrm{Cl}$ \\
\hline \multicolumn{13}{|l|}{ State surveys } \\
\hline Alabama & 38.1 & (32.9-43.7) & 43.5 & (38.5-48.6) & 41.0 & (36.8-45.2) & 22.0 & $(18.0-26.6)$ & 26.5 & $(22.9-30.4)$ & 24.5 & $(21.2-28.1)$ \\
\hline Alaska & 31.9 & $(28.3-35.8)$ & 39.7 & (34.2-45.3) & 36.1 & (32.7-39.7) & 15.0 & $(12.5-18.1)$ & 20.1 & $(16.5-24.1)$ & 17.7 & $(15.3-20.5)$ \\
\hline Arizona & 49.4 & $(44.3-54.5)$ & 53.7 & $(49.1-58.2)$ & 51.6 & $(47.4-55.8)$ & 25.4 & $(20.7-30.8)$ & 29.4 & $(25.2-34.1)$ & 27.5 & $(24.2-31.1)$ \\
\hline Arkansas & 39.7 & $(35.4-44.1)$ & 49.5 & $(44.1-54.9)$ & 44.7 & (41.4-47.9) & 21.7 & $(17.0-27.2)$ & 30.6 & $(24.7-37.2)$ & 26.4 & $(22.1-31.2)$ \\
\hline California & 43.0 & (36.9-49.4) & 46.6 & $(40.9-52.5)$ & 44.7 & $(39.2-50.3)$ & 18.6 & $(14.1-24.1)$ & 24.2 & $(19.4-29.9)$ & 21.4 & $(17.3-26.2)$ \\
\hline Connecticut & - & - & - & - & - & - & - & - & - & - & - & - \\
\hline Delaware & 38.3 & (34.8-42.0) & 42.2 & (38.2-46.2) & 40.5 & (37.8-43.4) & 20.2 & $(17.5-23.1)$ & 26.8 & $(23.2-30.7)$ & 23.5 & $(21.5-25.6)$ \\
\hline Florida & - & - & - & - & - & - & - & - & - & - & - & - \\
\hline Hawaii & 43.6 & $(40.3-46.9)$ & 46.5 & (43.2-49.9) & 45.1 & (42.5-47.8) & 22.0 & $(19.6-24.5)$ & 28.2 & $(26.0-30.4)$ & 25.1 & (23.3-26.9) \\
\hline Idaho & 45.0 & $(41.1-49.0)$ & 45.5 & $(39.0-52.1)$ & 45.2 & $(41.0-49.5)$ & 25.5 & $(22.1-29.1)$ & 24.3 & (20.2-28.9) & 24.8 & $(22.0-28.0)$ \\
\hline Illinois & 46.1 & $(41.6-50.6)$ & 48.0 & $(41.5-54.6)$ & 47.0 & $(43.1-51.0)$ & 23.1 & $(19.6-27.1)$ & 29.8 & $(24.0-36.3)$ & 26.6 & $(23.3-30.1)$ \\
\hline Indiana & 40.3 & $(35.3-45.6)$ & 47.3 & $(41.7-53.0)$ & 43.9 & (39.5-48.5) & 20.3 & $(17.2-23.7)$ & 27.2 & $(22.5-32.5)$ & 23.9 & $(20.6-27.7)$ \\
\hline Kentucky & 39.5 & (35.3-43.9) & 43.9 & (38.3-49.7) & 41.7 & $(37.6-46.0)$ & 23.3 & (19.3-27.8) & 23.4 & $(20.2-27.1)$ & 23.4 & $(20.5-26.5)$ \\
\hline Maine & 31.0 & $(28.9-33.1)$ & 34.2 & $(31.7-36.9)$ & 32.7 & $(30.6-34.8)$ & 14.5 & $(13.1-16.0)$ & 18.8 & $(17.0-20.8)$ & 16.8 & $(15.5-18.3)$ \\
\hline Maryland & 37.0 & $(36.1-38.0)$ & 38.0 & $(37.0-39.0)$ & 37.6 & $(36.8-38.3)$ & 18.7 & $(18.0-19.5)$ & 20.8 & $(20.1-21.5)$ & 20.0 & (19.4-20.6) \\
\hline Massachusetts & 41.2 & $(37.5-45.0)$ & 48.2 & $(44.7-51.6)$ & 44.8 & $(41.8-47.8)$ & 19.9 & $(16.9-23.3)$ & 27.3 & $(24.3-30.5)$ & 23.7 & $(21.1-26.5)$ \\
\hline Michigan & - & - & - & - & - & - & 19.7 & $(16.3-23.5)$ & 26.3 & $(22.4-30.7)$ & 23.0 & $(20.1-26.2)$ \\
\hline Mississippi & 38.3 & $(34.2-42.6)$ & 42.5 & $(37.1-48.1)$ & 40.3 & $(36.8-43.8)$ & 20.3 & $(17.6-23.3)$ & 25.2 & $(21.4-29.4)$ & 22.9 & (20.4-25.6) \\
\hline Missouri & 35.0 & $(29.4-41.0)$ & 46.7 & $(40.4-53.0)$ & 40.6 & (36.5-44.9) & 16.5 & (13.3-20.3) & 27.7 & $(23.5-32.4)$ & 22.0 & (18.7-25.7) \\
\hline Montana & 49.0 & $(46.2-51.8)$ & 53.1 & $(50.1-56.0)$ & 51.1 & $(48.7-53.4)$ & 27.2 & $(24.8-29.7)$ & 31.8 & $(29.0-34.7)$ & 29.5 & (27.4-31.7) \\
\hline Nebraska & 41.0 & $(36.6-45.5)$ & 35.3 & $(30.8-40.1)$ & 38.2 & (34.6-41.9) & 22.8 & $(19.4-26.6)$ & 21.2 & $(17.1-25.9)$ & 22.3 & $(19.3-25.6)$ \\
\hline Nevada & 52.9 & $(46.4-59.4)$ & 51.2 & $(45.4-56.9)$ & 52.0 & $(47.3-56.7)$ & 24.9 & $(21.2-29.0)$ & 26.3 & $(21.6-31.7)$ & 25.6 & $(22.9-28.5)$ \\
\hline New Hampshire & - & - & - & - & - & - & 23.4 & $(21.7-25.2)$ & 26.2 & $(24.5-28.1)$ & 25.0 & $(23.6-26.4)$ \\
\hline New Mexico & 52.9 & $(50.1-55.7)$ & 55.8 & $(53.2-58.3)$ & 54.4 & $(52.2-56.5)$ & 21.9 & $(19.5-24.4)$ & 26.0 & (24.4-27.7) & 24.0 & (22.5-25.6) \\
\hline New York & - & - & - & - & - & - & 21.6 & (18.9-24.6) & 21.4 & $(18.6-24.4)$ & 21.7 & $(19.5-24.0)$ \\
\hline North Carolina & 46.0 & $(40.6-51.5)$ & 52.7 & $(48.4-56.9)$ & 49.4 & $(45.1-53.7)$ & 24.6 & $(19.9-30.0)$ & 34.6 & $(30.3-39.1)$ & 29.6 & $(25.8-33.7)$ \\
\hline North Dakota & 39.0 & $(35.0-43.1)$ & 45.1 & $(41.3-49.0)$ & 42.1 & (39.1-45.3) & 19.1 & (16.3-22.2) & 25.3 & (22.2-28.8) & 22.3 & (19.8-25.0) \\
\hline Oklahoma & 43.1 & (37.8-48.5) & 48.6 & $(42.9-54.3)$ & 46.0 & $(41.7-50.3)$ & 19.2 & $(16.2-22.6)$ & 28.0 & $(22.7-34.0)$ & 23.8 & $(20.6-27.3)$ \\
\hline Pennsylvania & 39.5 & (35.4-43.8) & 42.0 & $(38.0-46.1)$ & 40.8 & (37.3-44.4) & 21.3 & $(18.0-25.0)$ & 26.9 & $(22.7-31.6)$ & 24.1 & $(21.0-27.6)$ \\
\hline Rhode Island & 39.5 & $(35.1-44.1)$ & 42.3 & $(37.4-47.3)$ & 40.9 & $(36.7-45.3)$ & 18.0 & $(14.8-21.8)$ & 20.2 & $(16.1-25.2)$ & 19.3 & $(16.1-22.8)$ \\
\hline South Carolina & 44.1 & (39.6-48.7) & 41.5 & (36.9-46.3) & 42.9 & (39.2-46.7) & 20.1 & $(16.0-25.0)$ & 19.1 & $(15.6-23.2)$ & 19.7 & $(16.2-23.8)$ \\
\hline South Dakota & 41.3 & $(33.2-50.0)$ & 40.6 & $(36.0-45.4)$ & 41.0 & (35.8-46.4) & 16.0 & $(12.3-20.5)$ & 18.4 & $(12.9-25.5)$ & 17.3 & $(13.0-22.6)$ \\
\hline Tennessee & 40.3 & (36.9-43.9) & 42.7 & $(39.4-46.0)$ & 41.6 & $(38.8-44.5)$ & 18.9 & $(16.5-21.6)$ & 24.2 & $(22.5-25.9)$ & 21.7 & (19.9-23.6) \\
\hline Vermont & 27.5 & $(26.6-28.4)$ & 32.9 & $(31.9-33.8)$ & 30.4 & $(29.7-31.0)$ & 12.8 & $(12.2-13.5)$ & 17.6 & $(16.9-18.3)$ & 15.3 & $(14.8-15.8)$ \\
\hline Virginia & - & - & - & - & - & - & 15.5 & (13.0-18.4) & 17.8 & (15.4-20.4) & 16.8 & $(14.7-19.1)$ \\
\hline West Virginia & 46.9 & $(42.2-51.8)$ & 51.1 & $(45.6-56.5)$ & 49.1 & $(45.0-53.3)$ & 29.6 & $(25.6-34.0)$ & 32.6 & $(28.5-37.1)$ & 31.2 & $(27.8-34.9)$ \\
\hline Wyoming & 46.4 & $(41.2-51.7)$ & 52.3 & $(47.8-56.8)$ & 49.4 & $(45.4-53.4)$ & 25.8 & $(22.3-29.6)$ & 33.2 & $(29.3-37.2)$ & 29.6 & $(26.9-32.4)$ \\
\hline Median & & 41.0 & & 45.5 & & 42.9 & & 20.3 & & 26.2 & & 23.5 \\
\hline Range & & . & & (.9-55.8) & & $0.4-54.4)$ & & .8-29.6) & & $7.6-34.6)$ & & $5.3-31.2)$ \\
\hline \multicolumn{13}{|c|}{ Large urban school district surveys } \\
\hline Baltimore, MD & 34.6 & $(29.4-40.2)$ & 32.3 & $(27.8-37.2)$ & 33.6 & (30.0-37.4) & 15.3 & $(12.1-19.1)$ & 18.5 & $(14.4-23.5)$ & 17.6 & $(14.8-20.8)$ \\
\hline Boston, MA & - & - & - & - & - & - & 14.3 & $(11.3-18.0)$ & 14.7 & $(12.3-17.6)$ & 14.5 & (12.4-16.9) \\
\hline Broward County, FL & 43.9 & $(39.2-48.8)$ & 46.3 & $(42.0-50.6)$ & 45.1 & $(41.6-48.6)$ & 20.4 & $(17.3-24.0)$ & 24.3 & $(20.3-28.8)$ & 22.4 & $(20.0-25.0)$ \\
\hline Cleveland, $\mathrm{OH}$ & 35.6 & $(31.3-40.3)$ & 37.6 & $(33.3-42.0)$ & 37.0 & $(33.6-40.6)$ & 18.0 & $(14.9-21.5)$ & 18.4 & $(15.5-21.7)$ & 19.1 & $(17.0-21.4)$ \\
\hline DeKalb County, GA & 37.7 & $(34.9-40.7)$ & 36.4 & $(32.3-40.8)$ & 37.2 & (34.6-39.8) & 16.5 & $(14.4-18.9)$ & 18.1 & $(15.4-21.1)$ & 17.4 & $(15.6-19.4)$ \\
\hline Detroit, MI & - & - & - & - & - & - & 12.2 & $(9.5-15.4)$ & 13.5 & (10.9-16.5) & 13.0 & $(10.9-15.4)$ \\
\hline District of Columbia & 34.3 & $(33.0-35.6)$ & 33.7 & $(32.2-35.1)$ & 34.1 & $(33.1-35.1)$ & 13.4 & $(12.5-14.4)$ & 13.1 & $(12.1-14.1)$ & 13.4 & $(12.7-14.1)$ \\
\hline Duval County, FL & 46.4 & $(43.3-49.6)$ & 41.7 & (38.7-44.7) & 44.3 & $(41.9-46.8)$ & 21.8 & $(19.3-24.5)$ & 21.3 & $(19.3-23.4)$ & 22.1 & $(20.3-23.9)$ \\
\hline Ft. Worth, TX & 39.7 & (36.6-42.8) & 45.4 & $(42.1-48.7)$ & 42.6 & $(40.3-44.8)$ & 18.7 & $(16.1-21.7)$ & 21.2 & $(18.7-24.0)$ & 19.9 & $(17.9-22.1)$ \\
\hline Houston, TX & 37.2 & (34.6-39.8) & 41.8 & (39.0-44.7) & 39.7 & (37.6-41.9) & 18.1 & $(16.2-20.2)$ & 22.2 & $(19.9-24.8)$ & 20.4 & $(18.7-22.2)$ \\
\hline Los Angeles, CA & 41.3 & (36.9-45.9) & 46.4 & $(42.7-50.1)$ & 43.7 & $(41.0-46.5)$ & 15.8 & $(13.4-18.6)$ & 17.8 & $(14.5-21.6)$ & 16.8 & $(14.8-19.1)$ \\
\hline Miami-Dade County, FL & 36.5 & $(33.0-40.2)$ & 42.1 & $(39.1-45.1)$ & 39.3 & $(36.6-42.0)$ & 16.7 & $(14.5-19.2)$ & 22.6 & $(20.0-25.5)$ & 19.6 & $(17.8-21.5)$ \\
\hline
\end{tabular}

See table footnotes on the next page. 
TABLE 42. (Continued) Percentage of high school students who ever used electronic vapor products* and who currently used electronic vapor products, ${ }^{*}+$ by sex — selected U.S. sites, Youth Risk Behavior Survey, 2015

\begin{tabular}{|c|c|c|c|c|c|c|c|c|c|c|c|c|}
\hline \multirow[b]{3}{*}{ Site } & \multicolumn{6}{|c|}{ Ever used electronic vapor products } & \multicolumn{6}{|c|}{ Current electronic vapor products use } \\
\hline & \multicolumn{2}{|c|}{ Female } & \multicolumn{2}{|r|}{ Male } & \multicolumn{2}{|r|}{ Total } & \multicolumn{2}{|c|}{ Female } & \multicolumn{2}{|r|}{ Male } & \multicolumn{2}{|r|}{ Total } \\
\hline & $\%$ & $\mathrm{Cl}^{\S}$ & $\%$ & $\mathrm{Cl}$ & $\%$ & $\mathrm{Cl}$ & $\%$ & $\mathrm{Cl}$ & $\%$ & $\mathrm{Cl}$ & $\%$ & $\mathrm{Cl}$ \\
\hline New York City, NY & - & - & - & - & - & - & 16.9 & $(15.3-18.5)$ & 14.8 & $(13.0-16.7)$ & 15.9 & $(14.3-17.5)$ \\
\hline Oakland, CA & 43.6 & (39.4-47.8) & 46.3 & $(42.0-50.5)$ & 45.1 & $(42.3-47.9)$ & 14.9 & (11.9-18.4) & 17.4 & $(14.7-20.5)$ & 16.2 & $(14.2-18.4)$ \\
\hline Orange County, FL & 38.6 & (34.9-42.4) & 42.0 & $(37.8-46.4)$ & 40.2 & $(37.5-43.0)$ & 19.0 & $(16.3-21.9)$ & 21.0 & $(17.6-24.9)$ & 20.1 & $(18.0-22.3)$ \\
\hline Palm Beach County, FL & 48.6 & $(44.6-52.7)$ & 51.1 & $(47.6-54.5)$ & 49.9 & $(46.8-53.0)$ & 25.3 & $(22.2-28.6)$ & 28.5 & $(25.2-32.0)$ & 27.4 & $(24.9-30.1)$ \\
\hline Philadelphia, PA & 40.3 & $(36.6-44.1)$ & 38.4 & $(33.6-43.3)$ & 39.3 & (36.1-42.7) & 17.6 & $(14.1-21.7)$ & 17.0 & $(14.1-20.5)$ & 17.4 & $(14.6-20.5)$ \\
\hline San Diego, CA & 41.9 & $(37.1-46.9)$ & 47.8 & $(44.0-51.7)$ & 44.9 & $(41.5-48.3)$ & 17.7 & $(14.8-21.2)$ & 25.4 & $(22.2-28.8)$ & 21.6 & $(19.1-24.4)$ \\
\hline San Francisco, CA & 35.1 & (30.8-39.6) & 29.2 & $(24.6-34.4)$ & 32.2 & $(28.3-36.3)$ & 13.0 & $(10.4-16.0)$ & 13.4 & $(10.1-17.5)$ & 13.3 & $(10.7-16.4)$ \\
\hline Median & \multicolumn{2}{|c|}{39.1} & \multicolumn{2}{|c|}{41.9} & \multicolumn{2}{|r|}{39.9} & \multicolumn{2}{|c|}{16.9} & \multicolumn{2}{|c|}{18.4} & \multicolumn{2}{|r|}{17.6} \\
\hline Range & \multicolumn{2}{|c|}{$(34.3-48.6)$} & \multicolumn{2}{|c|}{$(29.2-51.1)$} & \multicolumn{2}{|c|}{ (32.2-49.9) } & \multicolumn{2}{|c|}{$(12.2-25.3)$} & \multicolumn{2}{|c|}{$(13.1-28.5)$} & \multicolumn{2}{|c|}{$(13.0-27.4)$} \\
\hline
\end{tabular}

* Including e-cigarettes, e-cigars, e-pipes, vape pipes, vaping pens, e-hookahs, and hookah pens.

${ }^{\dagger}$ On at least 1 day during the 30 days before the survey.

$\S 95 \%$ confidence interval.

I Not available.

TABLE 43. Percentage of high school students who currently smoked cigarettes or cigars* and who currently used cigarettes, cigars, or smokeless tobacco, * by sex, race/ethnicity, and grade - United States, Youth Risk Behavior Survey, 2015

\begin{tabular}{|c|c|c|c|c|c|c|c|c|c|c|c|c|}
\hline \multirow[b]{3}{*}{ Category } & \multicolumn{6}{|c|}{ Current cigarette or cigar use } & \multicolumn{6}{|c|}{ Current cigarette, cigar, or smokeless tobacco use } \\
\hline & \multicolumn{2}{|c|}{ Female } & \multicolumn{2}{|r|}{ Male } & \multicolumn{2}{|r|}{ Total } & \multicolumn{2}{|c|}{ Female } & \multicolumn{2}{|r|}{ Male } & \multicolumn{2}{|r|}{ Total } \\
\hline & $\%$ & $\mathrm{Cl}^{\dagger}$ & $\%$ & $\mathrm{Cl}$ & $\%$ & $\mathrm{Cl}$ & $\%$ & $\mathrm{Cl}$ & $\%$ & $\mathrm{Cl}$ & $\%$ & $\mathrm{Cl}$ \\
\hline \multicolumn{13}{|c|}{ Race/Ethnicity } \\
\hline White $\S$ & 14.7 & (10.9-19.4) & 20.4 & $(17.6-23.4)$ & 17.5 & $(14.6-20.9)$ & 15.6 & $(11.8-20.3)$ & 26.7 & $(23.3-30.3)$ & 21.2 & $(17.8-25.0)$ \\
\hline Black $^{\S}$ & 10.1 & $(7.6-13.2)$ & 16.8 & $(13.1-21.3)$ & 13.9 & $(11.3-16.9)$ & 10.3 & $(7.8-13.4)$ & 18.6 & $(14.7-23.4)$ & 14.9 & $(12.1-18.2)$ \\
\hline Hispanic & 9.7 & $(7.6-12.4)$ & 16.5 & $(13.8-19.5)$ & 13.1 & $(11.0-15.5)$ & 10.3 & $(8.0-13.0)$ & 18.1 & $(14.9-21.8)$ & 14.2 & $(11.8-17.0)$ \\
\hline \multicolumn{13}{|l|}{ Grade } \\
\hline 9 & 8.8 & $(6.4-11.8)$ & 12.3 & $(10.3-14.6)$ & 10.7 & $(8.9-12.9)$ & 9.3 & $(6.9-12.4)$ & 15.8 & (13.4-18.7) & 12.8 & $(10.8-15.1)$ \\
\hline 10 & 12.2 & $(9.3-15.9)$ & 15.6 & $(12.5-19.2)$ & 13.9 & $(11.3-17.0)$ & 12.6 & $(9.6-16.2)$ & 19.6 & $(15.6-24.5)$ & 16.1 & (13.0-19.7) \\
\hline 11 & 13.0 & $(10.2-16.4)$ & 22.9 & (19.9-26.3) & 18.3 & $(15.8-21.0)$ & 14.2 & $(11.5-17.5)$ & 28.8 & (25.4-32.4) & 21.9 & (19.3-24.8) \\
\hline 12 & 17.2 & $(14.1-20.8)$ & 25.9 & $(21.2-31.3)$ & 21.6 & $(18.4-25.1)$ & 17.9 & $(14.6-21.8)$ & 29.9 & $(24.7-35.8)$ & 23.9 & $(20.5-27.8)$ \\
\hline Total & 12.7 & $(10.5-15.3)$ & 19.0 & (17.2-20.9) & 16.0 & $(14.2-18.0)$ & 13.4 & $(11.2-16.0)$ & 23.3 & $(21.0-25.8)$ & 18.5 & $(16.5-20.8)$ \\
\hline
\end{tabular}

* On at least 1 day during the 30 days before the survey.

$\dagger 95 \%$ confidence interval.

$\S$ Non-Hispanic. 
TABLE 44. Percentage of high school students who currently smoked cigarettes or cigars* and who currently used cigarettes, cigars, or smokeless tobacco, ${ }^{*}$ by sex — selected U.S. sites, Youth Risk Behavior Survey, 2015

\begin{tabular}{|c|c|c|c|c|c|c|c|c|c|c|c|c|}
\hline \multirow[b]{3}{*}{ Site } & \multicolumn{6}{|c|}{ Current cigarette or cigar use } & \multicolumn{6}{|c|}{ Current cigarette, cigar, or smokeless tobacco use } \\
\hline & \multicolumn{2}{|r|}{ Female } & \multicolumn{2}{|r|}{ Male } & \multicolumn{2}{|r|}{ Total } & \multicolumn{2}{|r|}{ Female } & \multicolumn{2}{|r|}{ Male } & \multicolumn{2}{|r|}{ Total } \\
\hline & $\%$ & $\mathrm{Cl}^{\dagger}$ & $\%$ & $\mathrm{Cl}$ & $\%$ & $\mathrm{Cl}$ & $\%$ & $\mathrm{Cl}$ & $\%$ & $\mathrm{Cl}$ & $\%$ & $\mathrm{Cl}$ \\
\hline \multicolumn{13}{|l|}{ State surveys } \\
\hline Alabama & 19.5 & $(16.1-23.4)$ & 21.7 & $(18.8-24.9)$ & 20.9 & (18.2-23.9) & 20.7 & $(17.0-25.1)$ & 30.8 & $(27.0-34.8)$ & 26.3 & $(22.6-30.2)$ \\
\hline Alaska & 10.1 & (7.4-13.7) & 18.8 & $(15.5-22.6)$ & 14.8 & (12.4-17.6) & 14.7 & $(11.5-18.5)$ & 26.1 & $(22.7-29.7)$ & 20.8 & $(17.8-24.0)$ \\
\hline Arizona & 12.9 & $(9.8-16.9)$ & 17.5 & $(14.2-21.5)$ & 15.3 & (12.4-18.7) & 14.7 & $(11.5-18.7)$ & 20.8 & $(16.7-25.7)$ & 17.9 & $(14.5-21.8)$ \\
\hline Arkansas & 19.4 & $(16.4-22.7)$ & 25.8 & $(20.9-31.3)$ & 22.7 & (19.3-26.4) & 20.3 & $(17.6-23.4)$ & 31.7 & (26.3-37.7) & 26.2 & $(22.7-30.1)$ \\
\hline California & 8.8 & $(5.7-13.2)$ & 12.4 & $(8.9-17.0)$ & 10.7 & $(7.8-14.6)$ & 9.1 & $(6.0-13.5)$ & 14.4 & (10.5-19.4) & 11.9 & $(8.7-15.9)$ \\
\hline Connecticut & — & - & - & - & - & - & - & - & - & - & - & - \\
\hline Delaware & 13.6 & $(10.0-18.2)$ & 17.1 & $(14.0-20.6)$ & 15.6 & (12.7-18.9) & 14.1 & $(10.4-18.7)$ & 19.8 & (16.4-23.7) & 17.1 & $(14.2-20.5)$ \\
\hline Florida & - & - & - & - & - & - & - & - & - & - & - & - \\
\hline Hawaii & - & - & - & - & - & - & - & - & - & - & - & - \\
\hline Idaho & 11.3 & $(8.6-14.6)$ & 15.5 & $(12.6-18.9)$ & 13.5 & $(11.3-15.9)$ & 12.4 & $(9.5-16.1)$ & 22.1 & $(17.7-27.2)$ & 17.4 & $(14.5-20.7)$ \\
\hline Illinois & 11.3 & $(9.4-13.5)$ & 18.5 & $(14.6-23.3)$ & 15.0 & $(13.1-17.2)$ & 12.2 & $(10.0-14.7)$ & 20.9 & $(17.0-25.3)$ & 16.7 & (14.7-18.9) \\
\hline Indiana & 14.0 & (11.0-17.6) & 20.7 & (15.2-27.4) & 17.6 & $(13.8-22.2)$ & 15.0 & (11.7-18.9) & 27.1 & (19.5-36.3) & 21.4 & $(16.3-27.6)$ \\
\hline Kentucky & 22.2 & $(17.9-27.2)$ & 24.1 & (20.7-27.9) & 23.4 & $(20.2-26.9)$ & 23.0 & (18.7-27.9) & 32.6 & $(28.5-37.1)$ & 28.0 & $(24.5-31.8)$ \\
\hline Maine & 12.7 & (11.3-14.2) & 17.7 & (15.9-19.6) & 15.4 & $(14.0-16.8)$ & 13.1 & (11.8-14.7) & 19.8 & (17.8-21.9) & 16.7 & $(15.2-18.3)$ \\
\hline Maryland & 12.1 & (11.4-12.8) & 16.0 & $(15.3-16.7)$ & 14.4 & $(13.8-15.0)$ & 13.1 & (12.4-13.9) & 19.0 & (18.2-19.8) & 16.4 & $(15.8-17.1)$ \\
\hline Massachusetts & 9.7 & $(7.7-12.1)$ & 18.8 & $(15.6-22.5)$ & 14.3 & $(12.0-17.0)$ & 10.2 & $(8.2-12.5)$ & 21.4 & $(18.1-25.1)$ & 15.9 & $(13.6-18.5)$ \\
\hline Michigan & 10.8 & $(8.0-14.3)$ & 20.1 & $(16.3-24.5)$ & 15.5 & $(12.9-18.5)$ & 11.6 & $(8.9-14.9)$ & 23.4 & $(19.3-28.0)$ & 17.6 & $(15.0-20.5)$ \\
\hline Mississippi & 21.0 & $(16.6-26.2)$ & 28.3 & $(24.2-32.9)$ & 24.8 & $(21.6-28.3)$ & 22.6 & (18.2-27.7) & 34.1 & $(29.9-38.7)$ & 28.6 & $(25.3-32.3)$ \\
\hline Missouri & 12.2 & $(8.8-16.6)$ & 22.8 & $(18.5-27.9)$ & 17.6 & $(13.8-22.1)$ & 14.5 & $(10.5-19.6)$ & 29.8 & $(24.5-35.7)$ & 22.1 & $(17.4-27.6)$ \\
\hline Montana & 17.1 & (15.0-19.4) & 22.8 & $(20.5-25.2)$ & 20.1 & $(18.2-22.1)$ & 19.1 & (16.6-21.8) & 31.0 & $(28.3-33.9)$ & 25.3 & $(23.0-27.8)$ \\
\hline Nebraska & 14.2 & $(10.6-18.8)$ & 18.6 & $(15.1-22.6)$ & 16.6 & $(13.6-20.1)$ & 15.7 & $(11.9-20.5)$ & 24.2 & $(20.0-28.9)$ & 20.1 & $(16.8-23.9)$ \\
\hline Nevada & 9.8 & $(7.2-13.2)$ & 11.9 & $(9.3-15.2)$ & 11.0 & $(8.9-13.4)$ & 10.6 & $(7.9-14.0)$ & 15.1 & $(12.6-17.9)$ & 13.0 & $(11.1-15.1)$ \\
\hline New Hampshire & 11.1 & $(10.1-12.2)$ & 19.8 & $(18.3-21.4)$ & 15.7 & $(14.7-16.9)$ & 11.6 & $(10.5-12.7)$ & 22.5 & $(20.6-24.5)$ & 17.4 & $(16.1-18.7)$ \\
\hline New Mexico & 11.9 & $(10.7-13.3)$ & 18.5 & (16.8-20.4) & 15.3 & $(14.1-16.6)$ & 13.1 & $(11.9-14.4)$ & 24.4 & $(21.9-27.2)$ & 18.9 & (17.4-20.6) \\
\hline New York & 11.1 & $(9.6-12.7)$ & 18.2 & $(14.7-22.4)$ & 15.0 & $(12.8-17.4)$ & 12.2 & $(10.7-13.9)$ & 20.6 & $(17.1-24.6)$ & 16.8 & $(14.7-19.1)$ \\
\hline North Carolina & - & - & - & - & - & - & - & - & - & - & - & - \\
\hline North Dakota & 13.0 & $(10.3-16.2)$ & 19.5 & $(16.8-22.5)$ & 16.3 & (14.3-18.7) & 14.0 & (11.3-17.4) & 27.3 & $(24.2-30.6)$ & 20.9 & $(18.6-23.5)$ \\
\hline Oklahoma & 12.3 & $(8.7-17.0)$ & 20.0 & $(15.9-24.7)$ & 16.2 & (13.1-19.8) & 12.9 & $(9.6-17.0)$ & 27.2 & $(22.4-32.7)$ & 20.0 & $(16.9-23.6)$ \\
\hline Pennsylvania & 13.5 & $(10.7-17.0)$ & 23.7 & $(19.8-28.1)$ & 18.7 & $(15.7-22.1)$ & 14.5 & (11.3-18.4) & 29.4 & $(24.5-34.8)$ & 22.1 & $(18.6-26.1)$ \\
\hline Rhode Island & 7.4 & $(6.0-9.2)$ & 14.4 & $(12.0-17.2)$ & 11.1 & $(9.7-12.8)$ & 8.8 & $(7.2-10.6)$ & 17.3 & $(14.2-21.0)$ & 13.3 & $(11.3-15.6)$ \\
\hline South Carolina & 15.9 & $(12.0-20.7)$ & 16.6 & $(12.1-22.5)$ & 16.4 & (12.8-20.7) & 17.4 & $(13.7-21.7)$ & 21.7 & $(16.5-27.9)$ & 19.7 & $(15.8-24.1)$ \\
\hline South Dakota & 13.4 & $(9.5-18.6)$ & 17.1 & $(12.7-22.5)$ & 15.4 & (11.3-20.6) & 15.9 & $(11.7-21.2)$ & 28.5 & $(22.6-35.3)$ & 22.4 & $(18.0-27.5)$ \\
\hline Tennessee & 14.2 & $(12.4-16.3)$ & 19.3 & (16.9-21.9) & 17.0 & (15.3-18.8) & 15.0 & $(13.1-17.1)$ & 27.7 & $(25.1-30.5)$ & 21.7 & $(19.9-23.7)$ \\
\hline Vermont & 12.4 & $(11.7-13.0)$ & 19.4 & $(18.7-20.2)$ & 16.1 & (15.6-16.6) & 13.2 & $(12.6-13.9)$ & 22.7 & $(21.9-23.6)$ & 18.2 & $(17.7-18.8)$ \\
\hline Virginia & 10.2 & $(8.3-12.5)$ & 12.9 & $(11.1-15.1)$ & 11.7 & $(10.0-13.5)$ & 11.0 & $(9.0-13.4)$ & 17.0 & $(14.8-19.5)$ & 14.1 & $(12.3-16.2)$ \\
\hline West Virginia & 22.0 & $(18.4-26.2)$ & 26.4 & $(22.5-30.8)$ & 24.2 & $(20.9-27.9)$ & 22.9 & $(19.1-27.2)$ & 35.4 & (30.5-40.6) & 29.2 & $(25.5-33.2)$ \\
\hline Wyoming & 19.5 & $(15.8-23.7)$ & 22.0 & $(18.3-26.4)$ & 20.8 & $(17.8-24.2)$ & 21.1 & $(17.4-25.3)$ & 28.8 & $(25.0-33.0)$ & 25.1 & $(22.1-28.5)$ \\
\hline Median & & 12.7 & & 18.8 & & 15.7 & & 14.1 & & 24.2 & & 19.7 \\
\hline Range & & 7.4-22.2) & & 1.9-28.3) & & $(0.7-24.8)$ & & $8.8-23.0)$ & & $4.4-35.4)$ & & $(1.9-29.2)$ \\
\hline \multicolumn{13}{|c|}{ Large urban school district surveys } \\
\hline Baltimore, MD & 16.2 & $(11.8-21.9)$ & 17.9 & $(13.1-24.1)$ & 18.1 & $(14.3-22.6)$ & 17.1 & $(12.6-22.9)$ & 21.1 & $(15.9-27.5)$ & 20.4 & $(16.3-25.1)$ \\
\hline Boston, MA & 6.3 & $(4.6-8.4)$ & 9.9 & $(7.5-13.0)$ & 8.2 & $(6.7-10.0)$ & 6.6 & $(4.8-9.1)$ & 11.0 & $(8.6-14.0)$ & 9.0 & $(7.4-10.8)$ \\
\hline Broward County, FL & 5.9 & $(4.2-8.4)$ & 12.4 & $(9.4-16.1)$ & 9.4 & $(7.4-11.7)$ & 6.4 & $(4.6-8.9)$ & 14.0 & $(10.9-17.7)$ & 10.5 & $(8.4-12.9)$ \\
\hline Cleveland, $\mathrm{OH}$ & - & - & - & - & - & - & - & - & - & - & - & - \\
\hline DeKalb County, GA & 10.0 & $(8.0-12.5)$ & 13.8 & $(11.5-16.5)$ & 12.0 & $(10.2-14.0)$ & 10.2 & $(8.2-12.7)$ & 15.3 & $(12.8-18.2)$ & 12.8 & $(11.0-14.9)$ \\
\hline Detroit, MI & 14.2 & $(10.1-19.5)$ & 18.6 & $(14.4-23.8)$ & 16.4 & $(12.6-20.9)$ & 14.9 & $(10.9-20.2)$ & 20.8 & $(16.7-25.7)$ & 18.0 & $(14.3-22.4)$ \\
\hline District of Columbia & - & - & - & - & - & - & - & - & - & - & - & - \\
\hline Duval County, FL & 12.8 & $(10.6-15.4)$ & 17.6 & $(14.5-21.1)$ & 15.7 & $(13.3-18.4)$ & 14.1 & $(11.8-16.8)$ & 20.7 & $(17.3-24.5)$ & 17.9 & $(15.3-20.7)$ \\
\hline Ft. Worth, TX & 11.0 & $(9.2-13.1)$ & 15.0 & $(12.5-17.9)$ & 12.9 & $(11.4-14.7)$ & 11.4 & $(9.6-13.6)$ & 16.6 & $(14.0-19.6)$ & 14.0 & $(12.3-15.8)$ \\
\hline Houston, TX & 16.0 & $(13.9-18.3)$ & 18.6 & $(16.3-21.2)$ & 17.7 & $(15.9-19.7)$ & 17.5 & (15.4-19.9) & 21.1 & $(18.8-23.6)$ & 19.8 & $(17.8-21.8)$ \\
\hline Los Angeles, CA & 5.0 & $(3.0-8.2)$ & 8.5 & $(6.1-11.9)$ & 6.8 & $(4.9-9.2)$ & 5.5 & (3.4-8.7) & 9.6 & $(7.1-13.0)$ & 7.5 & $(5.6-10.1)$ \\
\hline Miami-Dade County, FL & 5.7 & $(4.3-7.4)$ & 11.4 & $(9.3-13.9)$ & 8.5 & $(7.2-10.1)$ & 6.1 & $(4.8-7.7)$ & 12.2 & $(10.1-14.6)$ & 9.1 & $(7.8-10.6)$ \\
\hline
\end{tabular}

See table footnotes on the next page. 
TABLE 44. (Continued) Percentage of high school students who currently smoked cigarettes or cigars* and who currently used cigarettes, cigars, or smokeless tobacco,* by sex - selected U.S. sites, Youth Risk Behavior Survey, 2015

\begin{tabular}{|c|c|c|c|c|c|c|c|c|c|c|c|c|}
\hline \multirow[b]{3}{*}{ Site } & \multicolumn{6}{|c|}{ Current cigarette or cigar use } & \multicolumn{6}{|c|}{ Current cigarette, cigar, or smokeless tobacco use } \\
\hline & \multicolumn{2}{|c|}{ Female } & \multicolumn{2}{|r|}{ Male } & \multicolumn{2}{|r|}{ Total } & \multicolumn{2}{|r|}{ Female } & \multicolumn{2}{|r|}{ Male } & \multicolumn{2}{|r|}{ Total } \\
\hline & $\%$ & $\mathrm{Cl}^{\dagger}$ & $\%$ & $\mathrm{Cl}$ & $\%$ & $\mathrm{Cl}$ & $\%$ & $\mathrm{Cl}$ & $\%$ & $\mathrm{Cl}$ & $\%$ & $\mathrm{Cl}$ \\
\hline New York City, NY & 6.6 & $(5.6-7.7)$ & 11.1 & $(9.4-13.0)$ & 9.0 & $(7.8-10.4)$ & 7.4 & $(6.3-8.6)$ & 12.6 & $(10.9-14.5)$ & 10.2 & $(9.0-11.5)$ \\
\hline Oakland, CA & 7.1 & $(5.1-9.6)$ & 11.6 & $(9.2-14.5)$ & 9.6 & (7.9-11.7) & 7.5 & $(5.5-10.1)$ & 12.7 & $(9.9-16.0)$ & 10.4 & $(8.5-12.7)$ \\
\hline Orange County, FL & 7.7 & $(5.8-10.1)$ & 12.0 & $(9.2-15.3)$ & 9.9 & $(8.1-12.0)$ & 8.3 & $(6.4-10.7)$ & 14.0 & (11.0-17.8) & 11.2 & $(9.3-13.5)$ \\
\hline Palm Beach County, FL & 12.1 & $(9.7-15.0)$ & 18.3 & $(15.4-21.5)$ & 16.0 & $(13.9-18.4)$ & 13.0 & $(10.5-16.0)$ & 20.6 & $(17.4-24.3)$ & 17.8 & $(15.3-20.6)$ \\
\hline Philadelphia, PA & 12.5 & $(9.3-16.7)$ & 15.7 & $(11.9-20.5)$ & 14.3 & $(11.4-17.8)$ & 13.8 & $(10.2-18.3)$ & 18.9 & $(14.3-24.6)$ & 16.5 & $(13.3-20.3)$ \\
\hline San Diego, CA & - & - & - & - & - & - & - & - & - & - & - & - \\
\hline San Francisco, CA & - & - & - & - & - & - & - & - & - & - & - & - \\
\hline Median & \multirow{2}{*}{\multicolumn{2}{|c|}{$\begin{array}{c}10.0 \\
(5.0-16.2)\end{array}$}} & \multirow{2}{*}{\multicolumn{2}{|c|}{$\begin{array}{c}13.8 \\
(8.5-18.6)\end{array}$}} & \multirow{2}{*}{\multicolumn{2}{|c|}{$\begin{array}{c}12.0 \\
(6.8-18.1)\end{array}$}} & \multirow{2}{*}{\multicolumn{2}{|c|}{$\begin{array}{c}10.2 \\
(5.5-17.5)\end{array}$}} & \multirow{2}{*}{\multicolumn{2}{|c|}{$\begin{array}{c}15.3 \\
(9.6-21.1)\end{array}$}} & \multirow{2}{*}{\multicolumn{2}{|c|}{$\begin{array}{c}12.8 \\
(7.5-20.4)\end{array}$}} \\
\hline Range & & & & & & & & & & & & \\
\hline
\end{tabular}

* On at least 1 day during the 30 days before the survey.

$+95 \%$ confidence interval.

$\S$ Not available.

TABLE 45. Percentage of high school students who currently used tobacco,* by sex, race/ethnicity, and grade - United States, Youth Risk Behavior Survey, 2015

\begin{tabular}{|c|c|c|c|c|c|c|}
\hline \multirow[b]{2}{*}{ Category } & \multicolumn{2}{|c|}{ Female } & \multicolumn{2}{|c|}{ Male } & \multicolumn{2}{|c|}{ Total } \\
\hline & $\%$ & $\mathrm{Cl}^{\dagger}$ & $\%$ & $\mathrm{Cl}$ & $\%$ & $\mathrm{Cl}$ \\
\hline \multicolumn{7}{|c|}{ Race/Ethnicity } \\
\hline White ${ }^{\S}$ & 29.4 & $(24.6-34.8)$ & 36.6 & (32.8-40.5) & 32.9 & $(29.0-37.1)$ \\
\hline Black $^{\S}$ & 21.2 & $(17.7-25.1)$ & 30.6 & $(25.3-36.4)$ & 26.3 & $(22.7-30.3)$ \\
\hline Hispanic & 29.4 & $(25.6-33.6)$ & 34.2 & $(30.6-38.0)$ & 31.8 & $(28.7-35.1)$ \\
\hline \multicolumn{7}{|l|}{ Grade } \\
\hline 9 & 23.0 & $(19.7-26.7)$ & 26.9 & $(24.0-30.0)$ & 25.1 & $(22.7-27.8)$ \\
\hline 10 & 26.3 & $(22.8-30.2)$ & 31.7 & (26.7-37.2) & 28.9 & $(25.1-33.1)$ \\
\hline 11 & 30.0 & (25.9-34.4) & 39.4 & (36.5-42.4) & 34.9 & $(32.1-37.9)$ \\
\hline 12 & 32.2 & $(27.9-36.8)$ & 43.0 & $(36.7-49.5)$ & 37.5 & (33.4-41.9) \\
\hline Total & 27.7 & $(25.1-30.6)$ & 34.9 & $(32.2-37.7)$ & 31.4 & $(29.1-33.8)$ \\
\hline
\end{tabular}

* Cigarette, smokeless tobacco, cigar, or electronic vapor products use on at least 1 day during the 30 days before the survey.

† $95 \%$ confidence interval.

$\S$ Non-Hispanic. 
TABLE 46. Percentage of high school students who currently used tobacco,* by sex — selected U.S. sites, Youth Risk Behavior Survey, 2015

\begin{tabular}{|c|c|c|c|c|c|c|}
\hline \multirow[b]{2}{*}{ Site } & \multicolumn{2}{|c|}{ Female } & \multicolumn{2}{|c|}{ Male } & \multicolumn{2}{|c|}{ Total } \\
\hline & $\%$ & $\mathrm{Cl}^{\dagger}$ & $\%$ & $\mathrm{Cl}$ & $\%$ & $\mathrm{Cl}$ \\
\hline \multicolumn{7}{|l|}{ State surveys } \\
\hline Alabama & 30.6 & (25.4-36.4) & 39.6 & $(35.2-44.1)$ & 35.5 & (31.0-40.3) \\
\hline Alaska & 25.1 & $(21.5-29.2)$ & 35.9 & $(31.9-40.1)$ & 30.9 & $(27.5-34.5)$ \\
\hline Arizona & 31.5 & $(26.3-37.1)$ & 37.6 & $(32.6-42.8)$ & 34.7 & $(31.0-38.5)$ \\
\hline Arkansas & 31.2 & (26.4-36.4) & 42.1 & (35.6-48.9) & 36.9 & (32.7-41.2) \\
\hline California & 23.8 & $(18.2-30.6)$ & 30.7 & $(25.5-36.4)$ & 27.4 & $(22.5-32.9)$ \\
\hline Connecticut & —§ & - & - & - & - & - \\
\hline Delaware & 25.9 & $(22.1-30.0)$ & 33.7 & $(30.0-37.6)$ & 29.8 & $(27.2-32.6)$ \\
\hline Florida & - & - & - & - & - & - \\
\hline Hawaii & - & - & - & - & - & - \\
\hline Idaho & 27.7 & $(24.0-31.7)$ & 33.0 & $(27.4-39.2)$ & 30.4 & $(26.7-34.4)$ \\
\hline Illinois & 28.2 & $(24.8-31.8)$ & 37.0 & $(30.6-44.0)$ & 32.8 & $(29.3-36.5)$ \\
\hline Indiana & 26.1 & $(22.4-30.1)$ & 38.2 & $(31.1-45.8)$ & 32.4 & $(27.3-38.0)$ \\
\hline Kentucky & 31.6 & $(26.7-36.8)$ & 39.6 & (35.1-44.3) & 35.8 & $(31.8-40.0)$ \\
\hline Maine & 21.0 & $(19.5-22.7)$ & 27.6 & $(25.5-29.7)$ & 24.5 & $(23.0-26.2)$ \\
\hline Maryland & 25.0 & $(24.1-25.9)$ & 29.6 & $(28.7-30.5)$ & 27.6 & (26.9-28.4) \\
\hline Massachusetts & 23.8 & $(20.5-27.5)$ & 34.6 & $(30.9-38.5)$ & 29.3 & $(26.2-32.7)$ \\
\hline Michigan & 23.5 & (19.7-27.8) & 34.4 & $(30.5-38.5)$ & 29.1 & $(26.2-32.2)$ \\
\hline Mississippi & 32.5 & $(29.1-36.1)$ & 42.1 & (38.0-46.4) & 37.6 & (34.7-40.6) \\
\hline Missouri & 24.6 & $(20.0-29.8)$ & 39.5 & $(34.4-45.0)$ & 32.1 & $(27.4-37.2)$ \\
\hline Montana & 33.5 & $(30.4-36.6)$ & 43.2 & $(40.1-46.4)$ & 38.5 & $(35.8-41.2)$ \\
\hline Nebraska & 28.3 & $(24.3-32.7)$ & 32.2 & $(27.4-37.3)$ & 30.5 & $(26.9-34.4)$ \\
\hline Nevada & 29.4 & $(25.4-33.8)$ & 31.3 & $(26.4-36.6)$ & 30.4 & $(27.6-33.4)$ \\
\hline New Hampshire & 26.5 & $(24.7-28.3)$ & 33.7 & $(31.5-35.9)$ & 30.3 & $(28.8-32.0)$ \\
\hline New Mexico & 27.1 & $(24.9-29.5)$ & 37.0 & $(34.7-39.3)$ & 32.2 & (30.4-34.0) \\
\hline New York & 26.9 & $(24.2-29.9)$ & 30.1 & $(26.5-34.0)$ & 28.8 & $(26.3-31.5)$ \\
\hline North Carolina & - & - & - & - & - & - \\
\hline North Dakota & 24.9 & $(21.7-28.4)$ & 37.0 & $(33.3-40.9)$ & 31.1 & $(28.3-34.1)$ \\
\hline Oklahoma & 24.6 & $(20.7-29.0)$ & 37.8 & $(31.8-44.2)$ & 31.4 & $(27.7-35.3)$ \\
\hline Pennsylvania & 26.6 & $(22.8-30.7)$ & 37.8 & $(32.7-43.2)$ & 32.3 & $(28.4-36.6)$ \\
\hline Rhode Island & 21.4 & $(18.0-25.2)$ & 28.3 & $(24.5-32.5)$ & 25.1 & $(22.0-28.5)$ \\
\hline South Carolina & 28.2 & $(23.4-33.4)$ & 29.8 & $(24.3-35.9)$ & 29.1 & $(24.6-34.0)$ \\
\hline South Dakota & 25.0 & $(19.8-31.0)$ & 35.2 & $(28.9-42.0)$ & 30.3 & $(25.2-35.8)$ \\
\hline Tennessee & 26.3 & $(23.6-29.1)$ & 36.8 & $(34.3-39.3)$ & 31.9 & $(29.7-34.1)$ \\
\hline Vermont & 20.0 & $(19.2-20.8)$ & 28.9 & $(28.0-29.8)$ & 24.7 & $(24.1-25.3)$ \\
\hline Virginia & 20.5 & $(17.4-23.9)$ & 24.6 & $(21.9-27.6)$ & 22.7 & $(20.3-25.3)$ \\
\hline West Virginia & 36.2 & $(31.5-41.3)$ & 45.1 & $(40.3-50.0)$ & 40.8 & (36.8-44.9) \\
\hline Wyoming & 33.3 & $(28.5-38.4)$ & 43.1 & $(38.5-47.8)$ & 38.4 & (34.6-42.4) \\
\hline Median & & & & & & \\
\hline Range & & & & & & \\
\hline \multicolumn{7}{|c|}{ Large urban school district surveys } \\
\hline Baltimore, MD & 25.6 & $(20.9-31.0)$ & 30.5 & $(24.6-37.2)$ & 29.2 & $(25.3-33.5)$ \\
\hline Boston, MA & 18.7 & $(15.3-22.7)$ & 21.0 & $(18.2-24.1)$ & 19.9 & $(17.5-22.7)$ \\
\hline Broward County, FL & 22.8 & $(19.6-26.5)$ & 30.9 & $(26.3-36.0)$ & 27.1 & $(24.4-30.0)$ \\
\hline Cleveland, $\mathrm{OH}$ & - & - & - & - & - & - \\
\hline DeKalb County, GA & 22.0 & $(19.2-25.0)$ & 25.7 & $(22.1-29.6)$ & 23.9 & $(21.4-26.7)$ \\
\hline Detroit, MI & 23.4 & $(18.9-28.5)$ & 27.3 & $(23.0-32.0)$ & 25.5 & $(21.7-29.7)$ \\
\hline District of Columbia & - & - & - & - & - & - \\
\hline Duval County, FL & 27.6 & $(24.7-30.7)$ & 31.7 & $(28.5-35.1)$ & 30.4 & $(27.8-33.0)$ \\
\hline Ft. Worth, TX & 23.2 & $(20.5-26.1)$ & 28.2 & $(25.1-31.5)$ & 25.6 & $(23.4-28.0)$ \\
\hline Houston, TX & 27.5 & $(25.2-29.9)$ & 32.6 & $(29.9-35.5)$ & 30.5 & $(28.3-32.8)$ \\
\hline Los Angeles, CA & 18.9 & $(16.2-21.9)$ & 21.9 & $(17.9-26.5)$ & 20.4 & $(17.8-23.3)$ \\
\hline Miami-Dade County, FL & 19.1 & $(16.7-21.9)$ & 27.7 & $(24.8-30.7)$ & 23.3 & $(21.3-25.5)$ \\
\hline
\end{tabular}

See table footnotes on the next page. 
TABLE 46. (Continued) Percentage of high school students who currently used tobacco,* by sex — selected U.S. sites, Youth Risk Behavior Survey, 2015

\begin{tabular}{|c|c|c|c|c|c|c|}
\hline \multirow[b]{2}{*}{ Site } & \multicolumn{2}{|c|}{ Female } & \multicolumn{2}{|c|}{ Male } & \multicolumn{2}{|c|}{ Total } \\
\hline & $\%$ & $\mathrm{Cl}^{\dagger}$ & $\%$ & $\mathrm{Cl}$ & $\%$ & $\mathrm{Cl}$ \\
\hline New York City, NY & 20.7 & $(18.8-22.6)$ & 21.5 & $(19.6-23.5)$ & 21.3 & $(19.6-23.1)$ \\
\hline Oakland, CA & 19.4 & $(16.0-23.3)$ & 24.8 & $(21.4-28.6)$ & 22.4 & $(19.7-25.3)$ \\
\hline Orange County, FL & 22.4 & $(19.3-25.7)$ & 27.1 & $(22.9-31.8)$ & 24.8 & $(22.2-27.6)$ \\
\hline Palm Beach County, FL & 30.2 & $(26.8-33.9)$ & 36.8 & (32.9-41.0) & 34.3 & $(31.2-37.5)$ \\
\hline Philadelphia, PA & 25.6 & $(21.8-29.8)$ & 29.3 & $(24.2-35.1)$ & 27.6 & $(23.8-31.8)$ \\
\hline San Diego, CA & - & - & - & - & - & - \\
\hline San Francisco, CA & - & - & - & - & - & - \\
\hline Median & \multirow{2}{*}{\multicolumn{2}{|c|}{$\begin{array}{c}22.8 \\
(18.7-30.2)\end{array}$}} & \multirow{2}{*}{\multicolumn{2}{|c|}{$\begin{array}{c}27.7 \\
(21.0-36.8)\end{array}$}} & \multirow{2}{*}{\multicolumn{2}{|c|}{$\begin{array}{c}25.5 \\
(19.9-34.3)\end{array}$}} \\
\hline Range & & & & & & \\
\hline
\end{tabular}

* Cigarette, smokeless tobacco, cigar, or electronic vapor products use on at least 1 day during the 30 days before the survey.

† $95 \%$ confidence interval.

$\S$ Not available.

TABLE 47. Percentage of high school students who ever drank alcohol* and who drank alcohol ${ }^{\dagger}$ for the first time before age 13 years, by sex, race/ethnicity, and grade — United States, Youth Risk Behavior Survey, 2015

\begin{tabular}{|c|c|c|c|c|c|c|c|c|c|c|c|c|}
\hline \multirow[b]{3}{*}{ Category } & \multicolumn{6}{|c|}{ Ever drank alcohol } & \multicolumn{6}{|c|}{ Drank alcohol before age 13 years } \\
\hline & \multicolumn{2}{|c|}{ Female } & \multicolumn{2}{|r|}{ Male } & \multicolumn{2}{|r|}{ Total } & \multicolumn{2}{|c|}{ Female } & \multicolumn{2}{|r|}{ Male } & \multicolumn{2}{|r|}{ Total } \\
\hline & $\%$ & $\mathrm{Cl}^{\S}$ & $\%$ & $\mathrm{Cl}$ & $\%$ & $\mathrm{Cl}$ & $\%$ & $\mathrm{Cl}$ & $\%$ & $\mathrm{Cl}$ & $\%$ & $\mathrm{Cl}$ \\
\hline \multicolumn{13}{|c|}{ Race/Ethnicity } \\
\hline Whiteq & 66.7 & $(60.5-72.4)$ & 64.0 & $(60.9-67.0)$ & 65.3 & $(61.2-69.2)$ & 11.7 & $(9.2-14.8)$ & 17.3 & $(14.9-20.0)$ & 14.5 & $(12.6-16.6)$ \\
\hline Black & 57.9 & $(51.2-64.3)$ & 51.0 & $(44.8-57.2)$ & 54.4 & $(48.8-59.9)$ & 16.9 & $(13.4-21.1)$ & 18.7 & $(15.6-22.3)$ & 18.0 & $(16.1-20.2)$ \\
\hline Hispanic & 68.6 & $(65.1-71.9)$ & 63.4 & $(60.1-66.5)$ & 65.9 & $(63.1-68.7)$ & 19.0 & $(16.0-22.3)$ & 23.6 & $(21.3-26.0)$ & 21.3 & $(19.4-23.4)$ \\
\hline \multicolumn{13}{|l|}{ Grade } \\
\hline 9 & 53.0 & (49.9-56.1) & 48.9 & $(46.0-51.9)$ & 50.8 & (48.4-53.3) & 18.8 & $(16.8-21.0)$ & 21.5 & (18.4-24.9) & 20.3 & $(18.5-22.2)$ \\
\hline 10 & 62.7 & (51.8-72.5) & 58.8 & $(53.9-63.6)$ & 60.8 & (53.9-67.3) & 15.8 & $(12.1-20.3)$ & 21.3 & $(17.9-25.2)$ & 18.6 & (15.6-21.9) \\
\hline 11 & 72.1 & $(67.9-76.0)$ & 68.7 & $(65.7-71.6)$ & 70.3 & $(67.5-73.0)$ & 12.9 & $(10.9-15.3)$ & 17.5 & $(15.1-20.3)$ & 15.2 & $(13.6-17.1)$ \\
\hline 12 & 75.2 & $(71.7-78.4)$ & 71.5 & $(67.4-75.3)$ & 73.3 & $(70.3-76.1)$ & 9.9 & $(7.9-12.3)$ & 17.0 & $(13.8-20.8)$ & 13.5 & $(11.5-15.8)$ \\
\hline Total & 65.3 & $(61.5-69.0)$ & 61.4 & $(59.1-63.7)$ & 63.2 & $(60.6-65.8)$ & 14.6 & $(12.7-16.6)$ & 19.7 & (17.9-21.5) & 17.2 & $(16.0-18.4)$ \\
\hline
\end{tabular}

* At least one drink of alcohol on at least 1 day during their life.

† Other than a few sips.

$\S 95 \%$ confidence interval.

" Non-Hispanic. 
TABLE 48. Percentage of high school students who ever drank alcohol* and who drank alcohol ${ }^{\dagger}$ for the first time before age 13 years, by sex selected U.S. sites, Youth Risk Behavior Survey, 2015

\begin{tabular}{|c|c|c|c|c|c|c|c|c|c|c|c|c|}
\hline \multirow[b]{3}{*}{ Site } & \multicolumn{6}{|c|}{ Ever drank alcohol } & \multicolumn{6}{|c|}{ Drank alcohol before age 13 years } \\
\hline & \multicolumn{2}{|c|}{ Female } & \multicolumn{2}{|r|}{ Male } & \multicolumn{2}{|c|}{ Total } & \multicolumn{2}{|c|}{ Female } & \multicolumn{2}{|c|}{ Male } & \multicolumn{2}{|r|}{ Total } \\
\hline & $\%$ & $\mathrm{Cl}^{\S}$ & $\%$ & $\mathrm{Cl}$ & $\%$ & $\mathrm{Cl}$ & $\%$ & $\mathrm{Cl}$ & $\%$ & $\mathrm{Cl}$ & $\%$ & $\mathrm{Cl}$ \\
\hline \multicolumn{13}{|l|}{ State surveys } \\
\hline Alabama & 62.7 & $(57.7-67.4)$ & 56.7 & $(52.8-60.5)$ & 59.8 & $(56.5-63.0)$ & 16.8 & $(13.6-20.5)$ & 23.4 & $(20.6-26.4)$ & 20.4 & $(18.2-22.9)$ \\
\hline Alaska & 58.4 & $(54.8-61.9)$ & 50.4 & $(45.4-55.4)$ & 54.4 & $(50.9-57.9)$ & 10.8 & $(8.4-13.8)$ & 17.4 & $(14.3-20.9)$ & 14.3 & $(12.2-16.7)$ \\
\hline Arizona & - & - & - & - & - & - & 16.2 & $(12.6-20.5)$ & 16.5 & $(12.8-21.0)$ & 16.5 & $(13.6-19.8)$ \\
\hline Arkansas & 63.9 & $(58.9-68.6)$ & 58.4 & $(51.8-64.6)$ & 61.2 & $(57.7-64.5)$ & 16.5 & $(14.1-19.1)$ & 25.3 & $(21.3-29.6)$ & 21.0 & (19.4-22.8) \\
\hline California & 61.8 & $(55.7-67.5)$ & 56.0 & $(49.0-62.9)$ & 58.7 & $(52.6-64.5)$ & 16.3 & $(13.7-19.4)$ & 17.4 & $(13.9-21.6)$ & 16.8 & $(14.5-19.4)$ \\
\hline Connecticut & - & - & - & - & - & - & 8.1 & $(6.5-10.1)$ & 12.7 & (10.7-15.0) & 10.6 & $(9.1-12.5)$ \\
\hline Delaware & 62.4 & (57.9-66.8) & 55.2 & (50.8-59.5) & 58.8 & $(55.2-62.2)$ & 14.0 & (11.7-16.5) & 16.2 & (13.8-19.0) & 15.3 & $(13.7-17.2)$ \\
\hline Florida & - & - & - & - & - & - & 13.5 & $(12.1-15.1)$ & 17.5 & $(15.6-19.6)$ & 15.6 & $(14.2-17.1)$ \\
\hline Hawaii & - & - & - & - & - & - & 15.0 & $(12.9-17.3)$ & 18.6 & $(15.8-21.7)$ & 16.9 & $(14.6-19.4)$ \\
\hline Idaho & 60.8 & $(55.3-66.0)$ & 55.5 & $(49.4-61.4)$ & 58.1 & $(53.8-62.3)$ & 13.5 & $(10.4-17.5)$ & 16.9 & $(13.4-21.1)$ & 15.3 & $(12.5-18.7)$ \\
\hline Illinois & 64.3 & (59.5-68.8) & 57.5 & $(50.5-64.3)$ & 60.9 & (55.9-65.7) & 13.0 & $(10.4-16.1)$ & 18.7 & (15.4-22.4) & 15.8 & $(13.5-18.5)$ \\
\hline Indiana & 63.2 & $(58.3-67.8)$ & 61.3 & $(56.4-65.9)$ & 62.3 & $(58.3-66.1)$ & 13.3 & $(10.4-17.0)$ & 17.3 & $(14.5-20.5)$ & 15.4 & $(12.9-18.1)$ \\
\hline Kentucky & 62.3 & (58.9-65.5) & 51.2 & $(46.3-56.2)$ & 56.8 & (53.4-60.2) & 13.5 & $(11.2-16.3)$ & 17.9 & (15.1-21.0) & 15.9 & $(13.9-18.0)$ \\
\hline Maine & 56.2 & (53.9-58.5) & 50.1 & $(48.1-52.2)$ & 53.2 & $(51.2-55.1)$ & 11.7 & $(10.5-13.1)$ & 15.3 & (13.9-16.7) & 13.6 & $(12.5-14.8)$ \\
\hline Maryland & 56.6 & $(55.7-57.5)$ & 47.8 & (46.8-48.9) & 52.3 & $(51.5-53.2)$ & 15.9 & (15.3-16.5) & 18.3 & $(17.7-19.0)$ & 17.3 & $(16.8-17.7)$ \\
\hline Massachusetts & 63.6 & $(60.2-66.9)$ & 58.8 & (55.1-62.3) & 61.3 & (58.3-64.2) & 11.1 & (8.9-13.8) & 14.1 & (11.9-16.7) & 12.9 & (11.2-14.8) \\
\hline Michigan & 60.7 & $(54.9-66.3)$ & 56.6 & $(51.0-61.9)$ & 58.7 & $(54.0-63.2)$ & 13.7 & $(11.5-16.1)$ & 16.0 & $(13.5-18.9)$ & 14.8 & $(13.1-16.8)$ \\
\hline Mississippi & 62.2 & $(57.4-66.9)$ & 57.2 & $(52.5-61.8)$ & 59.9 & $(56.1-63.5)$ & 21.0 & (18.3-23.9) & 27.4 & $(23.6-31.5)$ & 24.5 & $(22.0-27.1)$ \\
\hline Missouri & - & - & - & - & - & - & 14.2 & $(10.7-18.5)$ & 20.5 & $(17.3-24.0)$ & 17.3 & (14.3-20.8) \\
\hline Montana & 72.7 & $(70.5-74.7)$ & 67.3 & $(64.8-69.7)$ & 69.9 & $(68.2-71.7)$ & 17.1 & $(15.0-19.5)$ & 21.8 & $(19.5-24.2)$ & 19.6 & $(17.8-21.5)$ \\
\hline Nebraska & 56.5 & $(51.8-61.2)$ & 46.6 & $(42.8-50.5)$ & 51.7 & $(48.4-55.0)$ & 11.8 & $(9.1-15.0)$ & 14.9 & (11.9-18.4) & 13.7 & $(11.5-16.1)$ \\
\hline Nevada & 68.6 & $(63.8-73.1)$ & 61.0 & $(56.6-65.2)$ & 64.8 & $(60.9-68.5)$ & 13.6 & $(10.6-17.2)$ & 20.0 & $(16.3-24.4)$ & 16.9 & $(14.2-20.0)$ \\
\hline New Hampshire & - & - & - & - & - & - & 8.6 & (7.7-9.7) & 12.6 & $(11.5-13.8)$ & 10.8 & $(10.0-11.5)$ \\
\hline New Mexico & - & - & - & - & - & - & 17.3 & $(15.6-19.1)$ & 22.8 & $(21.0-24.8)$ & 20.1 & $(18.8-21.6)$ \\
\hline New York & - & - & - & - & - & - & 14.8 & $(13.3-16.4)$ & 17.0 & $(14.6-19.8)$ & 16.1 & (14.5-17.7) \\
\hline North Carolina & - & - & - & - & - & - & 11.4 & $(8.7-14.8)$ & 16.4 & $(13.8-19.2)$ & 14.0 & $(12.0-16.3)$ \\
\hline North Dakota & 64.0 & $(59.6-68.2)$ & 60.4 & $(57.0-63.6)$ & 62.1 & $(59.3-64.9)$ & 9.2 & $(7.4-11.3)$ & 15.3 & $(12.8-18.2)$ & 12.4 & $(10.6-14.4)$ \\
\hline Oklahoma & 65.6 & $(60.8-70.1)$ & 61.7 & $(56.7-66.4)$ & 63.5 & $(59.6-67.3)$ & 12.0 & $(9.0-15.9)$ & 18.1 & (15.1-21.6) & 15.0 & $(12.7-17.7)$ \\
\hline Pennsylvania & 62.9 & $(58.6-67.1)$ & 58.9 & $(54.7-62.9)$ & 60.8 & $(57.0-64.6)$ & 11.4 & $(9.4-13.7)$ & 14.7 & $(12.5-17.2)$ & 13.1 & (11.4-15.0) \\
\hline Rhode Island & 58.1 & $(54.1-62.0)$ & 47.1 & (42.9-51.4) & 52.5 & (49.3-55.8) & 10.3 & $(7.6-13.8)$ & 12.4 & $(9.2-16.4)$ & 11.4 & $(8.6-15.0)$ \\
\hline South Carolina & 61.5 & $(56.3-66.5)$ & 49.7 & $(42.5-56.9)$ & 55.8 & $(51.3-60.2)$ & 15.0 & (10.6-20.8) & 20.7 & $(17.3-24.6)$ & 17.8 & $(14.8-21.3)$ \\
\hline South Dakota & 62.8 & $(56.8-68.4)$ & 57.8 & (49.2-65.9) & 60.3 & (53.7-66.4) & 16.3 & $(13.0-20.2)$ & 20.1 & $(14.4-27.4)$ & 18.4 & $(14.2-23.4)$ \\
\hline Tennessee & - & - & - & - & - & - & - & - & - & - & - & - \\
\hline Vermont & - & - & - & - & - & - & 10.0 & $(9.4-10.6)$ & 14.4 & $(13.7-15.1)$ & 12.3 & $(11.9-12.8)$ \\
\hline Virginia & 51.9 & $(48.3-55.6)$ & 49.6 & $(45.7-53.5)$ & 50.7 & $(47.5-53.9)$ & 12.4 & $(10.6-14.5)$ & 16.4 & $(14.2-18.9)$ & 14.6 & $(13.0-16.3)$ \\
\hline West Virginia & 67.0 & $(62.4-71.3)$ & 63.3 & $(58.3-67.9)$ & 65.1 & $(61.1-68.8)$ & 14.8 & $(13.0-16.9)$ & 21.8 & $(19.2-24.7)$ & 18.4 & $(16.5-20.4)$ \\
\hline Wyoming & 62.4 & $(57.6-67.1)$ & 59.6 & $(54.4-64.7)$ & 61.0 & $(56.6-65.3)$ & 16.4 & $(13.3-20.0)$ & 21.6 & $(19.1-24.3)$ & 19.2 & (16.8-21.9) \\
\hline Median & & 62.4 & & 56.9 & & 59.8 & & 13.5 & & 17.4 & & 15.7 \\
\hline Range & $(51$ & $.9-72.7)$ & & $6.6-67.3)$ & & $0.7-69.9)$ & & $1-21.0)$ & & (4-27.4) & & $0.6-24.5)$ \\
\hline \multicolumn{13}{|c|}{ Large urban school district surveys } \\
\hline Baltimore, MD & 54.0 & $(49.0-58.9)$ & 47.2 & $(41.6-52.8)$ & 51.1 & $(47.0-55.2)$ & 18.4 & $(15.0-22.4)$ & 21.0 & $(17.1-25.6)$ & 20.0 & $(17.5-22.8)$ \\
\hline Boston, MA & 57.8 & $(53.2-62.4)$ & 51.4 & $(46.6-56.2)$ & 54.5 & $(50.8-58.3)$ & 15.3 & $(12.5-18.6)$ & 17.5 & $(14.7-20.7)$ & 16.5 & (14.4-18.8) \\
\hline Broward County, FL & 64.2 & $(59.3-68.8)$ & 58.2 & $(53.5-62.8)$ & 61.2 & $(57.2-65.1)$ & 17.2 & $(14.5-20.3)$ & 18.8 & $(15.8-22.3)$ & 18.1 & $(16.2-20.2)$ \\
\hline Cleveland, $\mathrm{OH}$ & 60.5 & $(57.0-63.9)$ & 52.8 & $(48.7-56.9)$ & 56.8 & $(53.8-59.8)$ & 20.7 & $(18.0-23.8)$ & 25.2 & $(22.2-28.5)$ & 23.6 & $(21.4-26.0)$ \\
\hline DeKalb County, GA & 54.6 & $(49.4-59.7)$ & 47.9 & $(43.5-52.2)$ & 51.3 & $(47.5-55.0)$ & 13.5 & $(11.2-16.1)$ & 18.9 & $(15.9-22.3)$ & 16.2 & $(14.2-18.5)$ \\
\hline Detroit, MI & 59.6 & $(54.6-64.3)$ & 49.0 & $(44.2-53.8)$ & 54.9 & $(51.2-58.6)$ & 18.6 & $(15.4-22.3)$ & 19.8 & $(17.0-22.9)$ & 19.2 & $(16.8-21.9)$ \\
\hline District of Columbia & 51.1 & $(49.7-52.5)$ & 41.6 & $(40.1-43.1)$ & 46.6 & - & 18.0 & $(17.0-19.2)$ & 19.4 & $(18.2-20.7)$ & 18.8 & $(18.0-19.7)$ \\
\hline Duval County, FL & - & - & - & - & - & - & 18.3 & $(15.6-21.2)$ & 21.2 & $(18.1-24.8)$ & 20.1 & $(17.5-22.8)$ \\
\hline Ft. Worth, TX & 63.2 & $(59.9-66.5)$ & 56.2 & $(53.2-59.2)$ & 59.7 & $(57.3-62.0)$ & 12.8 & $(11.0-14.7)$ & 18.9 & $(16.4-21.7)$ & 15.9 & (14.4-17.5) \\
\hline Houston, TX & 58.9 & $(56.0-61.7)$ & 53.0 & $(50.0-56.1)$ & 56.1 & (53.8-58.4) & 16.4 & $(14.1-18.9)$ & 19.0 & $(16.7-21.4)$ & 17.8 & $(16.1-19.6)$ \\
\hline Los Angeles, CA & 55.7 & $(50.7-60.6)$ & 50.2 & $(44.0-56.4)$ & 53.0 & $(47.8-58.2)$ & 13.4 & $(10.4-17.1)$ & 17.2 & $(14.4-20.3)$ & 15.2 & $(13.0-17.7)$ \\
\hline Miami-Dade County, FL & 63.5 & $(60.1-66.7)$ & 62.3 & $(59.2-65.3)$ & 62.8 & $(60.3-65.2)$ & 15.0 & $(12.6-17.9)$ & 22.7 & $(20.0-25.6)$ & 18.8 & $(17.0-20.9)$ \\
\hline
\end{tabular}

See table footnotes on the next page. 
TABLE 48. (Continued) Percentage of high school students who ever drank alcohol* and who drank alcohol ${ }^{\dagger}$ for the first time before age 13 years, by sex — selected U.S. sites, Youth Risk Behavior Survey, 2015

\begin{tabular}{|c|c|c|c|c|c|c|c|c|c|c|c|c|}
\hline \multirow[b]{3}{*}{ Site } & \multicolumn{6}{|c|}{ Ever drank alcohol } & \multicolumn{6}{|c|}{ Drank alcohol before age 13 years } \\
\hline & \multicolumn{2}{|c|}{ Female } & \multicolumn{2}{|r|}{ Male } & \multicolumn{2}{|r|}{ Total } & \multicolumn{2}{|c|}{ Female } & \multicolumn{2}{|r|}{ Male } & \multicolumn{2}{|r|}{ Total } \\
\hline & $\%$ & $\mathrm{Cl}^{\S}$ & $\%$ & $\mathrm{Cl}$ & $\%$ & $\mathrm{Cl}$ & $\%$ & $\mathrm{Cl}$ & $\%$ & $\mathrm{Cl}$ & $\%$ & $\mathrm{Cl}$ \\
\hline New York City, NY & - & - & - & - & - & - & 17.4 & $(15.8-19.1)$ & 18.5 & $(16.8-20.4)$ & 18.2 & $(16.9-19.5)$ \\
\hline Oakland, CA & 55.1 & $(51.0-59.1)$ & 50.7 & $(46.4-55.0)$ & 53.1 & $(49.7-56.4)$ & 17.1 & $(14.1-20.6)$ & 22.2 & $(19.1-25.8)$ & 19.9 & $(17.8-22.3)$ \\
\hline Orange County, FL & 59.2 & $(55.2-63.1)$ & 54.9 & $(50.1-59.7)$ & 56.9 & $(53.4-60.2)$ & 13.0 & $(10.5-15.9)$ & 18.1 & $(14.9-21.9)$ & 15.5 & $(13.6-17.6)$ \\
\hline Palm Beach County, FL & 65.1 & $(61.0-69.0)$ & 59.1 & $(55.0-63.0)$ & 62.5 & (59.2-65.7) & 16.3 & $(13.9-19.0)$ & 20.5 & $(18.0-23.3)$ & 18.8 & $(16.8-21.0)$ \\
\hline Philadelphia, PA & 65.5 & $(60.0-70.6)$ & 54.2 & $(49.4-58.9)$ & 60.0 & $(56.5-63.4)$ & 16.8 & $(14.1-20.0)$ & 18.2 & $(14.5-22.6)$ & 17.6 & $(15.1-20.5)$ \\
\hline San Diego, CA & 60.8 & $(56.0-65.3)$ & 52.8 & $(47.7-57.9)$ & 56.8 & $(52.7-60.8)$ & 12.4 & (9.6-15.9) & 16.9 & $(14.7-19.4)$ & 14.8 & $(13.0-16.9)$ \\
\hline San Francisco, CA & 46.3 & $(41.7-50.9)$ & 40.7 & $(35.9-45.7)$ & 43.5 & (39.6-47.6) & 13.2 & $(10.6-16.3)$ & 16.3 & $(13.8-19.1)$ & 15.1 & $(13.1-17.3)$ \\
\hline Median & \multicolumn{2}{|c|}{59.2} & \multicolumn{2}{|c|}{52.8} & \multicolumn{2}{|c|}{56.1} & \multicolumn{2}{|c|}{16.4} & \multicolumn{2}{|c|}{18.9} & \multicolumn{2}{|c|}{18.1} \\
\hline Range & \multicolumn{2}{|c|}{$(46.3-65.5)$} & \multicolumn{2}{|c|}{$(40.7-62.3)$} & \multicolumn{2}{|c|}{$(43.5-62.8)$} & \multicolumn{2}{|c|}{$(12.4-20.7)$} & \multicolumn{2}{|c|}{$(16.3-25.2)$} & \multicolumn{2}{|c|}{$(14.8-23.6)$} \\
\hline
\end{tabular}

* At least one drink of alcohol on at least 1 day during their life.

† Other than a few sips.

$\S 95 \%$ confidence interval.

१ Not available.

TABLE 49. Percentage of high school students who currently drank alcohol* and who usually obtained the alcohol they drank by someone giving it to them, ${ }^{+}$by sex, race/ethnicity, and grade — United States, Youth Risk Behavior Survey, 2015

\begin{tabular}{|c|c|c|c|c|c|c|c|c|c|c|c|c|}
\hline \multirow[b]{3}{*}{ Category } & \multicolumn{6}{|c|}{ Current alcohol use } & \multicolumn{6}{|c|}{ Someone gave alcohol to them } \\
\hline & \multicolumn{2}{|c|}{ Female } & \multicolumn{2}{|r|}{ Male } & \multicolumn{2}{|r|}{ Total } & \multicolumn{2}{|c|}{ Female } & \multicolumn{2}{|r|}{ Male } & \multicolumn{2}{|r|}{ Total } \\
\hline & $\%$ & $\mathrm{Cl}^{\S}$ & $\%$ & $\mathrm{Cl}$ & $\%$ & $\mathrm{Cl}$ & $\%$ & $\mathrm{Cl}$ & $\%$ & $\mathrm{Cl}$ & $\%$ & $\mathrm{Cl}$ \\
\hline \multicolumn{13}{|c|}{ Race/Ethnicity } \\
\hline White & 35.3 & $(29.0-42.1)$ & 35.2 & $(32.4-38.0)$ & 35.2 & $(31.2-39.3)$ & 50.7 & $(47.0-54.4)$ & 41.6 & (36.3-47.0) & 46.1 & $(42.8-49.5)$ \\
\hline Black 9 & 25.9 & $(19.9-32.9)$ & 22.1 & $(16.7-28.7)$ & 23.8 & $(18.6-30.0)$ & 46.2 & $(37.8-54.9)$ & 40.2 & $(28.8-52.7)$ & 43.3 & (35.7-51.3) \\
\hline Hispanic & 35.6 & $(32.3-39.0)$ & 33.4 & $(30.6-36.2)$ & 34.4 & $(31.9-37.0)$ & 45.9 & $(41.7-50.3)$ & 36.5 & (31.9-41.4) & 41.3 & $(38.0-44.8)$ \\
\hline \multicolumn{13}{|l|}{ Grade } \\
\hline 9 & 24.9 & $(22.2-27.8)$ & 22.1 & $(18.5-26.2)$ & 23.4 & $(20.9-26.1)$ & 50.4 & $(43.7-57.1)$ & 39.4 & (32.8-46.3) & 44.9 & $(40.0-49.9)$ \\
\hline 10 & 28.8 & $(22.8-35.7)$ & 29.3 & $(24.5-34.7)$ & 29.0 & $(24.3-34.3)$ & 50.0 & $(44.7-55.3)$ & 34.5 & $(27.4-42.3)$ & 42.3 & (38.4-46.3) \\
\hline 11 & 38.3 & $(33.2-43.6)$ & 37.7 & $(34.4-41.1)$ & 38.0 & (34.6-41.4) & 49.7 & $(46.0-53.5)$ & 39.3 & $(32.6-46.4)$ & 44.3 & $(40.3-48.3)$ \\
\hline 12 & 43.2 & $(38.4-48.2)$ & 41.6 & $(36.0-47.3)$ & 42.4 & (38.4-46.4) & 45.3 & $(39.8-50.9)$ & 44.9 & $(38.9-51.0)$ & 45.1 & $(41.2-49.1)$ \\
\hline Total & 33.5 & $(29.8-37.5)$ & 32.2 & $(30.4-34.0)$ & 32.8 & $(30.4-35.2)$ & 48.5 & $(45.8-51.2)$ & 39.9 & $(36.8-43.2)$ & 44.1 & $(41.9-46.4)$ \\
\hline
\end{tabular}

* At least one drink of alcohol on at least 1 day during the 30 days before the survey.

† During the 30 days before the survey, among the $32.8 \%$ of students nationwide who currently drank alcohol.

$\S 95 \%$ confidence interval.

१ Non-Hispanic. 
TABLE 50. Percentage of high school students who currently drank alcohol* and who usually obtained the alcohol they drank by someone giving it to them, ${ }^{\dagger}$ by sex — selected U.S. sites, Youth Risk Behavior Survey, 2015

\begin{tabular}{|c|c|c|c|c|c|c|c|c|c|c|c|c|}
\hline \multirow[b]{3}{*}{ Site } & \multicolumn{6}{|c|}{ Current alcohol use } & \multicolumn{6}{|c|}{ Someone gave alcohol to them } \\
\hline & \multicolumn{2}{|c|}{ Female } & \multicolumn{2}{|r|}{ Male } & \multicolumn{2}{|r|}{ Total } & \multicolumn{2}{|c|}{ Female } & \multicolumn{2}{|r|}{ Male } & \multicolumn{2}{|r|}{ Total } \\
\hline & $\%$ & $\mathrm{Cl}^{\S}$ & $\%$ & $\mathrm{Cl}$ & $\%$ & $\mathrm{Cl}$ & $\%$ & $\mathrm{Cl}$ & $\%$ & $\mathrm{Cl}$ & $\%$ & $\mathrm{Cl}$ \\
\hline \multicolumn{13}{|l|}{ State surveys } \\
\hline Alabama & 32.3 & $(28.0-36.9)$ & 28.8 & $(24.3-33.8)$ & 30.7 & $(27.2-34.3)$ & 39.4 & (32.0-47.2) & 24.4 & (19.0-30.9) & 32.3 & $(28.5-36.4)$ \\
\hline Alaska & 23.6 & $(20.8-26.8)$ & 20.5 & $(17.0-24.5)$ & 22.0 & $(19.7-24.5)$ & 40.5 & $(33.2-48.3)$ & 28.0 & $(20.5-37.1)$ & 34.4 & $(28.2-41.2)$ \\
\hline Arizona & 36.7 & (30.8-43.1) & 33.0 & $(27.3-39.2)$ & 34.8 & (29.7-40.2) & 53.5 & $(48.0-58.9)$ & 34.2 & (26.7-42.7) & 44.4 & (39.5-49.3) \\
\hline Arkansas & 26.6 & $(23.1-30.4)$ & 28.7 & $(23.3-34.7)$ & 27.6 & (24.5-30.8) & 52.3 & $(41.0-63.4)$ & 37.3 & (32.0-43.0) & 44.6 & (39.5-49.8) \\
\hline California & 31.0 & $(25.7-36.8)$ & 26.9 & (21.1-33.6) & 28.9 & $(23.6-34.7)$ & 49.7 & (39.5-60.0) & 35.6 & (30.2-41.4) & 43.2 & $(37.6-49.1)$ \\
\hline Connecticut & 32.0 & $(28.3-36.0)$ & 28.0 & $(24.5-31.7)$ & 30.2 & $(27.2-33.4)$ & 37.1 & $(31.6-43.0)$ & 31.3 & $(26.7-36.3)$ & 34.0 & $(30.4-37.7)$ \\
\hline Delaware & 35.6 & (30.1-41.4) & 27.4 & $(23.8-31.4)$ & 31.4 & $(27.7-35.4)$ & 47.2 & $(41.6-52.9)$ & 30.6 & $(24.6-37.3)$ & 39.7 & (35.0-44.6) \\
\hline Florida & 33.1 & $(30.6-35.7)$ & 32.7 & $(30.6-34.9)$ & 33.0 & $(31.1-35.0)$ & -q & - & - & - & - & - \\
\hline Hawaii & 26.7 & $(24.0-29.7)$ & 23.2 & $(20.5-26.2)$ & 25.1 & $(23.2-27.2)$ & - & - & - & - & - & - \\
\hline Idaho & 29.5 & (24.9-34.5) & 27.0 & (21.9-32.9) & 28.3 & $(24.0-33.0)$ & 51.2 & $(45.3-57.0)$ & 33.2 & (25.8-41.5) & 42.2 & (37.2-47.3) \\
\hline Illinois & 31.8 & $(26.7-37.4)$ & 29.6 & $(23.6-36.5)$ & 30.7 & $(26.7-35.1)$ & 45.4 & $(37.8-53.1)$ & 35.7 & (32.3-39.3) & 40.8 & (36.5-45.3) \\
\hline Indiana & 30.4 & $(26.1-35.2)$ & 30.4 & $(24.7-36.9)$ & 30.5 & $(26.3-35.2)$ & 41.0 & (34.2-48.1) & 36.3 & (25.5-48.7) & 38.6 & (31.2-46.5) \\
\hline Kentucky & 31.2 & $(27.5-35.1)$ & 25.6 & $(21.8-29.8)$ & 28.5 & $(25.2-32.0)$ & 44.8 & $(37.7-52.2)$ & 24.2 & $(17.6-32.4)$ & 35.5 & (30.7-40.5) \\
\hline Maine & 25.7 & $(23.8-27.6)$ & 22.3 & (20.9-23.7) & 24.0 & $(22.6-25.4)$ & 45.4 & $(41.6-49.3)$ & 36.4 & (32.7-40.2) & 41.0 & (37.9-44.3) \\
\hline Maryland & 29.1 & $(28.2-30.2)$ & 23.0 & (22.1-23.9) & 26.1 & $(25.3-27.0)$ & 50.3 & $(48.9-51.7)$ & 39.3 & (37.9-40.8) & 45.3 & $(44.3-46.3)$ \\
\hline Massachusetts & 34.0 & (30.4-37.9) & 33.7 & $(30.4-37.1)$ & 33.9 & $(30.9-36.9)$ & - & - & - & - & - & - \\
\hline Michigan & 25.7 & $(20.9-31.3)$ & 26.0 & (22.0-30.5) & 25.9 & (22.5-29.7) & 42.5 & (33.9-51.6) & 33.7 & (26.0-42.3) & 38.0 & $(32.2-44.3)$ \\
\hline Mississippi & 33.7 & $(28.6-39.1)$ & 29.0 & $(25.5-32.8)$ & 31.5 & $(28.2-34.9)$ & 41.3 & (34.7-48.2) & 40.1 & $(32.2-48.6)$ & 40.6 & $(36.1-45.2)$ \\
\hline Missouri & 34.3 & (28.4-40.8) & 34.8 & (30.1-39.7) & 34.5 & $(30.2-39.1)$ & 44.2 & (36.8-51.8) & 35.4 & (28.0-43.5) & 39.9 & (34.8-45.2) \\
\hline Montana & 35.0 & $(32.3-37.9)$ & 33.4 & $(31.0-35.8)$ & 34.2 & $(32.2-36.3)$ & 37.8 & (33.1-42.8) & 32.0 & $(28.1-36.1)$ & 34.9 & $(31.6-38.4)$ \\
\hline Nebraska & 24.9 & (20.9-29.3) & 20.5 & $(16.8-24.6)$ & 22.7 & $(19.6-26.2)$ & 47.6 & $(39.1-56.2)$ & 30.6 & $(24.0-38.1)$ & 39.6 & $(34.3-45.1)$ \\
\hline Nevada & 39.0 & (33.3-45.0) & 28.0 & $(23.3-33.1)$ & 33.5 & $(28.8-38.5)$ & 38.5 & (32.4-45.0) & 32.2 & $(24.8-40.6)$ & 35.7 & (30.9-40.7) \\
\hline New Hampshire & 31.1 & (29.0-33.3) & 28.6 & $(26.7-30.5)$ & 29.9 & (28.2-31.7) & 47.7 & $(45.0-50.3)$ & 37.2 & (34.1-40.5) & 42.3 & $(40.2-44.4)$ \\
\hline New Mexico & 27.0 & $(24.7-29.5)$ & 25.1 & $(23.1-27.2)$ & 26.1 & $(24.4-27.9)$ & 45.2 & (41.0-49.5) & 33.1 & (29.5-37.0) & 39.3 & $(36.6-42.2)$ \\
\hline New York & 32.4 & $(28.9-36.2)$ & 26.8 & $(22.0-32.1)$ & 29.7 & (26.3-33.4) & 36.9 & (34.5-39.5) & 26.5 & $(22.3-31.1)$ & 32.2 & $(29.2-35.4)$ \\
\hline North Carolina & 31.2 & $(27.7-34.9)$ & 27.1 & $(22.6-32.0)$ & 29.2 & $(26.0-32.5)$ & 46.4 & $(41.6-51.2)$ & 27.2 & $(22.1-32.9)$ & 37.3 & $(32.8-42.0)$ \\
\hline North Dakota & 29.6 & (25.7-33.8) & 31.9 & $(27.8-36.2)$ & 30.8 & $(27.7-34.0)$ & 48.6 & $(43.2-54.0)$ & 34.9 & $(30.0-40.1)$ & 41.3 & $(37.8-44.9)$ \\
\hline Oklahoma & 26.7 & $(22.8-31.1)$ & 27.9 & $(22.7-33.9)$ & 27.3 & $(23.5-31.5)$ & 57.3 & $(48.9-65.3)$ & 33.2 & (24.8-42.8) & 45.2 & (38.7-51.8) \\
\hline Pennsylvania & 32.6 & $(28.6-36.8)$ & 28.6 & $(24.7-32.9)$ & 30.6 & $(27.4-34.0)$ & 49.8 & $(43.2-56.4)$ & 40.3 & (33.4-47.5) & 45.2 & $(40.6-49.9)$ \\
\hline Rhode Island & 30.0 & $(24.9-35.6)$ & 22.3 & (18.4-26.7) & 26.1 & (22.1-30.6) & 46.6 & $(40.4-52.9)$ & 29.7 & (24.4-35.7) & 39.2 & (34.1-44.6) \\
\hline South Carolina & 29.8 & $(25.3-34.8)$ & 19.1 & $(14.2-25.1)$ & 24.6 & $(21.2-28.2)$ & 40.0 & (31.2-49.6) & 35.1 & (23.1-49.3) & 38.5 & (31.0-46.5) \\
\hline South Dakota & 30.6 & $(24.3-37.9)$ & 25.3 & $(19.9-31.5)$ & 28.0 & $(23.1-33.6)$ & - & - & - & - & - & - \\
\hline Tennessee & - & - & - & - & - & - & - & - & - & - & - & - \\
\hline Vermont & 30.3 & $(29.4-31.2)$ & 29.5 & $(28.6-30.4)$ & 30.0 & $(29.3-30.6)$ & 46.3 & $(44.5-48.1)$ & 35.3 & $(33.6-37.2)$ & 40.8 & $(39.5-42.1)$ \\
\hline Virginia & 23.8 & (20.9-27.0) & 23.0 & $(20.3-25.9)$ & 23.4 & $(21.1-25.8)$ & 46.7 & $(40.4-53.1)$ & 41.0 & (34.7-47.5) & 43.9 & (39.4-48.6) \\
\hline West Virginia & 32.7 & $(28.2-37.5)$ & 29.4 & $(26.0-33.0)$ & 31.1 & $(28.1-34.2)$ & 44.2 & $(38.0-50.6)$ & 34.9 & $(28.7-41.6)$ & 39.7 & $(36.5-43.1)$ \\
\hline Wyoming & 32.2 & $(28.8-35.9)$ & 29.6 & $(25.1-34.6)$ & 31.0 & $(28.1-34.0)$ & 48.6 & $(42.0-55.1)$ & 32.8 & $(26.9-39.2)$ & 40.7 & $(36.2-45.4)$ \\
\hline Median & & 31.0 & & 27.9 & & 29.8 & & 45.8 & & 33.9 & & 39.8 \\
\hline Range & & .6-39.0) & $(19$ & $.1-34.8)$ & $(22$ & $.0-34.8)$ & $(36$ & $9-57.3)$ & & $2-41.0)$ & & $.2-45.3)$ \\
\hline \multicolumn{13}{|c|}{ Large urban school district surveys } \\
\hline Baltimore, MD & 23.4 & $(19.1-28.3)$ & 17.0 & $(12.6-22.6)$ & 20.5 & $(16.8-24.7)$ & - & - & - & - & 26.9 & $(19.9-35.2)$ \\
\hline Boston, MA & 28.4 & $(24.7-32.4)$ & 21.3 & $(17.9-25.1)$ & 24.8 & $(22.3-27.6)$ & 40.0 & $(32.9-47.5)$ & 28.9 & $(22.1-36.8)$ & 35.3 & $(30.1-40.8)$ \\
\hline Broward County, FL & 32.9 & $(28.4-37.8)$ & 28.0 & $(23.1-33.5)$ & 30.6 & $(27.1-34.3)$ & 40.6 & $(33.6-48.1)$ & 38.4 & $(29.1-48.7)$ & 39.6 & $(34.7-44.8)$ \\
\hline Cleveland, $\mathrm{OH}$ & 28.8 & $(25.2-32.7)$ & 26.2 & $(23.1-29.5)$ & 27.9 & $(25.5-30.5)$ & - & - & - & - & - & - \\
\hline DeKalb County, GA & 23.0 & $(20.0-26.3)$ & 18.5 & $(15.0-22.5)$ & 20.8 & $(18.1-23.7)$ & 40.9 & $(33.1-49.1)$ & 28.2 & $(21.8-35.6)$ & 35.1 & $(29.7-41.0)$ \\
\hline Detroit, MI & 25.1 & $(20.5-30.3)$ & 19.4 & $(15.2-24.4)$ & 22.6 & $(19.0-26.7)$ & 37.7 & $(28.2-48.1)$ & 39.6 & $(27.9-52.6)$ & 37.9 & $(29.7-46.9)$ \\
\hline District of Columbia & 22.5 & $(21.4-23.7)$ & 17.4 & $(16.2-18.6)$ & 20.1 & $(19.3-21.0)$ & - & - & - & - & - & - \\
\hline Duval County, FL & 33.1 & $(30.0-36.3)$ & 26.3 & $(23.7-29.0)$ & 30.2 & $(28.1-32.5)$ & 48.5 & $(43.7-53.4)$ & 31.7 & $(26.4-37.5)$ & 40.5 & $(36.7-44.5)$ \\
\hline Ft. Worth, TX & 30.3 & $(27.5-33.3)$ & 25.9 & $(23.2-28.7)$ & 28.1 & $(26.1-30.3)$ & 46.0 & $(40.2-51.9)$ & 32.1 & $(27.4-37.3)$ & 39.9 & $(35.9-44.1)$ \\
\hline Houston, TX & 29.5 & $(26.8-32.4)$ & 22.5 & $(20.0-25.2)$ & 26.1 & $(24.1-28.1)$ & 42.2 & (37.1-47.5) & 26.6 & $(21.2-32.8)$ & 35.0 & $(31.2-39.0)$ \\
\hline Los Angeles, CA & 23.4 & (20.4-26.6) & 20.0 & $(14.6-26.7)$ & 21.7 & $(18.0-26.0)$ & 30.7 & $(23.0-39.6)$ & 37.8 & $(30.8-45.4)$ & 33.7 & $(27.7-40.2)$ \\
\hline Miami-Dade County, FL & 41.0 & $(36.7-45.6)$ & 35.4 & $(32.4-38.4)$ & 38.2 & (35.2-41.4) & 45.5 & $(40.5-50.6)$ & 35.9 & (30.5-41.7) & 41.2 & (37.8-44.6) \\
\hline
\end{tabular}

See table footnotes on the next page. 
TABLE 50. (Continued) Percentage of high school students who currently drank alcohol* and who usually obtained the alcohol they drank by someone giving it to them, ${ }^{\dagger}$ by sex — selected U.S. sites, Youth Risk Behavior Survey, 2015

\begin{tabular}{|c|c|c|c|c|c|c|c|c|c|c|c|c|}
\hline \multirow[b]{3}{*}{ Site } & \multicolumn{6}{|c|}{ Current alcohol use } & \multicolumn{6}{|c|}{ Someone gave alcohol to them } \\
\hline & \multicolumn{2}{|c|}{ Female } & \multicolumn{2}{|r|}{ Male } & \multicolumn{2}{|r|}{ Total } & \multicolumn{2}{|c|}{ Female } & \multicolumn{2}{|r|}{ Male } & \multicolumn{2}{|r|}{ Total } \\
\hline & $\%$ & $\mathrm{Cl}^{\S}$ & $\%$ & $\mathrm{Cl}$ & $\%$ & $\mathrm{Cl}$ & $\%$ & $\mathrm{Cl}$ & $\%$ & $\mathrm{Cl}$ & $\%$ & $\mathrm{Cl}$ \\
\hline New York City, NY & 23.8 & $(21.2-26.7)$ & 17.6 & $(15.1-20.5)$ & 20.9 & $(18.5-23.4)$ & 33.3 & $(30.0-36.8)$ & 31.7 & $(27.3-36.5)$ & 32.4 & $(29.4-35.6)$ \\
\hline Oakland, CA & 22.4 & $(18.8-26.4)$ & 21.8 & $(18.4-25.6)$ & 22.1 & $(19.4-25.0)$ & - & - & - & - & - & - \\
\hline Orange County, FL & 32.5 & $(28.8-36.3)$ & 25.8 & $(22.1-29.9)$ & 29.1 & $(26.0-32.4)$ & 49.9 & $(43.5-56.4)$ & 38.3 & $(29.5-47.9)$ & 44.8 & (39.4-50.3) \\
\hline Palm Beach County, FL & 36.1 & $(32.2-40.1)$ & 32.5 & $(29.1-36.2)$ & 34.5 & $(31.4-37.7)$ & 43.2 & $(37.5-49.1)$ & 32.9 & $(27.2-39.3)$ & 37.9 & $(33.7-42.2)$ \\
\hline Philadelphia, PA & 30.4 & $(26.8-34.2)$ & 22.5 & $(18.6-27.0)$ & 26.6 & $(23.5-29.8)$ & 46.7 & $(41.3-52.1)$ & 32.7 & $(24.8-41.8)$ & 40.7 & $(36.4-45.2)$ \\
\hline San Diego, CA & 26.1 & $(22.9-29.6)$ & 23.6 & $(20.6-26.9)$ & 24.8 & $(22.3-27.5)$ & - & - & - & - & - & - \\
\hline San Francisco, CA & 21.2 & $(17.8-25.1)$ & 15.8 & $(12.6-19.6)$ & 18.4 & $(15.5-21.8)$ & - & - & - & - & - & - \\
\hline Median & \multicolumn{2}{|r|}{28.4} & \multicolumn{2}{|c|}{22.5} & \multicolumn{2}{|c|}{24.8} & \multicolumn{2}{|r|}{42.2} & \multicolumn{2}{|r|}{32.7} & \multicolumn{2}{|r|}{37.9} \\
\hline Range & \multicolumn{2}{|c|}{$(21.2-41.0)$} & \multicolumn{2}{|c|}{$(15.8-35.4)$} & \multicolumn{2}{|c|}{$(18.4-38.2)$} & \multicolumn{2}{|c|}{ (30.7-49.9) } & \multicolumn{2}{|c|}{$(26.6-39.6)$} & \multicolumn{2}{|c|}{ (26.9-44.8) } \\
\hline
\end{tabular}

* At least one drink of alcohol on at least 1 day during the 30 days before the survey.

${ }^{\dagger}$ During the 30 days before the survey, among students who currently drank alcohol.

$\S 95 \%$ confidence interval.

I Not available.

TABLE 51. Percentage of high school students who drank five or more drinks of alcohol in a row* and whose largest number of drinks in a row was 10 or more, ${ }^{\dagger}$ by sex, race/ethnicity, and grade - United States, Youth Risk Behavior Survey, 2015

\begin{tabular}{|c|c|c|c|c|c|c|c|c|c|c|c|c|}
\hline \multirow[b]{3}{*}{ Category } & \multicolumn{6}{|c|}{ Five or more drinks in a row } & \multicolumn{6}{|c|}{ Largest number of drinks in a row was 10 or more } \\
\hline & \multicolumn{2}{|c|}{ Female } & \multicolumn{2}{|c|}{ Male } & \multicolumn{2}{|r|}{ Total } & \multicolumn{2}{|c|}{ Female } & \multicolumn{2}{|c|}{ Male } & \multicolumn{2}{|c|}{ Total } \\
\hline & $\%$ & $\mathrm{Cl}^{\S}$ & $\%$ & $\mathrm{Cl}$ & $\%$ & $\mathrm{Cl}$ & $\%$ & $\mathrm{Cl}$ & $\%$ & $\mathrm{Cl}$ & $\%$ & $\mathrm{Cl}$ \\
\hline \multicolumn{13}{|c|}{ Race/Ethnicity } \\
\hline White & 18.6 & $(15.0-22.7)$ & 21.0 & $(18.3-24.0)$ & 19.7 & $(16.8-23.0)$ & 2.4 & $(1.6-3.7)$ & 6.6 & $(5.2-8.4)$ & 4.5 & $(3.5-5.8)$ \\
\hline Black ${ }^{q}$ & 9.9 & $(6.8-14.3)$ & 12.8 & $(9.7-16.6)$ & 11.4 & $(8.8-14.7)$ & 1.0 & $(0.6-1.6)$ & 3.2 & $(1.7-5.9)$ & 2.1 & $(1.3-3.3)$ \\
\hline Hispanic & 17.9 & $(15.4-20.7)$ & 17.5 & $(15.4-19.8)$ & 17.7 & $(15.8-19.7)$ & 3.6 & $(2.7-4.8)$ & 6.5 & $(4.9-8.6)$ & 5.1 & $(4.2-6.2)$ \\
\hline \multicolumn{13}{|l|}{ Grade } \\
\hline 9 & 10.5 & $(8.8-12.6)$ & 10.2 & $(8.6-12.1)$ & 10.4 & $(9.1-11.8)$ & 2.3 & $(1.5-3.4)$ & 2.4 & $(1.4-4.1)$ & 2.4 & $(1.7-3.3)$ \\
\hline 10 & 14.1 & $(10.9-18.1)$ & 16.2 & $(12.7-20.3)$ & 15.1 & $(12.2-18.6)$ & 2.2 & $(1.4-3.6)$ & 6.3 & $(4.7-8.5)$ & 4.2 & $(3.2-5.6)$ \\
\hline 11 & 19.6 & $(16.1-23.8)$ & 24.4 & $(21.1-28.1)$ & 22.1 & (19.6-24.7) & 2.5 & $(1.4-4.4)$ & 7.3 & $(5.6-9.4)$ & 5.0 & $(3.9-6.4)$ \\
\hline 12 & 23.8 & $(20.7-27.1)$ & 25.6 & $(21.3-30.4)$ & 24.6 & $(21.5-28.0)$ & 3.0 & $(2.0-4.6)$ & 8.8 & $(6.5-11.8)$ & 5.9 & $(4.5-7.9)$ \\
\hline Total & 16.8 & (14.4-19.6) & 18.6 & $(16.9-20.5)$ & 17.7 & (15.8-19.8) & 2.5 & $(1.9-3.3)$ & 6.1 & $(5.1-7.2)$ & 4.3 & $(3.6-5.1)$ \\
\hline
\end{tabular}

* Within a couple of hours on at least 1 day during the 30 days before the survey.

+ Within a couple of hours during the 30 days before the survey.

$\S 95 \%$ confidence interval.

I Non-Hispanic. 
TABLE 52. Percentage of high school students who drank five or more drinks of alcohol in a row* and whose largest number of drinks in a row was 10 or more, ${ }^{\dagger}$ by sex — selected U.S. sites, Youth Risk Behavior Survey, 2015

\begin{tabular}{|c|c|c|c|c|c|c|c|c|c|c|c|c|}
\hline \multirow[b]{3}{*}{ Site } & \multicolumn{6}{|c|}{ Five or more drinks in a row } & \multicolumn{6}{|c|}{ Largest number of drinks in a row was 10 or more } \\
\hline & \multicolumn{2}{|c|}{ Female } & \multicolumn{2}{|r|}{ Male } & \multicolumn{2}{|r|}{ Total } & \multicolumn{2}{|c|}{ Female } & \multicolumn{2}{|c|}{ Male } & \multicolumn{2}{|c|}{ Total } \\
\hline & $\%$ & $\mathrm{Cl}^{\S}$ & $\%$ & $\mathrm{Cl}$ & $\%$ & $\mathrm{Cl}$ & $\%$ & $\mathrm{Cl}$ & $\%$ & $\mathrm{Cl}$ & $\%$ & $\mathrm{Cl}$ \\
\hline \multicolumn{13}{|l|}{ State surveys } \\
\hline Alabama & 16.2 & $(13.5-19.3)$ & 15.8 & $(12.7-19.6)$ & 16.1 & $(13.5-19.1)$ & 2.8 & $(1.4-5.4)$ & 6.1 & $(4.1-9.0)$ & 4.4 & $(2.9-6.7)$ \\
\hline Alaska & 12.0 & $(9.6-14.9)$ & 12.9 & $(10.4-16.0)$ & 12.5 & $(10.8-14.5)$ & - & - & - & - & - & - \\
\hline Arizona & 19.7 & $(15.1-25.2)$ & 18.3 & $(13.9-23.6)$ & 19.0 & $(14.8-24.1)$ & - & - & - & - & - & - \\
\hline Arkansas & 14.8 & $(13.2-16.6)$ & 18.0 & $(14.6-21.9)$ & 16.3 & $(14.8-18.0)$ & 2.9 & $(2.1-4.0)$ & 9.0 & $(6.6-12.1)$ & 5.9 & $(4.6-7.6)$ \\
\hline California & 14.8 & $(11.3-19.0)$ & 15.7 & $(10.8-22.2)$ & 15.1 & $(11.3-19.9)$ & 2.1 & $(1.1-3.9)$ & 3.8 & $(2.2-6.4)$ & 2.9 & $(2.0-4.4)$ \\
\hline Connecticut & 14.1 & (11.0-17.9) & 13.6 & (11.0-16.7) & 14.0 & (11.6-16.8) & - & - & - & - & - & - \\
\hline Delaware & 15.2 & (12.4-18.5) & 14.2 & (11.5-17.5) & 14.8 & (12.6-17.3) & 1.1 & $(0.6-1.9)$ & 5.4 & $(3.6-7.8)$ & 3.4 & $(2.5-4.6)$ \\
\hline Florida & 13.4 & $(11.8-15.1)$ & 16.8 & $(15.2-18.6)$ & 15.3 & (13.9-16.8) & - & - & - & - & - & - \\
\hline Hawaii & 13.2 & $(11.7-14.9)$ & 13.5 & $(11.3-16.1)$ & 13.4 & $(12.2-14.8)$ & 1.7 & $(1.2-2.4)$ & 3.4 & $(2.4-4.9)$ & 2.6 & $(2.0-3.2)$ \\
\hline Idaho & 15.5 & $(12.8-18.7)$ & 15.5 & $(11.5-20.7)$ & 15.6 & $(12.5-19.3)$ & - & - & - & - & - & - \\
\hline Illinois & 16.2 & $(12.7-20.5)$ & 15.6 & (10.9-21.9) & 15.9 & $(13.0-19.4)$ & 1.9 & $(1.0-3.6)$ & 5.1 & $(2.4-10.6)$ & 3.5 & $(2.0-5.9)$ \\
\hline Indiana & 16.3 & $(13.2-19.9)$ & 18.4 & $(13.6-24.6)$ & 17.4 & $(14.0-21.5)$ & 1.7 & $(0.9-3.5)$ & 6.7 & $(4.8-9.4)$ & 4.3 & $(3.0-6.1)$ \\
\hline Kentucky & 18.0 & (15.4-20.9) & 17.5 & (13.8-22.0) & 17.7 & $(15.1-20.6)$ & 4.0 & $(2.5-6.4)$ & 5.5 & $(3.5-8.6)$ & 4.7 & $(3.3-6.7)$ \\
\hline Maine & 11.1 & $(9.7-12.6)$ & 12.1 & $(11.2-13.2)$ & 11.7 & $(10.8-12.7)$ & - & - & - & - & - & - \\
\hline Maryland & 13.1 & $(12.5-13.9)$ & 12.8 & $(12.1-13.4)$ & 13.1 & $(12.5-13.6)$ & - & - & - & - & - & - \\
\hline Massachusetts & 15.5 & $(13.3-17.9)$ & 19.9 & $(16.9-23.4)$ & 17.7 & (15.4-20.4) & - & - & - & - & - & - \\
\hline Michigan & 12.2 & $(9.2-16.0)$ & 12.8 & $(9.5-17.1)$ & 12.5 & $(9.9-15.7)$ & 1.1 & $(0.5-2.2)$ & 4.3 & $(2.8-6.4)$ & 2.7 & $(1.8-3.9)$ \\
\hline Mississippi & 16.3 & (12.4-21.0) & 14.0 & $(10.8-18.0)$ & 15.3 & $(12.6-18.4)$ & 1.2 & $(0.6-2.5)$ & 4.7 & $(2.8-7.7)$ & 2.9 & $(2.0-4.2)$ \\
\hline Missouri & 17.8 & $(13.6-23.0)$ & 21.3 & $(16.6-26.8)$ & 19.5 & $(16.2-23.2)$ & - & - & - & - & - & - \\
\hline Montana & 20.3 & (18.4-22.3) & 21.1 & $(19.2-23.2)$ & 20.7 & $(19.2-22.3)$ & 3.5 & $(2.7-4.7)$ & 8.4 & $(7.1-9.9)$ & 6.0 & $(5.1-7.1)$ \\
\hline Nebraska & 14.4 & $(10.6-19.1)$ & 14.4 & $(10.9-18.7)$ & 14.3 & (11.4-17.8) & 2.6 & $(1.4-4.6)$ & 4.1 & $(2.6-6.6)$ & 3.3 & $(2.3-4.9)$ \\
\hline Nevada & 18.5 & $(15.3-22.1)$ & 13.4 & $(10.8-16.5)$ & 15.8 & (13.5-18.5) & - & - & - & - & - & - \\
\hline New Hampshire & 16.1 & $(14.6-17.6)$ & 17.2 & $(15.9-18.6)$ & 16.8 & $(15.7-17.9)$ & - & - & - & - & - & - \\
\hline New Mexico & 13.8 & $(12.1-15.7)$ & 15.2 & $(13.8-16.8)$ & 14.6 & $(13.3-15.9)$ & 2.7 & $(2.1-3.4)$ & 4.6 & $(3.8-5.5)$ & 3.6 & $(3.1-4.2)$ \\
\hline New York & 15.6 & $(13.2-18.3)$ & 15.5 & $(11.9-19.9)$ & 15.6 & $(12.9-18.6)$ & 1.7 & $(1.1-2.8)$ & 4.4 & $(2.8-6.7)$ & 3.0 & $(2.2-4.2)$ \\
\hline North Carolina & 14.8 & $(11.7-18.5)$ & 13.0 & $(10.9-15.5)$ & 13.9 & $(12.1-15.8)$ & - & - & - & - & - & - \\
\hline North Dakota & 15.7 & $(12.9-19.0)$ & 19.3 & $(16.0-23.1)$ & 17.6 & $(15.3-20.1)$ & - & - & - & - & - & - \\
\hline Oklahoma & 14.2 & (11.5-17.3) & 18.9 & $(14.8-23.7)$ & 16.5 & (13.6-19.8) & 2.3 & (1.4-3.7) & 9.3 & $(6.4-13.2)$ & 5.8 & $(4.1-8.0)$ \\
\hline Pennsylvania & 14.4 & $(12.0-17.1)$ & 16.3 & $(13.2-20.0)$ & 15.4 & $(13.2-18.0)$ & - & - & - & - & - & - \\
\hline Rhode Island & 13.9 & $(10.0-19.0)$ & 11.7 & $(8.7-15.5)$ & 12.8 & $(9.7-16.7)$ & - & - & - & - & - & - \\
\hline South Carolina & 12.3 & $(9.4-15.8)$ & 10.7 & $(7.2-15.7)$ & 11.5 & $(9.1-14.4)$ & 1.4 & $(0.9-2.3)$ & 3.1 & $(1.6-5.9)$ & 2.3 & $(1.5-3.5)$ \\
\hline South Dakota & 13.8 & $(10.4-18.2)$ & 14.6 & $(10.1-20.6)$ & 14.3 & $(11.1-18.3)$ & - & - & - & - & - & - \\
\hline Tennessee & - & - & - & - & - & - & - & - & - & - & - & - \\
\hline Vermont & 14.6 & (13.9-15.3) & 17.2 & $(16.5-18.0)$ & 16.0 & (15.5-16.5) & 1.7 & $(1.5-2.0)$ & 5.5 & $(5.0-5.9)$ & 3.6 & $(3.4-3.9)$ \\
\hline Virginia & 9.8 & $(8.1-11.9)$ & 12.1 & $(10.2-14.3)$ & 11.0 & $(9.5-12.6)$ & 1.2 & $(0.7-2.0)$ & 2.8 & $(2.0-3.9)$ & 2.1 & $(1.6-2.6)$ \\
\hline West Virginia & 21.5 & $(17.8-25.7)$ & 18.0 & (15.4-21.0) & 19.8 & (17.8-21.9) & 6.9 & $(4.7-10.2)$ & 7.8 & $(5.6-10.8)$ & 7.4 & $(6.1-8.9)$ \\
\hline Wyoming & 19.0 & $(16.1-22.3)$ & 20.4 & $(16.9-24.3)$ & 19.7 & $(17.3-22.3)$ & 5.3 & $(3.7-7.5)$ & 8.9 & $(6.9-11.5)$ & 7.2 & $(5.8-8.8)$ \\
\hline Median & & 14.8 & & 15.5 & & 15.5 & & .0 & & 5.2 & & .5 \\
\hline Range & & $8-21.5)$ & & .7-21.3) & & $.0-20.7)$ & & $-6.9)$ & & -9.3) & & $-7.4)$ \\
\hline \multicolumn{13}{|c|}{ Large urban school district surveys } \\
\hline Baltimore, MD & 8.7 & $(6.6-11.4)$ & 7.6 & $(5.0-11.5)$ & 8.3 & $(6.4-10.7)$ & 1.2 & $(0.4-3.4)$ & 1.5 & $(0.6-3.6)$ & 1.5 & $(0.8-2.8)$ \\
\hline Boston, MA & 11.1 & $(8.7-14.1)$ & 10.8 & $(8.4-13.8)$ & 10.9 & $(9.2-12.9)$ & - & - & - & - & - & - \\
\hline Broward County, FL & 11.6 & $(9.1-14.7)$ & 11.3 & $(8.8-14.5)$ & 11.6 & $(9.6-13.9)$ & 1.8 & $(0.9-3.9)$ & 2.4 & $(1.3-4.4)$ & 2.2 & $(1.4-3.5)$ \\
\hline Cleveland, $\mathrm{OH}$ & 12.7 & $(10.4-15.5)$ & 13.5 & $(11.1-16.4)$ & 13.3 & $(11.6-15.2)$ & - & - & - & - & - & - \\
\hline DeKalb County, GA & 7.4 & $(5.8-9.4)$ & 8.7 & $(6.6-11.3)$ & 8.0 & $(6.6-9.7)$ & - & - & - & - & - & - \\
\hline Detroit, MI & 9.7 & $(7.5-12.4)$ & 7.9 & $(5.8-10.7)$ & 9.0 & $(7.1-11.2)$ & 0.4 & $(0.1-1.1)$ & 0.9 & $(0.4-2.3)$ & 0.7 & $(0.4-1.3)$ \\
\hline District of Columbia & 8.1 & $(7.4-8.9)$ & 8.3 & $(7.5-9.2)$ & 8.3 & $(7.8-8.9)$ & - & - & - & - & - & - \\
\hline Duval County, FL & 11.6 & $(9.8-13.6)$ & 12.4 & $(10.4-14.7)$ & 12.5 & $(11.0-14.2)$ & - & - & - & - & - & - \\
\hline Ft. Worth, TX & 14.1 & $(11.9-16.7)$ & 14.6 & $(12.4-17.1)$ & 14.5 & $(13.0-16.2)$ & 1.8 & $(1.2-2.9)$ & 5.1 & $(3.8-6.8)$ & 3.4 & $(2.7-4.4)$ \\
\hline Houston, TX & 12.9 & $(11.3-14.8)$ & 11.9 & $(10.1-13.8)$ & 12.5 & $(11.2-13.9)$ & 3.0 & $(2.1-4.3)$ & 3.3 & $(2.4-4.6)$ & 3.2 & $(2.4-4.1)$ \\
\hline Los Angeles, CA & 10.6 & $(8.2-13.5)$ & 9.7 & $(6.5-14.3)$ & 10.2 & $(8.0-12.9)$ & 1.3 & $(0.9-2.0)$ & 2.9 & $(1.7-5.0)$ & 2.1 & $(1.4-3.1)$ \\
\hline Miami-Dade County, FL & 15.7 & $(13.2-18.7)$ & 16.4 & $(14.0-19.2)$ & 16.0 & $(14.0-18.4)$ & 1.3 & $(0.9-2.1)$ & 4.5 & $(3.3-6.1)$ & 3.0 & $(2.3-3.8)$ \\
\hline
\end{tabular}

See table footnotes on the next page. 
TABLE 52. (Continued) Percentage of high school students who drank five or more drinks of alcohol in a row* and whose largest number of drinks in a row was 10 or more, ${ }^{\dagger}$ by sex — selected U.S. sites, Youth Risk Behavior Survey, 2015

\begin{tabular}{|c|c|c|c|c|c|c|c|c|c|c|c|c|}
\hline \multirow[b]{3}{*}{ Site } & \multicolumn{6}{|c|}{ Five or more drinks in a row } & \multicolumn{6}{|c|}{ Largest number of drinks in a row was 10 or more } \\
\hline & \multicolumn{2}{|c|}{ Female } & \multicolumn{2}{|r|}{ Male } & \multicolumn{2}{|r|}{ Total } & \multicolumn{2}{|c|}{ Female } & \multicolumn{2}{|c|}{ Male } & \multicolumn{2}{|c|}{ Total } \\
\hline & $\%$ & $\mathrm{Cl}^{\S}$ & $\%$ & $\mathrm{Cl}$ & $\%$ & $\mathrm{Cl}$ & $\%$ & $\mathrm{Cl}$ & $\%$ & $\mathrm{Cl}$ & $\%$ & $\mathrm{Cl}$ \\
\hline New York City, NY & 9.1 & $(7.6-10.8)$ & 7.8 & $(6.6-9.3)$ & 8.5 & $(7.3-9.9)$ & 1.2 & $(0.8-1.9)$ & 1.2 & $(0.7-1.8)$ & 1.2 & $(1.0-1.5)$ \\
\hline Oakland, CA & 8.9 & $(6.9-11.3)$ & 12.1 & $(9.6-15.2)$ & 10.7 & $(8.9-12.8)$ & 1.7 & $(1.0-2.9)$ & 2.7 & $(1.8-4.3)$ & 2.2 & $(1.6-3.1)$ \\
\hline Orange County, FL & 12.1 & $(9.8-14.7)$ & 12.4 & $(9.6-15.8)$ & 12.3 & $(10.3-14.5)$ & 0.9 & $(0.3-2.5)$ & 3.9 & $(2.5-6.0)$ & 2.4 & $(1.6-3.6)$ \\
\hline Palm Beach County, FL & 16.8 & $(14.2-19.7)$ & 15.6 & $(13.4-18.2)$ & 16.5 & $(14.5-18.7)$ & 2.5 & $(1.6-3.9)$ & 3.9 & $(2.7-5.8)$ & 3.3 & $(2.3-4.6)$ \\
\hline Philadelphia, PA & 10.7 & $(8.4-13.6)$ & 10.8 & $(8.1-14.2)$ & 10.8 & $(8.6-13.5)$ & - & - & - & - & - & - \\
\hline San Diego, CA & 11.1 & $(9.0-13.6)$ & 14.0 & $(11.8-16.4)$ & 12.6 & $(10.9-14.5)$ & 1.4 & $(0.8-2.4)$ & 4.3 & $(3.2-5.9)$ & 2.8 & $(2.2-3.7)$ \\
\hline San Francisco, CA & 9.7 & $(7.6-12.3)$ & 8.1 & $(6.2-10.5)$ & 8.8 & $(7.2-10.8)$ & 1.6 & $(0.9-3.0)$ & 0.7 & $(0.3-1.5)$ & 1.2 & $(0.7-1.9)$ \\
\hline Median & \multicolumn{2}{|r|}{11.1} & \multicolumn{2}{|r|}{11.3} & \multicolumn{2}{|r|}{10.9} & \multicolumn{2}{|c|}{1.4} & \multicolumn{2}{|r|}{2.9} & \multicolumn{2}{|c|}{2.2} \\
\hline Range & \multicolumn{2}{|c|}{ (7.4-16.8) } & \multicolumn{2}{|r|}{$(7.6-16.4)$} & \multicolumn{2}{|r|}{$(8.0-16.5)$} & \multicolumn{2}{|c|}{$(0.4-3.0)$} & \multicolumn{2}{|c|}{$(0.7-5.1)$} & \multicolumn{2}{|c|}{$(0.7-3.4)$} \\
\hline
\end{tabular}

* Within a couple of hours on at least 1 day during the 30 days before the survey.

† Within a couple of hours during the 30 days before the survey.

$\S 95 \%$ confidence interval.

In Not available.

TABLE 53. Percentage of high school students who ever used marijuana* and who tried marijuana for the first time before age 13 years, by sex, race/ethnicity, and grade - United States, Youth Risk Behavior Survey, 2015

\begin{tabular}{|c|c|c|c|c|c|c|c|c|c|c|c|c|}
\hline \multirow[b]{3}{*}{ Category } & \multicolumn{6}{|c|}{ Ever used marijuana } & \multicolumn{6}{|c|}{ Tried marijuana before age 13 years } \\
\hline & \multicolumn{2}{|c|}{ Female } & \multicolumn{2}{|r|}{ Male } & \multicolumn{2}{|r|}{ Total } & \multicolumn{2}{|c|}{ Female } & \multicolumn{2}{|r|}{ Male } & \multicolumn{2}{|c|}{ Total } \\
\hline & $\%$ & $\mathrm{Cl}^{\dagger}$ & $\%$ & $\mathrm{Cl}$ & $\%$ & $\mathrm{Cl}$ & $\%$ & $\mathrm{Cl}$ & $\%$ & $\mathrm{Cl}$ & $\%$ & $\mathrm{Cl}$ \\
\hline \multicolumn{13}{|c|}{ Race/Ethnicity } \\
\hline White ${ }^{\S}$ & 34.3 & (29.0-40.0) & 36.2 & (31.6-41.1) & 35.2 & $(30.9-39.8)$ & 4.2 & $(3.0-5.7)$ & 6.7 & $(5.2-8.7)$ & 5.4 & $(4.3-6.9)$ \\
\hline Black ${ }^{\S}$ & 40.5 & $(36.2-45.0)$ & 49.7 & $(45.5-53.9)$ & 45.5 & (42.4-48.6) & 7.4 & $(4.9-10.9)$ & 13.0 & $(9.6-17.5)$ & 10.6 & $(8.4-13.3)$ \\
\hline Hispanic & 45.3 & $(41.7-48.9)$ & 46.0 & $(42.4-49.8)$ & 45.6 & $(42.8-48.5)$ & 8.2 & $(6.8-9.8)$ & 13.6 & $(11.7-15.7)$ & 10.9 & $(9.5-12.6)$ \\
\hline \multicolumn{13}{|l|}{ Grade } \\
\hline 9 & 25.3 & $(21.5-29.4)$ & 26.5 & $(23.7-29.5)$ & 25.9 & $(23.2-28.9)$ & 6.8 & $(5.2-8.8)$ & 9.9 & $(7.5-12.9)$ & 8.5 & $(7.1-10.1)$ \\
\hline 10 & 33.8 & $(27.7-40.5)$ & 37.1 & (31.9-42.6) & 35.5 & $(30.3-41.0)$ & 7.2 & $(5.0-10.1)$ & 9.4 & $(7.2-12.3)$ & 8.3 & $(6.5-10.5)$ \\
\hline 11 & 43.6 & (38.6-48.8) & 46.9 & $(43.2-50.6)$ & 45.2 & (41.4-49.0) & 4.5 & $(3.2-6.2)$ & 8.9 & $(6.8-11.5)$ & 6.7 & $(5.3-8.4)$ \\
\hline 12 & 48.8 & $(44.7-52.9)$ & 50.9 & $(44.6-57.2)$ & 49.8 & $(45.9-53.6)$ & 3.7 & $(2.7-4.9)$ & 8.5 & $(6.5-11.1)$ & 6.1 & $(4.9-7.5)$ \\
\hline Total & 37.5 & (33.7-41.3) & 39.8 & $(36.5-43.1)$ & 38.6 & (35.5-41.8) & 5.6 & $(4.6-6.9)$ & 9.2 & $(7.7-11.0)$ & 7.5 & $(6.5-8.7)$ \\
\hline
\end{tabular}

* One or more times during their life.

† $95 \%$ confidence interval.

$\S$ Non-Hispanic. 
TABLE 54. Percentage of high school students who ever used marijuana* and who tried marijuana for the first time before age 13 years, by sex selected U.S. sites, Youth Risk Behavior Survey, 2015

\begin{tabular}{|c|c|c|c|c|c|c|c|c|c|c|c|c|}
\hline \multirow[b]{3}{*}{ Site } & \multicolumn{6}{|c|}{ Ever used marijuana } & \multicolumn{6}{|c|}{ Tried marijuana before age 13 years } \\
\hline & \multicolumn{2}{|c|}{ Female } & \multicolumn{2}{|r|}{ Male } & \multicolumn{2}{|r|}{ Total } & \multicolumn{2}{|c|}{ Female } & \multicolumn{2}{|r|}{ Male } & \multicolumn{2}{|r|}{ Total } \\
\hline & $\%$ & $\mathrm{Cl}^{\dagger}$ & $\%$ & $\mathrm{Cl}$ & $\%$ & $\mathrm{Cl}$ & $\%$ & $\mathrm{Cl}$ & $\%$ & $\mathrm{Cl}$ & $\%$ & $\mathrm{Cl}$ \\
\hline \multicolumn{13}{|l|}{ State surveys } \\
\hline Alabama & 31.0 & $(25.9-36.5)$ & 34.3 & $(30.0-38.9)$ & 32.9 & $(29.4-36.6)$ & 3.9 & $(2.4-6.4)$ & 9.9 & $(7.7-12.7)$ & 7.0 & $(5.6-8.8)$ \\
\hline Alaska & 35.4 & $(31.9-39.0)$ & 41.8 & $(37.4-46.3)$ & 38.8 & (35.6-42.2) & 6.1 & $(4.1-8.9)$ & 13.1 & $(10.4-16.3)$ & 9.9 & $(7.8-12.4)$ \\
\hline Arizona & 41.0 & $(34.9-47.4)$ & 44.4 & $(38.2-50.9)$ & 42.9 & $(37.1-48.8)$ & 8.9 & $(6.8-11.5)$ & 10.7 & $(8.6-13.4)$ & 10.0 & $(8.3-12.0)$ \\
\hline Arkansas & 33.2 & $(28.2-38.5)$ & 36.0 & (29.6-42.8) & 34.5 & $(31.2-38.0)$ & 6.9 & $(5.7-8.4)$ & 14.0 & (11.1-17.4) & 10.4 & $(8.6-12.6)$ \\
\hline California & 38.7 & (32.3-45.6) & 38.5 & (33.8-43.5) & 38.6 & (33.5-43.9) & 6.7 & $(4.7-9.6)$ & 8.3 & $(6.3-10.9)$ & 7.5 & $(5.8-9.7)$ \\
\hline Connecticut & 35.2 & $(30.8-39.9)$ & 35.3 & $(31.8-39.0)$ & 35.4 & $(32.2-38.8)$ & 3.1 & $(1.7-5.6)$ & 8.2 & $(6.4-10.5)$ & 5.8 & $(4.5-7.6)$ \\
\hline Delaware & 40.9 & $(36.0-46.0)$ & 41.6 & $(37.6-45.8)$ & 41.5 & $(37.5-45.5)$ & 4.7 & $(3.5-6.4)$ & 10.4 & $(8.1-13.2)$ & 7.8 & $(6.4-9.4)$ \\
\hline Florida & 35.6 & $(33.8-37.5)$ & 37.4 & $(34.7-40.2)$ & 36.6 & (34.8-38.6) & 6.1 & $(5.4-7.0)$ & 10.7 & $(9.3-12.3)$ & 8.6 & $(7.6-9.7)$ \\
\hline Hawaii & —§ & - & - & - & - & - & 7.2 & $(5.8-8.9)$ & 11.6 & $(9.5-14.1)$ & 9.5 & $(8.0-11.3)$ \\
\hline Idaho & 30.9 & $(26.8-35.4)$ & 32.0 & $(26.9-37.6)$ & 31.5 & $(27.9-35.5)$ & 4.1 & $(2.7-6.1)$ & 7.6 & $(5.4-10.6)$ & 6.0 & $(4.7-7.7)$ \\
\hline Illinois & 36.7 & (30.6-43.2) & 35.8 & (29.9-42.3) & 36.3 & (31.4-41.6) & 4.7 & $(3.5-6.3)$ & 9.8 & $(7.6-12.7)$ & 7.3 & $(6.1-8.8)$ \\
\hline Indiana & 34.3 & (29.4-39.5) & 35.6 & (30.6-40.9) & 35.1 & (31.0-39.5) & 5.2 & $(3.5-7.7)$ & 6.9 & $(5.8-8.2)$ & 6.2 & $(5.3-7.4)$ \\
\hline Kentucky & 32.6 & $(27.6-38.1)$ & 33.7 & $(29.4-38.3)$ & 33.1 & (29.9-36.5) & 6.3 & $(4.1-9.6)$ & 6.1 & $(4.1-9.1)$ & 6.3 & $(4.5-8.7)$ \\
\hline Maine & 35.6 & (33.7-37.5) & 33.5 & (31.4-35.7) & 34.7 & (33.1-36.3) & 5.8 & $(4.9-6.9)$ & 8.3 & $(7.3-9.4)$ & 7.2 & $(6.5-7.9)$ \\
\hline Maryland & 32.1 & $(31.1-33.2)$ & 32.6 & $(31.5-33.6)$ & 32.5 & (31.6-33.3) & 5.9 & $(5.5-6.3)$ & 9.8 & $(9.3-10.4)$ & 8.0 & $(7.6-8.4)$ \\
\hline Massachusetts & 40.2 & $(36.6-43.9)$ & 41.6 & $(37.3-46.1)$ & 40.9 & (37.6-44.4) & 4.0 & $(3.1-5.2)$ & 8.5 & $(6.8-10.5)$ & 6.3 & $(5.1-7.6)$ \\
\hline Michigan & 33.2 & $(29.4-37.2)$ & 34.6 & (30.6-38.9) & 33.9 & $(30.8-37.1)$ & 3.3 & $(2.0-5.5)$ & 8.5 & $(6.8-10.5)$ & 5.9 & $(4.8-7.4)$ \\
\hline Mississippi & 31.5 & $(26.0-37.6)$ & 38.6 & (34.7-42.7) & 35.1 & $(31.0-39.4)$ & 4.8 & $(3.5-6.6)$ & 13.7 & $(10.6-17.6)$ & 9.4 & $(7.7-11.3)$ \\
\hline Missouri & - & - & - & - & - & - & - & - & - & - & - & - \\
\hline Montana & 38.1 & (33.6-42.8) & 37.1 & (33.8-40.4) & 37.5 & (34.1-41.1) & 7.3 & $(5.7-9.2)$ & 8.8 & $(7.6-10.1)$ & 8.0 & $(6.9-9.4)$ \\
\hline Nebraska & 27.6 & $(23.5-32.1)$ & 25.2 & $(20.7-30.3)$ & 26.6 & $(22.9-30.6)$ & 5.6 & $(3.7-8.3)$ & 7.0 & $(4.7-10.3)$ & 6.3 & $(4.5-8.6)$ \\
\hline Nevada & 42.6 & $(37.3-48.1)$ & 39.2 & (32.9-45.8) & 40.9 & (36.2-45.8) & 6.4 & $(4.4-9.2)$ & 11.1 & $(8.5-14.3)$ & 8.8 & $(7.0-10.9)$ \\
\hline New Hampshire & - & - & - & - & - & - & 4.0 & $(3.1-5.1)$ & 8.0 & $(7.2-8.9)$ & 6.1 & $(5.6-6.8)$ \\
\hline New Mexico & - & - & - & - & - & - & 14.3 & $(12.4-16.3)$ & 18.6 & $(16.7-20.6)$ & 16.5 & $(14.9-18.2)$ \\
\hline New York & - & - & - & - & - & - & 3.8 & $(2.9-5.0)$ & 8.8 & $(7.3-10.5)$ & 6.5 & (5.6-7.6) \\
\hline North Carolina & 40.9 & (36.3-45.7) & 41.0 & (38.1-44.0) & 41.2 & (37.8-44.5) & 5.3 & $(3.9-7.1)$ & 11.1 & $(9.4-12.9)$ & 8.3 & $(7.3-9.5)$ \\
\hline North Dakota & - & - & - & - & - & - & 4.1 & $(2.7-6.4)$ & 6.4 & $(4.7-8.8)$ & 5.3 & (4.1-6.9) \\
\hline Oklahoma & 28.5 & $(24.3-33.1)$ & 36.2 & (30.3-42.5) & 32.4 & $(28.0-37.1)$ & 2.9 & $(1.8-4.5)$ & 11.9 & $(7.3-18.8)$ & 7.2 & $(4.8-10.8)$ \\
\hline Pennsylvania & 29.0 & $(24.7-33.7)$ & 34.0 & $(30.1-38.3)$ & 31.5 & $(27.9-35.4)$ & 4.1 & $(2.7-6.3)$ & 7.9 & $(6.1-10.1)$ & 6.1 & $(4.6-7.9)$ \\
\hline Rhode Island & 39.3 & $(36.3-42.5)$ & 37.8 & (35.2-40.5) & 38.7 & (36.2-41.2) & 4.9 & $(3.5-6.6)$ & 8.1 & $(5.8-11.2)$ & 6.7 & $(5.1-8.8)$ \\
\hline South Carolina & 34.9 & $(27.7-42.9)$ & 34.1 & (28.9-39.6) & 34.5 & (29.0-40.4) & 6.2 & (3.9-9.7) & 10.5 & $(7.6-14.3)$ & 8.4 & $(6.4-10.9)$ \\
\hline South Dakota & 26.2 & $(18.9-35.1)$ & 23.8 & (18.9-29.5) & 25.0 & (19.3-31.7) & 3.8 & $(1.8-7.7)$ & 8.2 & $(5.2-12.7)$ & 6.0 & $(4.0-9.0)$ \\
\hline Tennessee & - & - & - & - & - & - & - & - & - & - & - & - \\
\hline Vermont & - & - & - & - & - & - & 4.7 & $(4.3-5.1)$ & 8.0 & $(7.5-8.6)$ & 6.5 & $(6.2-6.8)$ \\
\hline Virginia & 29.6 & $(26.5-32.9)$ & 30.6 & $(27.7-33.7)$ & 30.1 & $(27.7-32.7)$ & 4.4 & $(3.3-6.0)$ & 7.7 & $(6.2-9.6)$ & 6.1 & $(4.9-7.6)$ \\
\hline West Virginia & 36.1 & $(31.2-41.3)$ & 33.2 & $(28.1-38.8)$ & 34.7 & $(30.2-39.4)$ & 7.4 & $(5.5-9.9)$ & 9.3 & $(6.3-13.7)$ & 8.4 & $(6.0-11.6)$ \\
\hline Wyoming & 33.1 & $(28.7-37.8)$ & 34.8 & (29.7-40.4) & 34.0 & $(29.6-38.7)$ & 6.9 & $(4.7-9.9)$ & 8.9 & $(6.9-11.4)$ & 7.9 & $(6.0-10.5)$ \\
\hline Median & & 34.9 & & 35.6 & & 34.7 & & 5.2 & & 8.8 & & 7.2 \\
\hline Range & & 6.2-42.6) & & $3.8-44.4)$ & & $5.0-42.9)$ & & $.9-14.3)$ & & $5.1-18.6)$ & & $5.3-16.5)$ \\
\hline \multicolumn{13}{|c|}{ Large urban school district surveys } \\
\hline Baltimore, MD & 45.3 & $(40.1-50.6)$ & 45.8 & $(39.8-51.9)$ & 45.8 & $(41.7-50.1)$ & 10.1 & $(7.8-13.1)$ & 21.2 & $(16.1-27.4)$ & 15.7 & $(12.7-19.4)$ \\
\hline Boston, MA & - & - & - & - & - & - & 4.6 & $(3.2-6.5)$ & 9.4 & $(7.2-12.4)$ & 7.0 & (5.5-8.9) \\
\hline Broward County, FL & 40.6 & $(36.5-45.0)$ & 39.8 & $(35.0-44.7)$ & 40.1 & (36.6-43.8) & 6.1 & $(4.5-8.3)$ & 9.3 & $(7.3-11.8)$ & 7.8 & $(6.4-9.3)$ \\
\hline Cleveland, $\mathrm{OH}$ & 41.5 & $(36.6-46.5)$ & 44.4 & $(39.9-49.1)$ & 43.2 & (39.6-46.8) & 8.7 & $(6.6-11.5)$ & 17.2 & $(14.2-20.6)$ & 13.2 & $(11.1-15.7)$ \\
\hline DeKalb County, GA & 40.5 & $(36.3-44.8)$ & 40.4 & $(36.1-44.9)$ & 40.5 & $(37.3-43.8)$ & 5.3 & $(4.0-7.0)$ & 14.0 & $(11.6-16.8)$ & 9.7 & $(8.2-11.4)$ \\
\hline Detroit, MI & 41.4 & $(37.4-45.6)$ & 41.5 & $(36.0-47.3)$ & 41.7 & $(38.0-45.6)$ & 9.3 & $(7.4-11.5)$ & 14.0 & $(11.0-17.8)$ & 11.5 & $(9.6-13.8)$ \\
\hline District of Columbia & - & - & - & - & - & - & 11.0 & $(10.2-12.0)$ & 20.0 & $(18.7-21.3)$ & 15.6 & $(14.8-16.3)$ \\
\hline Duval County, FL & - & - & - & - & - & - & 9.1 & $(7.2-11.4)$ & 13.0 & $(10.9-15.3)$ & 11.5 & $(9.9-13.3)$ \\
\hline Ft. Worth, TX & 37.3 & $(33.8-41.0)$ & 41.3 & $(38.2-44.5)$ & 39.3 & $(36.7-41.9)$ & 6.2 & $(4.8-7.8)$ & 11.8 & $(9.7-14.2)$ & 9.0 & $(7.7-10.5)$ \\
\hline Houston, TX & 37.6 & $(34.7-40.5)$ & 39.5 & $(36.7-42.4)$ & 38.7 & $(36.5-40.9)$ & 7.0 & $(5.7-8.6)$ & 11.7 & $(10.0-13.7)$ & 9.5 & $(8.2-10.8)$ \\
\hline Los Angeles, CA & 36.5 & $(31.6-41.8)$ & 32.7 & $(27.8-38.1)$ & 34.7 & $(30.5-39.2)$ & 6.4 & $(5.0-8.3)$ & 9.2 & $(6.7-12.3)$ & 7.8 & $(6.1-9.8)$ \\
\hline Miami-Dade County, FL & 30.4 & $(26.4-34.7)$ & 36.2 & $(31.9-40.8)$ & 33.3 & $(29.9-36.8)$ & 3.0 & $(2.1-4.2)$ & 8.5 & $(6.7-10.5)$ & 5.7 & $(4.7-6.9)$ \\
\hline
\end{tabular}

See table footnotes on the next page. 
TABLE 54. (Continued) Percentage of high school students who ever used marijuana* and who tried marijuana for the first time before age 13 years, by sex — selected U.S. sites, Youth Risk Behavior Survey, 2015

\begin{tabular}{|c|c|c|c|c|c|c|c|c|c|c|c|c|}
\hline \multirow[b]{3}{*}{ Site } & \multicolumn{6}{|c|}{ Ever used marijuana } & \multicolumn{6}{|c|}{ Tried marijuana before age 13 years } \\
\hline & \multicolumn{2}{|r|}{ Female } & \multicolumn{2}{|r|}{ Male } & \multicolumn{2}{|r|}{ Total } & \multicolumn{2}{|c|}{ Female } & \multicolumn{2}{|r|}{ Male } & \multicolumn{2}{|r|}{ Total } \\
\hline & $\%$ & $\mathrm{Cl}^{\dagger}$ & $\%$ & $\mathrm{Cl}$ & $\%$ & $\mathrm{Cl}$ & $\%$ & $\mathrm{Cl}$ & $\%$ & $\mathrm{Cl}$ & $\%$ & $\mathrm{Cl}$ \\
\hline New York City, NY & - & - & - & - & - & - & 4.8 & $(4.0-5.9)$ & 7.4 & $(6.1-9.0)$ & 6.2 & $(5.3-7.4)$ \\
\hline Oakland, CA & 45.6 & $(41.2-50.0)$ & 45.3 & $(40.7-49.9)$ & 45.6 & $(42.8-48.4)$ & - & - & - & - & - & - \\
\hline Orange County, FL & 31.8 & $(27.3-36.7)$ & 36.1 & $(31.6-40.9)$ & 33.9 & $(30.7-37.3)$ & 3.5 & $(2.4-5.2)$ & 10.9 & $(8.5-13.9)$ & 7.2 & $(5.7-9.0)$ \\
\hline Palm Beach County, FL & 40.2 & $(36.9-43.7)$ & 42.4 & (38.9-45.9) & 41.5 & (38.8-44.2) & 5.9 & $(4.6-7.6)$ & 12.8 & $(10.7-15.1)$ & 9.7 & $(8.2-11.6)$ \\
\hline Philadelphia, PA & 40.8 & $(33.4-48.6)$ & 40.1 & $(34.4-46.1)$ & 40.6 & $(34.5-47.0)$ & 6.4 & $(4.5-8.8)$ & 11.2 & $(8.9-13.9)$ & 8.8 & $(6.9-11.0)$ \\
\hline San Diego, CA & 34.2 & $(29.4-39.3)$ & 37.7 & $(34.1-41.4)$ & 35.9 & $(32.5-39.6)$ & 3.9 & $(2.7-5.6)$ & 11.1 & $(9.2-13.3)$ & 7.5 & $(6.4-8.9)$ \\
\hline San Francisco, CA & 30.7 & $(25.9-36.0)$ & 26.9 & $(22.6-31.6)$ & 28.7 & $(24.6-33.2)$ & 6.5 & $(4.6-9.1)$ & 6.2 & $(4.4-8.5)$ & 6.5 & $(4.9-8.4)$ \\
\hline Median & \multicolumn{2}{|c|}{40.2} & \multicolumn{2}{|c|}{40.1} & \multicolumn{2}{|c|}{40.1} & \multicolumn{2}{|c|}{6.3} & \multicolumn{2}{|c|}{11.4} & \multirow{2}{*}{\multicolumn{2}{|c|}{$\begin{array}{c}8.9 \\
(5.7-15.7)\end{array}$}} \\
\hline Range & & $30.4-45.6)$ & & 26.9-45.8) & & $8.7-45.8)$ & & $-11.0)$ & & $6.2-21.2)$ & & \\
\hline
\end{tabular}

* One or more times during their life.

† $95 \%$ confidence interval.

$\S$ Not available.

TABLE 55. Percentage of high school students who currently used marijuana* and who usually used marijuana by smoking it, ${ }^{\dagger}$ by sex, race/ ethnicity, and grade - United States, Youth Risk Behavior Survey, 2015

\begin{tabular}{|c|c|c|c|c|c|c|c|c|c|c|c|c|}
\hline \multirow[b]{3}{*}{ Category } & \multicolumn{6}{|c|}{ Current marijuana use } & \multicolumn{6}{|c|}{ Usually used marijuana by smoking it } \\
\hline & \multicolumn{2}{|c|}{ Female } & \multicolumn{2}{|r|}{ Male } & \multicolumn{2}{|r|}{ Total } & \multicolumn{2}{|c|}{ Female } & \multicolumn{2}{|c|}{ Male } & \multicolumn{2}{|r|}{ Total } \\
\hline & $\%$ & $\mathrm{Cl}^{\S}$ & $\%$ & $\mathrm{Cl}$ & $\%$ & $\mathrm{Cl}$ & $\%$ & $\mathrm{Cl}$ & $\%$ & $\mathrm{Cl}$ & $\%$ & $\mathrm{Cl}$ \\
\hline \multicolumn{13}{|c|}{ Race/Ethnicity } \\
\hline White & 18.7 & $(15.3-22.6)$ & 21.2 & $(17.2-26.0)$ & 19.9 & (16.8-23.5) & 91.9 & (88.6-94.4) & 90.5 & (86.3-93.5) & 91.2 & (88.5-93.2) \\
\hline Black ${ }^{\Uparrow}$ & 22.1 & $(19.4-25.0)$ & 31.3 & $(26.2-36.9)$ & 27.1 & $(24.0-30.4)$ & 93.4 & $(85.8-97.1)$ & 93.2 & $(86.5-96.7)$ & 92.2 & $(88.0-95.0)$ \\
\hline Hispanic & 23.5 & $(19.7-27.7)$ & 25.5 & $(22.7-28.6)$ & 24.5 & $(21.6-27.6)$ & 91.1 & $(85.1-94.8)$ & 88.5 & $(83.6-92.1)$ & 89.7 & $(85.1-93.1)$ \\
\hline \multicolumn{13}{|l|}{ Grade } \\
\hline 9 & 14.7 & $(12.1-17.7)$ & 15.5 & $(13.4-17.9)$ & 15.2 & $(13.3-17.3)$ & 89.5 & (79.6-94.9) & 89.5 & (82.4-93.9) & 88.7 & $(83.3-92.6)$ \\
\hline 10 & 17.5 & $(13.7-22.3)$ & 22.6 & (18.9-26.7) & 20.0 & $(16.5-24.1)$ & 91.4 & $(86.4-94.6)$ & 89.6 & (84.2-93.2) & 90.4 & $(87.5-92.6)$ \\
\hline 11 & 24.3 & (21.4-27.4) & 25.5 & (22.4-28.9) & 24.8 & (22.3-27.5) & 92.1 & $(85.6-95.8)$ & 91.3 & (85.9-94.8) & 90.6 & (85.9-93.8) \\
\hline 12 & 24.6 & $(21.2-28.3)$ & 30.5 & $(24.6-37.2)$ & 27.6 & $(23.8-31.6)$ & 96.7 & $(93.8-98.3)$ & 87.4 & (81.3-91.7) & 91.3 & $(87.1-94.2)$ \\
\hline Total & 20.1 & $(17.6-22.9)$ & 23.2 & $(20.4-26.3)$ & 21.7 & $(19.3-24.2)$ & 92.5 & $(90.1-94.3)$ & 89.0 & (85.9-91.4) & 90.0 & $(87.5-92.1)$ \\
\hline
\end{tabular}

* One or more times during the 30 days before the survey.

I In a joint, bong, pipe, or blunt during the 30 days before the survey, among the $21.7 \%$ of students who currently used marijuana.

$\S 95 \%$ confidence interval.

" Non-Hispanic. 
TABLE 56. Percentage of high school students who currently used marijuana, ${ }^{*}$ by sex — selected U.S. sites, Youth Risk Behavior Survey, 2015

\begin{tabular}{|c|c|c|c|c|c|c|}
\hline \multirow[b]{2}{*}{ Site } & \multicolumn{2}{|c|}{ Female } & \multicolumn{2}{|c|}{ Male } & \multicolumn{2}{|c|}{ Total } \\
\hline & $\%$ & $\mathrm{Cl}^{\dagger}$ & $\%$ & $\mathrm{Cl}$ & $\%$ & $\mathrm{Cl}$ \\
\hline \multicolumn{7}{|l|}{ State surveys } \\
\hline Alabama & 15.8 & $(12.9-19.0)$ & 18.6 & $(15.5-22.2)$ & 17.3 & $(15.2-19.6)$ \\
\hline Alaska & 16.5 & $(13.7-19.9)$ & 21.3 & $(18.0-24.9)$ & 19.0 & $(16.8-21.4)$ \\
\hline Arizona & 22.1 & $(17.3-27.8)$ & 24.1 & $(21.2-27.4)$ & 23.3 & (19.6-27.5) \\
\hline Arkansas & 16.5 & $(13.9-19.5)$ & 19.0 & $(15.8-22.7)$ & 17.8 & (15.9-19.7) \\
\hline California & 22.5 & $(17.3-28.8)$ & 23.0 & (18.1-28.7) & 22.9 & (18.5-27.9) \\
\hline Connecticut & 20.9 & $(17.3-25.0)$ & 19.7 & $(16.8-23.1)$ & 20.4 & (17.6-23.4) \\
\hline Delaware & 23.2 & $(18.8-28.4)$ & 23.1 & $(19.8-26.7)$ & 23.3 & (20.3-26.7) \\
\hline Florida & 20.1 & (18.6-21.8) & 22.5 & $(20.3-24.9)$ & 21.5 & $(20.0-23.2)$ \\
\hline Hawaii & 18.6 & $(16.1-21.3)$ & 20.0 & $(17.2-23.0)$ & 19.4 & (17.6-21.4) \\
\hline Idaho & 17.4 & $(13.9-21.4)$ & 16.6 & (12.8-21.3) & 17.0 & (14.1-20.5) \\
\hline Illinois & 19.6 & $(16.1-23.6)$ & 17.7 & $(13.9-22.3)$ & 18.7 & (15.9-21.8) \\
\hline Indiana & 15.9 & $(12.7-19.7)$ & 16.4 & (13.8-19.4) & 16.4 & (14.1-18.9) \\
\hline Kentucky & 16.9 & $(13.3-21.1)$ & 17.5 & $(14.6-20.8)$ & 17.2 & (14.7-20.0) \\
\hline Maine & 19.4 & $(17.9-21.0)$ & 20.2 & $(18.7-21.7)$ & 19.9 & (18.7-21.1) \\
\hline Maryland & 18.2 & (17.4-18.9) & 19.4 & $(18.6-20.2)$ & 18.8 & (18.2-19.5) \\
\hline Massachusetts & 23.0 & $(19.8-26.5)$ & 26.3 & $(23.2-29.7)$ & 24.5 & (21.8-27.5) \\
\hline Michigan & 17.9 & $(14.9-21.4)$ & 20.8 & $(17.1-24.9)$ & 19.3 & $(16.5-22.5)$ \\
\hline Mississippi & 17.7 & $(14.7-21.2)$ & 21.6 & $(18.5-25.0)$ & 19.7 & (17.3-22.3) \\
\hline Missouri & 12.6 & $(10.0-15.7)$ & 20.0 & $(16.7-23.9)$ & 16.3 & (13.6-19.3) \\
\hline Montana & 19.5 & $(16.7-22.6)$ & 19.6 & $(17.6-21.7)$ & 19.5 & (17.4-21.8) \\
\hline Nebraska & 14.4 & (10.8-18.8) & 12.7 & $(9.6-16.7)$ & 13.7 & $(10.8-17.2)$ \\
\hline Nevada & 20.9 & $(16.8-25.5)$ & 17.7 & $(14.5-21.4)$ & 19.3 & (16.3-22.7) \\
\hline New Hampshire & 20.0 & (18.4-21.7) & 23.9 & $(21.9-26.0)$ & 22.2 & (20.8-23.7) \\
\hline New Mexico & 24.9 & $(22.6-27.2)$ & 25.6 & $(23.9-27.5)$ & 25.3 & $(23.6-27.1)$ \\
\hline New York & 19.1 & $(16.4-22.2)$ & 18.9 & $(16.0-22.2)$ & 19.3 & $(16.9-21.8)$ \\
\hline North Carolina & 21.6 & $(17.3-26.7)$ & 22.8 & $(19.6-26.4)$ & 22.3 & $(20.1-24.7)$ \\
\hline North Dakota & 14.6 & $(11.8-17.8)$ & 15.8 & $(13.1-18.9)$ & 15.2 & (13.1-17.6) \\
\hline Oklahoma & 14.7 & $(11.4-18.8)$ & 20.7 & $(16.1-26.1)$ & 17.5 & (14.2-21.5) \\
\hline Pennsylvania & 15.9 & $(13.1-19.1)$ & 20.3 & $(17.3-23.8)$ & 18.2 & (15.9-20.7) \\
\hline Rhode Island & 22.7 & $(20.3-25.3)$ & 24.3 & $(22.2-26.5)$ & 23.6 & $(22.0-25.2)$ \\
\hline South Carolina & 19.1 & $(14.3-25.1)$ & 16.5 & $(12.7-21.1)$ & 17.8 & (14.3-21.9) \\
\hline South Dakota & 13.2 & $(8.8-19.2)$ & 11.5 & $(7.6-17.0)$ & 12.4 & $(8.4-17.7)$ \\
\hline Tennessee & —§ & - & - & - & - & - \\
\hline Vermont & 20.7 & $(19.9-21.5)$ & 23.8 & $(23.0-24.6)$ & 22.4 & $(21.8-22.9)$ \\
\hline Virginia & 14.9 & $(12.7-17.5)$ & 17.5 & $(15.1-20.1)$ & 16.2 & (14.4-18.2) \\
\hline West Virginia & 16.3 & $(13.0-20.3)$ & 16.7 & $(13.0-21.2)$ & 16.5 & (13.4-20.3) \\
\hline Wyoming & 17.4 & $(14.0-21.4)$ & 19.2 & $(16.2-22.6)$ & 18.3 & (15.4-21.6) \\
\hline Median & \multirow{2}{*}{\multicolumn{2}{|c|}{$\begin{array}{c}18.4 \\
(12.6-24.9)\end{array}$}} & \multirow{2}{*}{\multicolumn{2}{|c|}{$\begin{array}{c}19.8 \\
(11.5-26.3)\end{array}$}} & \multirow{2}{*}{\multicolumn{2}{|c|}{$\begin{array}{c}19.1 \\
(12.4-25.3)\end{array}$}} \\
\hline Range & & & & & & \\
\hline \multicolumn{7}{|c|}{ Large urban school district surveys } \\
\hline Baltimore, MD & 23.2 & $(18.8-28.3)$ & 22.9 & $(17.6-29.2)$ & 23.4 & $(19.4-28.0)$ \\
\hline Boston, MA & 21.7 & $(17.8-26.3)$ & 22.8 & $(19.3-26.7)$ & 22.3 & $(19.6-25.2)$ \\
\hline Broward County, FL & 22.6 & $(19.1-26.6)$ & 25.2 & $(21.3-29.6)$ & 24.0 & $(21.5-26.6)$ \\
\hline Cleveland, $\mathrm{OH}$ & 21.7 & $(17.9-26.0)$ & 26.3 & $(22.9-30.1)$ & 24.2 & $(21.4-27.1)$ \\
\hline DeKalb County, GA & 20.3 & $(17.5-23.4)$ & 23.9 & $(20.1-28.1)$ & 22.2 & $(19.6-25.0)$ \\
\hline Detroit, MI & 22.6 & $(19.0-26.7)$ & 22.5 & $(18.4-27.3)$ & 22.6 & $(19.5-26.0)$ \\
\hline District of Columbia & 29.1 & $(27.8-30.4)$ & 28.2 & $(26.8-29.6)$ & 28.7 & $(27.8-29.7)$ \\
\hline Duval County, FL & 23.8 & $(21.5-26.4)$ & 25.4 & $(22.9-28.0)$ & 25.0 & $(23.1-27.0)$ \\
\hline Ft. Worth, TX & 17.4 & $(14.9-20.2)$ & 20.7 & $(17.8-23.9)$ & 19.1 & $(17.1-21.2)$ \\
\hline Houston, TX & 17.5 & $(15.6-19.5)$ & 19.8 & $(17.6-22.3)$ & 18.8 & (17.2-20.5) \\
\hline Los Angeles, CA & 15.9 & $(12.4-20.1)$ & 17.4 & (13.5-21.9) & 16.6 & (14.1-19.4) \\
\hline Miami-Dade County, FL & 16.0 & $(13.2-19.2)$ & 22.5 & $(19.5-25.7)$ & 19.2 & (16.9-21.7) \\
\hline
\end{tabular}

See table footnotes on the next page. 
TABLE 56. (Continued) Percentage of high school students who currently used marijuana,* by sex — selected U.S. sites, Youth Risk Behavior Survey, 2015

\begin{tabular}{|c|c|c|c|c|c|c|}
\hline \multirow[b]{2}{*}{ Site } & \multicolumn{2}{|c|}{ Female } & \multicolumn{2}{|c|}{ Male } & \multicolumn{2}{|c|}{ Total } \\
\hline & $\%$ & $\mathrm{Cl}^{\dagger}$ & $\%$ & $\mathrm{Cl}$ & $\%$ & $\mathrm{Cl}$ \\
\hline New York City, NY & 15.8 & $(13.3-18.7)$ & 15.7 & $(13.7-18.0)$ & 15.9 & $(13.9-18.0)$ \\
\hline Oakland, CA & 25.4 & $(22.0-29.0)$ & 24.9 & $(21.5-28.5)$ & 25.3 & $(23.0-27.7)$ \\
\hline Orange County, FL & 17.0 & (14.0-20.5) & 21.5 & $(17.9-25.5)$ & 19.1 & $(16.8-21.7)$ \\
\hline Palm Beach County, FL & 20.5 & $(17.7-23.6)$ & 24.9 & $(21.9-28.1)$ & 23.0 & $(20.9-25.4)$ \\
\hline Philadelphia, PA & 21.1 & $(15.4-28.2)$ & 21.6 & $(17.8-25.9)$ & 21.6 & $(16.8-27.3)$ \\
\hline San Diego, CA & 15.3 & $(12.7-18.2)$ & 20.6 & $(17.9-23.6)$ & 18.0 & $(16.0-20.1)$ \\
\hline San Francisco, CA & 18.4 & $(14.4-23.3)$ & 16.3 & $(12.6-20.8)$ & 17.4 & $(13.9-21.5)$ \\
\hline $\begin{array}{l}\text { Median } \\
\text { Range }\end{array}$ & \multicolumn{2}{|c|}{$\begin{array}{c}20.5 \\
(15.3-29.1)\end{array}$} & \multicolumn{2}{|c|}{$\begin{array}{c}22.5 \\
(15.7-28.2)\end{array}$} & \multicolumn{2}{|c|}{$\begin{array}{c}22.2 \\
(15.9-28.7)\end{array}$} \\
\hline
\end{tabular}

* One or more times during the 30 days before the survey.

† $95 \%$ confidence interval.

$\S$ Not available.

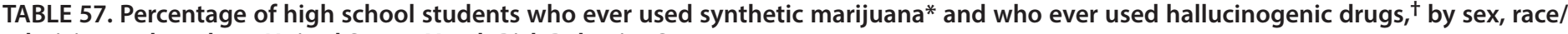
ethnicity, and grade - United States, Youth Risk Behavior Survey, 2015

\begin{tabular}{|c|c|c|c|c|c|c|c|c|c|c|c|c|}
\hline \multirow[b]{3}{*}{ Category } & \multicolumn{6}{|c|}{ Ever used synthetic marijuana } & \multicolumn{6}{|c|}{ Ever used hallucinogenic drugs } \\
\hline & \multicolumn{2}{|c|}{ Female } & \multicolumn{2}{|r|}{ Male } & \multicolumn{2}{|c|}{ Total } & \multicolumn{2}{|c|}{ Female } & \multicolumn{2}{|c|}{ Male } & \multicolumn{2}{|c|}{ Total } \\
\hline & $\%$ & $\mathrm{Cl}^{\S}$ & $\%$ & $\mathrm{Cl}$ & $\%$ & $\mathrm{Cl}$ & $\%$ & $\mathrm{Cl}$ & $\%$ & $\mathrm{Cl}$ & $\%$ & $\mathrm{Cl}$ \\
\hline \multicolumn{13}{|c|}{ Race/Ethnicity } \\
\hline White? & 7.6 & $(5.8-9.9)$ & 9.9 & $(8.2-11.9)$ & 8.8 & $(7.2-10.7)$ & 4.7 & $(3.4-6.5)$ & 8.1 & $(6.6-9.9)$ & 6.4 & $(5.0-8.1)$ \\
\hline Black? & 5.9 & $(4.1-8.5)$ & 8.9 & $(6.4-12.3)$ & 7.8 & $(6.0-9.9)$ & 1.9 & $(0.7-5.3)$ & 6.7 & $(4.4-10.1)$ & 4.7 & $(3.1-7.2)$ \\
\hline Hispanic & 10.3 & $(8.4-12.6)$ & 12.9 & $(10.7-15.6)$ & 11.7 & $(9.8-13.9)$ & 6.1 & $(4.7-7.8)$ & 7.4 & $(5.4-10.1)$ & 6.8 & $(5.3-8.7)$ \\
\hline \multicolumn{13}{|l|}{ Grade } \\
\hline 9 & 7.2 & $(5.7-9.0)$ & 6.7 & $(5.1-8.9)$ & 7.1 & $(5.7-8.7)$ & 3.1 & $(2.1-4.6)$ & 5.9 & $(4.6-7.5)$ & 4.7 & $(3.7-6.0)$ \\
\hline 10 & 7.9 & $(5.9-10.4)$ & 9.7 & $(7.5-12.5)$ & 8.8 & $(6.9-11.2)$ & 5.3 & $(3.7-7.5)$ & 7.0 & $(5.0-9.8)$ & 6.2 & $(4.6-8.2)$ \\
\hline 11 & 8.8 & $(7.0-11.0)$ & 11.1 & $(9.3-13.1)$ & 10.0 & $(8.4-11.9)$ & 5.9 & $(4.2-8.4)$ & 7.0 & $(5.6-8.7)$ & 6.7 & $(5.2-8.6)$ \\
\hline 12 & 7.6 & $(5.7-10.0)$ & 14.2 & $(11.0-18.1)$ & 11.0 & $(9.1-13.3)$ & 3.9 & $(2.6-5.6)$ & 12.0 & $(8.6-16.4)$ & 8.0 & $(6.0-10.6)$ \\
\hline Total & 7.9 & $(6.6-9.5)$ & 10.3 & $(8.8-12.0)$ & 9.2 & (7.9-10.8) & 4.6 & $(3.6-5.9)$ & 8.0 & $(6.7-9.5)$ & 6.4 & $(5.3-7.7)$ \\
\hline
\end{tabular}

* Also called "K2," "Spice," "fake weed," "King Kong," "Yucatan Fire,, "Skunk," or "Moon Rocks," one or more times during their life.

† Such as LSD, acid, PCP, angel dust, mescaline, or mushrooms, one or more times during their life.

$\S 95 \%$ confidence interval.

"Non-Hispanic. 
TABLE 58. Percentage of high school students who ever used synthetic marijuana, ${ }^{*}$ by sex — selected U.S. sites, Youth Risk Behavior Survey, 2015

\begin{tabular}{|c|c|c|c|c|c|c|}
\hline \multirow[b]{2}{*}{ Site } & \multicolumn{2}{|c|}{ Female } & \multicolumn{2}{|c|}{ Male } & \multicolumn{2}{|c|}{ Total } \\
\hline & $\%$ & $\mathrm{Cl}^{\dagger}$ & $\%$ & $\mathrm{Cl}$ & $\%$ & $\mathrm{Cl}$ \\
\hline \multicolumn{7}{|l|}{ State surveys } \\
\hline Alabama & 7.8 & $(5.8-10.4)$ & 12.2 & $(9.8-14.9)$ & 10.1 & $(8.4-12.1)$ \\
\hline Alaska & 5.1 & $(3.5-7.3)$ & 9.2 & $(7.1-11.8)$ & 7.5 & $(6.0-9.3)$ \\
\hline Arizona & —§ & - & - & - & - & - \\
\hline Arkansas & 9.2 & $(6.5-12.7)$ & 13.8 & $(9.8-19.1)$ & 11.7 & $(8.7-15.5)$ \\
\hline California & 8.1 & $(6.2-10.4)$ & 7.8 & (5.4-11.3) & 7.9 & $(6.1-10.2)$ \\
\hline Connecticut & 5.0 & $(3.6-7.0)$ & 7.9 & $(6.2-9.9)$ & 6.7 & (5.4-8.2) \\
\hline Delaware & 5.2 & $(3.8-7.2)$ & 8.3 & $(6.4-10.9)$ & 7.1 & $(5.8-8.7)$ \\
\hline Florida & - & - & - & - & - & - \\
\hline Hawaii & 5.7 & $(4.6-7.0)$ & 8.8 & $(7.5-10.2)$ & 7.5 & (6.4-8.8) \\
\hline Idaho & 7.2 & $(5.1-10.0)$ & 9.3 & $(7.1-12.2)$ & 8.4 & $(6.9-10.2)$ \\
\hline Illinois & - & - & - & - & - & - \\
\hline Indiana & 9.3 & $(6.6-12.9)$ & 12.1 & (8.7-16.4) & 10.8 & $(8.0-14.3)$ \\
\hline Kentucky & 11.6 & $(9.1-14.8)$ & 8.5 & $(6.5-11.2)$ & 10.3 & $(8.6-12.4)$ \\
\hline Maine & - & - & - & - & - & - \\
\hline Maryland & 7.5 & $(7.1-7.9)$ & 10.5 & $(9.9-11.2)$ & 9.2 & $(8.8-9.7)$ \\
\hline Massachusetts & 5.8 & $(4.4-7.6)$ & 9.5 & $(7.6-11.8)$ & 7.9 & (6.4-9.7) \\
\hline Michigan & 6.1 & $(4.6-8.1)$ & 9.4 & $(7.5-11.7)$ & 7.8 & $(6.3-9.6)$ \\
\hline Mississippi & 7.3 & $(5.7-9.3)$ & 11.7 & $(9.2-14.7)$ & 9.7 & $(8.0-11.6)$ \\
\hline Missouri & - & - & - & - & - & - \\
\hline Montana & 8.9 & $(7.1-11.0)$ & 10.1 & $(8.7-11.9)$ & 9.6 & $(8.1-11.2)$ \\
\hline Nebraska & 7.6 & $(5.6-10.4)$ & 6.9 & $(4.7-10.0)$ & 7.5 & $(5.6-9.9)$ \\
\hline Nevada & 9.6 & $(7.7-11.9)$ & 10.4 & $(8.2-13.2)$ & 10.0 & $(8.2-12.3)$ \\
\hline New Hampshire & 7.6 & $(6.7-8.6)$ & 10.5 & $(9.5-11.6)$ & 9.2 & $(8.4-10.0)$ \\
\hline New Mexico & - & - & - & - & - & - \\
\hline New York & 8.5 & $(6.5-11.0)$ & 10.8 & (8.4-13.6) & 10.0 & $(8.0-12.3)$ \\
\hline North Carolina & - & - & - & - & - & - \\
\hline North Dakota & - & - & - & - & - & - \\
\hline Oklahoma & 6.7 & $(4.7-9.3)$ & 12.0 & $(9.1-15.7)$ & 9.3 & $(7.2-11.8)$ \\
\hline Pennsylvania & 7.6 & $(6.0-9.7)$ & 10.8 & $(8.7-13.2)$ & 9.2 & (7.7-11.0) \\
\hline Rhode Island & 8.0 & $(5.5-11.5)$ & 9.2 & $(7.5-11.1)$ & 8.8 & $(7.3-10.5)$ \\
\hline South Carolina & 4.8 & $(2.6-8.5)$ & 7.9 & $(5.0-12.1)$ & 6.4 & (4.4-9.1) \\
\hline South Dakota & 4.4 & $(2.5-7.6)$ & 9.0 & $(5.4-14.7)$ & 6.8 & $(4.2-10.9)$ \\
\hline Tennessee & - & - & - & - & - & - \\
\hline Vermont & - & - & - & - & - & - \\
\hline Virginia & 5.6 & $(4.5-7.0)$ & 6.2 & $(5.1-7.5)$ & 5.9 & (5.1-6.9) \\
\hline West Virginia & 14.3 & $(11.4-17.7)$ & 14.8 & $(11.8-18.4)$ & 14.6 & $(12.3-17.3)$ \\
\hline Wyoming & 9.9 & $(7.7-12.8)$ & 10.5 & $(8.5-12.8)$ & 10.2 & $(8.5-12.2)$ \\
\hline Median & & & & & & \\
\hline Range & & & & & & \\
\hline \multicolumn{7}{|c|}{ Large urban school district surveys } \\
\hline Baltimore, MD & 8.8 & $(6.6-11.8)$ & 15.6 & $(11.6-20.5)$ & 13.3 & $(10.5-16.6)$ \\
\hline Boston, MA & 3.9 & $(2.7-5.6)$ & 8.0 & $(5.7-11.2)$ & 6.0 & $(4.7-7.7)$ \\
\hline Broward County, FL & 5.3 & $(3.8-7.4)$ & 8.6 & $(6.1-11.9)$ & 7.1 & $(5.6-9.0)$ \\
\hline Cleveland, $\mathrm{OH}$ & 8.1 & $(6.1-10.6)$ & 15.1 & $(12.3-18.4)$ & 12.5 & $(10.4-15.0)$ \\
\hline DeKalb County, GA & 2.9 & $(1.9-4.3)$ & 6.6 & $(4.7-9.1)$ & 4.9 & $(3.8-6.3)$ \\
\hline Detroit, MI & 4.4 & $(3.1-6.4)$ & 7.5 & $(5.6-10.1)$ & 6.1 & $(4.8-7.8)$ \\
\hline District of Columbia & 6.9 & $(6.2-7.7)$ & 9.9 & $(9.0-10.9)$ & 8.6 & $(8.0-9.2)$ \\
\hline Duval County, FL & 7.4 & $(5.9-9.2)$ & 11.3 & $(9.4-13.6)$ & 9.9 & $(8.5-11.5)$ \\
\hline Ft. Worth, TX & 8.7 & $(7.0-10.8)$ & 11.3 & $(9.3-13.6)$ & 9.9 & $(8.6-11.4)$ \\
\hline Houston, TX & 10.8 & $(9.2-12.6)$ & 13.0 & $(11.1-15.2)$ & 12.3 & $(10.8-13.9)$ \\
\hline Los Angeles, CA & 6.4 & $(5.1-8.1)$ & 6.4 & $(4.9-8.2)$ & 6.5 & $(5.5-7.7)$ \\
\hline Miami-Dade County, FL & 5.1 & $(3.8-6.9)$ & 8.0 & $(6.3-10.1)$ & 6.5 & $(5.4-8.0)$ \\
\hline
\end{tabular}

See table footnotes on the next page. 
TABLE 58. (Continued) Percentage of high school students who ever used synthetic marijuana,* by sex — selected U.S. sites, Youth Risk Behavior Survey, 2015

\begin{tabular}{|c|c|c|c|c|c|c|}
\hline \multirow[b]{2}{*}{ Site } & \multicolumn{2}{|c|}{ Female } & \multicolumn{2}{|c|}{ Male } & \multicolumn{2}{|c|}{ Total } \\
\hline & $\%$ & $\mathrm{Cl}^{\dagger}$ & $\%$ & $\mathrm{Cl}$ & $\%$ & $\mathrm{Cl}$ \\
\hline New York City, NY & 3.9 & $(3.1-4.8)$ & 6.5 & $(5.2-8.3)$ & 5.4 & $(4.6-6.3)$ \\
\hline Oakland, CA & - & - & - & - & - & - \\
\hline Orange County, FL & 7.7 & $(5.9-10.1)$ & 10.6 & $(8.1-13.8)$ & 9.2 & $(7.6-11.2)$ \\
\hline Palm Beach County, FL & 7.6 & $(5.8-9.8)$ & 12.1 & $(9.7-14.8)$ & 10.7 & $(9.0-12.6)$ \\
\hline Philadelphia, PA & 7.7 & $(5.7-10.4)$ & 12.4 & $(9.2-16.6)$ & 10.2 & $(7.8-13.2)$ \\
\hline San Diego, CA & 6.7 & $(5.0-8.8)$ & 9.8 & $(8.3-11.5)$ & 8.3 & (7.1-9.6) \\
\hline San Francisco, CA & - & - & - & - & - & - \\
\hline Median & \multirow{2}{*}{\multicolumn{2}{|c|}{$\begin{array}{c}6.9 \\
(2.9-10.8)\end{array}$}} & \multirow{2}{*}{\multicolumn{2}{|c|}{$\begin{array}{c}9.9 \\
(6.4-15.6)\end{array}$}} & \multicolumn{2}{|c|}{8.6} \\
\hline Range & & & & & & \\
\hline
\end{tabular}

* Also called "K2,,"Spice," "fake weed," "King Kong," "Yucatan Fire,,"Skunk," or "Moon Rocks," one or more times during their life.

† $95 \%$ confidence interval.

$\S$ Not available.

TABLE 59. Percentage of high school students who ever used cocaine* and who ever used ecstasy, ${ }^{\dagger}$ by sex, race/ethnicity, and grade — United States, Youth Risk Behavior Survey, 2015

\begin{tabular}{|c|c|c|c|c|c|c|c|c|c|c|c|c|}
\hline \multirow[b]{3}{*}{ Category } & \multicolumn{6}{|c|}{ Ever used cocaine } & \multicolumn{6}{|c|}{ Ever used ecstasy } \\
\hline & \multicolumn{2}{|c|}{ Female } & \multicolumn{2}{|c|}{ Male } & \multicolumn{2}{|c|}{ Total } & \multicolumn{2}{|c|}{ Female } & \multicolumn{2}{|c|}{ Male } & \multicolumn{2}{|c|}{ Total } \\
\hline & $\%$ & $\mathrm{Cl}^{\S}$ & $\%$ & $\mathrm{Cl}$ & $\%$ & $\mathrm{Cl}$ & $\%$ & $\mathrm{Cl}$ & $\%$ & $\mathrm{Cl}$ & $\%$ & $\mathrm{Cl}$ \\
\hline \multicolumn{13}{|c|}{ Race/Ethnicity } \\
\hline White & 3.3 & $(2.3-4.7)$ & 5.0 & $(3.9-6.3)$ & 4.1 & $(3.3-5.2)$ & 4.0 & $(2.9-5.4)$ & 4.7 & $(3.6-5.9)$ & 4.3 & (3.4-5.3) \\
\hline Black ${ }^{\Uparrow}$ & 1.8 & $(0.8-3.8)$ & 5.3 & $(3.6-7.8)$ & 3.8 & $(2.5-6.0)$ & 2.5 & $(1.6-4.0)$ & 5.9 & $(3.8-9.1)$ & 4.3 & $(3.0-6.1)$ \\
\hline Hispanic & 6.6 & $(5.2-8.2)$ & 9.4 & $(7.3-12.0)$ & 8.0 & $(6.6-9.7)$ & 4.1 & $(2.8-6.0)$ & 7.8 & $(6.5-9.4)$ & 6.1 & $(4.9-7.5)$ \\
\hline \multicolumn{13}{|l|}{ Grade } \\
\hline 9 & 3.3 & $(2.3-4.7)$ & 3.3 & $(2.2-5.0)$ & 3.4 & $(2.6-4.5)$ & 2.8 & $(1.9-4.0)$ & 3.6 & $(2.4-5.3)$ & 3.2 & $(2.5-4.1)$ \\
\hline 10 & 3.8 & $(2.6-5.6)$ & 6.4 & $(4.7-8.8)$ & 5.1 & $(3.8-6.8)$ & 3.7 & $(2.6-5.2)$ & 6.0 & $(4.5-8.1)$ & 4.9 & $(3.7-6.4)$ \\
\hline 11 & 3.3 & $(2.2-4.9)$ & 6.2 & $(4.7-8.2)$ & 5.0 & $(3.9-6.5)$ & 4.8 & (3.4-6.8) & 6.2 & $(4.6-8.2)$ & 5.7 & $(4.6-7.1)$ \\
\hline 12 & 4.5 & $(3.4-6.0)$ & 9.7 & $(7.1-13.2)$ & 7.2 & $(5.6-9.1)$ & 4.0 & $(2.8-5.6)$ & 8.0 & $(6.1-10.6)$ & 6.1 & $(4.8-7.7)$ \\
\hline Total & 3.8 & $(3.1-4.6)$ & 6.3 & $(5.1-7.9)$ & 5.2 & $(4.3-6.2)$ & 3.9 & $(3.1-4.7)$ & 6.0 & $(4.9-7.3)$ & 5.0 & $(4.3-5.8)$ \\
\hline
\end{tabular}

* Any form of cocaine, such as powder, crack, or freebase, one or more times during their life.

† Also called "MDMA," one or more times during their life.

$\S 95 \%$ confidence interval.

" Non-Hispanic. 
TABLE 60. Percentage of high school students who ever used cocaine* and who ever used ecstasy, ${ }^{\dagger}$ by sex - selected U.S. sites, Youth Risk Behavior Survey, 2015

\begin{tabular}{|c|c|c|c|c|c|c|c|c|c|c|c|c|}
\hline \multirow[b]{3}{*}{ Site } & \multicolumn{6}{|c|}{ Ever used cocaine } & \multicolumn{6}{|c|}{ Ever used ecstasy } \\
\hline & \multicolumn{2}{|c|}{ Female } & \multicolumn{2}{|c|}{ Male } & \multicolumn{2}{|c|}{ Total } & \multicolumn{2}{|c|}{ Female } & \multicolumn{2}{|c|}{ Male } & \multicolumn{2}{|r|}{ Total } \\
\hline & $\%$ & $\mathrm{Cl}^{\S}$ & $\%$ & $\mathrm{Cl}$ & $\%$ & $\mathrm{Cl}$ & $\%$ & $\mathrm{Cl}$ & $\%$ & $\mathrm{Cl}$ & $\%$ & $\mathrm{Cl}$ \\
\hline \multicolumn{13}{|l|}{ State surveys } \\
\hline Alabama & 3.5 & $(2.1-5.9)$ & 8.1 & $(6.1-10.8)$ & 6.1 & $(4.4-8.4)$ & 5.2 & (3.3-8.3) & 8.0 & $(5.3-12.0)$ & 7.0 & $(4.8-10.1)$ \\
\hline Alaska & 2.5 & $(1.5-4.3)$ & 6.0 & $(4.0-8.8)$ & 4.6 & (3.3-6.4) & 2.1 & $(1.2-3.4)$ & 5.5 & $(3.9-7.8)$ & 4.1 & $(3.0-5.7)$ \\
\hline Arizona & 7.5 & $(4.9-11.4)$ & 10.2 & $(8.3-12.5)$ & 9.2 & $(7.3-11.5)$ & -9 & - & - & - & - & - \\
\hline Arkansas & 4.7 & $(3.7-6.0)$ & 10.5 & $(7.3-14.9)$ & 7.7 & (5.7-10.4) & 4.2 & $(3.3-5.5)$ & 8.3 & $(5.5-12.4)$ & 6.5 & $(5.2-8.2)$ \\
\hline California & 5.3 & $(3.8-7.3)$ & 6.3 & $(3.7-10.5)$ & 5.8 & $(3.9-8.5)$ & 6.4 & $(4.7-8.6)$ & 7.0 & $(4.3-11.1)$ & 6.8 & $(4.8-9.7)$ \\
\hline Connecticut & 2.6 & $(1.7-3.9)$ & 6.1 & $(4.5-8.2)$ & 4.6 & $(3.5-6.0)$ & 3.2 & $(2.4-4.3)$ & 5.6 & $(4.5-7.0)$ & 4.6 & $(3.9-5.4)$ \\
\hline Delaware & 2.9 & $(1.9-4.5)$ & 5.2 & (3.7-7.4) & 4.4 & $(3.4-5.6)$ & 3.5 & $(2.4-5.1)$ & 5.5 & (3.9-7.7) & 4.7 & $(3.6-6.1)$ \\
\hline Florida & 4.3 & $(3.5-5.2)$ & 7.7 & $(6.6-9.1)$ & 6.2 & $(5.4-7.2)$ & - & - & - & - & - & - \\
\hline Hawaii & 4.1 & $(3.0-5.7)$ & 7.0 & $(5.7-8.5)$ & 5.7 & $(4.7-6.8)$ & 6.8 & $(5.3-8.7)$ & 8.5 & $(7.1-10.3)$ & 8.0 & $(6.7-9.5)$ \\
\hline Idaho & - & - & - & - & - & - & 4.2 & $(2.8-6.1)$ & 5.0 & $(3.7-6.7)$ & 4.7 & $(3.7-5.9)$ \\
\hline Illinois & 2.9 & $(2.2-3.8)$ & 7.9 & $(6.2-9.9)$ & 5.4 & $(4.4-6.7)$ & 4.2 & $(3.1-5.7)$ & 7.8 & $(6.0-10.1)$ & 6.1 & $(5.0-7.5)$ \\
\hline Indiana & 2.7 & $(1.7-4.2)$ & 5.2 & $(3.4-7.9)$ & 4.0 & $(2.9-5.7)$ & 3.3 & $(1.8-5.9)$ & 6.4 & $(3.9-10.5)$ & 5.0 & $(3.3-7.5)$ \\
\hline Kentucky & 4.2 & $(2.8-6.4)$ & 4.5 & $(3.4-5.8)$ & 4.6 & $(3.4-6.0)$ & 4.1 & $(2.6-6.4)$ & 5.8 & $(4.3-7.7)$ & 5.0 & $(3.9-6.5)$ \\
\hline Maine & - & - & - & - & - & - & - & - & - & - & - & - \\
\hline Maryland & 3.6 & $(3.3-4.0)$ & 6.7 & $(6.1-7.2)$ & 5.4 & $(5.1-5.8)$ & 4.3 & $(4.0-4.7)$ & 7.9 & $(7.4-8.5)$ & 6.4 & $(6.1-6.8)$ \\
\hline Massachusetts & 2.6 & $(1.7-4.0)$ & 6.1 & $(4.6-8.0)$ & 4.5 & $(3.3-6.0)$ & 2.5 & $(1.6-4.0)$ & 5.6 & $(4.3-7.3)$ & 4.3 & $(3.3-5.6)$ \\
\hline Michigan & 2.7 & $(1.5-4.9)$ & 5.5 & $(4.1-7.2)$ & 4.2 & $(3.1-5.5)$ & - & - & - & - & - & - \\
\hline Mississippi & 4.7 & $(3.2-6.8)$ & 7.9 & $(6.3-9.9)$ & 6.5 & $(5.3-8.0)$ & 4.9 & (3.3-7.3) & 10.1 & $(7.2-14.0)$ & 7.8 & $(6.0-10.1)$ \\
\hline Missouri & - & - & - & - & - & - & - & - & - & - & - & - \\
\hline Montana & 4.5 & $(3.7-5.6)$ & 5.7 & $(4.6-7.0)$ & 5.2 & $(4.4-6.1)$ & 4.6 & $(3.8-5.6)$ & 7.4 & $(6.2-8.9)$ & 6.1 & $(5.2-7.0)$ \\
\hline Nebraska & 5.2 & $(3.5-7.7)$ & 5.2 & (3.4-7.9) & 5.3 & $(4.0-7.1)$ & 5.0 & (3.7-6.7) & 5.2 & $(3.4-7.8)$ & 5.1 & $(3.9-6.8)$ \\
\hline Nevada & 6.9 & $(4.4-10.7)$ & 6.2 & $(3.9-9.7)$ & 6.6 & $(4.6-9.4)$ & 6.5 & $(4.2-10.1)$ & 7.8 & $(6.4-9.6)$ & 7.3 & $(6.0-8.9)$ \\
\hline New Hampshire & 3.1 & $(2.6-3.7)$ & 6.5 & (5.8-7.3) & 4.9 & $(4.4-5.5)$ & 3.4 & $(2.8-4.1)$ & 5.4 & $(4.8-6.1)$ & 4.5 & $(4.0-5.0)$ \\
\hline New Mexico & 6.4 & $(5.6-7.4)$ & 11.0 & $(10.0-12.2)$ & 8.8 & $(8.0-9.6)$ & 6.6 & $(5.6-7.6)$ & 9.2 & $(8.1-10.5)$ & 7.9 & $(7.2-8.8)$ \\
\hline New York & 5.4 & $(3.7-7.8)$ & 9.2 & $(7.5-11.3)$ & 7.6 & $(6.0-9.6)$ & - & - & - & - & - & - \\
\hline North Carolina & 3.5 & $(2.0-6.0)$ & 5.1 & $(4.0-6.4)$ & 4.5 & $(3.4-5.9)$ & - & - & - & - & - & - \\
\hline North Dakota & 3.1 & $(2.2-4.4)$ & 4.7 & $(3.4-6.4)$ & 3.9 & $(3.0-5.1)$ & 2.6 & $(1.7-3.8)$ & 4.7 & $(3.4-6.3)$ & 3.6 & $(2.8-4.8)$ \\
\hline Oklahoma & 1.7 & $(0.8-3.4)$ & 5.1 & $(3.8-6.8)$ & 3.4 & $(2.5-4.7)$ & 2.1 & $(1.2-3.6)$ & 5.7 & $(3.9-8.2)$ & 3.9 & $(2.7-5.6)$ \\
\hline Pennsylvania & 2.6 & $(1.8-3.7)$ & 4.6 & $(3.5-5.9)$ & 3.6 & $(3.0-4.4)$ & 3.1 & $(2.1-4.6)$ & 5.3 & $(3.8-7.4)$ & 4.2 & $(3.4-5.2)$ \\
\hline Rhode Island & 3.2 & $(1.9-5.1)$ & 6.0 & $(4.2-8.5)$ & 4.8 & $(3.3-6.9)$ & 3.8 & $(2.4-5.8)$ & 6.1 & $(4.0-9.2)$ & 5.1 & $(3.7-7.1)$ \\
\hline South Carolina & 2.6 & $(0.9-6.9)$ & 5.8 & $(3.7-9.0)$ & 4.2 & $(2.7-6.4)$ & 2.5 & $(0.9-6.7)$ & 4.2 & $(2.5-7.0)$ & 3.4 & $(2.0-5.6)$ \\
\hline South Dakota & - & - & - & - & - & - & - & - & - & - & - & - \\
\hline Tennessee & - & - & - & - & - & - & - & - & - & - & - & - \\
\hline Vermont & 3.5 & $(3.2-3.9)$ & 6.0 & $(5.5-6.5)$ & 4.8 & $(4.6-5.2)$ & - & - & - & - & - & - \\
\hline Virginia & 2.4 & $(1.8-3.2)$ & 5.1 & $(4.0-6.7)$ & 3.9 & $(3.1-4.9)$ & 2.9 & $(2.2-3.8)$ & 4.9 & $(3.9-6.1)$ & 4.0 & $(3.3-4.7)$ \\
\hline West Virginia & 2.8 & $(1.7-4.6)$ & 6.3 & $(3.7-10.6)$ & 4.6 & $(3.0-6.9)$ & 4.9 & $(3.3-7.2)$ & 8.4 & $(6.0-11.7)$ & 6.7 & $(5.0-9.0)$ \\
\hline Wyoming & 6.0 & $(4.1-8.5)$ & 8.7 & $(6.5-11.6)$ & 7.5 & $(5.8-9.7)$ & 6.2 & $(4.2-9.0)$ & 8.7 & $(6.6-11.3)$ & 7.4 & (5.7-9.7) \\
\hline Median & \multirow{2}{*}{\multicolumn{2}{|c|}{$\begin{array}{l}3.5 \\
.7-7.5)\end{array}$}} & \multirow{2}{*}{\multicolumn{2}{|c|}{$\begin{array}{c}6.1 \\
(4.5-11.0)\end{array}$}} & \multirow{2}{*}{\multicolumn{2}{|c|}{$\begin{array}{c}4.8 \\
(3.4-9.2)\end{array}$}} & \multirow{2}{*}{\multicolumn{2}{|c|}{$\begin{array}{c}4.2 \\
(2.1-6.8)\end{array}$}} & \multirow{2}{*}{\multicolumn{2}{|c|}{$\begin{array}{c}6.1 \\
(4.2-10.1)\end{array}$}} & & 5.1 \\
\hline Range & & & & & & & & & & & & 3.4-8.0) \\
\hline Large urban school dist & veys & & & & & & & & & & & \\
\hline Baltimore, MD & 2.8 & $(1.6-4.7)$ & 11.3 & $(8.2-15.3)$ & 8.0 & $(5.8-10.8)$ & 2.1 & $(1.1-4.0)$ & 9.4 & $(6.2-14.2)$ & 6.8 & $(4.7-9.9)$ \\
\hline Boston, MA & - & - & - & - & - & - & - & - & - & - & - & - \\
\hline Broward County, FL & 5.6 & $(4.0-7.8)$ & 7.0 & $(4.5-10.5)$ & 6.4 & $(4.9-8.4)$ & 4.6 & $(3.2-6.6)$ & 5.9 & $(4.3-8.0)$ & 5.5 & $(4.2-7.2)$ \\
\hline Cleveland, $\mathrm{OH}$ & - & - & - & - & - & - & - & - & - & - & - & - \\
\hline DeKalb County, GA & 1.9 & $(1.2-3.0)$ & 7.0 & $(5.2-9.4)$ & 4.5 & $(3.5-5.9)$ & 2.3 & $(1.4-3.6)$ & 7.1 & $(5.1-9.9)$ & 4.7 & $(3.6-6.1)$ \\
\hline Detroit, MI & 3.4 & $(2.2-5.1)$ & 6.8 & $(5.0-9.1)$ & 5.3 & $(4.0-6.9)$ & - & - & - & - & - & - \\
\hline District of Columbia & 4.2 & $(3.7-4.8)$ & 8.0 & (7.2-8.9) & 6.3 & $(5.8-6.8)$ & 3.0 & $(2.6-3.6)$ & 7.6 & $(6.8-8.5)$ & 5.5 & $(5.0-6.0)$ \\
\hline Duval County, FL & 5.8 & $(4.4-7.6)$ & 9.8 & $(7.7-12.4)$ & 8.5 & $(6.9-10.5)$ & 5.9 & $(4.3-8.1)$ & 9.8 & $(7.7-12.4)$ & 8.4 & $(6.7-10.5)$ \\
\hline Ft. Worth, TX & 3.8 & $(2.7-5.3)$ & 7.4 & $(5.9-9.4)$ & 5.6 & $(4.5-6.9)$ & 3.3 & $(2.3-4.6)$ & 7.6 & $(6.0-9.6)$ & 5.4 & $(4.4-6.7)$ \\
\hline Houston, TX & 7.4 & $(6.0-9.2)$ & 11.2 & $(9.5-13.3)$ & 9.6 & $(8.2-11.2)$ & 6.9 & $(5.5-8.7)$ & 9.6 & $(8.0-11.4)$ & 8.6 & $(7.3-10.2)$ \\
\hline Los Angeles, CA & 4.2 & $(2.8-6.3)$ & 5.8 & $(4.5-7.5)$ & 5.0 & $(3.9-6.5)$ & 3.9 & $(2.4-6.2)$ & 5.1 & $(3.9-6.5)$ & 4.5 & $(3.3-6.2)$ \\
\hline Miami-Dade County, FL & 3.4 & $(2.5-4.6)$ & 7.2 & $(5.4-9.4)$ & 5.3 & $(4.2-6.7)$ & 3.7 & $(2.7-5.1)$ & 8.8 & $(6.9-11.2)$ & 6.3 & $(5.0-7.8)$ \\
\hline
\end{tabular}

See table footnotes on the next page. 
TABLE 60. (Continued) Percentage of high school students who ever used cocaine* and who ever used ecstasy, ${ }^{\dagger}$ by sex - selected U.S. sites, Youth Risk Behavior Survey, 2015

\begin{tabular}{|c|c|c|c|c|c|c|c|c|c|c|c|c|}
\hline \multirow[b]{3}{*}{ Site } & \multicolumn{6}{|c|}{ Ever used cocaine } & \multicolumn{6}{|c|}{ Ever used ecstasy } \\
\hline & \multicolumn{2}{|c|}{ Female } & \multicolumn{2}{|c|}{ Male } & \multicolumn{2}{|c|}{ Total } & \multicolumn{2}{|c|}{ Female } & \multicolumn{2}{|r|}{ Male } & \multicolumn{2}{|r|}{ Total } \\
\hline & $\%$ & $\mathrm{Cl}^{\S}$ & $\%$ & $\mathrm{Cl}$ & $\%$ & $\mathrm{Cl}$ & $\%$ & $\mathrm{Cl}$ & $\%$ & $\mathrm{Cl}$ & $\%$ & $\mathrm{Cl}$ \\
\hline New York City, NY & 2.7 & $(2.0-3.7)$ & 5.7 & $(4.6-6.9)$ & 4.4 & $(3.6-5.3)$ & - & - & - & - & - & - \\
\hline Oakland, CA & - & - & - & - & - & - & - & - & - & - & - & - \\
\hline Orange County, FL & 3.0 & $(1.9-4.6)$ & 7.1 & $(4.6-10.7)$ & 5.0 & $(3.5-7.2)$ & 3.2 & $(2.0-5.1)$ & 8.2 & $(5.2-12.5)$ & 5.7 & $(4.1-7.9)$ \\
\hline Palm Beach County, FL & 4.5 & $(3.3-6.1)$ & 8.3 & $(6.5-10.5)$ & 7.0 & $(5.8-8.5)$ & 7.0 & $(4.9-9.8)$ & 9.4 & $(7.2-12.2)$ & 9.1 & (7.2-11.5) \\
\hline Philadelphia, PA & 3.7 & $(2.1-6.4)$ & 5.4 & $(2.8-10.2)$ & 4.6 & $(2.8-7.7)$ & 2.5 & $(1.4-4.4)$ & 5.6 & $(2.8-10.9)$ & 4.2 & $(2.5-7.1)$ \\
\hline San Diego, CA & 2.8 & $(1.9-4.1)$ & 7.8 & $(6.1-9.9)$ & 5.3 & $(4.3-6.6)$ & 5.5 & $(4.1-7.3)$ & 8.2 & $(6.5-10.3)$ & 6.9 & $(5.6-8.4)$ \\
\hline San Francisco, CA & 4.8 & $(3.1-7.2)$ & 5.1 & $(3.7-7.0)$ & 5.3 & $(3.9-7.1)$ & 3.9 & $(2.6-5.8)$ & 5.9 & $(4.2-8.1)$ & 5.1 & $(3.9-6.7)$ \\
\hline Median & \multicolumn{2}{|c|}{3.7} & \multicolumn{2}{|c|}{7.1} & \multicolumn{2}{|c|}{5.3} & \multicolumn{2}{|c|}{3.8} & \multicolumn{2}{|r|}{7.9} & \multicolumn{2}{|r|}{5.6} \\
\hline Range & \multicolumn{2}{|c|}{$(1.9-7.4)$} & \multicolumn{2}{|c|}{$(5.1-11.3)$} & \multicolumn{2}{|c|}{$(4.4-9.6)$} & \multicolumn{2}{|c|}{$(2.1-7.0)$} & \multicolumn{2}{|r|}{$(5.1-9.8)$} & \multicolumn{2}{|r|}{$(4.2-9.1)$} \\
\hline
\end{tabular}

* Any form of cocaine, such as powder, crack, or freebase, one or more times during their life.

† Also called "MDMA," one or more times during their life.

§ 95\% confidence interval.

१ Not available.

TABLE 61. Percentage of high school students who ever used heroin* and who ever used methamphetamines, ${ }^{\dagger}$ by sex, race/ethnicity, and grade - United States, Youth Risk Behavior Survey, 2015

\begin{tabular}{|c|c|c|c|c|c|c|c|c|c|c|c|c|}
\hline \multirow[b]{3}{*}{ Category } & \multicolumn{6}{|c|}{ Ever used heroin } & \multicolumn{6}{|c|}{ Ever used methamphetamines } \\
\hline & \multicolumn{2}{|c|}{ Female } & \multicolumn{2}{|c|}{ Male } & \multicolumn{2}{|c|}{ Total } & \multicolumn{2}{|c|}{ Female } & \multicolumn{2}{|c|}{ Male } & \multicolumn{2}{|c|}{ Total } \\
\hline & $\%$ & $\mathrm{Cl}^{\S}$ & $\%$ & $\mathrm{Cl}$ & $\%$ & $\mathrm{Cl}$ & $\%$ & $\mathrm{Cl}$ & $\%$ & $\mathrm{Cl}$ & $\%$ & $\mathrm{Cl}$ \\
\hline \multicolumn{13}{|c|}{ Race/Ethnicity } \\
\hline White & 0.8 & $(0.5-1.5)$ & 1.7 & $(1.2-2.4)$ & 1.3 & $(1.0-1.7)$ & 1.7 & $(1.1-2.7)$ & 2.5 & $(1.8-3.5)$ & 2.1 & $(1.5-2.8)$ \\
\hline Black ${ }^{\mathbb{1}}$ & 1.5 & $(0.6-3.6)$ & 3.8 & $(1.9-7.5)$ & 2.7 & $(1.3-5.6)$ & 1.4 & $(0.9-2.3)$ & 3.9 & $(2.1-7.4)$ & 2.8 & $(1.5-5.1)$ \\
\hline Hispanic & 1.9 & $(1.2-3.0)$ & 3.2 & $(2.1-5.0)$ & 2.6 & $(1.8-3.8)$ & 4.0 & $(2.9-5.5)$ & 4.7 & $(3.3-6.6)$ & 4.4 & $(3.3-5.9)$ \\
\hline \multicolumn{13}{|l|}{ Grade } \\
\hline 9 & 1.4 & $(0.9-2.3)$ & 2.0 & $(1.2-3.6)$ & 1.8 & $(1.2-2.6)$ & 2.2 & $(1.5-3.2)$ & 1.9 & $(1.1-3.1)$ & 2.0 & $(1.5-2.7)$ \\
\hline 10 & 1.5 & $(0.8-2.7)$ & 3.3 & $(2.2-5.0)$ & 2.4 & $(1.6-3.6)$ & 2.5 & $(1.5-4.2)$ & 4.2 & $(2.7-6.3)$ & 3.3 & $(2.3-4.9)$ \\
\hline 11 & 0.9 & $(0.5-1.7)$ & 2.3 & $(1.4-3.8)$ & 1.9 & $(1.1-3.0)$ & 2.3 & $(1.4-3.9)$ & 2.8 & $(1.8-4.2)$ & 2.8 & $(1.9-4.0)$ \\
\hline 12 & 1.0 & $(0.4-2.2)$ & 2.8 & $(1.6-4.6)$ & 1.9 & $(1.3-2.9)$ & 1.8 & $(1.2-2.8)$ & 5.6 & $(3.6-8.5)$ & 3.8 & $(2.7-5.3)$ \\
\hline Total & 1.2 & $(0.9-1.8)$ & 2.7 & $(1.9-3.8)$ & 2.1 & $(1.5-2.8)$ & 2.3 & $(1.7-3.0)$ & 3.6 & $(2.6-4.9)$ & 3.0 & $(2.4-3.8)$ \\
\hline
\end{tabular}

* Also called "smack," "junk," or "China White" one or more times during their life.

† Also called "speed," "crystal," "crank," or "ice" one or more times during their life.

$\S 95 \%$ confidence interval.

१ Non-Hispanic. 
TABLE 62. Percentage of high school students who ever used heroin* and who ever used methamphetamines, ${ }^{\dagger}$ by sex - selected U.S. sites, Youth Risk Behavior Survey, 2015

\begin{tabular}{|c|c|c|c|c|c|c|c|c|c|c|c|c|}
\hline \multirow[b]{3}{*}{ Site } & \multicolumn{6}{|c|}{ Ever used heroin } & \multicolumn{6}{|c|}{ Ever used methamphetamines } \\
\hline & \multicolumn{2}{|c|}{ Female } & \multicolumn{2}{|r|}{ Male } & \multicolumn{2}{|r|}{ Total } & \multicolumn{2}{|c|}{ Female } & \multicolumn{2}{|r|}{ Male } & \multicolumn{2}{|r|}{ Total } \\
\hline & $\%$ & $\mathrm{Cl}^{\S}$ & $\%$ & $\mathrm{Cl}$ & $\%$ & $\mathrm{Cl}$ & $\%$ & $\mathrm{Cl}$ & $\%$ & $\mathrm{Cl}$ & $\%$ & $\mathrm{Cl}$ \\
\hline \multicolumn{13}{|l|}{ State surveys } \\
\hline Alabama & 2.3 & $(1.2-4.3)$ & 6.9 & $(5.2-9.1)$ & 4.9 & $(3.7-6.6)$ & 4.8 & $(3.4-6.7)$ & 6.0 & $(3.3-10.6)$ & 5.7 & $(3.7-8.6)$ \\
\hline Alaska & 1.0 & $(0.4-2.4)$ & 2.9 & $(1.9-4.4)$ & 2.2 & $(1.3-3.5)$ & 1.6 & $(0.8-3.2)$ & 3.1 & $(2.0-4.7)$ & 2.6 & $(1.7-4.1)$ \\
\hline Arizona & 2.1 & $(1.3-3.4)$ & 3.4 & $(2.0-5.7)$ & 2.9 & $(2.1-4.0)$ & 2.7 & $(1.8-4.1)$ & 4.4 & $(2.5-7.6)$ & 3.8 & $(2.6-5.7)$ \\
\hline Arkansas & 2.9 & $(2.0-4.3)$ & 6.4 & $(4.2-9.7)$ & 4.9 & (3.8-6.4) & 3.5 & $(2.5-4.9)$ & 8.3 & $(4.9-13.7)$ & 6.0 & $(4.0-9.1)$ \\
\hline California & 1.6 & $(1.1-2.2)$ & 1.6 & $(0.8-3.1)$ & 1.6 & $(1.1-2.2)$ & 2.6 & $(1.6-4.2)$ & 3.1 & $(1.8-5.3)$ & 2.9 & $(1.9-4.3)$ \\
\hline Connecticut & 1.1 & $(0.6-2.2)$ & 3.0 & $(2.1-4.2)$ & 2.2 & $(1.6-3.1)$ & 1.9 & $(1.2-3.1)$ & 3.6 & $(2.8-4.6)$ & 3.0 & $(2.3-3.7)$ \\
\hline Delaware & 1.5 & $(0.7-3.1)$ & 3.3 & $(2.4-4.7)$ & 2.6 & $(1.9-3.6)$ & 2.3 & $(1.4-3.9)$ & 2.8 & $(1.8-4.4)$ & 2.7 & $(1.9-3.7)$ \\
\hline Florida & - & - & - & - & - & - & - & - & - & - & - & - \\
\hline Hawaii & 1.8 & $(1.3-2.4)$ & 4.4 & $(3.5-5.5)$ & 3.3 & $(2.7-4.1)$ & 2.4 & $(1.8-3.3)$ & 4.6 & $(3.7-5.7)$ & 3.8 & (3.1-4.7) \\
\hline Idaho & 1.0 & $(0.4-2.5)$ & 2.0 & $(1.1-3.7)$ & 1.6 & $(0.9-2.7)$ & 2.0 & $(1.1-3.5)$ & 2.4 & $(1.7-3.4)$ & 2.3 & $(1.7-3.1)$ \\
\hline Illinois & 1.6 & $(1.1-2.3)$ & 4.9 & $(3.3-7.1)$ & 3.4 & $(2.4-4.7)$ & 1.5 & $(1.0-2.2)$ & 5.4 & $(3.9-7.4)$ & 3.5 & $(2.6-4.6)$ \\
\hline Indiana & 1.4 & $(0.7-2.8)$ & 3.2 & $(1.5-6.6)$ & 2.4 & $(1.3-4.4)$ & 1.4 & $(0.8-2.6)$ & 4.1 & $(2.0-8.2)$ & 2.9 & $(1.5-5.4)$ \\
\hline Kentucky & 3.1 & $(1.9-5.1)$ & 3.6 & $(2.1-6.1)$ & 3.7 & $(2.5-5.4)$ & 3.1 & $(1.9-5.0)$ & 3.9 & $(2.6-5.8)$ & 3.9 & $(2.8-5.2)$ \\
\hline Maine & - & - & - & - & - & - & - & - & - & - & - & - \\
\hline Maryland & 2.4 & $(2.1-2.7)$ & 5.5 & $(5.0-6.0)$ & 4.2 & $(3.9-4.5)$ & 2.4 & $(2.2-2.8)$ & 5.4 & $(4.9-5.8)$ & 4.2 & $(3.9-4.5)$ \\
\hline Massachusetts & 0.8 & $(0.4-1.4)$ & 2.3 & $(1.7-3.1)$ & 1.7 & $(1.3-2.3)$ & 1.0 & $(0.5-1.8)$ & 2.8 & $(2.0-3.8)$ & 2.1 & $(1.6-2.8)$ \\
\hline Michigan & 1.2 & $(0.5-2.8)$ & 3.7 & $(2.4-5.7)$ & 2.5 & $(1.6-3.9)$ & 1.9 & $(1.0-3.8)$ & 4.4 & $(3.1-6.2)$ & 3.2 & $(2.2-4.6)$ \\
\hline Mississippi & 3.3 & $(2.1-5.1)$ & 7.9 & $(5.6-11.0)$ & 5.9 & $(4.4-7.8)$ & 4.1 & $(2.9-5.8)$ & 7.8 & $(5.5-10.8)$ & 6.0 & $(4.6-7.8)$ \\
\hline Missouri & - & - & - & - & - & - & - & - & - & - & - & - \\
\hline Montana & 1.1 & $(0.7-1.7)$ & 3.0 & $(2.2-4.0)$ & 2.1 & $(1.6-2.7)$ & 2.2 & $(1.6-3.1)$ & 3.8 & $(2.8-5.0)$ & 3.0 & $(2.3-3.9)$ \\
\hline Nebraska & 1.9 & $(0.9-4.2)$ & 2.8 & $(1.5-5.3)$ & 2.5 & $(1.4-4.3)$ & 3.8 & $(2.8-5.2)$ & 4.1 & $(2.5-6.8)$ & 4.2 & $(3.1-5.8)$ \\
\hline Nevada & 1.6 & $(0.9-2.9)$ & 3.5 & $(2.1-5.8)$ & 2.7 & $(2.0-3.8)$ & 3.5 & $(2.0-6.0)$ & 3.9 & $(2.3-6.6)$ & 3.8 & $(2.7-5.5)$ \\
\hline New Hampshire & 1.3 & $(1.0-1.9)$ & 3.2 & $(2.8-3.7)$ & 2.4 & $(2.1-2.8)$ & 1.4 & $(1.1-1.9)$ & 3.3 & $(2.8-3.9)$ & 2.5 & $(2.1-2.8)$ \\
\hline New Mexico & 1.7 & $(1.3-2.3)$ & 5.2 & $(4.2-6.3)$ & 3.5 & $(3.0-4.1)$ & 2.6 & $(2.0-3.3)$ & 6.1 & $(5.2-7.1)$ & 4.4 & $(3.9-5.0)$ \\
\hline New York & 2.6 & $(1.7-3.9)$ & 6.5 & $(5.0-8.4)$ & 4.8 & $(3.8-6.1)$ & - & - & - & - & - & - \\
\hline North Carolina & - & - & - & - & - & - & - & - & - & - & - & - \\
\hline North Dakota & 0.6 & $(0.3-1.1)$ & 1.7 & $(1.0-2.9)$ & 1.2 & $(0.8-1.8)$ & - & - & - & - & - & - \\
\hline Oklahoma & 0.3 & $(0.1-0.9)$ & 1.5 & $(0.8-2.8)$ & 0.9 & $(0.5-1.7)$ & 1.2 & $(0.7-2.4)$ & 2.1 & $(1.2-3.7)$ & 1.7 & $(1.0-2.7)$ \\
\hline Pennsylvania & 1.0 & $(0.6-1.9)$ & 2.9 & (1.8-4.5) & 2.0 & $(1.4-2.9)$ & 1.6 & $(0.9-2.6)$ & 3.0 & (2.0-4.5) & 2.3 & $(1.7-3.1)$ \\
\hline Rhode Island & 1.5 & $(0.8-2.9)$ & 5.3 & (3.6-7.8) & 3.6 & $(2.4-5.5)$ & - & - & - & - & - & - \\
\hline South Carolina & 1.1 & $(0.5-2.8)$ & 3.0 & $(1.6-5.7)$ & 2.1 & $(1.1-3.9)$ & 1.6 & $(0.6-4.4)$ & 4.0 & $(1.9-8.3)$ & 2.8 & $(1.4-5.6)$ \\
\hline South Dakota & 1.8 & $(0.8-4.3)$ & 3.8 & $(1.8-7.9)$ & 2.9 & $(1.5-5.5)$ & 2.8 & $(1.5-5.0)$ & 4.7 & $(2.4-8.9)$ & 3.8 & $(2.2-6.4)$ \\
\hline Tennessee & - & - & - & - & - & - & - & - & - & - & - & - \\
\hline Vermont & 1.4 & $(1.2-1.6)$ & 3.1 & $(2.8-3.5)$ & 2.3 & $(2.1-2.6)$ & 1.9 & $(1.6-2.2)$ & 3.4 & $(3.0-3.7)$ & 2.7 & $(2.5-2.9)$ \\
\hline Virginia & 1.2 & $(0.7-2.0)$ & 2.4 & $(1.8-3.3)$ & 1.8 & $(1.4-2.5)$ & 1.4 & $(0.9-2.3)$ & 3.3 & $(2.5-4.4)$ & 2.5 & $(1.9-3.3)$ \\
\hline West Virginia & 1.8 & $(1.1-2.9)$ & 5.0 & $(3.4-7.5)$ & 3.5 & $(2.4-5.1)$ & 2.6 & $(1.7-3.8)$ & 6.8 & $(4.1-10.9)$ & 4.7 & $(3.1-7.1)$ \\
\hline Wyoming & 2.3 & $(1.5-3.6)$ & 4.1 & $(2.8-5.8)$ & 3.3 & $(2.4-4.6)$ & 3.0 & $(1.8-5.0)$ & 5.1 & (3.4-7.5) & 4.2 & $(2.8-6.1)$ \\
\hline Median & \multirow{2}{*}{\multicolumn{2}{|c|}{$\begin{array}{c}1.5 \\
(0.3-3.3)\end{array}$}} & \multirow{2}{*}{\multicolumn{2}{|c|}{$\begin{array}{c}3.3 \\
(1.5-7.9)\end{array}$}} & \multirow{2}{*}{\multicolumn{2}{|c|}{$\begin{array}{c}2.5 \\
(0.9-5.9)\end{array}$}} & \multirow{2}{*}{\multicolumn{2}{|c|}{$\begin{array}{c}2.3 \\
(1.0-4.8)\end{array}$}} & \multirow{2}{*}{\multicolumn{2}{|c|}{$\begin{array}{c}4.0 \\
(2.1-8.3)\end{array}$}} & & 3.2 \\
\hline Range & & & & & & & & & & & & $1.7-6.0)$ \\
\hline Large urban school dist & veys & & & & & & & & & & & \\
\hline Baltimore, MD & 3.3 & $(2.0-5.4)$ & 11.0 & $(8.0-14.9)$ & 8.3 & $(6.1-11.1)$ & 2.9 & $(1.8-4.8)$ & 10.4 & $(7.6-14.0)$ & 7.9 & $(5.9-10.5)$ \\
\hline Boston, MA & 0.4 & $(0.1-1.3)$ & 3.0 & $(1.8-4.8)$ & 1.8 & $(1.2-2.7)$ & 1.1 & $(0.6-2.2)$ & 3.5 & $(2.1-5.5)$ & 2.4 & $(1.6-3.6)$ \\
\hline Broward County, FL & 2.4 & $(1.4-3.8)$ & 4.9 & $(3.2-7.5)$ & 4.0 & $(2.9-5.6)$ & 3.1 & $(2.0-4.7)$ & 5.4 & $(3.6-8.1)$ & 4.5 & $(3.3-6.0)$ \\
\hline Cleveland, $\mathrm{OH}$ & - & - & - & - & - & - & - & - & - & - & - & - \\
\hline DeKalb County, GA & 0.6 & $(0.3-1.2)$ & 4.2 & $(2.8-6.2)$ & 2.4 & $(1.6-3.5)$ & 1.0 & $(0.6-1.7)$ & 5.9 & $(4.2-8.2)$ & 3.5 & $(2.5-4.8)$ \\
\hline Detroit, MI & 2.7 & $(1.5-4.8)$ & 5.7 & $(4.0-8.2)$ & 4.3 & $(3.1-5.9)$ & 2.4 & $(1.5-4.0)$ & 4.7 & (3.2-6.9) & 3.7 & $(2.6-5.2)$ \\
\hline District of Columbia & 2.9 & $(2.5-3.5)$ & 6.0 & $(5.3-6.8)$ & 4.6 & $(4.2-5.1)$ & 2.5 & $(2.1-3.1)$ & 6.2 & $(5.5-7.0)$ & 4.5 & $(4.1-5.0)$ \\
\hline Duval County, FL & 3.6 & $(2.1-6.0)$ & 7.8 & $(5.5-11.0)$ & 6.3 & $(4.4-9.0)$ & 4.9 & $(2.9-8.0)$ & 8.6 & $(6.1-12.1)$ & 7.5 & $(5.3-10.5)$ \\
\hline Ft. Worth, TX & 0.5 & $(0.2-1.2)$ & 2.5 & $(1.6-4.0)$ & 1.5 & $(1.0-2.3)$ & 2.2 & $(1.4-3.4)$ & 3.3 & $(2.3-4.6)$ & 2.7 & $(2.0-3.6)$ \\
\hline Houston, TX & 4.6 & $(3.4-6.1)$ & 6.7 & $(5.2-8.6)$ & 6.1 & $(4.8-7.7)$ & 5.6 & $(4.3-7.3)$ & 6.7 & $(5.0-8.8)$ & 6.5 & $(5.2-8.2)$ \\
\hline Los Angeles, CA & 1.1 & $(0.6-2.1)$ & 2.8 & $(1.4-5.5)$ & 2.0 & $(1.1-3.7)$ & 2.2 & $(1.1-4.1)$ & 4.6 & $(2.9-7.3)$ & 3.4 & $(2.1-5.5)$ \\
\hline Miami-Dade County, FL & 1.4 & $(0.9-2.2)$ & 4.2 & $(2.8-6.2)$ & 2.8 & $(1.9-4.1)$ & 2.1 & $(1.4-3.2)$ & 4.2 & $(2.9-6.2)$ & 3.2 & $(2.2-4.6)$ \\
\hline
\end{tabular}

See table footnotes on the next page. 
TABLE 62. (Continued) Percentage of high school students who ever used heroin* and who ever used methamphetamines, ${ }^{\dagger}$ by sex - selected U.S. sites, Youth Risk Behavior Survey, 2015

\begin{tabular}{|c|c|c|c|c|c|c|c|c|c|c|c|c|}
\hline \multirow[b]{3}{*}{ Site } & \multicolumn{6}{|c|}{ Ever used heroin } & \multicolumn{6}{|c|}{ Ever used methamphetamines } \\
\hline & \multicolumn{2}{|c|}{ Female } & \multicolumn{2}{|c|}{ Male } & \multicolumn{2}{|r|}{ Total } & \multicolumn{2}{|c|}{ Female } & \multicolumn{2}{|r|}{ Male } & \multicolumn{2}{|r|}{ Total } \\
\hline & $\%$ & $\mathrm{Cl}^{\S}$ & $\%$ & $\mathrm{Cl}$ & $\%$ & $\mathrm{Cl}$ & $\%$ & $\mathrm{Cl}$ & $\%$ & $\mathrm{Cl}$ & $\%$ & $\mathrm{Cl}$ \\
\hline New York City, NY & 1.1 & $(0.8-1.6)$ & 3.6 & $(2.7-4.8)$ & 2.5 & $(1.9-3.3)$ & - & - & - & - & - & - \\
\hline Oakland, CA & - & - & - & - & - & - & - & - & - & - & - & - \\
\hline Orange County, FL & 2.2 & $(1.2-3.9)$ & 5.5 & $(3.1-9.6)$ & 3.9 & $(2.3-6.4)$ & 1.9 & $(1.1-3.2)$ & 4.5 & $(2.3-8.7)$ & 3.2 & $(1.8-5.6)$ \\
\hline Palm Beach County, FL & 3.2 & $(1.9-5.5)$ & 6.4 & $(4.4-9.3)$ & 5.7 & $(4.2-7.8)$ & 3.9 & $(2.5-6.2)$ & 6.2 & $(4.5-8.5)$ & 5.8 & $(4.4-7.7)$ \\
\hline Philadelphia, PA & 1.7 & $(0.9-3.0)$ & 4.5 & $(2.4-8.4)$ & 3.3 & $(1.8-6.0)$ & 1.8 & $(1.2-2.9)$ & 5.5 & $(2.9-10.2)$ & 3.8 & $(2.2-6.4)$ \\
\hline San Diego, CA & 0.8 & $(0.4-1.7)$ & 4.1 & $(3.0-5.5)$ & 2.5 & $(1.8-3.3)$ & 1.9 & $(1.1-3.1)$ & 5.2 & $(3.9-6.9)$ & 3.6 & $(2.7-4.7)$ \\
\hline San Francisco, CA & 1.9 & $(0.8-4.4)$ & 2.1 & $(1.3-3.4)$ & 2.4 & $(1.5-3.9)$ & 2.5 & $(1.5-4.1)$ & 4.4 & $(3.3-5.9)$ & 3.8 & $(2.8-5.2)$ \\
\hline Median & \multirow{2}{*}{\multicolumn{2}{|c|}{$\begin{array}{c}1.9 \\
(0.4-4.6)\end{array}$}} & \multirow{2}{*}{\multicolumn{2}{|c|}{$\begin{array}{c}4.5 \\
(2.1-11.0)\end{array}$}} & \multirow{2}{*}{\multicolumn{2}{|c|}{$\begin{array}{c}3.3 \\
(1.5-8.3)\end{array}$}} & \multirow{2}{*}{\multicolumn{2}{|c|}{$\begin{array}{c}2.3 \\
(1.0-5.6)\end{array}$}} & \multirow{2}{*}{\multicolumn{2}{|c|}{$\begin{array}{c}5.3 \\
(3.3-10.4)\end{array}$}} & \multirow{2}{*}{\multicolumn{2}{|c|}{$\begin{array}{c}3.7 \\
(2.4-7.9)\end{array}$}} \\
\hline Range & & & & & & & & & & & & \\
\hline
\end{tabular}

* Also called "smack," "junk," or "China White" one or more times during their life.

† Also called "speed," "crystal," "crank," or "ice" one or more times during their life.

$\S 95 \%$ confidence interval.

I Not available.

TABLE 63. Percentage of high school students who ever took steroids without a doctor's prescription* and who ever took prescription drugs without a doctor's prescription, ${ }^{\dagger}$ by sex, race/ethnicity, and grade — United States, Youth Risk Behavior Survey, 2015

\begin{tabular}{|c|c|c|c|c|c|c|c|c|c|c|c|c|}
\hline \multirow[b]{3}{*}{ Category } & \multicolumn{6}{|c|}{ Ever took steroids without a doctor's prescription } & \multicolumn{6}{|c|}{ Ever took prescription drugs without a doctor's prescription } \\
\hline & \multicolumn{2}{|c|}{ Female } & \multicolumn{2}{|c|}{ Male } & \multicolumn{2}{|c|}{ Total } & \multicolumn{2}{|c|}{ Female } & \multicolumn{2}{|r|}{ Male } & \multicolumn{2}{|r|}{ Total } \\
\hline & $\%$ & $\mathrm{Cl}^{\S}$ & $\%$ & $\mathrm{Cl}$ & $\%$ & $\mathrm{Cl}$ & $\%$ & $\mathrm{Cl}$ & $\%$ & $\mathrm{Cl}$ & $\%$ & $\mathrm{Cl}$ \\
\hline \multicolumn{13}{|c|}{ Race/Ethnicity } \\
\hline White & 1.8 & $(1.2-2.7)$ & 3.6 & $(2.8-4.6)$ & 2.7 & $(2.1-3.4)$ & 15.9 & $(14.0-18.2)$ & 17.1 & $(15.0-19.5)$ & 16.5 & $(14.7-18.5)$ \\
\hline Black ${ }^{\pi}$ & 3.6 & $(1.8-7.1)$ & 4.8 & $(2.6-8.7)$ & 4.5 & $(2.5-7.7)$ & 10.7 & $(8.4-13.6)$ & 18.1 & $(15.4-21.2)$ & 14.8 & $(12.8-17.1)$ \\
\hline Hispanic & 3.9 & $(2.8-5.4)$ & 4.1 & $(2.9-5.8)$ & 4.1 & $(3.1-5.4)$ & 16.5 & $(14.7-18.5)$ & 18.4 & $(15.8-21.4)$ & 17.5 & (15.6-19.7) \\
\hline \multicolumn{13}{|l|}{ Grade } \\
\hline 9 & 3.4 & $(2.3-5.0)$ & 3.5 & $(2.6-4.9)$ & 3.6 & $(2.8-4.6)$ & 12.5 & $(10.2-15.2)$ & 13.3 & $(10.2-17.2)$ & 13.0 & $(11.0-15.3)$ \\
\hline 10 & 3.4 & $(2.1-5.5)$ & 4.4 & $(3.0-6.4)$ & 3.9 & $(2.7-5.6)$ & 15.5 & $(12.5-19.1)$ & 15.1 & $(12.3-18.6)$ & 15.3 & $(12.7-18.4)$ \\
\hline 11 & 2.0 & $(1.4-2.8)$ & 2.8 & $(2.0-3.9)$ & 2.7 & $(2.0-3.6)$ & 16.0 & $(14.1-18.0)$ & 21.5 & (19.2-23.9) & 18.9 & $(17.4-20.6)$ \\
\hline 12 & 1.6 & $(0.9-2.6)$ & 4.8 & $(3.2-7.1)$ & 3.3 & $(2.4-4.5)$ & 18.8 & $(16.4-21.4)$ & 21.7 & $(17.9-26.1)$ & 20.3 & $(18.1-22.7)$ \\
\hline Total & 2.7 & $(2.1-3.5)$ & 4.0 & $(3.1-5.1)$ & 3.5 & $(2.8-4.3)$ & 15.6 & $(14.3-17.1)$ & 17.8 & $(16.1-19.6)$ & 16.8 & $(15.4-18.2)$ \\
\hline
\end{tabular}

* Pills or shots one or more times during their life.

† Such as Oxycontin, Percocet, Vicodin, codeine, Adderall, Ritalin, or Xanax, one or more times during their life.

$\S 95 \%$ confidence interval.

I Non-Hispanic. 
TABLE 64. Percentage of high school students who ever took steroids without a doctor's prescription* and who ever took prescription drugs without a doctor's prescription, ${ }^{\dagger}$ by sex — selected U.S. sites, Youth Risk Behavior Survey, 2015

\begin{tabular}{|c|c|c|c|c|c|c|c|c|c|c|c|c|}
\hline \multirow[b]{3}{*}{ Site } & \multicolumn{6}{|c|}{ Ever took steroids without a doctor's prescription } & \multicolumn{6}{|c|}{ Ever took prescription drugs without a doctor's prescription } \\
\hline & \multicolumn{2}{|c|}{ Female } & \multicolumn{2}{|r|}{ Male } & \multicolumn{2}{|r|}{ Total } & \multicolumn{2}{|c|}{ Female } & \multicolumn{2}{|r|}{ Male } & \multicolumn{2}{|r|}{ Total } \\
\hline & $\%$ & $\mathrm{Cl}^{\S}$ & $\%$ & $\mathrm{Cl}$ & $\%$ & $\mathrm{Cl}$ & $\%$ & $\mathrm{Cl}$ & $\%$ & $\mathrm{Cl}$ & $\%$ & $\mathrm{Cl}$ \\
\hline \multicolumn{13}{|l|}{ State surveys } \\
\hline Alabama & 3.7 & $(2.3-6.0)$ & 6.6 & $(4.2-10.1)$ & 5.6 & $(3.9-7.8)$ & 17.6 & $(14.3-21.5)$ & 20.0 & $(16.4-24.2)$ & 19.2 & $(16.7-22.1)$ \\
\hline Alaska & - & - & - & - & - & - & 13.1 & $(10.4-16.5)$ & 15.6 & $(12.8-19.0)$ & 14.6 & $(12.5-17.1)$ \\
\hline Arizona & 2.6 & $(1.9-3.5)$ & 6.4 & $(4.1-9.7)$ & 4.7 & (3.2-6.7) & - & - & - & - & - & - \\
\hline Arkansas & 4.1 & $(2.5-6.8)$ & 5.4 & $(3.3-8.7)$ & 4.9 & $(3.4-7.0)$ & 18.7 & (15.9-21.9) & 19.2 & $(15.5-23.4)$ & 19.1 & $(17.2-21.1)$ \\
\hline California & 2.0 & $(1.2-3.3)$ & 0.8 & $(0.3-1.9)$ & 1.4 & $(0.9-2.1)$ & 14.5 & $(12.0-17.3)$ & 14.0 & $(10.1-19.0)$ & 14.4 & $(11.4-18.0)$ \\
\hline Connecticut & - & - & - & - & - & - & 11.7 & $(10.1-13.5)$ & 11.9 & $(10.2-13.8)$ & 12.0 & $(10.7-13.4)$ \\
\hline Delaware & 1.4 & $(0.8-2.3)$ & 3.1 & $(2.1-4.7)$ & 2.5 & $(1.8-3.3)$ & 11.9 & $(9.5-14.8)$ & 12.7 & $(10.0-16.0)$ & 12.6 & $(10.7-14.7)$ \\
\hline Florida & 2.5 & $(1.9-3.3)$ & 5.1 & $(4.0-6.4)$ & 3.9 & $(3.2-4.9)$ & 11.8 & $(10.3-13.5)$ & 13.6 & (11.9-15.6) & 13.0 & $(11.6-14.5)$ \\
\hline Hawaii & - & - & - & - & - & - & 11.3 & $(8.7-14.5)$ & 12.9 & $(11.5-14.6)$ & 12.3 & $(10.6-14.4)$ \\
\hline Idaho & 2.4 & $(1.3-4.5)$ & 2.6 & $(1.6-4.2)$ & 2.5 & $(1.9-3.4)$ & 16.4 & (13.5-19.7) & 17.0 & $(13.5-21.1)$ & 16.8 & $(14.1-19.8)$ \\
\hline Illinois & 2.0 & $(1.2-3.3)$ & 6.0 & $(4.0-9.0)$ & 4.1 & $(2.9-5.7)$ & 13.0 & $(10.5-16.0)$ & 15.6 & $(12.3-19.6)$ & 14.4 & $(12.3-16.8)$ \\
\hline Indiana & 1.9 & $(1.0-3.4)$ & 4.1 & $(2.1-8.0)$ & 3.2 & $(1.9-5.5)$ & 15.7 & $(12.9-19.0)$ & 17.7 & $(14.7-21.1)$ & 16.8 & $(14.7-19.3)$ \\
\hline Kentucky & 3.0 & $(1.7-5.3)$ & 5.5 & $(3.7-8.2)$ & 4.5 & $(3.4-6.1)$ & 12.2 & $(9.8-15.1)$ & 13.0 & $(10.4-16.1)$ & 12.7 & $(10.9-14.7)$ \\
\hline Maine & - & - & - & - & - & - & 9.4 & $(8.4-10.5)$ & 11.8 & $(10.5-13.2)$ & 10.8 & $(10.0-11.7)$ \\
\hline Maryland & 2.9 & $(2.6-3.1)$ & 5.3 & $(4.9-5.7)$ & 4.3 & $(4.0-4.6)$ & 13.1 & $(12.6-13.7)$ & 14.9 & $(14.2-15.6)$ & 14.2 & $(13.7-14.8)$ \\
\hline Massachusetts & - & - & - & - & - & - & - & - & - & - & - & - \\
\hline Michigan & 1.7 & $(1.0-2.9)$ & 3.6 & $(2.6-5.2)$ & 2.7 & $(2.1-3.5)$ & 15.6 & (12.5-19.3) & 15.9 & (13.4-18.7) & 15.8 & (13.3-18.6) \\
\hline Mississippi & 4.3 & (3.2-5.8) & 7.7 & $(5.5-10.7)$ & 6.3 & $(4.9-8.1)$ & 12.8 & (10.4-15.6) & 21.3 & (17.4-25.7) & 17.2 & $(14.6-20.1)$ \\
\hline Missouri & - & - & - & - & - & - & 14.1 & $(11.6-17.1)$ & 21.0 & $(17.4-25.1)$ & 17.5 & $(15.0-20.4)$ \\
\hline Montana & 1.7 & $(1.2-2.5)$ & 3.2 & $(2.4-4.3)$ & 2.5 & $(1.9-3.2)$ & 15.4 & $(14.0-16.9)$ & 15.7 & $(13.8-17.9)$ & 15.6 & $(14.2-17.1)$ \\
\hline Nebraska & 3.4 & $(2.1-5.4)$ & 3.3 & $(2.0-5.4)$ & 3.5 & $(2.4-5.0)$ & 14.9 & $(11.8-18.7)$ & 11.9 & $(9.2-15.3)$ & 13.5 & $(11.1-16.4)$ \\
\hline Nevada & 4.0 & $(3.1-5.2)$ & 3.6 & $(2.2-6.0)$ & 3.9 & $(3.0-5.0)$ & 20.1 & $(16.7-23.9)$ & 17.0 & $(14.3-20.2)$ & 18.5 & $(16.1-21.2)$ \\
\hline New Hampshire & - & - & - & - & - & - & 12.5 & (11.5-13.5) & 14.0 & $(12.8-15.3)$ & 13.4 & $(12.5-14.4)$ \\
\hline New Mexico & - & - & - & - & - & - & 13.9 & (12.6-15.3) & 14.7 & (13.5-16.0) & 14.3 & $(13.4-15.3)$ \\
\hline New York & - & - & - & - & - & - & - & - & - & - & - & - \\
\hline North Carolina & 3.5 & (1.9-6.5) & 3.9 & $(2.5-6.1)$ & 3.9 & $(2.7-5.7)$ & 18.8 & $(15.1-23.0)$ & 16.5 & $(14.0-19.4)$ & 17.9 & $(15.2-20.9)$ \\
\hline North Dakota & - & - & - & - & - & - & 12.2 & (10.3-14.3) & 16.6 & (14.1-19.5) & 14.5 & $(12.8-16.3)$ \\
\hline Oklahoma & 2.1 & $(1.2-3.6)$ & 2.6 & (1.4-4.5) & 2.3 & $(1.5-3.5)$ & 13.5 & (11.0-16.5) & 14.1 & $(10.7-18.5)$ & 13.9 & $(11.5-16.7)$ \\
\hline Pennsylvania & 1.7 & $(1.0-3.0)$ & 3.2 & $(2.2-4.7)$ & 2.5 & $(1.8-3.4)$ & 12.1 & $(9.9-14.8)$ & 13.5 & $(11.6-15.7)$ & 12.8 & $(11.1-14.8)$ \\
\hline Rhode Island & 2.0 & $(1.0-3.9)$ & 5.1 & $(3.2-8.0)$ & 3.8 & $(2.3-6.2)$ & 11.4 & $(8.5-15.2)$ & 11.2 & $(8.3-14.9)$ & 11.6 & $(9.3-14.3)$ \\
\hline South Carolina & 1.7 & $(1.2-2.5)$ & 4.7 & $(2.8-7.8)$ & 3.3 & $(2.1-5.0)$ & 16.8 & $(12.5-22.1)$ & 16.0 & $(13.9-18.4)$ & 16.4 & $(13.8-19.4)$ \\
\hline South Dakota & - & - & - & - & - & - & 13.9 & $(10.1-18.9)$ & 12.2 & $(9.4-15.6)$ & 13.1 & $(10.2-16.7)$ \\
\hline Tennessee & - & - & - & - & - & - & - & - & - & - & - & - \\
\hline Vermont & - & - & - & - & - & - & - & - & - & - & - & - \\
\hline Virginia & 2.3 & $(1.5-3.3)$ & 3.6 & $(2.7-4.9)$ & 3.1 & $(2.4-4.0)$ & 14.0 & (11.8-16.6) & 16.9 & (14.6-19.6) & 15.6 & (13.9-17.5) \\
\hline West Virginia & 1.9 & $(1.0-3.9)$ & 7.2 & $(5.3-9.7)$ & 4.6 & $(3.5-6.2)$ & 15.0 & $(11.9-18.7)$ & 16.1 & $(12.1-21.0)$ & 15.5 & (12.4-19.2) \\
\hline Wyoming & 3.9 & $(2.6-5.8)$ & 5.4 & $(4.0-7.3)$ & 4.8 & $(3.6-6.3)$ & 14.0 & (11.2-17.3) & 13.8 & (11.7-16.3) & 14.0 & $(12.0-16.3)$ \\
\hline Median & & 2.3 & & 4.4 & & 3.8 & & 13.9 & & 15.2 & & 14.4 \\
\hline Range & & $4-4.3)$ & & $0.8-7.7)$ & & $1.4-6.3)$ & & $.4-20.1)$ & & $.2-21.3)$ & & $0.8-19.2)$ \\
\hline \multicolumn{13}{|c|}{ Large urban school district surveys } \\
\hline Baltimore, MD & 4.2 & $(2.5-6.9)$ & 10.9 & $(7.8-15.0)$ & 8.7 & $(6.4-11.7)$ & 11.2 & $(8.7-14.2)$ & 15.5 & $(11.6-20.4)$ & 14.1 & $(11.5-17.1)$ \\
\hline Boston, MA & - & - & - & - & - & - & 7.4 & $(5.6-9.8)$ & 8.3 & $(6.0-11.3)$ & 7.9 & $(6.4-9.7)$ \\
\hline Broward County, FL & 2.5 & $(1.6-3.9)$ & 4.7 & $(3.0-7.3)$ & 3.6 & $(2.5-5.2)$ & 12.4 & $(9.6-15.7)$ & 14.3 & $(11.7-17.4)$ & 13.5 & $(11.6-15.5)$ \\
\hline Cleveland, $\mathrm{OH}$ & - & - & - & - & - & - & 11.8 & $(9.5-14.5)$ & 18.7 & $(15.5-22.4)$ & 16.1 & $(13.7-18.8)$ \\
\hline DeKalb County, GA & 1.3 & $(0.8-2.2)$ & 5.1 & $(3.6-7.3)$ & 3.3 & $(2.4-4.4)$ & 13.1 & $(10.6-16.0)$ & 17.7 & $(14.6-21.2)$ & 15.4 & $(13.5-17.5)$ \\
\hline Detroit, Ml & 3.8 & $(2.6-5.5)$ & 6.6 & $(4.8-9.2)$ & 5.3 & $(4.0-7.0)$ & 10.3 & $(8.3-12.8)$ & 13.2 & $(10.8-16.1)$ & 11.9 & $(10.3-13.6)$ \\
\hline District of Columbia & - & - & - & - & - & - & 10.3 & $(9.4-11.2)$ & 16.4 & $(15.3-17.6)$ & 13.5 & $(12.8-14.2)$ \\
\hline Duval County, FL & - & - & - & - & - & - & - & - & - & - & - & - \\
\hline Ft. Worth, TX & 2.6 & $(1.8-3.7)$ & 3.5 & $(2.5-4.8)$ & 3.0 & $(2.4-3.8)$ & 12.1 & $(10.3-14.1)$ & 15.0 & $(12.8-17.6)$ & 13.6 & $(12.0-15.4)$ \\
\hline Houston, TX & 6.2 & $(4.9-7.6)$ & 6.4 & $(5.0-8.0)$ & 6.6 & $(5.5-7.9)$ & 14.4 & $(12.4-16.5)$ & 14.8 & $(12.9-16.9)$ & 14.9 & $(13.4-16.6)$ \\
\hline Los Angeles, CA & 1.8 & $(1.1-3.0)$ & 3.0 & $(2.1-4.2)$ & 2.5 & $(1.8-3.3)$ & 8.4 & $(6.5-10.8)$ & 10.7 & $(8.7-13.2)$ & 9.5 & $(7.8-11.6)$ \\
\hline Miami-Dade County, FL & 1.5 & $(0.9-2.3)$ & 4.6 & $(3.1-6.8)$ & 3.1 & $(2.1-4.4)$ & 10.1 & $(8.0-12.8)$ & 14.5 & $(12.1-17.3)$ & 12.3 & $(10.5-14.4)$ \\
\hline New York City, NY & - & - & - & - & - & - & - & - & - & - & - & - \\
\hline
\end{tabular}

See table footnotes on the next page. 
TABLE 64. (Continued) Percentage of high school students who ever took steroids without a doctor's prescription* and who ever took prescription drugs without a doctor's prescription, ${ }^{\dagger}$ by sex — selected U.S. sites, Youth Risk Behavior Survey, 2015

\begin{tabular}{|c|c|c|c|c|c|c|c|c|c|c|c|c|}
\hline \multirow[b]{3}{*}{ Site } & \multicolumn{6}{|c|}{ Ever took steroids without a doctor's prescription } & \multicolumn{6}{|c|}{ Ever took prescription drugs without a doctor's prescription } \\
\hline & \multicolumn{2}{|c|}{ Female } & \multicolumn{2}{|r|}{ Male } & \multicolumn{2}{|r|}{ Total } & \multicolumn{2}{|c|}{ Female } & \multicolumn{2}{|r|}{ Male } & \multicolumn{2}{|r|}{ Total } \\
\hline & $\%$ & $\mathrm{Cl}^{\S}$ & $\%$ & $\mathrm{Cl}$ & $\%$ & $\mathrm{Cl}$ & $\%$ & $\mathrm{Cl}$ & $\%$ & $\mathrm{Cl}$ & $\%$ & $\mathrm{Cl}$ \\
\hline Oakland, CA & - & - & - & - & - & - & 9.6 & $(7.6-12.0)$ & 13.6 & $(11.0-16.8)$ & 11.9 & $(10.1-13.9)$ \\
\hline Orange County, FL & 1.8 & $(1.0-3.2)$ & 4.9 & $(2.7-8.9)$ & 3.5 & $(2.0-5.8)$ & 11.0 & $(8.8-13.6)$ & 15.2 & $(12.7-18.1)$ & 13.1 & (11.4-14.9) \\
\hline Palm Beach County, FL & 2.7 & $(1.8-4.2)$ & 6.0 & $(4.0-8.8)$ & 5.0 & $(3.6-6.7)$ & 12.2 & $(10.1-14.6)$ & 14.6 & $(12.4-17.1)$ & 13.9 & $(12.2-15.8)$ \\
\hline Philadelphia, PA & 5.2 & $(3.4-7.9)$ & 6.3 & $(3.4-11.7)$ & 5.9 & $(3.5-9.8)$ & 12.3 & $(9.5-15.9)$ & 13.5 & $(9.6-18.6)$ & 13.1 & $(10.7-16.1)$ \\
\hline San Diego, CA & 1.9 & $(1.2-3.0)$ & 3.6 & $(2.7-4.8)$ & 2.7 & $(2.1-3.5)$ & 10.8 & $(8.6-13.5)$ & 15.9 & $(13.8-18.2)$ & 13.3 & $(11.6-15.3)$ \\
\hline San Francisco, CA & - & - & - & - & - & - & 11.6 & $(9.2-14.5)$ & 13.2 & $(10.4-16.7)$ & 12.7 & $(10.3-15.6)$ \\
\hline Median & \multirow{2}{*}{\multicolumn{2}{|c|}{$\begin{array}{l}2.5 \\
(13-62)\end{array}$}} & \multirow{2}{*}{\multicolumn{2}{|c|}{$\begin{array}{c}5.0 \\
(30-109)\end{array}$}} & \multirow{2}{*}{\multicolumn{2}{|c|}{$\begin{array}{c}3.5 \\
(2.5-8.7)\end{array}$}} & \multicolumn{2}{|r|}{11.2} & \multicolumn{2}{|r|}{14.6} & \multirow{2}{*}{\multicolumn{2}{|c|}{$\begin{array}{c}13.3 \\
(7.9-16.1)\end{array}$}} \\
\hline Range & & & & & & & & .4-14.4) & & $(8.3-18.7)$ & & \\
\hline
\end{tabular}

* Pills or shots one or more times during their life.

† Such as Oxycontin, Percocet, Vicodin, codeine, Adderall, Ritalin, or Xanax, one or more times during their life.

$\S 95 \%$ confidence interval.

" Not available.

TABLE 65. Percentage of high school students who ever used inhalants, ${ }^{*}$ by sex, race/ethnicity, and grade — United States, Youth Risk Behavior Survey, 2015

\begin{tabular}{|c|c|c|c|c|c|c|}
\hline \multirow[b]{2}{*}{ Category } & \multicolumn{2}{|c|}{ Female } & \multicolumn{2}{|c|}{ Male } & \multicolumn{2}{|c|}{ Total } \\
\hline & $\%$ & $\mathrm{Cl}^{\dagger}$ & $\%$ & $\mathrm{Cl}$ & $\%$ & $\mathrm{Cl}$ \\
\hline \multicolumn{7}{|c|}{ Race/Ethnicity } \\
\hline White ${ }^{\S}$ & 5.9 & $(4.5-7.7)$ & 6.9 & (5.9-8.2) & 6.4 & (5.4-7.6) \\
\hline Black $^{\S}$ & 5.9 & $(3.2-10.7)$ & 7.1 & $(4.7-10.4)$ & 6.8 & $(4.4-10.2)$ \\
\hline Hispanic & 8.3 & $(6.9-10.0)$ & 7.1 & $(5.7-8.9)$ & 7.8 & $(6.7-9.0)$ \\
\hline \multicolumn{7}{|l|}{ Grade } \\
\hline 9 & 8.5 & $(6.9-10.4)$ & 7.9 & $(5.8-10.7)$ & 8.3 & $(6.9-10.0)$ \\
\hline 10 & 7.8 & $(5.7-10.5)$ & 7.0 & $(5.6-8.8)$ & 7.5 & $(6.0-9.2)$ \\
\hline 11 & 4.6 & $(3.3-6.4)$ & 6.6 & $(5.1-8.5)$ & 5.9 & $(4.6-7.4)$ \\
\hline 12 & 4.9 & $(3.6-6.7)$ & 7.0 & $(5.1-9.5)$ & 6.0 & $(4.9-7.5)$ \\
\hline Total & 6.6 & $(5.5-7.9)$ & 7.2 & $(6.2-8.4)$ & 7.0 & $(6.2-8.0)$ \\
\hline
\end{tabular}

* Sniffed glue, breathed the contents of aerosol spray cans, or inhaled any paints or sprays to get high, one or more times during their life.

† $95 \%$ confidence interval.

$\S$ Non-Hispanic. 
TABLE 66. Percentage of high school students who ever used inhalants, ${ }^{*}$ by sex — selected U.S. sites, Youth Risk Behavior Survey, 2015

\begin{tabular}{|c|c|c|c|c|c|c|}
\hline \multirow[b]{2}{*}{ Site } & \multicolumn{2}{|c|}{ Female } & \multicolumn{2}{|c|}{ Male } & \multicolumn{2}{|c|}{ Total } \\
\hline & $\%$ & $\mathrm{Cl}^{\dagger}$ & $\%$ & $\mathrm{Cl}$ & $\%$ & $\mathrm{Cl}$ \\
\hline \multicolumn{7}{|l|}{ State surveys } \\
\hline Alabama & 11.1 & $(8.0-15.0)$ & 10.1 & $(8.1-12.6)$ & 10.9 & $(8.7-13.5)$ \\
\hline Alaska & 5.4 & $(4.1-7.0)$ & 6.6 & $(4.8-9.1)$ & 6.3 & $(5.0-7.9)$ \\
\hline Arizona & 8.5 & $(6.2-11.6)$ & 7.6 & $(5.0-11.6)$ & 8.1 & $(6.5-10.1)$ \\
\hline Arkansas & 8.1 & (6.7-9.8) & 11.7 & $(8.5-15.8)$ & 10.0 & $(7.8-12.7)$ \\
\hline California & 8.2 & $(6.6-10.2)$ & 5.7 & $(3.5-9.1)$ & 7.0 & $(5.1-9.5)$ \\
\hline Connecticut & 5.0 & $(3.8-6.7)$ & 7.2 & $(6.0-8.6)$ & 6.3 & (5.4-7.4) \\
\hline Delaware & 2.6 & $(1.6-4.4)$ & 3.2 & $(2.2-4.5)$ & 3.2 & $(2.5-4.2)$ \\
\hline Florida & —§ & - & - & - & - & - \\
\hline Hawaii & 6.2 & $(5.2-7.4)$ & 9.4 & $(7.9-11.1)$ & 8.0 & $(7.0-9.1)$ \\
\hline Idaho & 7.5 & (5.4-10.4) & 6.0 & $(4.6-7.8)$ & 6.8 & $(5.5-8.5)$ \\
\hline Illinois & 6.5 & $(5.3-7.9)$ & 7.1 & $(5.5-9.1)$ & 6.9 & $(5.9-8.1)$ \\
\hline Indiana & 6.6 & $(4.8-8.9)$ & 8.0 & $(5.3-11.8)$ & 7.4 & (5.4-9.9) \\
\hline Kentucky & 8.6 & $(7.1-10.5)$ & 5.7 & $(4.1-8.0)$ & 7.4 & $(6.1-8.8)$ \\
\hline Maine & 7.1 & $(6.4-7.8)$ & 8.1 & $(7.1-9.2)$ & 7.8 & (7.1-8.5) \\
\hline Maryland & 7.7 & $(7.3-8.2)$ & 8.7 & $(8.2-9.2)$ & 8.5 & $(8.1-8.9)$ \\
\hline Massachusetts & - & - & - & - & - & - \\
\hline Michigan & 7.1 & $(5.6-9.1)$ & 8.0 & $(6.1-10.4)$ & 7.7 & $(6.3-9.3)$ \\
\hline Mississippi & 9.4 & $(7.6-11.6)$ & 13.3 & $(10.2-17.3)$ & 11.6 & (9.7-13.8) \\
\hline Missouri & - & - & - & - & - & - \\
\hline Montana & 7.9 & $(6.7-9.3)$ & 8.2 & $(6.6-10.1)$ & 8.0 & (6.9-9.4) \\
\hline Nebraska & 9.3 & $(7.0-12.2)$ & 6.8 & (5.1-9.0) & 8.1 & $(6.6-10.0)$ \\
\hline Nevada & 8.4 & $(6.5-10.9)$ & 6.8 & $(4.8-9.5)$ & 7.7 & (6.0-9.8) \\
\hline New Hampshire & 6.0 & $(5.4-6.7)$ & 6.8 & $(6.0-7.6)$ & 6.4 & $(5.9-6.9)$ \\
\hline New Mexico & - & - & - & - & - & - \\
\hline New York & - & - & - & - & - & - \\
\hline North Carolina & 8.1 & $(5.9-11.1)$ & 7.3 & $(5.3-9.8)$ & 7.9 & $(6.2-10.0)$ \\
\hline North Dakota & - & - & - & - & - & - \\
\hline Oklahoma & 4.6 & $(3.1-6.9)$ & 5.0 & $(3.5-7.2)$ & 4.8 & $(3.6-6.3)$ \\
\hline Pennsylvania & 5.6 & $(4.2-7.5)$ & 6.5 & $(5.1-8.3)$ & 6.1 & $(5.0-7.5)$ \\
\hline Rhode Island & 5.2 & (3.9-6.8) & 6.9 & $(4.7-10.0)$ & 6.2 & $(4.7-8.2)$ \\
\hline South Carolina & 8.7 & $(6.3-11.9)$ & 7.0 & $(4.8-10.0)$ & 7.9 & $(5.8-10.6)$ \\
\hline South Dakota & 10.5 & $(7.7-14.3)$ & 9.3 & $(5.9-14.2)$ & 9.9 & $(7.5-13.0)$ \\
\hline Tennessee & - & - & - & - & - & - \\
\hline Vermont & 6.2 & $(5.8-6.7)$ & 6.7 & $(6.3-7.2)$ & 6.5 & $(6.2-6.9)$ \\
\hline Virginia & - & - & - & - & - & - \\
\hline West Virginia & 8.6 & $(6.6-11.1)$ & 10.1 & $(7.9-12.7)$ & 9.4 & $(7.5-11.6)$ \\
\hline Wyoming & 10.7 & $(8.4-13.6)$ & 10.3 & $(8.0-13.0)$ & 10.6 & $(8.6-13.0)$ \\
\hline Median & & & & & & \\
\hline Range & & & & & & \\
\hline \multicolumn{7}{|c|}{ Large urban school district surveys } \\
\hline Baltimore, MD & 10.2 & $(7.6-13.4)$ & 11.3 & $(7.9-15.8)$ & 11.6 & $(9.1-14.8)$ \\
\hline Boston, MA & 3.6 & $(2.2-5.8)$ & 5.6 & $(4.1-7.7)$ & 4.7 & $(3.5-6.2)$ \\
\hline Broward County, FL & 7.7 & $(5.7-10.3)$ & 7.3 & $(5.2-10.2)$ & 7.8 & $(6.1-9.9)$ \\
\hline Cleveland, $\mathrm{OH}$ & - & - & - & - & - & - \\
\hline DeKalb County, GA & 7.9 & $(6.3-9.9)$ & 9.8 & $(7.4-13.0)$ & 9.0 & $(7.5-10.7)$ \\
\hline Detroit, MI & 9.3 & $(7.5-11.3)$ & 8.3 & $(6.1-11.2)$ & 8.9 & $(7.4-10.7)$ \\
\hline District of Columbia & 11.5 & $(10.6-12.5)$ & 11.2 & $(10.2-12.2)$ & 11.5 & $(10.9-12.2)$ \\
\hline Duval County, FL & 11.0 & $(8.6-14.0)$ & 13.1 & $(10.2-16.7)$ & 12.7 & $(10.2-15.7)$ \\
\hline Ft. Worth, TX & 6.0 & $(4.6-7.6)$ & 5.5 & $(4.3-7.1)$ & 5.8 & $(4.8-6.9)$ \\
\hline Houston, TX & 8.2 & $(6.7-9.9)$ & 7.9 & $(6.4-9.9)$ & 8.4 & (7.1-9.9) \\
\hline Los Angeles, CA & 8.0 & $(6.0-10.5)$ & 6.4 & $(4.9-8.3)$ & 7.2 & $(6.0-8.6)$ \\
\hline Miami-Dade County, FL & 5.5 & $(4.2-7.2)$ & 6.6 & $(4.9-8.8)$ & 6.1 & $(4.9-7.5)$ \\
\hline
\end{tabular}

See table footnotes on the next page. 
TABLE 66. (Continued) Percentage of high school students who ever used inhalants, ${ }^{*}$ by sex — selected U.S. sites, Youth Risk Behavior Survey, 2015

\begin{tabular}{|c|c|c|c|c|c|c|}
\hline \multirow[b]{2}{*}{ Site } & \multicolumn{2}{|c|}{ Female } & \multicolumn{2}{|c|}{ Male } & \multicolumn{2}{|c|}{ Total } \\
\hline & $\%$ & $\mathrm{Cl}^{\dagger}$ & $\%$ & $\mathrm{Cl}$ & $\%$ & $\mathrm{Cl}$ \\
\hline New York City, NY & - & - & - & - & - & - \\
\hline Oakland, CA & - & - & - & - & - & - \\
\hline Orange County, FL & 7.1 & $(5.3-9.5)$ & 7.9 & $(5.3-11.6)$ & 7.6 & $(5.7-10.0)$ \\
\hline Palm Beach County, FL & 8.3 & $(6.2-11.2)$ & 11.1 & $(9.1-13.5)$ & 10.5 & $(8.7-12.7)$ \\
\hline Philadelphia, PA & 6.7 & $(5.2-8.7)$ & 7.7 & $(5.0-11.6)$ & 7.5 & $(5.5-10.0)$ \\
\hline San Diego, CA & 5.9 & $(4.4-8.0)$ & 7.1 & $(5.6-9.1)$ & 6.5 & $(5.3-8.0)$ \\
\hline San Francisco, CA & 5.1 & $(3.2-8.0)$ & 4.7 & $(3.6-6.1)$ & 5.1 & $(3.9-6.7)$ \\
\hline Median & \multirow{2}{*}{\multicolumn{2}{|c|}{$\begin{array}{c}7.8 \\
(3.6-11.5)\end{array}$}} & \multirow{2}{*}{\multicolumn{2}{|c|}{$\begin{array}{c}7.8 \\
(4.7-13.1)\end{array}$}} & \multirow{2}{*}{\multicolumn{2}{|c|}{$\begin{array}{c}7.7 \\
(4.7-12.7)\end{array}$}} \\
\hline Range & & & & & & \\
\hline
\end{tabular}

* Sniffed glue, breathed the contents of aerosol spray cans, or inhaled any paints or sprays to get high, one or more times during their life.

† $95 \%$ confidence interval.

$\S$ Not available.

TABLE 67. Percentage of high school students who ever injected any illegal drug* and who were offered, sold, or given an illegal drug on school property, ${ }^{\dagger}$ by sex, race/ethnicity, and grade - United States, Youth Risk Behavior Survey, 2015

\begin{tabular}{|c|c|c|c|c|c|c|c|c|c|c|c|c|}
\hline \multirow[b]{3}{*}{ Category } & \multicolumn{6}{|c|}{ Ever injected any illegal drug } & \multicolumn{6}{|c|}{ Offered, sold, or given an illegal drug on school property } \\
\hline & \multicolumn{2}{|c|}{ Female } & \multicolumn{2}{|c|}{ Male } & \multicolumn{2}{|c|}{ Total } & \multicolumn{2}{|c|}{ Female } & \multicolumn{2}{|c|}{ Male } & \multicolumn{2}{|c|}{ Total } \\
\hline & $\%$ & $\mathrm{Cl}^{\S}$ & $\%$ & $\mathrm{Cl}$ & $\%$ & $\mathrm{Cl}$ & $\%$ & $\mathrm{Cl}$ & $\%$ & $\mathrm{Cl}$ & $\%$ & $\mathrm{Cl}$ \\
\hline \multicolumn{13}{|c|}{ Race/Ethnicity } \\
\hline White & 0.7 & $(0.4-1.1)$ & 1.7 & $(1.2-2.4)$ & 1.2 & $(0.9-1.6)$ & 16.5 & $(13.3-20.3)$ & 23.2 & $(19.7-27.1)$ & 19.8 & $(16.7-23.4)$ \\
\hline Black ${ }^{9}$ & 1.3 & $(0.6-2.9)$ & 3.2 & $(1.5-6.6)$ & 2.5 & $(1.6-4.0)$ & 19.2 & $(13.9-25.8)$ & 22.0 & $(17.2-27.8)$ & 20.6 & (15.9-26.2) \\
\hline Hispanic & 1.6 & $(1.0-2.7)$ & 2.8 & $(1.8-4.4)$ & 2.2 & $(1.4-3.4)$ & 25.5 & $(22.3-29.0)$ & 28.9 & $(25.9-32.2)$ & 27.2 & $(24.8-29.8)$ \\
\hline \multicolumn{13}{|l|}{ Grade } \\
\hline 9 & 1.5 & $(0.9-2.3)$ & 1.5 & $(0.8-2.6)$ & 1.6 & $(1.1-2.3)$ & 20.0 & $(17.1-23.2)$ & 23.2 & $(19.9-26.9)$ & 21.6 & $(19.1-24.3)$ \\
\hline 10 & 0.7 & $(0.3-1.6)$ & 2.6 & $(1.7-3.8)$ & 1.6 & $(1.1-2.4)$ & 19.3 & $(15.3-24.1)$ & 24.6 & $(20.5-29.2)$ & 21.9 & $(18.2-26.2)$ \\
\hline 11 & 1.0 & $(0.6-1.8)$ & 1.9 & $(1.0-3.6)$ & 1.6 & $(1.0-2.7)$ & 20.4 & $(17.6-23.4)$ & 24.9 & (21.4-28.8) & 22.7 & $(20.0-25.7)$ \\
\hline 12 & 0.8 & $(0.4-1.7)$ & 3.4 & $(1.8-6.4)$ & 2.1 & $(1.3-3.5)$ & 16.3 & $(13.6-19.4)$ & 24.4 & $(21.0-28.1)$ & 20.3 & $(17.6-23.3)$ \\
\hline Total & 1.0 & $(0.7-1.5)$ & 2.3 & $(1.6-3.3)$ & 1.8 & $(1.3-2.3)$ & 19.1 & $(16.6-21.8)$ & 24.2 & $(21.7-26.9)$ & 21.7 & (19.4-24.2) \\
\hline
\end{tabular}

* Used a needle to inject any illegal drug into their body one or more times during their life.

${ }^{\dagger}$ During the 12 months before the survey.

$\S 95 \%$ confidence interval.

१ Non-Hispanic. 
TABLE 68. Percentage of high school students who ever injected any illegal drug* and who were offered, sold, or given an illegal drug on school property, ${ }^{\dagger}$ by sex — selected U.S. sites, Youth Risk Behavior Survey, 2015

\begin{tabular}{|c|c|c|c|c|c|c|c|c|c|c|c|c|}
\hline \multirow[b]{3}{*}{ Site } & \multicolumn{6}{|c|}{ Ever injected any illegal drug } & \multicolumn{6}{|c|}{ Offered, sold, or given an illegal drug on school property } \\
\hline & \multicolumn{2}{|c|}{ Female } & \multicolumn{2}{|c|}{ Male } & \multicolumn{2}{|c|}{ Total } & \multicolumn{2}{|c|}{ Female } & \multicolumn{2}{|c|}{ Male } & \multicolumn{2}{|c|}{ Total } \\
\hline & $\%$ & $\mathrm{Cl}^{\S}$ & $\%$ & $\mathrm{Cl}$ & $\%$ & $\mathrm{Cl}$ & $\%$ & $\mathrm{Cl}$ & $\%$ & $\mathrm{Cl}$ & $\%$ & $\mathrm{Cl}$ \\
\hline \multicolumn{13}{|l|}{ State surveys } \\
\hline Alabama & 2.4 & $(1.4-4.0)$ & 5.7 & $(3.6-8.9)$ & 4.2 & $(3.1-5.7)$ & 20.0 & $(16.1-24.7)$ & 29.3 & $(25.3-33.6)$ & 24.8 & (21.5-28.5) \\
\hline Alaska & - & - & - & - & - & - & - & - & - & - & - & - \\
\hline Arizona & - & - & - & - & - & - & 28.2 & $(25.7-31.0)$ & 30.6 & $(26.4-35.0)$ & 29.3 & $(26.6-32.0)$ \\
\hline Arkansas & 2.9 & $(1.9-4.3)$ & 5.2 & $(3.7-7.2)$ & 4.1 & $(3.3-5.0)$ & 23.9 & (21.3-26.8) & 30.3 & $(26.6-34.3)$ & 27.1 & $(24.1-30.3)$ \\
\hline California & 1.3 & $(0.8-2.0)$ & 1.3 & $(0.5-3.1)$ & 1.3 & $(0.7-2.2)$ & 24.2 & (20.9-27.9) & 27.4 & (22.8-32.6) & 26.1 & $(22.4-30.2)$ \\
\hline Connecticut & 1.1 & $(0.5-2.2)$ & 2.7 & $(1.6-4.6)$ & 2.0 & $(1.3-3.1)$ & 26.6 & $(23.4-30.2)$ & 30.3 & $(27.1-33.7)$ & 28.5 & $(25.8-31.3)$ \\
\hline Delaware & 1.0 & $(0.6-1.9)$ & 3.4 & $(2.2-5.1)$ & 2.4 & $(1.7-3.3)$ & 12.7 & $(10.6-15.1)$ & 18.1 & $(15.8-20.5)$ & 15.6 & $(14.0-17.3)$ \\
\hline Florida & - & - & - & - & - & - & 17.5 & $(16.1-19.0)$ & 19.2 & $(17.2-21.3)$ & 18.4 & $(17.1-19.8)$ \\
\hline Hawaii & 1.9 & $(1.3-2.8)$ & 4.5 & $(3.6-5.7)$ & 3.3 & $(2.8-4.0)$ & 24.9 & $(22.2-27.7)$ & 25.9 & $(23.8-28.1)$ & 25.4 & $(23.5-27.4)$ \\
\hline Idaho & 1.7 & $(1.2-2.5)$ & 1.6 & $(0.9-3.1)$ & 1.7 & $(1.2-2.4)$ & 20.2 & $(16.1-24.9)$ & 22.8 & $(19.3-26.6)$ & 21.5 & (18.8-24.5) \\
\hline Illinois & 1.9 & $(1.2-2.8)$ & 4.5 & $(2.9-6.9)$ & 3.3 & $(2.3-4.6)$ & 23.4 & (19.8-27.4) & 27.7 & $(24.0-31.7)$ & 25.6 & $(22.6-28.8)$ \\
\hline Indiana & 0.7 & $(0.3-1.6)$ & 3.5 & $(1.7-7.1)$ & 2.2 & $(1.1-4.3)$ & 22.8 & $(18.9-27.2)$ & 22.2 & (19.4-25.3) & 22.5 & $(20.3-24.9)$ \\
\hline Kentucky & - & - & - & - & - & - & 21.0 & $(18.0-24.4)$ & 20.5 & $(17.3-24.1)$ & 20.9 & (18.4-23.5) \\
\hline Maine & - & - & - & - & - & - & 12.8 & $(11.8-13.9)$ & 16.2 & $(14.6-17.9)$ & 14.7 & $(13.6-15.8)$ \\
\hline Maryland & 2.4 & $(2.1-2.7)$ & 4.4 & $(4.1-4.8)$ & 3.6 & $(3.3-3.8)$ & 24.1 & $(23.4-24.8)$ & 28.1 & $(27.4-28.9)$ & 26.2 & $(25.6-26.7)$ \\
\hline Massachusetts & - & - & - & - & - & - & 17.6 & $(15.6-19.7)$ & 22.9 & $(20.7-25.3)$ & 20.3 & $(18.6-22.2)$ \\
\hline Michigan & 1.4 & $(0.6-3.4)$ & 3.3 & $(2.2-4.7)$ & 2.4 & $(1.7-3.3)$ & 21.8 & $(18.3-25.7)$ & 28.9 & $(24.0-34.3)$ & 25.4 & $(22.1-29.1)$ \\
\hline Mississippi & 3.3 & $(2.2-5.0)$ & 7.3 & $(5.2-10.0)$ & 5.4 & $(4.2-7.1)$ & 21.9 & $(18.5-25.7)$ & 25.3 & (21.7-29.4) & 23.7 & $(20.9-26.6)$ \\
\hline Missouri & - & - & - & - & - & - & - & - & - & - & - & - \\
\hline Montana & 1.3 & $(0.8-2.0)$ & 2.1 & $(1.5-2.8)$ & 1.7 & $(1.3-2.2)$ & 19.8 & $(17.9-21.7)$ & 23.6 & $(21.6-25.7)$ & 21.7 & $(20.2-23.3)$ \\
\hline Nebraska & 3.1 & $(1.9-5.0)$ & 4.5 & $(2.7-7.4)$ & 4.0 & $(2.7-5.8)$ & 20.8 & (17.3-24.8) & 19.1 & $(15.4-23.5)$ & 19.9 & $(16.9-23.2)$ \\
\hline Nevada & 2.7 & $(1.6-4.5)$ & 2.9 & $(1.4-6.2)$ & 2.9 & $(1.9-4.5)$ & 30.3 & $(25.9-35.2)$ & 29.3 & $(26.0-32.9)$ & 29.8 & $(26.8-33.1)$ \\
\hline New Hampshire & - & - & - & - & - & - & 13.9 & $(12.6-15.3)$ & 19.0 & $(17.6-20.5)$ & 16.5 & $(15.6-17.5)$ \\
\hline New Mexico & 1.9 & $(1.4-2.6)$ & 4.4 & $(3.6-5.4)$ & 3.2 & $(2.7-3.8)$ & 25.1 & (23.3-26.9) & 29.9 & $(28.0-31.9)$ & 27.5 & $(25.9-29.2)$ \\
\hline New York & 2.2 & $(1.7-2.9)$ & 4.4 & $(3.0-6.4)$ & 3.4 & $(2.6-4.6)$ & - & - & - & - & - & - \\
\hline North Carolina & - & - & - & - & - & - & 19.9 & $(15.9-24.6)$ & 28.5 & $(25.5-31.8)$ & 24.5 & $(21.4-28.0)$ \\
\hline North Dakota & 0.5 & $(0.2-1.1)$ & 2.3 & $(1.5-3.6)$ & 1.4 & $(0.9-2.2)$ & 16.3 & (13.9-19.0) & 20.0 & $(17.5-22.8)$ & 18.2 & $(16.5-20.1)$ \\
\hline Oklahoma & 0.6 & $(0.2-1.7)$ & 1.5 & $(0.7-3.1)$ & 1.0 & $(0.5-2.0)$ & 12.4 & $(9.9-15.4)$ & 17.3 & $(14.1-20.9)$ & 15.0 & $(12.8-17.4)$ \\
\hline Pennsylvania & 0.9 & $(0.5-1.5)$ & 1.8 & $(1.1-3.0)$ & 1.3 & $(0.9-2.1)$ & 16.6 & $(14.3-19.2)$ & 22.0 & (19.5-24.8) & 19.4 & $(17.4-21.6)$ \\
\hline Rhode Island & - & - & - & - & - & - & - & - & - & - & - & - \\
\hline South Carolina & 0.4 & $(0.1-1.4)$ & 2.2 & $(1.1-4.2)$ & 1.3 & $(0.7-2.7)$ & 21.5 & $(16.8-26.9)$ & 24.0 & $(20.6-27.7)$ & 22.8 & $(19.9-26.0)$ \\
\hline South Dakota & 1.8 & $(0.6-5.0)$ & 2.6 & $(1.4-4.9)$ & 2.2 & $(1.1-4.5)$ & 19.6 & $(15.7-24.3)$ & 18.4 & $(14.4-23.2)$ & 19.0 & $(15.4-23.2)$ \\
\hline Tennessee & - & - & - & - & - & - & - & - & - & - & - & - \\
\hline Vermont & - & - & - & - & - & - & 15.0 & $(14.3-15.7)$ & 21.1 & $(20.3-21.9)$ & 18.1 & $(17.6-18.6)$ \\
\hline Virginia & 1.1 & $(0.6-1.8)$ & 2.0 & $(1.4-2.9)$ & 1.5 & $(1.1-2.1)$ & 14.4 & $(12.6-16.4)$ & 16.7 & $(14.8-18.8)$ & 15.6 & $(14.1-17.1)$ \\
\hline West Virginia & 2.1 & $(1.2-3.6)$ & 4.7 & $(3.0-7.4)$ & 3.5 & $(2.4-5.0)$ & 22.0 & $(18.1-26.5)$ & 29.6 & $(26.0-33.6)$ & 25.9 & $(22.9-29.2)$ \\
\hline Wyoming & 2.9 & $(1.6-5.1)$ & 3.7 & $(2.5-5.4)$ & 3.3 & $(2.2-5.0)$ & 21.2 & $(17.4-25.6)$ & 22.7 & $(20.0-25.7)$ & 22.0 & $(19.2-25.0)$ \\
\hline Median & & 1.8 & & 3.4 & & 2.4 & & 20.9 & & 23.2 & & 22.3 \\
\hline Range & & 4-3.3) & & $1.3-7.3)$ & & $0-5.4)$ & & 4-30.3) & & $5.2-30.6)$ & & (.7-29.8) \\
\hline \multicolumn{13}{|c|}{ Large urban school district surveys } \\
\hline Baltimore, MD & 3.1 & $(1.9-5.0)$ & 9.2 & $(6.2-13.4)$ & 6.6 & $(4.7-9.3)$ & 24.3 & $(20.8-28.1)$ & 26.1 & $(20.7-32.3)$ & 25.2 & $(21.8-28.9)$ \\
\hline Boston, MA & - & - & - & - & - & - & 19.3 & $(16.7-22.2)$ & 21.7 & $(18.7-25.1)$ & 20.5 & $(18.5-22.7)$ \\
\hline Broward County, FL & 3.2 & $(2.0-5.0)$ & 2.7 & $(1.6-4.7)$ & 3.0 & $(2.1-4.4)$ & 28.0 & $(24.4-31.8)$ & 33.2 & $(29.6-37.0)$ & 30.7 & $(28.0-33.5)$ \\
\hline Cleveland, $\mathrm{OH}$ & - & - & - & - & - & - & - & - & - & - & - & - \\
\hline DeKalb County, GA & 0.9 & $(0.4-1.8)$ & 3.4 & $(2.1-5.2)$ & 2.1 & $(1.4-3.2)$ & 27.7 & $(24.9-30.7)$ & 33.0 & $(28.8-37.4)$ & 30.3 & $(27.5-33.2)$ \\
\hline Detroit, MI & 3.7 & $(2.7-5.2)$ & 4.1 & $(2.6-6.2)$ & 4.0 & $(3.0-5.2)$ & 33.0 & $(29.8-36.4)$ & 36.5 & $(32.1-41.3)$ & 34.6 & $(31.6-37.6)$ \\
\hline District of Columbia & - & - & - & - & - & - & - & - & - & - & - & - \\
\hline Duval County, FL & - & - & - & - & - & - & 27.1 & $(24.8-29.4)$ & 31.0 & $(28.1-34.0)$ & 29.0 & $(27.2-30.8)$ \\
\hline Ft. Worth, TX & 0.8 & $(0.4-1.6)$ & 2.4 & $(1.6-3.6)$ & 1.6 & $(1.1-2.3)$ & 20.2 & $(17.8-22.8)$ & 22.7 & $(20.4-25.2)$ & 21.5 & $(19.8-23.4)$ \\
\hline Houston, TX & 3.2 & $(2.4-4.2)$ & 5.7 & $(4.4-7.3)$ & 4.7 & $(3.8-5.9)$ & 29.1 & $(26.5-31.8)$ & 31.2 & $(28.6-33.9)$ & 30.1 & $(28.2-32.0)$ \\
\hline Los Angeles, CA & 1.3 & $(0.7-2.2)$ & 2.6 & $(1.4-4.7)$ & 1.9 & $(1.2-3.2)$ & 24.7 & $(22.2-27.5)$ & 30.1 & $(26.7-33.7)$ & 27.5 & $(24.8-30.3)$ \\
\hline Miami-Dade County, FL & 0.9 & $(0.5-1.7)$ & 4.0 & $(2.7-5.9)$ & 2.4 & $(1.7-3.5)$ & 18.2 & $(15.3-21.6)$ & 25.7 & $(23.0-28.5)$ & 22.0 & $(19.9-24.2)$ \\
\hline
\end{tabular}

See table footnotes on the next page. 
TABLE 68. (Continued) Percentage of high school students who ever injected any illegal drug* and who were offered, sold, or given an illegal drug on school property, ${ }^{\dagger}$ by sex — selected U.S. sites, Youth Risk Behavior Survey, 2015

\begin{tabular}{|c|c|c|c|c|c|c|c|c|c|c|c|c|}
\hline \multirow[b]{3}{*}{ Site } & \multicolumn{6}{|c|}{ Ever injected any illegal drug } & \multicolumn{6}{|c|}{ Offered, sold, or given an illegal drug on school property } \\
\hline & \multicolumn{2}{|c|}{ Female } & \multicolumn{2}{|c|}{ Male } & \multicolumn{2}{|r|}{ Total } & \multicolumn{2}{|c|}{ Female } & \multicolumn{2}{|r|}{ Male } & \multicolumn{2}{|r|}{ Total } \\
\hline & $\%$ & $\mathrm{Cl}^{\S}$ & $\%$ & $\mathrm{Cl}$ & $\%$ & $\mathrm{Cl}$ & $\%$ & $\mathrm{Cl}$ & $\%$ & $\mathrm{Cl}$ & $\%$ & $\mathrm{Cl}$ \\
\hline New York City, NY & 1.3 & $(0.9-1.9)$ & 3.0 & $(2.1-4.3)$ & 2.2 & $(1.7-2.9)$ & - & - & - & - & - & - \\
\hline Oakland, CA & - & - & - & - & - & - & 30.3 & $(26.5-34.3)$ & 30.6 & (26.7-34.8) & 30.5 & $(27.8-33.3)$ \\
\hline Orange County, FL & 1.4 & $(0.7-2.9)$ & 5.1 & $(2.6-9.8)$ & 3.3 & $(1.8-5.9)$ & 18.9 & (15.9-22.4) & 22.7 & $(19.5-26.2)$ & 20.8 & $(18.7-23.0)$ \\
\hline Palm Beach County, FL & 3.2 & $(2.2-4.6)$ & 5.5 & $(4.0-7.7)$ & 4.9 & (3.8-6.4) & 23.4 & $(20.5-26.5)$ & 27.5 & $(24.4-30.9)$ & 25.4 & (22.9-28.1) \\
\hline Philadelphia, PA & 0.8 & $(0.3-2.0)$ & 4.0 & $(2.1-7.4)$ & 2.5 & $(1.4-4.2)$ & 26.8 & $(22.4-31.7)$ & 25.4 & $(21.1-30.4)$ & 26.1 & $(22.4-30.2)$ \\
\hline San Diego, CA & 0.6 & $(0.3-1.4)$ & 3.4 & $(2.4-4.9)$ & 2.1 & $(1.4-2.9)$ & 25.2 & $(22.2-28.6)$ & 29.7 & $(26.7-32.9)$ & 27.6 & $(25.7-29.5)$ \\
\hline San Francisco, CA & - & - & - & - & - & - & 22.5 & $(19.3-26.0)$ & 24.4 & $(20.8-28.5)$ & 23.8 & $(21.2-26.6)$ \\
\hline Median & \multirow{2}{*}{\multicolumn{2}{|c|}{$\begin{array}{c}1.3 \\
(0.6-3.7)\end{array}$}} & \multirow{2}{*}{\multicolumn{2}{|c|}{$\begin{array}{c}4.0 \\
(2.4-9.2)\end{array}$}} & \multirow{2}{*}{\multicolumn{2}{|c|}{$\begin{array}{c}2.5 \\
(1.6-6.6)\end{array}$}} & \multirow{2}{*}{\multicolumn{2}{|c|}{$\begin{array}{c}24.9 \\
(18.2-33.0)\end{array}$}} & \multirow{2}{*}{\multicolumn{2}{|c|}{$\begin{array}{c}28.6 \\
(21.7-36.5)\end{array}$}} & \multirow{2}{*}{\multicolumn{2}{|c|}{$\begin{array}{c}26.8 \\
(20.5-34.6)\end{array}$}} \\
\hline Range & & & & & & & & & & & & \\
\hline
\end{tabular}

* Used a needle to inject any illegal drug into their body one or more times during their life.

† During the 12 months before the survey.

$\S 95 \%$ confidence interval.

" Not available.

TABLE 69. Percentage of high school students who ever had sexual intercourse and who had sexual intercourse for the first time before age 13 years, by sex, race/ethnicity, and grade - United States, Youth Risk Behavior Survey, 2015

\begin{tabular}{|c|c|c|c|c|c|c|c|c|c|c|c|c|}
\hline \multirow[b]{3}{*}{ Category } & \multicolumn{6}{|c|}{ Ever had sexual intercourse } & \multicolumn{6}{|c|}{ Had first sexual intercourse before age 13 years } \\
\hline & \multicolumn{2}{|c|}{ Female } & \multicolumn{2}{|c|}{ Male } & \multicolumn{2}{|c|}{ Total } & \multicolumn{2}{|c|}{ Female } & \multicolumn{2}{|c|}{ Male } & \multicolumn{2}{|c|}{ Total } \\
\hline & $\%$ & $\mathrm{Cl}^{*}$ & $\%$ & $\mathrm{Cl}$ & $\%$ & $\mathrm{Cl}$ & $\%$ & $\mathrm{Cl}$ & $\%$ & $\mathrm{Cl}$ & $\%$ & $\mathrm{Cl}$ \\
\hline \multicolumn{13}{|c|}{ Race/Ethnicity } \\
\hline White ${ }^{\dagger}$ & 40.3 & (34.2-46.8) & 39.5 & (35.1-44.1) & 39.9 & $(35.0-45.0)$ & 1.6 & $(1.1-2.4)$ & 3.5 & $(2.5-4.9)$ & 2.5 & $(1.9-3.4)$ \\
\hline Black $^{\dagger}$ & 37.4 & (30.2-45.3) & 58.8 & $(52.8-64.5)$ & 48.5 & $(43.2-53.8)$ & 4.3 & $(2.4-7.4)$ & 12.1 & $(9.2-15.7)$ & 8.3 & $(6.4-10.8)$ \\
\hline Hispanic & 39.8 & $(35.1-44.7)$ & 45.1 & $(40.9-49.3)$ & 42.5 & $(38.6-46.5)$ & 3.1 & $(2.1-4.4)$ & 6.8 & $(5.3-8.7)$ & 5.0 & $(4.0-6.1)$ \\
\hline \multicolumn{13}{|l|}{ Grade } \\
\hline 9 & 20.7 & $(17.0-25.0)$ & 27.3 & $(23.1-31.9)$ & 24.1 & $(20.8-27.7)$ & 2.5 & $(1.6-3.7)$ & 4.6 & $(3.2-6.6)$ & 3.6 & $(2.6-4.8)$ \\
\hline 10 & 33.5 & $(26.4-41.5)$ & 37.9 & $(32.4-43.8)$ & 35.7 & $(29.9-42.0)$ & 2.7 & $(1.7-4.4)$ & 6.8 & $(5.3-8.7)$ & 4.7 & (3.7-6.1) \\
\hline 11 & 48.2 & $(42.1-54.4)$ & 51.2 & $(46.5-55.9)$ & 49.6 & $(45.1-54.1)$ & 1.6 & $(1.0-2.7)$ & 4.8 & $(3.4-6.6)$ & 3.2 & $(2.5-4.3)$ \\
\hline 12 & 57.2 & $(52.6-61.7)$ & 59.0 & $(53.7-64.1)$ & 58.1 & $(53.8-62.3)$ & 1.7 & $(0.9-3.0)$ & 5.5 & $(4.0-7.6)$ & 3.6 & $(2.7-4.8)$ \\
\hline Total & 39.2 & $(34.8-43.7)$ & 43.2 & $(39.4-47.0)$ & 41.2 & $(37.5-45.0)$ & 2.2 & $(1.6-3.0)$ & 5.6 & $(4.4-7.0)$ & 3.9 & $(3.2-4.8)$ \\
\hline
\end{tabular}

* $95 \%$ confidence interval.

† Non-Hispanic. 
TABLE 70. Percentage of high school students who ever had sexual intercourse and who had sexual intercourse for the first time before age 13 years, by sex — selected U.S. sites, Youth Risk Behavior Survey, 2015

\begin{tabular}{|c|c|c|c|c|c|c|c|c|c|c|c|c|}
\hline \multirow[b]{3}{*}{ Site } & \multicolumn{6}{|c|}{ Ever had sexual intercourse } & \multicolumn{6}{|c|}{ Had first sexual intercourse before age 13 years } \\
\hline & \multicolumn{2}{|c|}{ Female } & \multicolumn{2}{|r|}{ Male } & \multicolumn{2}{|r|}{ Total } & \multicolumn{2}{|c|}{ Female } & \multicolumn{2}{|c|}{ Male } & \multicolumn{2}{|c|}{ Total } \\
\hline & $\%$ & $\mathrm{Cl}^{*}$ & $\%$ & $\mathrm{Cl}$ & $\%$ & $\mathrm{Cl}$ & $\%$ & $\mathrm{Cl}$ & $\%$ & $\mathrm{Cl}$ & $\%$ & $\mathrm{Cl}$ \\
\hline \multicolumn{13}{|l|}{ State surveys } \\
\hline Alabama & 43.6 & (37.4-49.9) & 49.5 & $(43.7-55.4)$ & 46.3 & $(41.2-51.6)$ & 3.8 & $(2.4-6.0)$ & 10.4 & $(7.8-13.9)$ & 6.9 & $(5.4-9.0)$ \\
\hline Alaska & 35.0 & (30.4-40.0) & 36.7 & $(32.0-41.7)$ & 35.9 & $(32.3-39.8)$ & 2.0 & $(1.0-3.9)$ & 5.2 & $(3.5-7.8)$ & 3.7 & $(2.6-5.2)$ \\
\hline Arizona & 37.7 & (30.9-45.0) & 41.2 & (35.3-47.3) & 39.4 & (33.4-45.8) & 2.8 & $(1.7-4.6)$ & 3.5 & $(2.7-4.7)$ & 3.2 & $(2.5-4.2)$ \\
\hline Arkansas & 44.7 & $(36.5-53.2)$ & 47.3 & $(38.8-56.0)$ & 46.0 & (39.5-52.6) & 2.7 & $(1.5-4.7)$ & 9.3 & $(6.6-13.1)$ & 5.9 & $(4.2-8.0)$ \\
\hline California & 28.5 & $(24.9-32.4)$ & 36.0 & $(29.8-42.7)$ & 32.3 & $(28.0-37.0)$ & 2.6 & $(1.2-5.5)$ & 3.6 & $(2.4-5.6)$ & 3.1 & $(2.0-4.9)$ \\
\hline Connecticut & 31.3 & $(26.9-36.1)$ & 34.5 & $(29.8-39.5)$ & 33.0 & $(29.0-37.2)$ & 1.3 & $(0.7-2.4)$ & 4.0 & $(2.6-6.0)$ & 2.7 & $(1.9-3.8)$ \\
\hline Delaware & 43.5 & (38.3-48.9) & 49.2 & $(43.8-54.7)$ & 46.8 & $(42.1-51.5)$ & 2.4 & $(1.6-3.7)$ & 10.9 & $(8.1-14.6)$ & 6.8 & $(5.3-8.8)$ \\
\hline Florida & 35.7 & $(33.0-38.4)$ & 44.9 & (41.9-47.9) & 40.3 & (37.9-42.7) & 2.2 & $(1.6-2.9)$ & 9.1 & $(7.5-11.0)$ & 5.6 & $(4.7-6.7)$ \\
\hline Hawaii & $-^{\dagger}$ & - & - & - & - & - & 2.5 & $(1.8-3.5)$ & 4.4 & $(3.5-5.3)$ & 3.5 & $(2.9-4.2)$ \\
\hline Idaho & 35.7 & $(30.5-41.2)$ & 38.9 & (32.8-45.3) & 37.3 & (32.5-42.5) & 1.6 & $(1.0-2.6)$ & 4.8 & $(3.6-6.3)$ & 3.2 & $(2.4-4.3)$ \\
\hline Illinois & 35.8 & $(29.0-43.2)$ & 41.6 & (32.9-50.8) & 38.6 & (31.6-46.2) & 1.4 & $(0.8-2.5)$ & 5.2 & $(3.2-8.2)$ & 3.2 & $(2.1-4.9)$ \\
\hline Indiana & 41.7 & (36.0-47.7) & 41.6 & (34.2-49.4) & 41.7 & (36.0-47.7) & 2.5 & $(1.6-3.9)$ & 3.4 & $(2.1-5.3)$ & 3.0 & $(2.1-4.2)$ \\
\hline Kentucky & 41.4 & $(36.4-46.6)$ & 42.3 & $(36.4-48.4)$ & 41.7 & $(37.4-46.2)$ & 4.1 & $(3.0-5.6)$ & 5.4 & $(3.6-7.9)$ & 4.8 & $(3.7-6.2)$ \\
\hline Maine & 39.7 & (36.6-42.9) & 38.2 & (35.9-40.6) & 39.0 & (36.4-41.7) & 2.0 & $(1.6-2.4)$ & 3.6 & $(2.8-4.5)$ & 2.9 & $(2.5-3.4)$ \\
\hline Maryland & 29.8 & $(28.7-31.0)$ & 35.0 & $(33.7-36.3)$ & 32.4 & (31.3-33.4) & 2.4 & $(2.2-2.6)$ & 7.8 & (7.2-8.4) & 5.0 & $(4.7-5.4)$ \\
\hline Massachusetts & 34.5 & $(30.8-38.4)$ & 38.3 & $(33.3-43.6)$ & 36.4 & (32.4-40.6) & 1.6 & $(1.0-2.5)$ & 4.3 & $(3.2-5.9)$ & 2.9 & $(2.2-3.8)$ \\
\hline Michigan & 34.7 & $(29.5-40.3)$ & 36.8 & $(32.3-41.5)$ & 35.8 & $(31.9-39.8)$ & 2.0 & $(1.1-3.7)$ & 4.2 & $(2.8-6.2)$ & 3.1 & $(2.1-4.6)$ \\
\hline Mississippi & 44.0 & $(38.0-50.1)$ & 52.6 & $(47.7-57.4)$ & 48.0 & $(43.3-52.8)$ & 3.8 & $(2.5-5.6)$ & 13.3 & $(10.4-16.9)$ & 8.3 & $(6.7-10.2)$ \\
\hline Missouri & 35.1 & $(31.3-39.1)$ & 41.2 & $(36.6-46.0)$ & 37.7 & (34.2-41.4) & 2.9 & $(1.6-5.1)$ & 5.3 & (3.8-7.5) & 4.1 & $(3.1-5.3)$ \\
\hline Montana & 44.5 & $(41.0-48.0)$ & 43.6 & (40.4-46.8) & 44.0 & $(41.1-47.0)$ & 2.5 & $(1.8-3.4)$ & 3.6 & $(2.9-4.5)$ & 3.1 & $(2.5-3.8)$ \\
\hline Nebraska & 31.7 & (27.0-36.8) & 32.9 & (28.3-37.8) & 32.5 & $(28.6-36.6)$ & 2.1 & $(1.1-3.7)$ & 4.5 & $(2.9-7.0)$ & 3.3 & $(2.3-4.8)$ \\
\hline Nevada & 39.6 & (34.1-45.3) & 40.7 & (33.7-48.0) & 40.1 & (34.8-45.7) & 2.0 & $(1.2-3.3)$ & 4.0 & $(2.3-6.8)$ & 3.0 & $(2.0-4.5)$ \\
\hline New Hampshire & 38.7 & (36.2-41.4) & 39.8 & $(37.5-42.2)$ & 39.4 & (37.4-41.5) & 1.9 & $(1.5-2.4)$ & 3.6 & $(3.0-4.2)$ & 2.8 & $(2.4-3.2)$ \\
\hline New Mexico & 35.1 & $(32.6-37.6)$ & 39.0 & (36.4-41.8) & 37.1 & (34.9-39.2) & 2.7 & $(2.1-3.4)$ & 6.0 & $(5.2-7.0)$ & 4.3 & $(3.8-5.0)$ \\
\hline New York & 29.6 & $(24.3-35.5)$ & 31.5 & $(26.8-36.5)$ & 30.4 & $(26.2-35.1)$ & 1.5 & $(1.1-2.2)$ & 6.0 & $(4.3-8.4)$ & 3.7 & $(2.7-5.0)$ \\
\hline North Carolina & 41.4 & (37.4-45.4) & 45.8 & $(41.5-50.1)$ & 43.5 & (39.8-47.2) & 2.5 & $(1.4-4.4)$ & 7.8 & $(5.9-10.1)$ & 5.1 & (3.8-6.7) \\
\hline North Dakota & 38.6 & $(34.3-43.1)$ & 39.2 & $(35.5-43.1)$ & 38.9 & $(35.8-42.1)$ & 0.9 & $(0.5-1.6)$ & 4.3 & $(3.0-6.1)$ & 2.6 & $(1.9-3.6)$ \\
\hline Oklahoma & 40.8 & (35.3-46.6) & 46.3 & $(40.7-51.9)$ & 43.6 & (39.1-48.2) & 2.1 & $(1.3-3.3)$ & 5.7 & $(2.6-12.0)$ & 3.8 & $(2.1-6.7)$ \\
\hline Pennsylvania & 35.3 & $(30.5-40.3)$ & 37.3 & $(32.7-42.1)$ & 36.3 & $(32.0-40.8)$ & 2.4 & $(1.5-3.8)$ & 5.3 & $(4.0-7.0)$ & 3.8 & $(2.9-5.0)$ \\
\hline Rhode Island & 36.3 & $(32.6-40.0)$ & 37.0 & $(32.1-42.2)$ & 36.7 & (33.4-40.1) & 1.3 & $(0.7-2.6)$ & 4.4 & $(2.4-7.7)$ & 2.9 & $(1.7-4.7)$ \\
\hline South Carolina & 37.0 & $(29.8-44.8)$ & 43.6 & (35.2-52.4) & 40.3 & (33.1-47.9) & 2.4 & $(1.4-4.3)$ & 10.5 & $(7.2-15.1)$ & 6.3 & $(4.6-8.7)$ \\
\hline South Dakota & 34.9 & (27.3-43.3) & 39.4 & (30.4-49.1) & 37.2 & (30.2-44.8) & 2.6 & $(1.0-6.7)$ & 3.8 & $(2.0-7.1)$ & 3.3 & (1.7-6.3) \\
\hline Tennessee & - & - & - & - & - & - & - & - & - & - & - & - \\
\hline Vermont & - & - & - & - & - & - & 2.0 & $(1.7-2.3)$ & 4.4 & $(4.0-4.9)$ & 3.3 & $(3.0-3.5)$ \\
\hline Virginia & - & - & - & - & - & - & - & - & - & - & - & - \\
\hline West Virginia & 49.0 & $(42.1-56.0)$ & 44.4 & $(38.9-50.0)$ & 46.7 & $(41.0-52.5)$ & 4.1 & $(2.8-5.9)$ & 6.2 & $(4.3-8.9)$ & 5.1 & $(3.8-6.9)$ \\
\hline Wyoming & 42.5 & $(37.8-47.4)$ & 41.3 & $(37.3-45.3)$ & 41.9 & (38.5-45.4) & 3.1 & $(2.0-4.8)$ & 4.7 & $(3.3-6.7)$ & 3.9 & $(2.8-5.4)$ \\
\hline Median & & 37.0 & & 40.7 & & 39.0 & & 2.4 & & 4.8 & & 3.5 \\
\hline Range & & $8.5-49.0)$ & & $1.5-52.6)$ & & $0.4-48.0)$ & & 9-4.1) & & $4-13.3)$ & & $6-8.3)$ \\
\hline \multicolumn{13}{|c|}{ Large urban school district surveys } \\
\hline Baltimore, MD & 39.0 & $(31.2-47.3)$ & 62.0 & $(52.9-70.2)$ & 49.5 & $(42.9-56.0)$ & 4.9 & $(3.3-7.1)$ & 20.1 & $(15.5-25.7)$ & 12.2 & $(9.6-15.4)$ \\
\hline Boston, MA & 37.6 & $(32.0-43.5)$ & 46.3 & $(41.8-50.9)$ & 41.8 & $(37.7-46.0)$ & 2.2 & $(1.2-3.8)$ & 10.5 & $(8.1-13.6)$ & 6.2 & (4.9-7.9) \\
\hline Broward County, FL & 36.6 & (31.8-41.7) & 43.4 & (38.4-48.5) & 39.9 & (36.2-43.8) & 2.6 & $(1.5-4.3)$ & 8.2 & $(5.9-11.1)$ & 5.3 & $(4.1-6.8)$ \\
\hline Cleveland, $\mathrm{OH}$ & 44.6 & (39.4-50.0) & 57.4 & $(52.1-62.5)$ & 51.0 & $(46.6-55.4)$ & 3.7 & $(2.3-6.0)$ & 17.5 & $(14.1-21.5)$ & 10.5 & $(8.6-12.6)$ \\
\hline DeKalb County, GA & 33.7 & $(28.9-39.0)$ & 46.0 & $(42.2-49.8)$ & 39.6 & (35.9-43.5) & 2.8 & $(1.8-4.4)$ & 12.5 & $(10.3-15.2)$ & 7.4 & $(6.0-9.1)$ \\
\hline Detroit, MI & 34.2 & $(29.0-39.9)$ & 57.0 & $(50.4-63.4)$ & 44.1 & $(39.6-48.8)$ & 2.9 & $(1.9-4.3)$ & 17.1 & $(12.2-23.4)$ & 9.1 & $(6.8-12.1)$ \\
\hline District of Columbia & 32.7 & $(31.3-34.1)$ & 50.9 & $(49.2-52.7)$ & 40.9 & (39.8-42.0) & 3.5 & $(2.9-4.1)$ & 20.1 & $(18.7-21.6)$ & 11.0 & $(10.3-11.8)$ \\
\hline Duval County, FL & 34.0 & (30.7-37.4) & 39.8 & $(36.3-43.4)$ & 36.7 & (34.1-39.3) & 2.7 & $(1.9-3.8)$ & 8.7 & $(6.9-11.1)$ & 5.7 & $(4.6-6.9)$ \\
\hline Ft. Worth, TX & 35.1 & $(31.4-39.1)$ & 43.9 & $(39.7-48.1)$ & 39.5 & (36.2-42.8) & 1.7 & $(1.1-2.6)$ & 8.2 & $(6.3-10.6)$ & 4.9 & $(3.8-6.2)$ \\
\hline Houston, TX & 32.2 & $(28.9-35.7)$ & 42.0 & $(38.6-45.4)$ & 37.1 & $(34.4-39.9)$ & 2.2 & $(1.5-3.3)$ & 8.9 & $(7.2-11.0)$ & 5.5 & $(4.5-6.8)$ \\
\hline Los Angeles, CA & 22.9 & $(17.9-28.8)$ & 32.7 & $(28.0-37.7)$ & 27.6 & $(22.9-32.8)$ & 2.0 & $(1.1-3.6)$ & 5.7 & $(3.9-8.4)$ & 3.8 & $(2.8-5.0)$ \\
\hline Miami-Dade County, FL & 33.3 & $(29.0-37.9)$ & 43.8 & $(39.6-48.1)$ & 38.4 & $(34.7-42.2)$ & 1.1 & $(0.7-1.8)$ & 9.3 & $(7.6-11.4)$ & 5.2 & $(4.2-6.4)$ \\
\hline
\end{tabular}

See table footnotes on the next page. 
TABLE 70. (Continued) Percentage of high school students who ever had sexual intercourse and who had sexual intercourse for the first time before age 13 years, by sex — selected U.S. sites, Youth Risk Behavior Survey, 2015

\begin{tabular}{|c|c|c|c|c|c|c|c|c|c|c|c|c|}
\hline \multirow[b]{3}{*}{ Site } & \multicolumn{6}{|c|}{ Ever had sexual intercourse } & \multicolumn{6}{|c|}{ Had first sexual intercourse before age 13 years } \\
\hline & \multicolumn{2}{|c|}{ Female } & \multicolumn{2}{|r|}{ Male } & \multicolumn{2}{|r|}{ Total } & \multicolumn{2}{|c|}{ Female } & \multicolumn{2}{|r|}{ Male } & \multicolumn{2}{|c|}{ Total } \\
\hline & $\%$ & $\mathrm{Cl}^{*}$ & $\%$ & $\mathrm{Cl}$ & $\%$ & $\mathrm{Cl}$ & $\%$ & $\mathrm{Cl}$ & $\%$ & $\mathrm{Cl}$ & $\%$ & $\mathrm{Cl}$ \\
\hline New York City, NY & 24.3 & $(20.9-28.1)$ & 30.4 & $(26.0-35.2)$ & 27.2 & $(23.7-30.9)$ & 1.5 & $(1.0-2.2)$ & 7.5 & $(5.4-10.1)$ & 4.4 & $(3.3-5.8)$ \\
\hline Oakland, CA & 29.5 & $(24.2-35.3)$ & 44.6 & $(39.3-50.0)$ & 37.2 & (32.9-41.6) & 1.7 & $(0.9-3.0)$ & 12.7 & $(9.8-16.5)$ & 7.3 & (5.7-9.2) \\
\hline Orange County, FL & 31.6 & $(26.9-36.7)$ & 42.7 & $(37.2-48.3)$ & 37.1 & (32.9-41.5) & 1.3 & $(0.7-2.5)$ & 9.6 & $(6.9-13.3)$ & 5.3 & $(3.9-7.2)$ \\
\hline Palm Beach County, FL & 38.1 & $(34.0-42.4)$ & 44.6 & $(40.5-48.7)$ & 41.3 & $(37.9-44.8)$ & 3.3 & $(2.2-4.7)$ & 8.8 & $(7.0-11.1)$ & 6.1 & $(5.0-7.5)$ \\
\hline Philadelphia, PA & 47.9 & $(40.9-55.0)$ & 57.5 & $(49.5-65.0)$ & 52.4 & $(45.6-59.1)$ & 5.4 & $(3.4-8.6)$ & 14.5 & $(11.1-18.7)$ & 9.7 & $(7.9-11.9)$ \\
\hline San Diego, CA & 27.8 & $(23.0-33.1)$ & 36.0 & (31.4-40.9) & 32.0 & $(27.8-36.5)$ & 1.2 & $(0.7-2.1)$ & 5.6 & $(4.0-7.7)$ & 3.5 & $(2.6-4.6)$ \\
\hline San Francisco, CA & 26.1 & $(21.2-31.7)$ & 25.7 & $(21.2-30.7)$ & 25.9 & $(21.7-30.5)$ & 1.4 & $(0.7-2.5)$ & 3.9 & $(2.6-5.8)$ & 2.7 & $(1.9-3.8)$ \\
\hline Median & \multicolumn{2}{|c|}{33.7} & \multicolumn{2}{|c|}{43.9} & \multicolumn{2}{|c|}{39.5} & \multicolumn{2}{|c|}{2.2} & \multicolumn{2}{|c|}{$9.3^{(2.07}$} & \multicolumn{2}{|c|}{5.7} \\
\hline Range & \multicolumn{2}{|c|}{$(22.9-47.9)$} & \multicolumn{2}{|c|}{$(25.7-62.0)$} & \multicolumn{2}{|c|}{ (25.9-52.4) } & \multicolumn{2}{|c|}{$(1.1-5.4)$} & \multicolumn{2}{|c|}{$(3.9-20.1)$} & \multicolumn{2}{|c|}{$(2.7-12.2)$} \\
\hline
\end{tabular}

* $95 \%$ confidence interval.

${ }^{\dagger}$ Not available.

TABLE 71. Percentage of high school students who had sexual intercourse with four or more persons during their life and who were currently sexually active,* by sex, race/ethnicity, and grade - United States, Youth Risk Behavior Survey, 2015

\begin{tabular}{|c|c|c|c|c|c|c|c|c|c|c|c|c|}
\hline \multirow[b]{3}{*}{ Category } & \multicolumn{6}{|c|}{ Had sexual intercourse with four or more persons during their life } & \multicolumn{6}{|c|}{ Currently sexually active } \\
\hline & \multicolumn{2}{|c|}{ Female } & \multicolumn{2}{|r|}{ Male } & \multicolumn{2}{|r|}{ Total } & \multicolumn{2}{|c|}{ Female } & \multicolumn{2}{|c|}{ Male } & \multicolumn{2}{|r|}{ Total } \\
\hline & $\%$ & $\mathrm{Cl}^{\dagger}$ & $\%$ & $\mathrm{Cl}$ & $\%$ & $\mathrm{Cl}$ & $\%$ & $\mathrm{Cl}$ & $\%$ & $\mathrm{Cl}$ & $\%$ & $\mathrm{Cl}$ \\
\hline \multicolumn{13}{|c|}{ Race/Ethnicity } \\
\hline White ${ }^{\S}$ & 9.2 & $(7.1-11.9)$ & 10.6 & $(8.5-13.1)$ & 9.9 & $(8.1-12.0)$ & 31.4 & $(26.7-36.6)$ & 29.1 & $(26.3-32.1)$ & 30.3 & $(26.7-34.0)$ \\
\hline Black $^{\S}$ & 9.2 & $(6.5-12.8)$ & 28.2 & $(23.7-33.2)$ & 19.0 & (15.7-22.9) & 25.7 & $(20.3-31.9)$ & 40.0 & (34.7-45.5) & 33.1 & $(28.7-37.8)$ \\
\hline Hispanic & 6.7 & $(5.2-8.6)$ & 15.3 & $(12.8-18.1)$ & 11.0 & $(9.3-13.1)$ & 30.1 & $(26.2-34.2)$ & 30.5 & $(27.5-33.7)$ & 30.3 & $(27.3-33.5)$ \\
\hline \multicolumn{13}{|l|}{ Grade } \\
\hline 9 & 3.0 & $(2.1-4.4)$ & 6.7 & $(4.7-9.4)$ & 4.9 & $(3.7-6.5)$ & 14.0 & $(11.5-16.8)$ & 17.3 & $(14.6-20.4)$ & 15.7 & $(13.5-18.1)$ \\
\hline 10 & 6.0 & $(4.1-8.7)$ & 12.1 & $(9.8-14.8)$ & 9.0 & $(7.1-11.3)$ & 24.7 & $(19.6-30.6)$ & 26.4 & $(22.5-30.7)$ & 25.5 & (21.5-29.9) \\
\hline 11 & 10.7 & $(8.6-13.1)$ & 16.0 & $(12.8-19.9)$ & 13.4 & $(11.3-15.8)$ & 36.7 & (31.8-41.9) & 34.5 & $(30.7-38.6)$ & 35.5 & (31.7-39.4) \\
\hline 12 & 16.1 & (12.4-20.7) & 22.4 & $(18.4-27.0)$ & 19.2 & (16.2-22.6) & 46.5 & $(42.2-51.0)$ & 45.4 & $(41.1-49.7)$ & 46.0 & $(42.3-49.7)$ \\
\hline Total & 8.8 & $(7.2-10.6)$ & 14.1 & $(12.0-16.6)$ & 11.5 & $(9.9-13.3)$ & 29.8 & $(26.5-33.4)$ & 30.3 & $(27.9-32.9)$ & 30.1 & $(27.4-32.9)$ \\
\hline
\end{tabular}

* Had sexual intercourse with at least one person during the 3 months before the survey.

† $95 \%$ confidence interval.

$\S$ Non-Hispanic. 
TABLE 72. Percentage of high school students who had sexual intercourse with four or more persons during their life and who were currently sexually active,* by sex - selected U.S. sites, Youth Risk Behavior Survey, 2015

\begin{tabular}{|c|c|c|c|c|c|c|c|c|c|c|c|c|}
\hline \multirow[b]{3}{*}{ Site } & \multicolumn{6}{|c|}{$\begin{array}{c}\text { Had sexual intercourse with four or more persons } \\
\text { during their life }\end{array}$} & \multicolumn{6}{|c|}{ Currently sexually active } \\
\hline & \multicolumn{2}{|c|}{ Female } & \multicolumn{2}{|r|}{ Male } & \multicolumn{2}{|r|}{ Total } & \multicolumn{2}{|r|}{ Female } & \multicolumn{2}{|r|}{ Male } & \multicolumn{2}{|r|}{ Total } \\
\hline & $\%$ & $\mathrm{Cl}^{\dagger}$ & $\%$ & $\mathrm{Cl}$ & $\%$ & $\mathrm{Cl}$ & $\%$ & $\mathrm{Cl}$ & $\%$ & $\mathrm{Cl}$ & $\%$ & $\mathrm{Cl}$ \\
\hline \multicolumn{13}{|l|}{ State surveys } \\
\hline Alabama & 11.7 & $(9.2-14.8)$ & 19.5 & $(14.9-25.0)$ & 15.4 & $(12.7-18.6)$ & 34.9 & $(29.9-40.2)$ & 34.8 & $(29.9-40.1)$ & 34.9 & $(30.8-39.3)$ \\
\hline Alaska & 7.0 & $(4.8-10.1)$ & 10.4 & $(8.3-12.9)$ & 8.8 & $(7.0-11.0)$ & 26.5 & $(22.4-31.0)$ & 24.5 & $(20.1-29.6)$ & 25.6 & $(22.7-28.8)$ \\
\hline Arizona & 10.2 & $(6.6-15.5)$ & 10.8 & $(7.4-15.6)$ & 10.6 & $(7.6-14.5)$ & 28.9 & $(22.5-36.3)$ & 29.1 & $(24.3-34.4)$ & 29.0 & $(23.7-35.1)$ \\
\hline Arkansas & 12.9 & $(10.2-16.2)$ & 19.3 & $(14.7-25.0)$ & 16.0 & $(12.9-19.6)$ & 34.1 & $(27.8-40.9)$ & 34.1 & $(27.8-41.0)$ & 34.1 & $(29.5-39.0)$ \\
\hline California & 5.1 & $(3.9-6.8)$ & 10.4 & $(7.4-14.6)$ & 7.8 & $(5.8-10.5)$ & 23.1 & $(19.9-26.7)$ & 25.1 & $(18.7-32.9)$ & 24.2 & $(20.0-29.0)$ \\
\hline Connecticut & 5.3 & $(3.6-7.9)$ & 7.1 & $(5.3-9.5)$ & 6.2 & $(4.9-7.8)$ & 25.1 & $(21.5-29.1)$ & 21.4 & $(17.6-25.8)$ & 23.3 & $(20.1-26.9)$ \\
\hline Delaware & 8.8 & $(6.1-12.7)$ & 16.4 & $(12.8-20.8)$ & 12.9 & $(10.2-16.2)$ & 34.3 & $(29.2-39.8)$ & 31.6 & $(27.4-36.2)$ & 33.5 & $(29.4-37.9)$ \\
\hline Florida & 6.2 & $(5.3-7.4)$ & 16.5 & $(14.4-18.9)$ & 11.4 & $(10.1-12.9)$ & 25.5 & $(23.4-27.8)$ & 29.3 & $(26.8-31.9)$ & 27.5 & $(25.5-29.6)$ \\
\hline Hawaii & 7.0 & $(5.4-8.9)$ & 6.5 & (5.4-7.8) & 6.8 & $(5.6-8.2)$ & 25.4 & $(22.5-28.4)$ & 18.9 & $(16.2-22.0)$ & 22.3 & $(20.3-24.5)$ \\
\hline Idaho & —§ & - & - & - & - & - & 30.1 & $(25.2-35.5)$ & 29.5 & $(24.8-34.6)$ & 29.8 & $(25.8-34.1)$ \\
\hline Illinois & 7.0 & $(4.8-10.1)$ & 11.1 & $(7.5-16.2)$ & 9.0 & $(6.8-11.8)$ & 28.7 & $(23.1-35.1)$ & 30.6 & $(23.2-39.1)$ & 29.7 & $(24.2-35.8)$ \\
\hline Indiana & 8.0 & $(5.8-10.9)$ & 9.1 & $(6.3-13.0)$ & 8.7 & $(6.4-11.5)$ & 32.7 & $(27.3-38.7)$ & 30.7 & $(25.2-36.8)$ & 31.7 & $(27.1-36.7)$ \\
\hline Kentucky & 10.2 & $(7.4-14.0)$ & 10.6 & $(7.7-14.5)$ & 10.4 & $(8.2-13.1)$ & 29.6 & $(24.1-35.7)$ & 31.0 & $(26.3-36.2)$ & 30.3 & $(26.3-34.6)$ \\
\hline Maine & 8.1 & $(7.1-9.2)$ & 8.2 & $(7.0-9.6)$ & 8.2 & $(7.2-9.3)$ & 30.7 & $(28.0-33.5)$ & 26.2 & $(24.4-28.0)$ & 28.5 & $(26.5-30.6)$ \\
\hline Maryland & 5.9 & $(5.5-6.4)$ & 11.4 & $(10.8-12.2)$ & 8.6 & $(8.2-9.1)$ & 22.1 & $(21.2-23.0)$ & 23.0 & $(22.1-24.0)$ & 22.6 & $(21.8-23.4)$ \\
\hline Massachusetts & 6.0 & $(4.6-7.8)$ & 9.8 & $(7.5-12.7)$ & 7.9 & $(6.2-10.0)$ & 27.9 & $(24.6-31.4)$ & 27.6 & $(23.3-32.3)$ & 27.7 & $(24.4-31.3)$ \\
\hline Michigan & 8.2 & $(5.5-12.0)$ & 9.5 & $(7.2-12.4)$ & 8.8 & $(6.6-11.6)$ & 27.4 & $(22.5-33.0)$ & 23.8 & $(20.0-28.0)$ & 25.6 & $(22.2-29.4)$ \\
\hline Mississippi & 12.0 & $(8.8-16.2)$ & 19.4 & $(16.1-23.2)$ & 15.5 & $(13.1-18.1)$ & 32.7 & $(27.8-38.1)$ & 35.2 & $(31.4-39.2)$ & 33.8 & $(30.4-37.5)$ \\
\hline Missouri & - & - & - & - & - & - & 26.1 & $(22.3-30.2)$ & 29.2 & $(25.6-33.2)$ & 27.5 & $(24.3-30.9)$ \\
\hline Montana & 12.3 & $(10.5-14.2)$ & 14.6 & (12.0-17.7) & 13.4 & (11.7-15.4) & 34.9 & $(31.7-38.2)$ & 30.0 & $(27.5-32.6)$ & 32.3 & $(29.9-34.8)$ \\
\hline Nebraska & 7.1 & $(5.0-9.9)$ & 9.0 & $(6.5-12.4)$ & 8.0 & $(6.3-10.1)$ & 26.2 & $(22.2-30.7)$ & 23.4 & $(19.2-28.2)$ & 24.9 & $(21.4-28.7)$ \\
\hline Nevada & 8.7 & $(5.9-12.5)$ & 12.8 & $(9.1-17.6)$ & 10.7 & $(8.2-13.9)$ & 27.5 & $(22.9-32.7)$ & 26.4 & $(20.4-33.4)$ & 27.0 & $(22.3-32.2)$ \\
\hline New Hampshire & - & - & - & - & - & - & 32.0 & $(29.8-34.4)$ & 30.4 & $(28.4-32.4)$ & 31.3 & $(29.5-33.0)$ \\
\hline New Mexico & 6.5 & $(5.7-7.5)$ & 11.0 & $(9.7-12.5)$ & 8.7 & $(7.9-9.6)$ & 25.5 & $(23.4-27.6)$ & 24.6 & $(22.5-26.9)$ & 25.1 & $(23.4-26.9)$ \\
\hline New York & 5.9 & $(4.4-7.7)$ & 8.7 & $(6.3-12.0)$ & 7.2 & (5.5-9.4) & 24.3 & $(20.0-29.2)$ & 22.8 & $(19.1-27.0)$ & 23.6 & $(20.3-27.2)$ \\
\hline North Carolina & 9.1 & $(6.8-12.2)$ & 15.8 & $(11.7-21.0)$ & 12.5 & $(9.9-15.8)$ & 32.4 & $(29.5-35.5)$ & 33.0 & $(29.5-36.6)$ & 32.7 & $(30.3-35.2)$ \\
\hline North Dakota & - & - & - & - & - & - & 30.5 & $(26.5-34.8)$ & 28.9 & $(25.6-32.4)$ & 29.7 & $(26.8-32.7)$ \\
\hline Oklahoma & 8.4 & $(6.0-11.5)$ & 17.9 & $(14.2-22.4)$ & 13.1 & $(10.3-16.5)$ & 31.3 & $(26.3-36.8)$ & 31.0 & $(25.5-37.1)$ & 31.0 & $(26.7-35.7)$ \\
\hline Pennsylvania & 7.9 & $(5.9-10.5)$ & 12.8 & $(10.0-16.2)$ & 10.3 & $(8.2-12.8)$ & 26.5 & $(22.2-31.4)$ & 26.8 & $(22.4-31.8)$ & 26.7 & $(22.7-31.1)$ \\
\hline Rhode Island & 6.0 & $(4.5-7.9)$ & 8.8 & $(5.7-13.3)$ & 7.4 & $(6.0-8.9)$ & 28.3 & $(24.9-32.0)$ & 23.0 & $(20.6-25.6)$ & 25.7 & $(23.0-28.6)$ \\
\hline South Carolina & 4.9 & $(3.4-7.0)$ & 12.8 & $(8.4-19.2)$ & 8.7 & $(6.3-12.0)$ & 26.7 & $(20.5-34.1)$ & 28.2 & $(20.8-37.0)$ & 27.6 & $(21.7-34.4)$ \\
\hline South Dakota & 10.9 & $(6.9-17.0)$ & 14.2 & $(9.0-21.7)$ & 12.6 & $(8.8-17.8)$ & 26.6 & $(21.6-32.4)$ & 28.1 & $(20.6-37.2)$ & 27.5 & $(22.1-33.6)$ \\
\hline Tennessee & - & - & - & - & - & - & - & - & - & - & - & - \\
\hline Vermont & 9.5 & $(8.9-10.1)$ & 10.4 & $(9.8-11.0)$ & 10.0 & $(9.6-10.5)$ & 32.7 & $(31.8-33.7)$ & 29.6 & $(28.7-30.5)$ & 31.2 & $(30.6-31.9)$ \\
\hline Virginia & - & - & - & - & - & - & - & - & - & - & - & - \\
\hline West Virginia & 11.3 & $(8.5-14.9)$ & 15.4 & (12.2-19.4) & 13.4 & $(11.0-16.1)$ & 39.0 & $(33.0-45.4)$ & 31.8 & $(26.7-37.3)$ & 35.5 & $(30.4-40.9)$ \\
\hline Wyoming & 12.4 & $(9.4-16.2)$ & 13.3 & $(10.7-16.4)$ & 12.9 & (10.5-15.7) & 33.7 & $(29.2-38.5)$ & 29.1 & $(25.5-32.9)$ & 31.5 & $(28.2-34.9)$ \\
\hline Median & & 8.1 & & 11.1 & & 10.0 & & 28.7 & & 29.1 & & 28.5 \\
\hline Range & (4. & 9-12.9) & & $5-19.5)$ & & $.2-16.0)$ & & $.1-39.0)$ & & $9-35.2)$ & & $2.3-35.5)$ \\
\hline \multicolumn{13}{|c|}{ Large urban school district surveys } \\
\hline Baltimore, MD & 8.5 & $(5.5-13.1)$ & 27.5 & $(20.1-36.3)$ & 17.0 & $(12.2-23.0)$ & 28.8 & $(21.7-37.1)$ & 42.1 & $(32.6-52.3)$ & 34.9 & $(28.7-41.6)$ \\
\hline Boston, MA & 6.1 & $(4.2-8.9)$ & 17.8 & $(14.6-21.7)$ & 11.7 & $(9.8-14.0)$ & 28.6 & $(23.5-34.3)$ & 31.5 & $(27.2-36.1)$ & 29.9 & $(26.5-33.7)$ \\
\hline Broward County, FL & 7.3 & $(5.0-10.6)$ & 13.7 & $(10.7-17.4)$ & 10.6 & $(8.5-13.1)$ & 27.2 & $(22.8-32.1)$ & 27.8 & $(23.6-32.3)$ & 27.6 & $(24.2-31.2)$ \\
\hline Cleveland, $\mathrm{OH}$ & 9.3 & $(6.9-12.5)$ & 27.5 & $(23.1-32.3)$ & 18.2 & $(15.5-21.2)$ & 31.4 & $(26.6-36.6)$ & 38.9 & $(33.9-44.2)$ & 35.2 & $(31.3-39.3)$ \\
\hline DeKalb County, GA & 6.8 & $(5.2-8.8)$ & 17.1 & $(14.5-20.1)$ & 11.7 & $(9.8-13.8)$ & 22.7 & $(18.8-27.2)$ & 27.4 & $(23.8-31.4)$ & 25.0 & $(21.7-28.5)$ \\
\hline Detroit, Ml & 5.5 & $(4.0-7.6)$ & 21.6 & $(17.4-26.6)$ & 12.5 & $(10.4-15.0)$ & 23.9 & $(19.5-28.9)$ & 32.9 & $(27.6-38.5)$ & 27.8 & $(24.0-32.1)$ \\
\hline District of Columbia & 5.9 & $(5.2-6.6)$ & 23.4 & $(21.9-25.0)$ & 13.8 & $(13.0-14.6)$ & 24.2 & $(22.9-25.5)$ & 33.1 & (31.4-34.8) & 28.2 & $(27.2-29.3)$ \\
\hline Duval County, FL & 7.4 & $(5.8-9.4)$ & 13.6 & $(11.0-16.8)$ & 10.3 & $(8.7-12.1)$ & 24.2 & $(21.3-27.4)$ & 27.0 & $(23.5-30.7)$ & 25.6 & $(23.1-28.3)$ \\
\hline Ft. Worth, TX & 5.1 & $(3.9-6.6)$ & 16.5 & $(14.2-19.0)$ & 10.7 & $(9.3-12.2)$ & 25.3 & $(22.2-28.7)$ & 28.9 & $(25.9-32.1)$ & 27.1 & $(24.5-29.8)$ \\
\hline Houston, TX & 6.3 & $(4.8-8.3)$ & 16.2 & $(13.9-18.8)$ & 11.3 & $(9.6-13.2)$ & 22.3 & $(19.6-25.2)$ & 26.6 & $(23.8-29.5)$ & 24.4 & $(22.2-26.7)$ \\
\hline Los Angeles, CA & 2.3 & $(1.3-3.9)$ & 9.5 & $(6.9-13.0)$ & 5.7 & $(4.3-7.5)$ & 16.8 & $(13.2-21.1)$ & 21.0 & $(17.2-25.5)$ & 18.8 & $(15.3-23.0)$ \\
\hline Miami-Dade County, FL & 4.6 & $(3.4-6.2)$ & 17.5 & $(14.9-20.5)$ & 11.0 & $(9.2-13.0)$ & 24.2 & $(20.8-28.1)$ & 28.5 & $(24.9-32.4)$ & 26.3 & $(23.3-29.5)$ \\
\hline
\end{tabular}

See table footnotes on the next page. 
TABLE 72. (Continued) Percentage of high school students who had sexual intercourse with four or more persons during their life and who were currently sexually active,* by sex — selected U.S. sites, Youth Risk Behavior Survey, 2015

\begin{tabular}{|c|c|c|c|c|c|c|c|c|c|c|c|c|}
\hline \multirow[b]{3}{*}{ Site } & \multicolumn{6}{|c|}{$\begin{array}{l}\text { Had sexual intercourse with four or more persons } \\
\text { during their life }\end{array}$} & \multicolumn{6}{|c|}{ Currently sexually active } \\
\hline & \multicolumn{2}{|c|}{ Female } & \multicolumn{2}{|r|}{ Male } & \multicolumn{2}{|r|}{ Total } & \multicolumn{2}{|r|}{ Female } & \multicolumn{2}{|r|}{ Male } & \multicolumn{2}{|r|}{ Total } \\
\hline & $\%$ & $\mathrm{Cl}^{\dagger}$ & $\%$ & $\mathrm{Cl}$ & $\%$ & $\mathrm{Cl}$ & $\%$ & $\mathrm{Cl}$ & $\%$ & $\mathrm{Cl}$ & $\%$ & $\mathrm{Cl}$ \\
\hline New York City, NY & 4.3 & $(3.2-5.8)$ & 11.4 & $(8.7-14.7)$ & 7.6 & $(6.0-9.8)$ & 18.5 & $(15.4-22.0)$ & 19.1 & $(15.7-23.0)$ & 18.7 & $(15.9-21.9)$ \\
\hline Oakland, CA & 5.7 & $(3.9-8.3)$ & 16.5 & $(13.1-20.6)$ & 11.2 & $(9.1-13.6)$ & 20.8 & $(16.8-25.4)$ & 26.6 & $(22.3-31.5)$ & 23.9 & $(20.8-27.3)$ \\
\hline Orange County, FL & 4.2 & $(2.9-6.1)$ & 15.4 & $(11.9-19.7)$ & 9.7 & (7.7-12.1) & 22.6 & $(18.8-26.8)$ & 26.2 & $(22.0-30.9)$ & 24.4 & $(21.1-27.9)$ \\
\hline Palm Beach County, FL & 8.6 & $(6.7-10.9)$ & 15.8 & $(13.1-18.9)$ & 12.2 & $(10.4-14.2)$ & 30.0 & $(26.4-33.8)$ & 30.9 & $(27.1-35.0)$ & 30.6 & $(27.7-33.7)$ \\
\hline Philadelphia, PA & 11.3 & $(8.4-14.9)$ & 28.6 & $(22.4-35.8)$ & 19.4 & $(15.7-23.8)$ & 35.5 & $(29.8-41.7)$ & 39.1 & $(32.2-46.4)$ & 37.2 & $(31.5-43.2)$ \\
\hline San Diego, CA & 3.1 & $(2.1-4.4)$ & 10.5 & $(8.6-12.7)$ & 6.8 & (5.6-8.2) & 20.4 & $(16.4-25.0)$ & 24.4 & $(20.2-29.0)$ & 22.3 & $(18.9-26.2)$ \\
\hline San Francisco, CA & - & - & - & - & - & - & 20.1 & $(16.1-24.9)$ & 17.4 & $(13.6-21.9)$ & 18.7 & (15.2-22.9) \\
\hline Median & \multicolumn{2}{|c|}{6.0} & \multicolumn{2}{|r|}{16.5} & \multicolumn{2}{|r|}{11.2} & \multicolumn{2}{|r|}{24.2} & \multicolumn{2}{|r|}{27.8} & \multicolumn{2}{|r|}{26.3} \\
\hline Range & \multicolumn{2}{|c|}{$(2.3-11.3)$} & \multicolumn{2}{|r|}{$(9.5-28.6)$} & \multicolumn{2}{|c|}{$(5.7-19.4)$} & \multicolumn{2}{|c|}{ (16.8-35.5) } & \multicolumn{2}{|c|}{$(17.4-42.1)$} & \multicolumn{2}{|c|}{ (18.7-37.2) } \\
\hline
\end{tabular}

* Had sexual intercourse with at least one person during the 3 months before the survey.

† $95 \%$ confidence interval.

§ Not available.

TABLE 73. Percentage of high school students who used a condom during last sexual intercourse* and who used birth control pills before last sexual intercourse, ${ }^{*}+{ }^{\dagger}$ by sex, race/ethnicity, and grade - United States, Youth Risk Behavior Survey, 2015

\begin{tabular}{|c|c|c|c|c|c|c|c|c|c|c|c|c|}
\hline \multirow[b]{3}{*}{ Category } & \multicolumn{6}{|c|}{ Condom use } & \multicolumn{6}{|c|}{ Birth control pill use } \\
\hline & \multicolumn{2}{|c|}{ Female } & \multicolumn{2}{|c|}{ Male } & \multicolumn{2}{|c|}{ Total } & \multicolumn{2}{|c|}{ Female } & \multicolumn{2}{|r|}{ Male } & \multicolumn{2}{|r|}{ Total } \\
\hline & $\%$ & $\mathrm{Cl}^{\S}$ & $\%$ & $\mathrm{Cl}$ & $\%$ & $\mathrm{Cl}$ & $\%$ & $\mathrm{Cl}$ & $\%$ & $\mathrm{Cl}$ & $\%$ & $\mathrm{Cl}$ \\
\hline \multicolumn{13}{|c|}{ Race/Ethnicity } \\
\hline White & 55.9 & $(51.2-60.4)$ & 58.1 & $(52.8-63.3)$ & 56.8 & $(52.5-61.1)$ & 25.4 & $(21.2-30.3)$ & 21.4 & $(18.1-25.0)$ & 23.5 & (20.4-26.9) \\
\hline Black ${ }^{q}$ & 46.7 & $(38.7-54.8)$ & 73.6 & $(63.7-81.7)$ & 63.4 & $(56.5-69.7)$ & 9.0 & $(4.8-16.4)$ & 9.0 & $(6.0-13.2)$ & 9.0 & $(6.4-12.4)$ \\
\hline Hispanic & 48.3 & $(41.4-55.3)$ & 62.5 & $(57.1-67.7)$ & 55.6 & $(51.9-59.1)$ & 15.4 & $(11.0-21.2)$ & 8.1 & $(5.7-11.4)$ & 11.8 & $(8.8-15.7)$ \\
\hline \multicolumn{13}{|l|}{ Grade } \\
\hline 9 & 56.7 & (49.3-63.8) & 63.3 & $(54.8-71.1)$ & 60.5 & (54.8-65.9) & 11.2 & $(7.3-16.7)$ & 10.8 & $(6.9-16.7)$ & 10.9 & $(7.8-15.1)$ \\
\hline 10 & 54.0 & $(47.0-60.8)$ & 65.6 & $(57.8-72.7)$ & 59.9 & (54.2-65.4) & 20.2 & (15.9-25.3) & 11.6 & $(8.0-16.6)$ & 15.9 & $(13.0-19.2)$ \\
\hline 11 & 52.9 & $(46.8-59.0)$ & 62.5 & (55.9-68.7) & 57.7 & $(52.9-62.2)$ & 23.9 & (19.2-29.3) & 19.1 & $(15.2-23.6)$ & 21.5 & $(18.0-25.5)$ \\
\hline 12 & 48.8 & $(44.0-53.6)$ & 57.4 & $(51.6-63.0)$ & 52.9 & $(48.7-57.1)$ & 23.2 & $(17.9-29.5)$ & 16.9 & $(12.9-21.8)$ & 20.1 & (16.1-24.7) \\
\hline Total & 52.0 & $(48.9-55.2)$ & 61.5 & $(57.5-65.4)$ & 56.9 & $(53.9-59.8)$ & 21.3 & $(18.6-24.2)$ & 15.2 & $(12.9-17.8)$ & 18.2 & (16.1-20.5) \\
\hline
\end{tabular}

* Among the $30.1 \%$ of students nationwide who were currently sexually active.

$\dagger$ To prevent pregnancy.

$\S 95 \%$ confidence interval.

१ Non-Hispanic. 
TABLE 74. Percentage of high school students who used a condom during last sexual intercourse* and who used birth control pills before last sexual intercourse, ${ }^{* \dagger}+$ by sex — selected U.S. sites, Youth Risk Behavior Survey, 2015

\begin{tabular}{|c|c|c|c|c|c|c|c|c|c|c|c|c|}
\hline \multirow[b]{3}{*}{ Site } & \multicolumn{6}{|c|}{ Condom use } & \multicolumn{6}{|c|}{ Birth control pill use } \\
\hline & \multicolumn{2}{|r|}{ Female } & \multicolumn{2}{|r|}{ Male } & \multicolumn{2}{|r|}{ Total } & \multicolumn{2}{|r|}{ Female } & \multicolumn{2}{|r|}{ Male } & \multicolumn{2}{|r|}{ Total } \\
\hline & $\%$ & $\mathrm{Cl}^{\S}$ & $\%$ & $\mathrm{Cl}$ & $\%$ & $\mathrm{Cl}$ & $\%$ & $\mathrm{Cl}$ & $\%$ & $\mathrm{Cl}$ & $\%$ & $\mathrm{Cl}$ \\
\hline \multicolumn{13}{|l|}{ State surveys } \\
\hline Alabama & 42.8 & (33.3-53.0) & 58.9 & $(49.0-68.1)$ & 50.9 & $(43.1-58.7)$ & 24.3 & $(17.7-32.5)$ & 12.9 & $(8.8-18.4)$ & 18.8 & $(14.8-23.5)$ \\
\hline Alaska & 54.1 & $(46.1-62.0)$ & 69.8 & $(62.7-76.2)$ & 61.9 & (56.0-67.5) & 23.4 & $(17.5-30.5)$ & 14.4 & $(9.3-21.6)$ & 18.9 & $(14.7-24.0)$ \\
\hline Arizona & 51.8 & $(40.9-62.5)$ & 59.0 & $(48.7-68.5)$ & 55.1 & $(46.9-63.1)$ & 20.1 & $(12.3-30.9)$ & 21.1 & $(13.6-31.2)$ & 20.7 & $(13.5-30.2)$ \\
\hline Arkansas & 46.8 & $(40.8-52.9)$ & 59.6 & (44.8-72.8) & 52.7 & $(46.6-58.7)$ & 20.1 & (15.9-25.2) & 15.0 & $(8.9-24.1)$ & 17.8 & $(13.9-22.5)$ \\
\hline California & 55.8 & $(43.4-67.4)$ & 57.9 & $(47.5-67.5)$ & 56.8 & (50.4-62.9) & 14.3 & $(9.0-22.0)$ & 12.1 & $(8.0-17.9)$ & 13.1 & $(10.5-16.1)$ \\
\hline Connecticut & 53.6 & $(46.3-60.8)$ & 66.6 & $(59.5-72.9)$ & 59.4 & (53.4-65.1) & 30.8 & $(23.5-39.2)$ & 21.9 & $(16.2-29.0)$ & 26.8 & $(21.6-32.7)$ \\
\hline Delaware & 48.0 & $(39.9-56.2)$ & 66.3 & $(59.6-72.4)$ & 57.0 & $(51.6-62.3)$ & 23.1 & $(18.1-28.9)$ & 12.5 & $(8.8-17.5)$ & 18.0 & $(14.7-21.8)$ \\
\hline Florida & 56.2 & $(52.2-60.2)$ & 67.1 & $(62.3-71.6)$ & 61.7 & (58.4-64.9) & 15.8 & $(12.8-19.4)$ & 11.4 & $(9.6-13.5)$ & 13.6 & $(11.7-15.8)$ \\
\hline Hawaii & 44.8 & $(41.1-48.7)$ & 53.9 & $(43.8-63.7)$ & 48.4 & $(43.7-53.1)$ & 18.6 & $(14.2-23.9)$ & 13.8 & $(9.9-19.0)$ & 16.7 & (13.2-20.8) \\
\hline Idaho & 57.1 & $(48.9-64.9)$ & 59.8 & $(52.7-66.6)$ & 58.3 & $(52.9-63.5)$ & 一ा & - & - & - & - & - \\
\hline Illinois & 57.9 & (53.0-62.7) & 54.7 & $(48.4-60.9)$ & 56.2 & $(52.4-59.9)$ & 30.9 & $(19.6-45.1)$ & 17.4 & (12.0-24.5) & 24.0 & $(17.2-32.5)$ \\
\hline Indiana & 51.3 & $(44.3-58.2)$ & 55.6 & $(43.7-67.0)$ & 53.4 & $(45.9-60.8)$ & 26.3 & $(17.9-36.9)$ & 13.8 & (8.9-20.6) & 20.2 & $(14.2-27.8)$ \\
\hline Kentucky & 43.8 & $(35.6-52.3)$ & 64.6 & $(56.9-71.6)$ & 53.9 & $(47.4-60.3)$ & 26.1 & $(19.6-34.0)$ & 18.7 & $(14.3-24.1)$ & 22.4 & $(18.5-26.7)$ \\
\hline Maine & 54.0 & $(50.0-57.9)$ & 62.7 & $(59.5-65.8)$ & 57.9 & $(55.0-60.7)$ & 35.6 & $(32.9-38.3)$ & 28.9 & $(25.5-32.5)$ & 32.4 & (29.9-35.1) \\
\hline Maryland & 55.6 & (53.9-57.4) & 67.3 & $(65.5-68.9)$ & 61.3 & $(60.0-62.5)$ & 21.8 & $(20.4-23.3)$ & 13.3 & $(12.3-14.5)$ & 17.7 & (16.7-18.8) \\
\hline Massachusetts & 57.4 & $(51.6-63.0)$ & 67.6 & $(63.4-71.5)$ & 62.5 & (58.9-65.9) & 31.6 & $(26.3-37.4)$ & 25.2 & $(20.1-31.2)$ & 28.4 & $(24.5-32.6)$ \\
\hline Michigan & 53.5 & (43.9-62.8) & 62.0 & $(52.3-70.8)$ & 57.2 & $(50.6-63.6)$ & 22.7 & (15.8-31.4) & 24.0 & $(15.1-35.8)$ & 23.3 & $(18.1-29.4)$ \\
\hline Mississippi & 54.1 & $(45.3-62.7)$ & 57.6 & $(48.5-66.2)$ & 55.8 & $(49.9-61.6)$ & 19.9 & $(14.2-27.2)$ & 16.4 & $(11.2-23.3)$ & 18.3 & $(14.6-22.5)$ \\
\hline Missouri & 53.5 & $(45.0-61.9)$ & 59.8 & $(50.7-68.3)$ & 56.4 & $(48.5-64.0)$ & 27.1 & $(19.0-37.1)$ & 18.8 & $(10.9-30.6)$ & 22.9 & (17.4-29.4) \\
\hline Montana & 55.4 & $(51.5-59.3)$ & 63.6 & (57.9-68.9) & 59.2 & $(55.6-62.8)$ & 28.9 & $(25.6-32.4)$ & 25.0 & $(21.6-28.7)$ & 27.1 & $(24.3-30.1)$ \\
\hline Nebraska & 56.3 & $(48.0-64.3)$ & 58.4 & $(49.0-67.2)$ & 57.0 & $(49.9-63.8)$ & 24.6 & $(17.6-33.2)$ & 16.5 & $(10.8-24.4)$ & 20.7 & $(16.2-26.0)$ \\
\hline Nevada & 49.2 & $(40.4-58.1)$ & 58.5 & $(50.3-66.2)$ & 53.7 & $(47.2-60.1)$ & 23.9 & $(14.5-36.6)$ & 16.7 & $(10.0-26.7)$ & 20.4 & $(13.6-29.4)$ \\
\hline New Hampshire & 55.9 & $(52.9-58.9)$ & 65.1 & $(61.7-68.5)$ & 60.2 & (57.7-62.6) & 38.2 & $(34.8-41.7)$ & 27.1 & $(24.7-29.7)$ & 32.7 & (30.6-34.9) \\
\hline New Mexico & 45.4 & $(41.3-49.6)$ & 59.5 & $(55.6-63.2)$ & 52.1 & $(49.0-55.2)$ & 16.7 & $(13.9-19.9)$ & 13.0 & $(10.4-16.2)$ & 14.9 & $(13.0-17.0)$ \\
\hline New York & 55.6 & $(49.3-61.7)$ & 61.2 & $(57.1-65.2)$ & 58.1 & $(53.7-62.4)$ & 24.9 & (19.2-31.7) & 18.7 & $(13.5-25.3)$ & 22.1 & $(17.9-26.9)$ \\
\hline North Carolina & 55.5 & $(48.3-62.5)$ & 65.3 & $(57.1-72.7)$ & 60.5 & $(54.8-65.9)$ & 18.9 & $(12.3-27.8)$ & 19.0 & $(14.3-24.8)$ & 18.9 & $(15.1-23.4)$ \\
\hline North Dakota & 60.2 & $(53.3-66.7)$ & 62.5 & $(55.5-69.0)$ & 61.4 & $(56.5-66.0)$ & - & - & - & - & - & - \\
\hline Oklahoma & 56.7 & $(46.1-66.7)$ & 63.2 & $(52.5-72.8)$ & 59.9 & $(51.0-68.3)$ & 16.3 & $(10.1-25.4)$ & 13.4 & $(9.0-19.5)$ & 14.9 & (10.5-20.6) \\
\hline Pennsylvania & 54.8 & $(49.2-60.3)$ & 71.8 & $(64.2-78.3)$ & 63.3 & $(58.6-67.7)$ & 24.6 & $(18.9-31.2)$ & 15.2 & $(9.9-22.5)$ & 19.9 & $(15.3-25.5)$ \\
\hline Rhode Island & 57.7 & $(50.5-64.6)$ & 66.3 & $(57.7-73.9)$ & 61.4 & $(55.0-67.4)$ & 25.6 & $(19.8-32.6)$ & 28.8 & $(18.6-41.6)$ & 26.9 & $(19.7-35.6)$ \\
\hline South Carolina & 49.8 & $(36.8-62.8)$ & 68.0 & $(58.2-76.4)$ & 58.9 & $(49.5-67.7)$ & 22.6 & $(15.0-32.6)$ & 18.2 & $(11.5-27.6)$ & 20.3 & $(14.6-27.4)$ \\
\hline South Dakota & 61.8 & $(48.5-73.5)$ & 63.5 & $(47.9-76.7)$ & 62.7 & $(51.0-73.1)$ & 29.8 & $(21.9-39.0)$ & 25.3 & $(14.9-39.6)$ & 27.3 & $(20.5-35.4)$ \\
\hline Tennessee & - & - & - & - & - & - & - & - & - & - & - & - \\
\hline Vermont & 54.8 & $(53.0-56.5)$ & 62.0 & $(60.2-63.8)$ & 58.2 & $(57.0-59.5)$ & 38.5 & $(36.8-40.3)$ & 30.2 & $(28.5-32.0)$ & 34.6 & (33.4-35.9) \\
\hline Virginia & - & - & - & - & - & - & - & - & - & - & - & - \\
\hline West Virginia & 47.1 & $(41.7-52.6)$ & 57.3 & $(48.7-65.5)$ & 51.5 & $(46.3-56.6)$ & 34.5 & (28.9-40.6) & 19.9 & $(15.1-25.6)$ & 28.2 & $(23.7-33.2)$ \\
\hline Wyoming & 48.3 & $(40.9-55.7)$ & 58.0 & $(52.5-63.2)$ & 52.7 & $(48.0-57.3)$ & 25.7 & $(19.8-32.7)$ & 15.1 & $(11.0-20.4)$ & 20.9 & (16.4-26.2) \\
\hline Median & & 54.1 & & 62.0 & & 57.9 & & 24.6 & & 17.4 & & 20.7 \\
\hline Range & & (2.8-61.8) & & 3.9-71.8) & & $8.4-63.3)$ & & $4.3-38.5)$ & & $1.4-30.2)$ & & $3.1-34.6)$ \\
\hline \multicolumn{13}{|c|}{ Large urban school district surveys } \\
\hline Baltimore, MD & 54.2 & $(43.6-64.5)$ & 67.4 & $(58.7-75.0)$ & 61.1 & $(55.2-66.7)$ & 17.1 & $(10.5-26.5)$ & 12.3 & $(6.6-21.8)$ & 14.3 & $(9.8-20.5)$ \\
\hline Boston, MA & 48.9 & $(40.7-57.1)$ & 72.5 & $(65.3-78.8)$ & 60.6 & $(54.9-66.0)$ & 23.0 & $(16.7-30.7)$ & 14.3 & $(9.3-21.2)$ & 18.6 & $(14.5-23.6)$ \\
\hline Broward County, FL & 55.6 & $(47.7-63.3)$ & 67.9 & $(60.0-74.9)$ & 61.5 & $(55.2-67.3)$ & 13.2 & $(8.2-20.5)$ & 14.5 & $(9.7-21.2)$ & 13.8 & $(10.1-18.4)$ \\
\hline Cleveland, $\mathrm{OH}$ & 48.2 & $(40.0-56.6)$ & 61.9 & $(53.3-69.7)$ & 55.5 & $(48.9-61.8)$ & 16.4 & $(12.0-22.0)$ & 10.9 & $(7.1-16.4)$ & 13.4 & $(10.2-17.3)$ \\
\hline DeKalb County, GA & 53.6 & $(45.8-61.2)$ & 76.4 & $(68.0-83.2)$ & 65.0 & $(58.9-70.7)$ & 13.4 & $(9.2-19.1)$ & 11.1 & $(7.3-16.6)$ & 12.2 & $(9.2-16.0)$ \\
\hline Detroit, MI & 56.9 & $(47.9-65.5)$ & 80.8 & $(73.7-86.3)$ & 68.9 & $(63.1-74.2)$ & 10.2 & $(6.4-15.8)$ & 6.8 & $(3.8-11.9)$ & 8.7 & $(6.1-12.3)$ \\
\hline District of Columbia & 57.5 & $(54.3-60.6)$ & 75.2 & $(72.3-77.9)$ & 66.6 & $(64.4-68.7)$ & 8.0 & $(6.5-9.8)$ & 7.4 & $(5.9-9.3)$ & 7.7 & $(6.6-8.9)$ \\
\hline Duval County, FL & 53.2 & $(46.2-60.1)$ & 67.0 & $(61.2-72.2)$ & 59.8 & $(55.5-64.1)$ & 16.0 & $(12.1-21.0)$ & 13.3 & $(9.7-18.0)$ & 14.9 & $(12.1-18.1)$ \\
\hline Ft. Worth, TX & 43.7 & (36.9-50.7) & 65.9 & $(60.2-71.2)$ & 55.4 & $(51.1-59.7)$ & 12.1 & $(8.5-16.9)$ & 10.2 & $(6.9-14.7)$ & 11.1 & $(8.4-14.5)$ \\
\hline Houston, TX & 48.2 & $(41.5-55.0)$ & 67.5 & $(61.6-72.9)$ & 58.5 & $(54.3-62.6)$ & 8.5 & $(5.9-12.2)$ & 4.9 & $(2.7-8.9)$ & 6.8 & $(5.1-9.0)$ \\
\hline Los Angeles, CA & 54.4 & $(42.7-65.6)$ & 69.0 & $(61.9-75.4)$ & 62.3 & $(55.0-69.0)$ & 8.9 & $(4.2-17.9)$ & 5.8 & $(3.7-9.0)$ & 7.2 & $(4.1-12.3)$ \\
\hline Miami-Dade County, FL & 60.0 & $(52.7-67.0)$ & 68.0 & $(62.4-73.1)$ & 64.2 & $(59.6-68.5)$ & 9.6 & $(6.7-13.6)$ & 6.5 & $(4.3-9.7)$ & 8.0 & $(6.0-10.4)$ \\
\hline
\end{tabular}

See table footnotes on the next page. 
TABLE 74. (Continued) Percentage of high school students who used a condom during last sexual intercourse* and who used birth control pills before last sexual intercourse, ${ }^{*},+$ by sex - selected U.S. sites, Youth Risk Behavior Survey, 2015

\begin{tabular}{|c|c|c|c|c|c|c|c|c|c|c|c|c|}
\hline \multirow[b]{3}{*}{ Site } & \multicolumn{6}{|c|}{ Condom use } & \multicolumn{6}{|c|}{ Birth control pill use } \\
\hline & \multicolumn{2}{|c|}{ Female } & \multicolumn{2}{|r|}{ Male } & \multicolumn{2}{|r|}{ Total } & \multicolumn{2}{|r|}{ Female } & \multicolumn{2}{|r|}{ Male } & \multicolumn{2}{|r|}{ Total } \\
\hline & $\%$ & $\mathrm{Cl}^{\S}$ & $\%$ & $\mathrm{Cl}$ & $\%$ & $\mathrm{Cl}$ & $\%$ & $\mathrm{Cl}$ & $\%$ & $\mathrm{Cl}$ & $\%$ & $\mathrm{Cl}$ \\
\hline New York City, NY & 55.3 & $(48.8-61.7)$ & 69.9 & $(66.0-73.5)$ & 62.2 & $(58.0-66.3)$ & 13.4 & (11.0-16.4) & 11.5 & $(7.8-16.7)$ & 12.5 & $(10.2-15.2)$ \\
\hline Oakland, CA & 52.6 & $(43.1-61.9)$ & 67.1 & $(58.4-74.8)$ & 60.6 & $(53.9-66.9)$ & - & - & - & - & - & - \\
\hline Orange County, FL & 48.3 & $(40.3-56.2)$ & 66.5 & $(58.1-74.0)$ & 58.3 & $(52.0-64.4)$ & 14.0 & $(8.9-21.1)$ & 8.7 & $(4.8-15.2)$ & 11.1 & $(7.9-15.3)$ \\
\hline Palm Beach County, FL & 57.1 & $(50.8-63.1)$ & 64.2 & $(57.9-70.0)$ & 60.2 & $(55.8-64.5)$ & 15.6 & (11.4-20.9) & 15.3 & $(10.4-21.9)$ & 15.5 & $(11.9-19.8)$ \\
\hline Philadelphia, PA & 46.8 & $(39.6-54.1)$ & 65.3 & $(58.1-71.8)$ & 55.9 & $(50.9-60.8)$ & 13.5 & $(9.8-18.3)$ & 8.8 & $(5.5-13.9)$ & 11.2 & $(8.6-14.5)$ \\
\hline San Diego, CA & 59.2 & $(52.0-66.1)$ & 58.9 & $(50.9-66.5)$ & 59.0 & $(54.6-63.3)$ & 18.8 & $(13.4-25.7)$ & 16.1 & $(11.9-21.3)$ & 17.3 & $(13.6-21.7)$ \\
\hline San Francisco, CA & 47.6 & $(35.0-60.5)$ & 68.9 & $(57.9-78.1)$ & 57.7 & $(49.2-65.7)$ & 14.7 & $(8.6-23.9)$ & 8.6 & $(5.3-13.8)$ & 11.8 & $(7.7-17.7)$ \\
\hline Median & \multicolumn{2}{|r|}{53.6} & \multicolumn{2}{|r|}{67.5} & \multicolumn{2}{|r|}{60.6} & \multicolumn{2}{|r|}{13.4} & \multicolumn{2}{|r|}{10.5} & \multicolumn{2}{|r|}{12.0} \\
\hline Range & \multicolumn{2}{|c|}{$(43.7-60.0)$} & \multicolumn{2}{|c|}{ (58.9-80.8) } & \multicolumn{2}{|c|}{$(55.4-68.9)$} & \multicolumn{2}{|r|}{$(8.0-23.0)$} & \multicolumn{2}{|r|}{$(4.9-16.1)$} & \multicolumn{2}{|c|}{$(6.8-18.6)$} \\
\hline
\end{tabular}

* Among students who were currently sexually active.

† To prevent pregnancy.

$\S 95 \%$ confidence interval.

I Not available.

TABLE 75. Percentage of high school students who used an IUD* or implant ${ }^{\dagger}$ before last sexual intercourse ${ }^{\S}$ and who used a shot, ${ }^{\natural}$ patch, ${ }^{* *}$ or birth control ring ${ }^{\dagger \dagger}$ before last sexual intercourse, $\$$ by sex, race/ethnicity, and grade - United States, Youth Risk Behavior Survey, 2015

\begin{tabular}{|c|c|c|c|c|c|c|c|c|c|c|c|c|}
\hline \multirow[b]{3}{*}{ Category } & \multicolumn{6}{|c|}{ IUD or implant use } & \multicolumn{6}{|c|}{ Shot, patch, or birth control ring use } \\
\hline & \multicolumn{2}{|c|}{ Female } & \multicolumn{2}{|c|}{ Male } & \multicolumn{2}{|c|}{ Total } & \multicolumn{2}{|c|}{ Female } & \multicolumn{2}{|c|}{ Male } & \multicolumn{2}{|c|}{ Total } \\
\hline & $\%$ & $\mathrm{Cl}^{\S \S}$ & $\%$ & $\mathrm{Cl}$ & $\%$ & $\mathrm{Cl}$ & $\%$ & $\mathrm{Cl}$ & $\%$ & $\mathrm{Cl}$ & $\%$ & $\mathrm{Cl}$ \\
\hline \multicolumn{13}{|c|}{ Race/Ethnicity } \\
\hline White & 4.8 & $(3.0-7.5)$ & 2.9 & $(1.8-4.4)$ & 3.9 & $(2.8-5.4)$ & 8.9 & $(6.7-11.9)$ & 2.7 & $(1.8-4.2)$ & 6.0 & $(4.6-7.7)$ \\
\hline Black ๆๆ & 3.7 & $(1.8-7.3)$ & 1.1 & $(0.1-7.1)$ & 2.1 & $(1.0-4.4)$ & 7.8 & $(5.2-11.6)$ & 3.1 & $(1.4-6.9)$ & 4.9 & $(3.0-7.8)$ \\
\hline Hispanic & 4.0 & $(2.2-7.3)$ & 1.7 & $(0.7-4.1)$ & 2.9 & $(1.8-4.5)$ & 3.7 & $(2.4-5.7)$ & 2.5 & $(1.4-4.3)$ & 3.1 & $(2.0-4.8)$ \\
\hline \multicolumn{13}{|l|}{ Grade } \\
\hline 9 & 3.8 & $(1.2-11.8)$ & 0.8 & $(0.1-6.1)$ & 2.1 & $(0.8-5.3)$ & 5.8 & $(3.1-10.7)$ & 1.4 & $(0.5-4.4)$ & 3.3 & $(1.9-5.7)$ \\
\hline 10 & 4.1 & $(1.9-8.8)$ & 1.5 & $(0.6-3.6)$ & 2.8 & $(1.5-5.1)$ & 9.3 & $(6.4-13.3)$ & 2.4 & $(1.1-5.2)$ & 5.8 & $(4.1-8.0)$ \\
\hline 11 & 4.9 & (3.1-7.8) & 2.9 & $(1.4-5.7)$ & 3.9 & $(2.7-5.7)$ & 7.3 & $(4.5-11.6)$ & 3.5 & $(2.1-6.0)$ & 5.5 & $(3.7-8.0)$ \\
\hline 12 & 4.7 & $(2.9-7.7)$ & 2.8 & $(1.6-4.9)$ & 3.8 & $(2.5-5.6)$ & 8.3 & $(5.5-12.3)$ & 3.0 & $(1.8-5.1)$ & 5.7 & $(4.1-7.9)$ \\
\hline Total & 4.5 & (3.2-6.4) & 2.2 & $(1.5-3.2)$ & 3.3 & $(2.5-4.4)$ & 7.9 & $(6.1-10.1)$ & 2.7 & $(2.0-3.7)$ & 5.3 & $(4.3-6.6)$ \\
\hline
\end{tabular}

* Such as Mirena or ParaGard.

+ Such as Implanon or Nexplanon.

$\S$ To prevent pregnancy, among the $30.1 \%$ of students nationwide who were currently sexually active.

I Such as Depo-Provera.

** Such as OrthoEvra.

t+ Such as NuvaRing.

$\S \S 95 \%$ confidence interval.

ๆา Non-Hispanic. 
TABLE 76. Percentage of high school students who used an IUD* or implant ${ }^{\dagger}$ before last sexual intercourse ${ }^{\S}$ and who used a shot, ${ }^{\text {, }}$ patch, ${ }^{* *}$ or birth control ring ${ }^{\dagger+}$ before last sexual intercourse, ${ }^{\S}$ by sex — selected U.S. sites, Youth Risk Behavior Survey, 2015

\begin{tabular}{|c|c|c|c|c|c|c|c|c|c|c|c|c|}
\hline \multirow[b]{3}{*}{ Site } & \multicolumn{6}{|c|}{ IUD or implant use } & \multicolumn{6}{|c|}{ Shot, patch, or birth control ring use } \\
\hline & \multicolumn{2}{|c|}{ Female } & \multicolumn{2}{|c|}{ Male } & \multicolumn{2}{|c|}{ Total } & \multicolumn{2}{|c|}{ Female } & \multicolumn{2}{|c|}{ Male } & \multicolumn{2}{|r|}{ Total } \\
\hline & $\%$ & $\mathrm{Cl} \S$ & $\%$ & $\mathrm{Cl}$ & $\%$ & $\mathrm{Cl}$ & $\%$ & $\mathrm{Cl}$ & $\%$ & $\mathrm{Cl}$ & $\%$ & $\mathrm{Cl}$ \\
\hline \multicolumn{13}{|l|}{ State surveys } \\
\hline Alabama & 2.9 & $(1.2-6.5)$ & 1.2 & $(0.3-5.2)$ & 2.1 & $(1.0-4.2)$ & 11.9 & $(6.3-21.2)$ & 4.3 & $(1.5-11.7)$ & 8.7 & $(4.9-15.1)$ \\
\hline Alaska & 12.0 & $(8.0-17.7)$ & 6.7 & $(2.9-14.7)$ & 9.7 & $(6.6-14.2)$ & 4.8 & (2.4-9.3) & 8.4 & $(5.0-13.8)$ & 6.5 & (4.7-8.9) \\
\hline Arizona & 5.4 & $(2.0-13.8)$ & 3.2 & $(0.9-10.5)$ & 4.3 & $(1.9-9.4)$ & 3.5 & $(1.7-6.9)$ & 1.3 & $(0.4-4.4)$ & 2.4 & $(1.4-4.3)$ \\
\hline Arkansas & 2.4 & $(0.9-6.2)$ & 0.2 & $(0.1-1.0)$ & 1.4 & $(0.6-3.4)$ & 9.9 & $(5.4-17.6)$ & 7.7 & $(3.7-15.2)$ & 8.9 & $(5.8-13.4)$ \\
\hline California & 5.3 & $(2.4-11.3)$ & 1.1 & $(0.2-6.0)$ & 3.1 & $(1.4-6.6)$ & 5.6 & $(2.8-10.7)$ & 3.7 & $(1.1-11.1)$ & 4.6 & $(3.0-7.1)$ \\
\hline Connecticut & 3.8 & $(2.0-7.4)$ & 4.5 & $(2.2-9.0)$ & 4.1 & $(2.4-6.9)$ & 1.2 & $(0.3-5.3)$ & 2.1 & $(0.8-5.6)$ & 1.6 & $(0.8-3.3)$ \\
\hline Delaware & 4.3 & $(2.4-7.5)$ & 2.5 & $(1.2-5.0)$ & 3.3 & $(2.1-5.2)$ & 7.7 & $(4.8-12.3)$ & 4.5 & $(2.5-8.1)$ & 6.0 & $(4.0-9.0)$ \\
\hline Florida & 2.0 & $(1.1-3.5)$ & 0.6 & $(0.3-1.6)$ & 1.3 & $(0.8-2.2)$ & 3.8 & $(2.0-7.1)$ & 1.4 & $(0.7-2.8)$ & 2.5 & $(1.6-4.0)$ \\
\hline Hawaii & 6.1 & $(3.1-11.5)$ & 2.3 & $(1.3-4.2)$ & 4.6 & $(2.8-7.4)$ & 6.8 & $(4.3-10.5)$ & 3.6 & $(1.8-7.1)$ & 5.5 & $(4.0-7.5)$ \\
\hline Idaho & - & - & - & - & - & - & - & - & - & - & - & - \\
\hline Illinois & 4.8 & $(2.7-8.6)$ & 1.5 & $(0.6-3.8)$ & 3.1 & $(1.7-5.7)$ & 5.8 & $(2.9-11.3)$ & 2.2 & $(0.9-5.2)$ & 4.0 & $(2.5-6.3)$ \\
\hline Indiana & 5.0 & $(2.4-10.0)$ & 2.7 & $(0.8-8.4)$ & 3.9 & $(1.7-8.4)$ & 5.8 & $(2.5-13.2)$ & 5.4 & $(2.0-13.9)$ & 5.6 & $(2.7-11.3)$ \\
\hline Kentucky & 7.8 & $(3.9-15.1)$ & 2.6 & $(1.0-6.6)$ & 5.2 & $(2.6-10.0)$ & 10.5 & $(6.4-16.9)$ & 1.7 & $(0.9-3.0)$ & 6.1 & $(3.8-9.6)$ \\
\hline Maine & 6.4 & $(4.1-9.9)$ & 3.4 & $(2.1-5.3)$ & 5.0 & $(3.3-7.6)$ & 8.1 & $(6.3-10.3)$ & 4.2 & $(2.9-6.1)$ & 6.3 & $(5.0-8.0)$ \\
\hline Maryland & 2.6 & $(2.1-3.2)$ & 1.3 & $(1.0-1.8)$ & 2.0 & $(1.7-2.3)$ & 6.0 & $(5.2-7.0)$ & 2.0 & $(1.6-2.5)$ & 4.0 & $(3.6-4.6)$ \\
\hline Massachusetts & 6.0 & $(3.6-10.1)$ & 0.6 & $(0.2-2.6)$ & 3.4 & $(2.1-5.4)$ & 4.7 & $(2.7-7.9)$ & 2.9 & $(1.4-6.1)$ & 3.8 & $(2.5-5.8)$ \\
\hline Michigan & 3.7 & $(1.0-12.1)$ & 0.7 & $(0.1-4.3)$ & 2.3 & $(0.8-6.9)$ & 12.4 & $(7.1-20.6)$ & 2.6 & $(1.0-6.4)$ & 7.9 & $(4.9-12.5)$ \\
\hline Mississippi & 4.8 & $(2.6-8.8)$ & 3.1 & $(1.2-7.9)$ & 4.0 & $(2.4-6.7)$ & 10.9 & $(6.1-18.8)$ & 3.0 & $(1.1-7.7)$ & 7.2 & $(4.2-12.0)$ \\
\hline Missouri & 4.9 & $(2.5-9.2)$ & 4.1 & $(2.1-7.8)$ & 4.6 & $(2.9-7.2)$ & 8.7 & $(5.7-13.2)$ & 2.5 & $(1.1-5.7)$ & 5.6 & $(3.7-8.4)$ \\
\hline Montana & 5.6 & $(3.6-8.5)$ & 2.5 & $(1.4-4.5)$ & 4.2 & $(2.9-6.0)$ & 9.8 & $(7.8-12.4)$ & 2.8 & $(1.6-5.1)$ & 6.6 & (5.3-8.2) \\
\hline Nebraska & 5.0 & $(2.3-10.2)$ & 0.6 & $(0.1-4.3)$ & 3.3 & $(1.7-6.4)$ & 4.3 & $(2.4-7.6)$ & 2.9 & $(1.1-7.5)$ & 3.6 & $(2.2-5.8)$ \\
\hline Nevada & 2.7 & $(0.6-11.1)$ & 1.2 & $(0.3-4.6)$ & 1.9 & $(0.6-5.9)$ & 5.0 & $(2.3-10.4)$ & 1.1 & $(0.3-5.0)$ & 3.1 & $(1.5-6.3)$ \\
\hline New Hampshire & 6.6 & $(5.4-8.0)$ & 3.1 & $(2.5-4.0)$ & 4.9 & $(4.2-5.7)$ & 5.5 & $(4.2-7.0)$ & 2.4 & $(1.7-3.2)$ & 3.9 & $(3.2-4.8)$ \\
\hline New Mexico & 7.8 & $(6.0-10.2)$ & 5.1 & $(3.8-7.0)$ & 6.5 & $(5.3-7.9)$ & 9.0 & $(7.0-11.6)$ & 4.6 & $(3.2-6.7)$ & 7.0 & $(5.5-8.8)$ \\
\hline New York & 4.7 & $(2.1-10.5)$ & 3.4 & $(1.3-8.5)$ & 4.1 & $(2.1-7.9)$ & 5.4 & $(3.6-8.1)$ & 2.1 & $(1.1-3.8)$ & 3.9 & $(2.6-5.8)$ \\
\hline North Carolina & 1.9 & $(1.0-3.5)$ & 1.1 & $(0.3-4.8)$ & 1.5 & $(0.8-3.0)$ & 8.2 & $(5.7-11.5)$ & 3.6 & $(2.2-5.9)$ & 5.9 & (4.5-7.7) \\
\hline North Dakota & - & - & - & - & - & - & - & - & - & - & - & - \\
\hline Oklahoma & 3.8 & $(1.4-9.6)$ & 2.7 & $(1.0-6.8)$ & 3.3 & $(1.4-7.3)$ & 7.3 & $(4.5-11.6)$ & 5.3 & $(2.2-12.4)$ & 6.3 & $(3.9-10.1)$ \\
\hline Pennsylvania & 1.7 & $(0.8-3.7)$ & 1.7 & $(0.5-5.6)$ & 1.7 & $(0.7-4.0)$ & 4.4 & $(2.3-8.4)$ & 3.0 & $(1.6-5.4)$ & 3.7 & $(2.3-5.7)$ \\
\hline Rhode Island & 3.7 & $(1.6-8.1)$ & 0.6 & $(0.1-2.5)$ & 2.3 & $(1.0-5.1)$ & 5.5 & $(2.3-12.5)$ & 2.2 & $(0.8-6.1)$ & 4.0 & $(2.2-7.2)$ \\
\hline South Carolina & 6.5 & $(1.7-22.3)$ & 2.4 & $(0.7-7.7)$ & 4.4 & $(1.6-12.0)$ & 10.1 & $(5.4-18.0)$ & 3.3 & $(1.1-9.6)$ & 6.7 & $(4.3-10.3)$ \\
\hline South Dakota & 3.1 & $(1.3-7.2)$ & 2.2 & $(0.7-6.8)$ & 2.6 & $(1.2-5.5)$ & 5.3 & $(2.8-9.8)$ & 1.1 & $(0.2-5.3)$ & 3.0 & $(1.8-5.2)$ \\
\hline Tennessee & - & - & - & - & - & - & - & - & - & - & - & - \\
\hline Vermont & 8.4 & $(7.5-9.5)$ & 3.8 & $(3.1-4.6)$ & 6.2 & $(5.6-6.9)$ & 8.5 & $(7.5-9.5)$ & 4.1 & $(3.4-5.0)$ & 6.4 & $(5.8-7.1)$ \\
\hline Virginia & - & - & - & - & - & - & - & - & - & - & - & - \\
\hline West Virginia & 3.1 & $(1.3-7.0)$ & 3.9 & $(2.5-6.0)$ & 3.4 & $(2.2-5.4)$ & 7.2 & $(4.2-12.0)$ & 2.2 & $(0.8-6.0)$ & 5.0 & $(3.0-8.3)$ \\
\hline Wyoming & 6.5 & $(3.6-11.4)$ & 2.5 & $(1.2-4.9)$ & 4.6 & $(2.9-7.3)$ & 11.3 & $(7.0-17.6)$ & 7.5 & $(4.6-12.1)$ & 9.5 & $(6.4-13.8)$ \\
\hline Median & & 4.8 & & 2.5 & & 3.4 & & 6.8 & & 2.9 & & 5.6 \\
\hline Range & & 7-12.0) & & $.2-6.7)$ & & .3-9.7) & & $2-12.4)$ & & $1-8.4)$ & & $1.6-9.5)$ \\
\hline \multicolumn{13}{|c|}{ Large urban school district surveys } \\
\hline Baltimore, MD & 6.4 & $(2.4-15.7)$ & 0.0 & - & 2.8 & $(1.1-7.0)$ & 7.2 & $(3.9-12.8)$ & 9.1 & $(4.9-16.4)$ & 8.2 & $(5.5-12.0)$ \\
\hline Boston, MA & 9.7 & $(5.5-16.4)$ & 1.7 & $(0.6-4.6)$ & 5.7 & $(3.6-9.0)$ & 11.0 & $(6.4-18.5)$ & 2.6 & $(1.0-6.3)$ & 6.8 & $(4.0-11.3)$ \\
\hline Broward County, FL & 0.8 & $(0.1-5.7)$ & 0.0 & - & 0.6 & $(0.1-2.6)$ & 2.2 & $(0.7-7.3)$ & 0.0 & - & 1.1 & $(0.3-3.7)$ \\
\hline Cleveland, $\mathrm{OH}$ & 4.6 & $(2.3-9.0)$ & 1.3 & $(0.3-6.2)$ & 3.0 & $(1.6-5.6)$ & 16.0 & $(10.5-23.8)$ & 5.6 & $(3.2-9.7)$ & 10.5 & $(7.3-14.8)$ \\
\hline DeKalb County, GA & 3.9 & $(1.7-8.8)$ & 0.0 & - & 1.9 & $(0.8-4.4)$ & 6.0 & $(3.1-11.4)$ & 2.5 & $(1.0-6.2)$ & 4.2 & $(2.4-7.3)$ \\
\hline Detroit, MI & 2.5 & $(0.9-6.9)$ & 1.2 & $(0.2-8.4)$ & 1.8 & $(0.5-6.2)$ & 3.8 & $(1.7-8.0)$ & 0.0 & - & 2.1 & $(1.0-4.2)$ \\
\hline District of Columbia & 5.2 & $(4.0-6.9)$ & 1.7 & $(1.1-2.7)$ & 3.5 & $(2.7-4.4)$ & 9.5 & $(7.8-11.5)$ & 2.1 & $(1.4-3.2)$ & 5.7 & $(4.8-6.9)$ \\
\hline Duval County, FL & 2.9 & $(1.3-6.2)$ & 1.2 & $(0.3-4.3)$ & 2.3 & $(1.2-4.2)$ & 5.4 & $(3.1-9.2)$ & 2.7 & $(1.2-6.2)$ & 4.4 & $(2.8-6.7)$ \\
\hline Ft. Worth, TX & 2.0 & $(0.9-4.4)$ & 0.5 & $(0.1-1.8)$ & 1.2 & $(0.6-2.4)$ & 4.0 & $(2.0-8.0)$ & 1.1 & $(0.4-3.4)$ & 2.5 & $(1.4-4.5)$ \\
\hline Houston, TX & 3.4 & $(1.7-6.5)$ & 1.2 & $(0.4-3.2)$ & 2.2 & $(1.2-3.9)$ & 8.0 & $(5.3-12.0)$ & 1.2 & $(0.4-3.0)$ & 4.3 & $(3.1-6.1)$ \\
\hline Los Angeles, CA & 6.0 & $(2.7-12.7)$ & 0.9 & $(0.2-3.4)$ & 3.3 & $(1.5-6.9)$ & 6.7 & $(3.1-13.6)$ & 3.7 & $(1.5-8.8)$ & 5.5 & $(3.3-9.0)$ \\
\hline Miami-Dade County, FL & 0.4 & $(0.1-2.3)$ & 0.6 & $(0.2-1.9)$ & 0.5 & $(0.2-1.3)$ & 2.6 & $(1.2-5.5)$ & 1.4 & $(0.5-3.8)$ & 2.0 & $(1.0-4.0)$ \\
\hline
\end{tabular}

See table footnotes on the next page. 
TABLE 76. (Continued) Percentage of high school students who used an IUD* or implant ${ }^{\dagger}$ before last sexual intercourse ${ }^{\S}$ and who used a shot, patch, ${ }^{* *}$ or birth control ring ${ }^{\dagger \dagger}$ before last sexual intercourse, ${ }^{\S}$ by sex - selected U.S. sites, Youth Risk Behavior Survey, 2015

\begin{tabular}{|c|c|c|c|c|c|c|c|c|c|c|c|c|}
\hline \multirow[b]{3}{*}{ Site } & \multicolumn{6}{|c|}{ IUD or implant use } & \multicolumn{6}{|c|}{ Shot, patch, or birth control ring use } \\
\hline & \multicolumn{2}{|c|}{ Female } & \multicolumn{2}{|c|}{ Male } & \multicolumn{2}{|r|}{ Total } & \multicolumn{2}{|c|}{ Female } & \multicolumn{2}{|c|}{ Male } & \multicolumn{2}{|r|}{ Total } \\
\hline & $\%$ & $\mathrm{Cl} \S$ & $\%$ & $\mathrm{Cl}$ & $\%$ & $\mathrm{Cl}$ & $\%$ & $\mathrm{Cl}$ & $\%$ & $\mathrm{Cl}$ & $\%$ & $\mathrm{Cl}$ \\
\hline New York City, NY & 2.3 & $(1.2-4.5)$ & 0.9 & $(0.4-2.1)$ & 1.7 & $(1.0-2.8)$ & 5.6 & (3.4-9.0) & 4.0 & $(2.1-7.4)$ & 4.9 & $(3.2-7.3)$ \\
\hline Oakland, CA & - & - & - & - & - & - & - & - & - & - & - & - \\
\hline Orange County, FL & 2.8 & $(1.0-7.3)$ & 1.9 & $(0.5-6.4)$ & 2.5 & $(1.2-5.1)$ & 2.7 & $(1.1-7.0)$ & 0.0 & - & 1.3 & $(0.5-3.4)$ \\
\hline Palm Beach County, FL & 1.5 & $(0.5-4.6)$ & 0.0 & - & 0.7 & $(0.2-2.3)$ & 3.0 & $(1.2-7.2)$ & 1.5 & $(0.6-3.9)$ & 2.2 & $(1.1-4.3)$ \\
\hline Philadelphia, PA & 2.7 & $(0.9-7.4)$ & 1.3 & $(0.6-2.6)$ & 2.0 & $(0.9-4.2)$ & 12.0 & $(8.1-17.6)$ & 3.7 & $(1.6-8.0)$ & 8.0 & $(5.4-11.6)$ \\
\hline San Diego, CA & 2.3 & $(1.0-5.0)$ & 1.4 & $(0.3-6.0)$ & 1.8 & $(0.8-3.9)$ & 6.2 & (3.9-9.6) & 3.2 & $(1.2-8.1)$ & 4.5 & $(2.9-7.1)$ \\
\hline San Francisco, CA & 22.1 & $(15.2-31.1)$ & 11.0 & $(4.5-24.4)$ & 16.9 & $(11.1-24.9)$ & 14.4 & $(9.0-22.2)$ & 2.7 & $(0.7-9.6)$ & 8.9 & $(6.0-13.0)$ \\
\hline Median & \multicolumn{2}{|c|}{2.8} & \multicolumn{2}{|c|}{1.2} & \multicolumn{2}{|r|}{2.1} & \multicolumn{2}{|c|}{6.1} & \multicolumn{2}{|c|}{2.5} & \multicolumn{2}{|c|}{4.4} \\
\hline Range & \multicolumn{2}{|c|}{$(0.4-22.1)$} & \multicolumn{2}{|c|}{$(0.0-11.0)$} & \multicolumn{2}{|c|}{$(0.5-16.9)$} & \multicolumn{2}{|c|}{$(2.2-16.0)$} & \multicolumn{2}{|c|}{$(0.0-9.1)$} & \multicolumn{2}{|c|}{$(1.1-10.5)$} \\
\hline
\end{tabular}

* Such as Mirena or ParaGard.

† Such as Implanon or Nexplanon.

$\S$ To prevent pregnancy, among students who were currently sexually active.

I Such as Depo-Provera.

** Such as OrthoEvra.

t+ Such as NuvaRing.

$\S \S 95 \%$ confidence interval.

१ๆ Not available.

TABLE 77. Percentage of high school students who used birth control pills, an IUD* or implant, ${ }^{\dagger}$ or a shot, ${ }^{\S}$ patch, ${ }^{\mathbb{9}}$ or birth control ring** before last sexual intercourse ${ }^{\dagger \dagger}$ and who used both a condom during last sexual intercourse and birth control pills, an IUD* or implant, ${ }^{\dagger}$ or a shot, ${ }^{\S}$ patch, or birth control ring ${ }^{* *}$ before last sexual intercourse, ${ }^{\dagger \dagger}$ by sex, race/ethnicity, and grade — United States, Youth Risk Behavior Survey, 2015

\begin{tabular}{|c|c|c|c|c|c|c|c|c|c|c|c|c|}
\hline \multirow[b]{3}{*}{ Category } & \multicolumn{6}{|c|}{$\begin{array}{l}\text { Birth control pill use, IUD or implant, or shot, patch, or } \\
\text { birth control ring use }\end{array}$} & \multicolumn{6}{|c|}{$\begin{array}{l}\text { Condom use and birth control pill, IUD or implant, or } \\
\text { shot, patch, or birth control ring use }\end{array}$} \\
\hline & \multicolumn{2}{|c|}{ Female } & \multicolumn{2}{|r|}{ Male } & \multicolumn{2}{|c|}{ Total } & \multicolumn{2}{|c|}{ Female } & \multicolumn{2}{|c|}{ Male } & \multicolumn{2}{|c|}{ Total } \\
\hline & $\%$ & $\mathrm{Cl}^{\S \S}$ & $\%$ & $\mathrm{Cl}$ & $\%$ & $\mathrm{Cl}$ & $\%$ & $\mathrm{Cl}$ & $\%$ & $\mathrm{Cl}$ & $\%$ & $\mathrm{Cl}$ \\
\hline \multicolumn{13}{|c|}{ Race/Ethnicity } \\
\hline White & 39.2 & $(34.3-44.3)$ & 27.0 & $(23.3-30.9)$ & 33.3 & $(29.9-36.9)$ & 15.9 & $(12.5-20.1)$ & 7.7 & $(5.5-10.7)$ & 12.0 & $(9.7-14.7)$ \\
\hline Black ๆา & 20.5 & $(14.8-27.7)$ & 13.1 & $(9.2-18.5)$ & 15.9 & (12.4-20.3) & 5.7 & (3.4-9.4) & 4.1 & $(2.2-7.6)$ & 4.7 & $(3.1-7.0)$ \\
\hline Hispanic & 23.2 & (18.0-29.4) & 12.3 & $(8.7-17.3)$ & 17.8 & (13.9-22.5) & 4.8 & $(3.3-7.1)$ & 4.5 & $(2.7-7.2)$ & 4.7 & (3.4-6.5) \\
\hline \multicolumn{13}{|l|}{ Grade } \\
\hline 9 & 20.8 & $(15.0-28.1)$ & 13.1 & $(9.1-18.4)$ & 16.4 & $(12.7-20.9)$ & 9.4 & $(5.1-16.4)$ & 3.2 & $(1.4-7.2)$ & 5.8 & $(3.7-9.2)$ \\
\hline 10 & 33.6 & $(28.1-39.5)$ & 15.5 & (11.4-20.8) & 24.4 & (20.9-28.4) & 12.4 & $(8.9-17.1)$ & 4.3 & $(2.7-7.0)$ & 8.3 & $(6.0-11.3)$ \\
\hline 11 & 36.2 & (31.4-41.2) & 25.5 & $(20.5-31.2)$ & 30.9 & $(27.1-35.0)$ & 14.8 & (10.9-19.8) & 9.4 & $(5.7-15.3)$ & 12.2 & (9.5-15.6) \\
\hline 12 & 36.2 & $(30.2-42.7)$ & 22.7 & $(18.2-27.8)$ & 29.6 & $(25.5-33.9)$ & 9.9 & $(7.0-13.9)$ & 5.5 & $(3.8-7.9)$ & 7.7 & $(6.1-9.8)$ \\
\hline Total & 33.7 & $(30.3-37.4)$ & 20.2 & $(17.7-22.9)$ & 26.8 & (24.3-29.6) & 11.8 & $(9.6-14.5)$ & 5.9 & $(4.5-7.7)$ & 8.8 & $(7.3-10.6)$ \\
\hline
\end{tabular}

\footnotetext{
* Such as Mirena or ParaGard.

† Such as Implanon or Nexplanon.

§ Such as Depo-Provera.

I Such as OrthoEvra.

** Such as NuvaRing.

${ }^{t+}$ To prevent pregnancy, among the $30.1 \%$ of students nationwide who were currently sexually active.

$\S \S 95 \%$ confidence interval.

१ต Non-Hispanic.
} 
TABLE 78. Percentage of high school students who used birth control pills, an IUD* or implant, ${ }^{\dagger}$ or a shot, ${ }^{\S}$ patch, ${ }^{,}$or birth control ring ${ }^{* *}$ before last sexual intercourse ${ }^{\dagger \dagger}$ and who used both a condom during last sexual intercourse and birth control pills, an IUD* or implant, ${ }^{\dagger}$ or a shot, ${ }^{\S}$ patch, ${ }^{\text {ी }}$ or birth control ring ${ }^{* *}$ before last sexual intercourse, ${ }^{\dagger+}$ by sex — selected U.S. sites, Youth Risk Behavior Survey, 2015

\begin{tabular}{|c|c|c|c|c|c|c|c|c|c|c|c|c|}
\hline \multirow[b]{3}{*}{ Site } & \multicolumn{6}{|c|}{$\begin{array}{l}\text { Birth control pill use, IUD or implant, or shot, patch, or } \\
\text { birth control ring use }\end{array}$} & \multicolumn{6}{|c|}{$\begin{array}{l}\text { Condom use and birth control pill, IUD or implant, or } \\
\text { shot, patch, or birth control ring use }\end{array}$} \\
\hline & \multicolumn{2}{|c|}{ Female } & \multicolumn{2}{|r|}{ Male } & \multicolumn{2}{|r|}{ Total } & \multicolumn{2}{|c|}{ Female } & \multicolumn{2}{|r|}{ Male } & \multicolumn{2}{|r|}{ Total } \\
\hline & $\%$ & $\mathrm{Cl}^{\S \S}$ & $\%$ & $\mathrm{Cl}$ & $\%$ & $\mathrm{Cl}$ & $\%$ & $\mathrm{Cl}$ & $\%$ & $\mathrm{Cl}$ & $\%$ & $\mathrm{Cl}$ \\
\hline \multicolumn{13}{|l|}{ State surveys } \\
\hline Alabama & 39.0 & (30.6-48.2) & 18.4 & $(11.8-27.6)$ & 29.6 & $(23.1-37.0)$ & 9.9 & $(5.3-17.7)$ & 6.3 & (3.5-11.2) & 8.6 & $(5.5-13.3)$ \\
\hline Alaska & 40.2 & (32.2-48.8) & 29.5 & $(23.5-36.3)$ & 35.1 & $(30.4-40.1)$ & 17.6 & $(12.0-25.2)$ & 14.2 & (9.9-19.8) & 16.2 & (12.4-20.9) \\
\hline Arizona & 28.9 & (19.9-40.0) & 25.6 & $(16.7-37.0)$ & 27.4 & $(19.3-37.4)$ & 7.9 & $(3.8-15.8)$ & 6.4 & $(3.0-13.1)$ & 7.4 & $(4.4-12.3)$ \\
\hline Arkansas & 32.5 & $(24.6-41.6)$ & 22.9 & $(15.8-32.0)$ & 28.1 & $(25.2-31.2)$ & 11.4 & $(7.9-16.2)$ & 10.1 & $(4.6-20.5)$ & 10.8 & $(7.9-14.7)$ \\
\hline California & 25.1 & $(18.4-33.3)$ & 16.9 & $(10.8-25.6)$ & 20.7 & $(17.9-23.8)$ & 7.1 & $(3.9-12.6)$ & 4.6 & $(2.0-10.1)$ & 5.8 & (3.4-9.7) \\
\hline Connecticut & 35.9 & (27.8-44.8) & 28.5 & (20.3-38.5) & 32.5 & $(26.6-39.0)$ & 12.1 & $(8.2-17.6)$ & 9.8 & $(6.3-14.9)$ & 11.1 & $(7.9-15.3)$ \\
\hline Delaware & 35.1 & (29.8-40.8) & 19.5 & $(14.9-25.1)$ & 27.4 & $(23.6-31.5)$ & 9.3 & $(6.2-13.6)$ & 6.4 & (3.9-10.3) & 8.0 & (5.9-10.6) \\
\hline Florida & 21.6 & (17.9-25.9) & 13.5 & (11.4-15.8) & 17.5 & (15.3-19.9) & 9.2 & $(7.0-12.0)$ & 5.9 & $(4.4-7.9)$ & 7.5 & $(6.0-9.3)$ \\
\hline Hawaii & 31.4 & $(26.2-37.2)$ & 19.8 & (14.4-26.5) & 26.8 & $(23.2-30.7)$ & 7.3 & $(4.9-10.8)$ & 6.5 & (3.7-11.4) & 7.0 & $(5.5-9.0)$ \\
\hline Idaho & —าा & - & - & - & - & - & - & - & - & - & - & - \\
\hline Illinois & 41.5 & $(31.7-52.1)$ & 21.1 & $(14.8-29.1)$ & 31.1 & $(24.1-39.2)$ & 16.5 & $(9.9-26.3)$ & 6.9 & (3.8-12.2) & 11.6 & (7.5-17.5) \\
\hline Indiana & 37.1 & (27.1-48.4) & 21.8 & (14.9-30.7) & 29.7 & (21.9-38.8) & 13.9 & $(9.0-20.7)$ & 4.6 & (1.9-10.8) & 9.4 & $(6.0-14.6)$ \\
\hline Kentucky & 44.4 & (35.3-53.9) & 23.0 & $(17.7-29.2)$ & 33.6 & $(28.0-39.7)$ & 14.1 & $(9.9-19.7)$ & 9.1 & $(4.9-16.2)$ & 11.6 & $(8.5-15.7)$ \\
\hline Maine & 50.0 & $(46.4-53.7)$ & 36.5 & (32.3-40.8) & 43.8 & $(40.6-47.0)$ & 18.3 & (16.0-20.8) & 14.7 & $(12.2-17.6)$ & 16.7 & $(14.9-18.8)$ \\
\hline Maryland & 30.4 & $(29.1-31.9)$ & 16.6 & (15.5-17.9) & 23.7 & $(22.7-24.8)$ & 11.6 & $(10.6-12.6)$ & 5.8 & $(5.1-6.5)$ & 8.7 & $(8.1-9.4)$ \\
\hline Massachusetts & 42.3 & (36.2-48.5) & 28.8 & $(24.1-34.0)$ & 35.6 & (31.6-39.8) & 14.8 & $(11.1-19.6)$ & 11.2 & $(8.6-14.4)$ & 13.0 & $(11.0-15.3)$ \\
\hline Michigan & 38.7 & (30.1-48.0) & 27.2 & (17.3-40.0) & 33.5 & (26.8-40.9) & 13.3 & $(8.3-20.4)$ & 10.0 & $(4.6-20.6)$ & 11.8 & $(8.2-16.9)$ \\
\hline Mississippi & 35.7 & $(27.5-44.7)$ & 22.5 & (17.5-28.5) & 29.5 & $(24.9-34.5)$ & 13.2 & $(9.2-18.6)$ & 6.7 & $(3.7-12.1)$ & 10.1 & (7.4-13.7) \\
\hline Missouri & 40.7 & $(32.0-50.0)$ & 25.4 & $(16.3-37.3)$ & 33.0 & $(26.7-40.1)$ & 14.7 & (10.2-20.7) & 10.9 & $(6.2-18.5)$ & 12.7 & $(9.2-17.4)$ \\
\hline Montana & 44.3 & $(40.8-47.9)$ & 30.3 & $(26.6-34.3)$ & 37.9 & (35.0-40.8) & 17.6 & $(14.5-21.2)$ & 12.4 & $(9.7-15.8)$ & 15.2 & $(13.1-17.6)$ \\
\hline Nebraska & 33.8 & $(25.6-43.2)$ & 20.0 & (13.9-27.9) & 27.6 & $(22.5-33.2)$ & 11.3 & $(7.4-17.0)$ & 5.6 & $(2.9-10.8)$ & 8.6 & $(6.0-12.2)$ \\
\hline Nevada & 31.5 & (19.4-46.7) & 19.1 & $(11.6-29.7)$ & 25.4 & $(17.1-36.0)$ & 6.8 & $(3.4-13.2)$ & 5.2 & $(2.3-11.2)$ & 6.0 & $(3.5-10.1)$ \\
\hline New Hampshire & 50.2 & $(46.4-54.0)$ & 32.6 & $(30.1-35.2)$ & 41.6 & $(39.2-44.0)$ & 21.2 & $(18.4-24.3)$ & 13.5 & $(11.5-15.8)$ & 17.2 & $(15.5-19.1)$ \\
\hline New Mexico & 33.5 & $(29.8-37.5)$ & 22.8 & (19.3-26.8) & 28.4 & $(25.8-31.2)$ & 10.0 & $(7.9-12.6)$ & 7.8 & $(5.7-10.7)$ & 9.0 & (7.5-10.8) \\
\hline New York & 35.1 & $(28.8-42.0)$ & 24.2 & $(19.1-30.1)$ & 30.1 & $(26.2-34.2)$ & 13.8 & $(9.6-19.3)$ & 9.6 & $(6.1-14.7)$ & 11.9 & $(9.0-15.6)$ \\
\hline North Carolina & 28.9 & $(23.3-35.3)$ & 23.8 & $(19.6-28.5)$ & 26.3 & (23.2-29.6) & 11.6 & $(7.6-17.2)$ & 10.2 & (6.5-15.5) & 10.8 & $(8.6-13.6)$ \\
\hline North Dakota & - & - & - & - & - & - & - & - & - & - & - & - \\
\hline Oklahoma & 27.4 & $(20.7-35.3)$ & 21.4 & (14.8-29.9) & 24.5 & $(19.1-30.8)$ & 6.9 & $(3.9-12.0)$ & 6.7 & $(3.5-12.6)$ & 6.8 & $(4.7-9.9)$ \\
\hline Pennsylvania & 30.7 & $(25.3-36.5)$ & 19.9 & $(14.2-27.1)$ & 25.3 & $(20.7-30.5)$ & 8.4 & $(5.4-12.9)$ & 7.8 & $(4.7-12.5)$ & 8.1 & $(5.6-11.6)$ \\
\hline Rhode Island & 34.8 & $(30.3-39.5)$ & 31.6 & $(19.9-46.2)$ & 33.3 & (26.4-40.9) & 13.6 & $(8.9-20.3)$ & 13.2 & $(9.6-17.8)$ & 13.4 & $(10.7-16.6)$ \\
\hline South Carolina & 39.2 & (33.1-45.6) & 24.0 & $(15.7-34.7)$ & 31.4 & $(25.7-37.7)$ & 16.6 & (11.4-23.6) & 11.8 & $(7.5-18.0)$ & 14.1 & $(10.3-19.1)$ \\
\hline South Dakota & 38.2 & (29.3-47.9) & 28.6 & (18.0-42.3) & 33.0 & (25.7-41.3) & 18.5 & $(14.3-23.6)$ & 12.7 & $(4.5-31.0)$ & 15.3 & $(9.4-24.0)$ \\
\hline Tennessee & - & - & - & - & - & - & - & - & - & - & - & - \\
\hline Vermont & 55.4 & $(53.7-57.2)$ & 38.2 & (36.3-40.0) & 47.3 & (46.0-48.6) & 22.5 & $(21.0-24.0)$ & 14.5 & $(13.2-16.0)$ & 18.8 & $(17.8-19.8)$ \\
\hline Virginia & - & - & - & - & - & - & - & - & - & - & - & - \\
\hline West Virginia & 44.8 & $(36.3-53.6)$ & 26.0 & (19.7-33.4) & 36.6 & $(30.6-43.1)$ & 15.7 & (12.5-19.6) & 6.2 & (3.5-10.6) & 11.6 & $(9.1-14.6)$ \\
\hline Wyoming & 43.5 & $(37.3-49.9)$ & 25.1 & $(20.0-31.0)$ & 34.9 & (30.1-40.1) & 15.5 & (11.5-20.6) & 8.7 & $(5.6-13.2)$ & 12.4 & $(9.3-16.2)$ \\
\hline Median & & 35.9 & & 23.8 & & 30.1 & & 13.3 & & 8.7 & & 11.1 \\
\hline Range & & $1.6-55.4)$ & & $3.5-38.2)$ & & 7.5-47.3) & & .8-22.5) & & (.6-14.7) & & $5.8-18.8)$ \\
\hline \multicolumn{13}{|c|}{ Large urban school district s } \\
\hline Baltimore, MD & 30.6 & $(20.6-42.9)$ & 21.5 & $(13.1-33.1)$ & 25.3 & $(18.6-33.4)$ & 15.2 & $(8.6-25.6)$ & 6.0 & $(2.2-15.5)$ & 10.2 & $(6.6-15.4)$ \\
\hline Boston, MA & 43.7 & $(37.0-50.6)$ & 18.5 & $(13.0-25.7)$ & 31.1 & $(26.1-36.6)$ & 12.9 & $(8.5-19.1)$ & 5.9 & $(3.1-10.9)$ & 9.4 & $(6.6-13.3)$ \\
\hline Broward County, FL & 16.2 & $(10.9-23.5)$ & 14.5 & $(9.7-21.2)$ & 15.5 & $(11.7-20.2)$ & 9.1 & $(5.0-15.9)$ & 2.7 & $(0.9-7.7)$ & 6.0 & $(3.4-10.2)$ \\
\hline Cleveland, $\mathrm{OH}$ & 37.1 & $(29.4-45.4)$ & 17.8 & $(12.4-25.0)$ & 26.9 & $(21.3-33.3)$ & 12.2 & $(7.7-18.8)$ & 5.3 & $(2.8-9.6)$ & 8.6 & $(5.7-12.9)$ \\
\hline DeKalb County, GA & 23.3 & $(17.9-29.8)$ & 13.6 & $(9.1-20.0)$ & 18.3 & $(14.6-22.7)$ & 9.9 & $(5.8-16.4)$ & 7.1 & $(4.0-12.1)$ & 8.4 & $(5.8-12.1)$ \\
\hline Detroit, MI & 16.5 & $(11.5-23.1)$ & 8.0 & $(4.5-13.9)$ & 12.6 & $(9.2-17.0)$ & 7.2 & $(4.0-12.7)$ & 5.6 & $(2.6-11.7)$ & 6.6 & $(3.9-11.0)$ \\
\hline District of Columbia & 22.7 & $(20.2-25.5)$ & 11.3 & $(9.4-13.5)$ & 16.9 & $(15.2-18.6)$ & 9.5 & $(7.8-11.6)$ & 3.4 & $(2.4-4.9)$ & 6.4 & $(5.4-7.6)$ \\
\hline Duval County, FL & 24.3 & $(19.2-30.2)$ & 17.3 & $(13.0-22.6)$ & 21.5 & $(17.9-25.6)$ & 9.7 & $(6.6-14.1)$ & 5.2 & $(3.1-8.5)$ & 7.8 & $(5.6-10.9)$ \\
\hline Ft. Worth, TX & 18.0 & $(13.6-23.5)$ & 11.7 & $(8.2-16.5)$ & 14.8 & $(11.7-18.5)$ & 5.7 & $(3.3-9.7)$ & 5.7 & $(3.5-9.4)$ & 5.7 & $(3.8-8.4)$ \\
\hline Houston, TX & 19.9 & $(15.0-25.9)$ & 7.3 & $(4.5-11.6)$ & 13.3 & $(10.9-16.3)$ & 6.6 & $(4.2-10.2)$ & 3.7 & $(1.6-8.2)$ & 5.3 & $(3.6-7.7)$ \\
\hline Los Angeles, CA & 21.5 & $(13.7-32.1)$ & 10.4 & $(7.3-14.5)$ & 16.0 & $(11.1-22.5)$ & 6.1 & $(2.8-12.9)$ & 1.4 & $(0.5-3.7)$ & 4.1 & $(2.4-7.0)$ \\
\hline Miami-Dade County, FL & 12.6 & $(9.1-17.3)$ & 8.5 & $(5.7-12.5)$ & 10.4 & $(7.9-13.7)$ & 4.4 & $(2.5-7.6)$ & 1.3 & $(0.6-2.9)$ & 2.8 & $(1.7-4.4)$ \\
\hline
\end{tabular}

See table footnotes on the next page. 
TABLE 78. (Continued) Percentage of high school students who used birth control pills, an IUD* or implant, ${ }^{\dagger}$ or a shot, ${ }^{\S}$ patch, ${ }^{\natural}$ or birth control ring** before last sexual intercourse $^{\dagger \dagger}$ and who used both a condom during last sexual intercourse and birth control pills, an IUD* or implant, ${ }^{\dagger}$ or a shot, ${ }^{\S}$ patch, ${ }^{\uparrow}$ or birth control ring** before last sexual intercourse, ${ }^{+\dagger}$ by sex — selected U.S. sites, Youth Risk Behavior Survey, 2015

\begin{tabular}{|c|c|c|c|c|c|c|c|c|c|c|c|c|}
\hline \multirow[b]{3}{*}{ Site } & \multicolumn{6}{|c|}{$\begin{array}{l}\text { Birth control pill use, IUD or implant, or shot, patch, or } \\
\text { birth control ring use }\end{array}$} & \multicolumn{6}{|c|}{$\begin{array}{l}\text { Condom use and birth control pill, IUD or implant, or } \\
\text { shot, patch, or birth control ring use }\end{array}$} \\
\hline & \multicolumn{2}{|c|}{ Female } & \multicolumn{2}{|r|}{ Male } & \multicolumn{2}{|r|}{ Total } & \multicolumn{2}{|c|}{ Female } & \multicolumn{2}{|r|}{ Male } & \multicolumn{2}{|r|}{ Total } \\
\hline & $\%$ & $\mathrm{Cl} \S$ & $\%$ & $\mathrm{Cl}$ & $\%$ & $\mathrm{Cl}$ & $\%$ & $\mathrm{Cl}$ & $\%$ & $\mathrm{Cl}$ & $\%$ & $\mathrm{Cl}$ \\
\hline New York City, NY & 21.3 & $(18.1-24.9)$ & 16.4 & $(11.4-23.0)$ & 19.0 & $(15.9-22.6)$ & 6.3 & $(3.9-10.1)$ & 6.5 & $(3.6-11.3)$ & 6.4 & $(4.4-9.1)$ \\
\hline Oakland, CA & - & - & - & - & - & - & - & - & - & - & - & - \\
\hline Orange County, FL & 19.5 & $(13.1-28.0)$ & 10.5 & $(6.4-16.8)$ & 14.9 & (11.1-19.8) & 6.8 & $(3.5-12.5)$ & 3.1 & $(1.4-6.8)$ & 5.0 & $(3.1-8.1)$ \\
\hline Palm Beach County, FL & 20.0 & $(15.5-25.5)$ & 16.8 & $(11.8-23.2)$ & 18.4 & $(14.8-22.7)$ & 8.4 & $(5.4-12.9)$ & 6.4 & $(3.7-11.0)$ & 7.4 & $(5.3-10.1)$ \\
\hline Philadelphia, PA & 28.2 & $(21.5-36.0)$ & 13.7 & $(9.4-19.5)$ & 21.1 & $(16.5-26.6)$ & 10.7 & $(6.7-16.7)$ & 4.2 & $(2.1-8.1)$ & 7.5 & $(5.0-11.0)$ \\
\hline San Diego, CA & 27.3 & $(21.3-34.1)$ & 20.6 & $(15.6-26.8)$ & 23.7 & $(19.6-28.2)$ & 8.6 & $(5.3-13.5)$ & 2.9 & $(1.3-6.5)$ & 5.5 & $(3.9-7.7)$ \\
\hline San Francisco, CA & 51.2 & $(38.1-64.1)$ & 22.3 & $(15.1-31.5)$ & 37.6 & $(29.9-46.1)$ & 12.5 & $(7.6-19.8)$ & 3.2 & $(1.5-6.6)$ & 8.1 & $(5.5-11.8)$ \\
\hline Median & & 22.1 & & 14.1 & & 18.3 & & 8.8 & & 4.7 & & 6.5 \\
\hline Range & & $2.6-51.2)$ & & 7.3-22.3) & & $0.4-37.6)$ & & $4-15.2)$ & & 1.3-7.1) & & $8-10.2)$ \\
\hline
\end{tabular}

* Such as Mirena or ParaGard.

† Such as Implanon or Nexplanon.

$\S$ Such as Depo-Provera.

I Such as OrthoEvra.

** Such as NuvaRing.

†+ To prevent pregnancy, among students who were currently sexually active.

$\S \S 95 \%$ confidence interval.

१ศा Not available.

TABLE 79. Percentage of high school students who did not use any method to prevent pregnancy during last sexual intercourse* and who drank alcohol or used drugs before last sexual intercourse,* by sex, race/ethnicity, and grade - United States, Youth Risk Behavior Survey, 2015

\begin{tabular}{|c|c|c|c|c|c|c|c|c|c|c|c|c|}
\hline \multirow[b]{3}{*}{ Category } & \multicolumn{6}{|c|}{ Did not use any method to prevent pregnancy } & \multicolumn{6}{|c|}{ Drank alcohol or used drugs before last sexual intercourse } \\
\hline & \multicolumn{2}{|c|}{ Female } & \multicolumn{2}{|c|}{ Male } & \multicolumn{2}{|c|}{ Total } & \multicolumn{2}{|c|}{ Female } & \multicolumn{2}{|c|}{ Male } & \multicolumn{2}{|r|}{ Total } \\
\hline & $\%$ & $\mathrm{Cl}^{\dagger}$ & $\%$ & $\mathrm{Cl}$ & $\%$ & $\mathrm{Cl}$ & $\%$ & $\mathrm{Cl}$ & $\%$ & $\mathrm{Cl}$ & $\%$ & $\mathrm{Cl}$ \\
\hline \multicolumn{13}{|c|}{ Race/Ethnicity } \\
\hline White ${ }^{\S}$ & 10.2 & $(7.4-14.0)$ & 10.3 & $(7.8-13.6)$ & 10.4 & $(8.2-13.1)$ & 14.7 & $(11.7-18.3)$ & 24.4 & $(20.0-29.5)$ & 19.3 & $(17.1-21.7)$ \\
\hline Black ${ }^{\S}$ & 25.6 & $(18.3-34.5)$ & 9.9 & $(4.2-21.7)$ & 15.9 & $(11.0-22.6)$ & 19.0 & $(11.2-30.2)$ & 23.1 & $(12.5-38.7)$ & 21.8 & $(13.7-32.9)$ \\
\hline Hispanic & 22.7 & $(16.3-30.8)$ & 17.2 & $(14.0-20.9)$ & 20.0 & $(16.4-24.2)$ & 17.7 & $(13.6-22.6)$ & 27.7 & $(23.6-32.3)$ & 22.8 & $(19.7-26.3)$ \\
\hline \multicolumn{13}{|l|}{ Grade } \\
\hline 9 & 22.0 & $(16.5-28.6)$ & 12.1 & $(8.3-17.3)$ & 16.5 & $(12.8-20.9)$ & 16.8 & $(11.5-23.8)$ & 27.2 & $(21.6-33.8)$ & 22.7 & $(19.0-26.9)$ \\
\hline 10 & 12.9 & $(9.1-18.1)$ & 11.7 & $(8.6-15.8)$ & 12.3 & $(9.7-15.4)$ & 15.6 & $(11.5-20.9)$ & 23.3 & $(16.9-31.3)$ & 19.7 & $(16.0-23.9)$ \\
\hline 11 & 12.3 & $(9.1-16.4)$ & 9.9 & $(7.0-13.7)$ & 11.1 & $(8.8-13.9)$ & 16.7 & $(13.2-21.0)$ & 23.0 & $(17.6-29.3)$ & 19.8 & $(17.0-23.0)$ \\
\hline 12 & 16.2 & $(12.5-20.7)$ & 14.2 & (9.2-21.3) & 15.5 & (11.9-19.9) & 16.1 & $(12.4-20.6)$ & 25.6 & $(21.0-30.8)$ & 20.8 & $(17.9-24.0)$ \\
\hline Total & 15.2 & $(12.7-18.1)$ & 12.2 & $(9.5-15.4)$ & 13.8 & $(11.8-16.1)$ & 16.4 & (14.4-18.7) & 24.6 & $(21.2-28.4)$ & 20.6 & $(18.9-22.5)$ \\
\hline
\end{tabular}

* Among the $30.1 \%$ of students nationwide who were currently sexually active.

† $95 \%$ confidence interval.

$\S$ Non-Hispanic. 
TABLE 80. Percentage of high school students who did not use any method to prevent pregnancy during last sexual intercourse* and who drank alcohol or used drugs before last sexual intercourse, * by sex - selected U.S. sites, Youth Risk Behavior Survey, 2015

\begin{tabular}{|c|c|c|c|c|c|c|c|c|c|c|c|c|}
\hline \multirow[b]{3}{*}{ Site } & \multicolumn{6}{|c|}{ Did not use any method to prevent pregnancy } & \multicolumn{6}{|c|}{ Drank alcohol or used drugs before last sexual intercourse } \\
\hline & \multicolumn{2}{|c|}{ Female } & \multicolumn{2}{|c|}{ Male } & \multicolumn{2}{|r|}{ Total } & \multicolumn{2}{|c|}{ Female } & \multicolumn{2}{|r|}{ Male } & \multicolumn{2}{|r|}{ Total } \\
\hline & $\%$ & $\mathrm{Cl}^{\dagger}$ & $\%$ & $\mathrm{Cl}$ & $\%$ & $\mathrm{Cl}$ & $\%$ & $\mathrm{Cl}$ & $\%$ & $\mathrm{Cl}$ & $\%$ & $\mathrm{Cl}$ \\
\hline \multicolumn{13}{|l|}{ State surveys } \\
\hline Alabama & 18.5 & $(12.6-26.5)$ & 17.0 & $(11.1-25.2)$ & 17.7 & $(13.0-23.6)$ & 17.0 & $(12.0-23.5)$ & 22.4 & $(13.7-34.3)$ & 19.7 & $(13.9-27.0)$ \\
\hline Alaska & 11.5 & $(6.9-18.7)$ & 9.4 & $(5.4-15.7)$ & 10.4 & $(6.9-15.4)$ & 15.1 & $(9.7-22.7)$ & 15.6 & $(10.8-22.0)$ & 15.2 & $(11.2-20.4)$ \\
\hline Arizona & 15.3 & $(9.4-23.9)$ & 14.0 & $(9.6-19.9)$ & 14.6 & $(10.3-20.4)$ & 25.3 & $(16.2-37.2)$ & 21.4 & (16.2-27.7) & 23.5 & (17.9-30.2) \\
\hline Arkansas & 22.8 & $(18.2-28.3)$ & 16.6 & $(10.0-26.2)$ & 20.0 & $(16.2-24.4)$ & 11.9 & $(9.1-15.5)$ & 26.9 & $(20.1-34.8)$ & 18.9 & $(15.0-23.6)$ \\
\hline California & 12.4 & $(8.9-16.9)$ & 12.2 & $(6.6-21.2)$ & 12.4 & $(8.7-17.4)$ & 19.5 & $(12.5-29.2)$ & 23.3 & $(13.6-36.9)$ & 21.4 & $(14.5-30.2)$ \\
\hline Connecticut & 12.3 & $(7.8-18.7)$ & 10.5 & $(5.6-18.8)$ & 11.6 & $(7.8-17.1)$ & 19.1 & $(14.4-24.9)$ & 26.6 & $(21.8-32.0)$ & 22.4 & (18.9-26.4) \\
\hline Delaware & 14.1 & $(8.0-23.6)$ & 11.6 & $(8.0-16.7)$ & 13.3 & $(9.6-18.0)$ & 19.2 & $(14.8-24.4)$ & 26.4 & $(20.6-33.3)$ & 22.8 & $(19.3-26.8)$ \\
\hline Florida & 16.5 & $(14.0-19.3)$ & 11.4 & $(9.4-13.9)$ & 13.9 & $(12.1-15.9)$ & 18.0 & (15.4-20.9) & 28.3 & $(24.5-32.5)$ & 23.7 & $(21.3-26.3)$ \\
\hline Hawaii & 11.4 & $(7.8-16.4)$ & 15.8 & $(9.8-24.5)$ & 13.2 & $(8.9-19.2)$ & 18.6 & $(15.1-22.7)$ & 21.8 & $(17.2-27.2)$ & 19.9 & $(17.4-22.7)$ \\
\hline Idaho & —§ & - & - & - & - & - & 16.4 & $(11.7-22.5)$ & 14.9 & $(10.5-20.8)$ & 15.8 & $(12.4-20.1)$ \\
\hline Illinois & 11.8 & $(8.0-16.9)$ & 17.2 & $(14.6-20.1)$ & 14.5 & $(11.9-17.4)$ & 15.9 & $(9.7-25.0)$ & 23.8 & $(20.3-27.7)$ & 19.9 & $(16.8-23.5)$ \\
\hline Indiana & 16.3 & $(10.7-24.0)$ & 14.8 & $(6.7-29.6)$ & 15.5 & $(10.2-22.9)$ & 14.6 & $(9.6-21.5)$ & 20.4 & 13.7-29.2) & 17.5 & $(12.3-24.2)$ \\
\hline Kentucky & 17.5 & $(13.9-22.0)$ & 11.6 & $(7.9-16.9)$ & 14.5 & $(12.0-17.5)$ & 16.4 & $(10.5-24.6)$ & 17.3 & $11.3-25.6)$ & 17.1 & (13.4-21.7) \\
\hline Maine & 8.6 & $(6.9-10.7)$ & 9.7 & $(7.6-12.4)$ & 9.3 & $(8.0-10.8)$ & 14.0 & $(12.2-16.0)$ & 21.9 & $(18.9-25.2)$ & 17.7 & $(16.1-19.5)$ \\
\hline Maryland & 15.3 & $(14.0-16.7)$ & 13.8 & $(12.6-15.2)$ & 14.6 & $(13.7-15.7)$ & 21.2 & $(19.9-22.6)$ & 26.1 & $(24.7-27.4)$ & 23.7 & $(22.8-24.7)$ \\
\hline Massachusetts & 9.2 & $(6.7-12.6)$ & 8.0 & $(5.2-12.0)$ & 8.6 & $(6.5-11.4)$ & 19.4 & $(14.1-26.1)$ & 23.7 & (18.7-29.6) & 21.8 & $(18.1-26.0)$ \\
\hline Michigan & 11.5 & $(8.1-16.1)$ & 11.3 & $(5.6-21.3)$ & 11.4 & $(8.3-15.4)$ & 20.1 & (12.9-30.0) & 24.8 & $(17.6-33.6)$ & 22.2 & (17.4-27.8) \\
\hline Mississippi & 14.0 & $(8.5-22.2)$ & 15.7 & $(11.7-20.7)$ & 14.8 & $(10.6-20.3)$ & 13.3 & $(9.4-18.5)$ & 21.7 & $(16.1-28.6)$ & 17.4 & (13.7-21.7) \\
\hline Missouri & 10.5 & $(5.6-19.0)$ & 13.0 & $(8.5-19.4)$ & 12.1 & $(9.0-16.0)$ & 18.0 & $(12.9-24.5)$ & 22.4 & $(15.3-31.6)$ & 20.4 & (16.2-25.4) \\
\hline Montana & 9.1 & $(7.0-11.7)$ & 8.4 & $(6.0-11.8)$ & 8.8 & $(7.0-11.0)$ & 17.3 & $(14.7-20.2)$ & 22.5 & $(18.8-26.6)$ & 19.7 & $(17.4-22.3)$ \\
\hline Nebraska & 19.0 & (11.9-29.0) & 16.3 & $(10.6-24.2)$ & 17.8 & $(13.3-23.5)$ & 17.6 & $(11.6-25.8)$ & 17.7 & $(11.1-27.2)$ & 17.9 & (12.4-25.3) \\
\hline Nevada & 13.8 & $(9.7-19.1)$ & 11.0 & $(6.8-17.5)$ & 12.4 & $(10.0-15.4)$ & 17.6 & $(13.2-23.2)$ & 20.8 & $(15.3-27.7)$ & 19.2 & $(15.1-24.0)$ \\
\hline New Hampshire & 7.4 & $(6.0-9.0)$ & 8.0 & $(6.4-9.9)$ & 7.8 & $(6.7-9.1)$ & 16.1 & $(14.1-18.3)$ & 21.1 & $(19.0-23.3)$ & 18.9 & (17.4-20.5) \\
\hline New Mexico & 17.5 & $(14.2-21.2)$ & 11.7 & $(8.9-15.1)$ & 14.8 & (12.4-17.5) & 14.9 & (12.6-17.6) & 22.4 & $(19.1-26.2)$ & 18.6 & (16.5-20.9) \\
\hline New York & 13.6 & (10.9-16.9) & 16.9 & (12.9-21.8) & 15.1 & $(13.0-17.5)$ & 21.1 & (16.4-26.6) & 29.0 & 20.9-38.7) & 24.6 & (19.4-30.6) \\
\hline North Carolina & 15.4 & $(10.4-22.4)$ & 10.8 & $(7.5-15.3)$ & 13.1 & $(10.1-16.8)$ & 17.1 & $(10.1-27.4)$ & 16.3 & 11.9-22.0) & 17.0 & $(11.7-24.1)$ \\
\hline North Dakota & - & - & - & - & - & - & 15.1 & $(11.2-20.1)$ & 22.1 & $(16.5-28.9)$ & 18.7 & $(15.1-22.8)$ \\
\hline Oklahoma & 15.4 & $(9.8-23.3)$ & 9.9 & $(5.3-17.9)$ & 12.8 & -17.7) & 11.1 & $(6.6-18.0)$ & 17.5 & $(11.9-24.9)$ & 14.3 & $(10.5-19.3)$ \\
\hline Pennsylvania & 12.2 & $(8.7-16.8)$ & 9.9 & $(6.6-14.7)$ & 11.1 & $(8.3-14.6)$ & 13.6 & $(9.9-18.4)$ & 24.0 & $(17.3-32.2)$ & 18.8 & $(14.3-24.3)$ \\
\hline Rhode Island & 13.4 & $(9.3-19.1)$ & 8.6 & $(5.0-14.4)$ & 11.6 & (8.4-15.8) & - & - & - & - & - & - \\
\hline South Carolina & 14.2 & $(10.5-18.9)$ & 9.3 & $(5.5-15.1)$ & 11.7 & $(8.8-15.3)$ & 15.7 & $(10.0-23.8)$ & 21.8 & $(14.0-32.4)$ & 18.6 & $(14.5-23.6)$ \\
\hline South Dakota & 12.6 & $(7.4-20.5)$ & 11.9 & $(5.7-23.1)$ & 12.2 & $(6.8-20.9)$ & 8.4 & $(4.4-15.6)$ & 18.1 & $(10.3-29.9)$ & 13.5 & $(9.2-19.3)$ \\
\hline Tennessee & - & - & - & - & - & - & - & - & - & - & - & - \\
\hline Vermont & 7.3 & $(6.4-8.3)$ & 7.0 & $(6.1-8.1)$ & 7.2 & $(6.6-7.9)$ & 15.2 & $(14.0-16.5)$ & 22.3 & (20.8-23.9) & 18.7 & $(17.7-19.7)$ \\
\hline Virginia & - & - & - & - & - & - & - & - & - & - & - & - \\
\hline West Virginia & 11.3 & $(8.5-15.0)$ & 12.8 & $(7.7-20.6)$ & 12.0 & $(9.2-15.6)$ & 14.4 & (10.4-19.5) & 23.8 & $(18.2-30.3)$ & 18.5 & $(14.8-22.9)$ \\
\hline Wyoming & 12.9 & $(8.2-19.6)$ & 14.1 & (10.0-19.5) & 13.4 & $(9.7-18.3)$ & 18.8 & $(14.0-24.7)$ & 22.3 & (17.6-27.7) & 20.3 & $(16.7-24.6)$ \\
\hline Median & & 13.4 & & 11.6 & & 12.8 & & 16.7 & & 22.3 & & 18.9 \\
\hline Range & & $3-22.8)$ & & (0-17.2) & & .2-20.0) & & 4-25.3) & & (.9-29.0) & & $3.5-24.6)$ \\
\hline \multicolumn{13}{|c|}{ Large urban school district surveys } \\
\hline Baltimore, MD & 20.6 & $(13.0-31.1)$ & 12.6 & $(7.0-21.6)$ & 16.6 & $(11.3-23.6)$ & 18.7 & $(12.5-26.9)$ & 23.1 & $(16.4-31.5)$ & 21.0 & $(16.3-26.6)$ \\
\hline Boston, MA & 11.3 & $(7.3-17.0)$ & 9.8 & $(6.4-14.6)$ & 10.5 & $(7.7-14.3)$ & 15.7 & $(10.2-23.2)$ & 20.9 & $(14.6-29.0)$ & 18.3 & $(14.7-22.6)$ \\
\hline Broward County, FL & 15.8 & $(9.8-24.5)$ & 10.2 & $(6.2-16.3)$ & 12.9 & $(9.0-18.2)$ & 18.4 & $(12.7-25.8)$ & 19.7 & $(13.6-27.8)$ & 19.2 & $(15.1-23.9)$ \\
\hline Cleveland, $\mathrm{OH}$ & 22.1 & $(16.0-29.7)$ & 17.8 & $(12.2-25.3)$ & 19.7 & $(15.5-24.6)$ & 14.4 & $(10.4-19.8)$ & 23.6 & $(17.8-30.5)$ & 19.5 & $(15.6-24.0)$ \\
\hline DeKalb County, GA & 25.3 & $(19.3-32.3)$ & 10.4 & $(6.0-17.4)$ & 17.8 & $(14.0-22.5)$ & 16.9 & $(11.7-23.7)$ & 25.6 & $(18.8-33.8)$ & 21.3 & $(17.0-26.4)$ \\
\hline Detroit, MI & 19.0 & $(13.7-25.9)$ & 17.5 & $(12.0-24.8)$ & 18.1 & $(14.2-22.9)$ & 15.8 & $(11.5-21.4)$ & 18.0 & $(12.1-26.1)$ & 17.1 & $(13.0-22.1)$ \\
\hline District of Columbia & 24.7 & $(22.0-27.6)$ & 14.7 & $(12.5-17.2)$ & 19.6 & $(17.8-21.4)$ & 15.6 & $(13.5-18.0)$ & 20.3 & $(17.9-23.0)$ & 18.1 & $(16.4-19.8)$ \\
\hline Duval County, FL & 19.0 & $(14.9-23.9)$ & 15.7 & $(11.3-21.5)$ & 17.2 & $(13.7-21.4)$ & 17.0 & $(13.0-21.9)$ & 23.9 & $(18.9-29.8)$ & 20.2 & $(17.0-23.9)$ \\
\hline Ft. Worth, TX & 31.1 & $(24.8-38.1)$ & 13.8 & $(9.6-19.5)$ & 22.0 & $(18.0-26.6)$ & 14.1 & $(10.1-19.3)$ & 22.2 & $(17.3-27.9)$ & 18.2 & (15.2-21.7) \\
\hline Houston, TX & 26.3 & $(20.6-33.0)$ & 17.2 & $(13.7-21.5)$ & 21.5 & $(18.0-25.5)$ & 18.1 & $(14.1-22.9)$ & 23.9 & $(19.1-29.4)$ & 21.4 & $(18.1-25.1)$ \\
\hline Los Angeles, CA & 15.2 & $(9.8-23.0)$ & 16.2 & $(11.3-22.7)$ & 15.7 & $(11.7-20.6)$ & 17.4 & $(12.2-24.2)$ & 17.0 & $(13.3-21.5)$ & 17.2 & $(14.1-20.8)$ \\
\hline Miami-Dade County, FL & 14.6 & $(11.0-19.3)$ & 14.0 & $(9.9-19.4)$ & 14.4 & $(11.3-18.1)$ & 13.0 & $(9.0-18.4)$ & 27.7 & $(22.9-33.0)$ & 20.8 & $(17.6-24.5)$ \\
\hline
\end{tabular}

See table footnotes on the next page. 
TABLE 80. (Continued) Percentage of high school students who did not use any method to prevent pregnancy during last sexual intercourse* and who drank alcohol or used drugs before last sexual intercourse,* by sex - selected U.S. sites, Youth Risk Behavior Survey, 2015

\begin{tabular}{|c|c|c|c|c|c|c|c|c|c|c|c|c|}
\hline \multirow[b]{3}{*}{ Site } & \multicolumn{6}{|c|}{ Did not use any method to prevent pregnancy } & \multicolumn{6}{|c|}{ Drank alcohol or used drugs before last sexual intercourse } \\
\hline & \multicolumn{2}{|c|}{ Female } & \multicolumn{2}{|c|}{ Male } & \multicolumn{2}{|r|}{ Total } & \multicolumn{2}{|c|}{ Female } & \multicolumn{2}{|r|}{ Male } & \multicolumn{2}{|r|}{ Total } \\
\hline & $\%$ & $\mathrm{Cl}^{\dagger}$ & $\%$ & $\mathrm{Cl}$ & $\%$ & $\mathrm{Cl}$ & $\%$ & $\mathrm{Cl}$ & $\%$ & $\mathrm{Cl}$ & $\%$ & $\mathrm{Cl}$ \\
\hline New York City, NY & 22.5 & $(18.5-27.1)$ & 12.2 & $(9.7-15.3)$ & 17.7 & $(15.5-20.0)$ & 15.3 & $(10.5-22.0)$ & 22.3 & $(16.8-29.0)$ & 18.7 & $(13.8-24.8)$ \\
\hline Oakland, CA & - & - & - & - & - & - & 20.1 & $(13.0-29.9)$ & 22.1 & $(15.7-30.2)$ & 21.1 & $(15.7-27.8)$ \\
\hline Orange County, FL & 21.1 & $(15.0-28.9)$ & 12.7 & $(8.4-18.7)$ & 16.5 & $(12.7-21.2)$ & 17.5 & $(11.8-25.2)$ & 31.2 & $(22.9-40.8)$ & 24.7 & $(19.1-31.4)$ \\
\hline Palm Beach County, FL & 21.4 & $(16.8-26.9)$ & 12.7 & $(9.1-17.4)$ & 17.3 & $(14.0-21.3)$ & 19.1 & $(15.1-23.8)$ & 28.6 & $(23.1-34.9)$ & 24.0 & (20.5-27.9) \\
\hline Philadelphia, PA & 19.7 & $(14.2-26.7)$ & 14.5 & (11.6-17.9) & 17.1 & $(13.9-21.0)$ & 12.3 & $(8.4-17.6)$ & 14.1 & (10.8-18.3) & 13.2 & (10.4-16.6) \\
\hline San Diego, CA & 11.5 & $(7.2-17.8)$ & 13.1 & $(8.4-20.0)$ & 12.4 & (9.2-16.4) & 15.9 & $(12.1-20.7)$ & 23.1 & (18.3-28.7) & 19.8 & $(16.6-23.5)$ \\
\hline San Francisco, CA & 10.2 & $(6.0-16.8)$ & 11.5 & $(6.1-20.6)$ & 10.8 & $(6.8-16.8)$ & 21.0 & $(14.9-28.8)$ & 28.9 & $(21.2-38.1)$ & 24.8 & (19.7-30.6) \\
\hline Median & \multicolumn{2}{|c|}{20.1} & \multicolumn{2}{|r|}{13.4} & \multicolumn{2}{|r|}{17.1} & \multicolumn{2}{|c|}{16.9} & \multicolumn{2}{|r|}{23.1} & \multicolumn{2}{|r|}{19.8} \\
\hline Range & \multicolumn{2}{|c|}{$(10.2-31.1)$} & \multicolumn{2}{|c|}{$(9.8-17.8)$} & \multicolumn{2}{|c|}{$(10.5-22.0)$} & \multicolumn{2}{|c|}{$(12.3-21.0)$} & \multicolumn{2}{|c|}{$(14.1-31.2)$} & \multicolumn{2}{|c|}{$(13.2-24.8)$} \\
\hline
\end{tabular}

* Among students who were currently sexually active.

$\dagger 95 \%$ confidence interval.

$\S$ Not available.

TABLE 81. Percentage of high school students who were ever tested for human immunodeficiency virus (HIV), ${ }^{*}$ by sex, race/ethnicity, and grade United States, Youth Risk Behavior Survey, 2015

\begin{tabular}{|c|c|c|c|c|c|c|}
\hline \multirow[b]{2}{*}{ Category } & \multicolumn{2}{|c|}{ Female } & \multicolumn{2}{|c|}{ Male } & \multicolumn{2}{|c|}{ Total } \\
\hline & $\%$ & $\mathrm{Cl}^{\dagger}$ & $\%$ & $\mathrm{Cl}$ & $\%$ & $\mathrm{Cl}$ \\
\hline \multicolumn{7}{|c|}{ Race/Ethnicity } \\
\hline White ${ }^{\S}$ & 9.1 & $(7.4-11.2)$ & 7.0 & $(5.8-8.4)$ & 8.0 & $(7.1-9.1)$ \\
\hline Black ${ }^{\S}$ & 16.2 & $(11.6-22.0)$ & 17.1 & $(9.8-28.0)$ & 16.6 & $(11.2-23.9)$ \\
\hline Hispanic & 12.3 & $(10.1-14.8)$ & 10.1 & $(7.9-12.7)$ & 11.1 & $(9.4-13.1)$ \\
\hline \multicolumn{7}{|l|}{ Grade } \\
\hline 9 & 7.7 & $(5.7-10.2)$ & 7.9 & $(5.8-10.7)$ & 7.8 & $(6.3-9.5)$ \\
\hline 10 & 9.8 & $(7.2-13.3)$ & 9.8 & $(7.3-13.0)$ & 9.8 & $(7.6-12.5)$ \\
\hline 11 & 10.3 & $(8.7-12.2)$ & 8.8 & $(5.8-13.2)$ & 9.6 & $(7.7-12.0)$ \\
\hline 12 & 16.8 & $(14.0-20.1)$ & 10.9 & $(8.4-14.0)$ & 13.8 & $(11.6-16.4)$ \\
\hline Total & 11.1 & $(9.5-12.8)$ & 9.3 & (7.4-11.5) & 10.2 & (8.7-11.8) \\
\hline
\end{tabular}

* Does not count tests conducted when donating blood.

† $95 \%$ confidence interval.

$\S$ Non-Hispanic. 
TABLE 82. Percentage of high school students who were ever tested for human immunodeficiency virus (HIV),* by sex — selected U.S. sites, Youth Risk Behavior Survey, 2015

\begin{tabular}{|c|c|c|c|c|c|c|}
\hline \multirow[b]{2}{*}{ Site } & \multicolumn{2}{|c|}{ Female } & \multicolumn{2}{|c|}{ Male } & \multicolumn{2}{|c|}{ Total } \\
\hline & $\%$ & $\mathrm{Cl}^{\dagger}$ & $\%$ & $\mathrm{Cl}$ & $\%$ & $\mathrm{Cl}$ \\
\hline \multicolumn{7}{|l|}{ State surveys } \\
\hline Alabama & 18.5 & $(15.0-22.6)$ & 12.8 & $(10.1-16.2)$ & 15.8 & $(13.0-19.0)$ \\
\hline Alaska & $\_\S$ & - & - & - & - & - \\
\hline Arizona & - & - & - & - & - & - \\
\hline Arkansas & 17.7 & $(14.2-21.9)$ & 17.8 & $(13.5-23.1)$ & 17.9 & $(14.6-21.7)$ \\
\hline California & 9.3 & $(7.8-11.0)$ & 8.1 & $(5.9-11.0)$ & 8.7 & $(7.2-10.4)$ \\
\hline Connecticut & 11.3 & $(8.8-14.4)$ & 9.1 & $(7.2-11.6)$ & 10.3 & $(8.5-12.4)$ \\
\hline Delaware & 13.6 & $(11.1-16.7)$ & 12.2 & $(9.7-15.2)$ & 13.3 & $(11.1-16.0)$ \\
\hline Florida & 12.5 & $(11.1-14.2)$ & 12.5 & $(10.5-14.7)$ & 12.6 & $(11.1-14.2)$ \\
\hline Hawaii & - & - & - & - & - & - \\
\hline Idaho & 8.8 & $(6.9-11.2)$ & 7.8 & $(5.6-10.8)$ & 8.3 & $(6.8-10.1)$ \\
\hline Illinois & 13.6 & $(11.5-16.1)$ & 16.6 & $(14.0-19.7)$ & 15.1 & $(13.6-16.8)$ \\
\hline Indiana & 8.9 & $(6.7-11.6)$ & 8.8 & $(6.4-12.0)$ & 8.9 & $(7.0-11.2)$ \\
\hline Kentucky & 12.9 & $(10.2-16.3)$ & 10.1 & $(7.6-13.3)$ & 11.6 & $(9.6-14.0)$ \\
\hline Maine & - & - & - & - & - & - \\
\hline Maryland & 12.9 & $(12.2-13.6)$ & 14.2 & $(13.4-14.9)$ & 13.6 & $(13.0-14.2)$ \\
\hline Massachusetts & 9.6 & $(7.1-12.9)$ & 9.9 & $(8.1-12.0)$ & 9.9 & $(8.0-12.2)$ \\
\hline Michigan & 12.0 & $(9.0-15.8)$ & 13.0 & $(9.6-17.3)$ & 12.5 & $(9.5-16.2)$ \\
\hline Mississippi & 18.4 & $(14.4-23.1)$ & 16.2 & $(12.7-20.3)$ & 17.4 & (14.3-20.9) \\
\hline Missouri & - & - & - & - & - & - \\
\hline Montana & - & - & - & - & - & - \\
\hline Nebraska & 9.8 & $(7.8-12.2)$ & 8.8 & $(6.4-12.0)$ & 9.3 & $(7.8-11.0)$ \\
\hline Nevada & 11.3 & $(8.3-15.1)$ & 12.2 & $(9.8-15.1)$ & 12.1 & (10.7-13.5) \\
\hline New Hampshire & - & - & - & - & - & - \\
\hline New Mexico & 10.4 & $(9.2-11.7)$ & 10.4 & $(9.5-11.4)$ & 10.4 & $(9.6-11.2)$ \\
\hline New York & 15.8 & $(12.7-19.4)$ & 19.9 & $(17.0-23.3)$ & 18.0 & $(15.3-21.0)$ \\
\hline North Carolina & 11.0 & $(8.9-13.5)$ & 10.3 & $(7.5-14.1)$ & 10.6 & $(8.5-13.3)$ \\
\hline North Dakota & - & - & - & - & - & - \\
\hline Oklahoma & 9.8 & $(7.8-12.4)$ & 9.4 & $(6.6-13.2)$ & 9.5 & $(7.5-12.1)$ \\
\hline Pennsylvania & 11.3 & $(8.4-15.1)$ & 10.9 & $(7.8-15.1)$ & 11.1 & $(8.2-14.8)$ \\
\hline Rhode Island & 12.2 & $(9.5-15.5)$ & 14.8 & $(11.5-18.8)$ & 13.6 & $(10.9-16.9)$ \\
\hline South Carolina & 10.8 & $(6.9-16.7)$ & 9.9 & $(6.2-15.5)$ & 10.4 & $(7.5-14.3)$ \\
\hline South Dakota & 7.3 & $(4.3-12.2)$ & 7.3 & $(4.6-11.4)$ & 7.4 & $(4.8-11.2)$ \\
\hline Tennessee & - & - & - & - & - & - \\
\hline Vermont & 11.3 & $(10.7-11.9)$ & 9.3 & $(8.7-9.9)$ & 10.3 & $(9.9-10.7)$ \\
\hline Virginia & - & - & - & - & - & - \\
\hline West Virginia & 13.4 & $(10.2-17.4)$ & 12.9 & $(10.3-16.2)$ & 13.1 & $(10.9-15.7)$ \\
\hline Wyoming & 12.6 & $(10.1-15.6)$ & 10.2 & $(8.0-13.0)$ & 11.4 & $(9.5-13.7)$ \\
\hline Median & \multirow{2}{*}{\multicolumn{2}{|c|}{$\begin{array}{c}11.3 \\
(7.3-18.5)\end{array}$}} & \multirow{2}{*}{\multicolumn{2}{|c|}{$\begin{array}{c}10.4 \\
(7.3-19.9)\end{array}$}} & \multicolumn{2}{|c|}{$\begin{array}{c}11.4 \\
(7.4-18.0)\end{array}$} \\
\hline Range & & & & & & \\
\hline \multicolumn{7}{|c|}{ Large urban school district surveys } \\
\hline Baltimore, MD & 27.8 & $(22.9-33.3)$ & 30.8 & $(25.3-37.0)$ & 29.0 & $(25.2-33.1)$ \\
\hline Boston, MA & 20.5 & $(16.9-24.8)$ & 20.4 & $(17.2-24.0)$ & 20.4 & $(17.7-23.4)$ \\
\hline Broward County, FL & 17.1 & $(14.5-20.1)$ & 20.5 & $(17.1-24.3)$ & 18.9 & $(16.8-21.1)$ \\
\hline Cleveland, $\mathrm{OH}$ & 23.5 & $(19.6-27.9)$ & 26.0 & $(21.7-30.9)$ & 25.0 & $(21.6-28.8)$ \\
\hline DeKalb County, GA & 16.9 & $(14.3-20.0)$ & 19.4 & $(16.7-22.5)$ & 18.3 & $(16.3-20.4)$ \\
\hline Detroit, MI & 23.6 & $(20.1-27.5)$ & 24.9 & $(20.2-30.2)$ & 24.1 & $(21.3-27.2)$ \\
\hline District of Columbia & 37.0 & $(35.6-38.4)$ & 37.7 & $(36.1-39.3)$ & 37.4 & $(36.3-38.4)$ \\
\hline Duval County, FL & 16.7 & $(14.5-19.2)$ & 21.4 & $(18.8-24.3)$ & 18.9 & (17.1-20.9) \\
\hline Ft. Worth, TX & 7.8 & $(6.2-9.7)$ & 7.6 & $(6.1-9.3)$ & 7.7 & (6.4-9.2) \\
\hline Houston, $\mathrm{TX}$ & 19.4 & $(17.0-22.0)$ & 19.8 & $(17.5-22.2)$ & 19.6 & $(17.7-21.5)$ \\
\hline Los Angeles, CA & 12.1 & $(9.5-15.4)$ & 13.7 & $(12.0-15.6)$ & 12.8 & $(11.0-14.9)$ \\
\hline Miami-Dade County, FL & 13.8 & $(11.8-16.2)$ & 11.9 & $(9.8-14.5)$ & 13.0 & (11.4-14.7) \\
\hline
\end{tabular}

See table footnotes on the next page. 
TABLE 82. (Continued) Percentage of high school students who were ever tested for human immunodeficiency virus (HIV),* by sex — selected U.S. sites, Youth Risk Behavior Survey, 2015

\begin{tabular}{|c|c|c|c|c|c|c|}
\hline \multirow[b]{2}{*}{ Site } & \multicolumn{2}{|c|}{ Female } & \multicolumn{2}{|c|}{ Male } & \multicolumn{2}{|c|}{ Total } \\
\hline & $\%$ & $\mathrm{Cl}^{\dagger}$ & $\%$ & $\mathrm{Cl}$ & $\%$ & $\mathrm{Cl}$ \\
\hline New York City, NY & 20.7 & $(17.0-24.8)$ & 22.4 & $(18.4-27.1)$ & 21.6 & $(18.3-25.3)$ \\
\hline Oakland, CA & 27.9 & $(24.0-32.2)$ & 23.3 & $(19.9-27.0)$ & 25.5 & $(22.6-28.6)$ \\
\hline Orange County, FL & 6.9 & $(5.4-8.8)$ & 7.2 & $(5.5-9.5)$ & 7.0 & $(5.8-8.5)$ \\
\hline Palm Beach County, FL & 14.9 & $(12.3-17.8)$ & 16.4 & $(13.8-19.4)$ & 15.7 & $(13.7-17.9)$ \\
\hline Philadelphia, PA & 35.3 & $(28.6-42.7)$ & 35.0 & $(31.3-39.0)$ & 35.1 & $(30.3-40.2)$ \\
\hline San Diego, CA & 12.0 & $(9.6-14.9)$ & 11.9 & $(10.3-13.7)$ & 11.9 & (10.4-13.7) \\
\hline San Francisco, CA & 14.2 & $(11.5-17.5)$ & 14.1 & $(10.9-18.0)$ & 14.5 & (12.1-17.3) \\
\hline Median & \multirow{2}{*}{\multicolumn{2}{|c|}{$\begin{array}{c}17.1 \\
(6.9-37.0)\end{array}$}} & \multirow{2}{*}{\multicolumn{2}{|c|}{$\begin{array}{c}20.4 \\
(7.2-37.7)\end{array}$}} & \multirow{2}{*}{\multicolumn{2}{|c|}{$\begin{array}{c}18.9 \\
(7.0-37.4)\end{array}$}} \\
\hline Range & & & & & & \\
\hline
\end{tabular}

* Does not count tests conducted when donating blood.

† $95 \%$ confidence interval.

$\S$ Not available.

TABLE 83. Percentage of high school students who did not eat fruit or drink $100 \%$ fruit juices* and who ate fruit or drank $100 \%$ fruit juices one or more times/day, ${ }^{*}$ by sex, race/ethnicity, and grade — United States, Youth Risk Behavior Survey, 2015

\begin{tabular}{|c|c|c|c|c|c|c|c|c|c|c|c|c|}
\hline \multirow[b]{3}{*}{ Category } & \multicolumn{6}{|c|}{ Did not eat fruit or drink $100 \%$ fruit juices } & \multicolumn{6}{|c|}{ Ate fruit or drank $100 \%$ fruit juices one or more times/day } \\
\hline & \multicolumn{2}{|c|}{ Female } & \multicolumn{2}{|c|}{ Male } & \multicolumn{2}{|c|}{ Total } & \multicolumn{2}{|c|}{ Female } & \multicolumn{2}{|c|}{ Male } & \multicolumn{2}{|c|}{ Total } \\
\hline & $\%$ & $\mathrm{Cl}^{\dagger}$ & $\%$ & $\mathrm{Cl}$ & $\%$ & $\mathrm{Cl}$ & $\%$ & $\mathrm{Cl}$ & $\%$ & $\mathrm{Cl}$ & $\%$ & $\mathrm{Cl}$ \\
\hline \multicolumn{13}{|c|}{ Race/Ethnicity } \\
\hline White $\S$ & 4.3 & $(3.3-5.7)$ & 5.4 & $(4.2-6.9)$ & 4.9 & $(4.0-5.9)$ & 64.1 & $(59.8-68.1)$ & 62.1 & $(58.9-65.2)$ & 63.0 & $(60.0-65.9)$ \\
\hline Black $^{\S}$ & 5.2 & $(3.6-7.4)$ & 8.6 & $(5.7-12.7)$ & 6.9 & $(4.9-9.8)$ & 56.5 & $(51.8-61.2)$ & 67.0 & $(60.9-72.6)$ & 62.2 & (57.8-66.4) \\
\hline Hispanic & 4.0 & $(3.0-5.3)$ & 5.7 & $(4.3-7.6)$ & 4.9 & $(3.8-6.1)$ & 60.7 & $(58.0-63.2)$ & 67.3 & $(65.1-69.4)$ & 64.1 & $(62.5-65.7)$ \\
\hline \multicolumn{13}{|l|}{ Grade } \\
\hline 9 & 5.0 & (3.9-6.4) & 6.7 & $(4.9-9.1)$ & 6.0 & $(4.7-7.6)$ & 61.9 & $(59.3-64.4)$ & 65.0 & $(60.7-69.0)$ & 63.5 & $(60.7-66.3)$ \\
\hline 10 & 3.4 & $(2.3-5.1)$ & 6.7 & $(4.8-9.2)$ & 5.0 & $(4.0-6.3)$ & 62.7 & $(59.1-66.1)$ & 63.2 & (58.6-67.4) & 62.9 & (59.6-66.0) \\
\hline 11 & 3.7 & $(2.9-4.8)$ & 4.7 & $(3.3-6.5)$ & 4.4 & $(3.5-5.6)$ & 62.3 & $(58.8-65.6)$ & 66.7 & $(63.1-70.2)$ & 64.3 & $(61.8-66.8)$ \\
\hline 12 & 4.8 & $(3.5-6.5)$ & 5.5 & $(4.1-7.5)$ & 5.1 & $(4.1-6.5)$ & 62.3 & (57.9-66.4) & 63.8 & $(60.0-67.5)$ & 63.1 & $(59.8-66.2)$ \\
\hline Total & 4.3 & $(3.5-5.2)$ & 5.9 & $(4.8-7.3)$ & 5.2 & $(4.4-6.0)$ & 62.1 & (59.7-64.6) & 64.6 & $(62.2-66.9)$ & 63.3 & $(61.3-65.3)$ \\
\hline
\end{tabular}

* During the 7 days before the survey.

$\dagger 95 \%$ confidence interval.

$\S$ Non-Hispanic. 
TABLE 84. Percentage of high school students who did not eat fruit or drink $100 \%$ fruit juices* and who ate fruit or drank $100 \%$ fruit juices one or more times/day, ${ }^{*}$ by sex — selected U.S. sites, Youth Risk Behavior Survey, 2015

\begin{tabular}{|c|c|c|c|c|c|c|c|c|c|c|c|c|}
\hline \multirow[b]{3}{*}{ Site } & \multicolumn{6}{|c|}{ Did not eat fruit or drink $100 \%$ fruit juices } & \multicolumn{6}{|c|}{ Ate fruit or drank $100 \%$ fruit juices one or more times/day } \\
\hline & \multicolumn{2}{|c|}{ Female } & \multicolumn{2}{|r|}{ Male } & \multicolumn{2}{|r|}{ Total } & \multicolumn{2}{|c|}{ Female } & \multicolumn{2}{|r|}{ Male } & \multicolumn{2}{|r|}{ Total } \\
\hline & $\%$ & $\mathrm{Cl}^{\dagger}$ & $\%$ & $\mathrm{Cl}$ & $\%$ & $\mathrm{Cl}$ & $\%$ & $\mathrm{Cl}$ & $\%$ & $\mathrm{Cl}$ & $\%$ & $\mathrm{Cl}$ \\
\hline \multicolumn{13}{|l|}{ State surveys } \\
\hline Alabama & 9.9 & $(7.8-12.4)$ & 10.6 & $(8.2-13.5)$ & 10.2 & $(8.5-12.1)$ & 46.9 & $(41.7-52.1)$ & 51.4 & $(47.0-55.8)$ & 49.2 & $(45.8-52.7)$ \\
\hline Alaska & 5.0 & $(3.5-7.2)$ & 5.7 & $(4.2-7.8)$ & 5.3 & $(4.1-7.0)$ & 55.2 & $(51.1-59.3)$ & 57.6 & $(52.8-62.3)$ & 56.4 & (53.4-59.3) \\
\hline Arizona & 6.1 & $(4.7-7.9)$ & 7.2 & $(5.1-10.0)$ & 6.7 & $(5.2-8.6)$ & 59.4 & $(54.4-64.2)$ & 61.4 & $(57.7-64.9)$ & 60.5 & $(56.8-64.0)$ \\
\hline Arkansas & 9.4 & $(6.8-12.9)$ & 13.3 & $(11.1-15.9)$ & 11.4 & $(9.5-13.5)$ & 49.6 & $(46.0-53.2)$ & 54.6 & (48.9-60.3) & 52.1 & $(48.3-56.0)$ \\
\hline California & 2.8 & $(1.9-4.1)$ & 4.5 & (3.0-6.6) & 3.7 & $(2.8-4.9)$ & 66.1 & (61.9-70.1) & 69.1 & (63.9-73.8) & 67.7 & $(64.6-70.6)$ \\
\hline Connecticut & 3.8 & $(2.9-5.0)$ & 8.8 & $(7.0-11.0)$ & 6.3 & $(5.2-7.5)$ & 64.3 & $(60.4-68.0)$ & 59.5 & $(55.7-63.3)$ & 61.8 & $(58.6-65.0)$ \\
\hline Delaware & 4.6 & $(3.4-6.1)$ & 7.2 & $(5.6-9.3)$ & 6.3 & $(5.0-7.8)$ & 60.7 & $(57.4-64.0)$ & 63.5 & $(60.0-66.9)$ & 62.0 & $(59.3-64.7)$ \\
\hline Florida & 7.3 & $(6.3-8.4)$ & 7.8 & $(6.6-9.3)$ & 7.6 & $(6.6-8.7)$ & 57.7 & $(55.5-59.9)$ & 63.3 & $(61.1-65.5)$ & 60.6 & $(58.7-62.4)$ \\
\hline Hawaii & 6.9 & $(5.7-8.5)$ & 8.7 & $(7.3-10.4)$ & 7.8 & $(7.0-8.8)$ & 46.5 & $(43.5-49.6)$ & 52.0 & $(48.7-55.2)$ & 49.4 & $(46.7-52.1)$ \\
\hline Idaho & 3.1 & $(2.0-4.8)$ & 3.2 & $(2.4-4.3)$ & 3.2 & $(2.6-3.9)$ & 58.5 & $(55.3-61.7)$ & 62.7 & $(58.3-66.8)$ & 60.6 & $(58.1-63.1)$ \\
\hline Illinois & 5.0 & $(3.6-7.1)$ & 10.3 & $(8.8-12.1)$ & 7.7 & $(6.4-9.3)$ & 58.8 & (53.4-64.1) & 60.3 & $(56.8-63.7)$ & 59.6 & $(55.8-63.2)$ \\
\hline Indiana & 5.4 & $(3.5-8.3)$ & 7.5 & $(4.2-13.1)$ & 6.5 & $(4.2-10.1)$ & 58.2 & (54.4-61.9) & 62.9 & (58.3-67.3) & 60.5 & $(57.3-63.7)$ \\
\hline Kentucky & 5.8 & $(4.5-7.6)$ & 9.3 & $(7.0-12.3)$ & 7.7 & $(6.4-9.4)$ & 52.7 & $(48.3-57.0)$ & 52.7 & $(48.6-56.8)$ & 52.7 & $(49.4-56.0)$ \\
\hline Maine & 4.5 & $(4.0-5.1)$ & 6.5 & $(5.7-7.4)$ & 5.5 & $(5.0-6.2)$ & 61.5 & (58.4-64.5) & 60.3 & (58.1-62.4) & 60.9 & $(58.6-63.1)$ \\
\hline Maryland & 6.7 & $(6.3-7.2)$ & 9.1 & $(8.7-9.6)$ & 8.0 & $(7.6-8.3)$ & 54.5 & $(53.6-55.3)$ & 58.7 & $(57.9-59.5)$ & 56.6 & $(55.9-57.3)$ \\
\hline Massachusetts & 5.1 & $(3.9-6.6)$ & 5.9 & $(4.7-7.3)$ & 5.5 & $(4.7-6.5)$ & 60.2 & $(56.5-63.8)$ & 65.0 & $(61.8-68.2)$ & 62.6 & $(59.9-65.3)$ \\
\hline Michigan & 5.4 & $(3.5-8.3)$ & 7.7 & $(6.1-9.5)$ & 6.5 & $(5.2-8.2)$ & 55.8 & $(50.0-61.5)$ & 59.8 & $(56.0-63.5)$ & 57.9 & $(53.7-62.0)$ \\
\hline Mississippi & 10.4 & $(8.3-12.9)$ & 13.0 & $(10.1-16.5)$ & 11.7 & $(9.8-13.9)$ & 47.2 & $(43.2-51.3)$ & 51.1 & $(46.8-55.3)$ & 49.0 & $(46.3-51.8)$ \\
\hline Missouri & 5.7 & $(4.1-8.0)$ & 7.4 & $(5.1-10.8)$ & 6.6 & $(5.5-7.8)$ & 52.1 & (47.7-56.4) & 55.1 & $(51.7-58.5)$ & 53.6 & $(50.7-56.5)$ \\
\hline Montana & 3.6 & $(2.7-4.8)$ & 6.5 & $(5.4-7.8)$ & 5.1 & $(4.4-5.9)$ & 58.1 & (55.4-60.8) & 61.7 & (59.4-64.0) & 60.0 & $(57.9-62.1)$ \\
\hline Nebraska & 3.8 & $(2.4-6.0)$ & 7.4 & $(5.6-9.8)$ & 5.6 & $(4.4-7.2)$ & 58.7 & $(55.2-62.0)$ & 58.6 & $(54.1-63.0)$ & 58.7 & (55.7-61.6) \\
\hline Nevada & 3.7 & $(2.3-5.9)$ & 6.3 & $(4.3-9.3)$ & 5.2 & $(3.9-7.0)$ & 57.2 & $(52.5-61.8)$ & 63.0 & $(58.0-67.7)$ & 60.0 & $(56.9-63.0)$ \\
\hline New Hampshire & —§ & - & - & - & - & - & - & - & - & - & - & - \\
\hline New Mexico & 6.4 & $(5.3-7.6)$ & 8.5 & $(7.5-9.6)$ & 7.5 & $(6.6-8.4)$ & 52.3 & $(49.4-55.1)$ & 57.0 & (54.9-59.2) & 54.6 & $(52.8-56.4)$ \\
\hline New York & 5.5 & $(4.4-6.8)$ & 12.7 & $(10.2-15.9)$ & 9.1 & $(7.5-10.9)$ & 58.5 & $(56.1-60.8)$ & 54.2 & $(50.8-57.6)$ & 56.2 & $(54.3-58.1)$ \\
\hline North Carolina & 7.1 & $(5.2-9.5)$ & 10.4 & $(7.7-13.9)$ & 9.0 & $(7.0-11.6)$ & 58.3 & $(54.7-61.8)$ & 58.1 & $(53.7-62.3)$ & 58.0 & $(55.0-61.0)$ \\
\hline North Dakota & 3.1 & $(2.0-4.8)$ & 4.6 & $(3.5-6.1)$ & 3.9 & $(3.1-5.0)$ & 64.4 & $(61.7-67.1)$ & 60.7 & $(57.1-64.1)$ & 62.5 & $(60.2-64.8)$ \\
\hline Oklahoma & 7.4 & $(5.9-9.4)$ & 7.1 & $(5.4-9.2)$ & 7.4 & $(6.1-9.0)$ & 51.8 & $(47.2-56.4)$ & 58.5 & $(53.5-63.3)$ & 55.0 & $(52.5-57.5)$ \\
\hline Pennsylvania & 6.6 & $(4.8-8.9)$ & 7.5 & $(6.4-8.9)$ & 7.1 & $(6.0-8.4)$ & 55.6 & $(51.3-59.8)$ & 62.4 & $(59.8-65.0)$ & 59.0 & $(56.2-61.8)$ \\
\hline Rhode Island & 4.8 & $(3.7-6.4)$ & 7.8 & $(5.9-10.3)$ & 6.4 & $(5.3-7.6)$ & 59.7 & $(53.1-66.0)$ & 61.1 & $(55.5-66.4)$ & 60.5 & $(55.1-65.6)$ \\
\hline South Carolina & 9.0 & $(6.8-11.9)$ & 9.4 & $(7.8-11.3)$ & 9.2 & (7.7-10.9) & 42.8 & (39.6-46.1) & 55.6 & $(50.5-60.5)$ & 49.2 & $(46.1-52.3)$ \\
\hline South Dakota & 5.3 & $(3.5-8.0)$ & 10.8 & $(7.5-15.4)$ & 8.1 & $(5.9-11.1)$ & 54.5 & $(49.4-59.6)$ & 55.8 & $(50.2-61.3)$ & 55.2 & $(51.9-58.6)$ \\
\hline Tennessee & 9.3 & $(8.0-10.7)$ & 10.1 & $(8.8-11.6)$ & 9.8 & $(8.8-10.8)$ & 48.4 & $(45.8-51.0)$ & 56.3 & $(52.9-59.6)$ & 52.4 & $(50.2-54.5)$ \\
\hline Vermont & 3.4 & $(3.1-3.8)$ & 5.1 & $(4.7-5.5)$ & 4.3 & $(4.0-4.6)$ & 66.7 & $(65.8-67.7)$ & 67.8 & $(66.9-68.7)$ & 67.3 & $(66.7-68.0)$ \\
\hline Virginia & 6.4 & $(5.2-7.9)$ & 7.5 & $(6.3-9.0)$ & 7.0 & $(6.2-7.8)$ & 55.7 & $(52.8-58.5)$ & 61.5 & $(58.5-64.4)$ & 58.8 & $(56.2-61.3)$ \\
\hline West Virginia & 5.7 & $(4.1-8.0)$ & 10.4 & $(7.6-14.2)$ & 8.1 & $(6.4-10.3)$ & 56.2 & $(49.9-62.3)$ & 53.9 & $(49.9-57.8)$ & 55.1 & $(51.5-58.6)$ \\
\hline Wyoming & 4.6 & $(3.2-6.5)$ & 9.3 & $(7.6-11.3)$ & 7.0 & $(5.8-8.4)$ & 58.8 & $(55.7-61.7)$ & 61.6 & $(58.1-65.1)$ & 60.2 & $(57.9-62.5)$ \\
\hline Median & \multirow{2}{*}{\multicolumn{2}{|c|}{$\begin{array}{c}5.4 \\
(2.8-10.4)\end{array}$}} & \multirow{2}{*}{\multicolumn{2}{|c|}{$\begin{array}{c}7.7 \\
(3.2-13.3)\end{array}$}} & \multirow{2}{*}{\multicolumn{2}{|c|}{$\begin{array}{c}7.0 \\
(3.2-11.7)\end{array}$}} & \multirow{2}{*}{\multicolumn{2}{|c|}{$\begin{array}{c}57.4 \\
(42.8-66.7)\end{array}$}} & \multirow{2}{*}{\multicolumn{2}{|c|}{$\begin{array}{c}59.6 \\
(51.1-69.1)\end{array}$}} & & 58.7 \\
\hline Range & & & & & & & & & & & & 9.0-67.7) \\
\hline Large urban school dist & rveys & & & & & & & & & & & \\
\hline Baltimore, MD & 11.4 & $(7.9-16.2)$ & 11.5 & $(8.3-15.8)$ & 11.4 & $(8.8-14.7)$ & 50.6 & $(45.5-55.7)$ & 61.6 & $(55.9-67.1)$ & 55.9 & $(51.3-60.4)$ \\
\hline Boston, MA & 4.3 & $(3.1-5.9)$ & 8.4 & $(6.7-10.5)$ & 6.5 & $(5.4-7.8)$ & 53.2 & $(49.5-57.0)$ & 59.0 & $(55.4-62.5)$ & 56.0 & $(53.7-58.3)$ \\
\hline Broward County, FL & 5.8 & $(4.2-7.9)$ & 10.4 & $(7.7-14.1)$ & 8.2 & $(6.3-10.6)$ & 58.4 & $(54.0-62.7)$ & 61.5 & $(57.0-65.8)$ & 59.9 & $(56.4-63.3)$ \\
\hline Cleveland, $\mathrm{OH}$ & 6.6 & $(4.8-8.9)$ & 7.0 & $(5.1-9.6)$ & 6.8 & $(5.4-8.6)$ & 56.1 & $(52.2-60.0)$ & 57.2 & $(53.1-61.2)$ & 56.7 & $(53.6-59.7)$ \\
\hline DeKalb County, GA & 4.9 & $(3.5-6.7)$ & 6.0 & $(4.5-7.9)$ & 5.4 & $(4.3-6.8)$ & 59.6 & $(55.6-63.6)$ & 61.7 & $(58.6-64.7)$ & 60.6 & $(57.8-63.2)$ \\
\hline Detroit, MI & 7.6 & $(5.7-10.1)$ & 12.4 & $(9.5-16.0)$ & 9.7 & $(7.8-12.1)$ & 55.0 & $(50.9-59.1)$ & 57.4 & $(53.1-61.6)$ & 56.1 & $(52.8-59.3)$ \\
\hline District of Columbia & 7.7 & $(7.0-8.6)$ & 8.8 & $(7.9-9.8)$ & 8.3 & $(7.7-8.9)$ & 50.2 & $(48.8-51.7)$ & 57.3 & $(55.6-58.9)$ & 53.6 & $(52.6-54.7)$ \\
\hline Duval County, FL & 8.4 & $(7.1-9.9)$ & 13.5 & $(11.5-15.7)$ & 10.8 & $(9.5-12.2)$ & 51.7 & $(49.0-54.3)$ & 52.9 & $(49.8-55.9)$ & 52.4 & $(50.3-54.6)$ \\
\hline Ft. Worth, TX & 3.7 & $(2.6-5.2)$ & 6.3 & $(5.0-7.9)$ & 5.0 & $(4.1-6.1)$ & 61.7 & $(58.7-64.6)$ & 67.0 & $(64.3-69.6)$ & 64.4 & $(62.3-66.4)$ \\
\hline Houston, TX & 5.5 & $(4.3-7.0)$ & 8.1 & $(6.6-9.9)$ & 6.8 & $(5.8-7.9)$ & 54.9 & $(51.9-57.9)$ & 59.3 & $(56.5-62.1)$ & 57.3 & $(55.3-59.3)$ \\
\hline Los Angeles, CA & 3.6 & $(2.2-5.7)$ & 5.5 & $(4.1-7.4)$ & 4.6 & $(3.4-6.1)$ & 63.1 & $(58.4-67.6)$ & 65.4 & $(62.2-68.5)$ & 64.1 & $(61.4-66.8)$ \\
\hline Miami-Dade County, FL & 7.7 & $(6.0-9.9)$ & 7.6 & $(6.0-9.4)$ & 7.8 & $(6.5-9.4)$ & 56.5 & $(53.0-60.0)$ & 62.7 & $(59.6-65.7)$ & 59.6 & $(57.1-62.0)$ \\
\hline
\end{tabular}

See table footnotes on the next page. 
TABLE 84. (Continued) Percentage of high school students who did not eat fruit or drink $100 \%$ fruit juices* and who ate fruit or drank $100 \%$ fruit juices one or more times/day,* by sex — selected U.S. sites, Youth Risk Behavior Survey, 2015

\begin{tabular}{|c|c|c|c|c|c|c|c|c|c|c|c|c|}
\hline \multirow[b]{3}{*}{ Site } & \multicolumn{6}{|c|}{ Did not eat fruit or drink $100 \%$ fruit juices } & \multicolumn{6}{|c|}{ Ate fruit or drank $100 \%$ fruit juices one or more times/day } \\
\hline & \multicolumn{2}{|c|}{ Female } & \multicolumn{2}{|c|}{ Male } & \multicolumn{2}{|c|}{ Total } & \multicolumn{2}{|c|}{ Female } & \multicolumn{2}{|r|}{ Male } & \multicolumn{2}{|r|}{ Total } \\
\hline & $\%$ & $\mathrm{Cl}^{\dagger}$ & $\%$ & $\mathrm{Cl}$ & $\%$ & $\mathrm{Cl}$ & $\%$ & $\mathrm{Cl}$ & $\%$ & $\mathrm{Cl}$ & $\%$ & $\mathrm{Cl}$ \\
\hline New York City, NY & 7.0 & $(5.9-8.3)$ & 9.1 & $(7.6-10.9)$ & 8.1 & $(7.1-9.2)$ & 49.2 & $(46.6-51.7)$ & 56.0 & $(53.4-58.6)$ & 52.6 & $(50.6-54.6)$ \\
\hline Oakland, CA & - & - & - & - & - & - & - & - & - & - & - & - \\
\hline Orange County, FL & 4.5 & $(3.2-6.1)$ & 7.3 & $(5.5-9.6)$ & 5.9 & $(4.8-7.3)$ & 61.1 & $(57.8-64.4)$ & 65.1 & $(61.4-68.7)$ & 63.2 & $(60.4-65.9)$ \\
\hline Palm Beach County, FL & 6.7 & $(5.2-8.5)$ & 8.2 & $(6.7-10.1)$ & 7.5 & $(6.4-8.8)$ & 57.0 & $(53.8-60.0)$ & 58.4 & $(54.8-61.8)$ & 57.9 & $(55.8-60.0)$ \\
\hline Philadelphia, PA & 8.3 & $(6.3-10.9)$ & 8.7 & $(6.8-11.0)$ & 8.5 & $(7.1-10.2)$ & 54.2 & $(49.4-59.0)$ & 59.5 & $(55.3-63.6)$ & 56.9 & $(54.0-59.7)$ \\
\hline San Diego, CA & 3.5 & $(2.5-4.9)$ & 4.1 & $(3.0-5.6)$ & 3.8 & $(3.0-4.8)$ & 62.9 & $(58.7-66.8)$ & 68.0 & $(64.6-71.2)$ & 65.6 & (62.5-68.5) \\
\hline San Francisco, CA & 2.9 & $(1.9-4.4)$ & 6.2 & $(4.2-9.0)$ & 4.7 & (3.4-6.4) & 64.4 & $(60.7-67.9)$ & 69.4 & $(65.6-72.9)$ & 66.7 & $(64.2-69.1)$ \\
\hline Median & \multicolumn{2}{|r|}{6.2} & \multicolumn{2}{|r|}{8.1} & \multicolumn{2}{|r|}{7.1} & \multicolumn{2}{|r|}{56.3} & \multicolumn{2}{|r|}{60.5} & \multicolumn{2}{|c|}{57.6} \\
\hline Range & \multicolumn{2}{|c|}{$(2.9-11.4)$} & \multicolumn{2}{|c|}{$(4.1-13.5)$} & \multicolumn{2}{|c|}{$(3.8-11.4)$} & \multicolumn{2}{|c|}{$(49.2-64.4)$} & \multicolumn{2}{|c|}{ (52.9-69.4) } & \multicolumn{2}{|c|}{$(52.4-66.7)$} \\
\hline
\end{tabular}

* During the 7 days before the survey.

† $95 \%$ confidence interval.

$\S$ Not available.

TABLE 85. Percentage of high school students who ate fruit or drank $100 \%$ fruit juices two or more times/day* and who ate fruit or drank $100 \%$ fruit juices three or more times/day, ${ }^{*}$ by sex, race/ethnicity, and grade — United States, Youth Risk Behavior Survey, 2015

\begin{tabular}{|c|c|c|c|c|c|c|c|c|c|c|c|c|}
\hline \multirow[b]{3}{*}{ Category } & \multicolumn{6}{|c|}{ Ate fruit or drank $100 \%$ fruit juices two or more times/day } & \multicolumn{6}{|c|}{ Ate fruit or drank $100 \%$ fruit juices three or more times/day } \\
\hline & \multicolumn{2}{|c|}{ Female } & \multicolumn{2}{|r|}{ Male } & \multicolumn{2}{|r|}{ Total } & \multicolumn{2}{|c|}{ Female } & \multicolumn{2}{|r|}{ Male } & \multicolumn{2}{|r|}{ Total } \\
\hline & $\%$ & $\mathrm{Cl}^{\dagger}$ & $\%$ & $\mathrm{Cl}$ & $\%$ & $\mathrm{Cl}$ & $\%$ & $\mathrm{Cl}$ & $\%$ & $\mathrm{Cl}$ & $\%$ & $\mathrm{Cl}$ \\
\hline \multicolumn{13}{|c|}{ Race/Ethnicity } \\
\hline White ${ }^{\S}$ & 29.8 & $(27.1-32.6)$ & 29.9 & $(27.0-32.9)$ & 29.8 & $(27.3-32.3)$ & 16.0 & $(14.5-17.6)$ & 18.0 & (15.9-20.3) & 17.0 & $(15.5-18.6)$ \\
\hline Black $^{\S}$ & 27.8 & $(24.2-31.7)$ & 38.2 & (33.0-43.7) & 33.3 & (30.3-36.4) & 20.5 & $(16.9-24.7)$ & 29.1 & (25.0-33.6) & 25.1 & (22.9-27.5) \\
\hline Hispanic & 29.9 & $(27.2-32.8)$ & 36.7 & $(34.3-39.2)$ & 33.4 & $(31.5-35.3)$ & 20.5 & $(18.5-22.5)$ & 26.6 & $(23.8-29.5)$ & 23.6 & $(21.8-25.4)$ \\
\hline \multicolumn{13}{|l|}{ Grade } \\
\hline 9 & 30.0 & $(27.0-33.3)$ & 35.0 & (31.4-38.7) & 32.6 & $(30.0-35.3)$ & 16.9 & $(14.7-19.5)$ & 24.3 & $(21.2-27.8)$ & 20.8 & $(18.7-23.1)$ \\
\hline 10 & 28.7 & $(26.0-31.6)$ & 33.9 & $(29.3-38.7)$ & 31.2 & (28.8-33.7) & 17.4 & $(14.3-21.1)$ & 21.6 & $(18.5-25.1)$ & 19.5 & $(17.5-21.6)$ \\
\hline 11 & 30.3 & $(27.8-32.8)$ & 32.1 & $(28.8-35.7)$ & 31.1 & $(29.2-33.2)$ & 19.6 & $(17.6-21.7)$ & 20.9 & $(18.5-23.5)$ & 20.2 & $(18.6-21.9)$ \\
\hline 12 & 31.2 & $(27.8-34.7)$ & 31.0 & $(27.5-34.8)$ & 31.0 & $(29.0-33.1)$ & 18.2 & (15.9-20.8) & 20.5 & (18.0-23.3) & 19.4 & $(17.6-21.2)$ \\
\hline Total & 30.0 & $(28.5-31.5)$ & 33.1 & $(31.0-35.3)$ & 31.5 & $(30.1-33.0)$ & 18.0 & $(16.7-19.3)$ & 22.1 & $(20.6-23.6)$ & 20.0 & $(18.9-21.1)$ \\
\hline
\end{tabular}

* During the 7 days before the survey.

† $95 \%$ confidence interval.

$\S$ Non-Hispanic. 
TABLE 86. Percentage of high school students who ate fruit or drank $100 \%$ fruit juices two or more times/day* and who ate fruit or drank $100 \%$ fruit juices three or more times/day, ${ }^{*}$ by sex — selected U.S. sites, Youth Risk Behavior Survey, 2015

\begin{tabular}{|c|c|c|c|c|c|c|c|c|c|c|c|c|}
\hline \multirow[b]{3}{*}{ Site } & \multicolumn{6}{|c|}{ Ate fruit or drank $100 \%$ fruit juices two or more times/day } & \multicolumn{6}{|c|}{ Ate fruit or drank $100 \%$ fruit juices three or more times/day } \\
\hline & \multicolumn{2}{|r|}{ Female } & \multicolumn{2}{|r|}{ Male } & \multicolumn{2}{|r|}{ Total } & \multicolumn{2}{|c|}{ Female } & \multicolumn{2}{|r|}{ Male } & \multicolumn{2}{|r|}{ Total } \\
\hline & $\%$ & $\mathrm{Cl}^{\dagger}$ & $\%$ & $\mathrm{Cl}$ & $\%$ & $\mathrm{Cl}$ & $\%$ & $\mathrm{Cl}$ & $\%$ & $\mathrm{Cl}$ & $\%$ & $\mathrm{Cl}$ \\
\hline \multicolumn{13}{|l|}{ State surveys } \\
\hline Alabama & 22.9 & $(18.3-28.2)$ & 23.6 & $(20.0-27.6)$ & 23.4 & (21.0-25.9) & 13.9 & (10.2-18.5) & 16.2 & (13.3-19.5) & 15.0 & $(13.1-17.1)$ \\
\hline Alaska & 26.8 & $(23.6-30.2)$ & 30.3 & $(25.9-35.0)$ & 28.6 & (25.8-31.5) & 15.9 & (13.3-18.9) & 18.6 & $(15.1-22.7)$ & 17.3 & $(15.0-20.0)$ \\
\hline Arizona & 30.5 & $(25.8-35.6)$ & 29.4 & $(25.9-33.2)$ & 30.1 & (26.7-33.7) & 17.2 & $(12.9-22.7)$ & 19.3 & $(16.4-22.7)$ & 18.5 & $(15.5-21.8)$ \\
\hline Arkansas & 23.1 & $(19.6-27.1)$ & 29.5 & $(23.8-36.0)$ & 26.3 & (22.3-30.7) & 14.6 & $(12.2-17.3)$ & 18.6 & $(14.1-24.1)$ & 16.5 & $(13.8-19.8)$ \\
\hline California & 32.0 & $(29.1-35.0)$ & 34.7 & $(32.1-37.4)$ & 33.3 & (31.1-35.5) & 18.7 & $(16.3-21.4)$ & 21.4 & $(19.0-24.0)$ & 20.1 & $(18.4-22.0)$ \\
\hline Connecticut & 33.4 & $(29.8-37.2)$ & 27.7 & $(24.2-31.4)$ & 30.5 & $(27.7-33.5)$ & 18.7 & $(16.4-21.3)$ & 17.5 & (14.7-20.7) & 18.1 & $(16.1-20.3)$ \\
\hline Delaware & 28.8 & $(25.7-32.1)$ & 33.2 & $(29.9-36.6)$ & 31.0 & $(28.6-33.4)$ & 17.9 & $(15.6-20.5)$ & 22.5 & $(19.6-25.7)$ & 20.2 & $(18.3-22.1)$ \\
\hline Florida & 29.9 & $(28.2-31.7)$ & 36.0 & $(34.0-38.2)$ & 33.2 & (31.6-34.8) & 19.4 & $(17.8-21.2)$ & 25.1 & $(23.2-27.1)$ & 22.5 & $(21.2-23.9)$ \\
\hline Hawaii & 21.2 & (19.6-23.0) & 24.8 & $(22.7-27.1)$ & 23.2 & $(21.8-24.6)$ & 12.7 & (11.2-14.4) & 16.0 & (14.3-17.8) & 14.5 & (13.4-15.7) \\
\hline Idaho & 24.3 & $(22.1-26.7)$ & 27.0 & $(23.2-31.1)$ & 25.6 & $(23.1-28.3)$ & 12.7 & $(10.7-15.0)$ & 16.2 & $(13.4-19.6)$ & 14.5 & (12.4-16.9) \\
\hline Illinois & 27.7 & $(23.5-32.4)$ & 34.0 & (30.9-37.3) & 30.9 & $(28.2-33.7)$ & 17.3 & (14.5-20.5) & 21.1 & $(18.2-24.3)$ & 19.2 & $(16.9-21.8)$ \\
\hline Indiana & 23.6 & $(19.1-28.9)$ & 27.2 & $(23.3-31.5)$ & 25.5 & $(21.8-29.6)$ & 13.4 & $(10.3-17.3)$ & 14.4 & $(12.2-17.1)$ & 13.9 & $(12.1-15.9)$ \\
\hline Kentucky & 21.9 & $(18.9-25.1)$ & 20.1 & $(17.0-23.4)$ & 21.0 & $(19.3-22.9)$ & 13.0 & $(10.5-16.0)$ & 14.0 & $(11.7-16.7)$ & 13.6 & $(12.2-15.1)$ \\
\hline Maine & 29.7 & $(27.2-32.2)$ & 30.4 & $(29.1-31.8)$ & 30.1 & $(28.6-31.6)$ & 17.0 & (15.2-18.9) & 17.9 & $(16.7-19.1)$ & 17.4 & (16.3-18.6) \\
\hline Maryland & 27.3 & $(26.6-28.1)$ & 30.3 & $(29.6-31.1)$ & 28.8 & $(28.3-29.3)$ & 16.2 & (15.6-16.8) & 19.9 & (19.3-20.6) & 18.1 & $(17.6-18.5)$ \\
\hline Massachusetts & 30.6 & (26.9-34.6) & 31.9 & $(29.5-34.5)$ & 31.4 & (29.3-33.5) & 16.8 & (14.4-19.4) & 19.6 & $(17.2-22.3)$ & 18.3 & $(16.8-19.8)$ \\
\hline Michigan & 26.3 & $(22.7-30.3)$ & 28.7 & $(26.0-31.6)$ & 27.5 & $(24.9-30.3)$ & 14.3 & $(11.7-17.2)$ & 17.6 & $(15.6-19.8)$ & 15.9 & (14.1-17.9) \\
\hline Mississippi & 24.2 & $(20.3-28.5)$ & 26.4 & (23.1-29.9) & 25.2 & $(22.3-28.3)$ & 17.0 & $(13.5-21.1)$ & 19.3 & $(16.3-22.8)$ & 18.1 & $(15.4-21.1)$ \\
\hline Missouri & 23.4 & $(20.1-27.1)$ & 24.8 & $(21.6-28.2)$ & 24.1 & $(22.1-26.2)$ & 12.2 & $(8.8-16.7)$ & 15.4 & $(12.6-18.7)$ & 13.7 & $(11.2-16.7)$ \\
\hline Montana & 26.1 & (23.9-28.4) & 28.8 & $(26.3-31.4)$ & 27.5 & $(25.7-29.3)$ & 14.1 & $(12.7-15.7)$ & 17.2 & (14.8-19.9) & 15.7 & (14.3-17.3) \\
\hline Nebraska & 27.4 & $(23.9-31.2)$ & 26.2 & $(23.2-29.5)$ & 26.8 & $(24.6-29.2)$ & 15.8 & $(12.9-19.1)$ & 14.6 & $(12.2-17.4)$ & 15.2 & $(13.5-17.1)$ \\
\hline Nevada & 30.1 & $(24.4-36.5)$ & 28.6 & $(25.4-32.1)$ & 29.3 & $(26.1-32.7)$ & 17.8 & $(14.2-22.1)$ & 17.5 & (14.8-20.5) & 17.6 & $(15.1-20.4)$ \\
\hline New Hampshire & —§ & - & - & - & - & - & - & - & - & - & - & - \\
\hline New Mexico & 25.5 & (23.4-27.7) & 29.5 & $(27.7-31.3)$ & 27.5 & $(26.1-28.9)$ & 16.0 & (14.5-17.5) & 20.6 & $(19.1-22.2)$ & 18.3 & (17.2-19.5) \\
\hline New York & 32.2 & (29.4-35.2) & 28.8 & $(25.8-32.0)$ & 30.5 & $(28.3-32.7)$ & 19.4 & $(16.9-22.1)$ & 17.6 & (15.8-19.5) & 18.5 & $(16.7-20.4)$ \\
\hline North Carolina & 29.2 & $(25.8-32.8)$ & 25.5 & $(21.6-30.0)$ & 27.3 & (24.9-29.9) & 17.1 & $(13.7-21.1)$ & 16.9 & $(13.3-21.3)$ & 17.0 & $(14.3-20.1)$ \\
\hline North Dakota & 27.3 & $(24.0-30.8)$ & 27.8 & (24.3-31.6) & 27.6 & (24.9-30.4) & 14.0 & (11.7-16.6) & 16.0 & (13.7-18.6) & 15.1 & $(13.2-17.1)$ \\
\hline Oklahoma & 25.6 & (21.1-30.8) & 30.9 & $(27.4-34.6)$ & 28.2 & (26.0-30.5) & 12.9 & (9.9-16.6) & 21.8 & $(18.5-25.6)$ & 17.3 & (15.2-19.7) \\
\hline Pennsylvania & 24.7 & (22.0-27.6) & 32.4 & $(29.3-35.7)$ & 28.6 & $(26.1-31.1)$ & 11.5 & (9.7-13.7) & 21.2 & (19.0-23.5) & 16.4 & $(14.8-18.2)$ \\
\hline Rhode Island & 28.2 & (22.5-34.7) & 30.8 & $(27.0-34.9)$ & 29.6 & (25.3-34.3) & 15.5 & (11.7-20.3) & 17.3 & $(12.9-22.7)$ & 16.5 & $(12.7-21.1)$ \\
\hline South Carolina & 18.6 & $(14.4-23.7)$ & 29.1 & $(24.2-34.6)$ & 23.9 & $(20.1-28.1)$ & 13.7 & $(9.9-18.6)$ & 18.0 & $(14.2-22.4)$ & 15.9 & $(12.6-19.9)$ \\
\hline South Dakota & 22.2 & $(18.7-26.0)$ & 25.6 & $(20.8-31.1)$ & 24.0 & $(20.5-28.0)$ & 11.0 & $(7.2-16.3)$ & 15.0 & $(11.5-19.3)$ & 13.1 & $(10.1-16.7)$ \\
\hline Tennessee & 19.9 & $(18.2-21.7)$ & 26.2 & (23.3-29.4) & 23.1 & $(21.1-25.2)$ & 11.3 & $(9.7-13.1)$ & 16.4 & $(14.0-19.1)$ & 13.9 & $(12.2-15.8)$ \\
\hline Vermont & 33.5 & $(32.6-34.4)$ & 35.0 & $(34.1-36.0)$ & 34.3 & $(33.7-35.0)$ & 17.9 & $(17.2-18.7)$ & 21.6 & $(20.9-22.5)$ & 19.9 & $(19.3-20.4)$ \\
\hline Virginia & 26.5 & $(23.8-29.5)$ & 32.6 & $(29.6-35.7)$ & 29.8 & $(27.5-32.2)$ & 14.7 & $(12.6-17.1)$ & 19.0 & (16.9-21.3) & 17.0 & $(15.3-18.8)$ \\
\hline West Virginia & 26.8 & $(22.4-31.7)$ & 28.9 & $(26.3-31.6)$ & 27.9 & $(25.5-30.5)$ & 16.7 & $(13.3-20.8)$ & 19.8 & $(16.3-23.8)$ & 18.2 & $(16.1-20.7)$ \\
\hline Wyoming & 25.2 & $(22.8-27.7)$ & 28.0 & $(25.0-31.2)$ & 26.7 & $(24.5-29.0)$ & 14.3 & $(12.4-16.5)$ & 17.3 & $(14.7-20.2)$ & 15.9 & $(14.1-17.9)$ \\
\hline Median & & 26.6 & & 28.8 & & 27.7 & & 15.6 & & 17.7 & & 17.0 \\
\hline Range & & $8.6-33.5)$ & & $0.1-36.0)$ & & $1.0-34.3)$ & & 1.0-19.4) & & $4.0-25.1)$ & & $3.1-22.5)$ \\
\hline \multicolumn{13}{|c|}{ Large urban school district surveys } \\
\hline Baltimore, MD & 28.5 & $(24.2-33.3)$ & 35.7 & $(29.4-42.5)$ & 32.2 & $(28.1-36.6)$ & 20.9 & $(16.2-26.5)$ & 22.4 & $(17.8-27.8)$ & 21.5 & $(18.1-25.3)$ \\
\hline Boston, MA & 29.6 & $(26.8-32.5)$ & 35.1 & $(31.9-38.5)$ & 32.3 & $(30.2-34.4)$ & 18.6 & $(16.1-21.5)$ & 24.2 & $(21.1-27.6)$ & 21.4 & $(19.3-23.6)$ \\
\hline Broward County, FL & 32.9 & $(30.0-35.9)$ & 31.7 & $(27.3-36.5)$ & 32.4 & $(29.7-35.2)$ & 21.2 & $(18.6-24.1)$ & 21.9 & $(18.5-25.7)$ & 21.6 & $(19.5-23.9)$ \\
\hline Cleveland, $\mathrm{OH}$ & 28.5 & $(25.4-31.8)$ & 31.9 & $(28.3-35.7)$ & 30.0 & $(27.7-32.6)$ & 20.3 & $(17.2-23.7)$ & 23.1 & $(19.9-26.6)$ & 21.6 & $(19.3-24.0)$ \\
\hline DeKalb County, GA & 32.0 & $(28.9-35.3)$ & 35.1 & $(31.8-38.7)$ & 33.5 & $(31.1-36.0)$ & 20.8 & $(17.9-24.1)$ & 24.0 & $(21.3-26.9)$ & 22.3 & $(20.5-24.3)$ \\
\hline Detroit, MI & 22.7 & $(19.8-25.9)$ & 25.4 & $(22.0-29.2)$ & 24.0 & $(21.8-26.4)$ & 15.0 & $(12.8-17.6)$ & 19.9 & $(17.1-23.1)$ & 17.3 & $(15.7-19.1)$ \\
\hline District of Columbia & 25.4 & $(24.1-26.7)$ & 30.8 & $(29.4-32.4)$ & 28.0 & $(27.0-29.0)$ & 16.7 & $(15.6-17.8)$ & 21.9 & $(20.6-23.3)$ & 19.2 & $(18.3-20.1)$ \\
\hline Duval County, FL & 26.4 & $(24.3-28.7)$ & 26.7 & $(24.1-29.6)$ & 26.6 & $(24.7-28.5)$ & 18.2 & $(16.4-20.2)$ & 17.8 & $(15.7-20.2)$ & 18.0 & $(16.5-19.7)$ \\
\hline Ft. Worth, TX & 31.6 & $(28.6-34.8)$ & 37.4 & $(34.1-40.8)$ & 34.5 & $(32.3-36.8)$ & 22.0 & $(19.5-24.6)$ & 27.4 & $(24.5-30.4)$ & 24.6 & $(22.8-26.6)$ \\
\hline Houston, TX & 29.3 & $(27.0-31.7)$ & 29.7 & $(27.0-32.6)$ & 29.6 & $(27.8-31.5)$ & 18.5 & $(16.5-20.8)$ & 20.9 & $(18.4-23.6)$ & 19.8 & $(18.2-21.5)$ \\
\hline Los Angeles, CA & 30.0 & $(26.6-33.6)$ & 37.5 & (33.5-41.7) & 33.6 & $(31.5-35.7)$ & 17.3 & $(14.3-20.7)$ & 25.5 & $(22.0-29.5)$ & 21.3 & $(19.4-23.3)$ \\
\hline Miami-Dade County, FL & 31.4 & $(28.8-34.2)$ & 36.6 & $(33.5-39.8)$ & 34.1 & $(32.2-36.1)$ & 21.1 & $(18.7-23.8)$ & 26.5 & $(23.4-29.8)$ & 23.7 & $(21.9-25.6)$ \\
\hline
\end{tabular}

See table footnotes on the next page. 
TABLE 86. (Continued) Percentage of high school students who ate fruit or drank $100 \%$ fruit juices two or more times/day* and who ate fruit or drank $100 \%$ fruit juices three or more times/day, ${ }^{*}$ by sex — selected U.S. sites, Youth Risk Behavior Survey, 2015

\begin{tabular}{|c|c|c|c|c|c|c|c|c|c|c|c|c|}
\hline \multirow[b]{3}{*}{ Site } & \multicolumn{6}{|c|}{ Ate fruit or drank $100 \%$ fruit juices two or more times/day } & \multicolumn{6}{|c|}{ Ate fruit or drank $100 \%$ fruit juices three or more times/day } \\
\hline & \multicolumn{2}{|c|}{ Female } & \multicolumn{2}{|r|}{ Male } & \multicolumn{2}{|r|}{ Total } & \multicolumn{2}{|c|}{ Female } & \multicolumn{2}{|r|}{ Male } & \multicolumn{2}{|r|}{ Total } \\
\hline & $\%$ & $\mathrm{Cl}^{\dagger}$ & $\%$ & $\mathrm{Cl}$ & $\%$ & $\mathrm{Cl}$ & $\%$ & $\mathrm{Cl}$ & $\%$ & $\mathrm{Cl}$ & $\%$ & $\mathrm{Cl}$ \\
\hline New York City, NY & 26.3 & $(24.3-28.4)$ & 30.4 & $(27.4-33.5)$ & 28.4 & $(26.3-30.6)$ & 17.3 & $(15.7-19.1)$ & 20.3 & $(18.7-22.0)$ & 18.9 & $(17.6-20.2)$ \\
\hline Oakland, CA & - & - & - & - & - & - & - & - & - & - & - & - \\
\hline Orange County, FL & 30.9 & $(26.8-35.3)$ & 37.4 & $(33.6-41.4)$ & 34.2 & (30.8-37.6) & 17.6 & $(14.7-20.9)$ & 25.8 & $(22.2-29.7)$ & 21.8 & $(19.1-24.7)$ \\
\hline Palm Beach County, FL & 29.4 & $(26.6-32.4)$ & 32.3 & $(29.4-35.4)$ & 31.1 & $(29.0-33.3)$ & 17.5 & $(15.3-20.0)$ & 22.2 & $(19.3-25.4)$ & 20.1 & $(17.9-22.4)$ \\
\hline Philadelphia, PA & 30.7 & $(25.8-36.0)$ & 33.9 & $(31.1-36.8)$ & 32.2 & $(29.3-35.3)$ & 22.0 & $(17.1-27.9)$ & 24.3 & $(21.5-27.2)$ & 23.0 & (19.9-26.5) \\
\hline San Diego, CA & 31.1 & $(27.3-35.2)$ & 35.5 & $(32.0-39.2)$ & 33.4 & $(30.4-36.5)$ & 18.6 & $(15.6-22.1)$ & 22.4 & (19.4-25.6) & 20.6 & $(18.2-23.3)$ \\
\hline San Francisco, CA & 35.4 & $(31.7-39.3)$ & 40.0 & $(36.0-44.0)$ & 37.6 & (35.2-40.2) & 20.2 & $(17.0-23.8)$ & 24.9 & $(21.6-28.5)$ & 22.5 & (20.3-24.9) \\
\hline Median & & 29.8 & & 34.5 & & 32.2 & & 18.6 & & 22.7 & & 21.4 \\
\hline Range & & 2.7-35.4) & & $5.4-40.0)$ & & $4.0-37.6)$ & & $5.0-22.0)$ & & $7.8-27.4)$ & & 7.3-24.6) \\
\hline
\end{tabular}

* During the 7 days before the survey.

† $95 \%$ confidence interval.

$\S$ Not available.

TABLE 87. Percentage of high school students who did not eat vegetables*, ${ }^{\dagger}$ and who ate vegetables* one or more times/day, ${ }^{\dagger}$ by sex, race/ ethnicity, and grade - United States, Youth Risk Behavior Survey, 2015

\begin{tabular}{|c|c|c|c|c|c|c|c|c|c|c|c|c|}
\hline \multirow[b]{3}{*}{ Category } & \multicolumn{6}{|c|}{ Did not eat vegetables } & \multicolumn{6}{|c|}{ Ate vegetables one or more times/day } \\
\hline & \multicolumn{2}{|c|}{ Female } & \multicolumn{2}{|r|}{ Male } & \multicolumn{2}{|c|}{ Total } & \multicolumn{2}{|r|}{ Female } & \multicolumn{2}{|r|}{ Male } & \multicolumn{2}{|r|}{ Total } \\
\hline & $\%$ & $\mathrm{Cl}^{\S}$ & $\%$ & $\mathrm{Cl}$ & $\%$ & $\mathrm{Cl}$ & $\%$ & $\mathrm{Cl}$ & $\%$ & $\mathrm{Cl}$ & $\%$ & $\mathrm{Cl}$ \\
\hline \multicolumn{13}{|c|}{ Race/Ethnicity } \\
\hline White & 3.7 & $(2.9-4.7)$ & 6.0 & $(5.0-7.3)$ & 4.9 & $(4.2-5.8)$ & 64.5 & $(61.2-67.7)$ & 64.0 & $(61.4-66.6)$ & 64.2 & $(61.7-66.7)$ \\
\hline Black? & 8.8 & $(6.6-11.6)$ & 13.0 & $(10.6-15.8)$ & 10.9 & $(9.2-13.0)$ & 48.0 & $(41.1-55.0)$ & 56.5 & $(52.4-60.5)$ & 52.5 & $(48.6-56.4)$ \\
\hline Hispanic & 7.7 & $(6.2-9.5)$ & 9.2 & $(7.4-11.4)$ & 8.5 & $(7.3-9.7)$ & 55.3 & $(52.5-58.0)$ & 57.7 & $(55.0-60.3)$ & 56.5 & $(54.8-58.3)$ \\
\hline \multicolumn{13}{|l|}{ Grade } \\
\hline 9 & 6.0 & $(4.9-7.3)$ & 7.2 & (5.3-9.7) & 6.6 & $(5.4-8.1)$ & 57.3 & (53.9-60.5) & 59.7 & (54.9-64.4) & 58.6 & $(55.3-61.8)$ \\
\hline 10 & 5.9 & $(4.7-7.4)$ & 8.4 & $(6.3-11.3)$ & 7.1 & $(5.8-8.8)$ & 58.6 & $(55.1-62.0)$ & 60.9 & $(56.9-64.9)$ & 59.9 & $(56.9-62.7)$ \\
\hline 11 & 4.5 & $(3.3-6.0)$ & 8.0 & $(6.3-10.1)$ & 6.6 & $(5.6-7.8)$ & 61.3 & $(57.1-65.3)$ & 63.9 & $(60.6-67.0)$ & 62.5 & $(59.7-65.2)$ \\
\hline 12 & 5.7 & $(4.3-7.5)$ & 7.2 & $(5.5-9.3)$ & 6.4 & (5.2-7.9) & 63.1 & (59.4-66.6) & 63.9 & $(60.4-67.3)$ & 63.5 & $(60.9-66.0)$ \\
\hline Total & 5.6 & $(4.8-6.4)$ & 7.7 & $(6.6-8.9)$ & 6.7 & $(6.0-7.5)$ & 60.0 & $(57.5-62.4)$ & 62.0 & $(59.7-64.2)$ & 61.0 & $(58.9-63.0)$ \\
\hline
\end{tabular}

* Green salad, potatoes (excluding French fries, fried potatoes, or potato chips), carrots, or other vegetables.

$\dagger$ During the 7 days before the survey.

$\S 95 \%$ confidence interval.

" Non-Hispanic. 
TABLE 88. Percentage of high school students who did not eat vegetables ${ }^{*},+$ and who ate vegetables* one or more times/day, ${ }^{\dagger}$ by sex - selected U.S. sites, Youth Risk Behavior Survey, 2015

\begin{tabular}{|c|c|c|c|c|c|c|c|c|c|c|c|c|}
\hline \multirow[b]{3}{*}{ Site } & \multicolumn{6}{|c|}{ Did not eat vegetables } & \multicolumn{6}{|c|}{ Ate vegetables one or more times/day } \\
\hline & \multicolumn{2}{|c|}{ Female } & \multicolumn{2}{|r|}{ Male } & \multicolumn{2}{|r|}{ Total } & \multicolumn{2}{|c|}{ Female } & \multicolumn{2}{|r|}{ Male } & \multicolumn{2}{|r|}{ Total } \\
\hline & $\%$ & $\mathrm{Cl}^{\S}$ & $\%$ & $\mathrm{Cl}$ & $\%$ & $\mathrm{Cl}$ & $\%$ & $\mathrm{Cl}$ & $\%$ & $\mathrm{Cl}$ & $\%$ & $\mathrm{Cl}$ \\
\hline \multicolumn{13}{|l|}{ State surveys } \\
\hline Alabama & 10.0 & $(7.9-12.6)$ & 11.0 & $(8.7-13.9)$ & 10.4 & $(9.0-12.1)$ & 49.0 & $(45.2-52.8)$ & 52.8 & $(48.5-57.1)$ & 51.2 & $(47.9-54.6)$ \\
\hline Alaska & 4.0 & $(2.8-5.6)$ & 6.8 & $(5.2-8.8)$ & 5.4 & $(4.3-6.8)$ & 64.3 & $(60.3-68.2)$ & 62.7 & $(59.0-66.3)$ & 63.7 & $(60.6-66.7)$ \\
\hline Arizona & 6.1 & $(4.6-8.0)$ & 8.1 & $(5.8-11.3)$ & 7.1 & $(5.5-9.3)$ & 60.0 & $(54.5-65.2)$ & 57.9 & $(54.9-60.9)$ & 59.1 & $(55.5-62.6)$ \\
\hline Arkansas & 10.7 & $(8.8-13.0)$ & 11.9 & $(9.6-14.7)$ & 11.3 & $(10.0-12.8)$ & 53.9 & $(50.8-56.9)$ & 54.8 & $(51.3-58.3)$ & 54.5 & $(52.4-56.6)$ \\
\hline California & 6.4 & $(4.5-9.0)$ & 7.4 & $(4.0-13.2)$ & 6.9 & $(4.4-10.7)$ & 61.4 & $(56.4-66.2)$ & 61.7 & $(55.8-67.3)$ & 61.6 & $(56.7-66.3)$ \\
\hline Connecticut & 6.3 & $(4.7-8.3)$ & 7.7 & $(6.3-9.3)$ & 6.9 & $(5.8-8.3)$ & 66.3 & $(62.3-70.2)$ & 63.1 & $(59.5-66.6)$ & 64.8 & $(61.6-67.8)$ \\
\hline Delaware & — & - & - & - & - & - & - & - & - & - & - & - \\
\hline Florida & 8.1 & $(7.1-9.4)$ & 11.0 & $(9.8-12.3)$ & 9.6 & $(8.6-10.6)$ & 55.6 & $(53.1-58.1)$ & 58.1 & $(55.8-60.3)$ & 56.9 & $(54.9-58.8)$ \\
\hline Hawaii & - & - & - & - & - & - & - & - & - & - & - & - \\
\hline Idaho & 2.8 & $(2.0-4.1)$ & 3.9 & $(2.5-6.0)$ & 3.3 & $(2.4-4.7)$ & 66.1 & (61.7-70.2) & 63.9 & $(60.1-67.5)$ & 64.8 & $(62.5-67.1)$ \\
\hline Illinois & 8.4 & $(6.4-10.8)$ & 11.2 & $(9.1-13.7)$ & 9.8 & (8.4-11.4) & 56.2 & $(50.7-61.6)$ & 58.0 & $(53.3-62.6)$ & 57.1 & (53.5-60.6) \\
\hline Indiana & 5.9 & $(4.1-8.5)$ & 8.6 & $(6.2-11.9)$ & 7.3 & (5.4-9.9) & 58.9 & $(53.8-63.8)$ & 56.0 & $(51.5-60.4)$ & 57.5 & (53.9-61.1) \\
\hline Kentucky & 6.5 & $(4.5-9.1)$ & 10.4 & $(8.1-13.4)$ & 8.5 & $(6.9-10.5)$ & 58.8 & $(53.6-63.8)$ & 55.3 & $(51.8-58.7)$ & 57.1 & (54.3-59.9) \\
\hline Maine & - & - & - & - & - & - & - & - & - & - & - & - \\
\hline Maryland & 6.9 & $(6.5-7.3)$ & 9.8 & $(9.3-10.4)$ & 8.4 & $(8.0-8.8)$ & 58.3 & $(57.4-59.2)$ & 59.9 & $(59.1-60.8)$ & 59.2 & $(58.5-59.9)$ \\
\hline Massachusetts & 5.7 & $(4.3-7.5)$ & 6.3 & $(4.7-8.4)$ & 6.0 & $(4.7-7.6)$ & 61.8 & $(57.5-65.9)$ & 62.9 & $(58.9-66.7)$ & 62.4 & $(58.8-65.8)$ \\
\hline Michigan & 6.0 & $(4.2-8.6)$ & 6.7 & $(5.2-8.5)$ & 6.3 & $(4.9-8.0)$ & 58.3 & $(54.0-62.4)$ & 55.9 & $(51.2-60.5)$ & 57.1 & $(53.6-60.6)$ \\
\hline Mississippi & 9.2 & $(6.7-12.5)$ & 12.8 & $(10.5-15.5)$ & 10.9 & (9.3-12.9) & 51.3 & $(47.3-55.2)$ & 52.4 & $(49.1-55.6)$ & 51.9 & (49.2-54.6) \\
\hline Missouri & 5.5 & (3.3-8.9) & 6.8 & $(4.7-9.9)$ & 6.1 & $(4.4-8.3)$ & 56.1 & $(48.7-63.3)$ & 60.2 & $(55.6-64.6)$ & 58.0 & (53.1-62.8) \\
\hline Montana & 3.9 & $(3.2-4.9)$ & 6.3 & $(4.9-8.0)$ & 5.1 & $(4.2-6.3)$ & 65.5 & $(62.8-68.2)$ & 66.3 & $(64.1-68.5)$ & 66.0 & $(64.1-67.8)$ \\
\hline Nebraska & 4.3 & $(3.0-6.2)$ & 8.4 & $(6.4-11.0)$ & 6.4 & $(5.1-8.1)$ & 63.2 & $(59.8-66.5)$ & 64.1 & $(60.0-68.0)$ & 63.8 & $(60.9-66.5)$ \\
\hline Nevada & 5.2 & $(3.5-7.6)$ & 8.8 & $(5.9-12.9)$ & 7.2 & $(5.5-9.3)$ & 57.9 & $(52.0-63.6)$ & 60.3 & $(56.0-64.5)$ & 59.1 & (54.9-63.1) \\
\hline New Hampshire & - & - & - & - & - & - & - & - & - & - & - & - \\
\hline New Mexico & 6.1 & $(5.0-7.4)$ & 8.1 & $(7.1-9.4)$ & 7.1 & (6.4-7.9) & 57.4 & (55.0-59.8) & 61.5 & $(58.9-64.2)$ & 59.4 & (57.5-61.3) \\
\hline New York & - & - & - & - & - & - & - & - & - & - & - & - \\
\hline North Carolina & 7.1 & $(4.3-11.6)$ & 10.5 & (7.4-14.8) & 8.9 & $(6.8-11.7)$ & 60.0 & $(54.9-64.9)$ & 58.5 & $(52.5-64.2)$ & 59.2 & $(55.0-63.3)$ \\
\hline North Dakota & 3.5 & $(2.4-5.1)$ & 5.9 & $(4.4-7.9)$ & 4.7 & $(3.8-5.8)$ & 59.4 & $(55.9-62.9)$ & 57.6 & $(54.6-60.5)$ & 58.5 & (56.3-60.7) \\
\hline Oklahoma & 7.0 & $(4.9-9.8)$ & 8.9 & $(6.3-12.3)$ & 8.1 & $(6.4-10.2)$ & 56.2 & $(52.9-59.4)$ & 59.1 & $(55.3-62.7)$ & 57.6 & (55.4-59.8) \\
\hline Pennsylvania & 4.4 & $(2.9-6.4)$ & 7.7 & $(6.4-9.3)$ & 6.1 & $(5.1-7.2)$ & 60.6 & $(55.7-65.2)$ & 58.4 & $(54.9-61.8)$ & 59.5 & $(56.2-62.7)$ \\
\hline Rhode Island & 5.5 & $(3.3-9.1)$ & 8.6 & $(6.1-12.0)$ & 7.1 & $(4.9-10.2)$ & 60.4 & $(52.4-67.9)$ & 56.6 & $(51.7-61.4)$ & 58.6 & $(53.4-63.6)$ \\
\hline South Carolina & 8.9 & $(6.0-13.0)$ & 11.2 & $(8.5-14.6)$ & 10.0 & (7.3-13.5) & 45.6 & $(41.0-50.2)$ & 48.1 & $(39.4-56.8)$ & 46.7 & $(41.3-52.3)$ \\
\hline South Dakota & 6.1 & $(3.5-10.2)$ & 7.6 & $(6.0-9.8)$ & 6.9 & $(5.1-9.2)$ & 60.8 & $(53.3-67.8)$ & 57.7 & $(53.1-62.1)$ & 59.2 & $(55.3-63.1)$ \\
\hline Tennessee & 8.3 & $(7.0-9.9)$ & 11.1 & $(9.4-12.9)$ & 9.7 & $(8.5-11.1)$ & 50.0 & $(47.1-52.9)$ & 53.7 & $(50.5-56.9)$ & 51.9 & $(49.7-54.1)$ \\
\hline Vermont & 3.1 & $(2.8-3.4)$ & 5.5 & $(5.1-6.0)$ & 4.4 & $(4.1-4.6)$ & 73.4 & $(72.5-74.2)$ & 71.4 & $(70.5-72.3)$ & 72.3 & (71.7-72.9) \\
\hline Virginia & 5.6 & $(4.3-7.3)$ & 7.8 & $(6.4-9.4)$ & 6.7 & (5.6-8.1) & 59.7 & $(55.8-63.4)$ & 60.7 & $(57.2-64.0)$ & 60.1 & (57.0-63.2) \\
\hline West Virginia & 4.9 & $(3.1-7.7)$ & 11.7 & $(9.1-14.9)$ & 8.3 & $(6.2-11.1)$ & 57.7 & $(53.3-62.0)$ & 53.9 & $(49.4-58.3)$ & 55.8 & (52.3-59.2) \\
\hline Wyoming & 4.4 & $(3.0-6.3)$ & 8.7 & $(7.0-10.6)$ & 6.6 & $(5.5-7.9)$ & 66.0 & $(62.5-69.4)$ & 63.8 & $(60.6-66.8)$ & 64.9 & (62.4-67.2) \\
\hline Median & \multirow{2}{*}{\multicolumn{2}{|c|}{$(2.8-10.7)$}} & \multicolumn{2}{|c|}{8.5} & \multicolumn{2}{|c|}{7.1} & \multicolumn{2}{|c|}{59.1} & \multirow{2}{*}{\multicolumn{2}{|c|}{$\begin{array}{c}58.4 \\
(48.1-71.4)\end{array}$}} & & 59.1 \\
\hline Range & & & & $3.9-12.8)$ & & $3.3-11.3)$ & & $5.6-73.4)$ & & & & (6.7-72.3) \\
\hline Large urban school dis & rveys & & & & & & & & & & & \\
\hline Baltimore, MD & 11.3 & $(8.7-14.6)$ & 10.6 & $(7.3-15.1)$ & 10.8 & $(8.4-13.8)$ & 49.3 & $(44.5-54.2)$ & 58.1 & $(51.6-64.3)$ & 53.8 & $(50.1-57.4)$ \\
\hline Boston, MA & 9.2 & $(7.1-11.8)$ & 13.6 & $(11.1-16.6)$ & 11.4 & $(9.5-13.6)$ & 48.0 & $(44.0-52.0)$ & 50.6 & $(46.5-54.7)$ & 49.3 & $(46.2-52.5)$ \\
\hline Broward County, FL & 9.1 & $(7.0-11.8)$ & 12.2 & $(9.6-15.3)$ & 10.7 & $(9.0-12.7)$ & 53.6 & $(49.7-57.5)$ & 52.4 & $(48.2-56.5)$ & 53.0 & $(49.9-56.1)$ \\
\hline Cleveland, $\mathrm{OH}$ & 10.9 & $(8.8-13.6)$ & 11.6 & $(9.1-14.6)$ & 11.4 & $(9.6-13.4)$ & 48.5 & $(43.4-53.6)$ & 48.4 & $(43.6-53.2)$ & 48.7 & $(45.1-52.2)$ \\
\hline DeKalb County, GA & 8.0 & $(6.0-10.4)$ & 11.0 & $(8.8-13.7)$ & 9.5 & $(7.8-11.4)$ & 55.3 & $(51.3-59.2)$ & 54.6 & $(50.7-58.4)$ & 54.9 & $(52.0-57.8)$ \\
\hline Detroit, MI & 7.0 & $(5.5-8.9)$ & 11.5 & $(8.6-15.2)$ & 9.0 & $(7.3-11.0)$ & 54.2 & $(50.5-57.8)$ & 54.6 & $(49.9-59.3)$ & 54.5 & $(51.5-57.5)$ \\
\hline District of Columbia & 10.6 & $(9.7-11.6)$ & 12.0 & $(10.9-13.1)$ & 11.3 & $(10.6-12.0)$ & 48.2 & $(46.7-49.7)$ & 54.8 & $(53.2-56.5)$ & 51.3 & $(50.2-52.5)$ \\
\hline Duval County, FL & 9.1 & $(7.5-10.9)$ & 12.6 & $(10.8-14.5)$ & 10.7 & $(9.5-12.0)$ & 55.7 & $(52.9-58.5)$ & 56.0 & $(52.8-59.1)$ & 56.2 & $(54.1-58.2)$ \\
\hline Ft. Worth, TX & 8.9 & $(7.1-11.0)$ & 10.9 & $(9.0-13.3)$ & 9.9 & $(8.5-11.5)$ & 54.3 & $(50.8-57.9)$ & 56.0 & $(53.0-59.0)$ & 55.1 & $(52.9-57.4)$ \\
\hline Houston, TX & 10.2 & $(8.5-12.2)$ & 13.2 & $(11.6-15.0)$ & 11.6 & $(10.4-13.0)$ & 51.3 & $(48.9-53.8)$ & 51.1 & $(48.3-53.8)$ & 51.5 & $(49.5-53.4)$ \\
\hline Los Angeles, CA & 6.8 & $(5.0-9.2)$ & 9.4 & $(8.0-11.0)$ & 8.0 & $(7.0-9.1)$ & 53.7 & $(48.3-58.9)$ & 56.5 & $(52.2-60.8)$ & 55.0 & $(51.2-58.8)$ \\
\hline Miami-Dade County, FL & 10.0 & $(8.4-11.9)$ & 13.1 & $(11.0-15.5)$ & 11.7 & $(10.3-13.3)$ & 53.1 & $(49.9-56.3)$ & 54.9 & $(51.1-58.6)$ & 54.0 & $(51.1-56.8)$ \\
\hline
\end{tabular}

See table footnotes on the next page. 
TABLE 88. (Continued) Percentage of high school students who did not eat vegetables*, ${ }^{*}$ and who ate vegetables* one or more times/day, ${ }^{\dagger}$ by sex — selected U.S. sites, Youth Risk Behavior Survey, 2015

\begin{tabular}{|c|c|c|c|c|c|c|c|c|c|c|c|c|}
\hline \multirow[b]{3}{*}{ Site } & \multicolumn{6}{|c|}{ Did not eat vegetables } & \multicolumn{6}{|c|}{ Ate vegetables one or more times/day } \\
\hline & \multicolumn{2}{|c|}{ Female } & \multicolumn{2}{|r|}{ Male } & \multicolumn{2}{|r|}{ Total } & \multicolumn{2}{|c|}{ Female } & \multicolumn{2}{|r|}{ Male } & \multicolumn{2}{|r|}{ Total } \\
\hline & $\%$ & $\mathrm{Cl}^{\S}$ & $\%$ & $\mathrm{Cl}$ & $\%$ & $\mathrm{Cl}$ & $\%$ & $\mathrm{Cl}$ & $\%$ & $\mathrm{Cl}$ & $\%$ & $\mathrm{Cl}$ \\
\hline New York City, NY & - & - & - & - & - & - & - & - & - & - & - & - \\
\hline Oakland, CA & - & - & - & - & - & - & - & - & - & - & - & - \\
\hline Orange County, FL & 8.2 & $(6.2-10.8)$ & 13.7 & $(11.0-17.0)$ & 11.1 & $(9.3-13.3)$ & 54.4 & $(49.8-58.9)$ & 55.4 & $(50.9-59.8)$ & 54.8 & $(51.2-58.3)$ \\
\hline Palm Beach County, FL & 8.0 & $(6.4-10.1)$ & 11.6 & $(9.7-13.9)$ & 9.8 & $(8.6-11.2)$ & 55.5 & $(51.8-59.1)$ & 53.9 & $(51.0-56.7)$ & 54.9 & $(52.6-57.2)$ \\
\hline Philadelphia, PA & 8.9 & $(6.6-11.8)$ & 9.4 & $(7.6-11.4)$ & 9.1 & $(7.3-11.3)$ & 50.0 & $(45.5-54.5)$ & 56.2 & $(52.7-59.6)$ & 53.0 & $(49.8-56.3)$ \\
\hline San Diego, CA & 4.1 & $(2.7-6.0)$ & 7.1 & $(6.0-8.3)$ & 5.6 & $(4.7-6.6)$ & 61.7 & $(58.6-64.6)$ & 60.4 & $(57.1-63.6)$ & 61.1 & $(58.8-63.4)$ \\
\hline San Francisco, CA & - & - & - & - & - & - & - & - & - & - & - & - \\
\hline Median & \multirow{2}{*}{\multicolumn{2}{|c|}{$\begin{array}{c}9.0 \\
(4.1-113)\end{array}$}} & \multirow{2}{*}{\multicolumn{2}{|c|}{$\begin{array}{c}11.6 \\
(7.1-13.7)\end{array}$}} & \multirow{2}{*}{\multicolumn{2}{|c|}{$\begin{array}{c}10.7 \\
(5.6-11.7)\end{array}$}} & \multirow{2}{*}{\multicolumn{2}{|c|}{$\begin{array}{c}53.6 \\
(48.0-61.7)\end{array}$}} & \multirow{2}{*}{\multicolumn{2}{|c|}{$\begin{array}{c}54.8 \\
(48.4-60.4)\end{array}$}} & \multirow{2}{*}{\multicolumn{2}{|c|}{$\begin{array}{c}54.3 \\
(48.7-61.1)\end{array}$}} \\
\hline Range & & & & & & & & & & & & \\
\hline
\end{tabular}

* Green salad, potatoes (excluding French fries, fried potatoes, or potato chips), carrots, or other vegetables.

$\dagger$ During the 7 days before the survey.

$\S 95 \%$ confidence interval.

" Not available.

TABLE 89. Percentage of high school students who ate vegetables* two or more times/day ${ }^{\dagger}$ and who ate vegetables* three or more time/day, ${ }^{\dagger}$ by sex, race/ethnicity, and grade — United States, Youth Risk Behavior Survey, 2015

\begin{tabular}{|c|c|c|c|c|c|c|c|c|c|c|c|c|}
\hline \multirow[b]{3}{*}{ Category } & \multicolumn{6}{|c|}{ Ate vegetables two or more times/day } & \multicolumn{6}{|c|}{ Ate vegetables three or more times/day } \\
\hline & \multicolumn{2}{|c|}{ Female } & \multicolumn{2}{|r|}{ Male } & \multicolumn{2}{|r|}{ Total } & \multicolumn{2}{|c|}{ Female } & \multicolumn{2}{|r|}{ Male } & \multicolumn{2}{|r|}{ Total } \\
\hline & $\%$ & $\mathrm{Cl}^{\S}$ & $\%$ & $\mathrm{Cl}$ & $\%$ & $\mathrm{Cl}$ & $\%$ & $\mathrm{Cl}$ & $\%$ & $\mathrm{Cl}$ & $\%$ & $\mathrm{Cl}$ \\
\hline \multicolumn{13}{|c|}{ Race/Ethnicity } \\
\hline Whitef & 29.1 & $(26.1-32.4)$ & 28.2 & $(25.7-30.8)$ & 28.6 & $(26.4-31.0)$ & 13.1 & $(11.5-14.9)$ & 13.9 & $(12.4-15.7)$ & 13.5 & $(12.2-14.9)$ \\
\hline Black $^{\pi}$ & 17.9 & $(14.3-22.3)$ & 25.7 & $(21.1-30.9)$ & 22.0 & $(19.1-25.2)$ & 9.9 & $(6.2-15.4)$ & 16.1 & $(12.7-20.3)$ & 13.2 & (10.8-15.9) \\
\hline Hispanic & 23.8 & $(21.3-26.6)$ & 30.3 & $(27.6-33.2)$ & 27.2 & $(25.2-29.3)$ & 12.7 & $(10.7-15.0)$ & 18.8 & $(16.5-21.3)$ & 15.8 & $(14.2-17.5)$ \\
\hline \multicolumn{13}{|l|}{ Grade } \\
\hline 9 & 24.2 & $(21.6-26.9)$ & 28.9 & $(24.6-33.5)$ & 26.6 & $(23.9-29.6)$ & 11.6 & $(9.7-13.8)$ & 17.1 & $(13.9-20.7)$ & 14.5 & $(12.5-16.8)$ \\
\hline 10 & 25.5 & $(22.5-28.7)$ & 28.4 & $(25.5-31.4)$ & 26.9 & $(24.6-29.2)$ & 11.2 & $(9.2-13.5)$ & 15.9 & $(12.8-19.5)$ & 13.5 & $(12.0-15.1)$ \\
\hline 11 & 26.6 & $(22.8-30.7)$ & 30.9 & $(28.3-33.5)$ & 28.8 & $(26.6-31.1)$ & 13.9 & $(12.0-16.1)$ & 15.9 & $(13.5-18.8)$ & 15.0 & (13.4-16.8) \\
\hline 12 & 29.8 & $(26.1-33.8)$ & 30.0 & $(26.2-34.1)$ & 29.8 & $(27.0-32.8)$ & 14.9 & $(11.2-19.4)$ & 17.1 & $(14.7-19.8)$ & 16.0 & (13.7-18.5) \\
\hline Total & 26.5 & $(24.4-28.6)$ & 29.6 & $(27.6-31.7)$ & 28.0 & (26.4-29.7) & 12.9 & $(11.8-14.0)$ & 16.6 & $(15.2-18.0)$ & 14.8 & $(13.8-15.7)$ \\
\hline
\end{tabular}

* Green salad, potatoes (excluding French fries, fried potatoes, or potato chips), carrots, or other vegetables.

† During the 7 days before the survey.

$\S 95 \%$ confidence interval.

I Non-Hispanic. 
TABLE 90. Percentage of high school students who ate vegetables* two or more times/day ${ }^{\dagger}$ and who ate vegetables* three or more time/day, ${ }^{\dagger}$ by sex - selected U.S. sites, Youth Risk Behavior Survey, 2015

\begin{tabular}{|c|c|c|c|c|c|c|c|c|c|c|c|c|}
\hline \multirow[b]{3}{*}{ Site } & \multicolumn{6}{|c|}{ Ate vegetables two or more times/day } & \multicolumn{6}{|c|}{ Ate vegetables three or more times/day } \\
\hline & \multicolumn{2}{|c|}{ Female } & \multicolumn{2}{|r|}{ Male } & \multicolumn{2}{|r|}{ Total } & \multicolumn{2}{|c|}{ Female } & \multicolumn{2}{|r|}{ Male } & \multicolumn{2}{|r|}{ Total } \\
\hline & $\%$ & $\mathrm{Cl}^{\S}$ & $\%$ & $\mathrm{Cl}$ & $\%$ & $\mathrm{Cl}$ & $\%$ & $\mathrm{Cl}$ & $\%$ & $\mathrm{Cl}$ & $\%$ & $\mathrm{Cl}$ \\
\hline \multicolumn{13}{|l|}{ State surveys } \\
\hline Alabama & 16.7 & $(14.1-19.6)$ & 21.3 & $(17.6-25.5)$ & 19.3 & $(16.8-22.0)$ & 8.5 & $(6.5-10.9)$ & 11.5 & $(8.8-15.0)$ & 10.2 & $(8.5-12.3)$ \\
\hline Alaska & 28.5 & $(24.6-32.7)$ & 27.9 & (24.5-31.6) & 28.3 & $(25.8-30.9)$ & 15.0 & $(12.2-18.3)$ & 13.2 & (10.8-15.9) & 14.2 & $(12.4-16.1)$ \\
\hline Arizona & 27.6 & $(22.5-33.4)$ & 25.0 & $(21.8-28.5)$ & 26.4 & $(22.8-30.3)$ & 15.7 & $(13.0-18.8)$ & 13.7 & $(10.9-17.2)$ & 14.7 & $(12.3-17.6)$ \\
\hline Arkansas & 23.2 & (19.2-27.7) & 26.2 & (23.0-29.6) & 24.7 & $(23.2-26.4)$ & 12.0 & $(10.4-13.8)$ & 14.5 & $(11.8-17.8)$ & 13.4 & $(12.3-14.5)$ \\
\hline California & 28.8 & $(25.2-32.8)$ & 28.4 & $(24.1-33.1)$ & 28.8 & (25.9-31.9) & 15.3 & $(12.7-18.3)$ & 16.3 & (13.4-19.7) & 15.9 & $(14.0-18.0)$ \\
\hline Connecticut & 29.1 & $(24.5-34.2)$ & 28.3 & $(25.6-31.2)$ & 28.7 & $(25.9-31.6)$ & 12.3 & $(10.0-14.9)$ & 13.2 & $(11.1-15.7)$ & 12.8 & $(11.1-14.6)$ \\
\hline Delaware & - & - & - & - & - & - & - & - & - & - & - & - \\
\hline Florida & 25.0 & $(23.3-26.8)$ & 29.1 & $(27.4-30.8)$ & 27.2 & $(26.0-28.4)$ & 13.5 & $(12.2-14.8)$ & 17.3 & $(15.8-19.0)$ & 15.5 & $(14.4-16.7)$ \\
\hline Hawaii & - & - & - & - & - & - & - & - & - & - & - & - \\
\hline Idaho & 27.0 & $(23.6-30.7)$ & 25.6 & (22.1-29.3) & 26.2 & (24.2-28.3) & 11.0 & $(8.6-14.0)$ & 11.7 & $(9.6-14.1)$ & 11.3 & (9.7-13.2) \\
\hline Illinois & 22.2 & (18.3-26.6) & 27.3 & $(24.0-30.8)$ & 24.8 & (22.2-27.5) & 11.0 & (8.8-13.5) & 14.2 & (12.6-15.9) & 12.7 & $(11.3-14.2)$ \\
\hline Indiana & 22.0 & $(18.6-25.9)$ & 23.7 & (20.2-27.6) & 22.9 & (20.4-25.7) & 8.8 & $(6.4-12.0)$ & 10.8 & $(8.3-13.8)$ & 9.8 & $(8.0-11.9)$ \\
\hline Kentucky & 24.0 & (20.8-27.6) & 21.9 & (18.8-25.4) & 23.2 & $(21.0-25.5)$ & 11.1 & $(8.6-14.1)$ & 10.9 & $(8.7-13.7)$ & 11.1 & $(9.5-13.0)$ \\
\hline Maine & - & - & - & - & - & - & - & - & - & - & - & - \\
\hline Maryland & 24.1 & $(23.4-24.8)$ & 27.3 & $(26.7-27.9)$ & 25.8 & $(25.3-26.3)$ & 11.8 & $(11.2-12.3)$ & 14.8 & $(14.3-15.3)$ & 13.4 & $(13.0-13.7)$ \\
\hline Massachusetts & 24.7 & (21.4-28.3) & 26.3 & (22.9-29.9) & 25.7 & $(23.0-28.6)$ & 11.2 & $(9.2-13.7)$ & 12.6 & (10.9-14.6) & 12.0 & $(10.6-13.7)$ \\
\hline Michigan & 21.2 & $(18.1-24.8)$ & 23.2 & $(20.1-26.6)$ & 22.3 & $(20.2-24.6)$ & 10.5 & $(8.6-12.7)$ & 9.1 & $(7.2-11.5)$ & 9.8 & $(8.6-11.1)$ \\
\hline Mississippi & 20.5 & $(18.0-23.3)$ & 24.3 & $(21.3-27.5)$ & 22.6 & (20.5-24.8) & 11.1 & $(9.6-12.8)$ & 13.8 & $(11.4-16.6)$ & 12.4 & $(10.9-14.1)$ \\
\hline Missouri & 20.2 & $(17.3-23.5)$ & 23.5 & (20.6-26.7) & 21.8 & $(19.7-24.1)$ & 8.9 & $(5.9-13.2)$ & 12.1 & $(9.5-15.4)$ & 10.5 & $(8.7-12.6)$ \\
\hline Montana & 26.8 & (24.7-29.0) & 30.2 & $(27.8-32.6)$ & 28.5 & $(26.7-30.4)$ & 11.9 & $(10.5-13.5)$ & 14.8 & $(13.1-16.6)$ & 13.3 & $(12.2-14.6)$ \\
\hline Nebraska & 27.9 & $(24.5-31.5)$ & 26.8 & $(23.5-30.3)$ & 27.4 & $(24.7-30.3)$ & 13.3 & $(10.5-16.6)$ & 13.0 & (10.9-15.6) & 13.2 & (11.3-15.5) \\
\hline Nevada & 25.7 & $(21.0-31.1)$ & 25.7 & (23.0-28.7) & 25.8 & $(22.7-29.0)$ & 14.5 & $(10.9-19.1)$ & 13.0 & (10.6-15.9) & 13.8 & (11.5-16.5) \\
\hline New Hampshire & - & - & - & - & - & - & - & - & - & - & - & - \\
\hline New Mexico & 25.0 & (22.9-27.3) & 30.9 & (28.5-33.5) & 28.0 & $(26.1-29.9)$ & 13.4 & $(12.1-14.7)$ & 19.3 & $(17.3-21.6)$ & 16.4 & $(14.9-17.9)$ \\
\hline New York & - & - & - & - & - & - & - & - & - & - & - & - \\
\hline North Carolina & 26.3 & $(22.5-30.6)$ & 26.9 & (23.3-30.8) & 26.6 & (23.4-30.1) & 11.7 & (8.9-15.3) & 13.4 & $(10.5-17.1)$ & 12.5 & (10.1-15.5) \\
\hline North Dakota & 25.3 & (22.5-28.3) & 23.4 & (20.8-26.1) & 24.3 & (22.4-26.4) & 10.1 & $(8.4-12.0)$ & 12.1 & $(10.2-14.2)$ & 11.1 & $(9.8-12.6)$ \\
\hline Oklahoma & 18.9 & $(16.3-21.8)$ & 25.9 & (22.6-29.5) & 22.2 & $(19.8-24.8)$ & 9.3 & $(6.8-12.7)$ & 15.2 & $(12.8-18.0)$ & 12.1 & $(10.1-14.6)$ \\
\hline Pennsylvania & 23.7 & (20.9-26.8) & 24.9 & $(22.0-28.2)$ & 24.3 & (21.9-26.9) & 9.3 & (7.3-11.7) & 12.1 & $(9.8-14.7)$ & 10.7 & $(9.0-12.6)$ \\
\hline Rhode Island & 22.2 & (19.4-25.4) & 25.4 & (22.0-29.1) & 23.9 & (21.3-26.8) & 10.9 & $(9.1-13.0)$ & 12.9 & (10.7-15.4) & 12.0 & (10.9-13.3) \\
\hline South Carolina & 16.5 & $(14.4-18.8)$ & 20.7 & $(15.9-26.5)$ & 18.5 & $(16.1-21.2)$ & 7.5 & $(6.1-9.2)$ & 10.8 & $(8.7-13.4)$ & 9.1 & $(8.0-10.4)$ \\
\hline South Dakota & 21.8 & $(17.1-27.3)$ & 26.5 & (22.4-31.2) & 24.3 & (21.4-27.5) & 9.8 & (7.5-12.8) & 13.8 & (10.2-18.4) & 11.9 & $(9.6-14.7)$ \\
\hline Tennessee & 18.9 & $(16.9-21.1)$ & 23.0 & (20.7-25.6) & 21.1 & $(19.5-22.9)$ & 8.4 & (7.3-9.7) & 10.9 & $(9.3-12.6)$ & 9.7 & $(8.9-10.6)$ \\
\hline Vermont & 35.9 & $(35.0-36.9)$ & 36.5 & $(35.6-37.5)$ & 36.3 & $(35.6-36.9)$ & 17.4 & $(16.7-18.2)$ & 18.6 & $(17.8-19.3)$ & 18.1 & $(17.6-18.6)$ \\
\hline Virginia & 24.9 & (22.1-27.8) & 27.7 & $(24.8-30.7)$ & 26.4 & $(23.8-29.1)$ & 11.8 & $(9.9-13.8)$ & 15.8 & $(13.5-18.3)$ & 13.8 & (12.1-15.8) \\
\hline West Virginia & 24.2 & $(20.4-28.4)$ & 23.4 & $(19.6-27.6)$ & 23.8 & $(21.1-26.6)$ & 12.7 & $(10.8-15.0)$ & 13.0 & $(10.1-16.6)$ & 12.9 & $(11.3-14.7)$ \\
\hline Wyoming & 28.8 & $(25.8-32.0)$ & 31.3 & $(28.4-34.5)$ & 30.1 & $(27.9-32.4)$ & 13.1 & $(11.3-15.3)$ & 14.7 & $(12.6-17.1)$ & 13.9 & $(12.4-15.7)$ \\
\hline Median & & 24.4 & & 26.0 & & 25.2 & & 11.4 & & 13.2 & & 12.6 \\
\hline Range & & 5.5-35.9) & & $0.7-36.5)$ & & $8.5-36.3)$ & & $7.5-17.4)$ & & $.1-19.3)$ & & $9.1-18.1)$ \\
\hline \multicolumn{13}{|c|}{ Large urban school district surveys } \\
\hline Baltimore, MD & 20.6 & $(16.8-25.0)$ & 27.9 & $(22.4-34.1)$ & 24.4 & $(21.0-28.2)$ & 13.5 & $(10.0-18.1)$ & 15.3 & $(11.3-20.3)$ & 14.6 & $(11.7-18.1)$ \\
\hline Boston, MA & 21.3 & $(18.3-24.6)$ & 19.7 & $(16.8-23.0)$ & 20.5 & $(18.5-22.8)$ & 10.7 & $(8.5-13.3)$ & 9.8 & $(8.0-12.0)$ & 10.3 & $(8.9-12.0)$ \\
\hline Broward County, FL & 22.2 & $(19.2-25.6)$ & 23.9 & $(20.2-28.0)$ & 23.1 & $(20.6-25.9)$ & 12.1 & $(9.8-14.8)$ & 13.3 & $(10.6-16.5)$ & 12.8 & $(10.9-14.9)$ \\
\hline Cleveland, $\mathrm{OH}$ & 19.3 & $(16.5-22.4)$ & 23.6 & $(20.3-27.2)$ & 21.9 & $(19.5-24.5)$ & 10.6 & $(8.4-13.4)$ & 15.3 & $(12.5-18.6)$ & 13.5 & (11.7-15.5) \\
\hline DeKalb County, GA & 23.1 & $(20.0-26.5)$ & 26.8 & $(23.6-30.2)$ & 24.9 & $(22.8-27.1)$ & 12.7 & $(10.7-15.1)$ & 13.7 & $(11.6-16.2)$ & 13.2 & $(11.8-14.8)$ \\
\hline Detroit, MI & 19.2 & $(16.5-22.2)$ & 18.4 & $(14.9-22.5)$ & 19.0 & $(16.8-21.3)$ & 9.1 & $(7.4-11.3)$ & 9.6 & $(7.1-12.9)$ & 9.5 & $(7.9-11.4)$ \\
\hline District of Columbia & 19.3 & $(18.2-20.5)$ & 24.8 & (23.4-26.3) & 22.0 & $(21.1-22.9)$ & 10.4 & $(9.5-11.3)$ & 13.8 & $(12.7-15.0)$ & 12.1 & $(11.4-12.8)$ \\
\hline Duval County, FL & 22.7 & $(20.4-25.1)$ & 24.6 & $(22.1-27.2)$ & 24.1 & $(22.5-25.7)$ & 10.9 & $(9.4-12.7)$ & 12.7 & $(11.0-14.6)$ & 12.0 & $(10.9-13.2)$ \\
\hline Ft. Worth, TX & 24.4 & $(21.5-27.5)$ & 28.0 & $(25.1-31.1)$ & 26.2 & (23.9-28.7) & 14.8 & $(12.4-17.7)$ & 17.8 & $(15.4-20.5)$ & 16.4 & $(14.5-18.4)$ \\
\hline Houston, TX & 22.7 & $(20.6-24.9)$ & 20.4 & $(18.0-23.0)$ & 21.8 & $(20.1-23.6)$ & 12.2 & $(10.6-14.0)$ & 11.6 & $(9.8-13.6)$ & 12.2 & $(11.0-13.5)$ \\
\hline Los Angeles, CA & 19.6 & $(15.6-24.3)$ & 26.3 & $(23.7-28.9)$ & 22.8 & $(20.6-25.2)$ & 9.7 & $(8.2-11.4)$ & 13.9 & $(11.2-17.2)$ & 11.8 & $(10.2-13.6)$ \\
\hline Miami-Dade County, FL & 26.3 & $(23.7-29.1)$ & 28.3 & $(25.0-31.8)$ & 27.4 & (25.2-29.7) & 15.6 & (13.4-18.0) & 17.9 & $(15.5-20.5)$ & 16.8 & $(15.2-18.6)$ \\
\hline
\end{tabular}

See table footnotes on the next page. 
TABLE 90. (Continued) Percentage of high school students who ate vegetables* two or more times/day ${ }^{\dagger}$ and who ate vegetables* three or more time/day, ${ }^{\dagger}$ by sex — selected U.S. sites, Youth Risk Behavior Survey, 2015

\begin{tabular}{|c|c|c|c|c|c|c|c|c|c|c|c|c|}
\hline \multirow[b]{3}{*}{ Site } & \multicolumn{6}{|c|}{ Ate vegetables two or more times/day } & \multicolumn{6}{|c|}{ Ate vegetables three or more times/day } \\
\hline & \multicolumn{2}{|c|}{ Female } & \multicolumn{2}{|r|}{ Male } & \multicolumn{2}{|r|}{ Total } & \multicolumn{2}{|r|}{ Female } & \multicolumn{2}{|r|}{ Male } & \multicolumn{2}{|r|}{ Total } \\
\hline & $\%$ & $\mathrm{Cl}^{\S}$ & $\%$ & $\mathrm{Cl}$ & $\%$ & $\mathrm{Cl}$ & $\%$ & $\mathrm{Cl}$ & $\%$ & $\mathrm{Cl}$ & $\%$ & $\mathrm{Cl}$ \\
\hline New York City, NY & - & - & - & - & - & - & - & - & - & - & - & - \\
\hline Oakland, CA & - & - & - & - & - & - & - & - & - & - & - & - \\
\hline Orange County, FL & 27.2 & $(23.8-30.7)$ & 27.6 & $(23.1-32.5)$ & 27.2 & $(24.2-30.5)$ & 14.4 & $(11.9-17.2)$ & 18.3 & $(14.0-23.5)$ & 16.3 & $(13.6-19.4)$ \\
\hline Palm Beach County, FL & 21.6 & $(18.7-24.8)$ & 24.7 & $(21.7-27.9)$ & 23.4 & $(21.2-25.7)$ & 11.2 & $(9.2-13.6)$ & 13.5 & $(11.2-16.2)$ & 12.5 & $(10.8-14.3)$ \\
\hline Philadelphia, PA & 21.2 & $(18.5-24.1)$ & 24.4 & $(20.2-29.2)$ & 22.9 & $(20.4-25.6)$ & 10.2 & $(8.2-12.6)$ & 14.1 & $(10.8-18.1)$ & 12.3 & $(10.5-14.5)$ \\
\hline San Diego, CA & 26.4 & $(23.6-29.4)$ & 28.1 & $(25.2-31.1)$ & 27.2 & $(25.2-29.3)$ & 12.6 & $(10.2-15.5)$ & 14.0 & $(11.5-16.9)$ & 13.3 & $(11.3-15.5)$ \\
\hline San Francisco, CA & - & - & - & - & - & - & - & - & - & - & - & - \\
\hline Median & & 21.9 & & 24.7 & & 23.2 & & 11.6 & & 13.8 & & 12.6 \\
\hline Range & & 9.2-27.2) & & (8.4-28.3) & & (9.0-27.4) & & $9.1-15.6)$ & & $9.6-18.3)$ & & (9.5-16.8) \\
\hline
\end{tabular}

* Green salad, potatoes (excluding French fries, fried potatoes, or potato chips), carrots, or other vegetables.

† During the 7 days before the survey.

$\S 95 \%$ confidence interval.

" Not available.

TABLE 91. Percentage of high school students who did not drink milk* and who drank one or more glasses/day of milk, ${ }^{*}$ by sex, race/ethnicity, and grade - United States, Youth Risk Behavior Survey, 2015

\begin{tabular}{|c|c|c|c|c|c|c|c|c|c|c|c|c|}
\hline \multirow[b]{3}{*}{ Category } & \multicolumn{6}{|c|}{ Did not drink milk } & \multicolumn{6}{|c|}{ Drank one or more glasses/day of milk } \\
\hline & \multicolumn{2}{|r|}{ Female } & \multicolumn{2}{|r|}{ Male } & \multicolumn{2}{|r|}{ Total } & \multicolumn{2}{|c|}{ Female } & \multicolumn{2}{|r|}{ Male } & \multicolumn{2}{|r|}{ Total } \\
\hline & $\%$ & $\mathrm{Cl}^{\dagger}$ & $\%$ & $\mathrm{Cl}$ & $\%$ & $\mathrm{Cl}$ & $\%$ & $\mathrm{Cl}$ & $\%$ & $\mathrm{Cl}$ & $\%$ & $\mathrm{Cl}$ \\
\hline \multicolumn{13}{|c|}{ Race/Ethnicity } \\
\hline White ${ }^{\S}$ & 24.4 & $(20.6-28.7)$ & 12.1 & $(10.2-14.3)$ & 18.3 & $(15.9-21.1)$ & 32.0 & $(27.6-36.7)$ & 50.6 & $(45.7-55.5)$ & 41.2 & $(37.1-45.5)$ \\
\hline Black $^{\S}$ & 44.6 & (39.5-49.8) & 25.8 & $(23.0-28.8)$ & 34.7 & $(32.4-37.0)$ & 17.7 & $(14.5-21.5)$ & 31.8 & $(26.2-38.1)$ & 25.1 & $(22.2-28.2)$ \\
\hline Hispanic & 26.3 & $(23.7-29.1)$ & 13.0 & $(11.1-15.3)$ & 19.6 & $(17.7-21.6)$ & 27.2 & $(24.6-30.0)$ & 45.1 & $(42.6-47.7)$ & 36.2 & $(34.4-38.1)$ \\
\hline \multicolumn{13}{|l|}{ Grade } \\
\hline 9 & 27.0 & $(24.2-30.0)$ & 15.6 & $(13.1-18.5)$ & 20.9 & $(18.8-23.3)$ & 29.3 & $(25.5-33.4)$ & 47.0 & $(43.0-51.0)$ & 38.6 & $(35.2-42.1)$ \\
\hline 10 & 26.9 & $(21.2-33.4)$ & 14.1 & $(10.8-18.1)$ & 20.5 & $(16.9-24.6)$ & 30.1 & $(22.9-38.5)$ & 49.2 & $(42.5-55.9)$ & 39.6 & $(33.7-45.9)$ \\
\hline 11 & 31.7 & $(27.8-35.9)$ & 14.2 & $(12.1-16.6)$ & 22.8 & $(20.3-25.6)$ & 26.3 & $(21.9-31.4)$ & 44.7 & $(41.2-48.2)$ & 35.8 & $(32.2-39.6)$ \\
\hline 12 & 28.2 & $(24.8-32.0)$ & 16.6 & (13.9-19.7) & 22.4 & $(20.1-24.8)$ & 26.5 & $(23.4-29.9)$ & 43.8 & $(39.8-47.8)$ & 35.2 & (32.7-37.7) \\
\hline Total & 28.4 & $(25.5-31.5)$ & 15.1 & $(13.6-16.6)$ & 21.5 & (19.8-23.4) & 28.2 & $(25.1-31.6)$ & 46.2 & $(43.3-49.1)$ & 37.5 & $(34.8-40.2)$ \\
\hline
\end{tabular}

* During the 7 days before the survey.

$+95 \%$ confidence interval.

$\S$ Non-Hispanic. 
TABLE 92. Percentage of high school students who did not drink milk* and who drank one or more glasses/day of milk, by sex — selected U.S. sites, Youth Risk Behavior Survey, 2015

\begin{tabular}{|c|c|c|c|c|c|c|c|c|c|c|c|c|}
\hline \multirow[b]{3}{*}{ Site } & \multicolumn{6}{|c|}{ Did not drink milk } & \multicolumn{6}{|c|}{ Drank one or more glasses/day of milk } \\
\hline & \multicolumn{2}{|c|}{ Female } & \multicolumn{2}{|r|}{ Male } & \multicolumn{2}{|r|}{ Total } & \multicolumn{2}{|c|}{ Female } & \multicolumn{2}{|r|}{ Male } & \multicolumn{2}{|r|}{ Total } \\
\hline & $\%$ & $\mathrm{Cl}^{\dagger}$ & $\%$ & $\mathrm{Cl}$ & $\%$ & $\mathrm{Cl}$ & $\%$ & $\mathrm{Cl}$ & $\%$ & $\mathrm{Cl}$ & $\%$ & $\mathrm{Cl}$ \\
\hline \multicolumn{13}{|l|}{ State surveys } \\
\hline Alabama & 35.5 & (30.5-40.8) & 22.7 & $(19.6-26.0)$ & 29.0 & (25.4-32.9) & 18.9 & (15.1-23.5) & 32.9 & (30.4-35.4) & 26.1 & $(23.6-28.8)$ \\
\hline Alaska & — & - & - & - & - & - & - & - & - & - & - & - \\
\hline Arizona & - & - & - & - & - & - & - & - & - & - & - & - \\
\hline Arkansas & 33.3 & $(27.4-39.7)$ & 20.2 & $(18.0-22.6)$ & 26.7 & $(23.7-29.9)$ & 22.8 & $(18.4-28.0)$ & 37.1 & (32.8-41.7) & 29.9 & $(26.5-33.5)$ \\
\hline California & 30.0 & $(23.5-37.5)$ & 15.8 & $(13.0-19.1)$ & 22.8 & $(18.7-27.5)$ & 23.8 & (20.4-27.5) & 41.2 & (37.7-44.8) & 32.7 & $(29.8-35.6)$ \\
\hline Connecticut & - & - & - & - & - & - & - & - & - & - & - & - \\
\hline Delaware & 30.9 & $(27.5-34.6)$ & 18.3 & $(15.6-21.3)$ & 24.6 & $(22.3-27.0)$ & 23.7 & (21.2-26.5) & 41.8 & (38.7-45.0) & 32.9 & $(30.7-35.2)$ \\
\hline Florida & 29.2 & $(27.6-31.0)$ & 17.2 & (15.6-18.9) & 23.2 & (22.0-24.5) & 24.7 & (22.8-26.8) & 43.1 & $(40.8-45.3)$ & 33.9 & (32.4-35.5) \\
\hline Hawaii & 36.6 & (33.6-39.8) & 22.3 & $(20.3-24.4)$ & 29.6 & $(27.5-31.9)$ & 16.6 & $(14.4-19.1)$ & 29.6 & $(27.0-32.3)$ & 23.0 & $(21.0-25.0)$ \\
\hline Idaho & 19.7 & $(16.9-22.7)$ & 10.3 & $(8.0-13.1)$ & 14.8 & (13.0-16.9) & 30.4 & $(27.0-34.1)$ & 53.5 & (49.1-57.8) & 42.1 & $(39.2-45.1)$ \\
\hline Illinois & 31.9 & (28.4-35.6) & 16.5 & (13.4-20.3) & 24.1 & (21.5-26.8) & 25.0 & (21.9-28.3) & 42.4 & (38.3-46.7) & 33.8 & $(30.8-37.1)$ \\
\hline Indiana & 23.5 & $(20.2-27.1)$ & 16.5 & (12.9-20.9) & 20.0 & $(17.2-23.2)$ & 27.4 & $(23.2-32.0)$ & 43.0 & (38.4-47.6) & 35.3 & (32.3-38.4) \\
\hline Kentucky & 26.5 & $(22.3-31.0)$ & 18.5 & (15.4-22.0) & 22.5 & (19.9-25.4) & 22.3 & (19.1-25.9) & 37.9 & (33.6-42.4) & 30.3 & $(27.6-33.2)$ \\
\hline Maine & 23.4 & (22.0-24.8) & 14.2 & (13.0-15.6) & 18.8 & (17.9-19.7) & 34.1 & (32.8-35.4) & 50.2 & $(48.8-51.7)$ & 42.3 & (41.2-43.3) \\
\hline Maryland & - & - & - & - & - & - & - & - & - & - & - & - \\
\hline Massachusetts & 28.1 & $(24.7-31.7)$ & 13.8 & (11.8-16.2) & 21.0 & (18.8-23.3) & 27.6 & $(24.2-31.4)$ & 48.2 & $(45.0-51.5)$ & 38.0 & $(35.2-40.9)$ \\
\hline Michigan & 29.8 & $(25.8-34.1)$ & 17.1 & $(13.7-21.1)$ & 23.4 & (20.2-26.9) & 26.5 & (23.4-29.9) & 44.3 & (38.9-49.8) & 35.4 & $(31.5-39.5)$ \\
\hline Mississippi & 37.7 & (33.9-41.6) & 25.3 & $(21.6-29.3)$ & 31.7 & $(29.1-34.3)$ & 18.2 & $(15.2-21.7)$ & 29.0 & $(24.9-33.6)$ & 23.5 & $(20.5-26.8)$ \\
\hline Missouri & 26.3 & $(22.3-30.7)$ & 14.8 & $(12.0-18.2)$ & 20.8 & $(17.6-24.3)$ & 28.1 & $(24.2-32.3)$ & 44.8 & (41.9-47.7) & 36.0 & (33.4-38.7) \\
\hline Montana & 20.0 & (18.2-21.8) & 11.9 & $(10.2-13.7)$ & 15.8 & $(14.5-17.1)$ & 33.3 & $(30.8-36.0)$ & 49.1 & $(46.1-52.1)$ & 41.5 & $(39.0-44.0)$ \\
\hline Nebraska & 22.7 & (19.1-26.7) & 12.1 & $(9.6-15.1)$ & 17.3 & (14.9-19.9) & 33.9 & (30.7-37.4) & 49.1 & $(44.9-53.2)$ & 41.7 & (38.9-44.5) \\
\hline Nevada & 28.9 & $(25.1-33.0)$ & 17.9 & $(14.8-21.4)$ & 23.5 & $(20.9-26.2)$ & 24.6 & $(22.2-27.1)$ & 39.5 & $(36.0-43.2)$ & 32.2 & $(30.3-34.0)$ \\
\hline New Hampshire & - & - & - & - & - & - & - & - & - & - & - & - \\
\hline New Mexico & - & - & - & - & - & - & - & - & - & - & - & - \\
\hline New York & 34.2 & (32.2-36.2) & 26.9 & $(23.1-31.0)$ & 30.5 & $(28.4-32.7)$ & 21.4 & $(19.6-23.3)$ & 33.8 & (31.9-35.8) & 27.7 & $(26.0-29.5)$ \\
\hline North Carolina & - & - & - & - & - & - & - & - & - & - & - & - \\
\hline North Dakota & 15.9 & (13.5-18.5) & 12.0 & $(9.7-14.9)$ & 13.9 & (12.3-15.8) & 45.8 & (42.1-49.6) & 57.1 & (53.3-60.8) & 51.6 & $(48.7-54.4)$ \\
\hline Oklahoma & 26.7 & $(24.2-29.3)$ & 17.7 & $(14.8-21.1)$ & 22.4 & $(20.3-24.6)$ & 26.8 & $(22.3-32.0)$ & 42.3 & (38.3-46.3) & 34.2 & $(31.0-37.4)$ \\
\hline Pennsylvania & 25.8 & (22.7-29.2) & 15.6 & $(13.5-18.1)$ & 20.7 & (18.4-23.2) & 27.7 & (24.3-31.4) & 49.1 & $(45.2-52.9)$ & 38.4 & (35.3-41.7) \\
\hline Rhode Island & - & - & - & - & - & - & - & - & - & - & - & - \\
\hline South Carolina & 38.6 & $(32.3-45.4)$ & 21.4 & $(18.0-25.2)$ & 30.1 & $(26.5-34.0)$ & 17.6 & $(11.9-25.4)$ & 33.4 & $(26.8-40.8)$ & 25.4 & $(20.1-31.7)$ \\
\hline South Dakota & 18.9 & (14.4-24.3) & 13.7 & $(9.6-19.1)$ & 16.2 & (13.9-18.8) & 38.4 & (32.0-45.2) & 51.6 & $(48.1-55.1)$ & 45.2 & (40.9-49.5) \\
\hline Tennessee & 34.2 & (30.0-38.7) & 19.4 & $(16.5-22.6)$ & 26.6 & $(23.5-29.8)$ & 21.7 & $(19.1-24.7)$ & 36.3 & (32.9-39.8) & 29.3 & $(26.7-32.0)$ \\
\hline Vermont & - & - & - & - & - & - & - & - & - & - & - & - \\
\hline Virginia & 35.1 & $(32.0-38.3)$ & 20.8 & (18.3-23.5) & 27.7 & $(25.5-30.0)$ & 25.1 & $(22.3-28.2)$ & 43.1 & (39.6-46.7) & 34.4 & $(31.7-37.2)$ \\
\hline West Virginia & 24.6 & $(21.8-27.7)$ & 17.7 & (14.4-21.6) & 21.2 & (18.9-23.8) & 26.3 & $(22.9-30.1)$ & 41.2 & $(36.8-45.7)$ & 33.8 & (30.5-37.2) \\
\hline Wyoming & 23.1 & (20.4-26.1) & 16.2 & (13.2-19.8) & 19.6 & (17.4-22.1) & 33.0 & (29.7-36.6) & 46.1 & $(41.2-51.1)$ & 39.7 & $(36.7-42.7)$ \\
\hline Median & & 28.5 & & 17.1 & & 22.6 & & 25.7 & & 42.7 & & 34.0 \\
\hline Range & $(15$ & (9-38.6) & & .3-26.9) & & (.9-31.7) & & .6-45.8) & & $.0-57.1)$ & & $.0-51.6)$ \\
\hline \multicolumn{13}{|c|}{ Large urban school district surveys } \\
\hline Baltimore, MD & 41.7 & $(37.1-46.5)$ & 32.2 & $(25.9-39.3)$ & 36.9 & $(32.5-41.5)$ & 16.6 & $(12.6-21.7)$ & 24.7 & $(20.1-30.0)$ & 20.8 & $(17.4-24.7)$ \\
\hline Boston, MA & 32.9 & $(29.7-36.2)$ & 22.6 & $(19.1-26.5)$ & 27.7 & $(25.2-30.5)$ & 23.1 & $(20.2-26.3)$ & 37.1 & $(33.6-40.7)$ & 30.3 & $(27.8-32.9)$ \\
\hline Broward County, FL & 40.5 & $(36.4-44.7)$ & 28.0 & $(25.0-31.3)$ & 34.1 & $(31.3-36.9)$ & 16.5 & $(14.0-19.3)$ & 28.7 & $(25.3-32.4)$ & 22.8 & $(20.5-25.2)$ \\
\hline Cleveland, $\mathrm{OH}$ & - & - & - & - & - & - & - & - & - & - & - & - \\
\hline DeKalb County, GA & 43.3 & $(39.6-47.1)$ & 28.4 & $(25.2-32.0)$ & 35.9 & $(33.4-38.6)$ & 16.2 & $(13.7-19.1)$ & 27.1 & $(23.5-31.1)$ & 21.6 & $(19.2-24.3)$ \\
\hline Detroit, MI & 36.3 & $(32.4-40.3)$ & 29.9 & $(26.2-33.9)$ & 33.4 & $(30.5-36.5)$ & 13.7 & $(11.3-16.5)$ & 18.8 & $(15.9-22.1)$ & 16.2 & $(14.3-18.2)$ \\
\hline District of Columbia & - & - & - & - & - & - & - & - & - & - & - & - \\
\hline Duval County, FL & - & - & - & - & - & - & - & - & - & - & - & - \\
\hline Ft. Worth, TX & 28.2 & $(25.2-31.4)$ & 16.9 & $(14.5-19.7)$ & 22.5 & $(20.6-24.6)$ & 23.8 & $(21.3-26.4)$ & 39.9 & $(37.1-42.8)$ & 32.0 & $(29.9-34.1)$ \\
\hline Houston, TX & 33.2 & $(30.7-35.8)$ & 22.2 & $(20.0-24.5)$ & 27.5 & $(25.7-29.3)$ & 20.9 & $(18.8-23.2)$ & 30.3 & $(27.9-32.7)$ & 25.8 & $(24.2-27.5)$ \\
\hline Los Angeles, CA & 25.5 & $(21.4-30.1)$ & 15.5 & $(13.5-17.7)$ & 20.7 & $(18.1-23.6)$ & 21.9 & $(18.7-25.5)$ & 39.8 & (36.4-43.3) & 30.6 & $(28.0-33.2)$ \\
\hline Miami-Dade County, FL & 29.5 & $(26.6-32.6)$ & 19.3 & $(16.9-21.9)$ & 24.5 & $(22.8-26.3)$ & 25.6 & $(23.0-28.4)$ & 43.0 & $(39.2-46.8)$ & 34.1 & $(31.7-36.6)$ \\
\hline
\end{tabular}

See table footnotes on the next page. 
TABLE 92. (Continued) Percentage of high school students who did not drink milk* and who drank one or more glasses/day of milk, by sex selected U.S. sites, Youth Risk Behavior Survey, 2015

\begin{tabular}{|c|c|c|c|c|c|c|c|c|c|c|c|c|}
\hline \multirow[b]{3}{*}{ Site } & \multicolumn{6}{|c|}{ Did not drink milk } & \multicolumn{6}{|c|}{ Drank one or more glasses/day of milk } \\
\hline & \multicolumn{2}{|c|}{ Female } & \multicolumn{2}{|r|}{ Male } & \multicolumn{2}{|r|}{ Total } & \multicolumn{2}{|r|}{ Female } & \multicolumn{2}{|r|}{ Male } & \multicolumn{2}{|r|}{ Total } \\
\hline & $\%$ & $\mathrm{Cl}^{\dagger}$ & $\%$ & $\mathrm{Cl}$ & $\%$ & $\mathrm{Cl}$ & $\%$ & $\mathrm{Cl}$ & $\%$ & $\mathrm{Cl}$ & $\%$ & $\mathrm{Cl}$ \\
\hline New York City, NY & 39.8 & $(37.3-42.3)$ & 21.3 & $(19.5-23.3)$ & 30.8 & $(29.1-32.5)$ & 16.3 & $(14.4-18.5)$ & 28.5 & $(26.4-30.6)$ & 22.4 & $(20.8-24.0)$ \\
\hline Oakland, CA & - & - & - & - & - & - & - & - & - & - & - & - \\
\hline Orange County, FL & 33.2 & $(29.7-36.9)$ & 18.0 & $(14.9-21.7)$ & 25.7 & $(23.5-28.1)$ & 21.3 & $(18.6-24.2)$ & 38.7 & $(35.1-42.4)$ & 29.9 & $(27.2-32.8)$ \\
\hline Palm Beach County, FL & 38.1 & $(35.0-41.3)$ & 25.4 & $(22.6-28.3)$ & 31.5 & (29.5-33.6) & 18.7 & $(16.4-21.2)$ & 32.3 & $(29.5-35.2)$ & 25.4 & $(23.6-27.3)$ \\
\hline Philadelphia, PA & 32.2 & $(28.1-36.5)$ & 20.7 & $(17.3-24.5)$ & 26.6 & $(23.9-29.6)$ & 19.3 & $(15.5-23.8)$ & 33.3 & $(29.1-37.8)$ & 26.1 & $(22.8-29.7)$ \\
\hline San Diego, CA & 27.3 & $(24.2-30.6)$ & 16.6 & $(14.1-19.4)$ & 21.8 & $(19.7-24.1)$ & 22.5 & $(19.3-25.9)$ & 39.8 & $(36.9-42.7)$ & 31.3 & $(29.1-33.5)$ \\
\hline San Francisco, CA & 28.2 & $(24.6-32.0)$ & 18.6 & $(15.6-21.9)$ & 23.3 & $(21.1-25.8)$ & 22.8 & $(19.4-26.4)$ & 39.6 & $(35.4-44.0)$ & 31.2 & $(28.5-34.1)$ \\
\hline Median & & 33.2 & & 21.3 & & 27.5 & & 20.9 & & 33.3 & & 26.1 \\
\hline Range & & $5.5-43.3)$ & & (5.5-32.2) & & $0.7-36.9)$ & & (3.7-25.6) & & $(8.8-43.0)$ & & $(6.2-34.1)$ \\
\hline
\end{tabular}

* During the 7 days before the survey.

† $95 \%$ confidence interval.

$\S$ Not available.

TABLE 93. Percentage of high school students who drank two or more glasses/day of milk* and who drank three or more glasses/day of milk,* by sex, race/ethnicity, and grade - United States, Youth Risk Behavior Survey, 2015

\begin{tabular}{|c|c|c|c|c|c|c|c|c|c|c|c|c|}
\hline \multirow[b]{3}{*}{ Category } & \multicolumn{6}{|c|}{ Drank two or more glasses/day of milk } & \multicolumn{6}{|c|}{ Drank three or more glasses/day of milk } \\
\hline & \multicolumn{2}{|c|}{ Female } & \multicolumn{2}{|r|}{ Male } & \multicolumn{2}{|r|}{ Total } & \multicolumn{2}{|c|}{ Female } & \multicolumn{2}{|r|}{ Male } & \multicolumn{2}{|r|}{ Total } \\
\hline & $\%$ & $\mathrm{Cl}^{\dagger}$ & $\%$ & $\mathrm{Cl}$ & $\%$ & $\mathrm{Cl}$ & $\%$ & $\mathrm{Cl}$ & $\%$ & $\mathrm{Cl}$ & $\%$ & $\mathrm{Cl}$ \\
\hline \multicolumn{13}{|c|}{ Race/Ethnicity } \\
\hline White ${ }^{\S}$ & 17.8 & $(14.8-21.3)$ & 32.9 & $(28.5-37.6)$ & 25.3 & $(22.0-28.9)$ & 6.4 & $(5.1-7.9)$ & 16.1 & $(13.2-19.6)$ & 11.2 & $(9.5-13.3)$ \\
\hline Black $^{\S}$ & 5.8 & $(4.0-8.2)$ & 21.0 & $(15.7-27.5)$ & 13.8 & $(10.3-18.2)$ & 3.0 & $(2.0-4.5)$ & 10.3 & $(7.3-14.3)$ & 6.9 & $(5.0-9.3)$ \\
\hline Hispanic & 12.8 & $(10.8-15.1)$ & 28.3 & $(26.2-30.5)$ & 20.6 & $(19.2-22.1)$ & 4.9 & $(3.6-6.6)$ & 15.0 & $(13.3-16.8)$ & 10.0 & $(9.0-11.1)$ \\
\hline \multicolumn{13}{|l|}{ Grade } \\
\hline 9 & 16.7 & $(13.8-20.1)$ & 32.5 & $(29.1-36.2)$ & 25.1 & $(22.3-28.1)$ & 6.9 & $(5.1-9.3)$ & 15.7 & $(13.5-18.2)$ & 11.5 & $(9.8-13.4)$ \\
\hline 10 & 14.8 & (11.5-18.9) & 30.8 & $(25.6-36.6)$ & 22.9 & $(19.4-26.8)$ & 5.1 & $(4.0-6.5)$ & 17.0 & $(13.5-21.2)$ & 11.0 & $(9.3-13.0)$ \\
\hline 11 & 13.7 & $(10.8-17.2)$ & 29.2 & $(25.3-33.6)$ & 21.9 & $(18.7-25.4)$ & 4.6 & $(3.3-6.3)$ & 13.8 & $(11.3-16.8)$ & 9.4 & $(7.8-11.3)$ \\
\hline 12 & 12.5 & $(10.2-15.3)$ & 25.4 & $(21.8-29.4)$ & 19.0 & $(16.8-21.4)$ & 4.6 & $(3.2-6.5)$ & 12.1 & $(10.0-14.7)$ & 8.4 & $(7.2-9.8)$ \\
\hline Total & 14.6 & $(12.6-16.8)$ & 29.6 & $(27.0-32.4)$ & 22.4 & $(20.4-24.5)$ & 5.4 & $(4.5-6.4)$ & 14.8 & $(13.1-16.6)$ & 10.2 & $(9.3-11.3)$ \\
\hline
\end{tabular}

${ }^{*}$ During the 7 days before the survey.

† $95 \%$ confidence interval.

$\S$ Non-Hispanic. 
TABLE 94. Percentage of high school students who drank two or more glasses/day of milk* and who drank three or more glasses/day of milk, ${ }^{*}$ by sex - selected U.S. sites, Youth Risk Behavior Survey, 2015

\begin{tabular}{|c|c|c|c|c|c|c|c|c|c|c|c|c|}
\hline \multirow[b]{3}{*}{ Site } & \multicolumn{6}{|c|}{ Drank two or more glasses/day of milk } & \multicolumn{6}{|c|}{ Drank three or more glasses/day of milk } \\
\hline & \multicolumn{2}{|c|}{ Female } & \multicolumn{2}{|r|}{ Male } & \multicolumn{2}{|r|}{ Total } & \multicolumn{2}{|c|}{ Female } & \multicolumn{2}{|r|}{ Male } & \multicolumn{2}{|r|}{ Total } \\
\hline & $\%$ & $\mathrm{Cl}^{\dagger}$ & $\%$ & $\mathrm{Cl}$ & $\%$ & $\mathrm{Cl}$ & $\%$ & $\mathrm{Cl}$ & $\%$ & $\mathrm{Cl}$ & $\%$ & $\mathrm{Cl}$ \\
\hline \multicolumn{13}{|l|}{ State surveys } \\
\hline Alabama & 9.3 & $(6.6-12.7)$ & 20.7 & (18.2-23.5) & 15.1 & (13.1-17.5) & 5.0 & (3.3-7.6) & 11.5 & $(9.1-14.5)$ & 8.4 & $(6.8-10.4)$ \\
\hline Alaska & —§ & - & - & - & - & - & - & - & - & - & - & - \\
\hline Arizona & - & - & - & - & - & - & - & - & - & - & - & - \\
\hline Arkansas & 13.6 & $(11.1-16.4)$ & 23.8 & (18.9-29.5) & 18.6 & $(15.5-22.2)$ & 5.3 & $(4.1-6.9)$ & 13.1 & $(10.5-16.3)$ & 9.2 & $(7.7-11.0)$ \\
\hline California & 10.8 & $(8.2-14.1)$ & 24.5 & $(21.6-27.6)$ & 17.7 & $(15.7-19.9)$ & 4.0 & $(2.9-5.7)$ & 13.2 & (10.9-15.9) & 8.7 & $(7.5-10.0)$ \\
\hline Connecticut & - & - & - & - & - & - & - & - & - & - & - & - \\
\hline Delaware & 11.3 & $(9.5-13.4)$ & 25.6 & (22.5-28.9) & 18.4 & $(16.6-20.4)$ & 5.3 & $(3.9-7.1)$ & 12.4 & $(10.4-14.8)$ & 8.9 & $(7.7-10.4)$ \\
\hline Florida & 12.0 & $(10.6-13.5)$ & 26.7 & $(24.8-28.6)$ & 19.4 & (18.3-20.7) & 5.2 & $(4.3-6.3)$ & 12.5 & $(11.2-14.0)$ & 8.9 & $(8.1-9.8)$ \\
\hline Hawaii & 7.6 & $(6.3-9.2)$ & 17.6 & (15.9-19.5) & 12.6 & (11.5-13.7) & 2.7 & $(2.0-3.6)$ & 9.0 & $(7.7-10.5)$ & 5.8 & $(5.1-6.6)$ \\
\hline Idaho & 17.3 & $(14.0-21.2)$ & 39.4 & (35.6-43.2) & 28.6 & (25.9-31.4) & 7.3 & $(5.5-9.5)$ & 19.7 & (16.4-23.5) & 13.6 & $(11.7-15.7)$ \\
\hline Illinois & 13.2 & (11.0-15.7) & 27.0 & (23.3-31.0) & 20.1 & (17.8-22.6) & 6.0 & $(4.5-8.0)$ & 11.3 & $(8.7-14.6)$ & 8.7 & $(7.2-10.4)$ \\
\hline Indiana & 15.1 & (12.2-18.5) & 28.4 & (24.4-32.7) & 21.9 & (19.5-24.4) & 6.4 & $(4.7-8.7)$ & 12.9 & (10.5-15.8) & 9.9 & $(8.5-11.3)$ \\
\hline Kentucky & 11.6 & $(9.6-14.0)$ & 25.5 & (21.2-30.3) & 18.7 & $(16.3-21.2)$ & 3.9 & $(2.7-5.7)$ & 13.2 & $(10.6-16.4)$ & 8.7 & $(7.2-10.5)$ \\
\hline Maine & 19.4 & (18.3-20.4) & 33.1 & (31.6-34.8) & 26.4 & $(25.5-27.3)$ & 7.9 & $(7.1-8.9)$ & 16.9 & (15.6-18.3) & 12.5 & $(11.7-13.3)$ \\
\hline Maryland & - & - & - & - & - & - & - & - & - & - & - & - \\
\hline Massachusetts & 16.2 & $(13.5-19.3)$ & 30.9 & (27.5-34.5) & 23.6 & (20.9-26.6) & 6.4 & $(5.1-8.0)$ & 15.4 & (13.4-17.6) & 10.9 & $(9.5-12.6)$ \\
\hline Michigan & 15.4 & $(13.3-17.8)$ & 29.2 & $(24.8-34.1)$ & 22.3 & $(19.7-25.1)$ & 5.9 & $(4.2-8.0)$ & 12.6 & $(10.6-15.1)$ & 9.2 & $(7.8-10.9)$ \\
\hline Mississippi & 8.5 & $(6.3-11.5)$ & 16.7 & (14.0-19.9) & 12.6 & (10.4-15.1) & 4.5 & $(3.3-6.1)$ & 7.7 & (5.9-9.9) & 6.0 & $(4.8-7.6)$ \\
\hline Missouri & 15.8 & (13.3-18.7) & 29.0 & $(26.1-32.0)$ & 22.1 & $(20.2-24.2)$ & 7.1 & (5.4-9.3) & 13.8 & $(11.8-16.0)$ & 10.4 & $(9.5-11.4)$ \\
\hline Montana & 19.7 & $(18.1-21.4)$ & 34.7 & (31.8-37.8) & 27.4 & (25.5-29.4) & 8.5 & $(7.3-9.9)$ & 17.5 & (15.7-19.5) & 13.2 & $(12.1-14.3)$ \\
\hline Nebraska & 18.6 & $(15.9-21.6)$ & 35.2 & (31.3-39.4) & 27.1 & $(24.6-29.8)$ & 8.4 & $(6.5-10.9)$ & 17.6 & $(15.0-20.5)$ & 13.2 & $(11.5-15.1)$ \\
\hline Nevada & 15.1 & $(12.5-18.0)$ & 24.8 & $(21.5-28.4)$ & 19.9 & $(17.8-22.3)$ & 5.5 & $(3.6-8.2)$ & 11.9 & $(9.8-14.3)$ & 8.7 & $(7.2-10.4)$ \\
\hline New Hampshire & - & - & - & - & - & - & - & - & - & - & - & - \\
\hline New Mexico & - & - & - & - & - & - & - & - & - & - & - & - \\
\hline New York & 10.8 & $(9.3-12.5)$ & 19.8 & $(18.3-21.3)$ & 15.4 & $(14.0-16.8)$ & 4.3 & $(3.4-5.6)$ & 11.5 & $(9.5-13.8)$ & 7.9 & $(6.7-9.3)$ \\
\hline North Carolina & - & - & - & - & - & - & - & - & - & - & - & - \\
\hline North Dakota & 29.2 & $(26.0-32.6)$ & 42.0 & (38.8-45.3) & 35.8 & (33.4-38.2) & 11.1 & $(9.0-13.7)$ & 21.9 & (19.4-24.7) & 16.7 & $(15.0-18.5)$ \\
\hline Oklahoma & 13.7 & $(10.7-17.3)$ & 26.2 & (22.2-30.7) & 19.7 & (17.2-22.4) & 6.3 & $(4.9-8.0)$ & 11.0 & $(8.5-14.2)$ & 8.5 & $(7.3-10.0)$ \\
\hline Pennsylvania & 14.2 & (11.9-16.8) & 32.7 & (28.9-36.8) & 23.5 & (20.8-26.4) & 6.3 & (5.1-7.8) & 16.4 & (14.1-19.0) & 11.4 & $(9.9-13.1)$ \\
\hline Rhode Island & - & - & - & - & - & - & - & - & - & - & - & - \\
\hline South Carolina & 10.0 & $(6.9-14.2)$ & 23.5 & (18.3-29.6) & 16.6 & (13.2-20.6) & 4.8 & $(3.2-7.2)$ & 11.0 & $(7.8-15.4)$ & 7.8 & $(5.8-10.4)$ \\
\hline South Dakota & 24.0 & (17.7-31.6) & 35.8 & (31.4-40.5) & 30.0 & $(25.5-35.0)$ & 12.0 & $(8.2-17.3)$ & 23.5 & (20.9-26.3) & 17.9 & $(15.8-20.2)$ \\
\hline Tennessee & 11.0 & $(9.0-13.4)$ & 23.7 & (20.9-26.8) & 17.6 & $(15.3-20.1)$ & 4.6 & $(3.4-6.3)$ & 11.3 & $(10.0-12.8)$ & 8.1 & $(7.0-9.3)$ \\
\hline Vermont & - & - & - & - & - & - & - & - & - & - & - & - \\
\hline Virginia & 13.4 & $(11.5-15.7)$ & 25.3 & $(22.4-28.6)$ & 19.5 & $(17.6-21.7)$ & 5.8 & $(4.7-7.3)$ & 11.4 & $(9.5-13.6)$ & 8.7 & $(7.4-10.2)$ \\
\hline West Virginia & 14.4 & $(11.8-17.4)$ & 27.1 & (23.8-30.6) & 20.8 & (18.4-23.3) & 6.9 & $(5.3-9.0)$ & 12.8 & $(10.5-15.5)$ & 9.9 & $(8.5-11.4)$ \\
\hline Wyoming & 18.3 & $(15.9-21.0)$ & 31.7 & $(27.7-36.0)$ & 25.1 & (22.9-27.6) & 8.1 & $(6.6-9.9)$ & 17.7 & $(14.8-21.0)$ & 13.0 & $(11.3-14.9)$ \\
\hline Median & & 13.9 & & 26.8 & & 20.0 & & 5.9 & & 12.8 & & 9.0 \\
\hline Range & & 7.6-29.2) & & $6.7-42.0)$ & & $2.6-35.8)$ & & $-12.0)$ & & (.7-23.5) & & $5.8-17.9)$ \\
\hline \multicolumn{13}{|c|}{ Large urban school district surveys } \\
\hline Baltimore, MD & 10.2 & $(7.0-14.7)$ & 13.0 & $(9.7-17.2)$ & 11.8 & $(9.1-15.0)$ & 7.0 & $(4.4-11.1)$ & 8.6 & $(6.0-12.2)$ & 8.0 & $(5.9-10.8)$ \\
\hline Boston, MA & 11.2 & $(8.9-13.9)$ & 19.0 & $(16.6-21.7)$ & 15.2 & $(13.3-17.3)$ & 6.0 & $(4.7-7.6)$ & 9.5 & $(7.5-11.8)$ & 7.9 & $(6.8-9.1)$ \\
\hline Broward County, FL & 8.2 & $(6.3-10.6)$ & 17.2 & $(14.1-20.8)$ & 12.8 & $(11.0-14.8)$ & 3.3 & $(2.3-4.6)$ & 7.5 & $(5.8-9.5)$ & 5.4 & $(4.4-6.6)$ \\
\hline Cleveland, $\mathrm{OH}$ & - & - & - & - & - & - & - & - & - & - & - & - \\
\hline DeKalb County, GA & 7.5 & $(5.9-9.4)$ & 14.8 & $(12.3-17.7)$ & 11.1 & $(9.6-12.8)$ & 3.2 & $(2.2-4.4)$ & 6.9 & $(5.3-9.1)$ & 5.0 & $(4.0-6.3)$ \\
\hline Detroit, MI & 6.0 & $(4.5-8.0)$ & 10.5 & $(8.6-12.7)$ & 8.2 & $(6.8-9.8)$ & 2.5 & $(1.6-3.9)$ & 5.7 & $(4.3-7.6)$ & 4.1 & $(3.2-5.3)$ \\
\hline District of Columbia & - & - & - & - & - & - & - & - & - & - & - & - \\
\hline Duval County, FL & - & - & - & - & - & - & - & - & - & - & - & - \\
\hline Ft. Worth, TX & 12.5 & $(10.4-15.0)$ & 23.3 & $(20.7-26.0)$ & 18.0 & $(16.2-19.9)$ & 6.3 & $(4.9-8.0)$ & 12.0 & $(10.2-14.0)$ & 9.1 & (7.9-10.5) \\
\hline Houston, TX & 10.3 & $(8.7-12.1)$ & 18.4 & $(16.3-20.6)$ & 14.6 & $(13.2-16.1)$ & 4.9 & $(3.7-6.4)$ & 9.6 & $(8.2-11.3)$ & 7.4 & $(6.5-8.5)$ \\
\hline Los Angeles, CA & 10.7 & $(8.5-13.3)$ & 25.0 & $(20.8-29.8)$ & 17.6 & $(15.0-20.6)$ & 4.1 & $(2.9-5.7)$ & 10.3 & $(7.9-13.3)$ & 7.1 & $(5.6-9.0)$ \\
\hline Miami-Dade County, FL & 13.8 & $(12.0-15.9)$ & 26.8 & $(24.0-29.8)$ & 20.2 & $(18.5-22.0)$ & 6.3 & $(4.9-8.0)$ & 13.6 & $(11.4-16.1)$ & 9.9 & $(8.6-11.4)$ \\
\hline
\end{tabular}

See table footnotes on the next page. 
TABLE 94. (Continued) Percentage of high school students who drank two or more glasses/day of milk* and who drank three or more glasses/ day of milk,* by sex - selected U.S. sites, Youth Risk Behavior Survey, 2015

\begin{tabular}{|c|c|c|c|c|c|c|c|c|c|c|c|c|}
\hline \multirow[b]{3}{*}{ Site } & \multicolumn{6}{|c|}{ Drank two or more glasses/day of milk } & \multicolumn{6}{|c|}{ Drank three or more glasses/day of milk } \\
\hline & \multicolumn{2}{|c|}{ Female } & \multicolumn{2}{|r|}{ Male } & \multicolumn{2}{|r|}{ Total } & \multicolumn{2}{|c|}{ Female } & \multicolumn{2}{|r|}{ Male } & \multicolumn{2}{|r|}{ Total } \\
\hline & $\%$ & $\mathrm{Cl}^{\dagger}$ & $\%$ & $\mathrm{Cl}$ & $\%$ & $\mathrm{Cl}$ & $\%$ & $\mathrm{Cl}$ & $\%$ & $\mathrm{Cl}$ & $\%$ & $\mathrm{Cl}$ \\
\hline New York City, NY & 7.2 & $(6.2-8.5)$ & 15.9 & $(14.3-17.5)$ & 11.5 & $(10.3-12.9)$ & 2.9 & $(2.3-3.7)$ & 7.4 & $(6.3-8.5)$ & 5.1 & $(4.5-5.9)$ \\
\hline Oakland, CA & - & - & - & - & - & - & - & - & - & - & - & - \\
\hline Orange County, FL & 9.4 & $(7.5-11.6)$ & 26.4 & $(22.8-30.3)$ & 17.8 & $(15.5-20.5)$ & 4.4 & $(2.9-6.5)$ & 14.1 & $(10.9-18.0)$ & 9.3 & (7.2-11.9) \\
\hline Palm Beach County, FL & 7.5 & $(6.1-9.2)$ & 18.3 & $(16.2-20.5)$ & 12.8 & $(11.7-14.0)$ & 2.5 & $(1.7-3.8)$ & 8.8 & $(7.2-10.7)$ & 5.6 & $(4.7-6.7)$ \\
\hline Philadelphia, PA & 10.3 & $(7.8-13.5)$ & 18.4 & $(15.0-22.5)$ & 14.3 & $(11.9-17.1)$ & 5.4 & $(3.6-8.2)$ & 8.0 & $(5.5-11.5)$ & 6.7 & $(5.2-8.4)$ \\
\hline San Diego, CA & 9.3 & $(7.1-12.1)$ & 26.6 & $(23.9-29.4)$ & 18.1 & $(16.3-20.0)$ & 4.0 & $(2.7-6.0)$ & 12.0 & $(10.1-14.2)$ & 8.1 & $(6.9-9.6)$ \\
\hline San Francisco, CA & 10.9 & $(9.0-13.2)$ & 24.5 & $(21.1-28.2)$ & 17.7 & $(15.7-20.0)$ & 3.7 & $(2.6-5.2)$ & 9.8 & $(7.5-12.6)$ & 6.8 & $(5.4-8.4)$ \\
\hline Median & \multicolumn{2}{|r|}{10.2} & \multicolumn{2}{|c|}{18.4} & \multicolumn{2}{|r|}{14.6} & \multicolumn{2}{|c|}{4.1} & \multicolumn{2}{|r|}{9.5} & \multicolumn{2}{|r|}{7.1} \\
\hline Range & \multicolumn{2}{|c|}{$(6.0-13.8)$} & \multicolumn{2}{|c|}{$(10.5-26.8)$} & \multicolumn{2}{|c|}{$(8.2-20.2)$} & \multicolumn{2}{|c|}{$(2.5-7.0)$} & \multicolumn{2}{|c|}{$(5.7-14.1)$} & \multicolumn{2}{|c|}{$(4.1-9.9)$} \\
\hline
\end{tabular}

* During the 7 days before the survey.

$+95 \%$ confidence interval.

$\S$ Not available.

TABLE 95. Percentage of high school students who did not drink a can, bottle, or glass of soda or pop, ${ }^{*}+$ and who drank a can, bottle, or glass of soda or pop* one or more times/day, ${ }^{\dagger}$ by sex, race/ethnicity, and grade — United States, Youth Risk Behavior Survey, 2015

\begin{tabular}{|c|c|c|c|c|c|c|c|c|c|c|c|c|}
\hline \multirow[b]{3}{*}{ Category } & \multicolumn{6}{|c|}{ Did not drink soda or pop } & \multicolumn{6}{|c|}{ Drank soda or pop one or more times/day } \\
\hline & \multicolumn{2}{|c|}{ Female } & \multicolumn{2}{|r|}{ Male } & \multicolumn{2}{|r|}{ Total } & \multicolumn{2}{|r|}{ Female } & \multicolumn{2}{|r|}{ Male } & \multicolumn{2}{|r|}{ Total } \\
\hline & $\%$ & $\mathrm{Cl}^{\S}$ & $\%$ & $\mathrm{Cl}$ & $\%$ & $\mathrm{Cl}$ & $\%$ & $\mathrm{Cl}$ & $\%$ & $\mathrm{Cl}$ & $\%$ & $\mathrm{Cl}$ \\
\hline \multicolumn{13}{|c|}{ Race/Ethnicity } \\
\hline White & 33.3 & $(28.7-38.2)$ & 21.6 & $(19.7-23.7)$ & 27.5 & $(24.2-31.1)$ & 15.0 & $(11.5-19.2)$ & 24.5 & $(21.1-28.3)$ & 19.7 & $(16.4-23.4)$ \\
\hline Black" & 22.6 & $(18.3-27.7)$ & 23.1 & $(18.6-28.3)$ & 22.8 & $(19.5-26.5)$ & 21.6 & $(16.3-28.0)$ & 23.7 & $(19.1-29.0)$ & 22.7 & $(18.1-28.1)$ \\
\hline Hispanic & 26.3 & $(23.3-29.5)$ & 19.0 & $(16.5-21.7)$ & 22.6 & $(20.3-25.1)$ & 18.1 & $(15.3-21.3)$ & 25.1 & $(22.7-27.8)$ & 21.7 & $(19.5-24.0)$ \\
\hline \multicolumn{13}{|l|}{ Grade } \\
\hline 9 & 30.3 & (26.9-33.9) & 21.6 & $(19.0-24.3)$ & 25.8 & $(23.5-28.1)$ & 16.1 & $(13.3-19.5)$ & 22.4 & $(19.3-25.8)$ & 19.4 & $(16.9-22.2)$ \\
\hline 10 & 28.7 & $(24.2-33.8)$ & 20.5 & $(17.7-23.5)$ & 24.6 & $(21.2-28.5)$ & 16.2 & (12.9-20.2) & 25.4 & $(22.0-29.2)$ & 20.8 & $(17.8-24.1)$ \\
\hline 11 & 33.3 & $(29.3-37.6)$ & 19.8 & $(17.4-22.5)$ & 26.6 & $(23.8-29.6)$ & 15.1 & $(11.7-19.3)$ & 25.6 & $(22.9-28.4)$ & 20.5 & (17.7-23.6) \\
\hline 12 & 31.9 & $(27.1-37.2)$ & 23.6 & $(20.2-27.4)$ & 27.7 & $(24.0-31.8)$ & 17.9 & $(14.6-21.8)$ & 24.2 & $(20.4-28.4)$ & 21.0 & $(18.1-24.2)$ \\
\hline Total & 31.0 & $(27.4-34.8)$ & 21.4 & $(19.7-23.3)$ & 26.2 & $(23.6-28.9)$ & 16.4 & $(13.7-19.6)$ & 24.3 & $(22.0-26.9)$ & 20.4 & $(18.1-23.0)$ \\
\hline
\end{tabular}

* Not counting diet soda or diet pop.

t During the 7 days before the survey.

$\S 95 \%$ confidence interval.

"Non-Hispanic. 
TABLE 96. Percentage of high school students who did not drink a can, bottle, or glass of soda or pop ${ }^{*},+$ and who drank a can, bottle, or glass of soda or pop* one or more times/day, ${ }^{\dagger}$ by sex — selected U.S. sites, Youth Risk Behavior Survey, 2015

\begin{tabular}{|c|c|c|c|c|c|c|c|c|c|c|c|c|}
\hline \multirow[b]{3}{*}{ Site } & \multicolumn{6}{|c|}{ Did not drink soda or pop } & \multicolumn{6}{|c|}{ Drank soda or pop one or more times/day } \\
\hline & \multicolumn{2}{|c|}{ Female } & \multicolumn{2}{|r|}{ Male } & \multicolumn{2}{|r|}{ Total } & \multicolumn{2}{|c|}{ Female } & \multicolumn{2}{|r|}{ Male } & \multicolumn{2}{|r|}{ Total } \\
\hline & $\%$ & $\mathrm{Cl}^{\S}$ & $\%$ & $\mathrm{Cl}$ & $\%$ & $\mathrm{Cl}$ & $\%$ & $\mathrm{Cl}$ & $\%$ & $\mathrm{Cl}$ & $\%$ & $\mathrm{Cl}$ \\
\hline \multicolumn{13}{|l|}{ State surveys } \\
\hline Alabama & 17.5 & $(14.5-21.0)$ & 21.1 & (18.1-24.4) & 19.3 & $(17.3-21.5)$ & 28.6 & $(25.2-32.2)$ & 27.6 & $(24.6-30.8)$ & 28.2 & $(25.5-31.1)$ \\
\hline Alaska & 32.3 & $(28.6-36.2)$ & 19.7 & $(16.5-23.3)$ & 25.7 & $(22.9-28.6)$ & 14.5 & $(11.7-17.8)$ & 22.6 & $(19.5-26.0)$ & 18.8 & $(16.3-21.6)$ \\
\hline Arizona & 32.2 & $(28.2-36.4)$ & 23.6 & (18.7-29.4) & 27.8 & $(24.4-31.6)$ & 16.3 & $(12.8-20.5)$ & 22.5 & $(19.0-26.5)$ & 19.5 & $(16.3-23.2)$ \\
\hline Arkansas & 22.0 & $(19.2-25.1)$ & 21.0 & $(16.6-26.2)$ & 21.6 & $(18.4-25.2)$ & 26.1 & $(22.5-30.0)$ & 32.6 & $(27.7-38.0)$ & 29.3 & $(26.2-32.7)$ \\
\hline California & 33.0 & $(28.7-37.7)$ & 23.7 & (18.9-29.3) & 28.4 & $(25.0-32.2)$ & 12.3 & $(8.8-16.9)$ & 16.3 & $(14.3-18.6)$ & 14.2 & $(12.3-16.4)$ \\
\hline Connecticut & 37.3 & $(32.7-42.2)$ & 29.9 & $(26.6-33.4)$ & 33.6 & $(30.6-36.8)$ & 7.8 & $(5.8-10.4)$ & 16.0 & $(13.2-19.1)$ & 11.9 & $(10.1-14.0)$ \\
\hline Delaware & 31.2 & $(27.8-34.8)$ & 26.6 & $(24.0-29.5)$ & 29.0 & $(26.8-31.4)$ & 16.9 & $(14.3-20.0)$ & 20.7 & $(17.9-23.8)$ & 19.1 & $(17.0-21.3)$ \\
\hline Florida & 31.9 & $(29.7-34.3)$ & 25.0 & $(23.4-26.7)$ & 28.6 & $(27.1-30.0)$ & 17.0 & $(15.3-18.9)$ & 24.4 & $(22.4-26.5)$ & 20.8 & $(19.2-22.6)$ \\
\hline Hawaii & 39.2 & $(35.1-43.5)$ & 28.8 & $(25.8-31.9)$ & 34.2 & $(31.4-37.0)$ & 10.2 & $(8.8-12.0)$ & 16.7 & $(14.8-18.6)$ & 13.4 & $(12.3-14.7)$ \\
\hline Idaho & 32.7 & $(28.4-37.3)$ & 23.9 & $(21.2-26.9)$ & 28.1 & $(25.4-31.1)$ & 9.7 & $(7.3-12.8)$ & 16.9 & $(14.2-19.9)$ & 13.5 & $(11.5-15.7)$ \\
\hline Illinois & 35.9 & $(29.7-42.6)$ & 24.4 & $(21.8-27.1)$ & 30.1 & $(26.4-34.0)$ & 14.1 & $(11.0-17.9)$ & 22.3 & $(19.3-25.6)$ & 18.3 & $(16.0-20.8)$ \\
\hline Indiana & 28.5 & $(24.9-32.5)$ & 20.9 & $(17.6-24.5)$ & 24.6 & (21.6-27.8) & 15.7 & $(12.8-19.2)$ & 23.6 & (18.5-29.7) & 20.0 & $(16.5-24.1)$ \\
\hline Kentucky & 24.7 & $(21.0-28.8)$ & 22.7 & $(19.2-26.8)$ & 23.6 & $(20.8-26.7)$ & 31.6 & $(26.9-36.8)$ & 33.0 & $(28.1-38.3)$ & 32.4 & $(29.5-35.4)$ \\
\hline Maine & - & - & - & - & - & - & - & - & - & - & - & - \\
\hline Maryland & 30.9 & $(30.1-31.7)$ & 27.0 & $(26.2-27.9)$ & 29.0 & $(28.5-29.6)$ & 14.2 & $(13.5-14.9)$ & 19.1 & (18.4-19.7) & 16.6 & $(16.1-17.1)$ \\
\hline Massachusetts & 40.3 & $(36.2-44.5)$ & 24.6 & $(22.0-27.3)$ & 32.6 & $(29.7-35.5)$ & 9.7 & $(7.9-12.0)$ & 17.7 & $(14.8-21.0)$ & 13.8 & $(11.6-16.2)$ \\
\hline Michigan & 30.0 & $(26.7-33.6)$ & 22.6 & (19.2-26.5) & 26.3 & $(24.3-28.5)$ & 14.8 & $(12.3-17.6)$ & 23.0 & $(20.6-25.6)$ & 18.9 & $(17.1-20.9)$ \\
\hline Mississippi & 18.8 & $(15.9-22.0)$ & 18.4 & $(15.3-21.9)$ & 18.7 & (16.4-21.3) & 29.1 & $(26.9-31.5)$ & 29.9 & $(26.3-33.9)$ & 29.4 & (27.4-31.5) \\
\hline Missouri & 28.5 & $(22.4-35.5)$ & 20.5 & $(17.0-24.4)$ & 24.5 & $(20.4-29.0)$ & 17.1 & $(13.2-21.9)$ & 25.2 & $(21.8-28.9)$ & 21.1 & (18.1-24.4) \\
\hline Montana & 31.1 & $(28.6-33.7)$ & 20.6 & $(18.3-23.0)$ & 25.7 & $(23.8-27.6)$ & 12.9 & $(11.3-14.7)$ & 23.9 & $(22.2-25.7)$ & 18.6 & $(17.3-20.0)$ \\
\hline Nebraska & 26.9 & (23.4-30.6) & 21.8 & $(18.5-25.5)$ & 24.3 & $(22.0-26.9)$ & 15.8 & $(13.0-19.1)$ & 24.4 & $(21.3-27.8)$ & 20.4 & (18.1-22.8) \\
\hline Nevada & 31.7 & $(26.9-36.8)$ & 27.5 & $(24.7-30.4)$ & 29.6 & $(26.6-32.8)$ & 11.3 & $(8.8-14.5)$ & 16.1 & $(13.2-19.6)$ & 13.7 & $(11.7-16.0)$ \\
\hline New Hampshire & 46.1 & $(44.3-48.0)$ & 28.5 & $(26.7-30.4)$ & 37.0 & $(35.6-38.4)$ & 7.5 & (6.6-8.4) & 18.1 & $(16.8-19.5)$ & 13.0 & $(12.2-13.9)$ \\
\hline New Mexico & 25.5 & $(23.7-27.4)$ & 21.5 & $(20.0-23.1)$ & 23.5 & $(22.1-24.8)$ & 17.7 & $(16.4-19.0)$ & 24.3 & $(22.8-25.8)$ & 21.0 & $(19.9-22.1)$ \\
\hline New York & 38.0 & (34.8-41.2) & 30.2 & $(26.6-34.0)$ & 34.1 & $(32.2-36.1)$ & 11.3 & $(9.9-12.8)$ & 16.4 & (13.6-19.7) & 14.0 & (12.2-15.9) \\
\hline North Carolina & 26.8 & $(21.7-32.5)$ & 19.2 & $(16.1-22.8)$ & 23.1 & (19.8-26.8) & 23.9 & $(19.3-29.2)$ & 29.8 & $(24.6-35.5)$ & 26.8 & $(22.7-31.4)$ \\
\hline North Dakota & 31.5 & $(28.3-34.9)$ & 20.0 & $(17.7-22.4)$ & 25.6 & $(23.6-27.8)$ & 12.3 & $(9.8-15.4)$ & 24.7 & $(21.9-27.8)$ & 18.7 & (16.6-20.9) \\
\hline Oklahoma & 22.8 & $(19.3-26.7)$ & 20.3 & $(16.7-24.5)$ & 21.7 & $(19.0-24.7)$ & 25.3 & (21.4-29.6) & 33.4 & $(28.8-38.4)$ & 29.4 & $(25.9-33.2)$ \\
\hline Pennsylvania & 29.4 & $(25.4-33.6)$ & 24.5 & $(21.9-27.3)$ & 26.9 & $(24.6-29.3)$ & 12.9 & $(10.2-16.2)$ & 23.3 & $(20.2-26.8)$ & 18.2 & $(15.9-20.8)$ \\
\hline Rhode Island & 39.4 & $(32.2-47.1)$ & 29.5 & $(25.9-33.5)$ & 34.4 & $(29.7-39.4)$ & 9.5 & $(6.2-14.2)$ & 15.6 & (13.9-17.6) & 12.6 & (10.4-15.2) \\
\hline South Carolina & 22.7 & $(17.8-28.6)$ & 20.7 & $(17.1-25.0)$ & 21.7 & $(18.5-25.3)$ & 26.5 & $(21.3-32.5)$ & 26.6 & $(22.6-31.1)$ & 26.7 & $(23.6-30.0)$ \\
\hline South Dakota & 22.9 & (19.7-26.5) & 23.2 & $(18.1-29.2)$ & 23.1 & (19.3-27.3) & 17.4 & $(14.6-20.6)$ & 28.7 & $(22.3-36.1)$ & 23.2 & (19.4-27.4) \\
\hline Tennessee & 22.8 & $(19.9-26.1)$ & 20.7 & $(18.3-23.2)$ & 21.7 & $(19.6-24.0)$ & 26.0 & $(23.4-28.8)$ & 31.8 & $(28.7-35.1)$ & 29.0 & $(26.5-31.7)$ \\
\hline Vermont & 42.1 & $(41.2-43.1)$ & 24.8 & $(24.0-25.7)$ & 33.3 & $(32.7-34.0)$ & 9.5 & $(8.9-10.0)$ & 19.9 & (19.1-20.7) & 14.8 & (14.4-15.3) \\
\hline Virginia & 33.3 & $(30.2-36.6)$ & 27.0 & $(24.2-30.0)$ & 30.0 & $(27.4-32.7)$ & 14.2 & $(11.9-17.0)$ & 19.5 & $(16.5-23.0)$ & 17.0 & $(14.8-19.4)$ \\
\hline West Virginia & 24.0 & $(20.8-27.5)$ & 19.6 & $(16.0-23.8)$ & 21.8 & (19.4-24.5) & 26.4 & $(22.9-30.2)$ & 33.7 & $(30.3-37.4)$ & 30.1 & $(27.6-32.7)$ \\
\hline Wyoming & 28.8 & $(25.4-32.5)$ & 21.9 & $(18.4-26.0)$ & 25.4 & $(22.6-28.3)$ & 15.6 & $(13.2-18.2)$ & 26.6 & $(23.0-30.6)$ & 21.2 & $(18.9-23.6)$ \\
\hline Median & & 31.0 & & 22.9 & & 26.0 & & 15.2 & & 23.4 & & 19.0 \\
\hline Range & & $7.5-46.1)$ & & $8.4-30.2)$ & & (8.7-37.0) & & $.5-31.6)$ & & $5.6-33.7)$ & & $1.9-32.4)$ \\
\hline \multicolumn{13}{|c|}{ Large urban school district surveys } \\
\hline Baltimore, MD & 21.4 & $(17.5-25.8)$ & 18.5 & $(14.5-23.2)$ & 19.6 & $(17.0-22.4)$ & 28.2 & $(23.6-33.3)$ & 26.3 & $(21.7-31.5)$ & 27.5 & $(24.2-31.0)$ \\
\hline Boston, MA & 32.7 & $(29.3-36.4)$ & 26.3 & $(22.8-30.1)$ & 29.6 & $(27.3-32.0)$ & 14.3 & $(11.5-17.6)$ & 19.6 & $(16.9-22.7)$ & 16.9 & $(15.1-19.0)$ \\
\hline Broward County, FL & 32.9 & $(29.2-36.9)$ & 30.0 & $(26.0-34.3)$ & 31.3 & $(28.3-34.5)$ & 11.2 & $(8.8-14.1)$ & 19.3 & $(16.5-22.4)$ & 15.4 & (13.4-17.5) \\
\hline Cleveland, $\mathrm{OH}$ & 17.9 & $(14.8-21.4)$ & 24.7 & $(21.7-28.0)$ & 21.6 & $(19.5-24.0)$ & 25.9 & $(22.5-29.6)$ & 22.5 & $(19.1-26.3)$ & 24.2 & $(21.5-27.1)$ \\
\hline DeKalb County, GA & 28.9 & $(25.5-32.6)$ & 24.3 & $(20.9-28.0)$ & 26.6 & $(24.2-29.1)$ & 14.8 & $(12.6-17.2)$ & 17.6 & $(14.9-20.6)$ & 16.3 & $(14.5-18.1)$ \\
\hline Detroit, MI & 25.3 & $(22.5-28.5)$ & 26.8 & $(23.1-30.8)$ & 25.9 & $(23.5-28.4)$ & 25.1 & $(21.0-29.6)$ & 24.2 & $(19.6-29.3)$ & 24.7 & $(21.4-28.3)$ \\
\hline District of Columbia & 24.9 & $(23.7-26.2)$ & 25.9 & $(24.5-27.3)$ & 25.5 & $(24.6-26.5)$ & 18.4 & $(17.2-19.6)$ & 19.8 & $(18.5-21.2)$ & 19.1 & $(18.2-20.0)$ \\
\hline Duval County, FL & 28.9 & $(26.4-31.5)$ & 29.5 & $(26.3-32.9)$ & 29.2 & $(26.9-31.5)$ & 18.2 & $(16.0-20.6)$ & 20.3 & $(18.2-22.7)$ & 19.2 & $(17.7-20.8)$ \\
\hline Ft. Worth, TX & 19.3 & $(16.8-22.1)$ & 18.2 & $(16.0-20.7)$ & 18.9 & $(17.3-20.6)$ & 23.6 & $(21.1-26.2)$ & 29.0 & $(26.2-31.9)$ & 26.2 & $(24.2-28.3)$ \\
\hline Houston, TX & 24.3 & $(21.9-26.9)$ & 22.2 & (19.8-24.8) & 23.2 & $(21.4-25.2)$ & 18.4 & (16.2-20.9) & 20.9 & $(18.5-23.4)$ & 19.8 & (18.1-21.7) \\
\hline Los Angeles, CA & 31.8 & $(27.3-36.6)$ & 22.9 & $(19.6-26.5)$ & 27.4 & $(24.1-31.0)$ & 8.3 & $(6.3-10.8)$ & 14.5 & $(12.3-17.0)$ & 11.3 & $(9.6-13.4)$ \\
\hline Miami-Dade County, FL & 32.5 & $(30.0-35.1)$ & 22.7 & $(20.1-25.5)$ & 27.9 & $(26.0-29.8)$ & 15.8 & $(13.5-18.5)$ & 23.8 & $(21.3-26.6)$ & 19.8 & $(18.1-21.6)$ \\
\hline
\end{tabular}

See table footnotes on the next page. 
TABLE 96. (Continued) Percentage of high school students who did not drink a can, bottle, or glass of soda or pop*, ${ }^{*}$ and who drank a can, bottle, or glass of soda or pop* one or more times/day, ${ }^{\dagger}$ by sex — selected U.S. sites, Youth Risk Behavior Survey, 2015

\begin{tabular}{|c|c|c|c|c|c|c|c|c|c|c|c|c|}
\hline \multirow[b]{3}{*}{ Site } & \multicolumn{6}{|c|}{ Did not drink soda or pop } & \multicolumn{6}{|c|}{ Drank soda or pop one or more times/day } \\
\hline & \multicolumn{2}{|r|}{ Female } & \multicolumn{2}{|r|}{ Male } & \multicolumn{2}{|r|}{ Total } & \multicolumn{2}{|c|}{ Female } & \multicolumn{2}{|r|}{ Male } & \multicolumn{2}{|r|}{ Total } \\
\hline & $\%$ & $\mathrm{Cl}^{\S}$ & $\%$ & $\mathrm{Cl}$ & $\%$ & $\mathrm{Cl}$ & $\%$ & $\mathrm{Cl}$ & $\%$ & $\mathrm{Cl}$ & $\%$ & $\mathrm{Cl}$ \\
\hline New York City, NY & 30.9 & $(27.1-35.0)$ & 27.1 & $(25.3-28.9)$ & 29.0 & $(26.8-31.3)$ & 15.0 & $(12.7-17.7)$ & 16.5 & $(14.5-18.8)$ & 15.8 & $(14.1-17.7)$ \\
\hline Oakland, CA & - & - & - & - & - & - & - & - & - & - & - & - \\
\hline Orange County, FL & 34.2 & $(30.4-38.3)$ & 27.1 & $(23.8-30.6)$ & 30.7 & $(28.0-33.5)$ & 14.2 & $(11.2-17.8)$ & 22.8 & $(19.0-27.0)$ & 18.7 & $(15.9-21.8)$ \\
\hline Palm Beach County, FL & 35.5 & $(32.2-39.1)$ & 29.9 & $(26.4-33.6)$ & 33.0 & $(30.6-35.6)$ & 13.6 & $(11.7-15.7)$ & 17.7 & $(15.1-20.6)$ & 15.6 & $(13.8-17.6)$ \\
\hline Philadelphia, PA & 28.0 & $(23.1-33.5)$ & 21.0 & $(17.7-24.8)$ & 24.5 & $(21.6-27.8)$ & 18.3 & $(15.1-22.0)$ & 24.8 & $(21.4-28.4)$ & 21.6 & $(19.1-24.2)$ \\
\hline San Diego, CA & 41.6 & $(38.1-45.3)$ & 28.8 & $(26.3-31.5)$ & 35.0 & $(32.6-37.5)$ & 6.1 & $(4.6-8.1)$ & 13.0 & $(11.0-15.2)$ & 9.6 & $(8.2-11.2)$ \\
\hline San Francisco, CA & 46.1 & $(41.8-50.4)$ & 31.5 & $(28.4-34.7)$ & 39.0 & (36.1-41.9) & 8.8 & $(7.3-10.6)$ & 12.1 & $(9.9-14.9)$ & 10.5 & $(8.9-12.3)$ \\
\hline Median & & 29.9 & & 26.1 & & 27.6 & & 15.4 & & 20.0 & & 18.9 \\
\hline Range & & $7.9-46.1)$ & & $8.2-31.5)$ & & (8.9-39.0) & & $6.1-28.2)$ & & $(2.1-29.0)$ & & $9.6-27.5)$ \\
\hline
\end{tabular}

* Not counting diet soda or diet pop.

† During the 7 days before the survey.

$\S 95 \%$ confidence interval.

I Not available.

TABLE 97. Percentage of high school students who drank a can, bottle, or glass of soda or pop* two or more times/day ${ }^{\dagger}$ and who drank a can, bottle, or glass of soda or pop* three or more times/day, ${ }^{\dagger}$ by sex, race/ethnicity, and grade — United States, Youth Risk Behavior Survey, 2015

\begin{tabular}{|c|c|c|c|c|c|c|c|c|c|c|c|c|}
\hline \multirow[b]{3}{*}{ Category } & \multicolumn{6}{|c|}{ Drank soda or pop two or more times/day } & \multicolumn{6}{|c|}{ Drank soda or pop three or more times/day } \\
\hline & \multicolumn{2}{|c|}{ Female } & \multicolumn{2}{|c|}{ Male } & \multicolumn{2}{|c|}{ Total } & \multicolumn{2}{|c|}{ Female } & \multicolumn{2}{|c|}{ Male } & \multicolumn{2}{|c|}{ Total } \\
\hline & $\%$ & $\mathrm{Cl}^{\S}$ & $\%$ & $\mathrm{Cl}$ & $\%$ & $\mathrm{Cl}$ & $\%$ & $\mathrm{Cl}$ & $\%$ & $\mathrm{Cl}$ & $\%$ & $\mathrm{Cl}$ \\
\hline \multicolumn{13}{|c|}{ Race/Ethnicity } \\
\hline White & 9.1 & $(6.6-12.4)$ & 15.1 & $(12.5-18.2)$ & 12.1 & $(9.6-15.0)$ & 4.7 & $(3.1-6.9)$ & 7.2 & $(5.4-9.6)$ & 5.9 & $(4.4-8.0)$ \\
\hline Black? & 15.0 & $(10.2-21.5)$ & 15.8 & $(11.3-21.6)$ & 15.4 & $(11.4-20.6)$ & 9.9 & $(7.3-13.3)$ & 9.3 & $(6.3-13.4)$ & 9.5 & $(7.2-12.4)$ \\
\hline Hispanic & 11.4 & $(8.9-14.5)$ & 17.4 & $(15.2-19.8)$ & 14.4 & $(12.4-16.6)$ & 6.7 & $(5.1-8.8)$ & 9.4 & $(7.9-11.2)$ & 8.1 & $(6.8-9.5)$ \\
\hline \multicolumn{13}{|l|}{ Grade } \\
\hline 9 & 10.0 & $(8.0-12.6)$ & 14.6 & $(12.2-17.3)$ & 12.4 & (10.5-14.6) & 5.8 & $(4.3-8.0)$ & 7.5 & $(5.5-10.2)$ & 6.7 & (5.3-8.5) \\
\hline 10 & 10.2 & $(7.4-13.8)$ & 15.8 & $(13.1-18.9)$ & 13.0 & $(10.4-16.1)$ & 6.0 & $(4.3-8.4)$ & 9.1 & $(7.2-11.3)$ & 7.5 & $(6.0-9.4)$ \\
\hline 11 & 10.5 & (7.8-13.9) & 16.1 & (13.8-18.8) & 13.4 & $(11.2-16.0)$ & 5.8 & $(4.2-8.0)$ & 7.8 & $(6.0-10.2)$ & 7.0 & $(5.6-8.7)$ \\
\hline 12 & 10.5 & $(7.7-14.0)$ & 16.0 & $(13.0-19.6)$ & 13.2 & $(10.9-16.0)$ & 5.5 & $(3.8-7.9)$ & 8.4 & $(6.3-11.1)$ & 7.0 & $(5.4-9.0)$ \\
\hline Total & 10.4 & $(8.2-13.0)$ & 15.6 & (13.7-17.8) & 13.0 & $(11.1-15.2)$ & 5.9 & $(4.6-7.5)$ & 8.3 & $(6.8-10.0)$ & 7.1 & $(5.8-8.6)$ \\
\hline
\end{tabular}

* Not counting diet soda or diet pop.

${ }^{\dagger}$ During the 7 days before the survey.

\$ $95 \%$ confidence interval.

" Non-Hispanic. 
TABLE 98. Percentage of high school students who drank a can, bottle, or glass of soda or pop* two or more times/day ${ }^{\dagger}$ and who drank a can, bottle, or glass of soda or pop* three or more times/day, ${ }^{\dagger}$ by sex - selected U.S. sites, Youth Risk Behavior Survey, 2015

\begin{tabular}{|c|c|c|c|c|c|c|c|c|c|c|c|c|}
\hline \multirow[b]{3}{*}{ Site } & \multicolumn{6}{|c|}{ Drank soda or pop two or more times/day } & \multicolumn{6}{|c|}{ Drank soda or pop three or more times/day } \\
\hline & \multicolumn{2}{|r|}{ Female } & \multicolumn{2}{|r|}{ Male } & \multicolumn{2}{|r|}{ Total } & \multicolumn{2}{|c|}{ Female } & \multicolumn{2}{|r|}{ Male } & \multicolumn{2}{|r|}{ Total } \\
\hline & $\%$ & $\mathrm{Cl}^{\S}$ & $\%$ & $\mathrm{Cl}$ & $\%$ & $\mathrm{Cl}$ & $\%$ & $\mathrm{Cl}$ & $\%$ & $\mathrm{Cl}$ & $\%$ & $\mathrm{Cl}$ \\
\hline \multicolumn{13}{|l|}{ State surveys } \\
\hline Alabama & 19.8 & $(17.0-22.9)$ & 20.5 & $(17.9-23.3)$ & 20.1 & $(18.2-22.1)$ & 11.9 & $(9.9-14.2)$ & 13.0 & $(10.5-15.9)$ & 12.4 & $(10.8-14.2)$ \\
\hline Alaska & 10.8 & $(8.2-14.1)$ & 15.2 & $(11.8-19.4)$ & 13.1 & (10.4-16.4) & 6.1 & $(4.4-8.4)$ & 9.4 & $(6.9-12.7)$ & 7.8 & $(6.1-10.0)$ \\
\hline Arizona & 9.3 & $(6.9-12.4)$ & 12.0 & $(9.3-15.4)$ & 10.8 & $(8.7-13.4)$ & 4.9 & $(3.6-6.8)$ & 5.8 & $(4.3-7.8)$ & 5.4 & $(4.2-7.1)$ \\
\hline Arkansas & 19.0 & $(14.9-24.0)$ & 23.9 & (19.6-28.8) & 21.4 & $(18.0-25.2)$ & 11.7 & $(7.8-17.0)$ & 14.2 & (10.6-18.6) & 12.9 & $(9.9-16.6)$ \\
\hline California & 6.3 & $(4.4-8.9)$ & 9.0 & $(7.4-10.9)$ & 7.6 & $(6.5-8.9)$ & 3.3 & $(2.5-4.4)$ & 4.9 & $(3.4-7.2)$ & 4.1 & $(3.1-5.4)$ \\
\hline Connecticut & 5.1 & $(3.5-7.2)$ & 10.1 & $(8.0-12.7)$ & 7.6 & $(6.3-9.2)$ & 2.5 & $(1.6-4.0)$ & 5.9 & $(4.2-8.2)$ & 4.2 & $(3.1-5.6)$ \\
\hline Delaware & 10.8 & $(8.6-13.5)$ & 12.7 & $(10.4-15.3)$ & 11.8 & $(10.0-13.8)$ & 6.7 & $(5.2-8.6)$ & 8.1 & $(6.6-10.0)$ & 7.5 & (6.3-8.9) \\
\hline Florida & 11.3 & $(9.9-12.8)$ & 16.1 & $(14.2-18.2)$ & 13.7 & $(12.4-15.2)$ & 5.7 & $(4.8-6.7)$ & 9.0 & $(7.6-10.7)$ & 7.4 & $(6.5-8.5)$ \\
\hline Hawaii & 6.1 & $(4.9-7.5)$ & 10.0 & $(8.6-11.5)$ & 8.0 & (7.0-9.1) & 3.4 & $(2.5-4.6)$ & 6.2 & $(5.0-7.6)$ & 4.8 & $(3.9-5.8)$ \\
\hline Idaho & 5.2 & $(3.6-7.5)$ & 9.2 & $(7.0-12.1)$ & 7.3 & $(5.6-9.3)$ & 2.6 & $(1.6-4.3)$ & 5.4 & $(3.8-7.6)$ & 4.0 & $(2.9-5.6)$ \\
\hline Illinois & 8.9 & $(6.8-11.5)$ & 13.3 & (11.1-15.8) & 11.2 & $(9.6-13.0)$ & 5.6 & $(4.1-7.7)$ & 7.3 & $(5.7-9.4)$ & 6.6 & $(5.4-7.9)$ \\
\hline Indiana & 10.2 & $(7.9-13.1)$ & 16.0 & $(12.7-20.0)$ & 13.3 & $(11.1-15.9)$ & 4.5 & $(3.2-6.2)$ & 8.5 & $(6.5-11.2)$ & 6.6 & $(5.2-8.4)$ \\
\hline Kentucky & 22.5 & $(18.4-27.2)$ & 23.9 & $(19.8-28.7)$ & 23.3 & $(20.3-26.5)$ & 13.3 & $(10.0-17.4)$ & 13.0 & $(10.1-16.5)$ & 13.2 & $(11.0-15.8)$ \\
\hline Maine & -? & - & - & - & - & - & - & - & - & - & - & - \\
\hline Maryland & 9.5 & $(9.0-10.0)$ & 12.5 & $(11.9-13.1)$ & 11.0 & $(10.6-11.4)$ & 5.6 & $(5.1-6.0)$ & 7.5 & $(7.0-8.0)$ & 6.5 & $(6.2-6.9)$ \\
\hline Massachusetts & 6.7 & $(5.2-8.5)$ & 11.4 & $(9.0-14.3)$ & 9.1 & $(7.4-11.1)$ & 3.5 & $(2.5-4.9)$ & 6.6 & $(5.1-8.6)$ & 5.0 & $(3.9-6.5)$ \\
\hline Michigan & 9.0 & $(7.0-11.4)$ & 14.6 & $(12.1-17.5)$ & 11.8 & $(10.0-13.9)$ & 4.9 & $(3.7-6.6)$ & 7.5 & $(5.9-9.6)$ & 6.3 & $(5.1-7.7)$ \\
\hline Mississippi & 20.7 & $(18.4-23.1)$ & 20.4 & $(17.3-24.0)$ & 20.4 & $(18.5-22.5)$ & 11.3 & $(9.6-13.2)$ & 12.6 & $(9.5-16.6)$ & 11.9 & $(9.9-14.3)$ \\
\hline Missouri & 10.8 & $(7.7-15.0)$ & 17.1 & $(13.3-21.7)$ & 14.0 & $(11.2-17.2)$ & 5.0 & $(2.4-10.1)$ & 9.5 & $(7.3-12.2)$ & 7.2 & $(5.1-10.2)$ \\
\hline Montana & 6.7 & $(5.6-8.1)$ & 14.4 & $(13.0-15.9)$ & 10.7 & $(9.8-11.7)$ & 3.1 & $(2.5-3.9)$ & 7.2 & $(6.0-8.6)$ & 5.2 & $(4.5-6.1)$ \\
\hline Nebraska & 7.8 & $(5.8-10.4)$ & 15.6 & $(13.2-18.2)$ & 11.9 & $(10.2-13.8)$ & 3.3 & $(2.2-5.0)$ & 6.5 & $(5.0-8.5)$ & 5.1 & $(4.0-6.5)$ \\
\hline Nevada & 6.7 & $(4.9-9.1)$ & 9.3 & $(6.7-12.7)$ & 8.0 & $(6.4-9.9)$ & 4.5 & $(3.1-6.5)$ & 4.8 & $(3.2-7.2)$ & 4.7 & $(3.5-6.2)$ \\
\hline New Hampshire & 4.7 & $(4.1-5.5)$ & 13.7 & $(12.6-14.9)$ & 9.4 & $(8.7-10.2)$ & 2.6 & $(2.1-3.3)$ & 7.5 & $(6.8-8.3)$ & 5.2 & $(4.7-5.8)$ \\
\hline New Mexico & 10.3 & $(9.3-11.4)$ & 15.7 & $(14.3-17.2)$ & 13.0 & (12.1-13.9) & 5.3 & $(4.4-6.4)$ & 8.6 & $(7.5-9.8)$ & 7.0 & $(6.3-7.7)$ \\
\hline New York & 6.8 & $(5.6-8.3)$ & 10.7 & $(8.9-12.8)$ & 8.8 & $(7.7-10.1)$ & 3.9 & $(3.0-5.0)$ & 6.2 & (4.7-8.1) & 5.0 & (4.2-6.1) \\
\hline North Carolina & 16.6 & $(12.1-22.2)$ & 21.6 & $(16.8-27.4)$ & 19.1 & (15.3-23.6) & 11.2 & $(7.6-16.2)$ & 13.6 & $(11.0-16.6)$ & 12.3 & $(10.0-15.0)$ \\
\hline North Dakota & 7.1 & $(5.0-10.0)$ & 16.2 & $(13.9-18.9)$ & 11.7 & $(10.1-13.7)$ & 3.5 & $(2.2-5.7)$ & 8.0 & $(6.3-10.2)$ & 5.8 & (4.7-7.2) \\
\hline Oklahoma & 17.3 & $(14.2-20.8)$ & 21.9 & $(17.9-26.5)$ & 19.8 & $(16.8-23.0)$ & 7.7 & $(5.7-10.4)$ & 11.7 & $(9.0-15.2)$ & 9.7 & $(7.6-12.4)$ \\
\hline Pennsylvania & 8.9 & $(6.5-12.1)$ & 15.0 & $(12.5-17.9)$ & 12.0 & $(9.9-14.5)$ & 5.0 & $(3.4-7.3)$ & 8.2 & $(6.5-10.4)$ & 6.6 & $(5.2-8.4)$ \\
\hline Rhode Island & 6.1 & (3.7-9.8) & 10.5 & $(9.1-12.0)$ & 8.3 & $(6.8-10.1)$ & 3.5 & $(1.6-7.5)$ & 5.4 & $(4.1-7.2)$ & 4.5 & (3.1-6.4) \\
\hline South Carolina & 21.1 & (16.6-26.4) & 18.9 & $(16.0-22.2)$ & 20.1 & (17.2-23.4) & 13.1 & $(9.9-17.2)$ & 11.6 & $(8.2-16.1)$ & 12.5 & $(10.0-15.5)$ \\
\hline South Dakota & 11.0 & $(8.2-14.7)$ & 20.5 & $(15.3-26.9)$ & 15.9 & (12.6-19.7) & 4.7 & $(2.8-7.7)$ & 9.4 & $(6.0-14.4)$ & 7.1 & (5.4-9.2) \\
\hline Tennessee & 16.5 & $(14.5-18.6)$ & 22.8 & $(20.4-25.5)$ & 19.7 & $(17.8-21.8)$ & 9.2 & $(7.4-11.5)$ & 12.2 & $(10.6-14.0)$ & 10.8 & $(9.3-12.5)$ \\
\hline Vermont & 5.8 & $(5.4-6.3)$ & 13.7 & $(13.0-14.3)$ & 9.9 & $(9.5-10.3)$ & 3.2 & $(2.9-3.6)$ & 8.0 & $(7.5-8.5)$ & 5.7 & (5.4-6.0) \\
\hline Virginia & 7.7 & $(6.0-9.9)$ & 12.8 & $(10.6-15.5)$ & 10.4 & $(8.6-12.4)$ & 3.7 & $(2.6-5.2)$ & 7.6 & $(6.0-9.5)$ & 5.7 & $(4.5-7.2)$ \\
\hline West Virginia & 18.9 & $(15.8-22.4)$ & 25.2 & $(22.0-28.7)$ & 22.1 & $(19.8-24.6)$ & 11.5 & $(9.5-13.9)$ & 15.5 & $(13.3-18.0)$ & 13.5 & $(12.3-14.8)$ \\
\hline Wyoming & 8.8 & $(6.6-11.5)$ & 16.8 & $(13.3-21.0)$ & 12.9 & $(11.1-14.9)$ & 3.8 & $(2.4-6.0)$ & 9.3 & $(7.4-11.8)$ & 6.7 & (5.6-7.9) \\
\hline Median & & 9.1 & & 15.1 & & 11.8 & & 4.9 & & 8.0 & & 6.6 \\
\hline Range & & $4.7-22.5)$ & & $9.0-25.2)$ & & 7.3-23.3) & & $.5-13.3)$ & & (8-15.5) & & $4.0-13.5)$ \\
\hline \multicolumn{13}{|c|}{ Large urban school district surveys } \\
\hline Baltimore, MD & 21.5 & $(17.3-26.4)$ & 16.1 & $(12.5-20.6)$ & 19.2 & (16.4-22.4) & 15.2 & (11.7-19.6) & 11.6 & $(8.6-15.6)$ & 13.5 & $(10.9-16.6)$ \\
\hline Boston, MA & 9.8 & $(7.4-13.0)$ & 13.4 & $(11.2-15.9)$ & 11.6 & $(9.9-13.6)$ & 6.6 & $(4.7-9.2)$ & 9.0 & $(7.0-11.4)$ & 7.7 & $(6.3-9.4)$ \\
\hline Broward County, FL & 7.7 & $(5.8-10.2)$ & 11.5 & $(9.6-13.9)$ & 9.7 & $(8.2-11.5)$ & 4.8 & $(3.4-6.8)$ & 6.7 & $(5.1-8.7)$ & 5.8 & $(4.6-7.4)$ \\
\hline Cleveland, $\mathrm{OH}$ & 19.5 & $(16.6-22.9)$ & 17.1 & $(14.3-20.3)$ & 18.4 & $(16.2-20.8)$ & 14.2 & $(11.6-17.4)$ & 10.3 & $(7.9-13.4)$ & 12.4 & $(10.3-14.8)$ \\
\hline DeKalb County, GA & 9.9 & $(8.1-12.1)$ & 11.9 & $(9.6-14.7)$ & 10.9 & $(9.4-12.6)$ & 6.2 & $(4.7-8.3)$ & 6.8 & $(4.9-9.3)$ & 6.5 & (5.3-7.9) \\
\hline Detroit, MI & 18.7 & $(15.5-22.5)$ & 18.3 & $(14.1-23.5)$ & 18.6 & $(15.5-22.1)$ & 12.9 & $(10.0-16.3)$ & 11.7 & $(8.3-16.3)$ & 12.3 & $(9.7-15.6)$ \\
\hline District of Columbia & 12.8 & $(11.8-13.9)$ & 13.3 & $(12.2-14.5)$ & 13.0 & $(12.3-13.8)$ & 8.5 & $(7.6-9.4)$ & 8.1 & $(7.2-9.1)$ & 8.3 & $(7.6-8.9)$ \\
\hline Duval County, FL & 13.0 & $(11.2-15.0)$ & 13.2 & $(11.4-15.2)$ & 13.0 & $(11.8-14.3)$ & 8.3 & $(6.9-9.9)$ & 8.3 & $(6.8-10.1)$ & 8.3 & $(7.2-9.4)$ \\
\hline Ft. Worth, TX & 16.4 & $(14.1-18.9)$ & 19.2 & $(16.8-21.8)$ & 17.7 & $(15.9-19.8)$ & 10.0 & $(8.1-12.2)$ & 10.0 & $(8.4-11.9)$ & 10.0 & $(8.7-11.4)$ \\
\hline Houston, TX & 11.0 & $(9.4-12.9)$ & 12.4 & $(10.8-14.3)$ & 11.8 & $(10.5-13.2)$ & 5.8 & $(4.7-7.3)$ & 7.2 & $(5.9-8.6)$ & 6.6 & $(5.6-7.7)$ \\
\hline Los Angeles, CA & 4.8 & $(3.4-6.9)$ & 8.1 & $(6.4-10.2)$ & 6.4 & $(5.2-7.9)$ & 2.8 & $(2.0-3.9)$ & 3.7 & $(2.7-5.2)$ & 3.3 & $(2.5-4.2)$ \\
\hline Miami-Dade County, FL & 11.4 & $(9.3-13.7)$ & 16.9 & $(14.8-19.2)$ & 14.1 & $(12.6-15.8)$ & 6.1 & $(4.8-7.9)$ & 8.8 & (7.4-10.3) & 7.5 & $(6.5-8.6)$ \\
\hline
\end{tabular}

See table footnotes on the next page. 
TABLE 98. (Continued) Percentage of high school students who drank a can, bottle, or glass of soda or pop* two or more times/day ${ }^{\dagger}$ and who drank a can, bottle, or glass of soda or pop* three or more times/day, ${ }^{\dagger}$ by sex — selected U.S. sites, Youth Risk Behavior Survey, 2015

\begin{tabular}{|c|c|c|c|c|c|c|c|c|c|c|c|c|}
\hline \multirow[b]{3}{*}{ Site } & \multicolumn{6}{|c|}{ Drank soda or pop two or more times/day } & \multicolumn{6}{|c|}{ Drank soda or pop three or more times/day } \\
\hline & \multicolumn{2}{|c|}{ Female } & \multicolumn{2}{|r|}{ Male } & \multicolumn{2}{|r|}{ Total } & \multicolumn{2}{|c|}{ Female } & \multicolumn{2}{|r|}{ Male } & \multicolumn{2}{|r|}{ Total } \\
\hline & $\%$ & $\mathrm{Cl}^{\S}$ & $\%$ & $\mathrm{Cl}$ & $\%$ & $\mathrm{Cl}$ & $\%$ & $\mathrm{Cl}$ & $\%$ & $\mathrm{Cl}$ & $\%$ & $\mathrm{Cl}$ \\
\hline New York City, NY & 10.0 & $(8.3-12.0)$ & 10.7 & $(9.2-12.4)$ & 10.3 & $(9.1-11.7)$ & 5.5 & $(4.4-6.7)$ & 5.9 & $(4.9-7.2)$ & 5.7 & $(5.0-6.6)$ \\
\hline Oakland, CA & - & - & - & - & - & - & - & - & - & - & - & - \\
\hline Orange County, FL & 9.0 & $(7.0-11.5)$ & 15.2 & $(12.0-19.1)$ & 12.2 & $(10.0-14.7)$ & 5.4 & $(3.8-7.6)$ & 10.1 & $(7.7-13.1)$ & 7.8 & $(6.1-10.1)$ \\
\hline Palm Beach County, FL & 8.9 & $(7.4-10.6)$ & 11.7 & $(9.6-14.1)$ & 10.1 & $(8.7-11.7)$ & 4.5 & $(3.5-5.8)$ & 6.6 & $(5.3-8.2)$ & 5.5 & $(4.5-6.6)$ \\
\hline Philadelphia, PA & 13.7 & $(10.5-17.7)$ & 17.2 & $(13.9-21.2)$ & 15.5 & $(12.9-18.5)$ & 9.8 & $(6.9-13.7)$ & 10.5 & $(8.5-12.9)$ & 10.1 & $(8.2-12.4)$ \\
\hline San Diego, CA & 3.5 & $(2.4-4.9)$ & 8.4 & $(6.7-10.4)$ & 6.0 & $(4.9-7.2)$ & 1.8 & $(1.1-2.9)$ & 5.4 & $(4.1-7.1)$ & 3.6 & $(2.9-4.6)$ \\
\hline San Francisco, CA & 6.5 & $(5.1-8.3)$ & 6.2 & $(4.6-8.2)$ & 6.3 & $(5.2-7.7)$ & 2.6 & $(1.6-4.2)$ & 2.7 & $(1.6-4.4)$ & 2.6 & $(1.7-4.0)$ \\
\hline Median & \multicolumn{2}{|c|}{10.5} & \multicolumn{2}{|r|}{13.2} & \multicolumn{2}{|r|}{12.0} & \multicolumn{2}{|r|}{6.1} & \multicolumn{2}{|c|}{8.2} & \multirow{2}{*}{\multicolumn{2}{|c|}{$\begin{array}{c}7.6 \\
(2.6-13.5)\end{array}$}} \\
\hline Range & & $.5-21.5)$ & & $6.2-19.2)$ & & $6.0-19.2)$ & & $3-15.2)$ & & .7-11.7) & & \\
\hline
\end{tabular}

* Not counting diet soda or diet pop.

$\dagger$ During the 7 days before the survey.

$\S 95 \%$ confidence interval.

" Not available.

TABLE 99. Percentage of high school students who did not drink a can, bottle, or glass of a sports drink*, and who drank a can, bottle, or glass of a sports drink* one or more times/day, ${ }^{\dagger}$ by sex, race/ethnicity, and grade - United States, Youth Risk Behavior Survey, 2015

\begin{tabular}{|c|c|c|c|c|c|c|c|c|c|c|c|c|}
\hline \multirow[b]{3}{*}{ Category } & \multicolumn{6}{|c|}{ Did not drink sports drinks } & \multicolumn{6}{|c|}{ Drank sports drinks one or more times/day } \\
\hline & \multicolumn{2}{|c|}{ Female } & \multicolumn{2}{|r|}{ Male } & \multicolumn{2}{|r|}{ Total } & \multicolumn{2}{|c|}{ Female } & \multicolumn{2}{|r|}{ Male } & \multicolumn{2}{|r|}{ Total } \\
\hline & $\%$ & $\mathrm{Cl}^{\S}$ & $\%$ & $\mathrm{Cl}$ & $\%$ & $\mathrm{Cl}$ & $\%$ & $\mathrm{Cl}$ & $\%$ & $\mathrm{Cl}$ & $\%$ & $\mathrm{Cl}$ \\
\hline \multicolumn{13}{|c|}{ Race/Ethnicity } \\
\hline White & 55.8 & $(52.2-59.4)$ & 33.5 & (30.8-36.4) & 44.6 & $(41.3-47.9)$ & 6.7 & $(4.8-9.1)$ & 18.1 & $(15.9-20.5)$ & 12.4 & $(10.4-14.8)$ \\
\hline Black ${ }^{9}$ & 45.7 & $(37.0-54.7)$ & 25.9 & $(20.1-32.9)$ & 36.0 & $(30.6-41.8)$ & 14.3 & $(11.8-17.2)$ & 25.2 & $(19.0-32.5)$ & 19.7 & $(16.6-23.3)$ \\
\hline Hispanic & 45.2 & $(39.9-50.7)$ & 27.6 & $(23.2-32.4)$ & 36.2 & $(31.9-40.8)$ & 12.2 & $(9.8-15.1)$ & 19.0 & $(16.7-21.5)$ & 15.7 & $(13.5-18.0)$ \\
\hline \multicolumn{13}{|l|}{ Grade } \\
\hline 9 & 49.7 & $(44.7-54.7)$ & 31.3 & $(27.9-35.0)$ & 40.0 & (36.4-43.8) & 9.9 & $(7.8-12.6)$ & 18.5 & $(16.2-21.2)$ & 14.4 & $(12.5-16.6)$ \\
\hline 10 & 51.4 & $(46.8-56.0)$ & 31.2 & $(26.4-36.4)$ & 41.6 & $(37.3-46.1)$ & 9.6 & $(7.6-12.1)$ & 20.5 & $(17.3-24.1)$ & 14.9 & $(12.6-17.6)$ \\
\hline 11 & 53.4 & $(48.0-58.7)$ & 29.8 & $(25.3-34.6)$ & 41.5 & $(36.8-46.3)$ & 9.0 & $(6.4-12.4)$ & 21.1 & $(18.4-24.1)$ & 15.3 & $(12.8-18.1)$ \\
\hline 12 & 57.1 & $(53.0-61.1)$ & 36.9 & $(28.7-45.9)$ & 46.8 & $(42.0-51.6)$ & 6.5 & $(4.7-9.1)$ & 14.5 & $(10.9-18.9)$ & 10.6 & $(8.2-13.4)$ \\
\hline Total & 52.7 & $(48.9-56.6)$ & 32.3 & $(29.9-34.7)$ & 42.4 & (39.5-45.4) & 8.8 & $(7.1-11.0)$ & 18.7 & $(16.9-20.7)$ & 13.8 & (12.1-15.8) \\
\hline
\end{tabular}

* Not counting low calorie sports drinks.

${ }^{\dagger}$ During the 7 days before the survey.

$\S 95 \%$ confidence interval.

I Non-Hispanic. 
TABLE 100. Percentage of high school students who drank a can, bottle, or glass of a sports drink* two or more times/day ${ }^{\dagger}$ and who drank a can, bottle, or glass of a sports drink* three or more times/day, ${ }^{\dagger}$ by sex, race/ethnicity, and grade — United States, Youth Risk Behavior Survey, 2015

\begin{tabular}{|c|c|c|c|c|c|c|c|c|c|c|c|c|}
\hline \multirow[b]{3}{*}{ Category } & \multicolumn{6}{|c|}{ Drank sports drinks two or more times/day } & \multicolumn{6}{|c|}{ Drank sports drinks three or more times/day } \\
\hline & \multicolumn{2}{|c|}{ Female } & \multicolumn{2}{|c|}{ Male } & \multicolumn{2}{|r|}{ Total } & \multicolumn{2}{|c|}{ Female } & \multicolumn{2}{|c|}{ Male } & \multicolumn{2}{|c|}{ Total } \\
\hline & $\%$ & $\mathrm{Cl}^{\S}$ & $\%$ & $\mathrm{Cl}$ & $\%$ & $\mathrm{Cl}$ & $\%$ & $\mathrm{Cl}$ & $\%$ & $\mathrm{Cl}$ & $\%$ & $\mathrm{Cl}$ \\
\hline \multicolumn{13}{|c|}{ Race/Ethnicity } \\
\hline White? & 3.5 & $(2.5-5.1)$ & 9.0 & $(7.5-10.8)$ & 6.3 & $(5.0-7.9)$ & 1.9 & $(1.1-3.2)$ & 4.3 & $(3.1-5.9)$ & 3.1 & $(2.3-4.3)$ \\
\hline Blackף & 10.1 & $(7.5-13.5)$ & 21.4 & $(16.0-28.0)$ & 15.8 & $(13.2-18.7)$ & 5.1 & $(3.6-7.3)$ & 13.1 & $(8.7-19.1)$ & 9.2 & $(6.9-12.1)$ \\
\hline Hispanic & 7.9 & $(6.2-9.9)$ & 13.0 & $(10.9-15.3)$ & 10.5 & $(8.9-12.3)$ & 5.4 & $(3.9-7.5)$ & 8.7 & $(7.1-10.7)$ & 7.1 & $(5.8-8.7)$ \\
\hline \multicolumn{13}{|l|}{ Grade } \\
\hline 9 & 5.4 & $(3.9-7.5)$ & 10.6 & $(8.6-12.9)$ & 8.1 & $(6.7-9.9)$ & 3.3 & $(2.3-4.7)$ & 5.0 & $(3.5-7.1)$ & 4.2 & $(3.1-5.6)$ \\
\hline 10 & 5.8 & $(4.5-7.4)$ & 12.8 & $(10.3-15.9)$ & 9.2 & $(7.7-11.1)$ & 3.4 & $(2.2-5.3)$ & 8.3 & $(6.4-10.6)$ & 5.8 & $(4.5-7.5)$ \\
\hline 11 & 5.9 & $(4.1-8.4)$ & 12.4 & $(10.4-14.7)$ & 9.3 & $(7.6-11.3)$ & 3.6 & $(2.4-5.4)$ & 6.6 & $(5.0-8.8)$ & 5.2 & $(3.9-6.8)$ \\
\hline 12 & 4.1 & $(2.8-6.0)$ & 9.0 & $(6.6-12.1)$ & 6.6 & $(5.0-8.6)$ & 2.1 & $(1.3-3.6)$ & 5.5 & $(3.8-7.9)$ & 3.8 & $(2.8-5.3)$ \\
\hline Total & 5.4 & $(4.3-6.7)$ & 11.2 & $(9.7-13.0)$ & 8.3 & $(7.1-9.8)$ & 3.1 & $(2.4-4.2)$ & 6.3 & $(5.0-7.9)$ & 4.8 & $(3.9-5.9)$ \\
\hline
\end{tabular}

* Not counting low calorie sports drinks.

† During the 7 days before the survey.

$\S 95 \%$ confidence interval.

" Non-Hispanic.

TABLE 101. Percentage of high school students who did not drink water* and who drank one or more glasses/day of water,* by sex, race/ ethnicity, and grade — United States, Youth Risk Behavior Survey, 2015

\begin{tabular}{|c|c|c|c|c|c|c|c|c|c|c|c|c|}
\hline \multirow[b]{3}{*}{ Category } & \multicolumn{6}{|c|}{ Did not drink water } & \multicolumn{6}{|c|}{ Drank one or more glasses/day of water } \\
\hline & \multicolumn{2}{|c|}{ Female } & \multicolumn{2}{|c|}{ Male } & \multicolumn{2}{|c|}{ Total } & \multicolumn{2}{|c|}{ Female } & \multicolumn{2}{|r|}{ Male } & \multicolumn{2}{|r|}{ Total } \\
\hline & $\%$ & $\mathrm{Cl}^{\dagger}$ & $\%$ & $\mathrm{Cl}$ & $\%$ & $\mathrm{Cl}$ & $\%$ & $\mathrm{Cl}$ & $\%$ & $\mathrm{Cl}$ & $\%$ & $\mathrm{Cl}$ \\
\hline \multicolumn{13}{|c|}{ Race/Ethnicity } \\
\hline White ${ }^{\S}$ & 2.5 & $(1.6-3.8)$ & 2.9 & $(2.0-4.2)$ & 2.7 & $(2.0-3.7)$ & 77.2 & $(72.8-81.0)$ & 76.4 & (73.0-79.6) & 76.6 & $(73.2-79.7)$ \\
\hline Black $^{\S}$ & 9.0 & $(6.1-13.2)$ & 7.8 & $(5.1-11.8)$ & 8.7 & $(6.4-11.7)$ & 58.4 & $(50.5-65.9)$ & 62.0 & $(56.3-67.3)$ & 60.2 & $(54.8-65.2)$ \\
\hline Hispanic & 2.8 & $(2.0-3.8)$ & 3.8 & $(2.7-5.3)$ & 3.3 & $(2.5-4.3)$ & 70.6 & $(67.5-73.6)$ & 72.5 & $(69.3-75.4)$ & 71.6 & $(69.4-73.7)$ \\
\hline \multicolumn{13}{|l|}{ Grade } \\
\hline 9 & 2.9 & $(1.9-4.4)$ & 4.1 & $(2.7-6.3)$ & 3.7 & $(2.7-5.0)$ & 70.9 & $(66.5-74.9)$ & 71.6 & $(67.2-75.6)$ & 71.2 & $(67.3-74.8)$ \\
\hline 10 & 4.4 & $(2.9-6.5)$ & 2.7 & $(1.8-4.1)$ & 3.6 & $(2.6-5.0)$ & 71.8 & $(67.8-75.5)$ & 75.1 & (71.4-78.5) & 73.4 & $(70.3-76.2)$ \\
\hline 11 & 2.0 & $(1.2-3.4)$ & 3.1 & $(1.9-5.1)$ & 2.7 & $(1.9-3.9)$ & 76.0 & (72.1-79.6) & 74.7 & (70.0-78.9) & 75.0 & (71.4-78.4) \\
\hline 12 & 3.5 & $(2.2-5.4)$ & 4.1 & $(2.5-6.7)$ & 3.9 & $(2.6-5.8)$ & 75.4 & $(69.4-80.5)$ & 75.3 & $(72.1-78.2)$ & 75.3 & $(72.0-78.4)$ \\
\hline Total & 3.3 & $(2.4-4.3)$ & 3.5 & $(2.7-4.7)$ & 3.5 & $(2.8-4.3)$ & 73.4 & $(70.0-76.6)$ & 74.1 & $(71.5-76.5)$ & 73.6 & (70.9-76.2) \\
\hline
\end{tabular}

* During the 7 days before the survey.

† $95 \%$ confidence interval.

$\S$ Non-Hispanic. 
TABLE 102. Percentage of high school students who drank two or more glasses/day of water* and who drank three or more glasses/day of water, ${ }^{*}$ by sex, race/ethnicity, and grade - United States, Youth Risk Behavior Survey, 2015

\begin{tabular}{|c|c|c|c|c|c|c|c|c|c|c|c|c|}
\hline \multirow[b]{3}{*}{ Category } & \multicolumn{6}{|c|}{ Drank two or more glasses/day of water } & \multicolumn{6}{|c|}{ Drank three or more glasses/day of water } \\
\hline & \multicolumn{2}{|c|}{ Female } & \multicolumn{2}{|r|}{ Male } & \multicolumn{2}{|r|}{ Total } & \multicolumn{2}{|c|}{ Female } & \multicolumn{2}{|r|}{ Male } & \multicolumn{2}{|r|}{ Total } \\
\hline & $\%$ & $\mathrm{Cl}^{\dagger}$ & $\%$ & $\mathrm{Cl}$ & $\%$ & $\mathrm{Cl}$ & $\%$ & $\mathrm{Cl}$ & $\%$ & $\mathrm{Cl}$ & $\%$ & $\mathrm{Cl}$ \\
\hline \multicolumn{13}{|c|}{ Race/Ethnicity } \\
\hline White ${ }^{\S}$ & 65.7 & (61.4-69.8) & 67.2 & $(63.8-70.4)$ & 66.3 & $(63.0-69.5)$ & 49.7 & $(45.7-53.6)$ & 50.5 & $(47.5-53.5)$ & 49.9 & $(47.3-52.6)$ \\
\hline Black $^{\S}$ & 47.4 & $(40.4-54.4)$ & 54.1 & $(47.4-60.7)$ & 50.8 & $(46.1-55.4)$ & 35.7 & $(31.1-40.6)$ & 42.2 & $(34.3-50.6)$ & 39.1 & (34.2-44.3) \\
\hline Hispanic & 62.7 & $(59.8-65.4)$ & 64.7 & $(61.1-68.0)$ & 63.7 & $(61.3-66.1)$ & 47.9 & $(44.8-50.9)$ & 52.5 & $(49.0-56.0)$ & 50.3 & $(47.6-53.0)$ \\
\hline \multicolumn{13}{|l|}{ Grade } \\
\hline 9 & 61.3 & $(57.5-64.9)$ & 61.7 & (57.8-65.5) & 61.3 & (58.3-64.3) & 47.4 & $(44.2-50.6)$ & 50.8 & $(46.2-55.3)$ & 49.0 & $(45.8-52.3)$ \\
\hline 10 & 60.6 & $(57.0-64.0)$ & 67.5 & $(63.7-71.1)$ & 63.9 & (61.1-66.7) & 46.1 & (42.9-49.4) & 49.1 & $(46.2-52.0)$ & 47.6 & (45.3-49.9) \\
\hline 11 & 66.1 & $(62.3-69.7)$ & 66.1 & (62.1-69.8) & 65.8 & (62.7-68.9) & 51.4 & $(46.9-55.9)$ & 50.2 & $(45.7-54.7)$ & 50.6 & $(47.0-54.2)$ \\
\hline 12 & 65.4 & $(59.0-71.4)$ & 67.6 & $(63.8-71.2)$ & 66.6 & $(62.7-70.2)$ & 47.9 & $(43.4-52.4)$ & 53.6 & $(48.7-58.4)$ & 50.7 & $(47.3-54.1)$ \\
\hline Total & 63.2 & $(60.0-66.4)$ & 65.6 & (63.2-67.8) & 64.3 & (61.8-66.7) & 48.1 & $(45.6-50.6)$ & 51.0 & $(48.7-53.2)$ & 49.5 & $(47.5-51.5)$ \\
\hline
\end{tabular}

* During the 7 days before the survey.

$+95 \%$ confidence interval.

$\S$ Non-Hispanic.

TABLE 103. Percentage of high school students who did not eat breakfast* and who ate breakfast on all 7 days, ${ }^{*}$ by sex, race/ethnicity, and grade - United States, Youth Risk Behavior Survey, 2015

\begin{tabular}{|c|c|c|c|c|c|c|c|c|c|c|c|c|}
\hline \multirow[b]{3}{*}{ Category } & \multicolumn{6}{|c|}{ Did not eat breakfast } & \multicolumn{6}{|c|}{ Ate breakfast on all 7 days } \\
\hline & \multicolumn{2}{|r|}{ Female } & \multicolumn{2}{|r|}{ Male } & \multicolumn{2}{|r|}{ Total } & \multicolumn{2}{|c|}{ Female } & \multicolumn{2}{|r|}{ Male } & \multicolumn{2}{|r|}{ Total } \\
\hline & $\%$ & $\mathrm{Cl}^{\dagger}$ & $\%$ & $\mathrm{Cl}$ & $\%$ & $\mathrm{Cl}$ & $\%$ & $\mathrm{Cl}$ & $\%$ & $\mathrm{Cl}$ & $\%$ & $\mathrm{Cl}$ \\
\hline \multicolumn{13}{|c|}{ Race/Ethnicity } \\
\hline White $\S$ & 12.5 & $(10.2-15.3)$ & 11.3 & $(9.0-14.0)$ & 12.0 & $(10.1-14.3)$ & 34.8 & $(29.7-40.3)$ & 43.3 & (39.3-47.4) & 39.0 & (34.9-43.3) \\
\hline Black $^{\S}$ & 19.3 & $(14.6-25.2)$ & 16.8 & $(12.9-21.6)$ & 18.0 & $(15.5-20.7)$ & 24.7 & $(19.3-30.9)$ & 30.8 & $(25.8-36.2)$ & 27.9 & $(23.5-32.7)$ \\
\hline Hispanic & 15.5 & $(13.3-18.0)$ & 13.8 & $(11.5-16.5)$ & 14.7 & $(12.8-16.9)$ & 30.1 & $(27.5-32.8)$ & 39.5 & $(36.2-42.8)$ & 34.8 & $(32.5-37.2)$ \\
\hline \multicolumn{13}{|l|}{ Grade } \\
\hline 9 & 14.8 & (12.5-17.6) & 10.9 & $(8.9-13.2)$ & 12.8 & (10.9-14.8) & 31.9 & $(27.3-36.8)$ & 46.6 & $(42.6-50.7)$ & 39.6 & (35.6-43.7) \\
\hline 10 & 15.5 & $(12.8-18.6)$ & 13.6 & $(10.6-17.2)$ & 14.5 & $(12.2-17.1)$ & 31.1 & $(27.9-34.6)$ & 42.3 & $(38.4-46.4)$ & 36.6 & $(33.6-39.8)$ \\
\hline 11 & 12.7 & $(10.4-15.5)$ & 14.2 & $(11.6-17.1)$ & 13.8 & $(11.8-16.0)$ & 32.4 & $(28.0-37.1)$ & 37.1 & $(34.3-40.1)$ & 34.6 & $(31.6-37.7)$ \\
\hline 12 & 13.3 & $(10.7-16.5)$ & 14.8 & $(11.8-18.4)$ & 14.3 & $(12.2-16.6)$ & 32.9 & $(28.4-37.8)$ & 34.7 & $(30.9-38.9)$ & 33.8 & $(30.8-37.0)$ \\
\hline Total & 14.2 & $(12.5-16.2)$ & 13.3 & $(11.6-15.3)$ & 13.8 & (12.4-15.4) & 32.1 & (28.9-35.4) & 40.5 & (37.9-43.2) & 36.3 & (33.7-39.1) \\
\hline
\end{tabular}

* During the 7 days before the survey.

$+95 \%$ confidence interval.

$\S$ Non-Hispanic. 
TABLE 104. Percentage of high school students who did not eat breakfast* and who ate breakfast on all 7 days,* by sex — selected U.S. sites, Youth Risk Behavior Survey, 2015

\begin{tabular}{|c|c|c|c|c|c|c|c|c|c|c|c|c|}
\hline \multirow[b]{3}{*}{ Site } & \multicolumn{6}{|c|}{ Did not eat breakfast } & \multicolumn{6}{|c|}{ Ate breakfast on all 7 days } \\
\hline & \multicolumn{2}{|c|}{ Female } & \multicolumn{2}{|r|}{ Male } & \multicolumn{2}{|r|}{ Total } & \multicolumn{2}{|c|}{ Female } & \multicolumn{2}{|r|}{ Male } & \multicolumn{2}{|r|}{ Total } \\
\hline & $\%$ & $\mathrm{Cl}^{\dagger}$ & $\%$ & $\mathrm{Cl}$ & $\%$ & $\mathrm{Cl}$ & $\%$ & $\mathrm{Cl}$ & $\%$ & $\mathrm{Cl}$ & $\%$ & $\mathrm{Cl}$ \\
\hline \multicolumn{13}{|l|}{ State surveys } \\
\hline Alabama & 13.4 & $(10.3-17.2)$ & 17.5 & $(14.1-21.6)$ & 15.5 & $(12.7-18.7)$ & 25.6 & $(21.6-30.2)$ & 35.7 & (31.0-40.7) & 30.5 & $(26.9-34.3)$ \\
\hline Alaska & —§ & - & - & - & - & - & - & - & - & - & - & - \\
\hline Arizona & 15.5 & $(12.6-18.9)$ & 15.0 & $(13.0-17.4)$ & 15.2 & $(13.7-16.8)$ & 32.6 & $(27.8-37.9)$ & 38.6 & $(34.3-43.1)$ & 35.6 & $(32.0-39.3)$ \\
\hline Arkansas & 15.8 & $(11.6-21.2)$ & 17.4 & $(15.5-19.5)$ & 16.6 & $(14.2-19.3)$ & 29.7 & $(26.2-33.5)$ & 33.1 & $(27.5-39.2)$ & 31.3 & $(27.5-35.3)$ \\
\hline California & 11.5 & $(8.8-14.8)$ & 9.3 & $(7.2-11.8)$ & 10.3 & $(8.5-12.5)$ & 38.2 & $(34.9-41.7)$ & 45.9 & $(38.3-53.7)$ & 42.1 & $(37.2-47.2)$ \\
\hline Connecticut & 12.6 & (10.2-15.4) & 14.1 & (11.2-17.6) & 13.4 & (11.3-15.8) & 34.8 & (30.5-39.3) & 40.1 & $(36.1-44.2)$ & 37.4 & $(33.8-41.1)$ \\
\hline Delaware & 13.5 & $(10.5-17.1)$ & 12.4 & $(10.3-14.9)$ & 13.0 & $(11.2-15.1)$ & 35.4 & $(31.2-40.0)$ & 42.6 & $(39.3-46.1)$ & 39.0 & (35.9-42.1) \\
\hline Florida & 15.4 & $(14.1-16.9)$ & 14.3 & $(13.1-15.6)$ & 15.0 & $(13.9-16.1)$ & 34.8 & $(32.9-36.7)$ & 44.0 & $(41.4-46.5)$ & 39.4 & $(37.6-41.2)$ \\
\hline Hawaii & 10.9 & $(9.3-12.6)$ & 13.4 & $(11.6-15.3)$ & 12.1 & $(11.0-13.4)$ & 34.0 & $(31.1-37.0)$ & 38.4 & $(36.3-40.5)$ & 36.0 & $(34.0-38.0)$ \\
\hline Idaho & 12.0 & $(9.5-15.0)$ & 9.7 & $(7.4-12.6)$ & 10.9 & $(8.9-13.2)$ & 35.0 & $(32.2-38.0)$ & 41.9 & (38.6-45.3) & 38.5 & $(36.1-41.0)$ \\
\hline Illinois & 14.1 & (11.0-17.8) & 18.8 & $(14.5-24.0)$ & 16.4 & $(13.4-20.0)$ & 32.3 & (26.4-38.9) & 33.6 & $(28.0-39.7)$ & 32.9 & $(28.7-37.4)$ \\
\hline Indiana & 15.1 & $(11.7-19.4)$ & 14.8 & (10.0-21.4) & 15.0 & (11.2-19.7) & 27.7 & $(23.8-31.8)$ & 32.5 & $(27.8-37.4)$ & 30.1 & $(26.8-33.6)$ \\
\hline Kentucky & 12.4 & $(9.7-15.6)$ & 13.0 & (10.8-15.5) & 12.6 & (11.0-14.5) & 32.2 & (27.8-37.0) & 37.3 & (33.0-41.9) & 34.8 & (31.9-37.8) \\
\hline Maine & - & - & - & - & - & - & - & - & - & - & - & - \\
\hline Maryland & - & - & - & - & - & - & - & - & - & - & - & - \\
\hline Massachusetts & 14.3 & $(12.1-16.9)$ & 12.9 & $(11.0-15.0)$ & 13.7 & $(12.0-15.5)$ & 34.0 & (30.7-37.4) & 35.8 & $(32.7-39.0)$ & 34.9 & $(32.5-37.4)$ \\
\hline Michigan & 14.5 & $(11.7-17.9)$ & 19.2 & (15.9-23.0) & 16.8 & $(14.2-19.9)$ & 31.0 & $(24.9-37.9)$ & 33.8 & $(29.3-38.6)$ & 32.5 & $(27.9-37.4)$ \\
\hline Mississippi & 20.3 & $(17.0-24.1)$ & 18.7 & $(16.1-21.6)$ & 19.8 & $(17.4-22.3)$ & 23.5 & $(20.3-27.0)$ & 30.0 & $(26.9-33.4)$ & 26.6 & $(24.3-29.1)$ \\
\hline Missouri & 15.5 & $(12.5-19.0)$ & 15.6 & $(11.8-20.2)$ & 15.5 & $(12.6-18.9)$ & 30.5 & $(24.3-37.5)$ & 31.7 & $(27.4-36.4)$ & 30.9 & $(26.3-35.9)$ \\
\hline Montana & 11.5 & (10.2-13.0) & 13.4 & (11.7-15.3) & 12.5 & (11.4-13.8) & 36.0 & (33.4-38.7) & 40.4 & (37.9-42.8) & 38.2 & $(36.3-40.2)$ \\
\hline Nebraska & 13.3 & $(10.6-16.6)$ & 13.3 & $(10.8-16.3)$ & 13.3 & $(11.4-15.4)$ & 33.9 & $(30.4-37.5)$ & 38.8 & $(35.3-42.5)$ & 36.2 & (33.5-39.1) \\
\hline Nevada & 16.8 & $(13.7-20.3)$ & 16.3 & $(13.9-19.1)$ & 16.8 & $(14.7-19.0)$ & 30.8 & $(26.8-35.1)$ & 36.5 & $(32.0-41.3)$ & 33.6 & $(30.3-37.0)$ \\
\hline New Hampshire & - & - & - & - & - & - & - & - & - & - & - & - \\
\hline New Mexico & 15.1 & $(13.6-16.7)$ & 13.9 & (12.7-15.2) & 14.6 & $(13.6-15.7)$ & 30.2 & $(27.1-33.5)$ & 37.6 & (35.6-39.7) & 33.9 & $(31.8-36.1)$ \\
\hline New York & 14.7 & $(12.7-17.0)$ & 20.3 & (17.2-23.8) & 17.4 & (15.2-19.8) & 34.0 & $(31.2-36.8)$ & 35.6 & $(31.9-39.5)$ & 34.6 & $(32.1-37.3)$ \\
\hline North Carolina & 13.6 & $(10.2-17.9)$ & 14.0 & $(10.8-18.1)$ & 14.1 & (11.7-16.9) & 31.1 & $(27.5-34.9)$ & 40.2 & (37.3-43.1) & 35.5 & $(32.7-38.3)$ \\
\hline North Dakota & 10.0 & $(8.2-12.0)$ & 13.8 & (11.5-16.4) & 11.9 & (10.4-13.7) & 38.4 & (35.0-41.8) & 36.9 & (34.1-39.7) & 37.6 & $(35.2-40.1)$ \\
\hline Oklahoma & 15.5 & $(12.6-18.9)$ & 17.7 & $(14.1-22.0)$ & 16.5 & $(13.7-19.6)$ & 28.2 & $(24.6-32.1)$ & 33.6 & $(29.8-37.6)$ & 30.7 & (27.7-33.9) \\
\hline Pennsylvania & 14.3 & $(12.1-16.8)$ & 13.5 & (11.5-15.8) & 13.9 & $(12.3-15.7)$ & 31.4 & $(27.7-35.3)$ & 39.1 & (34.4-44.0) & 35.2 & $(31.6-39.1)$ \\
\hline Rhode Island & 14.8 & $(11.3-19.1)$ & 14.7 & $(11.7-18.3)$ & 14.9 & $(12.0-18.3)$ & 32.2 & $(27.1-37.7)$ & 35.2 & $(30.5-40.2)$ & 33.7 & $(29.2-38.5)$ \\
\hline South Carolina & 22.2 & $(18.6-26.1)$ & 17.8 & (13.7-22.7) & 20.1 & $(17.0-23.6)$ & 25.3 & (21.2-30.0) & 34.1 & $(29.1-39.4)$ & 29.5 & $(25.7-33.7)$ \\
\hline South Dakota & 15.0 & $(11.1-19.9)$ & 13.3 & (8.8-19.6) & 14.2 & $(11.1-18.0)$ & 31.5 & $(26.7-36.8)$ & 36.0 & $(30.5-41.9)$ & 33.8 & $(30.2-37.6)$ \\
\hline Tennessee & 15.7 & $(14.0-17.6)$ & 13.6 & $(11.8-15.7)$ & 14.6 & $(13.0-16.4)$ & 30.8 & $(27.4-34.5)$ & 39.9 & (37.4-42.4) & 35.4 & $(33.0-38.0)$ \\
\hline Vermont & 10.8 & (10.2-11.4) & 10.9 & (10.3-11.5) & 10.9 & $(10.5-11.3)$ & 38.1 & (37.2-39.0) & 42.9 & $(42.0-43.9)$ & 40.5 & $(39.8-41.1)$ \\
\hline Virginia & 14.9 & $(12.5-17.6)$ & 13.3 & $(11.4-15.6)$ & 14.1 & $(12.4-16.0)$ & 32.7 & $(29.3-36.3)$ & 42.5 & (39.4-45.7) & 37.7 & (35.1-40.4) \\
\hline West Virginia & 15.2 & $(12.0-19.1)$ & 14.3 & $(12.2-16.8)$ & 14.8 & $(12.3-17.6)$ & 29.5 & $(26.5-32.7)$ & 38.7 & $(34.7-42.9)$ & 34.3 & (31.7-36.9) \\
\hline Wyoming & 13.7 & $(11.0-17.0)$ & 15.6 & $(13.1-18.5)$ & 14.7 & $(12.5-17.3)$ & 31.9 & $(28.7-35.4)$ & 37.5 & $(34.8-40.2)$ & 34.6 & $(32.2-37.2)$ \\
\hline Median & & 14.5 & & 14.1 & & 14.6 & & 32.2 & & 37.5 & & 34.8 \\
\hline Range & & $0.0-22.2)$ & & $9.3-20.3)$ & & $0.3-20.1)$ & & $3.5-38.4)$ & & $0.0-45.9)$ & & $6.6-42.1)$ \\
\hline \multicolumn{13}{|c|}{ Large urban school district surveys } \\
\hline Baltimore, MD & 21.2 & $(17.5-25.6)$ & 19.3 & $(14.4-25.4)$ & 20.6 & $(17.8-23.8)$ & 23.1 & $(18.7-28.3)$ & 24.1 & $(19.6-29.4)$ & 23.2 & $(19.9-26.9)$ \\
\hline Boston, MA & 14.3 & $(11.9-17.0)$ & 15.1 & $(12.2-18.5)$ & 14.6 & $(12.6-16.9)$ & 27.2 & $(24.1-30.4)$ & 35.1 & $(30.6-40.0)$ & 31.1 & $(28.6-33.7)$ \\
\hline Broward County, FL & 15.3 & $(12.4-18.7)$ & 16.6 & $(13.1-20.9)$ & 16.0 & $(13.6-18.7)$ & 31.4 & $(27.6-35.4)$ & 31.9 & $(27.1-37.0)$ & 31.5 & $(28.3-34.8)$ \\
\hline Cleveland, $\mathrm{OH}$ & 23.2 & $(20.0-26.7)$ & 23.5 & $(20.1-27.2)$ & 23.8 & $(21.2-26.6)$ & 16.9 & $(14.3-19.9)$ & 22.7 & $(19.6-26.2)$ & 19.7 & $(17.5-22.1)$ \\
\hline DeKalb County, GA & 16.7 & $(14.0-19.9)$ & 17.6 & $(14.5-21.2)$ & 17.1 & $(15.1-19.4)$ & 26.8 & $(23.5-30.4)$ & 31.8 & $(27.9-36.1)$ & 29.3 & $(26.2-32.6)$ \\
\hline Detroit, MI & 20.8 & $(17.7-24.2)$ & 22.9 & $(17.8-28.8)$ & 21.7 & $(18.8-24.9)$ & 13.7 & $(10.9-17.0)$ & 19.4 & $(15.9-23.5)$ & 16.4 & $(14.1-18.9)$ \\
\hline District of Columbia & 17.4 & $(16.2-18.5)$ & 16.9 & $(15.7-18.2)$ & 17.2 & $(16.4-18.1)$ & 21.2 & $(20.0-22.4)$ & 28.4 & $(27.0-29.9)$ & 24.6 & $(23.6-25.5)$ \\
\hline Duval County, FL & - & - & - & - & - & - & - & - & - & - & - & - \\
\hline Ft. Worth, TX & 17.5 & $(15.1-20.2)$ & 18.3 & $(16.1-20.8)$ & 17.8 & $(16.1-19.6)$ & 28.7 & $(25.9-31.7)$ & 33.1 & $(30.2-36.2)$ & 31.0 & $(28.8-33.3)$ \\
\hline Houston, TX & 18.1 & $(16.1-20.4)$ & 19.9 & $(17.6-22.3)$ & 19.2 & $(17.6-20.9)$ & 27.9 & $(25.3-30.8)$ & 28.9 & $(26.3-31.7)$ & 28.3 & $(26.3-30.4)$ \\
\hline Los Angeles, CA & 13.1 & $(10.8-15.8)$ & 10.6 & $(8.5-13.2)$ & 11.8 & $(10.6-13.2)$ & 35.7 & $(32.9-38.7)$ & 41.3 & $(38.2-44.4)$ & 38.4 & $(36.3-40.6)$ \\
\hline Miami-Dade County, FL & 12.1 & $(10.1-14.4)$ & 11.0 & $(9.4-12.8)$ & 11.6 & $(10.3-13.1)$ & 39.2 & (35.4-43.2) & 50.0 & $(46.7-53.3)$ & 44.6 & $(42.1-47.1)$ \\
\hline
\end{tabular}

See table footnotes on the next page. 
TABLE 104. (Continued) Percentage of high school students who did not eat breakfast* and who ate breakfast on all 7 days, ${ }^{*}$ by sex - selected U.S. sites, Youth Risk Behavior Survey, 2015

\begin{tabular}{|c|c|c|c|c|c|c|c|c|c|c|c|c|}
\hline \multirow[b]{3}{*}{ Site } & \multicolumn{6}{|c|}{ Did not eat breakfast } & \multicolumn{6}{|c|}{ Ate breakfast on all 7 days } \\
\hline & \multicolumn{2}{|c|}{ Female } & \multicolumn{2}{|r|}{ Male } & \multicolumn{2}{|r|}{ Total } & \multicolumn{2}{|c|}{ Female } & \multicolumn{2}{|r|}{ Male } & \multicolumn{2}{|r|}{ Total } \\
\hline & $\%$ & $\mathrm{Cl}^{\dagger}$ & $\%$ & $\mathrm{Cl}$ & $\%$ & $\mathrm{Cl}$ & $\%$ & $\mathrm{Cl}$ & $\%$ & $\mathrm{Cl}$ & $\%$ & $\mathrm{Cl}$ \\
\hline New York City, NY & 15.4 & $(13.5-17.6)$ & 15.1 & $(13.6-16.7)$ & 15.3 & $(14.1-16.6)$ & 31.9 & $(28.7-35.2)$ & 38.4 & $(36.1-40.8)$ & 35.0 & $(32.5-37.6)$ \\
\hline Oakland, CA & 15.9 & $(12.9-19.5)$ & 19.0 & $(16.0-22.3)$ & 17.6 & $(15.4-20.0)$ & 27.6 & $(24.2-31.3)$ & 30.5 & $(27.1-34.0)$ & 28.9 & $(26.4-31.6)$ \\
\hline Orange County, FL & 12.6 & $(10.2-15.3)$ & 13.8 & $(11.4-16.6)$ & 13.1 & $(11.4-15.0)$ & 34.0 & $(29.7-38.5)$ & 44.6 & $(39.9-49.3)$ & 39.2 & $(35.7-42.8)$ \\
\hline Palm Beach County, FL & 15.5 & $(13.6-17.6)$ & 15.6 & $(13.0-18.6)$ & 15.7 & $(14.0-17.7)$ & 33.8 & $(30.9-36.9)$ & 37.5 & $(34.1-41.1)$ & 35.5 & $(33.3-37.7)$ \\
\hline Philadelphia, PA & 18.1 & $(14.6-22.2)$ & 15.7 & $(12.2-19.9)$ & 16.9 & $(15.0-19.0)$ & 22.7 & $(19.2-26.7)$ & 30.4 & $(26.7-34.3)$ & 26.3 & $(23.4-29.4)$ \\
\hline San Diego, CA & 11.5 & $(9.5-13.9)$ & 14.1 & $(12.2-16.1)$ & 12.8 & $(11.3-14.5)$ & 34.9 & $(31.1-38.9)$ & 39.2 & $(35.5-43.1)$ & 37.1 & $(34.5-39.7)$ \\
\hline San Francisco, CA & 12.6 & $(10.2-15.5)$ & 13.4 & $(10.6-16.7)$ & 13.4 & $(11.4-15.6)$ & 35.4 & $(31.8-39.1)$ & 45.5 & $(40.9-50.1)$ & 40.4 & $(37.3-43.6)$ \\
\hline Median & \multicolumn{2}{|c|}{15.7} & \multicolumn{2}{|c|}{16.1} & \multicolumn{2}{|c|}{16.4} & \multicolumn{2}{|c|}{28.3} & \multicolumn{2}{|c|}{32.5} & \multicolumn{2}{|c|}{31.0} \\
\hline Range & \multicolumn{2}{|c|}{$(11.5-23.2)$} & \multicolumn{2}{|c|}{$(10.6-23.5)$} & \multicolumn{2}{|c|}{$(11.6-23.8)$} & \multicolumn{2}{|c|}{ (13.7-39.2) } & \multicolumn{2}{|c|}{$(19.4-50.0)$} & \multicolumn{2}{|c|}{$(16.4-44.6)$} \\
\hline
\end{tabular}

* During the 7 days before the survey.

† $95 \%$ confidence interval.

$\S$ Not available.

TABLE 105. Percentage of high school students who did not participate in at least 60 minutes of physical activity on at least 1 day* and who were physically active at least 60 minutes/day on 5 or more days, * by sex, race/ethnicity, and grade — United States, Youth Risk Behavior Survey, 2015

\begin{tabular}{|c|c|c|c|c|c|c|c|c|c|c|c|c|}
\hline \multirow[b]{3}{*}{ Category } & \multicolumn{6}{|c|}{$\begin{array}{l}\text { Did not participate in at least } 60 \text { minutes of physical activity } \\
\text { on at least } 1 \text { day }\end{array}$} & \multicolumn{6}{|c|}{$\begin{array}{l}\text { Physically active at least } 60 \text { minutes/day } \\
\text { on } 5 \text { or more days }\end{array}$} \\
\hline & \multicolumn{2}{|c|}{ Female } & \multicolumn{2}{|r|}{ Male } & \multicolumn{2}{|r|}{ Total } & \multicolumn{2}{|c|}{ Female } & \multicolumn{2}{|r|}{ Male } & \multicolumn{2}{|r|}{ Total } \\
\hline & $\%$ & $\mathrm{Cl}^{\dagger}$ & $\%$ & $\mathrm{Cl}$ & $\%$ & $\mathrm{Cl}$ & $\%$ & $\mathrm{Cl}$ & $\%$ & $\mathrm{Cl}$ & $\%$ & $\mathrm{Cl}$ \\
\hline \multicolumn{13}{|c|}{ Race/Ethnicity } \\
\hline White ${ }^{\S}$ & 14.3 & $(11.5-17.6)$ & 8.8 & $(7.1-11.0)$ & 11.6 & $(9.7-13.8)$ & 43.5 & (38.8-48.2) & 62.0 & $(58.5-65.4)$ & 52.7 & $(49.3-56.2)$ \\
\hline Black $\S$ & 25.2 & $(21.3-29.6)$ & 16.2 & $(12.1-21.4)$ & 20.4 & (16.9-24.5) & 33.4 & (28.4-38.8) & 52.2 & $(46.1-58.2)$ & 43.5 & (38.9-48.2) \\
\hline Hispanic & 19.2 & $(17.3-21.2)$ & 11.9 & $(9.5-14.8)$ & 15.6 & $(13.7-17.6)$ & 33.1 & $(29.5-37.0)$ & 53.5 & $(50.9-56.2)$ & 43.4 & $(40.7-46.2)$ \\
\hline \multicolumn{13}{|l|}{ Grade } \\
\hline 9 & 14.7 & $(12.3-17.5)$ & 9.5 & $(7.0-12.6)$ & 12.0 & $(10.1-14.1)$ & 43.9 & (38.9-48.9) & 62.3 & $(58.5-66.1)$ & 53.7 & $(49.9-57.4)$ \\
\hline 10 & 15.8 & $(13.0-19.1)$ & 10.4 & $(8.0-13.5)$ & 13.1 & (10.8-15.8) & 41.9 & (36.7-47.2) & 58.7 & $(54.1-63.1)$ & 50.2 & $(46.1-54.2)$ \\
\hline 11 & 18.2 & $(15.8-20.8)$ & 12.4 & $(9.8-15.6)$ & 15.5 & (13.5-17.8) & 36.6 & (32.6-40.8) & 56.3 & (52.0-60.6) & 46.5 & (43.2-49.9) \\
\hline 12 & 21.4 & (18.4-24.9) & 12.4 & $(9.6-15.9)$ & 16.9 & (14.6-19.4) & 33.4 & $(29.5-37.6)$ & 53.3 & $(47.8-58.8)$ & 43.5 & $(40.2-46.8)$ \\
\hline Total & 17.5 & $(15.6-19.5)$ & 11.1 & $(9.5-12.9)$ & 14.3 & $(12.9-15.8)$ & 39.1 & (35.5-42.8) & 57.8 & $(55.0-60.5)$ & 48.6 & $(46.0-51.2)$ \\
\hline
\end{tabular}

* Doing any kind of physical activity that increased their heart rate and made them breathe hard some of the time during the 7 days before the survey.

† $95 \%$ confidence interval.

$\S$ Non-Hispanic.

NOTE: Because of changes in question context starting in 2011, national YRBS prevalence estimates derived from the 60 minutes of physical activity question in 2011 , 2013, and 2015 are not comparable to those reported in 2009 or earlier. On the 2005-2009 national YRBS questionnaire, physical activity was assessed with three questions (in the following order) that asked the number of days students participated in 1) at least 20 minutes of vigorous physical activity; 2) at least 30 minutes of moderate physical activity; and 3) at least 60 minutes of aerobic (moderate and vigorous) physical activity. On the 2011, 2013, and 2015 national YRBS questionnaires, only the 60 minutes of aerobic physical activity question was included. 
TABLE 106. Percentage of high school students who did not participate in at least 60 minutes of physical activity on at least 1 day* and who were physically active at least 60 minutes/day on 5 or more days,* by sex - selected U.S. sites, Youth Risk Behavior Survey, 2015

\begin{tabular}{|c|c|c|c|c|c|c|c|c|c|c|c|c|}
\hline \multirow[b]{3}{*}{ Site } & \multicolumn{6}{|c|}{$\begin{array}{l}\text { Did not participate in at least } 60 \text { minutes of physical activity } \\
\text { on at least } 1 \text { day }\end{array}$} & \multicolumn{6}{|c|}{$\begin{array}{l}\text { Physically active at least } 60 \text { minutes/day } \\
\text { on } 5 \text { or more days }\end{array}$} \\
\hline & \multicolumn{2}{|c|}{ Female } & \multicolumn{2}{|r|}{ Male } & \multicolumn{2}{|r|}{ Total } & \multicolumn{2}{|c|}{ Female } & \multicolumn{2}{|r|}{ Male } & \multicolumn{2}{|r|}{ Total } \\
\hline & $\%$ & $\mathrm{Cl}^{\dagger}$ & $\%$ & $\mathrm{Cl}$ & $\%$ & $\mathrm{Cl}$ & $\%$ & $\mathrm{Cl}$ & $\%$ & $\mathrm{Cl}$ & $\%$ & $\mathrm{Cl}$ \\
\hline \multicolumn{13}{|l|}{ State surveys } \\
\hline Alabama & 21.0 & $(16.7-26.0)$ & 14.4 & $(11.7-17.5)$ & 17.5 & (14.8-20.7) & 31.1 & $(27.0-35.6)$ & 51.6 & $(47.1-56.2)$ & 41.3 & (37.4-45.3) \\
\hline Alaska & 18.4 & (15.6-21.6) & 14.0 & $(11.3-17.2)$ & 16.1 & (14.1-18.4) & 38.8 & (35.0-42.6) & 49.3 & $(45.6-53.0)$ & 44.2 & $(41.4-47.0)$ \\
\hline Arizona & 17.3 & $(13.9-21.2)$ & 14.5 & $(11.3-18.3)$ & 15.9 & $(13.8-18.3)$ & 40.0 & $(35.1-45.0)$ & 52.7 & $(46.5-58.8)$ & 46.4 & $(41.5-51.4)$ \\
\hline Arkansas & 24.9 & (21.3-28.9) & 15.1 & $(12.6-18.0)$ & 20.0 & $(17.9-22.2)$ & 35.0 & $(29.6-40.7)$ & 51.9 & $(45.2-58.5)$ & 43.3 & (39.8-46.7) \\
\hline California & 14.7 & $(11.7-18.3)$ & 11.5 & $(7.8-16.7)$ & 13.1 & (10.9-15.7) & 41.4 & $(37.9-45.1)$ & 55.0 & $(50.7-59.2)$ & 48.1 & (45.2-51.0) \\
\hline Connecticut & 16.9 & $(14.1-20.1)$ & 11.7 & $(9.3-14.6)$ & 14.3 & $(12.0-16.9)$ & 39.9 & $(34.7-45.3)$ & 50.8 & $(45.2-56.3)$ & 45.3 & (41.4-49.3) \\
\hline Delaware & 22.9 & $(20.1-25.9)$ & 14.1 & (11.2-17.6) & 18.5 & $(16.5-20.7)$ & 33.3 & (30.2-36.6) & 53.1 & $(49.1-57.1)$ & 43.3 & $(40.5-46.2)$ \\
\hline Florida & 24.7 & $(22.4-27.1)$ & 15.1 & $(13.5-16.8)$ & 19.9 & $(18.4-21.5)$ & 30.6 & (28.4-32.9) & 53.1 & $(50.2-55.9)$ & 41.9 & (39.9-43.9) \\
\hline Hawaii & 21.4 & $(18.1-25.1)$ & 14.5 & $(12.8-16.3)$ & 18.0 & (15.9-20.3) & 30.7 & $(27.8-33.8)$ & 45.8 & $(43.2-48.3)$ & 38.1 & $(36.3-40.0)$ \\
\hline Idaho & 14.7 & (11.8-18.2) & 9.0 & $(6.5-12.2)$ & 11.7 & $(9.6-14.3)$ & 43.6 & (38.8-48.4) & 62.3 & (55.9-68.3) & 53.1 & $(48.9-57.4)$ \\
\hline Illinois & 14.3 & $(11.4-17.7)$ & 13.6 & $(11.6-15.8)$ & 13.9 & $(12.2-15.9)$ & 42.5 & (36.9-48.2) & 56.7 & (52.0-61.4) & 49.6 & $(46.2-53.0)$ \\
\hline Indiana & 17.8 & $(14.5-21.6)$ & 12.9 & $(9.4-17.4)$ & 15.4 & $(12.6-18.6)$ & 38.5 & $(34.8-42.2)$ & 54.5 & $(48.7-60.2)$ & 46.5 & $(42.6-50.5)$ \\
\hline Kentucky & 18.8 & (16.9-20.8) & 13.9 & $(11.8-16.2)$ & 16.5 & $(15.1-17.9)$ & 29.9 & $(25.7-34.5)$ & 44.1 & $(38.2-50.1)$ & 37.0 & (32.9-41.4) \\
\hline Maine & 18.1 & $(16.8-19.6)$ & 13.6 & $(12.5-14.8)$ & 15.9 & $(15.0-16.8)$ & 34.9 & $(32.9-37.0)$ & 47.4 & $(45.1-49.8)$ & 41.2 & $(39.6-42.8)$ \\
\hline Maryland & 22.8 & $(22.1-23.6)$ & 16.6 & $(16.0-17.3)$ & 19.8 & (19.2-20.4) & 30.1 & $(29.3-30.9)$ & 44.0 & $(42.8-45.2)$ & 36.9 & $(36.1-37.7)$ \\
\hline Massachusetts & 18.0 & $(14.7-21.9)$ & 12.0 & $(9.9-14.6)$ & 15.1 & $(12.7-17.8)$ & 37.4 & $(32.6-42.5)$ & 52.9 & $(49.0-56.8)$ & 45.2 & $(41.2-49.3)$ \\
\hline Michigan & 18.5 & $(14.7-23.0)$ & 13.4 & $(10.8-16.5)$ & 15.9 & $(13.4-18.8)$ & 42.0 & (34.9-49.4) & 50.1 & $(44.5-55.7)$ & 46.0 & (39.9-52.1) \\
\hline Mississippi & 26.6 & $(24.3-29.0)$ & 19.0 & $(16.3-22.1)$ & 22.9 & $(20.9-24.9)$ & 23.5 & $(19.8-27.6)$ & 45.1 & $(40.4-50.0)$ & 34.2 & $(30.7-37.8)$ \\
\hline Missouri & 18.8 & $(15.2-23.1)$ & 13.5 & $(10.4-17.2)$ & 16.4 & $(14.2-18.8)$ & 40.1 & $(36.0-44.3)$ & 51.9 & $(45.9-57.8)$ & 45.7 & $(41.6-49.8)$ \\
\hline Montana & 12.3 & (10.9-13.9) & 9.2 & $(7.8-10.7)$ & 10.7 & $(9.7-11.8)$ & 47.6 & $(44.8-50.3)$ & 60.1 & $(57.7-62.5)$ & 54.0 & $(52.3-55.7)$ \\
\hline Nebraska & 15.7 & $(12.9-18.9)$ & 12.7 & $(9.8-16.3)$ & 14.1 & $(11.9-16.6)$ & 46.3 & $(41.6-51.1)$ & 59.0 & $(54.5-63.3)$ & 52.8 & $(48.8-56.7)$ \\
\hline Nevada & 16.4 & $(13.2-20.1)$ & 9.4 & $(7.4-11.8)$ & 13.0 & (11.4-14.7) & 42.4 & $(37.4-47.6)$ & 58.9 & $(53.6-64.1)$ & 50.6 & $(47.1-54.1)$ \\
\hline New Hampshire & 15.1 & $(13.8-16.5)$ & 12.2 & $(11.1-13.4)$ & 13.6 & $(12.7-14.5)$ & 40.7 & $(37.9-43.6)$ & 52.9 & $(51.2-54.6)$ & 46.9 & $(45.1-48.7)$ \\
\hline New Mexico & 18.1 & $(16.5-19.8)$ & 11.0 & $(9.8-12.4)$ & 14.6 & $(13.5-15.7)$ & 44.3 & $(41.5-47.0)$ & 60.1 & $(57.3-62.7)$ & 52.2 & $(49.9-54.4)$ \\
\hline New York & 19.8 & $(17.6-22.1)$ & 17.6 & $(14.8-20.9)$ & 18.8 & $(16.6-21.2)$ & 37.3 & $(33.0-41.8)$ & 46.8 & $(42.8-50.8)$ & 41.8 & (38.4-45.3) \\
\hline North Carolina & 21.6 & $(19.4-24.0)$ & 13.5 & $(11.7-15.5)$ & 17.6 & $(16.2-19.1)$ & 33.7 & $(31.2-36.2)$ & 53.1 & $(48.9-57.1)$ & 43.4 & $(40.7-46.1)$ \\
\hline North Dakota & 13.5 & $(11.1-16.2)$ & 10.9 & $(9.0-13.1)$ & 12.1 & $(10.7-13.8)$ & 42.9 & (39.5-46.3) & 59.3 & $(55.9-62.6)$ & 51.3 & $(48.7-53.8)$ \\
\hline Oklahoma & 17.1 & (13.8-20.9) & 7.7 & $(5.9-10.0)$ & 12.4 & $(10.3-14.8)$ & 43.9 & $(40.4-47.5)$ & 64.2 & $(60.3-67.9)$ & 54.0 & $(51.0-56.9)$ \\
\hline Pennsylvania & 18.5 & $(15.5-22.1)$ & 12.5 & $(10.5-14.8)$ & 15.5 & (13.4-17.8) & 37.4 & $(34.3-40.6)$ & 53.8 & $(49.7-58.0)$ & 45.6 & $(42.5-48.8)$ \\
\hline Rhode Island & 17.5 & $(12.7-23.6)$ & 14.4 & $(12.6-16.4)$ & 16.0 & $(12.9-19.7)$ & 35.0 & $(29.4-41.2)$ & 52.2 & $(47.0-57.3)$ & 43.7 & (38.3-49.3) \\
\hline South Carolina & 26.8 & $(23.2-30.7)$ & 14.3 & $(10.6-19.0)$ & 20.6 & $(17.3-24.3)$ & 31.4 & $(26.9-36.3)$ & 53.7 & $(47.1-60.1)$ & 42.3 & (37.9-46.9) \\
\hline South Dakota & 16.4 & $(13.3-20.2)$ & 12.9 & $(9.0-18.1)$ & 14.7 & $(11.8-18.1)$ & 37.8 & $(33.0-42.9)$ & 56.4 & $(49.3-63.3)$ & 47.4 & (43.9-50.9) \\
\hline Tennessee & 21.2 & $(19.0-23.5)$ & 15.2 & $(13.7-16.9)$ & 18.2 & $(16.7-19.7)$ & 33.8 & $(31.1-36.6)$ & 51.3 & $(48.6-54.1)$ & 42.7 & $(40.6-44.9)$ \\
\hline Vermont & 16.9 & $(16.2-17.7)$ & 11.8 & $(11.1-12.4)$ & 14.4 & $(13.9-14.9)$ & 38.3 & $(37.4-39.3)$ & 53.3 & $(52.3-54.3)$ & 45.8 & $(45.2-46.5)$ \\
\hline Virginia & 21.6 & $(18.6-24.9)$ & 12.5 & $(10.5-14.7)$ & 16.9 & $(15.0-19.0)$ & 35.6 & $(32.2-39.0)$ & 54.2 & $(50.6-57.8)$ & 45.2 & $(42.2-48.2)$ \\
\hline West Virginia & 18.8 & $(15.7-22.4)$ & 15.5 & $(12.8-18.8)$ & 17.2 & $(14.8-19.8)$ & 39.2 & $(35.8-42.7)$ & 50.4 & $(44.7-56.1)$ & 44.9 & $(41.5-48.3)$ \\
\hline Wyoming & 14.3 & $(11.4-17.8)$ & 11.9 & $(9.8-14.2)$ & 13.0 & $(11.1-15.2)$ & 43.7 & $(39.4-48.1)$ & 57.4 & $(53.2-61.6)$ & 50.7 & $(47.3-54.1)$ \\
\hline Median & & 18.1 & & 13.5 & & 15.9 & & 38.3 & & 53.1 & & 45.3 \\
\hline Range & & $2.3-26.8)$ & & $7.7-19.0)$ & & $0.7-22.9)$ & & $3.5-47.6)$ & & $4.0-64.2)$ & & $4.2-54.0)$ \\
\hline \multicolumn{13}{|c|}{ Large urban school district surveys } \\
\hline Baltimore, MD & 28.5 & $(22.9-34.9)$ & 18.0 & $(14.3-22.4)$ & 23.3 & $(19.5-27.5)$ & 32.1 & $(26.4-38.4)$ & 41.8 & $(35.5-48.2)$ & 36.7 & $(32.3-41.4)$ \\
\hline Boston, MA & 29.5 & $(26.2-33.1)$ & 21.0 & $(18.0-24.2)$ & 25.4 & $(22.9-28.1)$ & 22.0 & $(19.2-25.2)$ & 38.0 & (34.3-41.8) & 30.1 & $(27.4-32.9)$ \\
\hline Broward County, FL & 29.6 & $(25.4-34.2)$ & 19.3 & $(16.1-23.0)$ & 24.4 & $(21.3-27.8)$ & 25.5 & $(21.9-29.4)$ & 41.9 & $(37.1-46.9)$ & 33.8 & (30.9-36.9) \\
\hline Cleveland, $\mathrm{OH}$ & 36.3 & $(31.6-41.3)$ & 24.1 & $(20.3-28.4)$ & 30.1 & $(27.1-33.3)$ & 24.3 & $(21.2-27.7)$ & 32.5 & $(28.2-37.2)$ & 28.3 & $(25.2-31.5)$ \\
\hline DeKalb County, GA & 25.9 & $(22.5-29.6)$ & 17.4 & $(14.3-21.1)$ & 21.6 & $(19.5-23.9)$ & 32.0 & $(28.4-35.8)$ & 49.1 & $(45.7-52.5)$ & 40.4 & $(37.9-43.1)$ \\
\hline Detroit, MI & 27.8 & $(25.1-30.6)$ & 20.9 & $(17.2-25.2)$ & 24.7 & $(22.3-27.2)$ & 25.8 & $(22.0-30.0)$ & 30.9 & $(26.9-35.3)$ & 28.1 & $(25.1-31.2)$ \\
\hline District of Columbia & 29.5 & $(28.2-30.9)$ & 21.5 & $(20.1-22.9)$ & 25.7 & $(24.8-26.7)$ & 24.8 & $(23.6-26.1)$ & 37.0 & $(35.4-38.6)$ & 30.5 & $(29.5-31.5)$ \\
\hline Duval County, FL & 27.7 & $(25.2-30.3)$ & 21.2 & $(18.6-24.0)$ & 24.7 & $(22.9-26.7)$ & 23.7 & $(21.5-26.0)$ & 36.4 & $(33.4-39.5)$ & 29.5 & $(27.6-31.5)$ \\
\hline Ft. Worth, TX & 19.8 & $(17.3-22.6)$ & 12.0 & $(9.8-14.5)$ & 15.8 & $(13.9-17.9)$ & 32.9 & $(29.9-36.0)$ & 51.1 & $(47.6-54.7)$ & 42.0 & $(39.6-44.5)$ \\
\hline Houston, TX & 25.2 & $(22.5-28.1)$ & 18.1 & $(16.1-20.4)$ & 21.6 & $(19.7-23.7)$ & 27.7 & $(23.5-32.4)$ & 40.7 & (37.9-43.6) & 34.3 & $(31.4-37.2)$ \\
\hline Los Angeles, CA & 16.1 & $(13.2-19.5)$ & 12.0 & $(10.1-14.2)$ & 14.1 & $(12.2-16.2)$ & 39.1 & $(35.0-43.4)$ & 54.6 & $(50.0-59.2)$ & 46.6 & $(42.9-50.4)$ \\
\hline Miami-Dade County, FL & 25.6 & $(22.3-29.3)$ & 13.6 & $(11.4-16.2)$ & 19.8 & $(17.6-22.2)$ & 27.1 & $(23.9-30.5)$ & 45.2 & $(41.9-48.5)$ & 36.1 & $(33.8-38.4)$ \\
\hline
\end{tabular}

See table footnotes on the next page. 
TABLE 106. (Continued) Percentage of high school students who did not participate in at least 60 minutes of physical activity on at least 1 day* and who were physically active at least 60 minutes/day on 5 or more days, * by sex - selected U.S. sites, Youth Risk Behavior Survey, 2015

\begin{tabular}{|c|c|c|c|c|c|c|c|c|c|c|c|c|}
\hline \multirow[b]{3}{*}{ Site } & \multicolumn{6}{|c|}{$\begin{array}{c}\text { Did not participate in at least } 60 \text { minutes of physical activity } \\
\text { on at least } 1 \text { day }\end{array}$} & \multicolumn{6}{|c|}{$\begin{array}{l}\text { Physically active at least } 60 \text { minutes/day } \\
\text { on } 5 \text { or more days }\end{array}$} \\
\hline & \multicolumn{2}{|c|}{ Female } & \multicolumn{2}{|r|}{ Male } & \multicolumn{2}{|r|}{ Total } & \multicolumn{2}{|r|}{ Female } & \multicolumn{2}{|r|}{ Male } & \multicolumn{2}{|r|}{ Total } \\
\hline & $\%$ & $\mathrm{Cl}^{\dagger}$ & $\%$ & $\mathrm{Cl}$ & $\%$ & $\mathrm{Cl}$ & $\%$ & $\mathrm{Cl}$ & $\%$ & $\mathrm{Cl}$ & $\%$ & $\mathrm{Cl}$ \\
\hline New York City, NY & 24.3 & $(21.6-27.2)$ & 16.3 & $(14.6-18.2)$ & 20.5 & $(18.7-22.3)$ & 32.3 & $(27.7-37.1)$ & 45.7 & $(43.4-48.0)$ & 38.7 & $(36.1-41.4)$ \\
\hline Oakland, CA & —ી & - & - & - & - & - & - & - & - & - & - & - \\
\hline Orange County, FL & 22.4 & $(19.5-25.7)$ & 15.3 & $(12.6-18.5)$ & 19.1 & $(17.0-21.4)$ & 31.3 & $(27.4-35.6)$ & 49.8 & $(45.7-54.0)$ & 40.5 & $(37.6-43.5)$ \\
\hline Palm Beach County, FL & 26.2 & $(22.9-29.8)$ & 16.5 & $(14.2-19.1)$ & 21.4 & $(19.3-23.8)$ & 26.5 & $(23.3-29.9)$ & 46.4 & $(43.2-49.7)$ & 36.2 & (33.7-38.9) \\
\hline Philadelphia, PA & 28.4 & $(23.9-33.3)$ & 20.0 & $(17.7-22.5)$ & 24.3 & $(21.7-27.2)$ & 26.3 & $(22.9-30.2)$ & 40.0 & $(35.3-44.9)$ & 32.9 & $(30.0-35.9)$ \\
\hline San Diego, CA & 15.6 & $(12.8-18.9)$ & 10.9 & $(9.3-12.8)$ & 13.2 & $(11.6-15.0)$ & 42.6 & $(38.0-47.4)$ & 59.0 & $(55.4-62.5)$ & 51.1 & $(47.5-54.6)$ \\
\hline San Francisco, CA & 20.0 & $(16.0-24.8)$ & 16.0 & $(13.1-19.5)$ & 18.2 & $(15.5-21.3)$ & 40.3 & $(35.3-45.4)$ & 46.7 & $(41.4-52.0)$ & 43.2 & (39.0-47.5) \\
\hline Median & & 26.0 & & 17.7 & & 21.6 & & 27.4 & & 43.5 & & 36.1 \\
\hline Range & $(15$ & $5.6-36.3)$ & & $0.9-24.1)$ & & $3.2-30.1)$ & & $2.0-42.6)$ & & $0.9-59.0)$ & & $8.1-51.1)$ \\
\hline
\end{tabular}

* Doing any kind of physical activity that increased their heart rate and made them breathe hard some of the time during the 7 days before the survey.

† $95 \%$ confidence interval.

I Not available.

TABLE 107. Percentage of high school students who were physically active at least 60 minutes/day on all 7 days* and who participated in muscle strengthening activities on 3 or more days, ${ }^{\dagger}$ by sex, race/ethnicity, and grade — United States, Youth Risk Behavior Survey, 2015

\begin{tabular}{|c|c|c|c|c|c|c|c|c|c|c|c|c|}
\hline \multirow[b]{3}{*}{ Category } & \multicolumn{6}{|c|}{ Physically active at least 60 minutes/day on all 7 days } & \multicolumn{6}{|c|}{ Participated in muscle strengthening activities } \\
\hline & \multicolumn{2}{|r|}{ Female } & \multicolumn{2}{|c|}{ Male } & \multicolumn{2}{|c|}{ Total } & \multicolumn{2}{|r|}{ Female } & \multicolumn{2}{|c|}{ Male } & \multicolumn{2}{|c|}{ Total } \\
\hline & $\%$ & $\mathrm{Cl}^{\S}$ & $\%$ & $\mathrm{Cl}$ & $\%$ & $\mathrm{Cl}$ & $\%$ & $\mathrm{Cl}$ & $\%$ & $\mathrm{Cl}$ & $\%$ & $\mathrm{Cl}$ \\
\hline \multicolumn{13}{|c|}{ Race/Ethnicity } \\
\hline White & 19.5 & $(17.6-21.6)$ & 38.5 & $(35.1-42.0)$ & 29.0 & $(26.6-31.6)$ & 46.1 & $(41.8-50.3)$ & 63.0 & $(59.6-66.3)$ & 54.5 & (51.4-57.7) \\
\hline Blackף & 16.6 & $(12.4-21.9)$ & 30.8 & $(25.8-36.3)$ & 24.2 & $(20.4-28.3)$ & 34.5 & $(28.4-41.1)$ & 69.8 & $(63.1-75.6)$ & 52.3 & $(48.0-56.6)$ \\
\hline Hispanic & 14.7 & $(11.8-18.1)$ & 34.2 & $(31.5-37.1)$ & 24.6 & $(22.1-27.3)$ & 39.9 & $(35.5-44.5)$ & 64.4 & $(60.9-67.7)$ & 52.4 & $(49.3-55.4)$ \\
\hline \multicolumn{13}{|l|}{ Grade } \\
\hline 9 & 20.9 & $(18.5-23.5)$ & 40.1 & (36.5-43.8) & 31.0 & $(28.5-33.7)$ & 48.2 & $(43.7-52.8)$ & 64.9 & $(60.9-68.7)$ & 56.9 & $(53.3-60.4)$ \\
\hline 10 & 19.0 & $(16.3-22.1)$ & 36.7 & $(33.3-40.2)$ & 27.8 & (25.4-30.4) & 43.0 & $(39.1-47.0)$ & 67.3 & $(63.2-71.2)$ & 54.9 & $(51.8-58.0)$ \\
\hline 11 & 16.0 & $(14.3-18.0)$ & 34.3 & $(30.6-38.2)$ & 25.3 & $(23.1-27.6)$ & 39.3 & $(35.1-43.7)$ & 62.5 & $(59.0-65.8)$ & 51.1 & $(48.0-54.2)$ \\
\hline 12 & 14.3 & $(12.0-17.0)$ & 32.6 & $(27.4-38.4)$ & 23.5 & $(20.6-26.8)$ & 39.9 & $(34.1-45.9)$ & 59.9 & $(55.9-63.9)$ & 50.0 & $(46.3-53.7)$ \\
\hline Total & 17.7 & $(16.2-19.2)$ & 36.0 & (33.6-38.5) & 27.1 & (25.4-28.8) & 42.7 & $(39.5-46.0)$ & 63.7 & $(61.2-66.1)$ & 53.4 & $(51.1-55.6)$ \\
\hline
\end{tabular}

* Doing any kind of physical activity that increased their heart rate and made them breathe hard some of the time during the 7 days before the survey.

† Such as, push-ups, sit-ups, or weight lifting, during the 7 days before the survey.

$\S 95 \%$ confidence interval.

"Non-Hispanic.

NOTE: Because of changes in question context starting in 2011, national YRBS prevalence estimates derived from the 60 minutes of physical activity question in 2011 , 2013, and 2015 are not comparable to those reported in 2009 or earlier. On the 2005-2009 national YRBS questionnaire, physical activity was assessed with three questions (in the following order) that asked the number of days students participated in 1) at least 20 minutes of vigorous physical activity; 2 ) at least 30 minutes of moderate physical activity; and 3) at least 60 minutes of aerobic (moderate and vigorous) physical activity. On the 2011, 2013, and 2015 national YRBS questionnaires, only the 60 minutes of aerobic physical activity question was included. 
TABLE 108. Percentage of high school students who were physically active at least 60 minutes/day on all 7 days, ${ }^{*}$ by sex - selected U.S. sites, Youth Risk Behavior Survey, 2015

\begin{tabular}{|c|c|c|c|c|c|c|}
\hline \multirow[b]{2}{*}{ Site } & \multicolumn{2}{|c|}{ Female } & \multicolumn{2}{|c|}{ Male } & \multicolumn{2}{|c|}{ Total } \\
\hline & $\%$ & $\mathrm{Cl}^{\dagger}$ & $\%$ & $\mathrm{Cl}$ & $\%$ & $\mathrm{Cl}$ \\
\hline \multicolumn{7}{|l|}{ State surveys } \\
\hline Alabama & 15.2 & $(12.0-19.2)$ & 35.5 & (31.4-39.9) & 25.4 & $(22.3-28.7)$ \\
\hline Alaska & 17.0 & $(14.5-19.7)$ & 24.7 & $(21.5-28.3)$ & 20.9 & (18.8-23.3) \\
\hline Arizona & 19.3 & (16.9-21.9) & 32.1 & $(27.4-37.2)$ & 26.0 & (22.7-29.5) \\
\hline Arkansas & 20.7 & $(17.1-24.9)$ & 36.7 & (31.9-41.8) & 28.6 & $(25.5-31.8)$ \\
\hline California & 18.9 & $(16.2-21.8)$ & 31.8 & $(27.0-37.1)$ & 25.3 & $(21.7-29.2)$ \\
\hline Connecticut & 18.1 & $(15.4-21.2)$ & 32.6 & $(28.4-37.1)$ & 25.3 & $(22.7-28.1)$ \\
\hline Delaware & 17.6 & $(15.1-20.4)$ & 31.4 & $(28.0-35.1)$ & 24.7 & $(22.5-27.0)$ \\
\hline Florida & 14.3 & $(12.8-15.9)$ & 34.0 & $(31.7-36.4)$ & 24.1 & $(22.6-25.7)$ \\
\hline Hawaii & 14.2 & $(12.2-16.6)$ & 26.7 & $(23.7-30.0)$ & 20.3 & (18.7-21.9) \\
\hline Idaho & 21.1 & $(17.4-25.3)$ & 37.9 & (33.0-43.0) & 29.6 & $(26.5-32.9)$ \\
\hline Illinois & 19.9 & $(16.1-24.2)$ & 33.8 & $(29.6-38.2)$ & 26.8 & $(24.2-29.6)$ \\
\hline Indiana & 16.5 & $(13.7-19.7)$ & 33.8 & $(29.4-38.4)$ & 25.3 & $(22.9-28.0)$ \\
\hline Kentucky & 12.9 & $(9.8-16.7)$ & 27.3 & $(22.7-32.4)$ & 20.2 & $(17.1-23.7)$ \\
\hline Maine & 15.6 & $(14.1-17.3)$ & 27.4 & $(25.3-29.7)$ & 21.6 & (20.4-22.9) \\
\hline Maryland & 14.1 & (13.6-14.7) & 25.0 & $(24.2-25.9)$ & 19.5 & $(19.0-20.0)$ \\
\hline Massachusetts & 17.6 & $(15.3-20.2)$ & 30.4 & $(26.9-34.3)$ & 24.1 & $(21.4-27.0)$ \\
\hline Michigan & 19.3 & $(15.7-23.5)$ & 30.0 & $(25.9-34.4)$ & 24.6 & $(21.1-28.6)$ \\
\hline Mississippi & 13.9 & $(10.8-17.7)$ & 28.6 & $(25.1-32.3)$ & 21.2 & (18.6-23.9) \\
\hline Missouri & 19.0 & $(16.3-22.1)$ & 33.5 & $(28.1-39.2)$ & 26.0 & $(22.0-30.3)$ \\
\hline Montana & 20.7 & $(18.7-22.8)$ & 36.3 & $(33.6-39.1)$ & 28.7 & $(27.0-30.4)$ \\
\hline Nebraska & 23.6 & $(20.5-27.1)$ & 35.6 & $(31.4-40.0)$ & 29.7 & $(26.9-32.7)$ \\
\hline Nevada & 21.2 & $(18.3-24.3)$ & 35.9 & $(31.6-40.5)$ & 28.6 & $(25.8-31.5)$ \\
\hline New Hampshire & 15.1 & (13.8-16.4) & 29.0 & $(27.5-30.5)$ & 22.3 & $(21.2-23.4)$ \\
\hline New Mexico & 23.4 & (21.4-25.4) & 38.3 & (36.0-40.7) & 30.9 & $(29.1-32.7)$ \\
\hline New York & 19.0 & (16.0-22.5) & 27.8 & $(24.2-31.7)$ & 23.3 & $(20.9-25.8)$ \\
\hline North Carolina & 14.8 & $(12.3-17.8)$ & 33.8 & $(32.3-35.4)$ & 24.3 & $(22.9-25.8)$ \\
\hline North Dakota & 15.7 & $(13.2-18.7)$ & 34.5 & (31.4-37.9) & 25.4 & $(23.2-27.7)$ \\
\hline Oklahoma & 21.5 & $(18.2-25.1)$ & 43.6 & $(38.3-49.1)$ & 32.2 & $(29.4-35.2)$ \\
\hline Pennsylvania & 17.3 & $(15.3-19.5)$ & 32.4 & $(28.8-36.1)$ & 24.8 & (22.4-27.4) \\
\hline Rhode Island & 12.6 & (10.8-14.8) & 27.7 & $(24.5-31.2)$ & 20.3 & $(18.0-22.7)$ \\
\hline South Carolina & 13.6 & $(10.2-17.9)$ & 34.0 & $(27.5-41.2)$ & 23.6 & $(20.2-27.4)$ \\
\hline South Dakota & 18.8 & $(15.8-22.1)$ & 36.8 & $(31.2-42.9)$ & 28.1 & $(24.3-32.1)$ \\
\hline Tennessee & 17.6 & $(15.8-19.5)$ & 33.9 & $(31.5-36.3)$ & 25.9 & $(24.2-27.7)$ \\
\hline Vermont & 15.6 & $(14.9-16.3)$ & 30.3 & $(29.5-31.2)$ & 23.1 & $(22.5-23.7)$ \\
\hline Virginia & 17.6 & $(15.6-19.8)$ & 32.3 & $(29.3-35.5)$ & 25.1 & $(22.9-27.5)$ \\
\hline West Virginia & 18.3 & $(15.2-21.8)$ & 33.0 & $(29.0-37.2)$ & 25.8 & $(23.2-28.5)$ \\
\hline Wyoming & 20.5 & $(17.7-23.5)$ & 33.5 & $(30.2-36.9)$ & 27.1 & $(24.8-29.6)$ \\
\hline Median & \multirow{2}{*}{\multicolumn{2}{|c|}{$\begin{array}{c}17.6 \\
(12.6-23.6)\end{array}$}} & \multirow{2}{*}{\multicolumn{2}{|c|}{$\begin{array}{c}33.0 \\
(24.7-43.6)\end{array}$}} & \multirow{2}{*}{\multicolumn{2}{|c|}{$\begin{array}{c}25.3 \\
(19.5-32.2)\end{array}$}} \\
\hline Range & & & & & & \\
\hline \multicolumn{7}{|c|}{ Large urban school district surveys } \\
\hline Baltimore, MD & 16.4 & $(12.3-21.7)$ & 25.6 & $(20.0-32.3)$ & 20.8 & $(17.1-25.0)$ \\
\hline Boston, MA & 9.6 & $(7.8-11.7)$ & 21.9 & $(18.8-25.5)$ & 15.9 & $(13.9-18.1)$ \\
\hline Broward County, FL & 11.4 & $(9.1-14.3)$ & 24.1 & $(19.7-29.2)$ & 17.9 & (15.4-20.6) \\
\hline Cleveland, $\mathrm{OH}$ & 14.1 & $(11.8-16.7)$ & 19.1 & $(16.0-22.6)$ & 16.4 & $(14.3-18.7)$ \\
\hline DeKalb County, GA & 16.0 & $(13.2-19.2)$ & 31.5 & $(29.0-34.2)$ & 23.7 & $(21.5-25.9)$ \\
\hline Detroit, MI & 14.8 & $(12.3-17.7)$ & 17.0 & $(13.6-21.1)$ & 15.8 & $(13.5-18.3)$ \\
\hline District of Columbia & 11.7 & $(10.7-12.7)$ & 20.9 & $(19.6-22.2)$ & 16.0 & $(15.2-16.8)$ \\
\hline Duval County, FL & 11.4 & $(9.6-13.4)$ & 21.2 & $(18.7-23.9)$ & 15.9 & (14.3-17.6) \\
\hline Ft. Worth, TX & 16.5 & $(14.4-18.9)$ & 32.5 & $(29.4-35.8)$ & 24.5 & $(22.5-26.7)$ \\
\hline Houston, TX & 14.7 & $(11.6-18.3)$ & 24.9 & $(22.4-27.6)$ & 19.8 & $(17.6-22.3)$ \\
\hline Los Angeles, CA & 16.5 & $(13.7-19.7)$ & 30.3 & $(26.8-34.1)$ & 23.2 & $(20.9-25.7)$ \\
\hline Miami-Dade County, FL & 12.7 & $(10.8-14.9)$ & 26.7 & $(23.6-30.0)$ & 19.5 & $(17.9-21.3)$ \\
\hline
\end{tabular}

\footnotetext{
See table footnotes on the next page.
} 
TABLE 108. (Continued) Percentage of high school students who were physically active at least 60 minutes/day on all 7 days, ${ }^{*}$ by sex — selected U.S. sites, Youth Risk Behavior Survey, 2015

\begin{tabular}{|c|c|c|c|c|c|c|}
\hline \multirow[b]{2}{*}{ Site } & \multicolumn{2}{|c|}{ Female } & \multicolumn{2}{|c|}{ Male } & \multicolumn{2}{|c|}{ Total } \\
\hline & $\%$ & $\mathrm{Cl}^{\dagger}$ & $\%$ & $\mathrm{Cl}$ & $\%$ & $\mathrm{Cl}$ \\
\hline New York City, NY & 15.9 & $(13.7-18.4)$ & 26.2 & $(23.7-28.9)$ & 20.9 & $(19.4-22.5)$ \\
\hline Oakland, CA & — & - & - & - & - & - \\
\hline Orange County, FL & 13.7 & $(11.2-16.5)$ & 30.5 & $(26.7-34.6)$ & 22.1 & $(19.6-24.9)$ \\
\hline Palm Beach County, FL & 11.0 & $(8.8-13.7)$ & 30.1 & $(27.1-33.3)$ & 20.4 & $(18.3-22.7)$ \\
\hline Philadelphia, PA & 14.5 & $(12.3-17.0)$ & 25.2 & $(22.6-27.9)$ & 19.6 & $(18.0-21.3)$ \\
\hline San Diego, CA & 18.3 & $(15.1-22.0)$ & 35.5 & $(31.3-39.8)$ & 27.0 & $(23.8-30.6)$ \\
\hline San Francisco, CA & 14.4 & $(11.7-17.6)$ & 22.9 & $(19.3-26.9)$ & 18.6 & $(16.0-21.5)$ \\
\hline Median & \multicolumn{2}{|c|}{14.4} & \multirow{2}{*}{\multicolumn{2}{|c|}{$\begin{array}{c}25.4 \\
(17.0-35.5)\end{array}$}} & \multirow{2}{*}{\multicolumn{2}{|c|}{$\begin{array}{c}19.7 \\
(15.8-27.0)\end{array}$}} \\
\hline Range & & & & & & \\
\hline
\end{tabular}

* Doing any kind of physical activity that increased their heart rate and made them breathe hard some of the time during the 7 days before the survey.

† $95 \%$ confidence interval.

१ Not available.

TABLE 109. Percentage of high school students who played video or computer games or used a computer* for 3 or more hours/day ${ }^{\dagger}$ and who watched 3 or more hours/day of television, ${ }^{\dagger}$ by sex, race/ethnicity, and grade — United States, Youth Risk Behavior Survey, 2015

\begin{tabular}{|c|c|c|c|c|c|c|c|c|c|c|c|c|}
\hline \multirow[b]{3}{*}{ Category } & \multicolumn{6}{|c|}{ Used computers 3 or more hours/day } & \multicolumn{6}{|c|}{ Watched television 3 or more hours/day } \\
\hline & \multicolumn{2}{|c|}{ Female } & \multicolumn{2}{|r|}{ Male } & \multicolumn{2}{|r|}{ Total } & \multicolumn{2}{|c|}{ Female } & \multicolumn{2}{|c|}{ Male } & \multicolumn{2}{|r|}{ Total } \\
\hline & $\%$ & $\mathrm{Cl}^{\S}$ & $\%$ & $\mathrm{Cl}$ & $\%$ & $\mathrm{Cl}$ & $\%$ & $\mathrm{Cl}$ & $\%$ & $\mathrm{Cl}$ & $\%$ & $\mathrm{Cl}$ \\
\hline \multicolumn{13}{|c|}{ Race/Ethnicity } \\
\hline Whitef & 38.3 & (33.2-43.7) & 38.9 & (35.5-42.4) & 38.6 & (34.8-42.5) & 18.8 & $(15.8-22.1)$ & 21.4 & $(18.2-24.9)$ & 20.0 & $(17.3-23.1)$ \\
\hline Black & 48.4 & $(41.7-55.1)$ & 41.2 & $(36.4-46.3)$ & 44.6 & $(40.2-49.1)$ & 41.5 & $(37.0-46.1)$ & 37.0 & $(30.2-44.4)$ & 39.2 & $(34.1-44.5)$ \\
\hline Hispanic & 47.4 & $(44.1-50.7)$ & 45.1 & $(42.5-47.7)$ & 46.2 & $(44.3-48.1)$ & 29.2 & $(26.3-32.3)$ & 27.4 & $(24.8-30.1)$ & 28.2 & $(26.0-30.6)$ \\
\hline \multicolumn{13}{|l|}{ Grade } \\
\hline 9 & 48.7 & $(45.8-51.6)$ & 42.5 & (37.8-47.2) & 45.4 & (42.2-48.7) & 25.3 & (21.9-29.0) & 26.3 & (22.9-29.9) & 25.7 & $(23.0-28.6)$ \\
\hline 10 & 43.3 & $(40.2-46.5)$ & 43.4 & $(39.8-47.0)$ & 43.4 & $(40.6-46.2)$ & 24.1 & $(20.0-28.8)$ & 24.6 & (20.9-28.7) & 24.5 & $(21.0-28.4)$ \\
\hline 11 & 38.1 & (34.4-41.9) & 36.1 & (32.4-39.9) & 37.2 & (34.3-40.1) & 22.4 & $(20.1-24.8)$ & 24.6 & $(21.3-28.1)$ & 23.6 & (21.4-25.9) \\
\hline 12 & 40.4 & $(34.8-46.2)$ & 40.8 & $(36.3-45.5)$ & 40.5 & $(36.8-44.3)$ & 25.9 & $(22.4-29.7)$ & 24.4 & $(21.6-27.5)$ & 25.1 & $(22.7-27.7)$ \\
\hline Total & 42.8 & (39.9-45.7) & 40.6 & (37.7-43.7) & 41.7 & (39.3-44.2) & 24.4 & $(22.2-26.8)$ & 25.0 & $(22.6-27.5)$ & 24.7 & $(22.7-26.9)$ \\
\hline
\end{tabular}

* For something that was not school work.

† On an average school day.

$\S 95 \%$ confidence interval.

" Non-Hispanic. 
TABLE 110. Percentage of high school students who played video or computer games or used a computer* for 3 or more hours/day ${ }^{\dagger}$ and who watched $\geq 3$ hours/day of television, ${ }^{\dagger}$ by sex — selected U.S. sites, Youth Risk Behavior Survey, 2015

\begin{tabular}{|c|c|c|c|c|c|c|c|c|c|c|c|c|}
\hline \multirow[b]{3}{*}{ Site } & \multicolumn{6}{|c|}{ Used computers 3 or more hours/day } & \multicolumn{6}{|c|}{ Watched television 3 or more hours/day } \\
\hline & \multicolumn{2}{|c|}{ Female } & \multicolumn{2}{|r|}{ Male } & \multicolumn{2}{|r|}{ Total } & \multicolumn{2}{|r|}{ Female } & \multicolumn{2}{|r|}{ Male } & \multicolumn{2}{|r|}{ Total } \\
\hline & $\%$ & $\mathrm{Cl}^{\S}$ & $\%$ & $\mathrm{Cl}$ & $\%$ & $\mathrm{Cl}$ & $\%$ & $\mathrm{Cl}$ & $\%$ & $\mathrm{Cl}$ & $\%$ & $\mathrm{Cl}$ \\
\hline \multicolumn{13}{|l|}{ State surveys } \\
\hline Alabama & 40.8 & (36.7-45.0) & 36.2 & (31.5-41.1) & 38.2 & (35.1-41.5) & 33.5 & $(27.1-40.7)$ & 28.0 & $(24.4-32.0)$ & 30.8 & $(26.6-35.2)$ \\
\hline Alaska & 32.8 & $(28.5-37.5)$ & 35.4 & $(31.6-39.3)$ & 34.3 & $(31.2-37.5)$ & 20.1 & $(17.1-23.6)$ & 24.1 & $(20.9-27.6)$ & 22.2 & $(20.2-24.5)$ \\
\hline Arizona & 41.5 & $(35.1-48.1)$ & 39.6 & $(35.4-44.0)$ & 40.5 & $(35.7-45.5)$ & 26.2 & $(22.6-30.0)$ & 22.8 & $(18.8-27.2)$ & 24.7 & $(21.2-28.5)$ \\
\hline Arkansas & 40.8 & (35.8-45.9) & 42.0 & (38.2-45.9) & 41.2 & (37.2-45.4) & 30.8 & $(25.4-36.7)$ & 31.2 & $(24.3-39.1)$ & 31.0 & $(25.7-36.9)$ \\
\hline California & 43.5 & $(38.0-49.1)$ & 40.7 & (33.5-48.4) & 42.1 & (36.5-47.9) & 22.3 & $(18.2-27.0)$ & 23.7 & (20.9-26.8) & 23.1 & $(20.2-26.1)$ \\
\hline Connecticut & 38.2 & (33.9-42.8) & 37.3 & $(33.7-41.1)$ & 37.8 & $(34.6-41.1)$ & 22.3 & $(19.8-25.1)$ & 21.6 & $(18.3-25.4)$ & 21.9 & $(19.7-24.3)$ \\
\hline Delaware & 32.7 & $(29.1-36.4)$ & 38.3 & (34.4-42.4) & 35.6 & $(32.8-38.5)$ & 27.2 & $(24.2-30.4)$ & 28.1 & $(25.0-31.4)$ & 27.9 & $(25.7-30.2)$ \\
\hline Florida & 42.1 & (40.4-43.9) & 42.2 & (39.9-44.6) & 42.2 & $(40.6-43.8)$ & 27.8 & (25.3-30.4) & 28.8 & (26.2-31.4) & 28.2 & $(26.1-30.5)$ \\
\hline Hawaii & 42.1 & (38.8-45.5) & 39.3 & (35.4-43.2) & 40.6 & $(39.2-42.0)$ & 21.8 & $(19.5-24.2)$ & 21.8 & (19.9-23.7) & 21.8 & $(20.2-23.5)$ \\
\hline Idaho & 32.5 & $(29.7-35.6)$ & 34.8 & $(29.6-40.4)$ & 33.7 & $(30.6-37.0)$ & 20.6 & $(17.5-24.0)$ & 19.1 & $(16.1-22.5)$ & 19.7 & $(17.1-22.6)$ \\
\hline Illinois & 36.6 & $(30.5-43.1)$ & 37.3 & (33.2-41.5) & 36.9 & (33.3-40.7) & 21.6 & $(18.0-25.7)$ & 22.0 & $(17.5-27.2)$ & 21.8 & $(18.5-25.5)$ \\
\hline Indiana & 37.4 & (33.9-41.1) & 39.5 & (33.9-45.3) & 38.4 & (34.5-42.5) & 22.2 & $(17.6-27.4)$ & 22.2 & (18.6-26.3) & 22.3 & $(19.7-25.1)$ \\
\hline Kentucky & 41.9 & (37.6-46.3) & 38.5 & (34.7-42.4) & 40.1 & (37.1-43.2) & 25.4 & (22.3-28.7) & 25.3 & (21.6-29.4) & 25.5 & $(23.1-28.0)$ \\
\hline Maine & 37.2 & $(35.3-39.1)$ & 39.3 & $(36.7-42.1)$ & 38.3 & (36.4-40.3) & 22.7 & (21.0-24.5) & 23.5 & (21.8-25.3) & 23.1 & $(21.6-24.6)$ \\
\hline Maryland & 37.9 & (37.1-38.6) & 38.9 & (38.0-39.8) & 38.3 & $(37.7-39.0)$ & 27.1 & $(26.3-28.0)$ & 26.4 & (25.4-27.3) & 26.7 & $(26.0-27.5)$ \\
\hline Massachusetts & 43.3 & (40.2-46.4) & 42.9 & (39.7-46.3) & 43.2 & (40.8-45.7) & -9 & - & - & - & - & - \\
\hline Michigan & 40.0 & $(36.0-44.1)$ & 41.4 & $(37.2-45.7)$ & 40.6 & (37.3-44.0) & 20.9 & $(17.6-24.6)$ & 22.4 & (18.4-27.0) & 21.7 & $(18.3-25.4)$ \\
\hline Mississippi & 35.2 & $(31.5-39.1)$ & 33.3 & (29.9-36.8) & 34.1 & $(31.6-36.7)$ & 36.1 & $(31.1-41.4)$ & 30.9 & $(26.6-35.6)$ & 33.4 & $(29.8-37.2)$ \\
\hline Missouri & 38.3 & $(32.9-43.9)$ & 37.1 & $(32.2-42.2)$ & 37.4 & (33.5-41.5) & 23.9 & $(18.7-30.1)$ & 18.1 & $(14.8-22.1)$ & 21.2 & $(17.6-25.3)$ \\
\hline Montana & 32.4 & $(30.1-34.7)$ & 36.0 & $(33.8-38.3)$ & 34.2 & $(32.5-35.9)$ & 22.0 & $(19.9-24.1)$ & 21.9 & $(19.4-24.7)$ & 21.9 & $(20.0-24.0)$ \\
\hline Nebraska & 31.9 & $(27.6-36.5)$ & 31.1 & $(27.1-35.5)$ & 31.5 & $(28.4-34.9)$ & 19.1 & $(16.3-22.4)$ & 21.1 & $(18.5-24.0)$ & 20.1 & $(18.0-22.4)$ \\
\hline Nevada & 36.7 & $(31.6-42.2)$ & 36.9 & (30.9-43.3) & 36.7 & (33.1-40.5) & 22.1 & $(19.1-25.4)$ & 22.2 & $(17.5-27.7)$ & 22.1 & $(19.2-25.2)$ \\
\hline New Hampshire & 37.0 & (35.3-38.8) & 40.5 & $(38.9-42.1)$ & 38.9 & $(37.7-40.1)$ & 18.6 & (17.1-20.3) & 19.7 & (18.6-20.9) & 19.1 & $(18.2-20.1)$ \\
\hline New Mexico & 39.1 & $(37.1-41.2)$ & 38.6 & (35.9-41.4) & 38.9 & (37.0-40.8) & 24.9 & (23.2-26.6) & 24.6 & (23.0-26.4) & 24.7 & $(23.4-26.1)$ \\
\hline New York & 37.4 & $(33.6-41.2)$ & 37.2 & (33.2-41.3) & 37.2 & (34.3-40.3) & 27.0 & (24.3-29.8) & 21.7 & $(19.3-24.3)$ & 24.2 & $(22.2-26.3)$ \\
\hline North Carolina & 42.3 & $(36.3-48.5)$ & 42.2 & (36.2-48.4) & 42.3 & (38.2-46.4) & 30.3 & $(26.5-34.4)$ & 31.0 & $(27.8-34.3)$ & 30.5 & $(27.7-33.4)$ \\
\hline North Dakota & 37.4 & (34.2-40.8) & 39.7 & (36.2-43.3) & 38.6 & (36.5-40.7) & 16.7 & $(14.1-19.7)$ & 20.9 & (18.7-23.4) & 18.9 & $(17.1-20.7)$ \\
\hline Oklahoma & 44.6 & $(41.6-47.6)$ & 46.5 & $(41.1-51.9)$ & 45.6 & (42.7-48.6) & 28.2 & $(25.0-31.7)$ & 29.9 & $(26.5-33.5)$ & 28.9 & $(26.4-31.5)$ \\
\hline Pennsylvania & 42.3 & $(38.6-46.0)$ & 43.9 & $(40.3-47.6)$ & 43.0 & (40.5-45.6) & 27.2 & $(23.8-30.9)$ & 28.3 & $(25.2-31.7)$ & 27.8 & $(25.3-30.4)$ \\
\hline Rhode Island & 40.3 & (33.6-47.3) & 40.0 & (35.0-45.2) & 40.0 & $(35.0-45.2)$ & 21.9 & $(16.7-28.2)$ & 22.6 & (18.3-27.5) & 22.2 & $(17.6-27.5)$ \\
\hline South Carolina & 42.7 & (39.4-46.0) & 36.3 & $(30.2-42.9)$ & 39.5 & $(36.2-42.9)$ & 29.1 & $(22.7-36.4)$ & 27.8 & (21.6-34.8) & 28.4 & $(23.2-34.2)$ \\
\hline South Dakota & 39.5 & (34.9-44.3) & 36.3 & $(32.1-40.7)$ & 37.8 & (33.9-41.9) & 21.7 & $(17.8-26.3)$ & 21.1 & (15.6-28.0) & 21.5 & $(18.2-25.2)$ \\
\hline Tennessee & 42.6 & $(39.8-45.4)$ & 44.1 & $(41.3-47.0)$ & 43.4 & $(41.3-45.6)$ & 29.6 & $(27.9-31.3)$ & 28.9 & $(25.3-32.8)$ & 29.2 & $(27.1-31.5)$ \\
\hline Vermont & - & - & - & - & - & - & - & - & - & - & - & - \\
\hline Virginia & 42.3 & (38.7-45.9) & 41.4 & (39.1-43.8) & 41.9 & (39.4-44.3) & 23.5 & (20.4-27.0) & 24.2 & $(21.3-27.5)$ & 23.9 & $(21.3-26.6)$ \\
\hline West Virginia & 41.9 & $(36.7-47.3)$ & 44.9 & $(40.7-49.2)$ & 43.4 & $(39.3-47.5)$ & 28.6 & $(25.1-32.3)$ & 25.0 & $(20.4-30.2)$ & 26.8 & $(23.6-30.2)$ \\
\hline Wyoming & 29.7 & $(26.2-33.4)$ & 30.3 & $(27.4-33.4)$ & 30.1 & $(27.8-32.4)$ & 22.0 & $(18.6-25.8)$ & 19.8 & $(17.6-22.2)$ & 21.0 & $(18.7-23.5)$ \\
\hline Median & & 39.3 & & 39.1 & & 38.7 & & 23.5 & & 23.5 & & 23.1 \\
\hline Range & $(29$ & $.7-44.6)$ & & $.3-46.5)$ & & $.1-45.6)$ & & $7-36.1)$ & & $1-31.2)$ & & $.9-33.4)$ \\
\hline \multicolumn{13}{|c|}{ Large urban school district surveys } \\
\hline Baltimore, MD & 34.0 & $(30.1-38.2)$ & 37.1 & (30.4-44.3) & 35.3 & $(31.0-39.9)$ & 36.9 & $(32.3-41.8)$ & 35.4 & $(28.4-43.1)$ & 35.9 & (32.0-40.0) \\
\hline Boston, MA & 45.4 & $(41.3-49.6)$ & 43.5 & $(39.9-47.3)$ & 44.4 & $(41.4-47.3)$ & 28.8 & $(25.6-32.2)$ & 33.1 & $(29.6-36.8)$ & 30.9 & $(28.7-33.3)$ \\
\hline Broward County, FL & 42.6 & $(38.5-46.9)$ & 39.2 & $(34.9-43.6)$ & 40.9 & $(38.0-43.9)$ & 28.3 & $(25.1-31.7)$ & 27.7 & $(23.7-32.1)$ & 28.1 & $(25.1-31.3)$ \\
\hline Cleveland, $\mathrm{OH}$ & 42.4 & $(37.3-47.6)$ & 43.2 & $(38.2-48.4)$ & 42.5 & $(38.7-46.3)$ & 38.0 & $(34.3-41.9)$ & 33.7 & $(29.0-38.8)$ & 35.5 & $(32.4-38.7)$ \\
\hline DeKalb County, GA & 36.3 & (32.8-40.0) & 36.5 & (32.3-40.8) & 36.4 & $(33.7-39.1)$ & 29.9 & $(26.3-33.8)$ & 31.8 & $(28.6-35.1)$ & 30.9 & $(28.2-33.7)$ \\
\hline Detroit, MI & 35.1 & $(31.1-39.4)$ & 32.0 & $(28.0-36.3)$ & 33.8 & $(30.3-37.4)$ & 31.9 & $(28.7-35.3)$ & 29.7 & $(25.5-34.2)$ & 30.9 & $(28.2-33.7)$ \\
\hline District of Columbia & 38.2 & $(36.8-39.7)$ & 38.0 & $(36.4-39.6)$ & 38.1 & $(37.0-39.1)$ & 32.6 & $(31.2-34.0)$ & 31.9 & $(30.4-33.5)$ & 32.2 & $(31.2-33.3)$ \\
\hline Duval County, FL & 35.9 & $(33.4-38.6)$ & 36.6 & (33.9-39.3) & 36.2 & $(34.3-38.0)$ & 29.7 & $(27.3-32.2)$ & 26.8 & (24.2-29.6) & 28.2 & $(26.3-30.2)$ \\
\hline Ft. Worth, TX & 43.5 & $(40.3-46.7)$ & 42.3 & $(38.3-46.4)$ & 42.9 & $(40.3-45.6)$ & 30.9 & $(28.0-34.0)$ & 32.5 & $(29.6-35.5)$ & 31.7 & $(29.5-33.9)$ \\
\hline Houston, TX & 34.7 & $(31.7-37.8)$ & 35.4 & $(32.6-38.3)$ & 34.8 & $(32.6-37.1)$ & 29.9 & $(27.1-32.9)$ & 26.1 & $(23.4-28.9)$ & 28.1 & $(26.0-30.2)$ \\
\hline Los Angeles, CA & 41.7 & $(37.7-45.9)$ & 41.7 & $(38.8-44.6)$ & 41.7 & $(38.7-44.7)$ & 27.5 & $(24.7-30.4)$ & 26.8 & $(23.9-30.0)$ & 27.1 & $(25.1-29.1)$ \\
\hline Miami-Dade County, FL & 42.5 & $(38.7-46.4)$ & 41.4 & (38.2-44.6) & 42.0 & $(39.7-44.4)$ & 29.4 & $(26.5-32.5)$ & 27.9 & $(24.9-31.1)$ & 28.7 & $(26.4-31.0)$ \\
\hline
\end{tabular}

See table footnotes on the next page. 
TABLE 110. (Continued) Percentage of high school students who played video or computer games or used a computer* for 3 or more hours/day ${ }^{\dagger}$ and who watched $\geq 3$ hours/day of television, ${ }^{\dagger}$ by sex — selected U.S. sites, Youth Risk Behavior Survey, 2015

\begin{tabular}{|c|c|c|c|c|c|c|c|c|c|c|c|c|}
\hline \multirow[b]{3}{*}{ Site } & \multicolumn{6}{|c|}{ Used computers 3 or more hours/day } & \multicolumn{6}{|c|}{ Watched television 3 or more hours/day } \\
\hline & \multicolumn{2}{|c|}{ Female } & \multicolumn{2}{|r|}{ Male } & \multicolumn{2}{|r|}{ Total } & \multicolumn{2}{|r|}{ Female } & \multicolumn{2}{|r|}{ Male } & \multicolumn{2}{|r|}{ Total } \\
\hline & $\%$ & $\mathrm{Cl}^{\S}$ & $\%$ & $\mathrm{Cl}$ & $\%$ & $\mathrm{Cl}$ & $\%$ & $\mathrm{Cl}$ & $\%$ & $\mathrm{Cl}$ & $\%$ & $\mathrm{Cl}$ \\
\hline New York City, NY & 44.5 & $(41.2-47.7)$ & 46.8 & $(43.9-49.7)$ & 45.6 & $(43.1-48.1)$ & 31.5 & $(28.8-34.4)$ & 26.2 & $(23.4-29.2)$ & 28.9 & $(26.6-31.3)$ \\
\hline Oakland, CA & 41.4 & (36.3-46.8) & 42.2 & (38.3-46.1) & 41.7 & (38.2-45.3) & 28.4 & $(24.6-32.7)$ & 31.0 & $(26.7-35.7)$ & 29.7 & $(26.8-32.7)$ \\
\hline Orange County, FL & 42.5 & $(38.4-46.8)$ & 44.7 & $(40.4-49.0)$ & 43.8 & (40.6-46.9) & 28.4 & $(24.0-33.2)$ & 29.5 & $(25.3-34.1)$ & 29.0 & (25.6-32.7) \\
\hline Palm Beach County, FL & 38.9 & $(35.5-42.4)$ & 36.0 & $(32.8-39.2)$ & 37.7 & (35.0-40.4) & 26.2 & $(23.1-29.6)$ & 26.4 & $(23.8-29.1)$ & 26.0 & $(23.9-28.2)$ \\
\hline Philadelphia, PA & 46.6 & $(41.9-51.4)$ & 49.3 & $(44.1-54.5)$ & 47.7 & $(44.7-50.8)$ & 34.3 & $(27.8-41.5)$ & 34.1 & $(29.0-39.5)$ & 34.2 & $(30.1-38.6)$ \\
\hline San Diego, CA & 46.4 & $(43.0-49.8)$ & 43.3 & $(40.0-46.7)$ & 44.8 & $(42.5-47.2)$ & 19.5 & $(17.1-22.2)$ & 19.9 & $(17.7-22.4)$ & 19.7 & $(18.1-21.4)$ \\
\hline San Francisco, CA & - & - & - & - & - & - & - & - & - & - & - & - \\
\hline Median & & 42.0 & & 41.5 & & 41.7 & & 29.8 & & 29.6 & & 29.3 \\
\hline Range & & $4.0-46.6)$ & & (2.0-49.3) & & $3.8-47.7)$ & & (9.5-38.0) & & 9.9-35.4) & & $9.7-35.9)$ \\
\hline
\end{tabular}

* For something that was not school work.

† On an average school day.

$\S 95 \%$ confidence interval.

१ Not available.

TABLE 111. Percentage of high school students who attended physical education (PE) classes on $\geq 1$ days* and who attended PE classes on all 5 days, * by sex, race/ethnicity, and grade - United States, Youth Risk Behavior Survey, 2015

\begin{tabular}{|c|c|c|c|c|c|c|c|c|c|c|c|c|}
\hline \multirow[b]{3}{*}{ Category } & \multicolumn{6}{|c|}{ Attended PE classes } & \multicolumn{6}{|c|}{ Attended PE classes daily } \\
\hline & \multicolumn{2}{|c|}{ Female } & \multicolumn{2}{|r|}{ Male } & \multicolumn{2}{|r|}{ Total } & \multicolumn{2}{|c|}{ Female } & \multicolumn{2}{|r|}{ Male } & \multicolumn{2}{|r|}{ Total } \\
\hline & $\%$ & $\mathrm{Cl}^{\dagger}$ & $\%$ & $\mathrm{Cl}$ & $\%$ & $\mathrm{Cl}$ & $\%$ & $\mathrm{Cl}$ & $\%$ & $\mathrm{Cl}$ & $\%$ & $\mathrm{Cl}$ \\
\hline \multicolumn{13}{|c|}{ Race/Ethnicity } \\
\hline White ${ }^{\S}$ & 45.9 & $(38.1-54.0)$ & 51.0 & $(42.7-59.3)$ & 48.4 & $(41.1-55.8)$ & 21.1 & $(13.8-30.8)$ & 29.6 & $(23.1-37.1)$ & 25.4 & $(18.8-33.3)$ \\
\hline Black $^{\S}$ & 52.2 & $(44.3-60.1)$ & 65.4 & $(60.2-70.2)$ & 59.2 & $(53.8-64.4)$ & 32.2 & $(26.0-39.1)$ & 38.9 & $(31.2-47.2)$ & 35.8 & $(29.3-42.7)$ \\
\hline Hispanic & 50.1 & $(43.4-56.8)$ & 60.5 & $(53.1-67.6)$ & 55.4 & $(49.0-61.6)$ & 33.0 & $(26.4-40.4)$ & 42.4 & (34.5-50.6) & 37.7 & (30.7-45.3) \\
\hline \multicolumn{13}{|l|}{ Grade } \\
\hline 9 & 70.4 & $(62.6-77.2)$ & 72.2 & $(62.6-80.1)$ & 71.4 & $(63.6-78.1)$ & 39.5 & $(31.2-48.4)$ & 44.6 & $(35.4-54.1)$ & 42.2 & $(34.0-50.9)$ \\
\hline 10 & 53.9 & $(45.1-62.5)$ & 61.3 & $(52.1-69.7)$ & 57.5 & $(49.1-65.5)$ & 27.0 & $(18.8-37.0)$ & 36.1 & $(29.2-43.7)$ & 31.5 & $(24.3-39.7)$ \\
\hline 11 & 34.6 & $(25.7-44.7)$ & 42.2 & $(34.8-49.9)$ & 38.5 & $(31.6-46.0)$ & 18.1 & $(12.6-25.4)$ & 25.2 & $(19.2-32.4)$ & 21.8 & (16.7-27.9) \\
\hline 12 & 29.1 & $(22.5-36.8)$ & 42.9 & $(36.2-49.9)$ & 36.1 & $(30.1-42.5)$ & 16.0 & $(11.4-21.9)$ & 27.9 & $(22.1-34.6)$ & 21.9 & $(17.3-27.3)$ \\
\hline Total & 47.8 & $(41.4-54.1)$ & 55.3 & $(48.7-61.7)$ & 51.6 & $(45.9-57.4)$ & 25.5 & $(19.6-32.6)$ & 33.8 & $(27.5-40.6)$ & 29.8 & $(24.0-36.2)$ \\
\hline
\end{tabular}

* In an average week when they were in school.

† 95\% confidence interval.

$\S$ Non-Hispanic. 
TABLE 112. Percentage of high school students who attended physical education (PE) classes on $\geq 1$ days* and who attended PE classes on all 5 days, ${ }^{*}$ by sex — selected U.S. sites, Youth Risk Behavior Survey, 2015

\begin{tabular}{|c|c|c|c|c|c|c|c|c|c|c|c|c|}
\hline \multirow[b]{3}{*}{ Site } & \multicolumn{6}{|c|}{ Attended PE classes } & \multicolumn{6}{|c|}{ Attended PE classes daily } \\
\hline & \multicolumn{2}{|c|}{ Female } & \multicolumn{2}{|r|}{ Male } & \multicolumn{2}{|r|}{ Total } & \multicolumn{2}{|c|}{ Female } & \multicolumn{2}{|r|}{ Male } & \multicolumn{2}{|r|}{ Total } \\
\hline & $\%$ & $\mathrm{Cl}^{\dagger}$ & $\%$ & $\mathrm{Cl}$ & $\%$ & $\mathrm{Cl}$ & $\%$ & $\mathrm{Cl}$ & $\%$ & $\mathrm{Cl}$ & $\%$ & $\mathrm{Cl}$ \\
\hline \multicolumn{13}{|l|}{ State surveys } \\
\hline Alabama & 41.1 & $(33.5-49.1)$ & 57.1 & $(50.8-63.1)$ & 49.0 & $(43.0-55.0)$ & 25.2 & (18.2-33.8) & 39.6 & (33.5-46.0) & 32.2 & $(26.0-39.0)$ \\
\hline Alaska & 34.9 & (29.8-40.4) & 51.1 & $(45.4-56.7)$ & 43.2 & (38.4-48.1) & 13.2 & $(10.3-16.7)$ & 18.5 & $(14.3-23.5)$ & 15.9 & $(13.0-19.3)$ \\
\hline Arizona & 34.0 & (27.0-41.9) & 47.3 & $(41.0-53.6)$ & 40.9 & (34.5-47.7) & 21.5 & (14.4-30.9) & 30.6 & $(23.1-39.2)$ & 26.3 & (18.9-35.2) \\
\hline Arkansas & 34.2 & $(29.3-39.6)$ & 46.0 & $(39.1-53.2)$ & 40.1 & $(37.2-43.1)$ & 25.2 & $(21.4-29.5)$ & 32.2 & $(24.2-41.5)$ & 28.6 & $(24.2-33.4)$ \\
\hline California & 54.7 & $(46.8-62.4)$ & 56.7 & $(48.5-64.6)$ & 55.9 & $(48.3-63.3)$ & 36.9 & $(28.8-45.9)$ & 40.1 & $(30.8-50.1)$ & 38.8 & $(30.4-48.0)$ \\
\hline Connecticut & 58.2 & $(49.1-66.9)$ & 66.4 & $(57.7-74.1)$ & 62.3 & $(53.8-70.2)$ & 10.5 & $(6.1-17.6)$ & 12.6 & $(8.6-18.1)$ & 11.5 & $(7.6-17.1)$ \\
\hline Delaware & 36.2 & $(30.3-42.5)$ & 40.9 & $(34.9-47.2)$ & 38.5 & (33.2-44.2) & 9.6 & $(7.0-13.1)$ & 12.3 & $(9.5-15.7)$ & 11.1 & (8.9-13.7) \\
\hline Florida & 31.6 & $(29.0-34.4)$ & 50.9 & $(47.7-54.0)$ & 41.2 & (38.7-43.7) & 14.1 & $(12.0-16.6)$ & 30.3 & $(27.0-33.8)$ & 22.2 & $(19.7-24.8)$ \\
\hline Hawaii & 36.8 & (33.2-40.5) & 48.3 & $(44.0-52.6)$ & 42.4 & (39.2-45.7) & 5.0 & (3.8-6.4) & 8.5 & $(7.0-10.2)$ & 6.7 & $(5.6-7.8)$ \\
\hline Idaho & 38.0 & (29.9-46.8) & 53.9 & $(47.6-60.1)$ & 46.1 & (39.6-52.8) & 16.9 & $(11.1-24.7)$ & 25.7 & $(16.8-37.1)$ & 21.4 & $(14.6-30.3)$ \\
\hline Illinois & 77.7 & $(66.1-86.1)$ & 79.3 & $(72.0-85.1)$ & 78.5 & $(70.1-85.0)$ & 59.8 & $(45.0-73.0)$ & 61.8 & $(50.9-71.5)$ & 60.7 & $(48.7-71.5)$ \\
\hline Indiana & 32.6 & $(25.1-41.1)$ & 49.6 & $(41.2-58.0)$ & 41.2 & (33.2-49.7) & 21.6 & $(17.1-26.9)$ & 34.1 & $(26.7-42.4)$ & 28.0 & $(22.5-34.3)$ \\
\hline Kentucky & 31.1 & $(24.5-38.5)$ & 44.2 & $(38.3-50.3)$ & 37.8 & $(32.6-43.3)$ & 19.3 & $(13.5-26.9)$ & 25.2 & $(19.5-31.9)$ & 22.3 & $(17.6-27.8)$ \\
\hline Maine & 35.4 & $(31.9-39.0)$ & 42.6 & (38.4-46.8) & 39.0 & (35.6-42.6) & 4.9 & (3.2-7.6) & 6.5 & $(4.3-9.8)$ & 5.8 & $(3.9-8.5)$ \\
\hline Maryland & 30.6 & $(29.2-32.0)$ & 44.3 & (42.9-45.8) & 37.6 & (36.4-38.8) & 12.6 & $(11.6-13.8)$ & 18.7 & $(17.3-20.2)$ & 15.6 & $(14.5-16.8)$ \\
\hline Massachusetts & 52.4 & $(45.3-59.5)$ & 57.6 & $(51.5-63.5)$ & 55.0 & $(49.0-61.0)$ & 18.4 & $(13.7-24.3)$ & 22.1 & $(17.5-27.4)$ & 20.1 & $(15.8-25.3)$ \\
\hline Michigan & 23.7 & (18.5-29.9) & 39.3 & (32.2-46.8) & 31.5 & $(26.5-37.0)$ & 16.1 & (11.9-21.4) & 28.8 & (22.0-36.7) & 22.4 & $(17.7-28.1)$ \\
\hline Mississippi & 39.1 & (33.7-44.8) & 49.1 & $(42.9-55.3)$ & 44.3 & $(39.5-49.2)$ & 24.3 & $(19.8-29.5)$ & 25.8 & $(20.6-31.7)$ & 25.0 & $(21.1-29.4)$ \\
\hline Missouri & 36.9 & $(27.9-46.9)$ & 55.6 & $(47.8-63.1)$ & 46.0 & $(38.5-53.8)$ & 18.2 & $(11.1-28.2)$ & 28.2 & $(20.4-37.7)$ & 23.0 & (16.2-31.8) \\
\hline Montana & 49.9 & $(45.0-54.8)$ & 59.5 & $(55.4-63.4)$ & 54.9 & $(50.7-58.9)$ & 28.3 & $(24.2-32.9)$ & 36.8 & $(32.4-41.3)$ & 32.7 & $(28.7-37.0)$ \\
\hline Nebraska & 41.1 & (36.2-46.2) & 56.6 & $(52.8-60.3)$ & 49.0 & $(45.1-52.9)$ & 26.9 & $(23.0-31.3)$ & 38.9 & (34.4-43.6) & 32.8 & $(29.2-36.7)$ \\
\hline Nevada & 50.3 & $(41.2-59.4)$ & 67.6 & $(58.8-75.4)$ & 59.1 & $(51.2-66.5)$ & 27.6 & $(19.8-37.1)$ & 30.7 & $(21.7-41.6)$ & 29.1 & $(21.9-37.5)$ \\
\hline New Hampshire & —§ & - & - & - & - & - & - & - & - & - & - & - \\
\hline New Mexico & 44.0 & (40.1-48.0) & 55.6 & (51.9-59.2) & 49.8 & $(46.5-53.2)$ & 23.4 & (19.8-27.5) & 30.8 & $(27.1-34.7)$ & 27.1 & $(23.6-30.9)$ \\
\hline New York & 91.1 & (87.9-93.5) & 84.4 & (80.2-87.8) & 87.8 & (84.5-90.4) & 18.7 & $(14.8-23.5)$ & 17.8 & $(14.1-22.2)$ & 18.4 & $(15.1-22.2)$ \\
\hline North Carolina & 31.8 & $(25.2-39.1)$ & 47.3 & $(38.7-56.1)$ & 39.8 & (33.0-47.0) & 19.9 & (13.5-28.2) & 30.4 & (23.7-37.9) & 25.2 & $(19.7-31.6)$ \\
\hline North Dakota & 46.5 & $(41.0-52.1)$ & 51.6 & $(45.7-57.5)$ & 49.2 & $(44.3-54.0)$ & 34.6 & $(29.3-40.3)$ & 37.9 & $(33.6-42.3)$ & 36.3 & $(32.3-40.4)$ \\
\hline Oklahoma & 30.4 & $(25.5-35.8)$ & 46.0 & (37.9-54.2) & 37.8 & (32.4-43.6) & 26.8 & $(22.7-31.3)$ & 41.3 & (34.5-48.5) & 33.7 & $(29.2-38.6)$ \\
\hline Pennsylvania & 57.6 & $(47.4-67.1)$ & 61.2 & $(53.6-68.2)$ & 59.4 & $(51.0-67.4)$ & 15.0 & $(10.6-20.9)$ & 22.1 & $(17.2-27.8)$ & 18.5 & $(14.2-23.8)$ \\
\hline Rhode Island & 73.5 & (63.7-81.4) & 72.9 & $(67.7-77.5)$ & 73.2 & $(66.0-79.4)$ & 20.0 & $(10.0-36.1)$ & 16.7 & $(9.8-27.0)$ & 18.4 & $(10.1-31.2)$ \\
\hline South Carolina & 25.3 & $(17.5-35.1)$ & 48.9 & $(39.1-58.8)$ & 37.0 & $(28.0-47.0)$ & 15.2 & $(9.6-23.3)$ & 33.2 & $(26.8-40.2)$ & 24.2 & $(18.0-31.6)$ \\
\hline South Dakota & 25.4 & $(17.2-35.8)$ & 34.1 & (22.2-48.3) & 29.9 & (20.1-41.9) & 11.0 & $(6.0-19.1)$ & 15.4 & $(10.1-22.7)$ & 13.2 & $(8.5-19.8)$ \\
\hline Tennessee & 34.9 & $(30.8-39.2)$ & 47.3 & $(41.9-52.7)$ & 41.3 & (36.9-45.8) & 21.6 & $(17.9-25.9)$ & 28.9 & $(23.9-34.5)$ & 25.3 & $(21.3-29.8)$ \\
\hline Vermont & - & - & - & - & - & - & - & - & - & - & - & - \\
\hline Virginia & 44.3 & $(38.2-50.6)$ & 55.3 & $(50.8-59.6)$ & 50.0 & $(45.3-54.7)$ & 11.8 & $(8.4-16.4)$ & 14.7 & $(10.5-20.3)$ & 13.3 & (9.7-17.9) \\
\hline West Virginia & 33.1 & $(26.5-40.5)$ & 40.1 & (31.9-48.9) & 36.8 & $(29.8-44.3)$ & 24.7 & $(18.6-32.0)$ & 25.6 & $(18.0-35.0)$ & 25.2 & $(19.0-32.6)$ \\
\hline Wyoming & 45.8 & $(39.9-51.9)$ & 52.8 & $(47.9-57.6)$ & 49.4 & $(44.4-54.4)$ & 21.2 & $(16.7-26.6)$ & 21.6 & $(17.0-27.2)$ & 21.4 & $(17.2-26.3)$ \\
\hline Median & & 36.9 & & 51.1 & & 44.3 & & 19.9 & & 28.2 & & 23.0 \\
\hline Range & & .7-91.1) & & $(1-84.4)$ & $(29$ & $.9-87.8)$ & & 9-59.8) & & $5-61.8)$ & & $8-60.7)$ \\
\hline \multicolumn{13}{|c|}{ Large urban school district surveys } \\
\hline Baltimore, MD & 28.8 & $(21.3-37.8)$ & 37.2 & $(31.2-43.7)$ & 33.4 & $(27.5-39.9)$ & 15.4 & $(8.5-26.1)$ & 16.6 & $(12.4-22.0)$ & 15.7 & $(10.9-22.2)$ \\
\hline Boston, MA & 32.1 & $(24.5-40.8)$ & 42.1 & (35.1-49.6) & 37.2 & (30.3-44.5) & 7.6 & $(5.4-10.6)$ & 9.6 & $(6.7-13.5)$ & 8.6 & $(6.4-11.5)$ \\
\hline Broward County, FL & 27.1 & $(22.3-32.4)$ & 47.9 & $(41.3-54.5)$ & 37.7 & $(33.0-42.5)$ & 16.1 & $(12.7-20.2)$ & 30.5 & $(24.1-37.7)$ & 23.4 & $(19.6-27.6)$ \\
\hline Cleveland, $\mathrm{OH}$ & - & - & - & - & - & - & - & - & - & - & - & - \\
\hline DeKalb County, GA & 36.9 & $(30.5-43.9)$ & 45.3 & $(40.2-50.6)$ & 41.1 & $(36.0-46.4)$ & 24.8 & $(19.7-30.6)$ & 27.4 & $(23.0-32.3)$ & 26.1 & $(22.0-30.6)$ \\
\hline Detroit, MI & 42.5 & $(37.9-47.2)$ & 47.7 & $(42.1-53.3)$ & 45.0 & $(40.7-49.4)$ & 15.5 & $(12.4-19.3)$ & 18.1 & $(14.7-22.0)$ & 16.6 & $(13.7-20.0)$ \\
\hline District of Columbia & - & - & - & - & - & - & - & - & - & - & - & - \\
\hline Duval County, FL & - & - & - & - & - & - & - & - & - & - & - & - \\
\hline Ft. Worth, TX & 49.7 & $(44.9-54.5)$ & 55.1 & $(51.2-58.9)$ & 52.4 & $(48.8-55.9)$ & 33.5 & $(29.6-37.6)$ & 40.3 & $(36.3-44.5)$ & 36.9 & $(33.7-40.1)$ \\
\hline Houston, TX & 53.5 & $(48.6-58.3)$ & 54.8 & $(51.3-58.3)$ & 54.4 & $(50.8-58.0)$ & 22.6 & $(17.9-28.1)$ & 22.5 & $(19.3-26.0)$ & 22.5 & $(19.1-26.3)$ \\
\hline Los Angeles, CA & 60.8 & $(51.8-69.1)$ & 61.1 & $(50.6-70.6)$ & 61.1 & $(51.9-69.5)$ & 39.2 & $(27.5-52.4)$ & 34.0 & $(24.8-44.6)$ & 36.7 & $(26.5-48.2)$ \\
\hline Miami-Dade County, FL & 36.5 & $(31.0-42.4)$ & 51.3 & $(45.5-57.0)$ & 43.7 & (38.8-48.7) & 6.4 & $(4.5-9.0)$ & 11.2 & $(8.9-14.1)$ & 8.8 & $(7.1-10.7)$ \\
\hline
\end{tabular}

See table footnotes on the next page. 
TABLE 112. (Continued) Percentage of high school students who attended physical education (PE) classes on $\geq 1$ days* and who attended PE classes on all 5 days, ${ }^{*}$ by sex — selected U.S. sites, Youth Risk Behavior Survey, 2015

\begin{tabular}{|c|c|c|c|c|c|c|c|c|c|c|c|c|}
\hline \multirow[b]{3}{*}{ Site } & \multicolumn{6}{|c|}{ Attended PE classes } & \multicolumn{6}{|c|}{ Attended PE classes daily } \\
\hline & \multicolumn{2}{|c|}{ Female } & \multicolumn{2}{|r|}{ Male } & \multicolumn{2}{|r|}{ Total } & \multicolumn{2}{|r|}{ Female } & \multicolumn{2}{|r|}{ Male } & \multicolumn{2}{|r|}{ Total } \\
\hline & $\%$ & $\mathrm{Cl}^{\dagger}$ & $\%$ & $\mathrm{Cl}$ & $\%$ & $\mathrm{Cl}$ & $\%$ & $\mathrm{Cl}$ & $\%$ & $\mathrm{Cl}$ & $\%$ & $\mathrm{Cl}$ \\
\hline New York City, NY & 85.5 & $(78.8-90.3)$ & 82.6 & (78.2-86.3) & 84.0 & $(79.0-88.1)$ & 35.8 & $(27.7-44.8)$ & 36.3 & $(29.3-43.9)$ & 36.0 & $(29.0-43.6)$ \\
\hline Oakland, CA & - & - & - & - & - & - & - & - & - & - & - & - \\
\hline Orange County, FL & 34.2 & $(27.3-41.8)$ & 57.8 & $(51.2-64.2)$ & 46.0 & (39.4-52.8) & 19.4 & $(15.2-24.6)$ & 40.5 & (35.6-45.5) & 29.9 & $(25.7-34.5)$ \\
\hline Palm Beach County, FL & 32.1 & $(27.4-37.2)$ & 50.1 & $(45.0-55.2)$ & 41.5 & $(37.0-46.1)$ & 10.4 & $(7.7-13.9)$ & 17.8 & $(15.0-21.1)$ & 14.1 & $(11.8-16.7)$ \\
\hline Philadelphia, PA & 45.3 & $(36.7-54.3)$ & 45.8 & $(39.3-52.4)$ & 45.7 & $(38.6-52.9)$ & 17.5 & $(11.0-26.8)$ & 17.6 & $(12.2-24.6)$ & 17.5 & $(12.1-24.5)$ \\
\hline San Diego, CA & 57.1 & $(47.8-66.0)$ & 60.4 & $(53.0-67.3)$ & 58.8 & $(50.9-66.3)$ & 39.8 & $(32.6-47.6)$ & 45.2 & $(39.1-51.4)$ & 42.7 & $(36.4-49.2)$ \\
\hline San Francisco, CA & - & - & - & - & - & - & - & - & - & - & - & - \\
\hline Median & & 39.7 & & 50.7 & & 45.3 & & 18.4 & & 24.9 & & 22.9 \\
\hline Range & & 7.1-85.5) & & 7.2-82.6) & & 3.4-84.0) & & $(6.4-39.8)$ & & $9.6-45.2)$ & & $(8.6-42.7)$ \\
\hline
\end{tabular}

* In an average week when they were in school.

† $95 \%$ confidence interval.

$\S$ Not available.

TABLE 113. Percentage of high school students who played on at least one sports team,* by sex, race/ethnicity, and grade - United States, Youth Risk Behavior Survey, 2015

\begin{tabular}{|c|c|c|c|c|c|c|}
\hline \multirow[b]{2}{*}{ Category } & \multicolumn{2}{|c|}{ Female } & \multicolumn{2}{|c|}{ Male } & \multicolumn{2}{|c|}{ Total } \\
\hline & $\%$ & $\mathrm{Cl}^{\dagger}$ & $\%$ & $\mathrm{Cl}$ & $\%$ & $\mathrm{Cl}$ \\
\hline \multicolumn{7}{|c|}{ Race/Ethnicity } \\
\hline White $\S$ & 60.7 & $(51.6-69.2)$ & 64.4 & $(60.6-68.0)$ & 62.4 & (56.6-67.9) \\
\hline Black ${ }^{\S}$ & 47.7 & $(41.8-53.8)$ & 66.5 & $(60.3-72.2)$ & 57.6 & (52.8-62.2) \\
\hline Hispanic & 40.7 & $(37.3-44.1)$ & 56.3 & $(52.3-60.3)$ & 48.5 & (46.1-50.9) \\
\hline \multicolumn{7}{|l|}{ Grade } \\
\hline 9 & 57.6 & $(52.6-62.6)$ & 68.1 & $(62.7-73.0)$ & 63.0 & (58.6-67.2) \\
\hline 10 & 55.1 & $(47.2-62.8)$ & 63.5 & $(59.5-67.3)$ & 59.2 & (53.8-64.3) \\
\hline 11 & 51.7 & $(45.6-57.8)$ & 62.3 & $(57.5-66.8)$ & 57.0 & (52.6-61.3) \\
\hline 12 & 46.9 & $(39.9-54.0)$ & 54.6 & $(48.5-60.7)$ & 50.8 & (46.4-55.3) \\
\hline Total & 53.0 & $(47.3-58.6)$ & 62.2 & (59.5-64.9) & 57.6 & $(54.0-61.2)$ \\
\hline
\end{tabular}

* Run by their school or community groups during the 12 months before the survey.

† $95 \%$ confidence interval.

$\S$ Non-Hispanic. 
TABLE 114. Percentage of high school students who played on at least one sports team,* by sex — selected U.S. sites, Youth Risk Behavior Survey, 2015

\begin{tabular}{|c|c|c|c|c|c|c|}
\hline \multirow[b]{2}{*}{ Site } & \multicolumn{2}{|c|}{ Female } & \multicolumn{2}{|c|}{ Male } & \multicolumn{2}{|c|}{ Total } \\
\hline & $\%$ & $\mathrm{Cl}^{\dagger}$ & $\%$ & $\mathrm{Cl}$ & $\%$ & $\mathrm{Cl}$ \\
\hline \multicolumn{7}{|l|}{ State surveys } \\
\hline Alabama & 47.7 & $(42.6-52.8)$ & 56.7 & $(52.6-60.7)$ & 52.1 & $(48.4-55.9)$ \\
\hline Alaska & 61.9 & $(56.7-66.9)$ & 63.4 & $(59.5-67.1)$ & 62.7 & $(59.2-66.1)$ \\
\hline Arizona & 45.8 & $(40.5-51.2)$ & 52.4 & $(47.2-57.6)$ & 49.2 & $(45.2-53.3)$ \\
\hline Arkansas & 45.4 & $(38.9-52.0)$ & 58.4 & $(53.0-63.5)$ & 51.7 & $(48.1-55.3)$ \\
\hline California & 50.6 & $(43.8-57.4)$ & 61.5 & $(58.3-64.5)$ & 56.0 & $(52.1-59.8)$ \\
\hline Connecticut & — & - & - & - & - & - \\
\hline Delaware & 50.3 & $(45.7-54.9)$ & 58.5 & $(54.1-62.9)$ & 54.7 & $(51.0-58.5)$ \\
\hline Florida & 43.7 & $(41.4-46.0)$ & 54.3 & $(51.7-56.9)$ & 49.0 & $(47.0-51.0)$ \\
\hline Hawaii & 48.6 & $(45.8-51.4)$ & 55.8 & $(52.5-59.0)$ & 52.2 & $(50.2-54.2)$ \\
\hline Idaho & 55.7 & $(51.2-60.1)$ & 60.7 & $(53.5-67.4)$ & 58.1 & $(53.3-62.8)$ \\
\hline Illinois & 53.0 & $(48.2-57.6)$ & 61.5 & $(56.4-66.4)$ & 57.3 & $(53.1-61.4)$ \\
\hline Indiana & 58.6 & $(53.5-63.6)$ & 62.2 & $(56.5-67.5)$ & 60.4 & $(55.7-64.8)$ \\
\hline Kentucky & 48.2 & $(44.1-52.3)$ & 53.3 & $(49.1-57.4)$ & 50.8 & $(48.1-53.5)$ \\
\hline Maine & - & - & - & - & - & - \\
\hline Maryland & - & - & - & - & - & - \\
\hline Massachusetts & 59.0 & $(54.5-63.4)$ & 62.6 & $(58.1-66.9)$ & 60.6 & $(56.6-64.4)$ \\
\hline Michigan & - & - & - & - & - & - \\
\hline Mississippi & 43.4 & $(40.5-46.4)$ & 53.9 & $(50.5-57.2)$ & 48.7 & $(46.1-51.3)$ \\
\hline Missouri & 56.4 & $(49.2-63.4)$ & 56.2 & $(52.3-60.0)$ & 56.3 & $(51.5-61.0)$ \\
\hline Montana & 60.4 & $(58.0-62.8)$ & 64.1 & $(61.5-66.7)$ & 62.4 & $(60.5-64.2)$ \\
\hline Nebraska & 61.5 & $(56.4-66.3)$ & 66.7 & $(62.7-70.5)$ & 64.3 & $(60.9-67.5)$ \\
\hline Nevada & 50.3 & $(45.5-55.1)$ & 58.4 & $(53.2-63.4)$ & 54.3 & $(50.5-58.1)$ \\
\hline New Hampshire & - & - & - & - & - & - \\
\hline New Mexico & - & - & - & - & - & - \\
\hline New York & - & - & - & - & - & - \\
\hline North Carolina & - & - & - & - & - & 一 \\
\hline North Dakota & - & - & - & - & - & - \\
\hline Oklahoma & 52.6 & $(47.6-57.4)$ & 56.4 & $(51.8-60.9)$ & 54.8 & $(51.8-57.7)$ \\
\hline Pennsylvania & 60.3 & $(56.5-63.9)$ & 62.8 & $(58.9-66.6)$ & 61.6 & $(58.7-64.3)$ \\
\hline Rhode Island & - & - & - & - & - & - \\
\hline South Carolina & 42.8 & $(37.5-48.2)$ & 54.6 & $(50.7-58.4)$ & 48.6 & $(46.1-51.2)$ \\
\hline South Dakota & - & - & - & - & - & - \\
\hline Tennessee & 44.1 & $(41.2-47.2)$ & 56.0 & $(52.6-59.4)$ & 50.2 & $(47.6-52.8)$ \\
\hline Vermont & - & - & - & - & - & - \\
\hline Virginia & 51.3 & $(47.8-54.8)$ & 60.0 & $(56.5-63.3)$ & 55.8 & $(53.0-58.5)$ \\
\hline West Virginia & 49.7 & $(45.3-54.2)$ & 53.6 & $(48.9-58.2)$ & 51.7 & $(48.0-55.5)$ \\
\hline Wyoming & 59.6 & $(55.1-63.9)$ & 64.5 & $(60.4-68.3)$ & 62.0 & $(58.4-65.5)$ \\
\hline Median & & & & & & \\
\hline Range & & 61.9) & & & & 4.3) \\
\hline \multicolumn{7}{|c|}{ Large urban school district surveys } \\
\hline Baltimore, MD & 41.2 & (35.3-47.4) & 60.8 & $(53.8-67.4)$ & 50.8 & $(46.0-55.5)$ \\
\hline Boston, MA & 41.4 & $(37.9-45.1)$ & 52.6 & $(48.7-56.5)$ & 47.0 & $(44.1-49.9)$ \\
\hline Broward County, FL & 40.5 & $(36.8-44.4)$ & 53.8 & $(49.2-58.4)$ & 47.3 & $(43.9-50.7)$ \\
\hline Cleveland, $\mathrm{OH}$ & 40.0 & $(35.4-44.8)$ & 52.6 & $(48.4-56.8)$ & 46.6 & $(43.3-50.0)$ \\
\hline DeKalb County, GA & 50.3 & $(45.8-54.8)$ & 57.9 & $(54.3-61.5)$ & 54.1 & $(50.8-57.4)$ \\
\hline Detroit, MI & - & - & - & - & - & - \\
\hline District of Columbia & 49.7 & $(48.2-51.2)$ & 60.7 & $(59.1-62.4)$ & 54.9 & $(53.8-56.0)$ \\
\hline Duval County, FL & 44.5 & $(41.7-47.5)$ & 53.0 & $(50.1-55.8)$ & 48.7 & $(46.6-50.9)$ \\
\hline Ft. Worth, TX & 49.1 & $(45.2-52.9)$ & 60.1 & $(57.0-63.1)$ & 54.8 & $(52.1-57.5)$ \\
\hline Houston, TX & 41.4 & $(37.8-45.2)$ & 51.5 & $(48.6-54.4)$ & 46.9 & $(44.4-49.4)$ \\
\hline Los Angeles, CA & 45.7 & $(41.0-50.5)$ & 53.8 & $(50.9-56.7)$ & 49.6 & $(46.6-52.6)$ \\
\hline Miami-Dade County, FL & 34.6 & $(31.8-37.5)$ & 54.7 & $(51.3-58.0)$ & 44.7 & $(42.2-47.3)$ \\
\hline
\end{tabular}

\footnotetext{
See table footnotes on the next page.
} 
TABLE 114. (Continued) Percentage of high school students who played on at least one sports team,* by sex — selected U.S. sites, Youth Risk Behavior Survey, 2015

\begin{tabular}{|c|c|c|c|c|c|c|}
\hline \multirow[b]{2}{*}{ Site } & \multicolumn{2}{|c|}{ Female } & \multicolumn{2}{|c|}{ Male } & \multicolumn{2}{|c|}{ Total } \\
\hline & $\%$ & $\mathrm{Cl}^{\dagger}$ & $\%$ & $\mathrm{Cl}$ & $\%$ & $\mathrm{Cl}$ \\
\hline New York City, NY & - & - & - & - & - & - \\
\hline Oakland, CA & - & - & - & - & - & - \\
\hline Orange County, FL & 43.8 & $(40.4-47.3)$ & 57.5 & $(53.0-61.9)$ & 50.7 & $(47.8-53.6)$ \\
\hline Palm Beach County, FL & 41.2 & (37.7-44.8) & 54.8 & $(51.8-57.9)$ & 48.5 & $(46.0-51.0)$ \\
\hline Philadelphia, PA & 42.7 & $(37.5-47.9)$ & 55.5 & $(52.3-58.7)$ & 48.9 & $(45.6-52.3)$ \\
\hline San Diego, CA & 48.7 & $(44.6-52.9)$ & 62.1 & $(58.7-65.4)$ & 55.6 & $(52.8-58.4)$ \\
\hline San Francisco, CA & - & - & - & - & - & - \\
\hline Median & \multirow{2}{*}{\multicolumn{2}{|c|}{$\begin{array}{c}42.7 \\
(34.6-50.3)\end{array}$}} & \multirow{2}{*}{\multicolumn{2}{|c|}{$\begin{array}{c}54.8 \\
(51.5-62.1)\end{array}$}} & \multirow{2}{*}{\multicolumn{2}{|c|}{$\begin{array}{c}48.9 \\
(44.7-55.6)\end{array}$}} \\
\hline Range & & & & & & \\
\hline
\end{tabular}

* Run by their school or community groups during the 12 months before the survey.

$+95 \%$ confidence interval.

$\S$ Not available.

TABLE 115. Percentage of high school students who had obesity* and who were overweight, ${ }^{\dagger}$ by sex, race/ethnicity, and grade - United States, Youth Risk Behavior Survey, 2015

\begin{tabular}{|c|c|c|c|c|c|c|c|c|c|c|c|c|}
\hline \multirow[b]{3}{*}{ Category } & \multicolumn{6}{|c|}{ Obesity } & \multicolumn{6}{|c|}{ Overweight } \\
\hline & \multicolumn{2}{|c|}{ Female } & \multicolumn{2}{|r|}{ Male } & \multicolumn{2}{|r|}{ Total } & \multicolumn{2}{|c|}{ Female } & \multicolumn{2}{|r|}{ Male } & \multicolumn{2}{|r|}{ Total } \\
\hline & $\%$ & $\mathrm{Cl}^{\S}$ & $\%$ & $\mathrm{Cl}$ & $\%$ & $\mathrm{Cl}$ & $\%$ & $\mathrm{Cl}$ & $\%$ & $\mathrm{Cl}$ & $\%$ & $\mathrm{Cl}$ \\
\hline \multicolumn{13}{|l|}{ Race/Ethnicity } \\
\hline White? & 9.1 & $(6.6-12.4)$ & 15.6 & $(13.4-18.1)$ & 12.4 & $(10.5-14.6)$ & 14.6 & $(13.1-16.2)$ & 15.9 & $(14.1-17.8)$ & 15.2 & $(14.1-16.5)$ \\
\hline Blackף & 15.2 & $(11.7-19.5)$ & 18.2 & $(15.2-21.5)$ & 16.8 & $(14.2-19.6)$ & 21.2 & $(16.6-26.7)$ & 13.6 & (10.4-17.4) & 17.2 & $(15.1-19.5)$ \\
\hline Hispanic & 13.3 & $(11.6-15.2)$ & 19.4 & $(17.0-22.0)$ & 16.4 & $(14.8-18.2)$ & 20.0 & $(17.9-22.2)$ & 17.0 & $(14.8-19.4)$ & 18.4 & $(17.0-19.9)$ \\
\hline \multicolumn{13}{|l|}{ Grade } \\
\hline 9 & 10.3 & $(8.8-12.0)$ & 15.4 & $(13.3-17.8)$ & 13.0 & $(11.7-14.4)$ & 17.6 & $(14.9-20.7)$ & 16.0 & $(13.4-19.1)$ & 16.8 & $(14.8-19.0)$ \\
\hline 10 & 12.1 & $(9.4-15.5)$ & 18.2 & $(15.2-21.7)$ & 15.2 & $(13.0-17.6)$ & 16.3 & $(14.4-18.4)$ & 14.8 & $(12.1-17.9)$ & 15.5 & $(13.8-17.4)$ \\
\hline 11 & 10.2 & $(8.1-12.8)$ & 18.4 & $(15.5-21.8)$ & 14.5 & $(12.6-16.6)$ & 15.3 & $(13.4-17.4)$ & 16.4 & $(14.2-18.8)$ & 15.9 & $(14.5-17.4)$ \\
\hline 12 & 10.5 & $(8.4-12.9)$ & 15.0 & (12.0-18.7) & 12.7 & $(10.7-15.1)$ & 17.0 & $(14.3-20.0)$ & 15.0 & $(12.4-18.0)$ & 16.0 & $(13.8-18.4)$ \\
\hline Total & 10.8 & $(9.3-12.5)$ & 16.8 & $(14.8-19.0)$ & 13.9 & $(12.5-15.5)$ & 16.6 & $(15.3-17.9)$ & 15.5 & $(14.2-17.0)$ & 16.0 & $(15.2-16.9)$ \\
\hline
\end{tabular}

* Students who were $\geq 95$ th percentile for body mass index, based on sex- and age-specific reference data from the 2000 CDC growth charts.

+ Students who were $\geq 85$ th percentile but $<95$ th percentile for body mass index, based on sex-and age-specific reference data from the 2000 CDC growth charts.

$\S 95 \%$ confidence interval.

ๆ Non-Hispanic. 
TABLE 116. Percentage of high school students who had obesity* and who were overweight, ${ }^{\dagger}$ by sex — selected U.S. sites, Youth Risk Behavior Survey, 2015

\begin{tabular}{|c|c|c|c|c|c|c|c|c|c|c|c|c|}
\hline \multirow[b]{3}{*}{ Site } & \multicolumn{6}{|c|}{ Obesity } & \multicolumn{6}{|c|}{ Overweight } \\
\hline & \multicolumn{2}{|c|}{ Female } & \multicolumn{2}{|r|}{ Male } & \multicolumn{2}{|r|}{ Total } & \multicolumn{2}{|c|}{ Female } & \multicolumn{2}{|r|}{ Male } & \multicolumn{2}{|r|}{ Total } \\
\hline & $\%$ & $\mathrm{Cl}^{\S}$ & $\%$ & $\mathrm{Cl}$ & $\%$ & $\mathrm{Cl}$ & $\%$ & $\mathrm{Cl}$ & $\%$ & $\mathrm{Cl}$ & $\%$ & $\mathrm{Cl}$ \\
\hline \multicolumn{13}{|l|}{ State surveys } \\
\hline Alabama & 13.5 & $(9.9-18.2)$ & 18.6 & $(16.0-21.6)$ & 16.1 & (13.5-19.1) & 17.0 & (14.4-20.0) & 18.0 & (14.9-21.5) & 17.5 & $(15.3-20.0)$ \\
\hline Alaska & 10.9 & $(8.4-14.1)$ & 16.9 & (14.3-19.9) & 14.0 & (11.9-16.5) & 18.1 & (14.8-21.8) & 15.5 & (13.4-18.0) & 16.7 & $(14.7-19.0)$ \\
\hline Arizona & 7.0 & $(4.7-10.4)$ & 14.5 & $(11.5-18.2)$ & 10.9 & $(8.8-13.4)$ & 14.2 & $(12.1-16.7)$ & 15.1 & $(12.9-17.6)$ & 14.7 & $(13.0-16.5)$ \\
\hline Arkansas & 14.7 & $(11.8-18.1)$ & 21.3 & $(18.6-24.4)$ & 18.0 & $(16.1-20.1)$ & 18.5 & $(16.3-20.9)$ & 17.6 & $(13.2-23.0)$ & 18.0 & $(15.3-21.2)$ \\
\hline California & 9.2 & $(7.3-11.6)$ & 18.5 & $(15.2-22.3)$ & 13.9 & $(11.4-16.9)$ & 15.8 & (13.5-18.4) & 17.1 & $(12.8-22.5)$ & 16.5 & (13.7-19.7) \\
\hline Connecticut & 9.3 & $(6.9-12.5)$ & 15.2 & $(12.8-17.9)$ & 12.3 & $(10.2-14.7)$ & 14.1 & $(11.3-17.4)$ & 14.4 & (12.4-16.6) & 14.3 & $(12.4-16.4)$ \\
\hline Delaware & 13.3 & (11.1-15.8) & 18.1 & (15.9-20.6) & 15.8 & $(14.1-17.5)$ & 17.3 & $(14.8-20.2)$ & 14.3 & $(12.1-16.9)$ & 15.8 & $(13.9-17.8)$ \\
\hline Florida & 8.7 & $(7.5-10.0)$ & 15.8 & $(14.4-17.3)$ & 12.3 & (11.2-13.4) & 15.5 & $(13.9-17.2)$ & 13.6 & $(12.3-14.9)$ & 14.5 & (13.5-15.6) \\
\hline Hawaii & 8.1 & $(6.6-9.9)$ & 17.6 & $(14.6-20.9)$ & 12.9 & (11.0-15.2) & 15.1 & (13.0-17.4) & 15.6 & $(14.1-17.2)$ & 15.3 & $(14.0-16.8)$ \\
\hline Idaho & 7.4 & $(5.5-10.0)$ & 14.5 & $(11.7-17.9)$ & 11.1 & $(9.1-13.5)$ & 14.3 & $(12.1-16.8)$ & 16.2 & $(13.3-19.5)$ & 15.3 & (13.4-17.3) \\
\hline Illinois & 8.4 & $(6.3-11.2)$ & 16.7 & $(14.5-19.1)$ & 12.6 & $(10.9-14.6)$ & 14.6 & $(11.7-18.1)$ & 16.1 & (14.0-18.5) & 15.4 & (14.1-16.8) \\
\hline Indiana & 9.7 & $(7.3-12.8)$ & 17.5 & $(14.8-20.5)$ & 13.6 & $(11.8-15.7)$ & 18.1 & $(14.6-22.3)$ & 16.5 & (13.1-20.5) & 17.3 & $(14.5-20.5)$ \\
\hline Kentucky & 16.2 & $(13.6-19.3)$ & 20.6 & $(17.6-24.1)$ & 18.5 & (16.4-20.8) & 19.2 & $(15.9-23.1)$ & 14.9 & $(12.1-18.2)$ & 17.0 & (14.5-19.9) \\
\hline Maine & 9.1 & $(7.6-11.0)$ & 17.2 & $(15.7-18.9)$ & 13.3 & $(11.9-14.9)$ & 14.8 & (13.4-16.3) & 14.9 & $(14.0-16.0)$ & 14.9 & $(14.0-15.8)$ \\
\hline Maryland & 8.8 & $(8.3-9.4)$ & 14.1 & $(13.5-14.7)$ & 11.5 & $(11.1-12.0)$ & 15.4 & (14.8-16.1) & 14.5 & (13.9-15.1) & 14.9 & $(14.5-15.4)$ \\
\hline Massachusetts & 7.2 & $(5.6-9.1)$ & 14.7 & $(12.1-17.6)$ & 11.0 & (9.4-12.8) & 14.7 & (12.4-17.3) & 16.0 & (14.2-17.8) & 15.3 & $(13.6-17.2)$ \\
\hline Michigan & 11.5 & $(9.5-13.9)$ & 17.0 & $(15.2-19.0)$ & 14.3 & $(12.6-16.2)$ & 16.2 & $(13.2-19.7)$ & 15.8 & $(13.8-18.0)$ & 16.0 & (14.2-17.9) \\
\hline Mississippi & 16.2 & $(14.0-18.5)$ & 21.7 & $(18.6-25.2)$ & 18.9 & $(17.0-21.0)$ & 19.2 & $(16.5-22.3)$ & 14.9 & $(12.7-17.4)$ & 17.1 & $(15.7-18.5)$ \\
\hline Missouri & 10.3 & $(6.5-16.0)$ & 15.9 & $(13.3-19.1)$ & 13.1 & $(9.9-17.0)$ & 12.4 & $(9.9-15.5)$ & 14.3 & $(11.4-17.7)$ & 13.3 & $(11.2-15.8)$ \\
\hline Montana & 7.4 & $(6.2-8.7)$ & 13.0 & $(11.5-14.8)$ & 10.3 & $(9.2-11.5)$ & 14.9 & $(13.3-16.7)$ & 15.1 & $(13.7-16.6)$ & 15.0 & $(14.0-16.2)$ \\
\hline Nebraska & 9.3 & $(7.1-12.2)$ & 16.5 & (13.9-19.5) & 13.0 & (11.1-15.2) & 16.4 & (14.0-19.1) & 17.3 & (14.5-20.7) & 16.9 & (15.1-18.8) \\
\hline Nevada & 6.3 & $(4.5-8.9)$ & 17.8 & $(14.4-21.8)$ & 12.2 & $(10.3-14.4)$ & 14.1 & (10.7-18.4) & 15.9 & (13.1-19.0) & 15.0 & $(12.8-17.6)$ \\
\hline New Hampshire & 7.9 & (7.1-8.8) & 16.3 & $(15.0-17.6)$ & 12.2 & $(11.5-13.0)$ & 14.4 & (13.3-15.6) & 14.6 & $(13.1-16.2)$ & 14.5 & (13.4-15.6) \\
\hline New Mexico & 11.6 & (10.3-13.0) & 19.5 & (18.2-20.8) & 15.6 & (14.8-16.5) & 16.6 & (15.2-18.0) & 15.9 & $(14.6-17.2)$ & 16.2 & (15.2-17.3) \\
\hline New York & 10.1 & $(8.3-12.3)$ & 16.1 & $(14.3-18.1)$ & 13.1 & (11.6-14.8) & 13.9 & $(11.8-16.2)$ & 13.9 & (11.7-16.6) & 13.9 & $(12.5-15.5)$ \\
\hline North Carolina & 12.5 & $(9.5-16.3)$ & 20.2 & $(17.1-23.7)$ & 16.4 & (13.8-19.5) & 17.2 & (13.5-21.7) & 14.6 & (11.9-17.9) & 15.9 & (13.4-18.8) \\
\hline North Dakota & 8.6 & $(7.2-10.2)$ & 19.1 & $(16.8-21.6)$ & 14.0 & (12.5-15.7) & 17.1 & $(14.7-19.9)$ & 12.3 & $(10.4-14.7)$ & 14.7 & $(13.2-16.3)$ \\
\hline Oklahoma & 12.9 & $(9.2-17.8)$ & 22.0 & $(18.1-26.4)$ & 17.3 & (14.4-20.6) & 16.7 & $(13.7-20.2)$ & 13.9 & $(10.9-17.5)$ & 15.3 & (13.1-17.8) \\
\hline Pennsylvania & 12.4 & (10.4-14.9) & 15.4 & (12.8-18.4) & 14.0 & (12.3-15.8) & 16.7 & (14.6-19.1) & 15.0 & $(12.7-17.6)$ & 15.8 & $(14.3-17.5)$ \\
\hline Rhode Island & 7.6 & $(5.2-10.9)$ & 16.2 & $(13.8-18.9)$ & 12.0 & $(9.8-14.5)$ & 14.1 & $(10.8-18.1)$ & 15.2 & $(14.3-16.3)$ & 14.7 & $(13.0-16.5)$ \\
\hline South Carolina & 13.4 & $(10.9-16.3)$ & 19.0 & $(15.1-23.7)$ & 16.3 & $(14.5-18.2)$ & 17.5 & $(15.1-20.1)$ & 18.8 & $(16.3-21.6)$ & 18.2 & $(17.1-19.3)$ \\
\hline South Dakota & 10.3 & $(6.7-15.6)$ & 18.8 & $(16.7-21.2)$ & 14.7 & $(12.2-17.6)$ & 13.3 & $(10.9-16.1)$ & 15.7 & $(11.9-20.5)$ & 14.5 & $(12.1-17.4)$ \\
\hline Tennessee & 14.2 & $(12.0-16.7)$ & 22.7 & $(20.1-25.4)$ & 18.6 & $(16.7-20.7)$ & 17.8 & $(15.9-19.8)$ & 16.4 & $(14.6-18.3)$ & 17.1 & $(15.9-18.3)$ \\
\hline Vermont & 8.6 & $(8.0-9.2)$ & 16.1 & $(15.4-16.9)$ & 12.4 & $(11.9-12.9)$ & 13.9 & $(13.2-14.6)$ & 14.1 & $(13.4-14.8)$ & 14.0 & $(13.5-14.5)$ \\
\hline Virginia & 11.4 & $(9.0-14.3)$ & 14.5 & $(12.6-16.5)$ & 13.0 & $(11.3-14.9)$ & 15.7 & $(13.5-18.1)$ & 14.6 & $(12.7-16.7)$ & 15.1 & $(13.6-16.8)$ \\
\hline West Virginia & 12.2 & $(9.0-16.4)$ & 23.4 & (20.3-26.8) & 17.9 & $(15.1-21.2)$ & 17.1 & $(14.2-20.5)$ & 16.8 & $(13.8-20.2)$ & 17.0 & $(15.0-19.1)$ \\
\hline Wyoming & 8.0 & $(6.6-9.6)$ & 13.8 & $(11.4-16.6)$ & 11.0 & $(9.5-12.6)$ & 13.9 & $(12.0-16.1)$ & 15.2 & $(12.3-18.6)$ & 14.6 & $(13.1-16.2)$ \\
\hline Median & & 9.7 & & 17.0 & & 13.3 & & 15.7 & & 15.2 & & 15.3 \\
\hline Range & & 5.3-16.2) & & $3.0-23.4)$ & & $0.3-18.9)$ & & 2.4-19.2) & & 2.3-18.8) & & $3.3-18.2)$ \\
\hline \multicolumn{13}{|c|}{ Large urban school district surveys } \\
\hline Baltimore, MD & 17.9 & $(13.9-22.7)$ & 16.2 & $(12.5-20.8)$ & 17.0 & $(14.4-20.0)$ & 17.3 & $(13.4-22.1)$ & 17.5 & $(13.7-22.1)$ & 17.4 & $(14.2-21.1)$ \\
\hline Boston, MA & 11.7 & $(9.5-14.4)$ & 17.2 & $(14.2-20.7)$ & 14.6 & $(12.5-16.9)$ & 22.3 & $(19.5-25.4)$ & 17.0 & $(13.6-20.9)$ & 19.5 & $(17.2-22.2)$ \\
\hline Broward County, FL & 8.8 & $(6.7-11.6)$ & 11.6 & $(9.4-14.3)$ & 10.3 & $(8.7-12.1)$ & 15.2 & $(11.8-19.4)$ & 12.1 & $(9.7-15.0)$ & 13.6 & $(11.4-16.2)$ \\
\hline Cleveland, $\mathrm{OH}$ & 17.6 & $(14.8-20.8)$ & 18.2 & $(16.0-20.7)$ & 17.9 & $(16.2-19.8)$ & 21.0 & $(18.1-24.1)$ & 16.1 & $(13.9-18.6)$ & 18.4 & $(16.7-20.3)$ \\
\hline DeKalb County, GA & 12.4 & $(10.2-15.1)$ & 11.0 & $(9.0-13.5)$ & 11.7 & $(10.2-13.4)$ & 16.1 & $(13.4-19.2)$ & 13.4 & $(11.0-16.3)$ & 14.7 & $(12.7-17.0)$ \\
\hline Detroit, MI & 21.2 & $(18.4-24.2)$ & 24.2 & $(20.7-28.0)$ & 22.5 & $(20.1-25.2)$ & 25.0 & $(22.3-27.9)$ & 17.5 & $(14.7-20.7)$ & 21.6 & $(19.5-23.8)$ \\
\hline District of Columbia & 14.4 & $(13.4-15.5)$ & 15.9 & $(14.7-17.0)$ & 15.1 & (14.4-15.9) & 20.8 & $(19.6-22.0)$ & 15.0 & $(13.9-16.1)$ & 17.9 & $(17.0-18.7)$ \\
\hline Duval County, FL & 10.2 & $(8.6-12.1)$ & 16.1 & $(14.1-18.4)$ & 13.1 & $(11.8-14.5)$ & 15.6 & $(13.9-17.6)$ & 13.8 & $(11.8-16.0)$ & 14.7 & $(13.4-16.2)$ \\
\hline Ft. Worth, TX & 14.0 & $(12.0-16.3)$ & 21.6 & $(18.9-24.6)$ & 17.9 & $(16.1-19.8)$ & 21.2 & $(18.5-24.2)$ & 16.7 & $(14.4-19.2)$ & 18.9 & $(17.1-20.8)$ \\
\hline Houston, TX & 14.4 & $(12.3-16.7)$ & 19.0 & $(17.0-21.2)$ & 16.8 & $(15.3-18.3)$ & 19.9 & $(17.8-22.1)$ & 17.2 & $(15.3-19.4)$ & 18.5 & $(17.1-20.0)$ \\
\hline Los Angeles, CA & 10.1 & $(7.3-13.8)$ & 16.6 & $(14.3-19.2)$ & 13.3 & $(10.9-16.1)$ & 23.8 & $(21.8-25.8)$ & 15.9 & $(13.2-19.0)$ & 19.9 & $(17.9-22.0)$ \\
\hline Miami-Dade County, FL & 9.6 & $(7.9-11.6)$ & 13.6 & $(11.0-16.8)$ & 11.6 & $(10.0-13.5)$ & 16.1 & $(13.7-18.8)$ & 16.6 & $(14.3-19.2)$ & 16.3 & $(14.8-18.0)$ \\
\hline
\end{tabular}

See table footnotes on the next page. 
TABLE 116. (Continued) Percentage of high school students who had obesity* and who were overweight, ${ }^{\dagger}$ by sex - selected U.S. sites, Youth Risk Behavior Survey, 2015

\begin{tabular}{|c|c|c|c|c|c|c|c|c|c|c|c|c|}
\hline \multirow[b]{3}{*}{ Site } & \multicolumn{6}{|c|}{ Obesity } & \multicolumn{6}{|c|}{ Overweight } \\
\hline & \multicolumn{2}{|c|}{ Female } & \multicolumn{2}{|r|}{ Male } & \multicolumn{2}{|r|}{ Total } & \multicolumn{2}{|c|}{ Female } & \multicolumn{2}{|r|}{ Male } & \multicolumn{2}{|r|}{ Total } \\
\hline & $\%$ & $\mathrm{Cl}^{\S}$ & $\%$ & $\mathrm{Cl}$ & $\%$ & $\mathrm{Cl}$ & $\%$ & $\mathrm{Cl}$ & $\%$ & $\mathrm{Cl}$ & $\%$ & $\mathrm{Cl}$ \\
\hline New York City, NY & 10.6 & $(9.0-12.4)$ & 14.2 & $(12.6-15.9)$ & 12.4 & (11.3-13.7) & 17.1 & $(14.7-19.8)$ & 14.0 & $(12.5-15.7)$ & 15.5 & $(13.8-17.4)$ \\
\hline Oakland, CA & 10.8 & $(8.4-13.8)$ & 20.5 & $(17.3-24.2)$ & 16.1 & (13.8-18.7) & 23.3 & (19.6-27.5) & 11.4 & $(9.1-14.3)$ & 16.9 & $(14.8-19.2)$ \\
\hline Orange County, FL & 7.8 & $(5.9-10.1)$ & 14.0 & $(11.5-17.0)$ & 10.9 & $(9.2-12.9)$ & 15.8 & $(13.2-18.9)$ & 15.2 & $(12.5-18.3)$ & 15.5 & $(13.4-17.8)$ \\
\hline Palm Beach County, FL & 9.2 & $(7.5-11.1)$ & 12.8 & $(10.7-15.2)$ & 11.0 & $(9.6-12.6)$ & 12.0 & $(10.0-14.3)$ & 15.7 & $(13.5-18.2)$ & 13.9 & $(12.2-15.8)$ \\
\hline Philadelphia, PA & 13.6 & $(11.0-16.6)$ & 13.9 & $(10.2-18.5)$ & 13.7 & $(12.1-15.5)$ & 20.0 & $(17.4-22.9)$ & 13.6 & $(11.4-16.0)$ & 16.8 & $(15.3-18.3)$ \\
\hline San Diego, CA & 7.5 & (5.8-9.8) & 15.3 & $(13.0-17.9)$ & 11.5 & $(10.0-13.2)$ & 17.9 & $(15.6-20.3)$ & 15.3 & $(12.7-18.3)$ & 16.6 & $(14.7-18.6)$ \\
\hline San Francisco, CA & 5.9 & $(4.3-8.0)$ & 13.6 & $(11.4-16.2)$ & 9.9 & $(8.4-11.6)$ & 13.7 & $(10.9-17.0)$ & 12.7 & $(9.9-16.3)$ & 13.2 & $(10.9-15.8)$ \\
\hline Median & \multicolumn{2}{|c|}{10.8} & \multicolumn{2}{|c|}{15.9} & \multicolumn{2}{|r|}{13.3} & \multicolumn{2}{|r|}{17.9} & \multicolumn{2}{|r|}{15.3} & \multicolumn{2}{|r|}{16.8} \\
\hline Range & \multicolumn{2}{|c|}{$(5.9-21.2)$} & \multicolumn{2}{|c|}{$(11.0-24.2)$} & \multicolumn{2}{|c|}{ (9.9-22.5) } & \multicolumn{2}{|c|}{$(12.0-25.0)$} & \multicolumn{2}{|c|}{ (11.4-17.5) } & \multicolumn{2}{|c|}{$(13.2-21.6)$} \\
\hline
\end{tabular}

* Students who were $\geq 95$ th percentile for body mass index, based on sex- and age-specific reference data from the 2000 CDC growth charts.

+ Students who were $\geq 85$ th percentile but $<95$ th percentile for body mass index, based on sex- and age-specific reference data from the 2000 CDC growth charts.

$\S 95 \%$ confidence interval.

TABLE 117. Percentage of high school students who described themselves as slightly or very overweight and who were trying to lose weight, by sex, race/ethnicity, and grade — United States, Youth Risk Behavior Survey, 2015

\begin{tabular}{|c|c|c|c|c|c|c|c|c|c|c|c|c|}
\hline \multirow[b]{3}{*}{ Category } & \multicolumn{6}{|c|}{ Described themselves as overweight } & \multicolumn{6}{|c|}{ Were trying to lose weight } \\
\hline & \multicolumn{2}{|c|}{ Female } & \multicolumn{2}{|r|}{ Male } & \multicolumn{2}{|r|}{ Total } & \multicolumn{2}{|c|}{ Female } & \multicolumn{2}{|r|}{ Male } & \multicolumn{2}{|r|}{ Total } \\
\hline & $\%$ & $\mathrm{Cl}^{*}$ & $\%$ & $\mathrm{Cl}$ & $\%$ & $\mathrm{Cl}$ & $\%$ & $\mathrm{Cl}$ & $\%$ & $\mathrm{Cl}$ & $\%$ & $\mathrm{Cl}$ \\
\hline \multicolumn{13}{|c|}{ Race/Ethnicity } \\
\hline White $^{\dagger}$ & 35.7 & $(32.3-39.3)$ & 24.9 & $(22.5-27.5)$ & 30.3 & $(28.6-32.2)$ & 59.5 & $(56.1-62.7)$ & 28.8 & $(26.1-31.7)$ & 44.1 & $(41.8-46.4)$ \\
\hline Black $^{\dagger}$ & 34.9 & $(29.2-41.1)$ & 20.0 & $(17.3-23.0)$ & 27.0 & $(24.2-30.1)$ & 54.2 & $(48.6-59.8)$ & 26.2 & $(23.4-29.1)$ & 39.4 & (35.7-43.2) \\
\hline Hispanic & 45.3 & $(43.1-47.4)$ & 28.0 & $(24.9-31.3)$ & 36.4 & $(34.2-38.8)$ & 66.4 & $(63.4-69.3)$ & 40.0 & $(37.0-43.1)$ & 53.1 & (50.2-55.9) \\
\hline \multicolumn{13}{|l|}{ Grade } \\
\hline 9 & 35.8 & $(32.9-38.9)$ & 25.5 & $(23.2-27.9)$ & 30.3 & $(28.4-32.2)$ & 59.4 & $(56.6-62.1)$ & 31.1 & $(28.0-34.4)$ & 44.3 & $(41.7-47.0)$ \\
\hline 10 & 38.9 & $(34.1-43.9)$ & 25.2 & $(21.9-28.8)$ & 32.0 & $(29.5-34.7)$ & 59.3 & $(54.0-64.4)$ & 32.0 & $(29.3-34.7)$ & 45.7 & $(43.1-48.4)$ \\
\hline 11 & 38.5 & (35.4-41.7) & 25.2 & $(22.4-28.2)$ & 31.6 & $(29.3-34.0)$ & 61.7 & $(57.7-65.5)$ & 31.0 & $(27.4-34.8)$ & 45.7 & $(42.3-49.2)$ \\
\hline 12 & 39.6 & $(36.1-43.3)$ & 25.0 & $(21.4-28.9)$ & 32.3 & $(29.3-35.4)$ & 62.6 & $(58.6-66.5)$ & 31.9 & $(29.2-34.8)$ & 47.3 & $(44.6-50.0)$ \\
\hline Total & 38.2 & $(36.2-40.2)$ & 25.3 & $(23.4-27.3)$ & 31.5 & $(30.2-32.9)$ & 60.6 & (58.7-62.5) & 31.4 & $(29.5-33.5)$ & 45.6 & $(44.0-47.3)$ \\
\hline
\end{tabular}

* $95 \%$ confidence interval.

${ }^{\dagger}$ Non-Hispanic. 
TABLE 118. Percentage of high school students who described themselves as slightly or very overweight and who were trying to lose weight, by sex - selected U.S. sites, Youth Risk Behavior Survey, 2015

\begin{tabular}{|c|c|c|c|c|c|c|c|c|c|c|c|c|}
\hline \multirow[b]{3}{*}{ Site } & \multicolumn{6}{|c|}{ Described themselves as overweight } & \multicolumn{6}{|c|}{ Were trying to lose weight } \\
\hline & \multicolumn{2}{|r|}{ Female } & \multicolumn{2}{|r|}{ Male } & \multicolumn{2}{|r|}{ Total } & \multicolumn{2}{|c|}{ Female } & \multicolumn{2}{|r|}{ Male } & \multicolumn{2}{|r|}{ Total } \\
\hline & $\%$ & $\mathrm{Cl}^{*}$ & $\%$ & $\mathrm{Cl}$ & $\%$ & $\mathrm{Cl}$ & $\%$ & $\mathrm{Cl}$ & $\%$ & $\mathrm{Cl}$ & $\%$ & $\mathrm{Cl}$ \\
\hline \multicolumn{13}{|l|}{ State surveys } \\
\hline Alabama & 36.0 & (32.4-39.8) & 21.6 & (18.9-24.5) & 28.8 & $(26.6-31.1)$ & 56.2 & $(52.4-59.9)$ & 32.5 & $(28.1-37.2)$ & 44.3 & $(41.3-47.4)$ \\
\hline Alaska & 37.9 & $(33.6-42.4)$ & 26.3 & $(22.8-30.2)$ & 31.9 & $(29.4-34.4)$ & $\underline{-}^{\dagger}$ & - & - & - & - & - \\
\hline Arizona & 35.0 & $(31.1-39.2)$ & 26.1 & $(23.1-29.3)$ & 30.4 & $(28.1-32.8)$ & 61.1 & $(56.1-66.0)$ & 36.4 & $(31.7-41.4)$ & 48.5 & $(46.0-50.9)$ \\
\hline Arkansas & 38.4 & $(34.6-42.4)$ & 25.4 & $(21.6-29.5)$ & 31.9 & $(28.9-35.1)$ & 61.2 & $(58.3-64.0)$ & 35.1 & $(29.8-40.9)$ & 48.3 & $(44.8-51.9)$ \\
\hline California & 37.9 & (34.2-41.9) & 29.4 & $(26.0-33.0)$ & 33.4 & $(30.9-35.9)$ & 59.2 & $(54.1-64.1)$ & 35.6 & $(31.5-39.9)$ & 46.9 & $(43.0-50.9)$ \\
\hline Connecticut & 33.4 & (29.7-37.4) & 26.6 & (23.0-30.5) & 30.0 & $(27.0-33.2)$ & 60.5 & $(56.7-64.2)$ & 34.2 & (29.8-38.9) & 47.2 & $(44.1-50.3)$ \\
\hline Delaware & 37.7 & $(34.0-41.5)$ & 25.6 & $(22.5-29.0)$ & 31.5 & $(29.2-34.0)$ & 59.5 & $(56.2-62.7)$ & 29.6 & $(26.7-32.7)$ & 44.8 & $(42.3-47.4)$ \\
\hline Florida & 33.0 & $(30.9-35.2)$ & 23.7 & $(21.8-25.7)$ & 28.3 & $(26.9-29.8)$ & 56.7 & $(54.6-58.8)$ & 29.0 & $(26.8-31.3)$ & 42.8 & $(41.5-44.1)$ \\
\hline Hawaii & 36.1 & (33.8-38.5) & 27.8 & $(25.3-30.5)$ & 32.0 & $(30.5-33.6)$ & - & - & - & - & - & - \\
\hline Idaho & 36.9 & (33.2-40.7) & 23.8 & $(20.6-27.2)$ & 30.1 & $(27.5-32.9)$ & 63.1 & $(57.9-67.9)$ & 25.9 & $(22.1-30.1)$ & 44.0 & $(39.9-48.3)$ \\
\hline Illinois & 35.1 & (31.6-38.9) & 25.7 & (22.9-28.7) & 30.3 & $(28.0-32.7)$ & 58.0 & $(53.2-62.7)$ & 34.5 & $(30.3-39.0)$ & 46.1 & $(42.5-49.8)$ \\
\hline Indiana & 37.2 & (33.3-41.3) & 26.1 & $(22.2-30.3)$ & 31.6 & $(29.0-34.2)$ & 61.0 & $(57.5-64.4)$ & 33.2 & $(29.6-37.1)$ & 46.9 & $(44.1-49.7)$ \\
\hline Kentucky & 38.8 & (34.9-42.9) & 25.5 & (22.1-29.2) & 32.0 & $(28.9-35.3)$ & 61.2 & $(58.1-64.2)$ & 32.9 & (30.1-35.7) & 46.9 & $(44.4-49.4)$ \\
\hline Maine & - & - & - & - & - & - & - & - & - & - & - & - \\
\hline Maryland & 30.8 & (30.1-31.5) & 21.6 & (20.9-22.2) & 26.2 & $(25.7-26.7)$ & - & - & - & - & - & - \\
\hline Massachusetts & 35.9 & $(32.6-39.3)$ & 27.3 & $(24.7-30.0)$ & 31.5 & $(29.7-33.4)$ & 59.5 & $(56.8-62.0)$ & 32.7 & $(29.9-35.6)$ & 46.0 & $(43.8-48.3)$ \\
\hline Michigan & 39.3 & (34.9-43.9) & 26.1 & (23.4-29.0) & 32.7 & $(30.4-35.0)$ & 64.0 & $(60.4-67.4)$ & 32.9 & $(29.1-36.9)$ & 48.4 & $(45.4-51.5)$ \\
\hline Mississippi & 35.9 & (32.4-39.6) & 22.5 & $(20.2-24.8)$ & 29.1 & $(27.3-31.0)$ & 57.5 & $(52.0-62.8)$ & 36.3 & (33.3-39.3) & 46.8 & $(43.7-49.8)$ \\
\hline Missouri & - & - & - & - & - & - & 58.8 & $(53.7-63.7)$ & 29.0 & $(25.0-33.4)$ & 44.6 & $(40.6-48.5)$ \\
\hline Montana & 35.6 & (33.1-38.2) & 23.8 & (21.9-25.9) & 29.5 & $(28.1-31.0)$ & 54.6 & $(51.8-57.5)$ & 27.7 & $(25.8-29.7)$ & 40.7 & $(38.8-42.7)$ \\
\hline Nebraska & 35.6 & $(31.5-39.9)$ & 25.5 & $(22.7-28.6)$ & 30.3 & $(27.9-32.9)$ & 55.2 & $(50.9-59.5)$ & 32.1 & $(27.8-36.6)$ & 43.2 & $(40.1-46.4)$ \\
\hline Nevada & 34.0 & (30.2-38.0) & 27.7 & (24.4-31.2) & 30.8 & $(27.5-34.2)$ & 59.9 & $(55.8-63.9)$ & 35.9 & (32.6-39.3) & 47.8 & $(44.2-51.4)$ \\
\hline New Hampshire & - & - & - & - & - & - & 61.2 & $(59.1-63.3)$ & 27.6 & $(26.2-29.1)$ & 44.1 & $(42.7-45.4)$ \\
\hline New Mexico & - & - & - & - & - & - & - & - & - & - & - & - \\
\hline New York & - & - & - & - & - & - & - & - & - & - & - & - \\
\hline North Carolina & 32.1 & $(28.8-35.6)$ & 23.3 & $(20.5-26.3)$ & 27.6 & $(25.7-29.6)$ & 58.3 & $(55.3-61.2)$ & 33.5 & $(29.7-37.5)$ & 45.7 & $(43.6-47.8)$ \\
\hline North Dakota & 38.0 & (34.6-41.5) & 26.7 & $(24.1-29.5)$ & 32.2 & $(30.0-34.4)$ & 60.7 & $(57.2-64.0)$ & 29.5 & $(26.8-32.4)$ & 44.7 & $(42.5-46.8)$ \\
\hline Oklahoma & 40.2 & (35.9-44.6) & 24.0 & (20.4-28.1) & 32.2 & $(28.9-35.7)$ & 62.8 & $(58.7-66.7)$ & 31.9 & $(28.3-35.8)$ & 47.6 & $(44.3-50.8)$ \\
\hline Pennsylvania & 36.3 & (33.1-39.6) & 25.7 & (22.4-29.4) & 30.9 & $(28.7-33.2)$ & 56.9 & (53.9-59.9) & 31.4 & (28.6-34.4) & 44.0 & $(42.0-46.0)$ \\
\hline Rhode Island & 31.8 & (29.3-34.4) & 25.0 & $(23.0-27.1)$ & 28.3 & $(26.5-30.2)$ & 58.3 & $(53.6-62.9)$ & 33.8 & (29.4-38.5) & 45.8 & $(43.0-48.7)$ \\
\hline South Carolina & 34.2 & $(28.7-40.1)$ & 25.1 & (21.9-28.7) & 29.6 & $(27.0-32.4)$ & 55.6 & $(51.2-59.8)$ & 30.1 & $(26.2-34.5)$ & 42.9 & $(40.0-45.8)$ \\
\hline South Dakota & 39.9 & (34.3-45.8) & 25.4 & (21.9-29.3) & 32.5 & $(29.3-35.8)$ & - & - & - & - & - & - \\
\hline Tennessee & 36.5 & $(34.1-38.9)$ & 24.9 & $(22.4-27.6)$ & 30.5 & $(28.6-32.5)$ & 55.5 & $(53.1-57.9)$ & 32.2 & $(29.4-35.1)$ & 43.4 & $(41.5-45.4)$ \\
\hline Vermont & 36.3 & $(35.4-37.3)$ & 26.1 & $(25.2-26.9)$ & 31.1 & $(30.4-31.7)$ & 56.2 & $(55.3-57.2)$ & 27.9 & $(27.1-28.8)$ & 41.9 & $(41.2-42.5)$ \\
\hline Virginia & 35.1 & $(32.0-38.3)$ & 25.1 & $(22.6-27.7)$ & 30.0 & $(28.1-31.9)$ & 56.2 & $(52.8-59.5)$ & 32.7 & $(29.7-35.8)$ & 44.1 & $(41.5-46.6)$ \\
\hline West Virginia & 36.2 & $(32.9-39.6)$ & 29.2 & $(26.3-32.3)$ & 32.7 & $(30.4-35.0)$ & 59.8 & $(56.5-63.0)$ & 39.6 & $(36.1-43.1)$ & 49.5 & $(47.1-51.9)$ \\
\hline Wyoming & 34.9 & $(31.8-38.0)$ & 23.2 & $(20.5-26.2)$ & 28.9 & $(26.7-31.1)$ & 57.7 & $(54.1-61.2)$ & 27.9 & $(25.2-30.7)$ & 42.4 & $(40.3-44.5)$ \\
\hline Median & & 36.0 & & 25.5 & & 30.6 & & 59.0 & & 32.6 & & 45.2 \\
\hline Range & & $0.8-40.2)$ & & 1.6-29.4) & & $6.2-33.4)$ & & $4.6-64.0)$ & & 5.9-39.6) & & $0.7-49.5)$ \\
\hline \multicolumn{13}{|c|}{ Large urban school district surveys } \\
\hline Baltimore, MD & 29.3 & $(23.2-36.3)$ & 19.3 & $(15.5-24.0)$ & 24.2 & $(20.3-28.6)$ & 51.5 & $(46.7-56.3)$ & 30.4 & $(25.4-35.9)$ & 41.1 & $(37.5-44.8)$ \\
\hline Boston, MA & 36.1 & $(32.7-39.7)$ & 27.0 & $(23.8-30.5)$ & 31.6 & $(29.1-34.1)$ & 60.9 & $(57.2-64.6)$ & 37.4 & $(33.1-41.9)$ & 49.1 & $(46.1-52.1)$ \\
\hline Broward County, FL & 35.8 & $(32.3-39.5)$ & 24.0 & $(20.5-27.8)$ & 29.9 & $(27.5-32.4)$ & 57.3 & $(53.4-61.1)$ & 31.8 & $(27.7-36.1)$ & 44.5 & $(41.4-47.7)$ \\
\hline Cleveland, $\mathrm{OH}$ & 39.1 & $(35.2-43.1)$ & 26.0 & $(23.2-29.0)$ & 32.4 & $(30.0-34.9)$ & 59.7 & $(55.8-63.5)$ & 36.1 & $(32.7-39.6)$ & 47.5 & $(44.8-50.2)$ \\
\hline DeKalb County, GA & 33.2 & $(29.8-36.8)$ & 16.6 & $(13.9-19.6)$ & 24.8 & $(22.2-27.6)$ & 56.4 & $(53.4-59.4)$ & 29.4 & $(26.3-32.6)$ & 43.0 & $(40.3-45.7)$ \\
\hline Detroit, MI & 32.0 & $(28.9-35.3)$ & 17.3 & $(13.7-21.7)$ & 25.5 & $(22.7-28.6)$ & 58.6 & $(54.9-62.2)$ & 39.6 & $(34.5-45.0)$ & 49.9 & $(46.6-53.3)$ \\
\hline District of Columbia & 31.0 & $(29.7-32.4)$ & 18.4 & $(17.2-19.7)$ & 25.0 & $(24.1-25.9)$ & 54.8 & $(53.4-56.3)$ & 33.4 & $(31.9-35.0)$ & 44.7 & $(43.6-45.8)$ \\
\hline Duval County, FL & 33.1 & $(30.7-35.7)$ & 20.5 & $(18.3-22.9)$ & 27.1 & $(25.3-29.0)$ & 54.7 & $(51.9-57.5)$ & 30.0 & $(27.4-32.8)$ & 42.8 & $(40.8-44.8)$ \\
\hline Ft. Worth, TX & 39.7 & $(36.9-42.6)$ & 28.3 & $(25.5-31.4)$ & 34.1 & $(32.0-36.3)$ & 61.3 & $(58.3-64.2)$ & 41.2 & $(37.7-44.9)$ & 51.3 & $(48.8-53.8)$ \\
\hline Houston, TX & 36.8 & $(34.3-39.3)$ & 26.0 & $(23.9-28.2)$ & 31.2 & $(29.4-33.0)$ & 58.5 & $(55.2-61.7)$ & 41.9 & $(39.2-44.5)$ & 49.8 & $(48.0-51.7)$ \\
\hline Los Angeles, CA & 40.7 & $(35.6-46.0)$ & 31.0 & $(27.2-35.1)$ & 35.9 & $(31.9-40.2)$ & 66.6 & $(64.1-69.1)$ & 42.8 & $(38.7-47.0)$ & 55.1 & $(52.5-57.6)$ \\
\hline Miami-Dade County, FL & 34.8 & $(31.8-38.0)$ & 24.9 & $(21.8-28.4)$ & 29.8 & $(27.6-32.2)$ & 58.0 & $(55.0-61.0)$ & 34.0 & $(31.2-36.9)$ & 46.1 & $(44.1-48.2)$ \\
\hline
\end{tabular}

See table footnotes on the next page. 
TABLE 118. (Continued) Percentage of high school students who described themselves as slightly or very overweight and who were trying to lose weight, by sex — selected U.S. sites, Youth Risk Behavior Survey, 2015

\begin{tabular}{|c|c|c|c|c|c|c|c|c|c|c|c|c|}
\hline \multirow[b]{3}{*}{ Site } & \multicolumn{6}{|c|}{ Described themselves as overweight } & \multicolumn{6}{|c|}{ Were trying to lose weight } \\
\hline & \multicolumn{2}{|c|}{ Female } & \multicolumn{2}{|r|}{ Male } & \multicolumn{2}{|r|}{ Total } & \multicolumn{2}{|c|}{ Female } & \multicolumn{2}{|r|}{ Male } & \multicolumn{2}{|r|}{ Total } \\
\hline & $\%$ & $\mathrm{Cl}^{*}$ & $\%$ & $\mathrm{Cl}$ & $\%$ & $\mathrm{Cl}$ & $\%$ & $\mathrm{Cl}$ & $\%$ & $\mathrm{Cl}$ & $\%$ & $\mathrm{Cl}$ \\
\hline New York City, NY & - & - & - & - & - & - & - & - & - & - & - & - \\
\hline Oakland, CA & 38.7 & (34.3-43.2) & 24.3 & $(20.9-28.0)$ & 31.2 & (28.4-34.3) & - & - & - & - & - & - \\
\hline Orange County, FL & 31.0 & $(26.9-35.5)$ & 23.3 & $(20.1-26.9)$ & 27.1 & $(24.2-30.2)$ & 57.2 & $(53.2-61.2)$ & 30.5 & $(27.3-33.8)$ & 43.8 & $(41.2-46.5)$ \\
\hline Palm Beach County, FL & 30.6 & $(27.3-34.1)$ & 21.1 & $(18.7-23.7)$ & 25.8 & $(23.7-28.1)$ & 54.0 & $(51.1-56.9)$ & 32.0 & $(29.2-34.8)$ & 42.6 & $(40.6-44.8)$ \\
\hline Philadelphia, PA & 31.3 & $(28.3-34.6)$ & 20.9 & $(17.1-25.1)$ & 26.2 & $(24.0-28.6)$ & 55.8 & $(51.5-59.9)$ & 33.8 & $(29.3-38.6)$ & 45.1 & $(42.1-48.2)$ \\
\hline San Diego, CA & 37.8 & (34.1-41.7) & 27.2 & $(24.2-30.3)$ & 32.4 & (30.2-34.7) & 60.5 & $(57.0-63.8)$ & 34.9 & $(31.3-38.7)$ & 47.5 & $(44.7-50.2)$ \\
\hline San Francisco, CA & 40.8 & (37.0-44.8) & 23.1 & $(19.7-26.9)$ & 31.5 & $(28.7-34.5)$ & 58.4 & $(54.3-62.4)$ & 33.8 & $(30.3-37.5)$ & 45.9 & $(43.0-48.9)$ \\
\hline Median & & 35.3 & & 23.6 & & 29.8 & & 58.0 & & 33.8 & & 45.9 \\
\hline Range & & $9.3-40.8)$ & & $6.6-31.0)$ & & $4.2-35.9)$ & & $1.5-66.6)$ & & 9.4-42.8) & & $(1.1-55.1)$ \\
\hline
\end{tabular}

* $95 \%$ confidence interval.

† Not available.

TABLE 119. Percentage of high school students who had ever been told by a doctor or nurse that they had asthma and who saw a dentist, ${ }^{*}$ by sex, race/ethnicity, and grade — United States, Youth Risk Behavior Survey, 2015

\begin{tabular}{|c|c|c|c|c|c|c|c|c|c|c|c|c|}
\hline \multirow[b]{3}{*}{ Category } & \multicolumn{6}{|c|}{ Had asthma } & \multicolumn{6}{|c|}{ Saw a dentist } \\
\hline & \multicolumn{2}{|r|}{ Female } & \multicolumn{2}{|r|}{ Male } & \multicolumn{2}{|r|}{ Total } & \multicolumn{2}{|c|}{ Female } & \multicolumn{2}{|c|}{ Male } & \multicolumn{2}{|r|}{ Total } \\
\hline & $\%$ & $\mathrm{Cl}^{\dagger}$ & $\%$ & $\mathrm{Cl}$ & $\%$ & $\mathrm{Cl}$ & $\%$ & $\mathrm{Cl}$ & $\%$ & $\mathrm{Cl}$ & $\%$ & $\mathrm{Cl}$ \\
\hline \multicolumn{13}{|c|}{ Race/Ethnicity } \\
\hline White $\S$ & 23.7 & (21.9-25.6) & 20.4 & (18.4-22.6) & 22.1 & $(20.7-23.5)$ & 80.9 & (77.7-83.7) & 81.3 & (77.9-84.3) & 81.0 & (78.2-83.6) \\
\hline Black $^{\S}$ & 27.3 & $(22.0-33.3)$ & 28.4 & $(24.3-32.8)$ & 27.8 & $(24.6-31.2)$ & 64.2 & (59.7-68.4) & 61.1 & $(55.4-66.4)$ & 62.5 & (58.5-66.3) \\
\hline Hispanic & 22.0 & $(19.1-25.2)$ & 23.0 & $(20.3-26.0)$ & 22.5 & $(20.0-25.2)$ & 68.1 & $(64.5-71.4)$ & 64.8 & $(62.1-67.4)$ & 66.4 & (64.4-68.3) \\
\hline \multicolumn{13}{|l|}{ Grade } \\
\hline 9 & 23.5 & $(20.8-26.3)$ & 22.6 & $(19.2-26.5)$ & 23.0 & $(20.8-25.3)$ & 75.1 & (71.4-78.5) & 76.5 & (72.6-79.9) & 75.6 & (72.6-78.3) \\
\hline 10 & 22.7 & $(20.3-25.3)$ & 22.6 & $(19.5-26.1)$ & 22.7 & $(20.6-25.0)$ & 76.4 & $(71.3-80.8)$ & 72.1 & $(67.4-76.3)$ & 74.2 & (69.9-78.2) \\
\hline 11 & 23.6 & $(20.4-27.2)$ & 22.8 & $(20.6-25.1)$ & 23.2 & $(21.2-25.2)$ & 75.8 & $(72.7-78.6)$ & 75.8 & $(70.1-80.7)$ & 75.6 & (71.9-79.0) \\
\hline 12 & 23.6 & $(20.4-27.2)$ & 20.9 & $(17.6-24.8)$ & 22.3 & $(19.7-25.1)$ & 75.0 & $(69.8-79.5)$ & 70.5 & $(66.9-74.0)$ & 72.7 & (68.9-76.2) \\
\hline Total & 23.3 & $(21.8-24.9)$ & 22.2 & $(20.5-24.0)$ & 22.8 & $(21.5-24.1)$ & 75.5 & $(72.6-78.1)$ & 73.7 & (70.4-76.8) & 74.4 & (71.6-77.1) \\
\hline
\end{tabular}

* For a check-up, exam, teeth cleaning, or other dental work during the 12 months before the survey.

† $95 \%$ confidence interval.

$\S$ Non-Hispanic. 
TABLE 120. Percentage of high school students who had ever been told by a doctor or nurse that they had asthma and who saw a dentist,* by sex selected U.S. sites, Youth Risk Behavior Survey, 2015

\begin{tabular}{|c|c|c|c|c|c|c|c|c|c|c|c|c|}
\hline \multirow[b]{3}{*}{ Site } & \multicolumn{6}{|c|}{ Had asthma } & \multicolumn{6}{|c|}{ Saw a dentist } \\
\hline & \multicolumn{2}{|c|}{ Female } & \multicolumn{2}{|r|}{ Male } & \multicolumn{2}{|r|}{ Total } & \multicolumn{2}{|c|}{ Female } & \multicolumn{2}{|r|}{ Male } & \multicolumn{2}{|r|}{ Total } \\
\hline & $\%$ & $\mathrm{Cl}^{\dagger}$ & $\%$ & $\mathrm{Cl}$ & $\%$ & $\mathrm{Cl}$ & $\%$ & $\mathrm{Cl}$ & $\%$ & $\mathrm{Cl}$ & $\%$ & $\mathrm{Cl}$ \\
\hline \multicolumn{13}{|l|}{ State surveys } \\
\hline Alabama & 22.0 & $(18.4-26.0)$ & 28.7 & $(23.9-33.9)$ & 25.2 & (21.9-28.9) & 73.5 & (69.2-77.4) & 68.0 & (64.0-71.7) & 70.4 & $(67.1-73.5)$ \\
\hline Alaska & 20.6 & $(17.7-23.9)$ & 18.3 & (15.6-21.4) & 19.4 & (17.4-21.6) & 71.1 & $(66.0-75.7)$ & 69.6 & $(65.1-73.8)$ & 70.3 & $(66.5-73.8)$ \\
\hline Arizona & —§ & - & - & - & - & - & - & - & - & - & - & - \\
\hline Arkansas & 24.7 & (20.3-29.6) & 27.1 & $(23.8-30.7)$ & 25.9 & (23.3-28.7) & 72.1 & $(68.4-75.6)$ & 66.6 & $(62.1-70.9)$ & 69.4 & $(67.6-71.2)$ \\
\hline California & 21.4 & $(18.1-25.3)$ & 19.7 & (16.9-22.8) & 20.4 & (18.3-22.8) & 74.5 & (70.9-77.9) & 72.7 & $(67.2-77.7)$ & 73.6 & $(70.2-76.8)$ \\
\hline Connecticut & - & - & - & - & - & - & 81.5 & (77.6-84.9) & 80.4 & (76.9-83.5) & 80.8 & $(78.0-83.3)$ \\
\hline Delaware & 24.4 & $(21.8-27.2)$ & 26.5 & $(24.0-29.2)$ & 25.4 & $(23.6-27.3)$ & 77.2 & $(74.1-80.0)$ & 70.0 & $(65.2-74.5)$ & 73.3 & $(69.9-76.5)$ \\
\hline Florida & 22.8 & $(21.2-24.3)$ & 23.5 & $(21.8-25.2)$ & 23.2 & $(22.0-24.5)$ & 67.7 & $(64.7-70.6)$ & 64.1 & $(61.4-66.7)$ & 65.7 & $(63.2-68.1)$ \\
\hline Hawaii & 30.4 & $(28.4-32.5)$ & 32.2 & $(29.9-34.6)$ & 31.3 & $(29.5-33.1)$ & 72.4 & $(68.3-76.1)$ & 69.4 & $(64.6-73.7)$ & 70.8 & $(66.6-74.6)$ \\
\hline Idaho & 18.2 & $(15.2-21.7)$ & 20.3 & $(17.2-23.8)$ & 19.3 & (17.4-21.3) & 81.7 & $(78.8-84.4)$ & 77.5 & $(72.8-81.6)$ & 79.6 & (77.1-81.9) \\
\hline Illinois & 21.1 & (18.7-23.8) & 21.6 & $(18.9-24.6)$ & 21.4 & (19.3-23.6) & 78.9 & $(74.3-82.9)$ & 75.3 & (72.1-78.3) & 77.1 & $(74.1-79.8)$ \\
\hline Indiana & 25.7 & $(21.8-29.9)$ & 22.3 & $(19.1-25.9)$ & 23.9 & (21.2-26.8) & 75.3 & $(69.9-80.0)$ & 73.3 & (66.1-79.4) & 74.3 & $(69.1-78.9)$ \\
\hline Kentucky & 25.6 & $(22.6-29.0)$ & 25.3 & (22.4-28.4) & 25.5 & $(23.1-28.1)$ & 71.5 & $(67.0-75.5)$ & 68.2 & $(64.2-71.9)$ & 69.8 & $(66.5-72.9)$ \\
\hline Maine & 24.0 & (22.5-25.5) & 24.6 & $(23.1-26.1)$ & 24.3 & (23.2-25.4) & - & - & - & - & - & - \\
\hline Maryland & 25.2 & $(24.5-25.9)$ & 27.4 & $(26.6-28.2)$ & 26.3 & (25.8-26.9) & 77.5 & (76.6-78.4) & 75.2 & (74.3-76.0) & 76.1 & $(75.4-76.9)$ \\
\hline Massachusetts & - & - & - & - & - & - & - & - & - & - & - & - \\
\hline Michigan & 24.0 & $(21.2-27.1)$ & 26.7 & $(23.1-30.5)$ & 25.3 & $(22.6-28.1)$ & 75.2 & (71.5-78.6) & 72.3 & $(68.8-75.5)$ & 73.7 & (70.7-76.5) \\
\hline Mississippi & 26.0 & $(22.2-30.3)$ & 24.6 & $(21.5-28.1)$ & 25.6 & (22.6-28.8) & - & - & - & - & - & - \\
\hline Missouri & 20.6 & $(16.2-25.8)$ & 23.5 & $(20.2-27.1)$ & 22.0 & $(18.7-25.7)$ & 73.2 & $(67.5-78.3)$ & 65.4 & $(60.4-70.1)$ & 69.2 & $(65.7-72.6)$ \\
\hline Montana & 23.2 & $(21.5-25.0)$ & 21.3 & $(18.8-23.9)$ & 22.2 & $(20.7-23.9)$ & 77.0 & $(73.7-80.0)$ & 73.0 & (70.4-75.5) & 74.9 & $(72.6-77.0)$ \\
\hline Nebraska & 19.3 & (16.5-22.5) & 20.5 & $(17.3-24.2)$ & 20.0 & (17.5-22.8) & 77.3 & $(73.5-80.7)$ & 73.6 & $(69.8-77.2)$ & 75.2 & $(72.4-77.8)$ \\
\hline Nevada & - & - & - & - & - & - & 70.0 & $(66.9-73.0)$ & 67.8 & $(64.2-71.2)$ & 68.9 & $(66.1-71.4)$ \\
\hline New Hampshire & 23.1 & (21.7-24.6) & 21.5 & $(20.2-22.8)$ & 22.3 & (21.3-23.4) & 84.1 & $(82.8-85.2)$ & 81.6 & $(80.2-83.0)$ & 82.7 & $(81.8-83.7)$ \\
\hline New Mexico & 24.2 & $(22.7-25.7)$ & 26.5 & $(24.7-28.4)$ & 25.3 & (24.2-26.5) & 75.4 & (73.7-77.1) & 71.7 & $(69.7-73.7)$ & 73.5 & $(72.0-75.0)$ \\
\hline New York & 25.2 & (23.4-27.1) & 25.8 & $(22.3-29.7)$ & 25.6 & $(23.6-27.8)$ & 74.0 & (70.2-77.4) & 72.2 & $(68.6-75.5)$ & 73.0 & $(69.8-75.9)$ \\
\hline North Carolina & 24.5 & (20.6-28.9) & 27.5 & $(24.0-31.3)$ & 25.9 & (23.2-28.9) & - & - & - & - & - & - \\
\hline North Dakota & - & - & - & - & - & - & 78.6 & $(75.3-81.6)$ & 75.5 & (72.5-78.2) & 77.0 & $(74.7-79.2)$ \\
\hline Oklahoma & 24.1 & (20.8-27.7) & 24.3 & (21.4-27.4) & 24.5 & $(22.1-27.0)$ & 75.8 & (71.4-79.7) & 67.3 & (61.8-72.4) & 71.6 & $(67.5-75.4)$ \\
\hline Pennsylvania & 23.1 & (20.5-25.9) & 26.3 & $(23.6-29.3)$ & 24.7 & (22.9-26.6) & 83.6 & (79.8-86.7) & 79.8 & (76.0-83.2) & 81.6 & $(78.5-84.4)$ \\
\hline Rhode Island & - & - & - & - & - & - & 80.2 & (72.4-86.2) & 76.0 & $(69.3-81.5)$ & 78.0 & $(71.4-83.4)$ \\
\hline South Carolina & 21.1 & (17.9-24.8) & 24.2 & (18.9-30.3) & 22.6 & (18.8-26.9) & 69.2 & $(62.8-74.9)$ & 69.9 & $(64.5-74.9)$ & 69.6 & $(64.3-74.5)$ \\
\hline South Dakota & - & - & - & - & - & - & 81.0 & $(76.4-84.9)$ & 72.8 & $(66.0-78.7)$ & 76.8 & $(72.3-80.8)$ \\
\hline Tennessee & 21.7 & $(19.6-24.1)$ & 21.6 & $(18.7-24.8)$ & 21.8 & (19.8-23.9) & 70.1 & $(67.6-72.5)$ & 66.9 & $(63.9-69.9)$ & 68.5 & $(66.0-70.8)$ \\
\hline Vermont & - & - & - & - & - & - & - & - & - & - & - & - \\
\hline Virginia & 22.3 & (20.3-24.5) & 22.4 & (20.1-24.9) & 22.4 & $(20.7-24.1)$ & 77.1 & (73.6-80.3) & 74.2 & (71.3-76.9) & 75.6 & (72.7-78.3) \\
\hline West Virginia & 24.9 & $(21.9-28.1)$ & 27.1 & $(23.1-31.4)$ & 25.9 & (23.1-29.0) & 74.3 & $(69.6-78.6)$ & 72.3 & (67.6-76.6) & 73.3 & $(69.2-77.0)$ \\
\hline Wyoming & 23.1 & (19.9-26.8) & 23.5 & (20.7-26.5) & 23.4 & (21.2-25.8) & 76.9 & (74.0-79.6) & 73.1 & $(69.8-76.1)$ & 74.9 & $(72.4-77.1)$ \\
\hline Median & & 23.2 & & 24.3 & & 24.3 & & 75.4 & & 72.3 & & 73.6 \\
\hline Range & & $8.2-30.4)$ & & $8.3-32.2)$ & & $9.3-31.3)$ & & 7.7-84.1) & & 4.1-81.6) & & 5.7-82.7) \\
\hline \multicolumn{13}{|c|}{ Large urban school district surveys } \\
\hline Baltimore, MD & 32.4 & $(29.0-36.0)$ & 35.3 & $(30.3-40.6)$ & 34.4 & (31.1-37.8) & 69.8 & (65.5-73.8) & 55.3 & $(49.9-60.6)$ & 62.6 & $(59.4-65.7)$ \\
\hline Boston, MA & - & - & - & - & - & - & 70.7 & $(67.2-74.0)$ & 71.4 & $(68.2-74.4)$ & 71.1 & $(68.7-73.5)$ \\
\hline Broward County, FL & 21.0 & $(18.0-24.3)$ & 26.0 & $(22.4-30.0)$ & 23.7 & $(21.2-26.4)$ & 64.5 & $(60.3-68.5)$ & 67.1 & $(62.6-71.4)$ & 65.7 & $(62.5-68.8)$ \\
\hline Cleveland, $\mathrm{OH}$ & - & - & - & - & - & - & - & - & - & - & - & - \\
\hline DeKalb County, GA & 26.5 & $(23.6-29.6)$ & 29.8 & $(26.4-33.4)$ & 28.1 & $(25.9-30.3)$ & 62.1 & $(58.4-65.7)$ & 63.1 & $(58.9-67.1)$ & 62.5 & $(59.6-65.4)$ \\
\hline Detroit, MI & 26.2 & $(23.3-29.3)$ & 32.2 & $(28.6-36.1)$ & 28.9 & (26.4-31.5) & 56.1 & $(52.0-60.1)$ & 54.0 & $(48.9-58.9)$ & 55.1 & $(51.6-58.6)$ \\
\hline District of Columbia & 30.4 & $(29.1-31.8)$ & 31.0 & $(29.4-32.5)$ & 30.8 & $(29.8-31.9)$ & - & - & - & - & - & - \\
\hline Duval County, FL & 25.7 & $(23.5-28.0)$ & 27.2 & $(24.6-30.0)$ & 26.2 & $(24.5-28.0)$ & 67.5 & $(64.9-70.0)$ & 64.2 & $(61.1-67.1)$ & 65.7 & $(63.7-67.7)$ \\
\hline Ft. Worth, TX & 19.4 & $(17.0-21.9)$ & 18.6 & $(16.2-21.3)$ & 19.0 & (17.3-20.7) & 66.6 & $(64.0-69.1)$ & 64.5 & $(61.0-67.9)$ & 65.6 & $(63.4-67.8)$ \\
\hline Houston, TX & 21.4 & $(19.1-23.9)$ & 23.3 & $(20.8-26.1)$ & 22.4 & (20.5-24.4) & 62.2 & (59.1-65.2) & 59.1 & $(56.0-62.1)$ & 60.4 & $(58.2-62.6)$ \\
\hline Los Angeles, CA & 20.6 & $(17.3-24.4)$ & 18.7 & $(15.6-22.4)$ & 19.7 & $(17.1-22.5)$ & 71.0 & $(67.2-74.5)$ & 68.5 & $(64.9-72.0)$ & 69.6 & $(66.2-72.9)$ \\
\hline Miami-Dade County, FL & 20.8 & $(18.7-23.1)$ & 24.9 & $(22.3-27.6)$ & 22.8 & $(21.0-24.8)$ & 65.9 & $(62.2-69.4)$ & 63.6 & $(59.5-67.6)$ & 64.8 & $(61.5-67.9)$ \\
\hline
\end{tabular}

See table footnotes on the next page. 
TABLE 120. (Continued) Percentage of high school students who had ever been told by a doctor or nurse that they had asthma and who saw a dentist, ${ }^{*}$ by sex — selected U.S. sites, Youth Risk Behavior Survey, 2015

\begin{tabular}{|c|c|c|c|c|c|c|c|c|c|c|c|c|}
\hline \multirow[b]{3}{*}{ Site } & \multicolumn{6}{|c|}{ Had asthma } & \multicolumn{6}{|c|}{ Saw a dentist } \\
\hline & \multicolumn{2}{|c|}{ Female } & \multicolumn{2}{|r|}{ Male } & \multicolumn{2}{|r|}{ Total } & \multicolumn{2}{|r|}{ Female } & \multicolumn{2}{|r|}{ Male } & \multicolumn{2}{|r|}{ Total } \\
\hline & $\%$ & $\mathrm{Cl}^{\dagger}$ & $\%$ & $\mathrm{Cl}$ & $\%$ & $\mathrm{Cl}$ & $\%$ & $\mathrm{Cl}$ & $\%$ & $\mathrm{Cl}$ & $\%$ & $\mathrm{Cl}$ \\
\hline New York City, NY & 22.8 & $(20.2-25.6)$ & 25.7 & $(22.8-28.8)$ & 24.2 & $(22.0-26.6)$ & 68.4 & $(65.9-70.8)$ & 71.3 & $(68.7-73.8)$ & 69.7 & $(68.1-71.3)$ \\
\hline Oakland, CA & 23.8 & $(20.2-27.7)$ & 23.2 & $(19.6-27.4)$ & 23.8 & $(21.3-26.5)$ & 66.8 & $(62.5-70.9)$ & 65.9 & $(62.0-69.6)$ & 66.2 & $(62.9-69.3)$ \\
\hline Orange County, FL & 22.8 & $(19.9-26.0)$ & 19.6 & $(16.7-22.8)$ & 21.0 & $(19.2-23.1)$ & 65.2 & $(61.3-68.9)$ & 58.3 & $(53.1-63.3)$ & 61.6 & $(58.1-65.0)$ \\
\hline Palm Beach County, FL & 20.7 & $(17.8-23.9)$ & 25.8 & $(23.0-28.8)$ & 23.4 & $(21.3-25.6)$ & 65.2 & $(61.7-68.6)$ & 64.6 & $(61.7-67.4)$ & 64.5 & (62.1-66.9) \\
\hline Philadelphia, PA & 28.7 & $(26.6-31.0)$ & 28.4 & $(25.0-32.0)$ & 28.5 & $(26.3-30.8)$ & 70.4 & $(65.5-75.0)$ & 65.2 & $(58.9-71.0)$ & 67.8 & $(63.1-72.3)$ \\
\hline San Diego, CA & 17.7 & $(15.2-20.6)$ & 20.5 & $(17.9-23.3)$ & 19.2 & $(17.4-21.2)$ & 72.5 & $(68.7-75.9)$ & 68.6 & $(65.8-71.3)$ & 70.5 & $(68.0-72.9)$ \\
\hline San Francisco, CA & 19.9 & $(17.2-22.8)$ & 20.0 & $(17.0-23.4)$ & 20.0 & $(17.9-22.4)$ & 73.4 & $(69.4-77.1)$ & 72.2 & $(68.6-75.5)$ & 72.6 & $(70.3-74.9)$ \\
\hline Median & \multicolumn{2}{|r|}{22.8} & \multicolumn{2}{|r|}{25.7} & \multicolumn{2}{|r|}{23.7} & \multicolumn{2}{|r|}{66.8} & \multicolumn{2}{|r|}{64.6} & \multicolumn{2}{|r|}{65.7} \\
\hline Range & \multicolumn{2}{|c|}{$(17.7-32.4)$} & \multicolumn{2}{|c|}{$(18.6-35.3)$} & \multicolumn{2}{|c|}{$(19.0-34.4)$} & \multicolumn{2}{|c|}{$(56.1-73.4)$} & \multicolumn{2}{|c|}{$(54.0-72.2)$} & \multicolumn{2}{|c|}{ (55.1-72.6) } \\
\hline
\end{tabular}

* For a check-up, exam, teeth cleaning, or other dental work during the 12 months before the survey.

† $95 \%$ confidence interval.

$\S$ Not available.

TABLE 121. Percentage of high school students who had 8 or more hours of sleep,* by sex, race/ethnicity, and grade - United States, Youth Risk Behavior Survey, 2015

\begin{tabular}{|c|c|c|c|c|c|c|}
\hline \multirow[b]{2}{*}{ Category } & \multicolumn{2}{|c|}{ Female } & \multicolumn{2}{|c|}{ Male } & \multicolumn{2}{|c|}{ Total } \\
\hline & $\%$ & $\mathrm{Cl}^{\dagger}$ & $\%$ & $\mathrm{Cl}$ & $\%$ & $\mathrm{Cl}$ \\
\hline \multicolumn{7}{|l|}{ Race/Ethnicity } \\
\hline White ${ }^{\S}$ & 24.9 & $(22.5-27.4)$ & 31.1 & $(27.9-34.6)$ & 28.0 & $(25.6-30.5)$ \\
\hline Black ${ }^{\S}$ & 20.6 & $(17.0-24.6)$ & 25.6 & $(21.1-30.7)$ & 23.5 & $(20.1-27.2)$ \\
\hline Hispanic & 26.8 & $(23.2-30.6)$ & 32.9 & $(29.1-36.9)$ & 29.8 & (26.4-33.4) \\
\hline \multicolumn{7}{|l|}{ Grade } \\
\hline 9 & 29.1 & $(26.0-32.5)$ & 39.3 & (35.2-43.4) & 34.4 & $(31.5-37.4)$ \\
\hline 10 & 23.1 & $(20.0-26.5)$ & 33.8 & $(31.2-36.6)$ & 28.4 & $(26.0-30.9)$ \\
\hline 11 & 23.0 & $(19.6-26.8)$ & 22.9 & $(18.8-27.5)$ & 22.9 & $(19.7-26.5)$ \\
\hline 12 & 22.2 & $(19.6-25.1)$ & 22.6 & $(18.8-26.8)$ & 22.4 & $(19.8-25.3)$ \\
\hline Total & 24.4 & $(22.3-26.7)$ & 30.1 & $(27.2-33.0)$ & 27.3 & $(25.1-29.6)$ \\
\hline
\end{tabular}

* On an average school night.

† $95 \%$ confidence interval.

$\S$ Non-Hispanic. 
TABLE 122. Percentage of high school students who had 8 or more hours of sleep,* by sex — selected U.S. sites, Youth Risk Behavior Survey, 2015

\begin{tabular}{|c|c|c|c|c|c|c|}
\hline \multirow[b]{2}{*}{ Site } & \multicolumn{2}{|c|}{ Female } & \multicolumn{2}{|c|}{ Male } & \multicolumn{2}{|c|}{ Total } \\
\hline & $\%$ & $\mathrm{Cl}^{\dagger}$ & $\%$ & $\mathrm{Cl}$ & $\%$ & $\mathrm{Cl}$ \\
\hline \multicolumn{7}{|l|}{ State surveys } \\
\hline Alabama & 25.3 & $(21.0-30.1)$ & 31.2 & $(27.5-35.2)$ & 28.1 & $(25.4-31.0)$ \\
\hline Alaska & —§ & - & - & - & - & - \\
\hline Arizona & - & - & - & - & - & - \\
\hline Arkansas & 29.1 & $(24.4-34.2)$ & 29.7 & $(25.0-34.9)$ & 29.5 & $(25.3-34.0)$ \\
\hline California & 26.5 & $(20.6-33.2)$ & 31.5 & $(26.4-37.2)$ & 29.0 & $(23.7-34.9)$ \\
\hline Connecticut & 17.5 & $(14.9-20.4)$ & 22.1 & $(19.4-25.1)$ & 19.9 & $(18.1-21.7)$ \\
\hline Delaware & 22.0 & $(19.2-25.2)$ & 26.6 & $(23.2-30.3)$ & 24.2 & $(21.9-26.7)$ \\
\hline Florida & 21.1 & $(19.3-23.1)$ & 25.0 & $(23.1-26.9)$ & 23.1 & $(21.7-24.6)$ \\
\hline Hawaii & 22.5 & $(19.2-26.3)$ & 27.0 & $(24.6-29.5)$ & 24.7 & $(22.2-27.3)$ \\
\hline Idaho & - & - & - & - & - & - \\
\hline Illinois & 21.6 & (18.5-25.0) & 25.4 & $(21.2-30.1)$ & 23.5 & $(21.0-26.1)$ \\
\hline Indiana & 19.2 & $(16.0-22.9)$ & 23.5 & $(20.7-26.6)$ & 21.4 & $(19.2-23.8)$ \\
\hline Kentucky & 21.8 & $(18.7-25.2)$ & 26.8 & $(22.8-31.2)$ & 24.3 & $(21.5-27.3)$ \\
\hline Maine & - & - & - & - & - & - \\
\hline Maryland & 22.1 & $(21.3-22.9)$ & 25.7 & $(24.9-26.5)$ & 23.8 & $(23.2-24.5)$ \\
\hline Massachusetts & 19.1 & $(16.9-21.6)$ & 25.0 & $(21.9-28.3)$ & 22.0 & $(19.8-24.3)$ \\
\hline Michigan & 18.5 & $(15.4-22.1)$ & 22.0 & $(18.9-25.5)$ & 20.3 & (17.9-23.1) \\
\hline Mississippi & - & - & - & - & - & - \\
\hline Missouri & 25.5 & $(21.4-30.1)$ & 29.4 & $(25.9-33.2)$ & 27.4 & $(24.0-31.0)$ \\
\hline Montana & 29.3 & $(27.5-31.2)$ & 35.8 & $(33.0-38.6)$ & 32.6 & (30.9-34.4) \\
\hline Nebraska & 28.9 & $(24.5-33.8)$ & 35.1 & $(31.1-39.3)$ & 31.9 & $(28.6-35.5)$ \\
\hline Nevada & 22.7 & $(19.3-26.6)$ & 25.6 & $(22.2-29.3)$ & 24.1 & $(21.6-26.8)$ \\
\hline New Hampshire & 24.6 & $(22.5-26.9)$ & 31.8 & $(30.0-33.6)$ & 28.4 & (26.9-29.9) \\
\hline New Mexico & 28.0 & $(26.1-30.0)$ & 35.5 & (33.6-37.5) & 31.7 & $(30.2-33.3)$ \\
\hline New York & 21.2 & $(19.1-23.5)$ & 22.4 & (19.5-25.6) & 21.9 & $(19.8-24.3)$ \\
\hline North Carolina & 20.8 & $(16.4-26.0)$ & 29.3 & $(24.2-34.9)$ & 25.0 & $(21.7-28.5)$ \\
\hline North Dakota & 28.1 & $(24.9-31.5)$ & 30.9 & $(27.4-34.5)$ & 29.5 & $(27.0-32.2)$ \\
\hline Oklahoma & 24.7 & $(20.8-29.1)$ & 31.9 & $(28.1-36.0)$ & 28.2 & $(25.1-31.5)$ \\
\hline Pennsylvania & 22.3 & $(19.4-25.5)$ & 29.1 & $(26.3-32.1)$ & 25.6 & $(23.3-28.1)$ \\
\hline Rhode Island & - & - & - & - & - & - \\
\hline South Carolina & 26.2 & $(22.1-30.8)$ & 29.8 & $(24.2-36.1)$ & 27.9 & $(24.2-32.0)$ \\
\hline South Dakota & 34.7 & (28.6-41.4) & 41.5 & (37.6-45.6) & 38.2 & (34.2-42.3) \\
\hline Tennessee & 29.1 & $(26.0-32.4)$ & 29.7 & $(27.2-32.3)$ & 29.4 & $(27.8-31.0)$ \\
\hline Vermont & - & - & - & - & - & - \\
\hline Virginia & 25.1 & $(22.9-27.4)$ & 30.0 & $(27.2-33.0)$ & 27.6 & (25.4-29.9) \\
\hline West Virginia & 16.2 & $(12.9-20.2)$ & 18.9 & $(15.7-22.5)$ & 17.5 & (14.7-20.7) \\
\hline Wyoming & 28.3 & $(24.9-31.9)$ & 32.4 & $(29.4-35.6)$ & 30.3 & $(28.3-32.3)$ \\
\hline Median & \multirow{2}{*}{\multicolumn{2}{|c|}{$\begin{array}{c}23.6 \\
(16.2-34.7)\end{array}$}} & \multirow{2}{*}{\multicolumn{2}{|c|}{$\begin{array}{c}29.3 \\
(18.9-41.5)\end{array}$}} & \multirow{2}{*}{\multicolumn{2}{|c|}{$\begin{array}{c}26.5 \\
(17.5-38.2)\end{array}$}} \\
\hline Range & & & & & & \\
\hline \multicolumn{7}{|c|}{ Large urban school district surveys } \\
\hline Baltimore, MD & 23.5 & $(19.2-28.4)$ & 24.4 & $(18.9-30.7)$ & 23.9 & $(20.3-27.9)$ \\
\hline Boston, MA & 16.3 & $(13.6-19.4)$ & 18.6 & $(15.5-22.2)$ & 17.6 & (15.3-20.3) \\
\hline Broward County, FL & 13.7 & $(11.0-17.0)$ & 15.2 & $(12.3-18.7)$ & 14.4 & (12.4-16.6) \\
\hline Cleveland, $\mathrm{OH}$ & 19.5 & $(16.1-23.3)$ & 20.3 & $(17.3-23.6)$ & 20.1 & $(18.0-22.3)$ \\
\hline DeKalb County, GA & 18.0 & $(15.5-20.8)$ & 21.4 & $(18.8-24.3)$ & 19.6 & $(17.6-21.8)$ \\
\hline Detroit, Ml & 15.9 & $(13.5-18.5)$ & 14.6 & $(11.8-18.1)$ & 15.3 & $(13.3-17.6)$ \\
\hline District of Columbia & 28.0 & $(26.8-29.3)$ & 29.0 & $(27.6-30.3)$ & 28.4 & $(27.5-29.4)$ \\
\hline Duval County, FL & 16.8 & $(14.5-19.5)$ & 21.1 & $(18.8-23.6)$ & 19.0 & $(17.2-21.0)$ \\
\hline Ft. Worth, TX & 30.4 & $(27.4-33.5)$ & 32.1 & $(29.0-35.5)$ & 31.3 & $(28.8-33.8)$ \\
\hline Houston, TX & 25.3 & $(22.7-28.2)$ & 23.4 & $(20.6-26.5)$ & 24.4 & $(22.4-26.6)$ \\
\hline Los Angeles, CA & 27.5 & $(22.6-33.1)$ & 33.0 & $(29.5-36.8)$ & 30.2 & $(26.8-33.8)$ \\
\hline Miami-Dade County, FL & 17.4 & $(14.9-20.3)$ & 21.9 & $(19.0-25.2)$ & 19.6 & $(17.3-22.0)$ \\
\hline
\end{tabular}

See table footnotes on the next page. 
TABLE 122. (Continued) Percentage of high school students who had 8 or more hours of sleep,* by sex — selected U.S. sites, Youth Risk Behavior Survey, 2015

\begin{tabular}{|c|c|c|c|c|c|c|}
\hline \multirow[b]{2}{*}{ Site } & \multicolumn{2}{|c|}{ Female } & \multicolumn{2}{|c|}{ Male } & \multicolumn{2}{|c|}{ Total } \\
\hline & $\%$ & $\mathrm{Cl}^{\dagger}$ & $\%$ & $\mathrm{Cl}$ & $\%$ & $\mathrm{Cl}$ \\
\hline New York City, NY & 23.1 & $(20.5-25.9)$ & 27.3 & (23.9-31.0) & 25.2 & $(22.9-27.6)$ \\
\hline Oakland, CA & 28.6 & $(23.8-33.9)$ & 30.3 & $(26.1-34.9)$ & 29.4 & $(25.8-33.3)$ \\
\hline Orange County, FL & 16.8 & $(14.1-19.9)$ & 24.5 & $(20.5-29.0)$ & 20.6 & (17.9-23.7) \\
\hline Palm Beach County, FL & 16.8 & $(14.2-19.6)$ & 20.3 & $(17.6-23.3)$ & 18.5 & (16.4-20.8) \\
\hline Philadelphia, PA & 20.5 & $(17.7-23.7)$ & 18.7 & $(14.1-24.3)$ & 19.7 & $(16.8-22.8)$ \\
\hline San Diego, CA & 23.7 & $(20.8-26.8)$ & 32.3 & $(28.5-36.5)$ & 28.1 & $(25.2-31.3)$ \\
\hline San Francisco, CA & 22.7 & (19.5-26.4) & 27.2 & $(23.7-31.0)$ & 24.8 & $(22.1-27.7)$ \\
\hline Median & \multirow{2}{*}{\multicolumn{2}{|c|}{$\begin{array}{c}20.5 \\
(13.7-30.4)\end{array}$}} & \multirow{2}{*}{\multicolumn{2}{|c|}{$\begin{array}{c}23.4 \\
(14.6-33.0)\end{array}$}} & \multirow{2}{*}{\multicolumn{2}{|c|}{$\begin{array}{c}20.6 \\
(14.4-31.3)\end{array}$}} \\
\hline Range & & & & & & \\
\hline
\end{tabular}

* On an average school night.

† $95 \%$ confidence interval.

$\S$ Not available.

TABLE 123. Percentage of high school students who used an indoor tanning device* and who had a sunburn, ${ }^{\dagger}$ by sex, race/ethnicity, and grade United States, Youth Risk Behavior Survey, 2015

\begin{tabular}{|c|c|c|c|c|c|c|c|c|c|c|c|c|}
\hline \multirow[b]{2}{*}{ Category } & \multicolumn{2}{|c|}{ Female } & \multicolumn{2}{|c|}{ Male } & \multicolumn{2}{|c|}{ Total } & \multicolumn{2}{|c|}{ Female } & \multicolumn{2}{|r|}{ Male } & \multicolumn{2}{|r|}{ Total } \\
\hline & $\%$ & $\mathrm{Cl}^{\S}$ & $\%$ & $\mathrm{Cl}$ & $\%$ & $\mathrm{Cl}$ & $\%$ & $\mathrm{Cl}$ & $\%$ & $\mathrm{Cl}$ & $\%$ & $\mathrm{Cl}$ \\
\hline \multicolumn{13}{|c|}{ Race/Ethnicity } \\
\hline White & 15.2 & $(11.7-19.5)$ & 3.7 & $(2.7-5.2)$ & 9.4 & $(7.5-11.6)$ & 77.7 & $(72.1-82.4)$ & 67.6 & $(64.0-71.0)$ & 72.5 & $(68.3-76.4)$ \\
\hline Black ${ }^{\text {I }}$ & 2.1 & $(1.1-3.8)$ & 5.3 & $(3.0-9.1)$ & 3.7 & $(2.5-5.4)$ & 16.2 & $(12.4-21.1)$ & 13.4 & $(9.4-18.6)$ & 15.0 & (12.1-18.4) \\
\hline Hispanic & 5.8 & $(3.9-8.5)$ & 3.7 & $(2.7-5.1)$ & 4.7 & $(3.5-6.3)$ & 43.8 & $(39.4-48.4)$ & 38.0 & $(34.1-42.0)$ & 40.8 & (37.4-44.4) \\
\hline \multicolumn{13}{|l|}{ Grade } \\
\hline 9 & 6.0 & $(4.5-7.9)$ & 2.7 & $(1.7-4.3)$ & 4.2 & $(3.3-5.4)$ & 60.0 & $(54.7-65.1)$ & 50.4 & $(43.9-56.9)$ & 54.9 & $(49.8-60.0)$ \\
\hline 10 & 7.1 & $(4.4-11.3)$ & 3.5 & $(2.4-5.2)$ & 5.3 & $(3.8-7.5)$ & 58.9 & $(52.2-65.3)$ & 52.9 & $(47.2-58.5)$ & 55.9 & $(50.0-61.7)$ \\
\hline 11 & 14.0 & (9.9-19.3) & 3.9 & $(2.4-6.4)$ & 9.0 & $(6.6-12.1)$ & 60.7 & $(55.4-65.9)$ & 52.4 & $(47.5-57.3)$ & 56.4 & (52.1-60.6) \\
\hline 12 & 16.2 & $(12.4-20.8)$ & 5.8 & $(4.2-8.1)$ & 10.9 & $(8.6-13.5)$ & 59.5 & $(51.7-66.8)$ & 52.2 & $(47.5-57.0)$ & 55.8 & $(50.5-61.0)$ \\
\hline Total & 10.6 & $(8.4-13.4)$ & 4.0 & $(3.2-5.0)$ & 7.3 & $(6.0-8.9)$ & 59.8 & $(54.1-65.2)$ & 52.0 & $(47.9-56.0)$ & 55.8 & (51.2-60.3) \\
\hline
\end{tabular}

* Such as a sunlamp, sunbed, or tanning booth (not counting getting a spray-on tan) one or more times during the 12 months before the survey.

† One or more times during the 12 months before the survey, counting even a small part of the skin turning red or hurting for 12 hours or more after being outside in the sun or after using a sunlamp or other indoor tanning device.

$\S 95 \%$ confidence interval.

१ Non-Hispanic. 
TABLE 124. Percentage of high school students who avoided foods because eating the food could cause an allergic reaction, ${ }^{*}$ by sex, race/ ethnicity, and grade - United States, Youth Risk Behavior Survey, 2015

\begin{tabular}{|c|c|c|c|c|c|c|}
\hline \multirow[b]{2}{*}{ Category } & \multicolumn{2}{|c|}{ Female } & \multicolumn{2}{|c|}{ Male } & \multicolumn{2}{|c|}{ Total } \\
\hline & $\%$ & $\mathrm{Cl}^{\dagger}$ & $\%$ & $\mathrm{Cl}$ & $\%$ & $\mathrm{Cl}$ \\
\hline \multicolumn{7}{|c|}{ Race/Ethnicity } \\
\hline White ${ }^{\S}$ & 19.5 & $(17.6-21.4)$ & 11.3 & $(9.0-14.1)$ & 15.4 & $(13.5-17.5)$ \\
\hline Black ${ }^{\S}$ & 22.3 & $(17.4-28.1)$ & 18.8 & $(13.2-26.1)$ & 20.7 & $(17.1-24.8)$ \\
\hline Hispanic & 18.2 & (15.9-20.8) & 10.2 & $(8.3-12.5)$ & 14.1 & $(12.7-15.7)$ \\
\hline \multicolumn{7}{|l|}{ Grade } \\
\hline 9 & 24.3 & $(21.3-27.7)$ & 13.5 & (10.9-16.6) & 18.7 & $(16.7-20.8)$ \\
\hline 10 & 17.6 & $(15.1-20.4)$ & 12.5 & $(10.0-15.5)$ & 15.2 & $(13.5-17.1)$ \\
\hline 11 & 19.0 & (16.9-21.3) & 10.7 & $(8.3-13.8)$ & 14.8 & $(13.0-16.8)$ \\
\hline 12 & 18.8 & $(16.6-21.2)$ & 11.3 & $(8.3-15.2)$ & 14.9 & $(12.9-17.1)$ \\
\hline Total & 19.9 & (18.6-21.4) & 12.0 & (10.5-13.8) & 16.0 & $(14.8-17.2)$ \\
\hline
\end{tabular}

* Such as skin rashes, swelling, itching, vomiting, coughing, or trouble breathing.

† $95 \%$ confidence interval.

$\S$ Non-Hispanic. 
TABLE 125. National health objectives and leading health indicators from Healthy People 2020, measured by the Youth Risk Behavior Survey, 2015.

\begin{tabular}{|c|c|c|c|c|c|}
\hline \multirow[b]{2}{*}{ Topic Area } & \multirow[b]{2}{*}{$\begin{array}{l}\text { Objective } \\
\text { number* }\end{array}$} & \multirow[b]{2}{*}{ Objective } & \multirow[b]{2}{*}{ Behavior description } & \multicolumn{2}{|c|}{$\begin{array}{l}\% \text { students in } \\
\text { grades } 9-12\end{array}$} \\
\hline & & & & $\begin{array}{l}\text { HP2020 } \\
\text { target }\end{array}$ & $\begin{array}{l}2015 \\
\text { YRBS }\end{array}$ \\
\hline Adolescent Health & $\mathrm{AH}-7$ & $\begin{array}{l}\text { Reduce the proportion of adolescents who } \\
\text { have been offered, sold, or given an illegal } \\
\text { drug on school property }\end{array}$ & $\begin{array}{l}\text { Were offered, sold, or given an illegal drug on } \\
\text { school property during the past } 12 \text { months }\end{array}$ & 20.4 & 21.7 \\
\hline Cancer & C-20.3 & $\begin{array}{l}\text { Reduce the proportion of adolescents in grades } \\
9 \text { through } 12 \text { who report using artificial } \\
\text { sources of ultraviolet light for tanning }\end{array}$ & $\begin{array}{l}\text { Used an indoor tanning device, such as a sunlamp, } \\
\text { sunbed, or tanning booth one or more times } \\
\text { during the } 12 \text { months before the survey }\end{array}$ & 14.0 & 7.3 \\
\hline Cancer & C-20.5 & $\begin{array}{l}\text { Increase the proportion of adolescents in } \\
\text { grades } 9 \text { through } 12 \text { who follow protective } \\
\text { measures that may reduce the risk of } \\
\text { skin cancer }\end{array}$ & $\begin{array}{l}\text { Most of the time or always wore sunscreen with } \\
\text { an SPF of } 15 \text { or higher when outside for more } \\
\text { than } 1 \text { hour on a sunny day }\end{array}$ & 11.2 & $\mathrm{NA}^{\dagger}$ \\
\hline $\begin{array}{l}\text { Injury and Violence } \\
\text { Prevention }\end{array}$ & IVP-34 & Reduce physical fighting among adolescents & $\begin{array}{l}\text { In a physical fight one or more times during the } \\
12 \text { months before the survey }\end{array}$ & 28.4 & 22.6 \\
\hline $\begin{array}{l}\text { Injury and Violence } \\
\text { Prevention }\end{array}$ & IVP-35 & Reduce bullying among adolescents & $\begin{array}{l}\text { Bullied on school property during the } 12 \text { months } \\
\text { before the survey }\end{array}$ & 17.9 & 20.2 \\
\hline $\begin{array}{l}\text { Injury and Violence } \\
\text { Prevention }\end{array}$ & IVP-36 & $\begin{array}{l}\text { Reduce weapon carrying by adolescents on } \\
\text { school property }\end{array}$ & $\begin{array}{l}\text { Carried a weapon (e.g., a gun, knife, or club) on } \\
\text { school property on at least } 1 \text { day during the } 30 \\
\text { days before the survey }\end{array}$ & 4.6 & 4.1 \\
\hline $\begin{array}{l}\text { Mental Health and } \\
\text { Mental Disorders }\end{array}$ & MHMD-2 & Reduce suicide attempts by adolescents & $\begin{array}{l}\text { Made a suicide attempt during the } 12 \text { months } \\
\text { before the survey that resulted in an injury, } \\
\text { poisoning, or overdose that had to be treated by } \\
\text { a doctor or nurse }\end{array}$ & 1.7 & 2.8 \\
\hline $\begin{array}{l}\text { Mental Health and } \\
\text { Mental Disorders }\end{array}$ & MHMD-3 & $\begin{array}{l}\text { Reduce the proportion of adolescents who } \\
\text { engage in disordered eating behaviors in an } \\
\text { attempt to control their weight }\end{array}$ & $\begin{array}{l}\text { Did not eat for } 24 \text { or more hours; took diet pills, } \\
\text { powders, or liquids without a doctor's advice; or } \\
\text { vomited or took laxatives to lose weight to keep } \\
\text { from gaining weight during the } 30 \text { days before } \\
\text { the survey }\end{array}$ & 12.9 & NA \\
\hline Physical Activity & PA-3.1 & $\begin{array}{l}\text { Increase the proportion of adolescents who } \\
\text { meet current Federal physical activity } \\
\text { guidelines for aerobic physical activity }\end{array}$ & $\begin{array}{l}\text { Were physically active doing any kind of physical } \\
\text { activity that increased their heart rate and made } \\
\text { them breathe hard some of the time for a total of } \\
\text { at least } 60 \text { minutes per day on each of the } 7 \text { days } \\
\text { before the survey }\end{array}$ & $20.2^{\S}$ & 27.1 \\
\hline Physical Activity & PA-3.2 & $\begin{array}{l}\text { Increase the proportion of adolescents who } \\
\text { meet current Federal physical activity } \\
\text { guidelines for muscle-strengthening activity }\end{array}$ & $\begin{array}{l}\text { Participated in muscle strengthening activities, } \\
\text { such as push-ups, sit-ups or weight lifting on } 3 \text { or } \\
\text { more days during the } 7 \text { days before the survey }\end{array}$ & None set & 53.4 \\
\hline Physical Activity & PA-3.3 & $\begin{array}{l}\text { Increase the proportion of adolescents who } \\
\text { meet current Federal physical activity } \\
\text { guidelines for aerobic physical activity and for } \\
\text { muscle-strengthening activity }\end{array}$ & $\begin{array}{l}\text { Were physically active doing any kind of physical } \\
\text { activity that increased their heart rate and made } \\
\text { them breathe hard some of the time for a total of } \\
\text { at least } 60 \text { minutes per day on each of the } 7 \text { days } \\
\text { before the survey and who participated in } \\
\text { muscle strengthening activities, such as } \\
\text { push-ups, sit-ups or weight lifting on } 3 \text { or more } \\
\text { days during the } 7 \text { days before the survey }\end{array}$ & None set & 20.5 \\
\hline Physical Activity & PA-5 & $\begin{array}{l}\text { Increase the proportion of adolescents who } \\
\text { participate in daily school physical education }\end{array}$ & $\begin{array}{l}\text { Went to physical education classes } 5 \text { days in an } \\
\text { average week when they are in school }\end{array}$ & 36.6 & 29.8 \\
\hline Physical Activity & PA-8.2.3 & $\begin{array}{l}\text { Increase the proportion of adolescents in } \\
\text { grades } 9 \text { through } 12 \text { who view television, } \\
\text { videos, or play video games for no more than } \\
2 \text { hours a day }\end{array}$ & $\begin{array}{l}\text { Watched television for no more than } 2 \text { hours per } \\
\text { day on an average school day }\end{array}$ & 73.9 & 75.3 \\
\hline Physical Activity & PA-8.3.3 & $\begin{array}{l}\text { Increase the proportion of adolescents in } \\
\text { grades } 9 \text { through } 12 \text { who use a computer or } \\
\text { play computer games outside of school (for } \\
\text { nonschool work) for no more than } 2 \text { hours } \\
\text { a day }\end{array}$ & $\begin{array}{l}\text { Played video or computer games or used a } \\
\text { computer for something that was not school } \\
\text { work for no more than } 2 \text { hours per day on an } \\
\text { average school day }\end{array}$ & 82.6 & 58.3 \\
\hline
\end{tabular}

See table footnotes on the next page. 
TABLE 125. (Continued) National health objectives and leading health indicators from Healthy People 2020,* measured by the Youth Risk Behavior Survey, 2015.

\begin{tabular}{|c|c|c|c|c|c|}
\hline \multirow[b]{2}{*}{ Topic Area } & \multirow[b]{2}{*}{$\begin{array}{l}\text { Objective } \\
\text { number* }\end{array}$} & \multirow[b]{2}{*}{ Objective } & \multirow[b]{2}{*}{ Behavior description } & \multicolumn{2}{|c|}{$\begin{array}{l}\% \text { students in } \\
\text { grades } 9-12\end{array}$} \\
\hline & & & & $\begin{array}{l}\text { HP2020 } \\
\text { target }\end{array}$ & $\begin{array}{l}2015 \\
\text { YRBS }\end{array}$ \\
\hline Sleep Health & $\mathrm{SH}-3$ & $\begin{array}{l}\text { Increase the proportion of students in grades } 9 \\
\text { through } 12 \text { who get sufficient sleep }\end{array}$ & $\begin{array}{l}\text { Had } 8 \text { or more hours of sleep on an average } \\
\text { school night }\end{array}$ & 33.2 & 27.3 \\
\hline Substance Abuse & SA-1 & $\begin{array}{l}\text { Reduce the proportion of adolescents who } \\
\text { report that they rode, during the previous } 30 \\
\text { days, with a driver who had been drinking } \\
\text { alcohol }\end{array}$ & $\begin{array}{l}\text { Rode in a car or other vehicle one or more times } \\
\text { driven by someone who had been drinking } \\
\text { alcohol during the } 30 \text { days before the survey }\end{array}$ & 25.5 & 20.0 \\
\hline Tobacco Use & TU-2.1 & $\begin{array}{l}\text { Reduce the proportion of adolescents who use } \\
\text { tobacco products (past } 30 \text { days) }\end{array}$ & $\begin{array}{l}\text { Smoked cigarettes; used chewing tobacco, snuff, } \\
\text { or dip; or smoked cigars, cigarillos, or little cigars } \\
\text { on at least one day during the } 30 \text { days before the } \\
\text { survey }\end{array}$ & 21.0 & 17.0 \\
\hline Tobacco Use & TU-2.2" & $\begin{array}{l}\text { Reduce the proportion of adolescents who use } \\
\text { cigarettes (past } 30 \text { days) }\end{array}$ & $\begin{array}{l}\text { Currently smoked cigarettes on at least one day } \\
\text { during the } 30 \text { days before the survey }\end{array}$ & 16.0 & 10.8 \\
\hline Tobacco Use & TU-2.3 & $\begin{array}{l}\text { Reduce the proportion of adolescents who use } \\
\text { smokeless tobacco products (past } 30 \text { days) }\end{array}$ & $\begin{array}{l}\text { Currently used chewing tobacco, snuff, or dip on } \\
\text { at least one day during the } 30 \text { days before the } \\
\text { survey }\end{array}$ & 6.9 & 7.3 \\
\hline Tobacco Use & TU-2.4 & $\begin{array}{l}\text { Reduce the proportion of adolescents who use } \\
\text { cigars (past } 30 \text { days) }\end{array}$ & $\begin{array}{l}\text { Currently smoked cigars, cigarillos, or little cigars } \\
\text { on at least one day during the } 30 \text { days before the } \\
\text { survey }\end{array}$ & 8.0 & 10.3 \\
\hline Tobacco Use & TU-7 & $\begin{array}{l}\text { Increase smoking cessation attempts by } \\
\text { adolescent smokers }\end{array}$ & $\begin{array}{l}\text { Tried to quit smoking cigarettes, among students } \\
\text { who ever smoked cigarettes daily during the } 12 \\
\text { months before the survey }\end{array}$ & 64.0 & NA \\
\hline
\end{tabular}

\footnotetext{
* Source: Adapted from U.S. Department of Health and Human Services and Office of Disease Prevention Health Promotion, Healthy People 2020. Washington, DC. Available at http://www.healthypeople.gov. Accessed January 17, 2016.

† Not available from the 2015 national YRBS.

$\S$ The target setting method for this objective was a 10\% improvement from the baseline; the baseline source was the 2009 national YRBS. However, because of changes in question context starting in 2011, national YRBS prevalence estimates derived from the 60 minutes of physical activity question in 2011, 2013, and 2015 are not comparable to those reported in 2009 or earlier. On the 2005-2009 national YRBS questionnaire, physical activity was assessed with three questions (in the following order) that asked the number of days students participated in 1) at least 20 minutes of vigorous physical activity; 2) at least 30 minutes of moderate physical activity; and 3) at least 60 minutes of aerobic (moderate and vigorous) physical activity. On the 2011, 2013, and 2015 national YRBS questionnaire, only the 60 minutes of aerobic physical activity question was included.

" Leading Health Indicator.
} 



The Morbidity and Mortality Weekly Report (MMWR) Series is prepared by the Centers for Disease Control and Prevention (CDC) and is available free of charge in electronic format. To receive an electronic copy each week, visit MMWR's free subscription page at http://www.cdc.gov/mmwr/mmwrsubscribe.html. Paper copy subscriptions are available through the Superintendent of Documents, U.S. Government Printing Office, Washington, DC 20402; telephone 202-512-1800.

Readers who have difficulty accessing this PDF file may access the HTML file at http://www.cdc.gov/mmwr/volumes/65/ss/ss6506a1.htm?s_cid=ss6506a1_w. Address all inquiries about the $M M W R$ Series, including material to be considered for publication, to Executive Editor, MMWR Series, Mailstop E-90, CDC, 1600 Clifton Rd., N.E., Atlanta, GA 30329-4027 or to mmwrq@cdc.gov.

All material in the MMWR Series is in the public domain and may be used and reprinted without permission; citation as to source, however, is appreciated. Use of trade names and commercial sources is for identification only and does not imply endorsement by the U.S. Department of Health and Human Services.

References to non-CDC sites on the Internet are provided as a service to $M M W R$ readers and do not constitute or imply endorsement of these organizations or their programs by CDC or the U.S. Department of Health and Human Services. CDC is not responsible for the content of these sites. URL addresses listed in $M M W R$ were current as of the date of publication.

ISSN: 1546-0738 (Print) 